\title{
Electrohydraulic Forming of Near-Net Shape Automotive Panels
}

\author{
DOE Award Number: DE-FG36-08G018128
}

Project Period: September 2008 through September 2013

Sergey F. Golovashchenko, phone: (313) 337-3738; sgolovas@ford.com

Ford Motor Company, MD3135, 2101 Village Rd., Dearborn, MI 48121

Other project team member organizations:

\author{
United States Steel \\ Pacific Northwest National Laboratory \\ Troy Tooling Technologies
}




\section{Executive Summary}

\section{Project Objective:}

The objective of this project was to develop the electrohydraulic forming (EHF) process as a near-net shape automotive panel manufacturing technology that simultaneously reduces the energy embedded in vehicles and the energy consumed while producing automotive structures. EHF is an electro-dynamic process based upon high-voltage discharge of capacitors between two electrodes positioned in a fluid-filled chamber. The electrical discharge results in the vaporization of a small volume of the liquid, which creates a high-intensity, high-velocity shock wave in the liquid. The shock wave is directed toward a sheet metal workpiece, which is ultimately driven into a die. This process is extremely fast, uses lower-cost single-sided tooling, and potentially derives significantly increased formability from many sheet metal materials due to the elevated strain rate. The technical objectives of the proposed project included developing: 1) a predictive numerical design tool for EHF processes, 2) an electrode and discharge chamber equipment design suitable for automotive panel manufacturing, 3) a laboratory prototyping system and using the system for process development, 4) enhanced applications of the EHF process by demonstrating the ability to trim and calibrate formed parts, and 5) demonstrate one full-scale automotive prototype using the developed EHF process.

\section{Project Scope}

As part of Collaborative Energy Efficiency and Carbon Footprint Research and Demonstration for the U.S. Manufacturing Industry, addressing Energy Intensive Processes, Sustainable Manufacturing Platform, Net and Near-Net Shape Design and Manufacturing Focus Area, "EHF of Near-Net Shape Automotive Panels" project developed the technology that has the following energy, environment, and economic benefits: uses substantially smaller amounts of tooling materials and energy compared to conventional stamping; enables significant down-gauging of steel panels in automotive structures, which substantially reduces the energy embedded in vehicles and their carbon footprint.

Major results obtained during the project include:

- $\quad$ developing numerical model of the electrohydraulic forming process of Advanced High Strength Steels (AHSS);

- $\quad$ quantifying formability of AHSS in pulsed forming conditions and comparing formability of selected AHSS (DP500, DP600, DP780 and DP980) in conventional forming and in EHF, which demonstrated that technical benefits of using EHF are higher for higher strength materials correlating with the general demand to reduce consumption of steel by using higher strength materials;

- $\quad$ developing the electrode design suitable for high volume production conditions;

- $\quad$ understanding the limitations on loads on the die in pulsed forming conditions;

- $\quad$ developing an automated fully computer controlled EHF cell incorporating all necessary elements of a high volume system such as air and water management system, blank delivery to the stamping zone and removal of the formed panel; 
- validation of performance of major components of EHF pulse generator in cyclic loading conditions;

- demonstration of electrohydraulic springback calibration and electrohydraulic trimming of stamped panels;

- full scale demonstration of a hybrid conventional and EHF forming process

\section{Conclusions}

1. Developed numerical model of the EHF process was validated experimentally at both coupon and component level. The numerical model was successfully applied to the design of the electrode system and multi-electrode EHF chamber for full scale validation of the process. The numerical model predicting stresses in the dies for pulsed forming was validated by the experimental study of the die insert failure for corner filling operation.

2. Electrohydraulic forming process and its major subsystems including durable electrodes, EHF chamber, water/air management system, pulse generator and developed process controls were validated to be capable to operate in fully automated computer controlled mode.

3. Novel processes of electrohydraulic trimming and electrohydraulic calibration have been demonstrated at the component reduced scale level.

4. Full scale demonstration of a hybrid conventional forming-EHF forming process demonstrated that one of the most complex sheet metal automotive parts, the dash panel, was formed in laboratory conditions from DP600 steel providing factor of two increase in material strength and enabling potential down-gauging of the panel by $25 \%$.

5. The economic feasibility of the developed EHF processes was demonstrated by developing a cost model of the process comparing it to the conventional stamping process. The cost model demonstrated that the developed process is cost neutral with the accuracy of accepted assumptions to the conventional stamping process but reduces the consumption of raw materials by $25 \%$ which has potential to reduce carbon footprint of produced cars and trucks when the technology gets implemented by reducing the energy embedded in vehicles and the energy consumed while producing automotive structures as well as the weight reduction of cars and trucks which is required to reduce fuel consumption.

\section{Commercialization plan}

Project DE-FG36-08G018128 is included in the official project portfolio of Ford Research \& Advanced Engineering (R\&AE). All Ford R\&AE projects are monitored and tracked via the Global Technology Development System (GTDS) to effectively manage the implementation process. At this moment, the internal Ford project is being continued jointly with Amino North America. After full completion of the Ford internal study, the project will be reviewed by the Management team, and the decision regarding the future of the EHF technology will be made. If the technology goes for production implementation, a specific panel from a new vehicle program will be specified for the EHF process, and the technology will be further developed from the Concept Readiness stage to Application Readiness and Implementation Readiness stages. 


\section{Acknowledgment:}

This report is based upon work supported by the U. S. Department of Energy under Award No. DE-FG36-08GO18128.

\section{Disclaimer:}

Any findings, opinions, and conclusions or recommendations expressed in this report are those of the authors and do not necessarily reflect the views of the Department of Energy. 


\section{Table of Contents}

Executive Summary

Page 2

Table of Contents

Page 5

List of Figures

Page 6

List of Tables

Page 18

Introduction

Page 19

Background

Page 21

Task 1: Predictive modeling of the EHF process

Page 26

Task 2: Electrode system and discharge chamber design and development

Page 63

Task 3: Demonstration of EHF process automation and equipment at production rates .....Page 88

Task 4: Electrohydraulic sheet metal forming process enhancement and optimization $\quad$...Page 110

Task 5: Demonstration of EHF for production conditions

Page 135

Task 6: New applications for EH stamping technology

Page 149

Task 7: Demonstration of EHF of a full scale automotive panel application Page 186

Accomplishments

Page 211

Conclusions

Page 217

Recommendations

Page 219

References / Bibliography

Page 220 


\section{List of Figures}

Figure 1.1.1.

Figure 1.1.2.

Figure 1.1.3.

Figure 1.1.4.

Figure 1.1.5.

Figure 1.1.6.

Figure 1.1.7.

Figure 1.1.8.

Figure 1.1.9.

Figure 1.1.10.

Figure 1.1.11.

Figure 1.1.12.

Figure 1.1.13.

Figure 1.1.14.

Figure 1.1.15.

Figure 1.1.16.

Figure 1.1.17.

Figure 1.1.18.
Schematic of sheet metal forming with an EHF chamber and die.

Schematic of electrical circuit at high-voltage discharge in a liquid (C capacitance of the battery, $L$ - total inductance of the system, $R 0$ - resistance of the circuit external to the discharge channel, $R_{v}$ - resistance of the discharge channel).

Adopted tensile specimen design for Hopkinson-bar tensile tests; L/W = 1.5.

$\mathrm{L} / \mathrm{W}=1.5$, Engineering stress-strain curves for 3 tests for each strain rate (1000/s, 2000/s, 3000/s) (a) Rolling direction, (b) Transverse direction.

Effect of strain rate (quasi-static and high rate) on stress-strain behavior.

Johnson-Cook model predictions vs. experiments for strain rates 1000-3000/s.

Stress-strain curve of DP980 steel in the rolling direction at two different strainrates.

Cylindrical discharge chamber: $\mathrm{D}=100 \mathrm{~mm}, \mathrm{~d}=2 \mathrm{~mm}$.

Dimensionless function of power deposition in a discharge channel; (No = $\left.6.35 \cdot 10^{7} \mathrm{~W}, \mathrm{~T}_{0}=154 \mu \mathrm{s}\right)$.

Evolution of shape and progression of major strains in the blank of DP600 sheet while completely filling the $38^{\circ} \mathrm{V}$-shape cavity.

Evolution of strain rate along the DP 600 blank while completely filling the Vshape die.

Channel shape, pressure distribution, and blank geometry versus time for EHF without prior hydroforming.

Channel shape, pressure distribution, and blank geometry versus time for EHF after preliminary hydroforming step.

Strain history for cone-filling: a - single-pulse EHF, b - after a quasi-static preforming step.

Evolution of strain rates during cone-filling: a - single-pulse EHF, b - after a quasi-static preforming step.

The shape of the blank to be formed illustrating the most critical areas requiring the highest pressure to fill the corners of the die cavity, marked as 1, 2 and 3 .

EHF chamber configuration for forming of the license plate area: $a$ - initial, $b$ modified.

Results of numerical simulation for critical area 1 ( $a$ - initial design, $b-$ new design). 
Figure 1.1.19.

Figure 1.1.20.

Figure 1.1.21.

Figure 1.1.22.

Figure 1.1.23.

Figure 1.1.24.

Figure 1.2.1.

Figure 1.3.2.

Figure 1.3.3.

Figure 1.3.4.

Figure 1.3.5.

Figure 1.3.6.

Figure 1.3.7.

Figure 1.3.8.

Figure 1.3.9.

Figure 1.3.10.

Figure 1.3.11.

Figure 1.3.12.

Figure 1.3.13.

Figure 1.3.14.

Figure 1.3.15.

Figure 1.3.16.
Geometry of the die (a) and the chamber (b).

Channel expansion and deformation of the blank during the first stage of EHF process.

Progression of blank geometry.

Schematic of electrohydraulic trimming.

Channel expansion during $\mathrm{EH}$ trimming.

Deformation of a blank during $\mathrm{EH}$ trimming. The red line shows the cutting edge.

Comparison of modeling results of one-step (left) and two-step (right) forming processes.

A simulation of the $\mathrm{EH}$ forming into an elastically deformable die.

Von Mises stress (MPa) distribution in the die.

Von Mises stress (MPa) contour. Die cross-section through the highly loaded element at the moment of impact.

Distribution of effective stress in $38^{\circ}$ plane strain insert for EHF of $1 \mathrm{~mm}$ DP980 blank material.

Cracks in $38^{\circ}$ plane strain insert.

The model for contact algorithms evaluation in LS-DYNA.

Sheet plate to bar numerical simulation model.

3D plain strain $34^{\circ} \mathrm{V}$-shaped die simulation.

Development of contact between the blank and the die.

Normal to the die surface component of velocity of a typical blank node.

Bar impact into a rigid wall

Stress values in elastic bar impacting a rigid wall: red line - stress in the center of the contact surface; green line - stress on the side surface of the bar. See elements in Figure 1.3.12.

Stress values in elastoplastic bar impacting a rigid wall: red line - stress in the center of the contact surface; green line - stress on the bar's side surface. See elements in Figure 1.3.12.

Bar-to-bar impact schematic.

Two impacting elastic bars, both meshed with solid elements and different size mesh. 
Figure 1.3.17.

Figure 1.3.18.

Figure 1.3.19.

Figure 1.3.20.

Figure 1.3.21.

Figure 2-1.

Figure 2-2.

Figure 2-3.

Figure 2-4.

Figure 2-5.

Figure 2-6.

Figure 2-7.

Figure 2-8.

Figure 2-9.

Figure 2-10.

Figure 2-11.

Figure 2-12.
Simulation of electrohydraulic forming of a DP-500 blank into a V-shape die.

Stresses in the elements shown in Figure 1.3.17 along the contact surface in the V-die during EHF.

View of tool steel B V-shape die after a series of EHF forming cycles.

$3 \mathrm{D}$ plain strain $38^{\circ} \mathrm{V}$-shaped die simulation.

Contact stresses at the two stress peaks in the corner element.

Early Electrode System Design.

Electrode Failure Modes: (a) asymmetric erosion; (b) bending; and (c) erosion.

Cross section of 1.8 liter electrode testing chamber. A thick steel plate covers the top during testing. Not shown are the braces which anchor the electrode ends to the $\mathrm{G}-10$ base plate and prevent electrode ejection.

Electrode designs: (a) old and (b) threaded

Typical $11 \mathrm{kV}(12.1 \mathrm{~kJ})$ electrical pulse showing initiation of the plasma channel at $\mathrm{t}=0$.

Brittle failures in the threaded region of the electrode external to the EHF chamber: a) tungsten failure and first molybdenum failure at end of turned machining center: $\mathrm{c}$ ); second Mo failure showing fracture surface melting.

Volumetric erosion rate per discharge for electrode materials. Tungsten erosion after a single discharge was not measureable.

Linear and volumetric erosion rates of steel electrodes with different initial tip shapes. The graph at a) shows the volumetric loss rate and b) shows the data plotted against lost electrode length.

Initial and final electrode profiles after 120 pulses. With the exception of the "Old Hemi" electrode design from Figure 2-4a, each electrode is shown in two orientations $\left(0^{\circ} \& 90^{\circ}\right)$.

Digitized final electrode profiles after 120 discharges.

Electrode tip profiles: a) average and extremes, b) the fitted energized electrode equation, averaged energized and ground data, and averaged natural tip (energized and ground) data, and c) natural tip data (raw and maximum/minimum).

Effects of entrained air on electrode erosion over 120 discharges in the 1.8 liter chamber. 
Figure 2-13.

Figure 2-14.

Figure 2-15.

Figure 2-16.

Figure 2-17.

Figure 2-18.

Figure 2-19.

Figure 2-20.

Figure 2-21.

Figure 2-22.

Figure 2-23.

Figure 2-24.

Figure 2-25.

Figure 3.1.1.

Figure 3.1.2.
Electrical response of EHF discharges as a function of inter-electrode gap.

Typical (polyurethane) insulation damage observed on electrodes with square insulation profiles after 120 pulses. Note both chipping and separation of insulator from electrode shaft.

Typical (polyurethane) insulation damage observed on polyurethane electrodes with conical insulation profiles. After 120 pulses no significant damage was noted. This failure occurred after 650 pulses were applied. Note relative lack of insulation chipping. Failure observed in both electrodes includes circumferential cracking of insulation just behind the conical region, separation of insulator from electrode shaft, and final longitudinal failure of insulation.

Exploded view of split electrode design.

Cross-sectional view of assembled split electrode design.

Half nuts welded to conical insulation tip electrodes, as seen before testing. Note that the welds were placed on the electrode tip side (not the insulation side) and some damage occurred to the insulation as a result of welding.

Results of test of electrodes with welded nuts as insulator protection - weld erosion and failure after 480 pulses. Both nuts were loose as significant damage occurred to threads after weld failure.

Results of electrodes test with machined insulation protection tip. Weld placed behind protector and insulation cast on after welding, as seen at left. Photo at right shows the electrodes in the chamber suspended after 1000 pulses were applied.

Detail of damage observed in test of machined and welded insulation electrode protection tip. Note general insulation erosion, chipping, and longitudinal crack formation.

Results of durability testing on electrode with integrated protective shield. Electrode system lasted 2000 pulses - a life sufficient for the electrodes to survive a single day of production at high volume.

Cross-section of new electrode system showing the new system inserted into the chamber wall.

Detail of the moment of discharge.

Graph showing full analysis of EHF electrical signals, including $t_{\text {init }}, V_{\max }, V_{\text {charge }}$, $V_{\text {discharge }}, I_{\max }$, end of pulse, $100 \%$ rise time, pulse width and asymmetry factor.

Fluid Management System (FHS) circuit diagram.

Automation room layout with the Fluid Management System (FHS). 
Figure 3.1.3.

Figure 3.1.4.

Figure 3.1.5.

Figure 3.1.6.

Figure 3.1.7.

Figure 3.2-1.

Figure 3.2-2.

Figure 3.2-3.

Figure 3.2-4.

Figure 3.2-5.

Figure 3.2-6.

Figure 3.2-7.

Figure 3.2-8.

Figure 3.2-9.

Figure 3.2-10.

Figure 3.2-11.

Figure 3.3.1.

Figure 3.3.2.

Figure 3.3.3.

Figure 3.3.4.
The two-skid, primary portion of the Fluid Handling System.

The third Fluid Handling System skid with the drain pump, fill manifold and valves for vacuum, high pressure fluid, low pressure fluid, air pressure, and vacuum.

Fluid samples from FHS: Bottle 1 - 50 pulses, Bottle 2 - 7 pulses, and Bottle 3 7000 pulses with filtration.

The magnetic rod in the center of a cellulose bag filter that collects the ferrous electrode erosion.

Edison Pro dehumidifier capable of removing 270 pints of water per day.

Round seal simulated using explicit code LS-Dyna.

Round seal simulated using implicit code MARC and employing the MooneyRivlin material model.

Diagram of the testing fixture for cyclic loading of sealing.

Actual fixture for cyclic loading of seals.

Groove plate carrying two rubber seals for wear testing.

Neoprene seal wear expected under EHF forming conditions.

Hybrid metal seal with $9 / 32$ inch metal seal atop $1 / 4$ inch rubber seal on bottom in a 0.30 inch wide groove.

Hybrid metal seal model indicating a contact force of $29 \mathrm{MPa}$ at the sheet interface.

Results of unlubricated and lubricated testing of hybrid seal at a variety of compression loads.

Tool for testing seals under hydroforming pressures.

Extrusion damage during pressure testing of the monolithic neoprene seal.

Shows the Master PLC and subsystem control diagram.

Material Handling Gantry. Input blanks are placed on wood pallet and finished parts are placed on the stack in the pan.

Original press now upgraded with new pump, manifold, and controls. See Figure 3.3.6 for a picture of upgraded press.

Blank a) as-clamped and b) conventionally but incompletely hydroformed at 300 psi. 
Figure 3.3.5.

Figure 3.3.6.

Figure 3.3.7.

Figure 3.3.8.

Figure 4.1.1.

Figure 4.1.2.

Figure 4.1.3.

Figure 4.1.4.

Figure 4.1.5.

Figure 4.1.6.

Figure 4.1.7.

Figure 4.1.8.
EHF Automation Cell.

Flow chart for the EHF cell showing automation control steps.

EHF Automation Part Production.

Blank shown a) as-clamped in the press, and b) fully formed by EHF automated cell.

Round pressure vessel and accompanying die for forming conical samples in a state of biaxial strain: (a) CAD design of the round pressure vessel, (b) the fabricated vessel, and (c) the existing conical die (shown upside down) which mates with the round pressure vessel.

Rectangular pressure vessel and accompanying die for forming elongated channel samples in a state of plane strain: (a) CAD design of the rectangular pressure vessel, (b) the fabricated vessel, and (c) the existing elongated channel die (shown upside down) which mates with the rectangular pressure vessel.

Test specimens of DP980, $1.0 \mathrm{~mm}$, used in pre-forming experiments. These specimens were formed into a conical die in biaxial strain. The specimen on the left was formed using only quasi-static water pressure at 5000 psi. The specimen on the right was pre-formed at 5000 psi and then finally formed using one EHF pulse at $10.5 \mathrm{kV}(17 \mathrm{~kJ})$.

Test specimens of DP980, $1.0 \mathrm{~mm}$, used in pre-forming experiments. These specimens were formed into a v-shaped channel die in plane strain. The specimen on the left was formed using only quasi-static water pressure at 5000 psi. The specimen on the right was pre-formed at 5000 psi and then finally formed using one EHF pulse at $10 \mathrm{kV}(16 \mathrm{~kJ})$.

Test specimens of DP780, $1.0 \mathrm{~mm}$, used in pre-forming experiments. These specimens were formed into a conical die in biaxial strain. The specimen on the left was formed using only quasi-static water pressure at 4000 psi. The specimen on the right was pre-formed at $4000 \mathrm{psi}$ and then finally formed using one EHF pulse at $10.5 \mathrm{kV}(17 \mathrm{~kJ})$.

Test specimens of DP780, $1.0 \mathrm{~mm}$, used in pre-forming experiments. These specimens were formed into a v-shaped channel die in plane strain. The specimen on the left was formed using only quasi-static water pressure at 4000 psi. The specimen on the right was pre-formed at 4000 psi and then finally formed using one EHF pulse at $10 \mathrm{kV}(16 \mathrm{~kJ})$.

Test specimens of HSLA 350, $1.0 \mathrm{~mm}$, used in pre-forming experiments. These specimens were formed into a conical die in biaxial strain. The specimen on the left was formed using only quasi-static water pressure at 4000 psi. The specimen on the right was pre-formed at $4000 \mathrm{psi}$ and then finally formed using one EHF pulse at $7.5 \mathrm{kV}(9 \mathrm{~kJ})$.

Test specimens of HSLA 350, $1.0 \mathrm{~mm}$, used in pre-forming experiments. These specimens were formed into a v-shaped channel die in plane strain. The specimen on the left was formed using only quasi-static water pressure at 4000 psi. The specimen on the right was pre-formed at 4000 psi and then finally formed using one EHF pulse at $8 \mathrm{kV}(10 \mathrm{~kJ})$. 
Figure 4.1.9.

Figure 4.1.10.

Figure 4.1.11.

Figure 4.2.1.

Figure 4.2.2.

Figure 4.2.3.

Figure 4.2.4.

Figure 4.2.5.

Figure 4.2.6.

Figure 4.2.7.

Figure 4.2.8.

Figure 4.2.9.

Figure 4.2.10.

Figure 4.2.11.

Figure 4.2.12.

Figure 4.2.13.

Figure 4.2.14.

Figure 4.2.15.
Experimental results of the effect of static pre-forming on formability of DP980, $1.0 \mathrm{~mm}$, in EHF.

Experimental results of the effect of static pre-forming on formability of DP780, $1.0 \mathrm{~mm}$, in EHF.

Experimental results of the effect of static pre-forming on formability of HSLA $350,1.0 \mathrm{~mm}$, in EHF.

Tooling used to evaluate die materials in EHF conditions. (a) die inserts fabricated from D6510, S0050A, DC53, and SLD-Magic, (b) insert holder, and (c) EHF chamber.

Results of microhardness measurements for D6510 nodular iron.

Optical micrographs for D6510 nodular iron; a) 100X, b) 500X, c) 1000X, d) 3000X SEM image; $2 \%$ Nital etch.

View of D6510 nodular iron V-shape die after a series of 16 EHF forming cycles. The first pulse in the series had an energy of $8 \mathrm{~kJ}$, and the final pulse had an energy of $26 \mathrm{~kJ}$.

Results of microhardness measurements for S0050A steel.

Optical micrographs for S0050A steel; a) 100X, b) 500X, c) $1000 \mathrm{X}$, d) $3000 \mathrm{X}$ SEM image; $2 \%$ Nital etch.

View of S0050A steel V-shape die after series of $17 \mathrm{EHF}$ forming cycles. The first pulse in the series had an energy of $8 \mathrm{~kJ}$, and the final pulse had an energy of 28 $\mathrm{kJ}$.

Results of microhardness measurements for DC53 tool steel.

Optical micrograph for DC53 tool steel; a) 100X, b) 500X, c) 1000X, d) $3000 \mathrm{X}$ SEM image; $2 \%$ Nital etch. Note the presence of large clusters of carbides.

View of DC53 tool steel V-shape die after a series of ten EHF forming cycles. The first pulse in the series had an energy of $8 \mathrm{~kJ}$, and the final pulse had an energy of $17 \mathrm{~kJ}$.

Results of microhardness measurements for SLD Magic tool steel.

Optical micrograph for SLD Magic tool steel; a) 100X, b) 500X, c) 1000X, d) 3000X SEM image; $2 \%$ Nital etch. Note: carbides well dispersed.

View of SLD Magic tool steel V-shape die after a series of 11 EHF forming cycles. The first pulse in the series had an energy of $8 \mathrm{~kJ}$, and the final pulse had an energy of $18 \mathrm{~kJ}$.

The EHF chamber (a) and die (b) used for lubricant testing. The die cavity is in the shape of an elongated U-channel, which allows for material to be drawn in near the central portion of the die.

Photographs of blanks before and after forming. The draw-in length for each formed specimen was computed by subtracting the final width (measured at the middle of the blank) from the initial width. 
Figure 4.2.16.

Figure 4.2.17.

Figure 4.2.18.

Figure 5.1.1.

Figure 5.1.2.

Figure 5.1.3.

Figure 5.1.4.

Figure 5.1.5.

Figure 5.1.6.

Figure 5.1.7.

Figure 5.1.8.

Figure 5.1.9.

Figure 5.1.10.

Figure 5.1.11.

Figure 5.1.12.

Figure 5.1.13.

Figure 5.1.14.
Photographs of blanks with splits arising due to inadequate lubrication: (a) full view of the blank, (b) close-up view of the split area.

Summary of lubricant test results at a clamping force of 150 tons. Draw-in length was measured at the middle of each specimen after forming.

Summary of lubricant test results at a clamping force of 75 tons. Draw-in length was measured at the middle of each specimen after forming.

New FEA-designed chamber incorporating electrode system, sealing system, and water management design improvements.

The new chamber optimized to decrease cycle time by reducing the volume from 19 to 11 liters and enlarged porting.

Typical cycle time based on the timing collected with the data acquisition system.

Initial Finite Element Models with 8.9 million (left) and 4.9 million elements (right).

Working Finite Element model with 2.9 million elements. The side view shows the die closed on the sheet metal blank and chamber.

New License Pocket Chamber simulations showing the voltage (V) performance in the ungrounded (left) and grounded conditions (right).

New License Pocket Chamber simulations showing the electric field distribution $(\mathrm{V} / \mathrm{m})$ in the ungrounded (left) and grounded conditions (right).

Photograph showing arcing witness marks on both chamber wall and chamber floor. The electrodes in this photo were offset from center to eliminate wall arcing which, while successful as a local strategy, was replaced by arcing to the floor.

New License Pocket Chamber simulations showing the space charge density $\left(\mathrm{C} / \mathrm{m}^{3}\right)$ distributions immediately surrounding the electrodes in the ungrounded (left) and grounded conditions (right).

Three dimensional view showing accumulation of negative charge on the surface of the positive electrode in the grounded chamber and running into the chamber wall.

Bad discharge in license pocket chamber: Current profile (left) and voltage profile (right). Note lack of current returning through the negative electrode.

Bad pulse current leaving through actuator rod.

All-steel negative electrode with large cross-section installed in chamber.

Discharge using all steel negative electrode. Current profile on the left and voltage profile on the right. Note asymmetric waveform shape. 
Figure 5.2.1.

Figure 5.2.2.

Figure 5.2.3.

Figure 5.2.4.

Figure 5.2.5.

Figure 5.2.6.

Figure 6.1.1.

Figure 6.1.2.

Figure 6.1.3.

Figure 6.1.4.

Figure 6.1.5.

Figure 6.1.6.

Figure 6.1.7.

Figure 6.1.8.

Figure 6.1.9.

Figure 6.1.10.
Labview based data acquisition system for capturing data from the automation cell.

Over 1200 formed parts from the automation cell.

NWL Transformer's $50 \mathrm{kV}$ charger is designed for continuous pulsing, and according to the manufacturer, has extended life exceeding significantly the life of other components.

Capacitor bank of one of the two modules connected to individual spark gap switches and pulsed in parallel.

The high voltage spark gap switch ( $25 \mathrm{kV}$ to $50 \mathrm{kV}$ ) and trigger box.

Erosion on the electrodes of the high voltage spark gap switch that carries up to $50 \mathrm{kV}$ and $75 \mathrm{kA}$.

A cross-sectional, schematic view of the channel chamber connected to the pulsed-current generator during electrohydraulic trimming.

Laboratory tool for electrohydraulic trimming: (a) CAD model of the assembled tool; (b) channel chamber with three electrodes; (c) cross-section of the assembled tool; (d) upper half of the tool with sharp trimming edge and offal guiding support.

Schematic of EH-trimming tool with three different offal guiding insert designs.

Electrohydraulic trimming of $1 \mathrm{~mm}$ thick DP590 blanks using a pulse energy of 9 $\mathrm{kJ}$ at $7.5 \mathrm{kV}$ : (a) sample trimmed with offal guiding insert A shown in Figure 6.1.3a; (b) sample trimmed with offal guiding insert B shown in Figure 6.1.3b; (c) sample trimmed with offal guiding insert $C$ shown in Figure 6.1.3c.

The effect of reflected pressure waves in $\mathrm{EH}$ trimming, illustrated by the results of trimming $2 \mathrm{~mm}$ thick 4130 steel: (a) schematic sectional view of the EH trimming chamber, showing one central electrode and the direction of pressure wave propagation after the discharge; (b) trimming results using a pulse energy of 40 $\mathrm{kJ}$ at $20 \mathrm{kV}$; (c) trimming results using a pulse energy of $50.6 \mathrm{~kJ}$ at $22.5 \mathrm{kV}$.

Candidate part selected for development and testing of EH-trimming. The part was at $50 \%$ scale.

Conventional stamping tool for forming of the C346 floor side inner at $50 \%$ scale; (a) upper tool, including male punch, binder surface, gas springs, and guide pins, and (b) lower tool, including a female die, guides for blank positioning, guide pin bushings, and spacers for adjusting tool contact pressure and material drawing.

EH-trimming tool for the C346 floor side inner at 50\% scale. (a) Upper tool, including trim knives, scrap support, and guide pin bushings. (b) Lower tool, including chamber with sub-channels, electrodes, and guide pins.

EH trimming tool, lower half, including four fluid sub-channels.

Upper half of $\mathrm{EH}$ trimming tool, including trim blades. 
Figure 6.1.11.

Figure 6.1.12.

Figure 6.1.13.

Figure 6.1.14.

Figure 6.1.15.

Figure 6.1.16.

Figure 6.1.17.

Figure 6.1.18.

Figure 6.2.1.

Figure 6.2.2.

Figure 6.2.3.

Figure 6.2.4.

Figure 6.2.5.

Figure 6.2.6.

Figure 6.2.7.

Figure 6.2.8.

Figure 6.2.9.

Figure 6.2.10.

Figure 6.2.11.
Close-up view of one of the fluid sub-channels. The lower edge in the image - the edge closer to the smaller seal groove - corresponds to the trim line of the panel.

Close-up view of the edge of the trim blade (shown protruding above the offal guiding area or offal space). This region of the trim line corresponds to the central region of the fluid channel shown in Figure 6.1.11.

Front floor side inner panel, trimmed using $\mathrm{EH}$ trimming.

The offal resulting from EH trimming of the floor side inner panel.

A stack of panels trimmed using $\mathrm{EH}$ trimming, revealing the absence of any burrs and a clean trim edge matching the fine details of the part geometry.

The front nose of the floor side inner panel partially trimmed using $\mathrm{EH}$-trimming. The mechanism of EH-trimming can be seen in the photograph: the part-side of the metal is clamped in place, and the scrap-side of the metal is deformed upward and outward, against the sharp trim edge and away from the part-side.

A small area of chipping on the sharp trim edge in the large $\mathrm{EH}$ trim tool.

Two small areas of chipping on the sharp trim edge in the large EH trim tool.

Bending of a flat strip on a sheet metal brake where the sample is clamped against a tool with a 1 " or $1 / 2$ " radius, bent to $90^{\circ}$, then released from the tool and allowed to springback.

Comparison of DP980 samples before and after flattening in a 1.5 MN hydraulic clamping press. As seen in the Figure, the hydraulic press was not able to remove all of the springback from the strip.

EH calibration chamber with electrodes connected by an aluminum bridge wire.

Comparison of a strip of DP980, $1 \mathrm{~mm}$ thick, bent over a 1" radius and then flattened by a $1.5 \mathrm{MN}$ clamping press, to a strip then calibrated by one $\mathrm{EH}$ pulse at $7 \mathrm{kV}$ and $7.8 \mathrm{~kJ}$.

Experimental results of EH calibration of DP980 strips, in terms of springback angle as a function of discharge energy, using an initial bending radius of 1 ".

Experimental results of EH calibration of DP980 strips, in terms of springback angle as a function of discharge energy, using an initial bending radius of 0.5 ".

Experimental results of EH calibration of DP980 strips of $1 \mathrm{~mm}$ thickness, in terms of springback angle as a function of discharge energy.

Experimental results of EH calibration of DP980 strips of $1.4 \mathrm{~mm}$ thickness, in terms of springback angle as a function of discharge energy.

Geometry of the chamber for the single-cavity chamber concept (a) and for the multiple sub-chamber concept (b).

Points of observation on the surface of the blank.

Pressure history after the discharge in the single-cavity EH calibration chamber. 
Figure 6.2.12.

Figure.6.2.13.

Figure 6.2.14.

Figure 6.2.15.

Figure 6.2.16.

Figure 6.2.17.

Figure 6.2.18.

Figure 6.2.19.

Figure 7.1.

Figure 7.2.

Figure 7.3.

Figure 7.4.

Figure 7.5.

Figure 7.6.

Figure 7.7.

Figure 7.8.

Figure 7.9.

Figure 7.10.

Figure 7.11.

Figure 7.12.
Pressure history during the $\mathrm{EH}$ discharge in the multi chamber design.

Propagation of pressure inside the first sub-chamber during the $\mathrm{EH}$ calibration process.

Optimized design of the chamber which was selected for fabrication.

The lower half of the EH calibration tool, showing three sub-chambers and three electrodes.

Close-up view of the two sub-chambers near the back end of the $\mathrm{EH}$ calibration tool, showing the position of the energized electrodes and the ground electrodes.

Overall view of the upper half of the EH calibration tool, which consists of a female die surface cut to the desired part geometry.

The stamped panel before (a) and after (b) the EH calibration process was applied.

The stamped panel before (a) and after (b) the $\mathrm{EH}$ calibration process was applied.

Final die with female beads.

Final incoming blank shape.

Blank location after gravity phase. 2 pins control the blank location.

Blank location after the binder closing is completed.

Final formability of the deformed blank after steps 1,2 and 3.

Preformed blank with EHF chamber layout.

The EHF chamber final design.

The shape of the discharge channel and deformation of the blank during the first discharge in sub-chamber \#1.

Evolution of the shape of the blank in the central area of the dash panel after each forming step and discharge.

Formability map of the central cavity after pulsed pressure was applied, and the process was completed.

The dash panel pre-forming die with center punch driven by nitrogen cylinders and two side punches attached to the upper die shoe.

The preforming die in the assembled configuration, with the flat blank positioned by locating pins. 
Figure 7.13.

Figure 7.14.

Figure 7.15.

Figure 7.16.

Figure 7.17.

Figure 7.18.

Figure 7.19.

Figure 7.20.

Figure 7.21.

Figure 7.22.

Figure 7.23.

Figure 7.24.

Figure 7.25.

Figure 7.26.

Figure 7.27.
Individual flat blanks laser trimmed from $1.5 \mathrm{~mm}$ thick DP600 sheet.

Upper binder of the preforming die with two rows of drawbeads enabling two different modes of the material flow during the stage of central punch drawing and during the stage of side punch drawing.

Assembled upper preforming die in upside down position.

Assembled lower preforming die.

The assembled preforming die installed in a 3000 ton, double action hydraulic press at Amino NA during the tryout process.

Preformed blank positioned inside the lower die during measurements of the material inflow into the die cavity.

Final dash panel forming tool with the EHF fluid chamber on top and the lower die machined to the final part shape.

EHF die providing final shape forming.

EHF chamber prior to the installation of electrodes.

EHF tooling in open position during the tryout process at the Ford Norwood lab.

EHF tooling in closed position during the tryout process at the Ford Norwood lab.

The fully formed dash panel (1.5 mm DP600) after EHF forming. a) Viewed from the EHF chamber side. b) Viewed from the die side

EHF-assisted conventional stamping (left) and conventional stamping processes as employed in cost models.

Output from conventional stamping cost model.

Output from EHF-assisted stamping cost model. 


\section{List of Tables}

Table 1.1.1. Johnson-Cook constitutive model parameters for DP 780.

Table 1.3.1. Convergence of the V-Die corner element von-Mises Stress.

Table 2-1. $\quad$ Electrode equation best fit parameters to energized electrode tip profile.

Table 2-2. Insulation Testing Results.

Table 4.2.1. Summary of mechanical properties of the die materials under evaluation.

Table 4.2.2. Summary of the chemical composition of the die materials under evaluation.

Table 4.2.3. Sequence of EHF pulses used to evaluate each die material.

Table 7.1. Typical Cost Model Inputs.

Table A1. Description of Tasks and Milestones.

Table A2. Task/Subtask Schedule. 


\section{Introduction}

\section{Brief description of Electrohydraulic Forming}

The technical objective is to develop and demonstrate proof of commercial feasibility of the EHF process within the Energy Intensive Processes, Near Net-Shape technology platform identified by DOE-AMO. EHF is a high-rate forming process based upon high-voltage discharge of capacitors between two electrodes positioned in a fluid-filled chamber. The shockwave in the liquid, initiated by the expansion of the plasma channel formed between two electrodes, propagates towards the blank and causes the blank to be deformed into a one-sided die cavity. In order to prevent a short circuit between the two electrodes (usually made out of steel), they must be electrically isolated from the chamber. The insulation material should be able to withstand the maximum process voltage. Also, a proper hydraulic seal must be maintained, especially in the site where electrodes are inserted into the chamber and between the blank and the chamber. Water leaks can cause substantial loss of efficiency. The cavity between the blank and the die should be vacuumed to avoid the high compressibility of air as the blank fills the die cavity. This process is extremely fast, uses lower-cost single-sided tooling, and derives significantly increased formability from many sheet metal materials due to the elevated strain rate.

Specific problems being addressed

The primary method of stamping body panels and structural parts is via a two sided die installed in a single transfer press or through a sequence of them in a line of presses. During the era of low oil prices, most automotive parts were stamped from low carbon mild steel. Formability of Advanced High Strength Steels (AHSS) is substantially lower. Furthermore, AHSS greatly increase springback - defined as the elastic relaxation of a stamped blank that occurs after release from the stamping die due to higher strength of AHSS compared to mild steel. EHF deforms the material under relatively high strain rates into single-sided tools and increases maximum elongations that AHSS can withstand without fracture approximately factor of two. It also eliminates the elastic springback by propagating dynamic loads through material thickness at the end of the EHF process.

\section{Target industries}

The project is targeting automotive industry including OEMs and Tier 1 and Tier 2 suppliers. It also targets heavy truck and aerospace industry, where similar issues of insufficient formability of sheet metal blanks and springback of stamped panels exist.

\section{Potential Energy Savings}

The objective of this project is to develop and deploy the EHF process as a near-net shape automotive panel manufacturing technology that simultaneously reduces the energy embedded in vehicles and the energy consumed while producing vehicles. The specific potential energy savings of the project result from EHF processing that:

- Uses half the tooling materials and energy compared to conventional stamping 
- Enables the down-gauging of steel panels in automotive structures by more than $25 \%$, which substantially reduces the energy embedded in vehicles and their carbon footprint by reducing the amount of embedded steel in the vehicle

- Consolidates multiple conventional forming, blanking, and punching processes into a single EHF process, thereby eliminating machines through process consolidation.

Given these benefits, developing and commercializing EHF technology has the potential to yield an energy savings of $16.38 \mathrm{TBtu} /$ year, these calculations do not include the substantial fuel economy improvements achieved by reducing the weight of transportation vehicles by downgauging steel sheet.

\section{Economic viability}

Very detailed cost analysis of the developed EHF process was produced in comparison with conventional stamping technology. It should be noted that the conventional stamping cannot produce designated parts from AHSS. Current industrial practice is to use mild steels, such as Extra Deep Drawing Quality (EDDQ) steel currently used in production for the dash panel selected for full scale demonstration. The developed EHF process is capable of using the material with factor of two higher strength and potentially provides $25 \%$ of downgauging. The detailed cost analysis illustrated that the EHF process is almost cost neutral to the conventional stamping process within the accuracy of accepted assumptions. The initially expected substantial high economic benefits due to downgauging and using less steel were significantly reduced due to increased cost of the AHSS steels. Current cost differential between $1 \mathrm{lb}$ of AHSS DP 600 steel and EDDQ is approximately 25\%. In 2008, this cost differential was expected to be only $5 \%$, as it was predicted by the steel producers. Since the cost of the sheet metal blank is the major component of the cost of the stamping, the economic benefit is getting neutralized. However, OEMs today are paying approximately $\$ 3$ per pound of weight saved. From this perspective having almost $\$ 0$ cost of weight saving represents a very strong economic incentive.

\section{Commercialization status and plans}

Project DE-FG36-08G018128 is included in the official project portfolio of Ford Research \& Advanced Engineering ( $R \& A E)$. All Ford $R \& A E$ projects are monitored and tracked via the Global Technology Development System (GTDS) to effectively manage the implementation process. At this moment, the internal Ford project is being continued jointly with Amino North America Corporation. After full completion of the Ford internal study, the project will be reviewed by the Management team, and the decision regarding the future of the EHF technology will be made. If the technology is approved for production implementation, a specific panel from a new vehicle program will be specified for the EHF process, and the technology will be further developed from the Concept Readiness stage to Application Readiness and Implementation Readiness stages of the GTDS process. 


\section{Background}

\section{State of the art}

The electrohydraulic effect is created via a shockwave generated by the discharge of high voltage capacitors through a pair of electrodes in a liquid filled chamber. The shockwave in the liquid, initiated by the expansion of the plasma channel formed between two electrodes, propagates towards the blank and causes the blank to be deformed into a one-sided die cavity. A schematic of the process is shown in Figure B-1. The figure illustrates that electrical energy is stored in high voltage capacitors employing a transformer and a set of diodes. A special switch/discharging device (ignitron, vacuum discharger, or solid state switch) closes the circuit and delivers the high voltage stored in capacitors to the electrodes. At the beginning of the discharge process, the electrical resistance of the ionized channel between the two electrodes drops by several orders of magnitude, and the electric current grows sharply due to increase of temperature and expansion of the plasma channel. Due to the significant amount of electric energy pumped through the small ionized channel, the temperature may reach $10^{4} \mathrm{~K}$, and the pressure inside the channel may grow to $10^{4} \mathrm{MPa}$ for a short time [B1]. Driven by such high pressure, the discharge channel quickly expands at a speed of up to $1500 \mathrm{~m} / \mathrm{sec}$, and thus creates a shock wave.
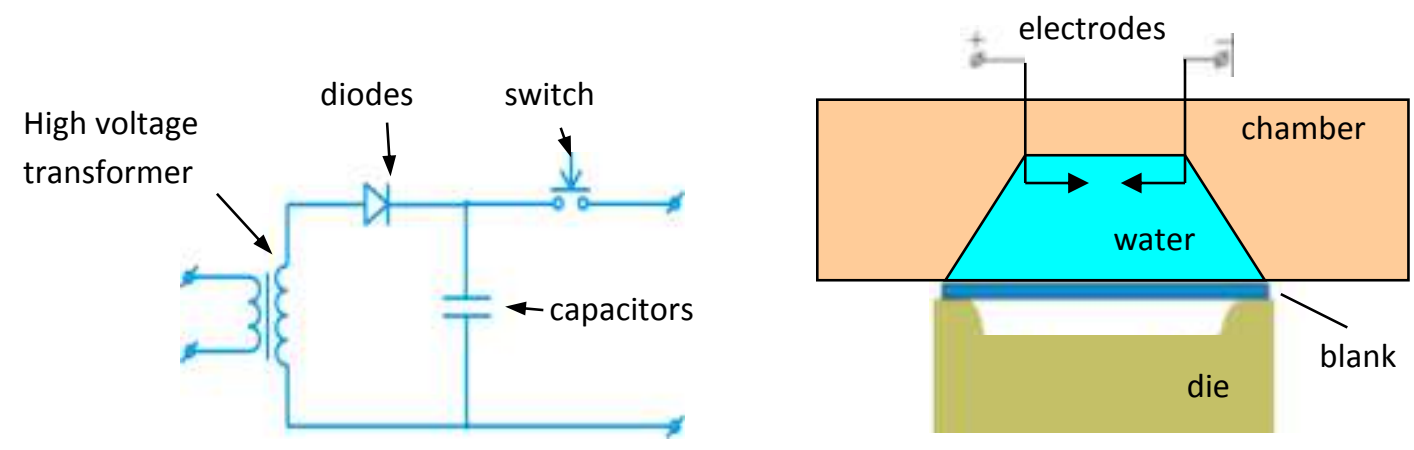

Figure B-1. Schematic of the electrohydraulic forming process

In order to prevent a short circuit between the two electrodes (usually made out of steel), they must be electrically isolated from the chamber. The insulation material should be able to withstand the maximum process voltage. Also, a proper hydraulic seal must be maintained, especially in the site where electrodes are inserted into the chamber and between the blank and the chamber. Water leaks can cause substantial loss of efficiency. The cavity between the blank and the die should be vacuumed to avoid the high compressibility of air as the blank fills the die cavity.

The electrohydraulic effect was discovered in early experiments [B2] where strong mechanical forces were observed during electric discharge in water. The development of practical applications of the EH effect, including its use in sheet metal forming, started in 1950s [B3]. To some extent EHF was considered similar to explosive forming, developed earlier. In the 1960s 
and 1970s a number of patents were submitted and research studies published, for example [B4, B5 and B6], mostly related to design of discharge electrodes, methods of discharge initiation between them, and, in particular, methods of wire delivery to connect the two electrodes prior to the electric discharge of capacitors.

EHF has had very limited industrial usage - mostly in experimental facilities or in very low volume production in 1960s and 1970s. The reasons for this were the lack of advanced equipment capable of delivering the high voltage discharge in a timely manner, the erosion of electrodes, the need to deliver a wire in the clearance between two electrodes, dealing with water as a transmitting media (and attendant sealing issues) and the lack of economical drivers: the automotive industry at that time was using mild steels with high formability. The majority of practical applications of EHF at that time were limited to the aerospace industry where, typically, rather small parts of less than 300-400 mm were stamped. The advantage of EHF was realized mostly through the reduction of capital investment, since EHF requires one-sided dies rather than two-sided, and this reduction aligned well with low volume of aerospace applications. According to reference [B7], the Cincinnati Shaper Co. in Ohio built machines ranging in size from about 35 to $150 \mathrm{~kJ}$ output power. However, their production was discontinued by 1980 . After a few attempts at industrial usage in the US, Great Britain and Japan, EHF was mostly used in the former Soviet Union [B8] for variety of low volume sheet metal stamping applications. EHF has never been seen in automotive applications anywhere.

In the 1990s only a few EHF related research studies on sheet metal blanks were published, mostly concentrating on formability aspects of sheet material under high-rate forming conditions. In the early 1990 s G. Daehn and his research group investigated the formability of sheet metal at high strain rates, and they observed an improvement in formability of up to five times, when measuring the maximum strain in the fracture zone after a static bulging test and comparing to that obtained after EHF into a conical die [B9]. Similar study of sheet metal blank formation into an open round window and into a conical die was performed in more recent publications [B10, B11]. A very detailed report on pulsed forming state of the art mostly related to electromagnetic forming technology rather than EHF was presented in [B12].

\section{Specific area being addressed}

EHF process as well as other pulsed forming processes demonstrated capability to substantially extend maximum elongation, which sheet metal material can withstand without fracture. In this project, the EHF process was applied to forming of AHSS materials which have strong potential for energy savings and reducing weight of cars and trucks to reduce fuel consumption. The EHF process was developed from forming small scale coupons to 1)an automated EHF cell where a fragment of the panel was formed in a computer controlled fully automated regime; 2)full scale hybrid laboratory process of conventional forming - EHF forming of a full scale panel. The following important enablers for implementing the laboratory process in industry have been developed: a) durable electrode system; b) durable seal system of the EHF chamber; c) numerical model of the process enabling the process design. 


\section{Project Objectives}

The objective of this project is to develop the electrohydraulic forming (EHF) process as a nearnet shape automotive panel manufacturing technology that simultaneously reduces the energy embedded in vehicles and the energy consumed while producing automotive structures. The technical objectives of the proposed project include developing: 1) a predictive numerical design tool for EHF processes, 2) an electrode and discharge chamber equipment design suitable for automotive panel manufacturing, 3) a laboratory prototyping system and using the system for process development, and 4) one full scale automotive prototype using the developed EHF process.

\section{Approach}

A. Numerical models. A semi-empirical model of the discharge channel was built based upon measurements of the electric parameters as a function of time. A hydrodynamic model based upon an explicit integration procedure modeled pressure delivery from the discharge channel to the blank. Numerical models of the discharge channel and pressure generation were coupled with the model of the sheet metal blank deformation being in contact with the deformable die. The blank/die interactions were modeled based upon a solid elasto-plastic dynamic deformation model solved by an explicit integration method. Based upon the stress-strain history of the die obtained through finite element modeling, stamping die life predictive capability was developed based upon continuously accumulated fatigue damage along the loading history.

Process modeling tools were utilized to predict processing parameters and model tooling designs for a full scale automotive panel. The analytical models were used to predict forming energy levels, electrode placement, and tooling chamber design required to efficiently form a full scale automotive panel. Tooling and operating parameters were optimized using the modeling capabilities.

An analytical model was developed to provide a method for defining a preform shape which can be then reshaped in the next forming operation into part shape that cannot be formed directly from flat sheet in a single EHF step

B. Electrode development. An electrode erosion study was conducted employing an experimental electrohydraulic chamber. Parameters of electric discharge were registered during cyclic testing of the electrode system for each discharge. Measurement of the electrode erosion were conducted optically by temporarily removing electrode from the chamber and registering changes in its shape, dimensions, and weight loss. A correlation between the parameters of the discharge and the electrode system performance was established. The variation of the discharge parameters was examined in conjunction with changing conditions inside the chamber, and based on this understanding a criterion was developed for electrode adjustment. 
C. Water/ Air management system. Water flow was measured while filling and evacuating the chamber. An efficient system design and measurement techniques was developed to reduce the time for filling and evacuating the water from the closed volume of the chamber.

Various sealing concepts were explored. Testing in pulsed cycling conditions was conducted to identify the appropriate sealing materials and schedule for sealing adjustment. Vacuum system was developed to remove air from the chamber to increase the process efficiency as well as from the area between the blank and the die to eliminate air compression while forming the final features of the targeted shape.

D. Quasistatic preforming process. Successful demonstration of a preforming process reduced the number of electrohydraulic discharges and the cycle time necessary to form the final shape of the part. The extent to which the final shape cavity should be filled during the preforming process was understood based upon numerical modeling of the forming process to maximize the amount of metal in the die cavity before the material undergoes substantial stretching.

E. EHF cell. The material handling and placement system, high-rate charging of the capacitor bank, filling and sealing of the electrode chamber, and pulsed sheet forming process necessary for commercial implementation of the EHF process into an automotive stamping application were demonstrated. To demonstrate these required technologies, this a relatively small model part was selected. The automated functions that were incorporated in the demonstration EHF system included: 1) a blank handling device capable of installing a blank into the die, and transporting a stamped blank from the die to the stacking area, 2) rapid cycling of the chamber water management system, 3) capacitor charging and discharging. Automated experiments were conducted for the specified number of hours. The parameters of the process, including waveforms of electric parameters and chamber fluid parameters were monitored through the testing procedure.

F. Die material and lubrication. A number of inserts from die material candidates were fabricated and tested in EHF forming conditions for AHSS steels. As a result the die material approved for high volume stamping applications was selected as a main candidate for EHF dies. Several surface hardening techniques have been evaluated from die performance without any plastic deformation. A number of lubricants among approved for high volume production in stamping operations were tested in EHF forming conditions. The lubricant-candidate was selected based upon best performance of delivering more metal into the die cavity, which served as an indicator of minimized friction.

G. Electrohydraulic trimming and calibration. The novel EH trimming process employing only one sided trim die was demonstrated initially on flat coupons and then on a production panel at $50 \%$ scale. During the discharge, the shock wave was generated and transmitted to the scrap surface, shearing off the scrap along the sharp edge of the one-sided trim die.

A critical new application for $\mathrm{EH}$ technology was the $\mathrm{EH}$ springback calibration - a manufacturing method for eliminating the majority of elastic strains in stamped blanks. The 
proposed proprietary concept of EH calibration was validated using AHSS sheet stamped into a U-channel and then for a $50 \%$ reduced scale component.

H. Full scale demonstration of the EHF process. The candidate for the full scale demonstration was selected based upon preliminary analysis of potential candidate sheet metal parts with high weight saving potential. Based upon intensive numerical simulation, the hybrid process including initial quasistatic preforming followed by the final EHF forming process was developed. Cost analysis of the EH processes elements compared to conventional stamping processes was performed using established cost models employed for analysis of production parts at Ford.

Relevant qualifications and past experience of the project team members.

Ford $R \& A E$ has extensive experience in commercialization of new manufacturing technologies, and has had a good track record in moving new technologies from laboratory and pilot-scale levels to fully commercialized manufacturing. US Steel has research staff dedicated to develop new materials and processes for commercialization within the automotive industry, and the Pacific Northwest National Laboratory has more the fifteen years of research, development, and deployment experience with Ford Motor Company and within the domestic automotive manufacturing industry. 


\section{Task 1. Predictive modeling of the EHF process}

\section{Sub-task 1.1 Numerical model of pulsed pressure generation process}

The Electro-Hydraulic Forming (EHF) process forms a blank into the die with a pressure wave created inside a water filled chamber. This pressure wave is created by an electrical discharge between two electrodes inside the chamber. Water must be delivered to the chamber, and air must be eliminated from the volume between the blank and die before the blank is formed. Optimum performance is achieved when the electrode chamber is completely filled with water, and all air bubbles are eliminated.

A typical chamber consists of a thick-walled, hollow cavity through which electrically isolated electrodes penetrate, as in Figure 1.1.1. The cavity is filled with water, fully immersing the electrodes. A sheet metal blank is placed on top, and above that, facing downward, is placed a one-sided die. The remaining air is evacuated from both sides of the blank. A capacitor bank charged anywhere from 5 to $50 \mathrm{kV}$ is discharged through the electrodes. About a millisecond after the voltage is applied to the electrodes, a high temperature plasma channel forms, and current from the capacitors drives and expands the plasma channel. The region surrounding the plasma channel is filled with gas in the form of superheated steam which transitions to a steam/water interface. Because the chamber is filled with a slightly

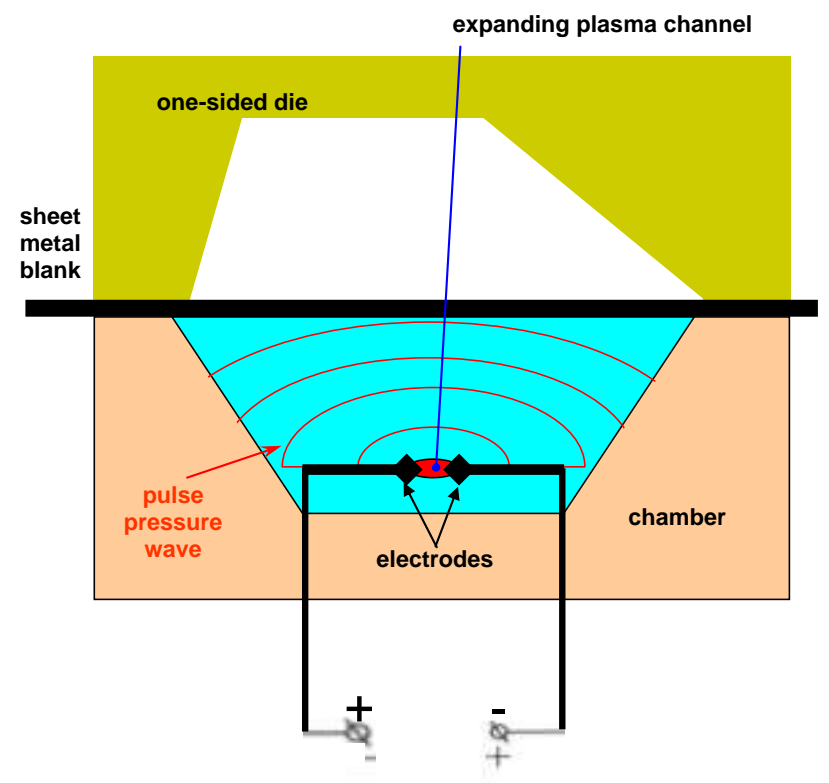

Figure 1.1.1. Schematic of sheet metal forming with an EHF chamber and die. compressible fluid, water, and all air is evacuated, immense pressures rapidly build up, and the sheet metal blank is explosively driven into the die.

In order to develop a numerical model of the EHF process, the following models need to be developed and later on coupled: 1) Pressure generation in the discharge channel; 2) Vaportemperature-pressure gradient around the discharge channel; 3) Compressible liquid as a pressure transmitting media; 4) Deformation of the chamber and blank in contact with the deformable die. 
The energy dissipated in the discharge channel

The discharge channel is a plasma bubble which rapidly expands due to the high pressure. The source of this pressure is additional internal energy which is created by electrical current going through the channel.

There are a few approaches which can be used to determine the energy dissipated in the channel. One is a simulation of the discharge electrical circuit.

The differential equation for current $i(t)$ in the circuit in Figure 1.1.2 includes variable resistance of discharge channel $R_{v}$, cumulative resistance of pulsed forming machine and connecting cables $R_{0}$, and inductance of discharge circuit $L$. It can be written in the following form:

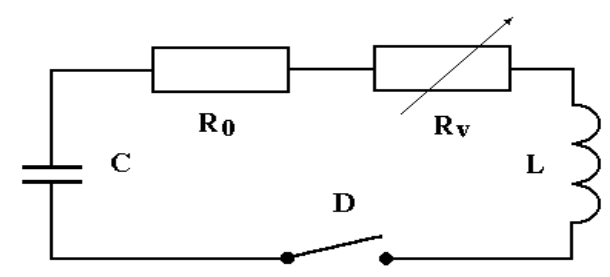

Figure 1.1.2. Schematic of electrical circuit at high-voltage discharge in a liquid $(\mathrm{C}$ - capacitance of the battery, $L$ - total inductance of the system, Ro - resistance of the circuit external to the discharge channel, $R_{v}$ - resistance of

$$
\frac{d^{2} i}{d t^{2}}+\frac{R_{v}(i, t)+R_{0}}{L} \cdot \frac{d i}{d t}+\frac{1}{L C} i=0,
$$

with the following initial conditions

$$
i(0)=0,\left.\quad \frac{d i}{d t}\right|_{t=0}=-\frac{U_{0}}{L},
$$

where $U_{0}-$ initial voltage of capacitor battery.

The electric power being pumped through the discharge channel can be defined by the following equation:

$$
N=i^{2} R_{v}(i, t)
$$

Integrating this equation by time defines the energy deposition in the discharge channel $E_{c h}(t)$ through the discharge process:

$$
E_{c h}=\int_{0}^{t} i^{2}(t) R_{v}(i, t) d t
$$

To use this approach, the resistance of the channel as a function of time is required, and this is a separate and difficult simulation problem. 
Another more practical experimental approach can be used to obtain the energy dissipated in the channel. In this approach the energy for the specific configuration is obtained from experimentally measured electrical current and voltage. The electric power can be calculated simply as:

$$
N=i(t) \cdot U(t)
$$

where $i(t)$ and $U(t)$ are the current and voltage between the electrodes measured by an oscilloscope, respectively.

\section{The model of the plasma channel}

The plasma channel is modeled in LS DYNA as an adiabatically expanding volume of gas. This is possible because the energy exchange between the plasma and the surrounding water is significantly less than the energy exchange inside plasma bubble, which was shown in [1.1.1]. The electric energy is assumed to be introduced uniformly through the channel volume. Pressure in each finite element of the plasma channel can be calculated using the following equation of state of ideal gas as substantiated in [1.1.2].

$$
p_{c h}=(\gamma-1)\left(\rho / \rho_{0}\right) E
$$

where

$\mathrm{p}_{\mathrm{ch}}$ - pressure at a given point of the discharge channel;

$\rho$ - current mass density of gas inside the channel;

$\rho_{0}-$ initial mass density of gas inside the channel;

$E$ - portion of the energy produced inside the channel;

$\gamma$-adiabatic coefficient for plasma produced from tap water inside the channel.

In LS-DYNA, the material with equation of state (1.1.6) can be simulated using the material model MAT_NULL, and with the additional EOS card EOS_LINEAR_POLYNOMIAL_WITH _ENERGY_LEAK which defines pressure in the following form [1.1.3]:

$$
p_{c h}=C_{0}+C_{1} \mu^{2}+C_{2} \mu^{3}+C_{3} \mu+\left(C_{4}+C_{5} \mu+C_{6} \mu^{2}\right) E^{\prime}
$$

where $C_{0}, C_{1}, C_{2}, C_{3}, C_{4}, C_{5}$, and $C_{6}$ are user defined constants; $\mu=1 / V^{\prime}-1 ; V^{\prime}=\rho_{0} / \rho$ is the relative volume; $\mathrm{E}^{\prime}$ is the internal energy per initial volume and increased according to a user defined energy deposition rate versus time curve. The energy deposition rate can be calculated analytically or measured experimentally as described above. Initial plasma density was $\rho_{0}=$ $1000 \mathrm{~kg} / \mathrm{m}^{3}$.

Model of liquid

The model of the liquid was used from LS DYNA as an ideal compressible liquid with the specific cavitations threshold using MAT_ELASTIC_FLUID (MAT_ELASTIC with option FLUID) model. The list of necessary parameters includes the initial mass density, compression modulus 
and negative pressure threshold corresponding to the beginning of cavitations. The following material properties are employed: density $\rho_{0}=1000 \mathrm{~kg} / \mathrm{m}^{3}$, bulk modulus $\mathrm{k}=2.35^{\star} 10^{9} \mathrm{~Pa}$.

\section{Meshing technique and choice of solver}

The simulation of the expanding discharge channel interacting with the liquid, and the moving liquid interacting with a deformable blank creates an issue of substantial mesh distortion. When using the traditional Lagrangian approach, the deformation of the elements makes it almost impossible to continue a simulation longer than 100-200 $\mu \mathrm{s}$, depending on configuration. To eliminate the issue of mesh distortion, the Arbitrary Lagrange Eulerian (ALE) solver with MultiMaterial capability was used which is available in LS-DYNA.

\section{Model of sheet metal blank}

Modeling of sheet metal blank formation into a designated shape can be conducted using two different approaches. Both of them will be employed in this project: 1) model of elastic-plastic shell incorporating bending stiffness and being in contact with a rigid die is more economical for prediction of the strain distributions and also for potential fracture in the blank; 2) elastic-plastic solid in contact with a deformable die is defined as an elastic-plastic solid which accounts for loads on the die and provides more accuracy in sharp corner filling operations. Both of these models are available in LS-DYNA.

The influence of strain rate on the hardening law may be accounted for using the Cowper and Symonds model which scales the yield stress with the following factor:

$$
k_{d}=1+\left(\frac{\dot{\varepsilon}}{C}\right)^{1 / p}
$$

where $k_{d}$ is the scaling coefficient, $\dot{\varepsilon}$ is the strain rate, and $C$ and $p$ are strain-rate parameters. The Cowper and Symonds model has the advantage in LS-DYNA that it can be used together with two material models which are commonly used in metal-forming simulations: *MAT_PIECEWISE_LINEAR_PLASTICITY and *MAT_POWER_LAW_PLASTICITY. In the first case the yield stress is defined by a user-defined curve and in addition is multiplied by the $k_{d}$ value. If the power law material model is used, then the yield stress is defined by following expression:

$$
\sigma_{y}=K \varepsilon^{m}\left(1+\left(\frac{\dot{\varepsilon}}{C}\right)^{1 / p}\right),
$$

where $\varepsilon$ is the elastic strain, $\mathrm{K}$ is the strength coefficient, $\mathrm{m}$ is the hardening exponent, $\dot{\varepsilon}$ is the strain rate, and $C$ and $p$ are strain-rate parameters. The DP600 steel has following parameters: $K=932.1 \mathrm{MPa}, m=0.12, C=458.0^{*} 10^{6}, p=8.413$.

\section{High-Strain-Rate Tensile Tests}

Since there is no standard for the specimen design to be used in tensile split Hopkinson bar tests, different specimen geometries with different $L$ to $W$ ratios were tested. A ratio of 1.5 was determined to be appropriate for high-rate tests, i.e. strain-to-failure didn't change much by 
further increasing the L/W ratio. The final specimen geometry adopted and used in all the tests discussed in the following sections is represented in Figure 1.1.3.

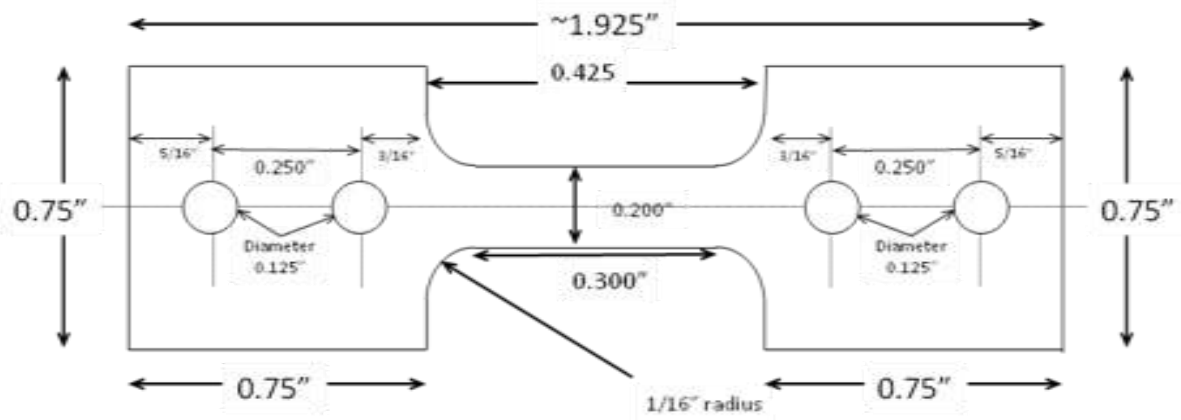

Figure 1.1.3. Adopted tensile specimen design for Hopkinson-bar tensile tests; L/W = 1.5.

Three tests were performed for each strain rate (1000/s, 2000/s, and 3000/s) in rolling (RD) and transverse directions (TD). All the stress-strain curves are represented in Figures 1.1.4 (a) and 1.1.4 (b) in rolling and transverse direction, respectively. We observe very little difference in the strength levels in the RD and TD directions in the strain-rate range 1000-3000/s. In both rolling and transverse directions and for the three strain rates, the stress-strain curve suggests strain localization at $\sim 12-15 \%$ strain. Strains-to-failure in the strain-rate range $1000-3000 / \mathrm{s}$ are on the order of 23 to $26 \%$. Figure 1.1 .5 summarizes the stress-strain data for quasi-static and high rates.

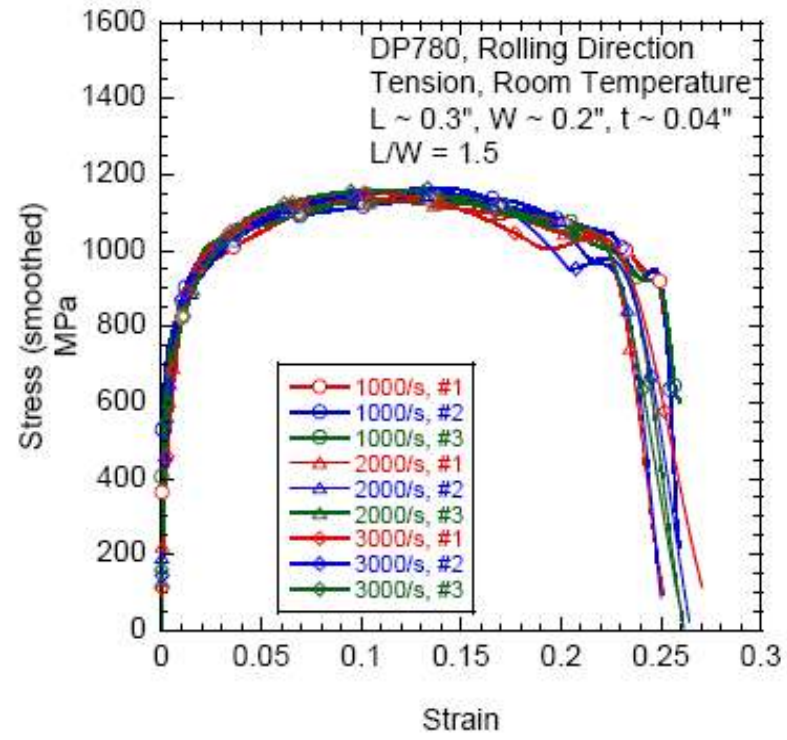

(a)

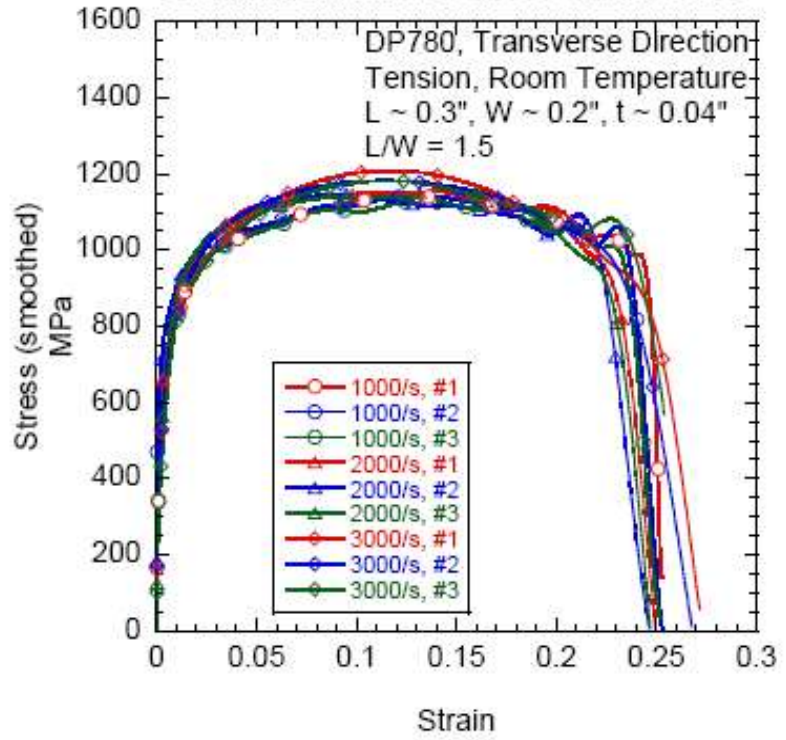

(b)

Figure 1.1.4. $L / W=1.5$, Engineering stress-strain curves for 3 tests for each strain rate $(1000 / \mathrm{s}, 2000 / \mathrm{s}$, 


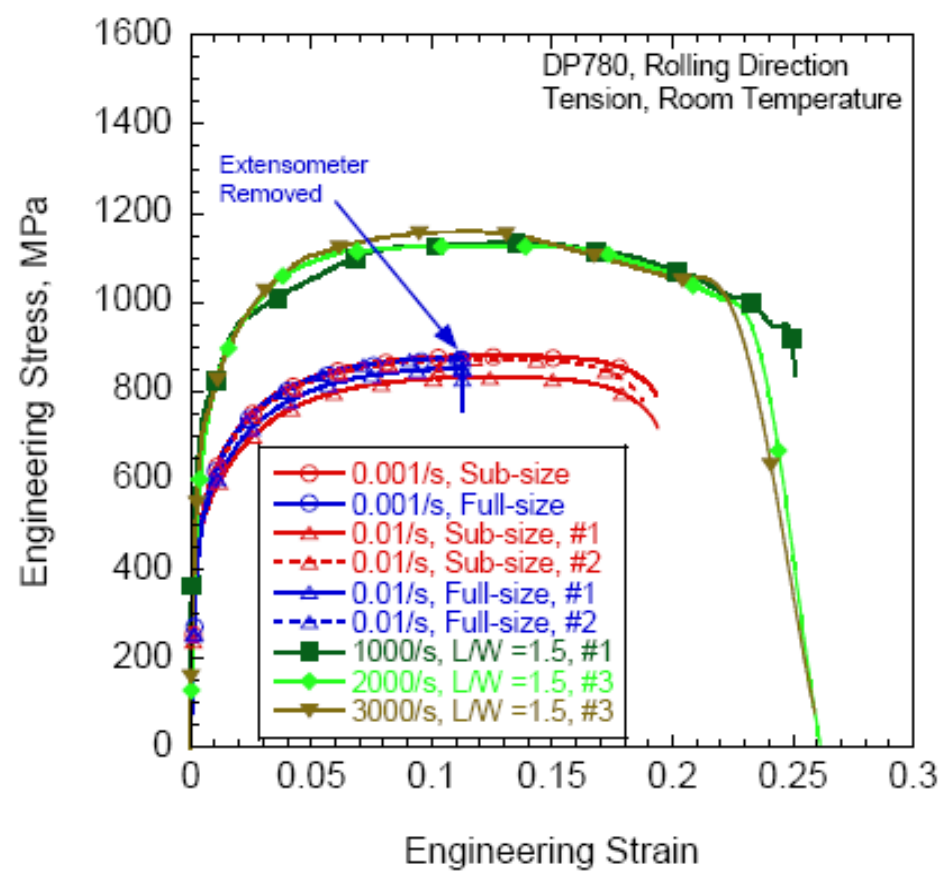

Figure 1.1.5. Effect of strain rate (quasi-static and high rate) on stress-strain behavior.

Comparing the high-rate data from Hopkinson-bar tests with quasi-static data leads to the following observations:

(i) Flow stress at $1000-3000 / \mathrm{s}$ is $\sim 35 \%$ higher than at quasi-static strain rates $(0.001$ and $0.01 / \mathrm{s}$ ). The Ultimate Tensile Strength (UTS) in high-rate conditions is $\sim 1200 \mathrm{MPa}$ and less than $900 \mathrm{MPa}$ in quasi-static conditions.

(ii) In the strain-rate range 1000-3000/s, the stress-strain curve suggests strain localization $\sim 12-15 \%$ strain. At quasi-static rates, the localization occurs closer to $\sim 17 \%$ strain.

(iii) Strain-to-failure is not dramatically greater at $1000-3000 / \mathrm{s}(\sim 24 \%)$ relative to $0.001-0.01$ /s ( 19\%). The results need to be confirmed by performing the quasi-static tests with the same specimen geometry as the high-rate tests.

The constitutive model used to predict the behavior of DP780 in high rates is the Johnson-Cook model. The equation relating the stress to the strain, strain-rate, and temperature is written as follows:

$$
\sigma=\left(A+B \varepsilon_{p}^{n}\right)\left(1+C \ln \dot{\varepsilon}_{p}^{*}\right)\left(1-T_{*}^{m}\right),
$$

with the equivalent plastic strain $\varepsilon_{p}$, the dimensionless plastic strain rate $\dot{\varepsilon}_{p}^{*}=\dot{\varepsilon}_{p} / \dot{\varepsilon}_{0}$ and the homologous temperature $T_{*}^{m}$. The Model's parameters were calibrated to best fit the experimental stress-strain curves. Their values are reported in Table 1.1.1. 
Table 1.1.1. Johnson-Cook constitutive model parameters for DP 780.

\begin{tabular}{|c|c|c|c|c|c|}
\hline & $A$ & $B$ & $C$ & $n$ & $m$ \\
\hline DP 780 & 550 & 840 & 0.042 & 0.25 & 1.230 \\
\hline
\end{tabular}

Since we do not use any damage criterion at this time, we are not able to predict necking and ultimately failure. Figure 1.1.6 represents a comparison between the model prediction and the experiments. Before necking (strain less than $\sim 12-15 \%$ ) the model is in good correlation with the experiments. Other models need to be investigated and more effort will be made in the future in order to predict damage. Figure 1.1.7 shows the experimental results obtained for DP980 steel at quasi-static rates $(0.001 / \mathrm{s})$ and high-strain rate $(2400 / \mathrm{s})$.

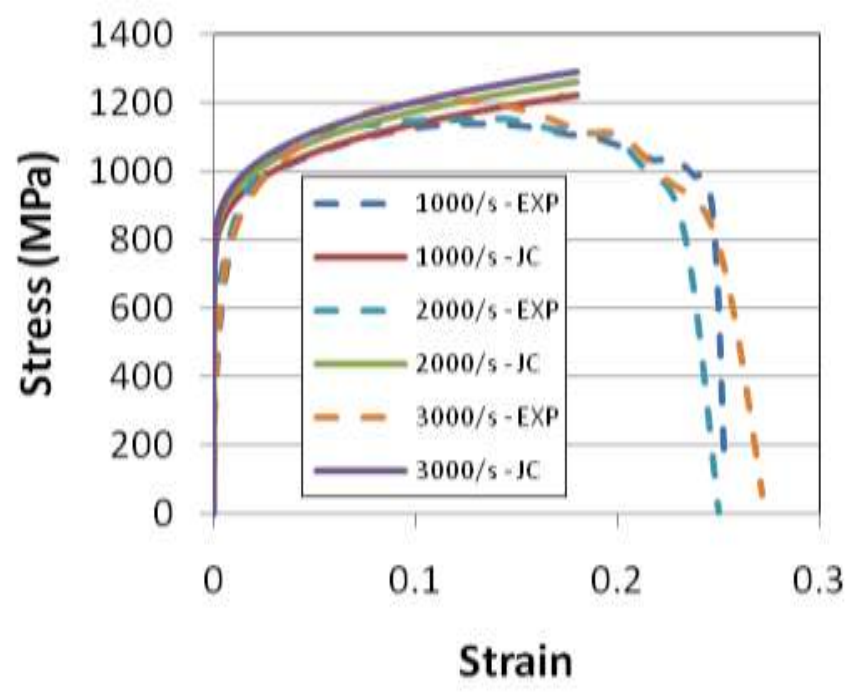

Figure 1.1.6. Johnson-Cook model predictions vs. experiments for strain rates 1000-3000/s.

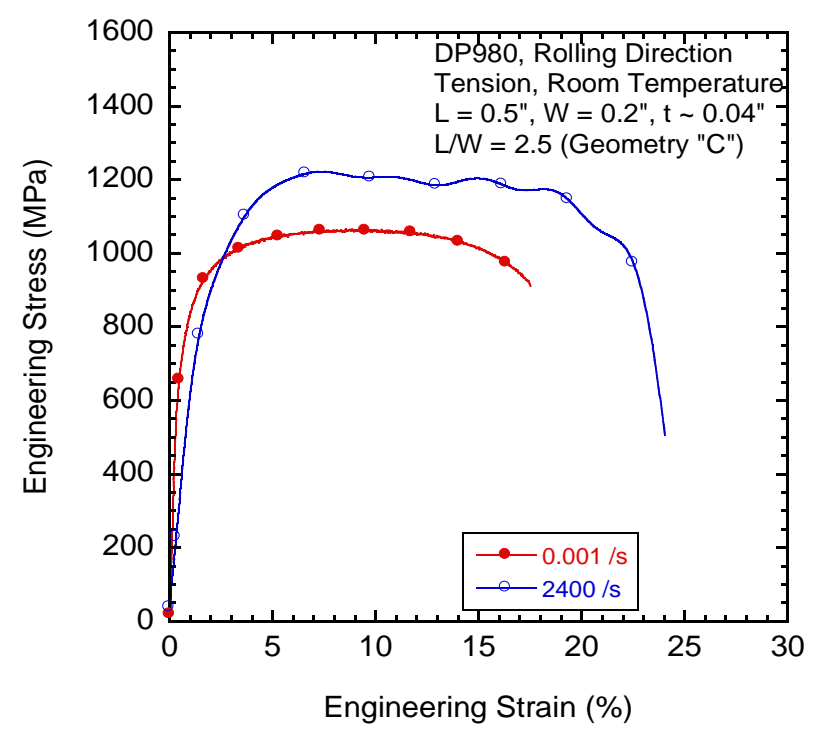

Figure 1.1.7. Stress-strain curve of DP980 steel in the rolling direction at two different strain-rates. 
Experimental validation of the numerical model of the EHF process

Initial validation of the numerical model is done by comparison of the measured and simulated pressure in the simple cylindrical chamber shown in figure 1.1.8. The experimental curve of power deposition inside the chamber is shown in Figure 1.1.9.

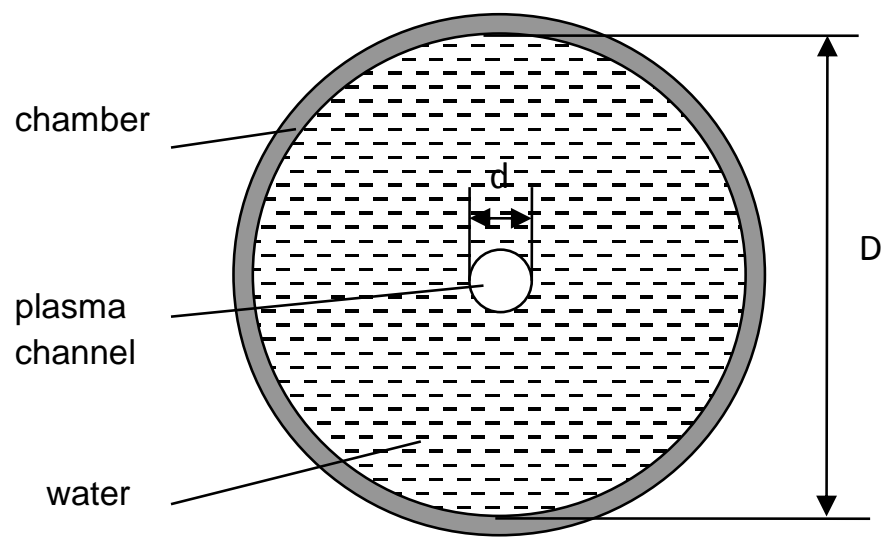

Figure 1.1.8 Cylindrical discharge chamber: $D=100 \mathrm{~mm}, \mathrm{~d}=2 \mathrm{~mm}$.

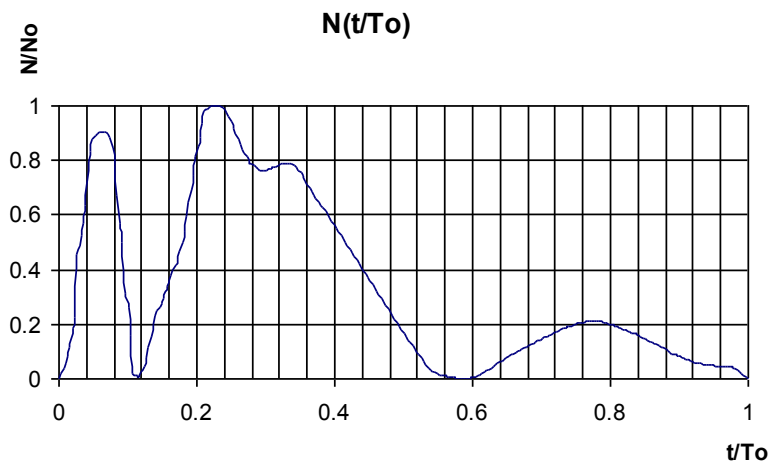

Figure 1.1.9. Dimensionless function of power deposition in a discharge channel; (No $=6.35 \cdot 10^{7} \mathrm{~W}, \mathrm{~T}_{0}=$ $154 \mu \mathrm{s})$.

The maximum pressure on the wall was predicted as $67 \mathrm{MPa}$. The maximum pressure on the wall measured by a sensor was $76 \mathrm{MPa}$, which is in reasonable agreement with the numerically predicted value.

Using the developed numerical technique, a number of studies was performed. One study was conducted to develop a fundamental understanding of the forming conditions during filling the 
corners at high speed, which is a typical situation during EHF. Corner filling processes can be represented by sheet formation into the V-shape die.

Figure 1.1.10 shows the mechanics of the blank's formation while partially filling the V-shape die and the progression of major strains in the blank as it is being formed in plane strain. Increasing the discharge energy to a level that completely fills the $\mathrm{V}$-shape die causes a change in the strain distribution and also elevates the strain rate. The maximum strain in this case is located near the apex of the V-shape, even though the simulation indicates that it is still at some distance from the apex. The strain rate for the majority of the sidewall area exceeds 15,000 /sec, and the maximum strain rate is above 20,000 /sec, as shown in Figure 1.1.11.

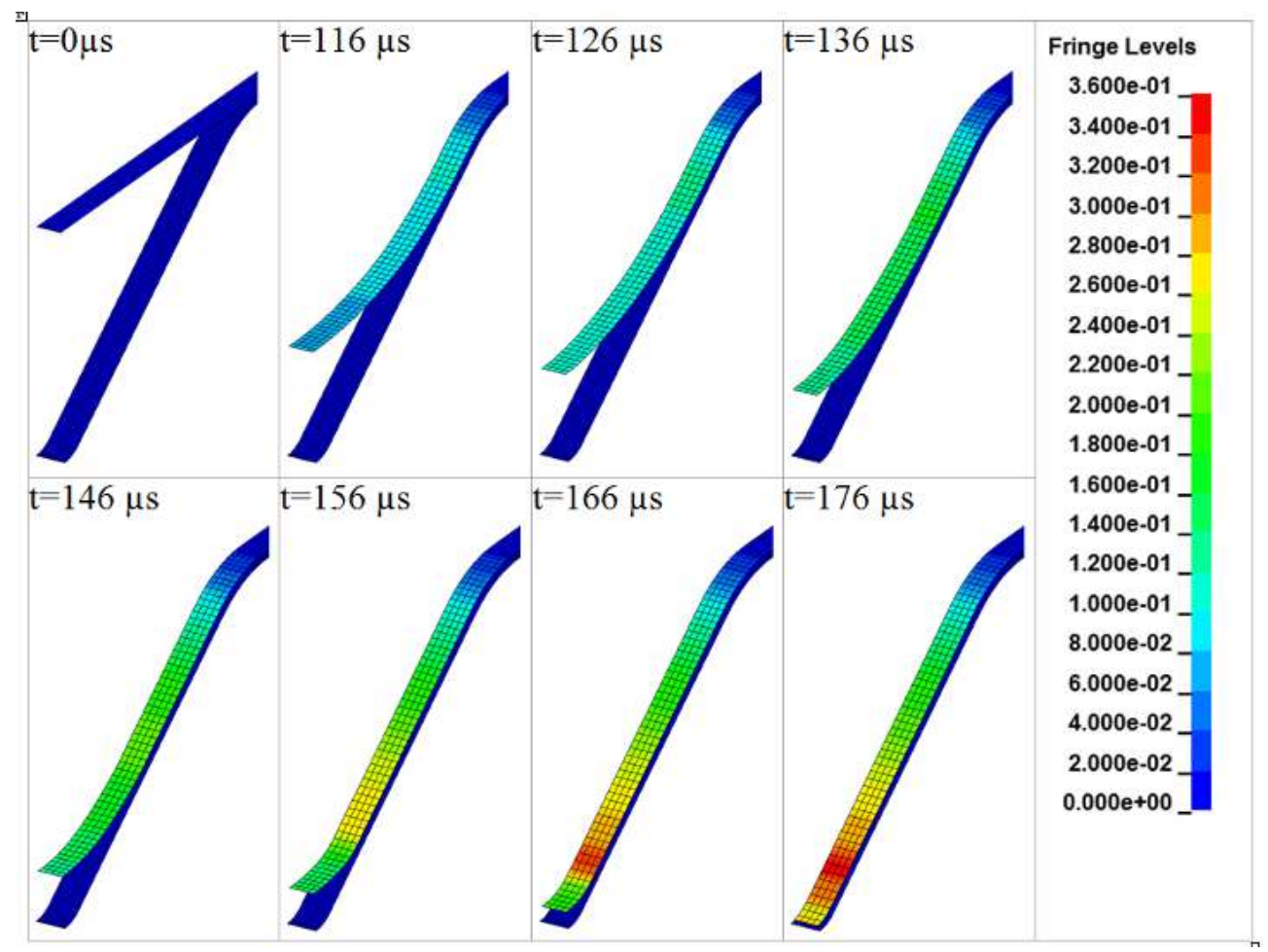

Figure 1.1.10. Evolution of shape and progression of major strains in the blank of DP600 sheet while completely filling the $38^{\circ} \mathrm{V}$-shape cavity. 

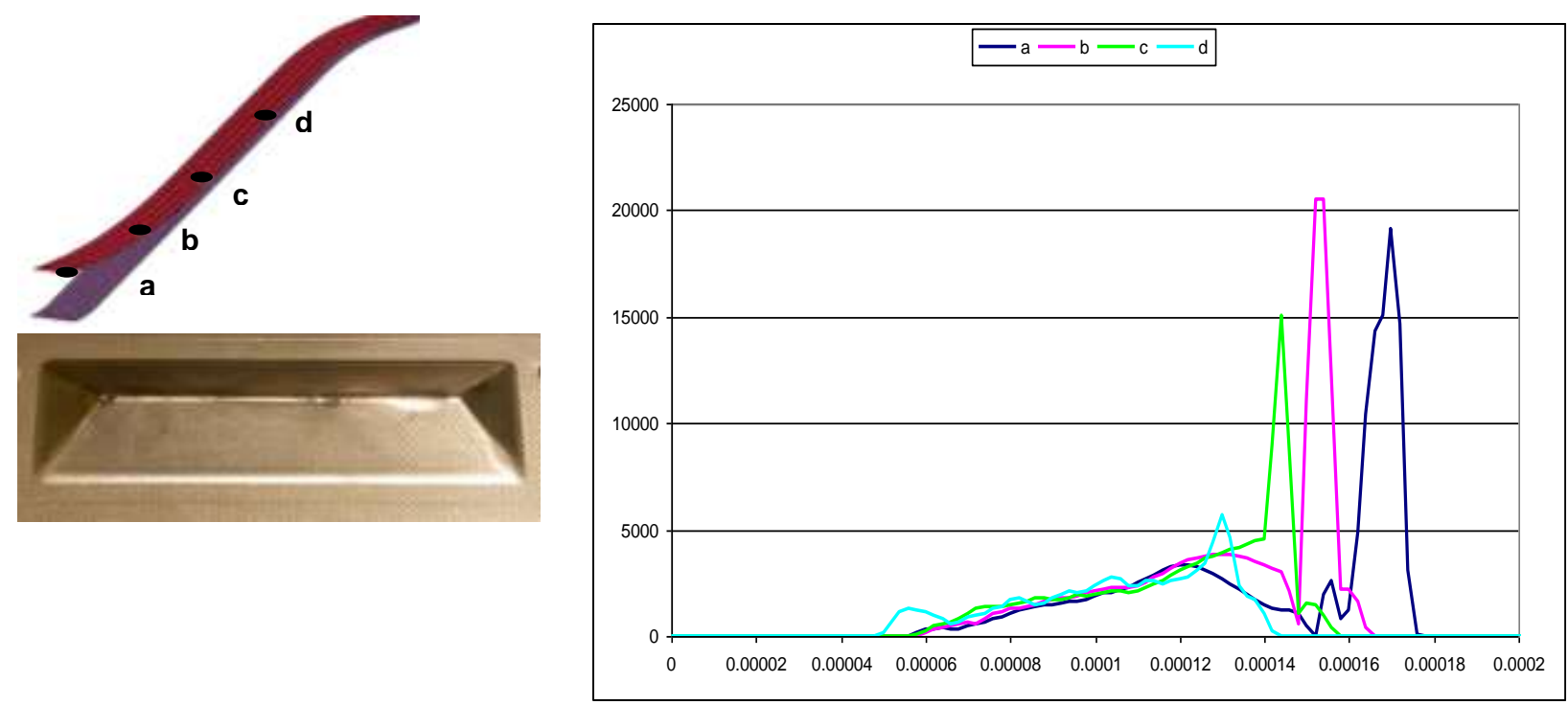

Figure 1.1.11. Evolution of strain rate along the DP 600 blank while completely filling the V-shape die.

Such a substantial increase in strain rates and the corresponding velocity of impact between the blank and the die leads to enhanced material elongation before fracture, as observed in experimental results. Obviously, there is some limit of elongation that a material can withstand for a given stress, strain and strain rate path. When the limit is reached, fracture occurs even though the blank may still be flying with some considerable velocity and is still being deformed due to inertial forces. In such cases, the splits may open rather broadly.

The lesson learned from this study was that in order to form a part without splits, the blank should get into the corner filling process before it gets very close to its quasistatic formability limit. In this case the benefit of EHF can be fully utilized.

Another study was made to analyze combined static preforming followed by EHF forming. Such a configuration in many cases is very favorable in terms of getting the maximum amount of sheet metal into the die cavity rather than aggressively stretching it. Such an approach also helps to minimize the loads on the stamping die as well as the loads on the electrode system. The major question is to define whether the benefits of EHF technology can be retained when the initial forming stage is done quasi-statically.

The numerical experiment was performed for the $\mathrm{EH}$ forming into the $34^{\circ}$ conical die. The conical die was selected as an example of a shape which is very difficult to fill in conventional forming. For this reason, this geometry was also very popular in the research literature [1.1.41.1.7]. The material of the blank was DP780, $1 \mathrm{~mm}$. The impulse energy was adjusted such that it would provide stable filling of the die cavity without excessive impact. The first case simulated was the pure EHF process, which begins with a flat blank that is then formed from the undeformed state using EHF only. The results of this simulation (channel shape, pressure distribution, and blank deformation) are shown in Figure 1.1.12. The second case was EH 
forming after the preliminary hydrostatic preforming step applied with a quasi-static pressure of $4000 \mathrm{psi}(27.6 \mathrm{MPa})$. The results of this simulation (channel shape, pressure distribution, and deformation of the blank) are shown in Figure 1.1.13. A comparison of the strains in a few points of the blank (Figure 1.1.14) and a comparison of strain rates in the same points (Figure 1.1.15) show no significant difference in the maximum levels of strains and strain rates between the two cases. Although the duration of the process is shorter in the case of EHF with hydrostatic preforming, there is evidently still enough time and enough open space between the blank and the die for the blank to accelerate to the same velocity as that in the case of the pure EHF process.

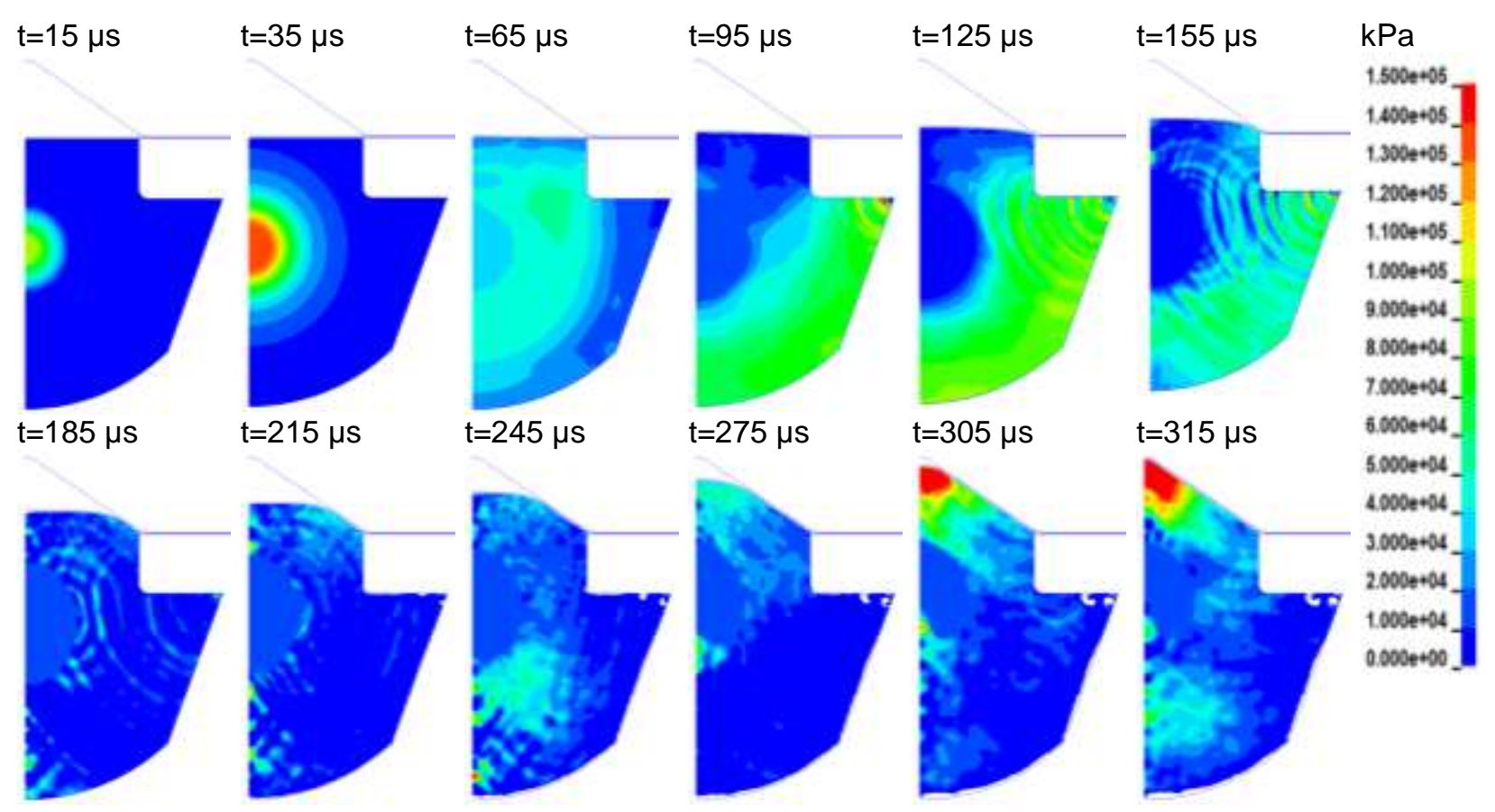

Figure 1.1.12. Channel shape, pressure distribution, and blank geometry versus time for EHF without prior hydroforming. 
$\mathrm{t}=15 \mu \mathrm{s}$

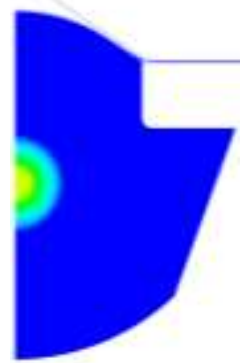

$\mathrm{t}=120 \mu \mathrm{s}$

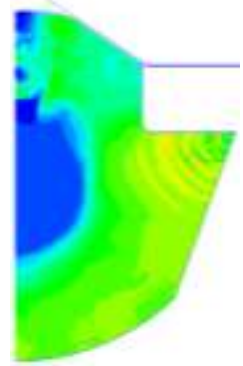

$\mathrm{t}=30 \mu \mathrm{s}$

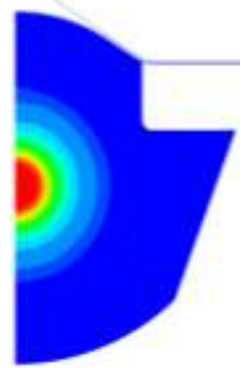

$\mathrm{t}=150 \mu \mathrm{s}$

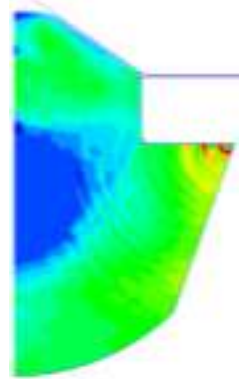

$t=60 \mu \mathrm{s}$

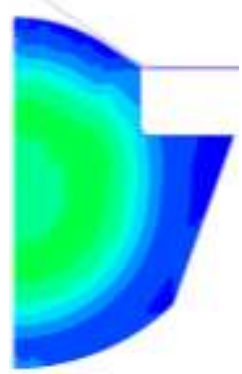

$\mathrm{t}=180 \mu \mathrm{s}$

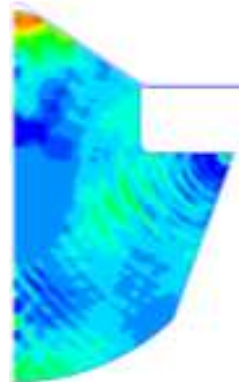

$t=90 \mu s$

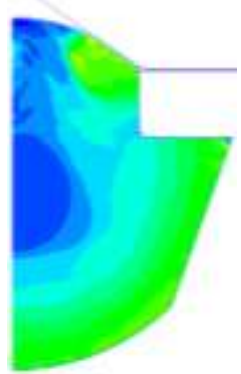

$\mathrm{t}=200 \mu \mathrm{s}$

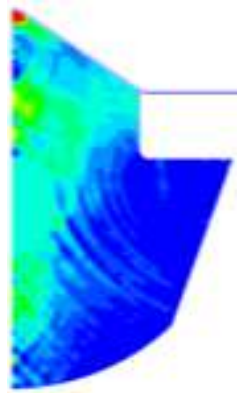

$\mathrm{kPa}$

t. soletess 1.6000+15 t.3olewas 12006 e-15_ 1.104ens. 1000ents a $00010+14$ $3.000 \mathrm{e}-14$ 7 . Bolet 14 t. soberits s.600e-14 4.50lend4 2.6014064 2.00leste 1.800ense a.600e+10

Figure 1.1.13. Channel shape, pressure distribution, and blank geometry versus time for EHF after preliminary hydroforming step.

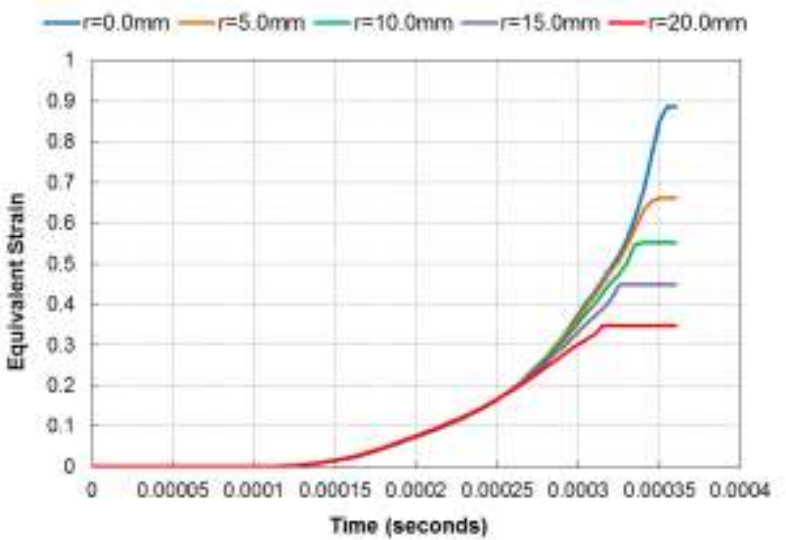

a)

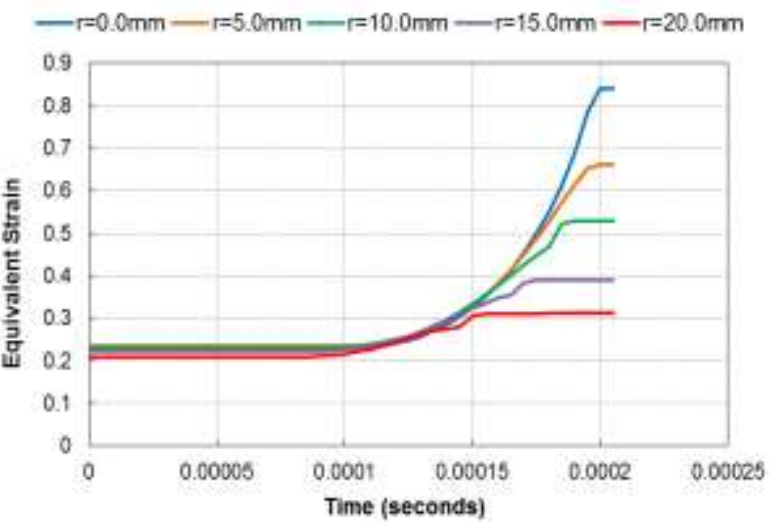

b)

Figure 1.1.14. Strain history for cone-filling: a - single-pulse EHF, b - after a quasi-static preforming step. 


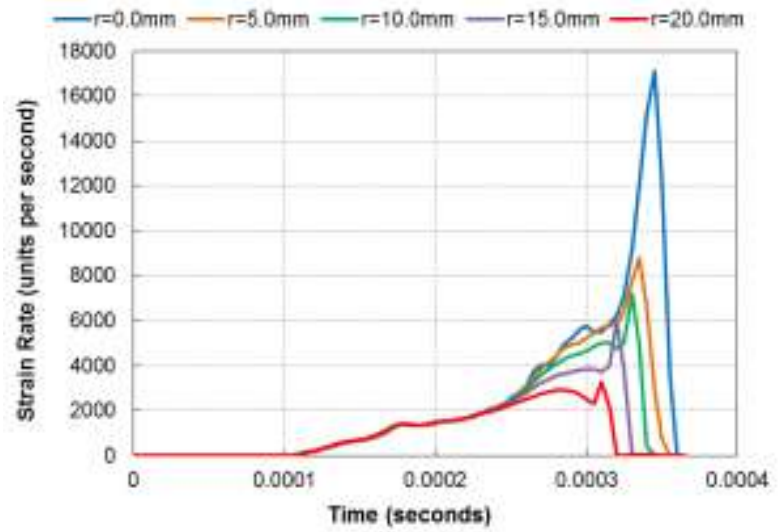

a)

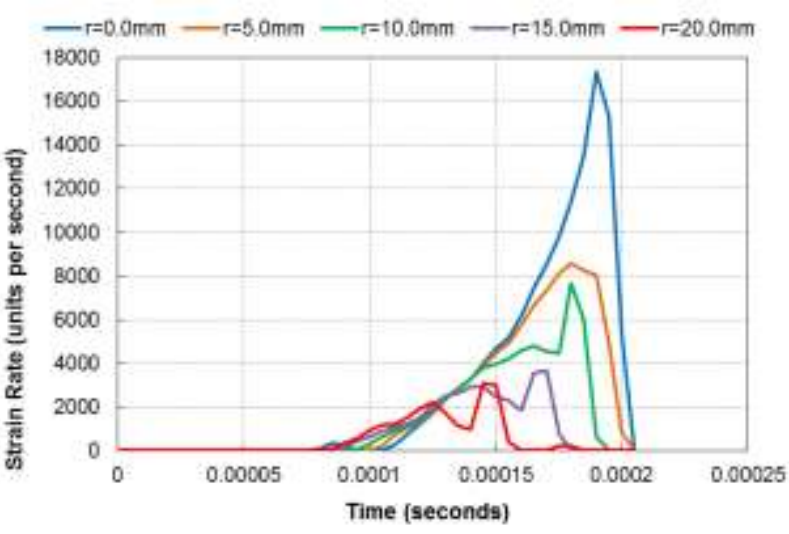

b)

Figure 1.1.15. Evolution of strain rates during cone-filling: a - single-pulse EHF, b - after a quasi-static preforming step.

The developed numerical technique was used to simulate the processes of forming exterior and interior closure panels. One of the examples is the simulation of the deck lid part which was used for demonstration in EHF automated cell. The first simulation was performed to analyze the distribution of pulse pressure during the calibration step, to recognize the critical areas, and to optimize the shape and the volume of the discharge channel. The shape of the blank and the critical areas are shown in figure 1.1.16. The original and modified chamber geometry are shown in figure 1.1.17. The effect from reducing the volume for one of the critical areas is

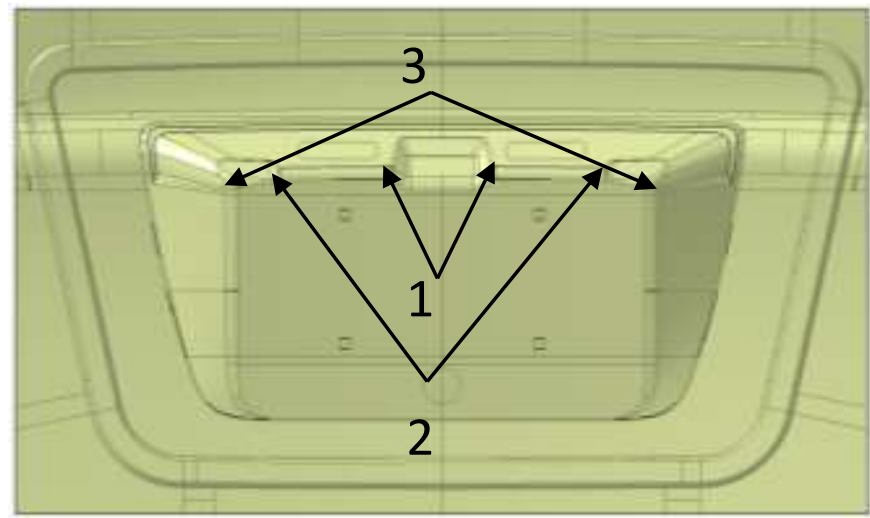

Figure 1.1.16. The shape of the blank to be formed illustrating the most critical areas requiring the highest pressure to fill the corners of the die cavity, marked as 1, 2 and 3. shown in figure 1.1.18.

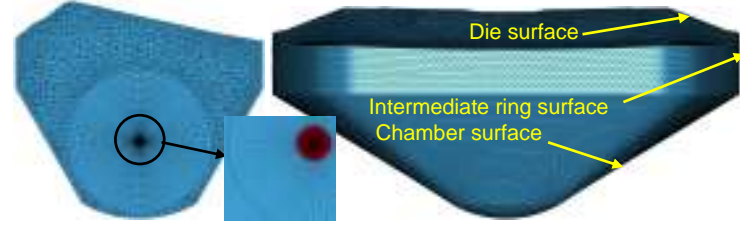

a)

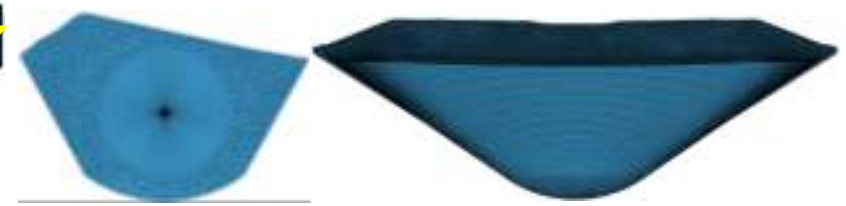

b)

Figure 1.1.17. EHF chamber configuration for forming of the license plate area: $a$ - initial, $b$ - modified. 


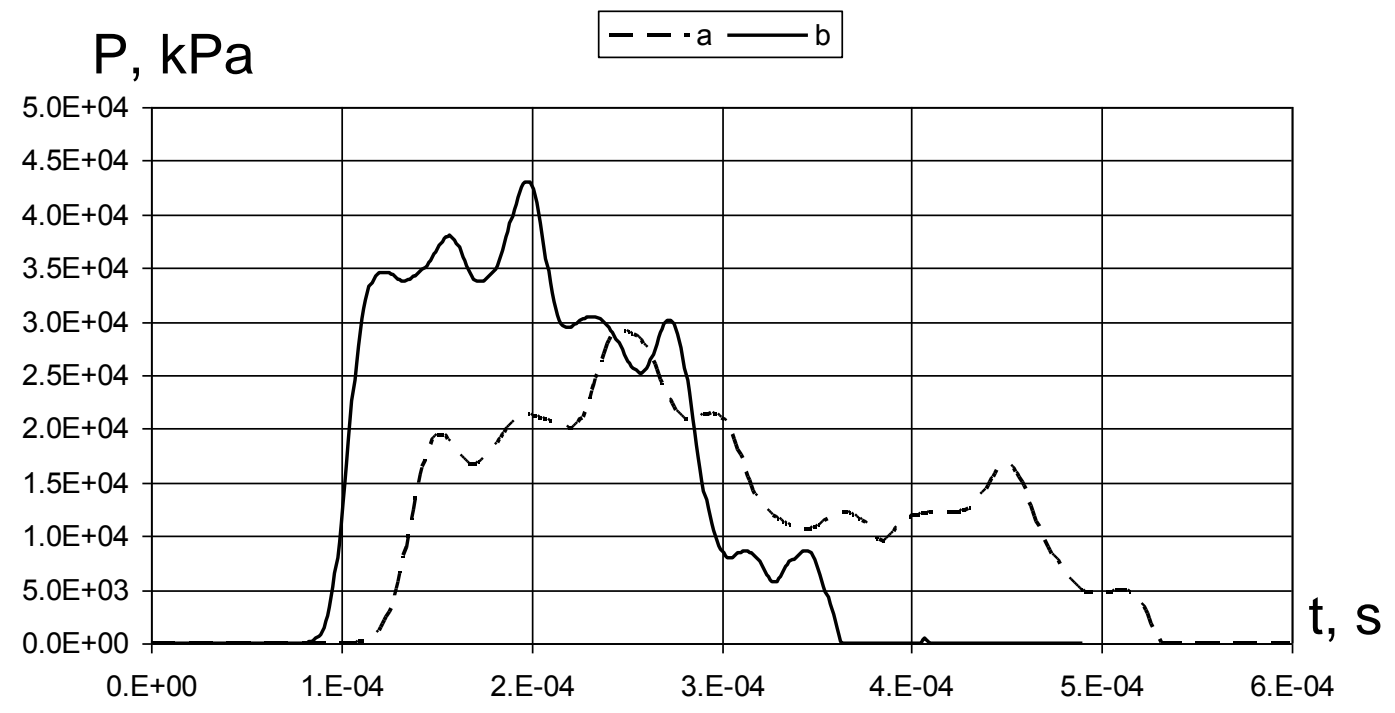

Figure 1.1.18. Results of numerical simulation for critical area 1 (a - initial design, $b-$ new design).

The geometry of the chamber is shown in figure 1.1.19. The shape of discharge channel and deformation of the blank during the first discharge is shown in figure 1.1.20, and the blank after each discharge is shown in figure 1.1.21.

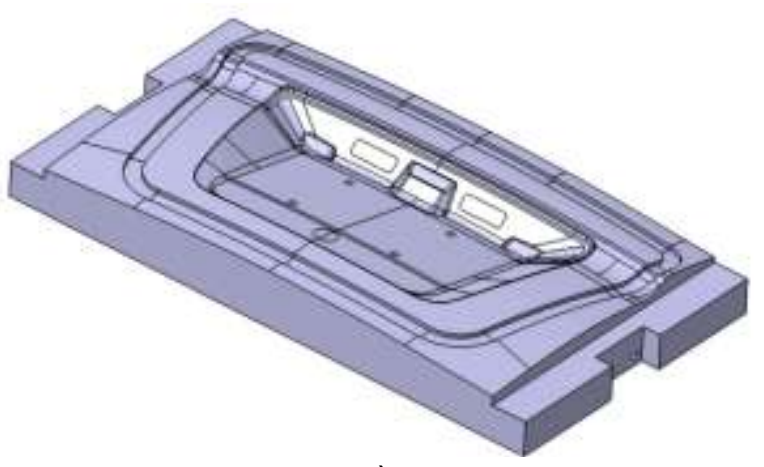

(a)

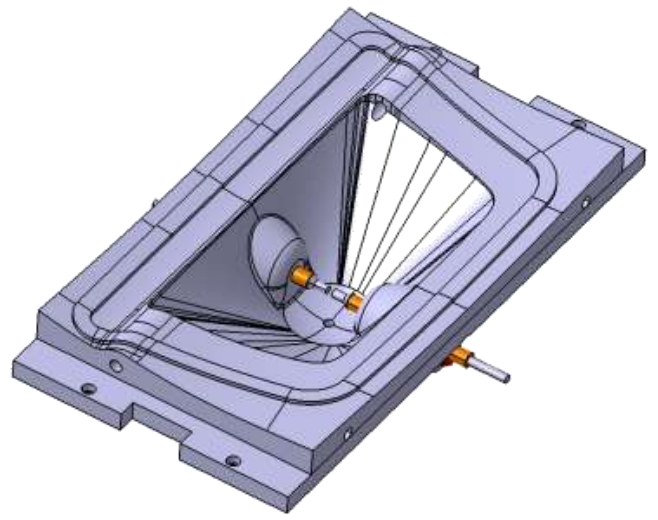

(b)

Figure 1.1.19. Geometry of the die (a) and the chamber (b). 

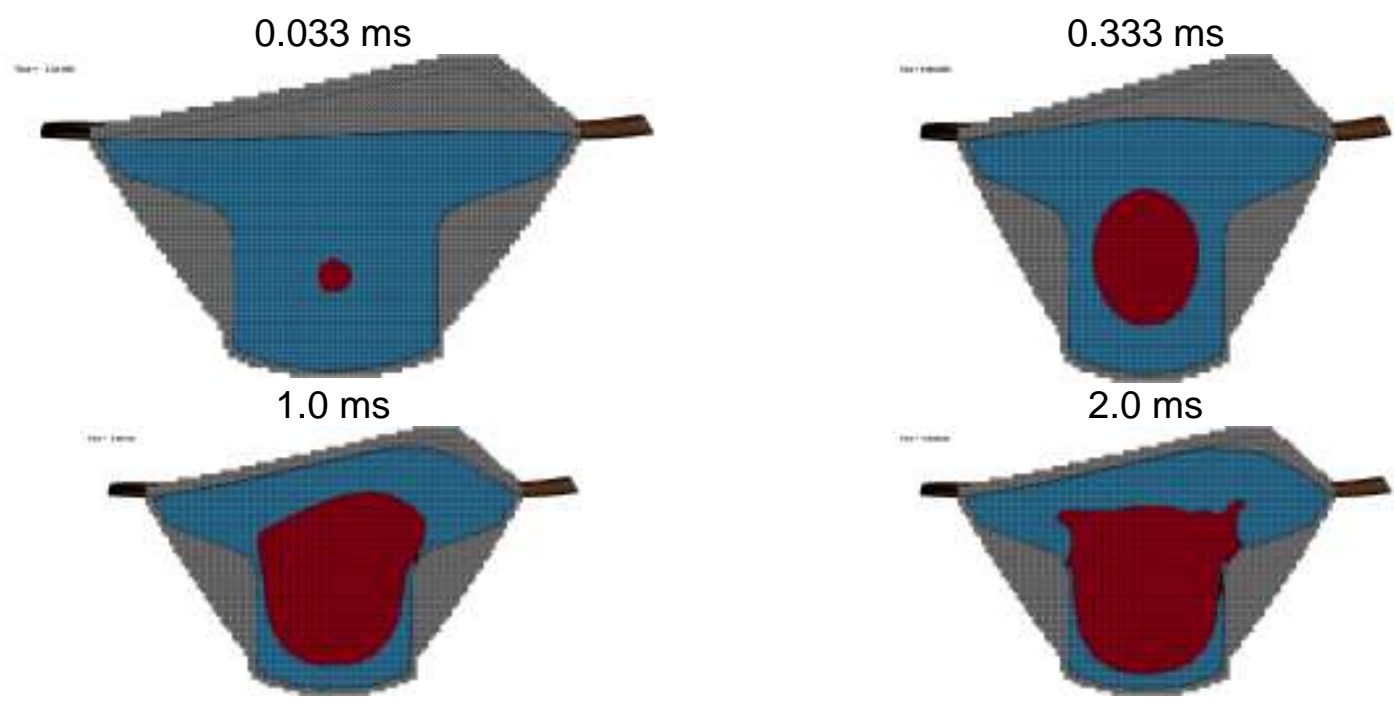

Figure 1.1.20. Channel expansion and deformation of the blank during the first stage of EHF process.
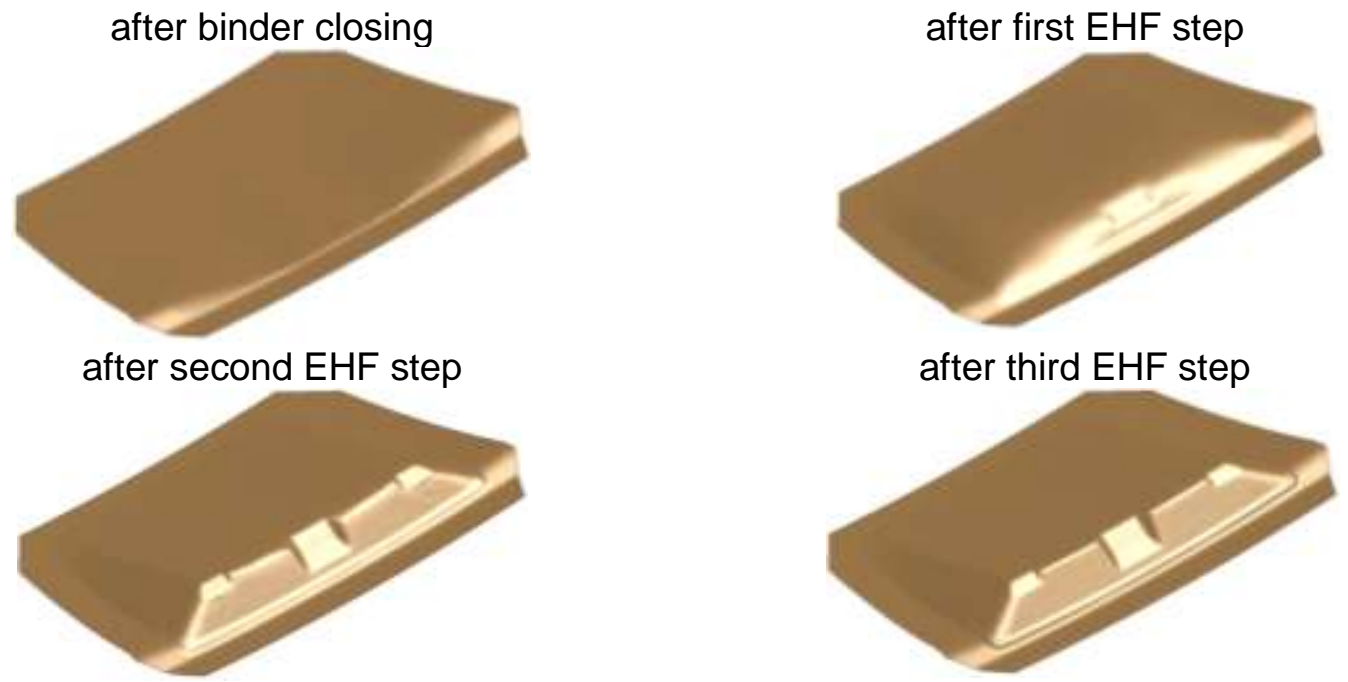

Figure 1.1.21. Progression of blank geometry 
The simulation of the EH trimming process was also performed. A schematic of the EH trimming process is shown in Figure 1.1.22. After the discharge occurs, the wave of pulsed pressure forms the blank into a relatively narrow cavity of the die. Unlike a traditional trimming process, separation along a sharp edge of a die occurs primarily due to the tearing mechanism. The strain state at the shearing edge can be considered as a plane strain state. The assumption was made that the fracture mechanism is the same as in the EH forming, i.e. the tearing due to an excessive stretching deformation when the first major strain $\varepsilon_{1}$ reaches the limit corresponding to the plane strain point of Forming limit Diagram (FLD), which is usually the lowest point of the FLD diagram. In LS-DYNA the fracture strain can be directly used as a fault criteria in FAUL field of the *MAT_PIECEWISE_LINEAR_PLASTICITY card. Based on existing FLD data for DP500 and our recent studies on extended formability in high rate forming, the fracture strain limit was assumed to be 0.5 . The shape of the discharge channel during the trimming process is shown in figure 1.1.23 and the progress of blank deformation and trimming is shown in figure 1.1.24.

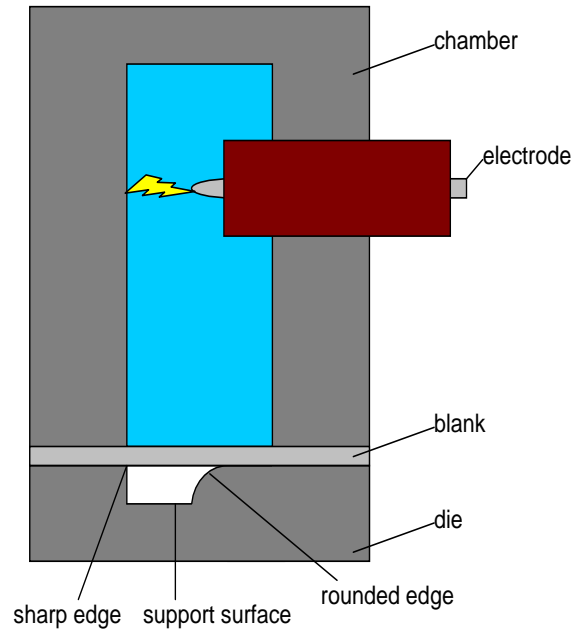

Figure 1.1.22. Schematic of electrohydraulic trimming

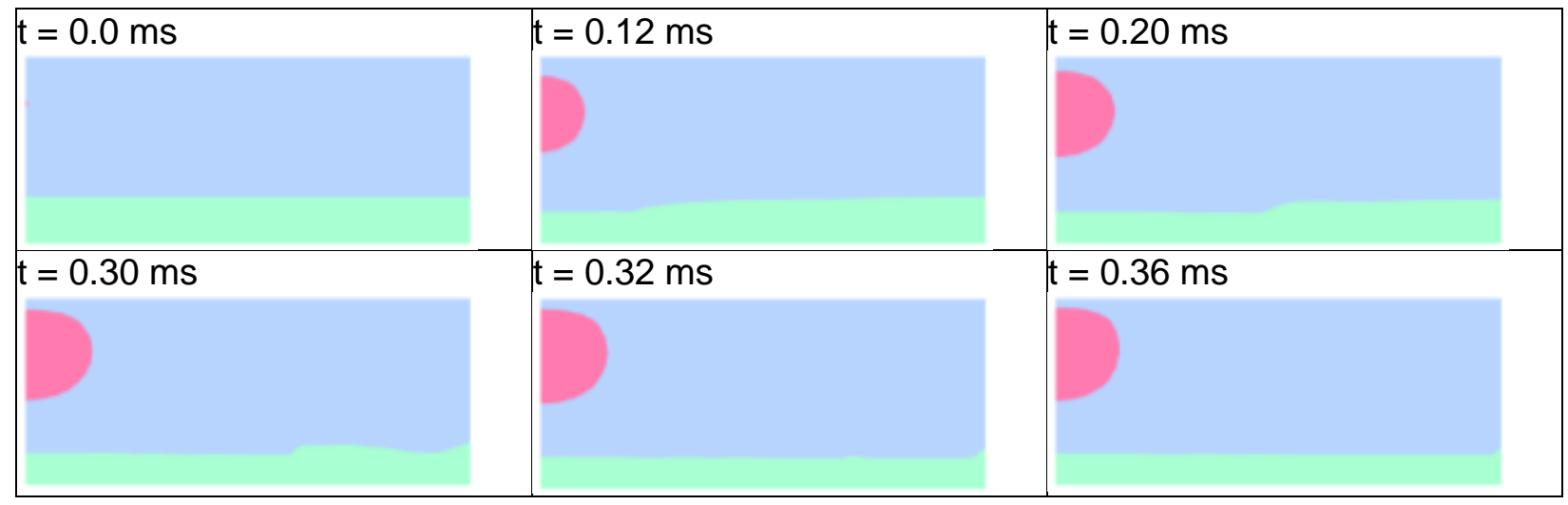

Figure 1.1.23. Channel expansion during EH trimming.

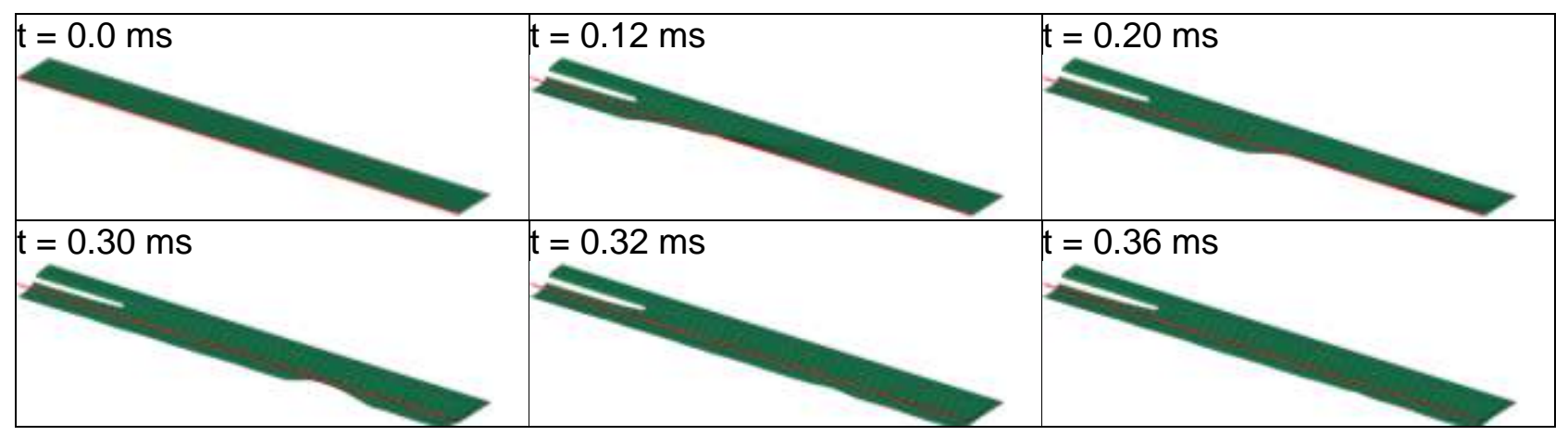

Figure 1.1.24. Deformation of a blank during $\mathrm{EH}$ trimming. The red line shows the cutting edge. 
Sub-task 1.2. Optimal distribution of strains in the blank in two-step forming process.

A two-step forming method was developed in order to expand the capabilities of EHF technology even further. A method is designated for forming a part from a sheet metal blank into a final design shape that has critical areas. The method comprises drawing the blank into an intermediate shape that includes a bulge adjacent to the critical region. The bulge (or bulges) accumulates the material which is later used to fill the critical areas. The intermediate shaped part is then formed into the final design shape. In this particular case, the final forming step is conducted using the electrohydraulic forming process.

Critical regions are the areas of the final design shape that require either stretching the blank into a sharp corner, or drawing the blank around a relatively sharp corner to a depth that exceeds the material forming limits. In the drawing step, the blank is drawn to a depth that is within the forming limits of the blank. Then, the forming step results in the formation of a final part that would exceed the forming limits, if a single step forming process was used. This becomes possible due to distributing the strains over a larger area than it would normally happen in a one-step forming process. The method involves producing an intermediate shaped part that has a bulge adjacent to the critical region. This intermediate shape should have a generous entry radius into the die cavity, allowing more material to be drawn into the die cavity during drawing. The intermediate shaped part has substantially the same surface area as that of the final part shape. It is important to emphasize that an intermediate cavity shape is confined within a boundary of the final cavity, thus allowing the drawn preform shape blank to be positioned inside the final die cavity. According to the method, the drawing operation may be conducted using either a stamping die set or a single sided electrohydraulic forming die. The final forming operation can then be performed employing EHF.

A method of designing a part to be formed from a sheet metal blank is provided according to the following steps. The final part shape is defined in a computer-aided design model. It is analyzed using finite element analysis from the perspective of stamping the part in a one-step process. If the part cannot be stamped in one step from the candidate sheet metal material, the critical regions are identified in the final geometry where the material's forming limits are approached or exceeded. Below is an explanation how the intermediate preform shape can be identified. The intention is to locally reduce the strain applied to the blank by developing an intermediate part shape based upon the shape of the final part. The surface area of the intermediate part shape and the final part shape should be nearly the same. If it is appreciably smaller, a risk of cracks arises, and if it is appreciably larger, wrinkles become a problem. Optimal result will probably be attained if some relatively small stretching is allowed during final forming.

The key is in defining a preform part shape that is within the boundaries of the final die cavity. This step is accomplished by virtual reverse deformation - take the final shape backwards into the preform shape. Finite element analysis is used to determine the force distribution in the critical regions of the part which needs to be imposed in order to transform the final shape into the intermediate shape. The final part shape is deformed to develop the intermediate part 
shape, so that the surface area relationship of the intermediate part shape and the final part shape is maintained, and the intermediate shape is confined within the final die cavity.

The method of designing a two-step forming process includes designing a bulge into the preform that then provides material to fill a critical region, such as a sharp corner, in the final forming step. Development of the pre-form's shape is an iterative process. It is necessary to verify that the forming limits are not exceeded during the preforming step and then, again, in the final forming step, an actual die set is fabricated. For this verification step, an accurate finite element formulation should be employed. However, for the design step described above, simplified formulations of finite element method may be employed in order to conduct the described reverse deformation process. Clearly, the most straightforward approach is to use the full 3D or shell element formulations available in commercial codes. However, this approach is very labor intensive, since a large number of runs may be necessary to identify an appropriate preform shape. In order to address this issue, a simplified model was developed, which enabled the reverse deformation process to be efficiently modeled (within several minutes on a personal computer). The proposed simplified approach is described below.

The simplified model can be presented as a set of triangles with masses located in their vertices, while the ribs of the triangles form elastic springs. External force can be applied to each node in the mesh. The initial state of the system may be free of forces or have preexisting internal forces. Some of the nodes can be permanently or temporarily fixed or be restricted in certain displacements. Below is the explanation of the design sequence of the preforming shape enabling a more smooth distribution of strains through the blank volume.

The first step in the design process is to develop a numerical mesh of the final shape of the part. Computer-aided design (CAD) math data representing the surface of the part is loaded into a meshing software system. It can be done using Altair Hypermesh software.

The second step is to develop a strategy of what should be done with the final shape in order to transform it into a preform shape friendly for both preforming and final shape forming processes. Typical areas of major concern include the following: sharp radii at the entrance into the die cavity; deep cavity with sharp radius at the bottom; and the area of trihedral corners. Based on practical experience in the area of sheet metal forming, two major requirements to the preform shape can be formulated as: 1) it should have a rather smooth entry into the die cavity; 2) it should accumulate metal sufficient for further forming the final shape with rather moderate stretching as an additional pocket of metal. This can be accomplished as stretching the bottom of the final shape into rather smooth "hill" geometry. Obviously, special attention should be paid to filling the trihedral corners, where the most strain occurs.

The third step is to identify boundary conditions and to understand the mechanism of blank backward deformation which should lead to formation of the stamping-friendly preform shape. If the part has a symmetry plane, a symmetry line should be applied: the nodes originally belonging to the symmetry line always stay on the symmetry plane. Also, the final shape of the blank serves as a rigid surface which cannot be penetrated through during the blank backward 
deformation process. This requirement is defined by the necessity to position the blank after preforming into the final shape die. Even though the general trend is that the blank will move away from the final shape, some areas can move through this surface if non-penetration boundary condition is not applied. The desired mechanism of blank deformation during its backward motion is that the sharp entry radii would become much larger, the sharp corner at the bottom of the cavity would become much more generous and smooth; the material coming out of sharp corners would move into the "hill" - material pocket formed in the center of the bottom area and on the sides.

The fourth step is the most critical and labor intensive: it requires identifying the appropriate distribution of external forces that would deform the blank with applied boundary conditions into a smooth, stamping-friendly preform shape. This step is typically conducted through a number of iterations, since the distribution of forces is unknown at the beginning and is being identified through numerical experiments.

The fifth step is in verifying whether the obtained preform shape can be formed from the flat sheet. In a production environment such a preforming step can be conducted as one operation by having either a three-action press or by having nitrogen cylinders to be mounted inside the tool on a double action press. The first action of the press should be dedicated to creating the binder force, the second action would be applied to the drawing punch, while the third action would be forming the "hill" on the bottom of the drawn preform.

The sixth step is to verify whether the final shape part can be formed with electrohydraulic forming technology. This verification step can be conducted using the numerical model of the electrohydraulic forming process. However, it can be accomplished using the simplified approach employing the LS-DYNA commercial code and validating that the maximum strains do not exceed the material formability level available for electrohydraulic forming.

An example of benefits of the developed two step-forming process vs. a single-step process is illustrated in Figure 1.2.1. As a result of the two-step forming process, the maximum true strain level was lowered from 1.2 (splitting) to 0.37. 


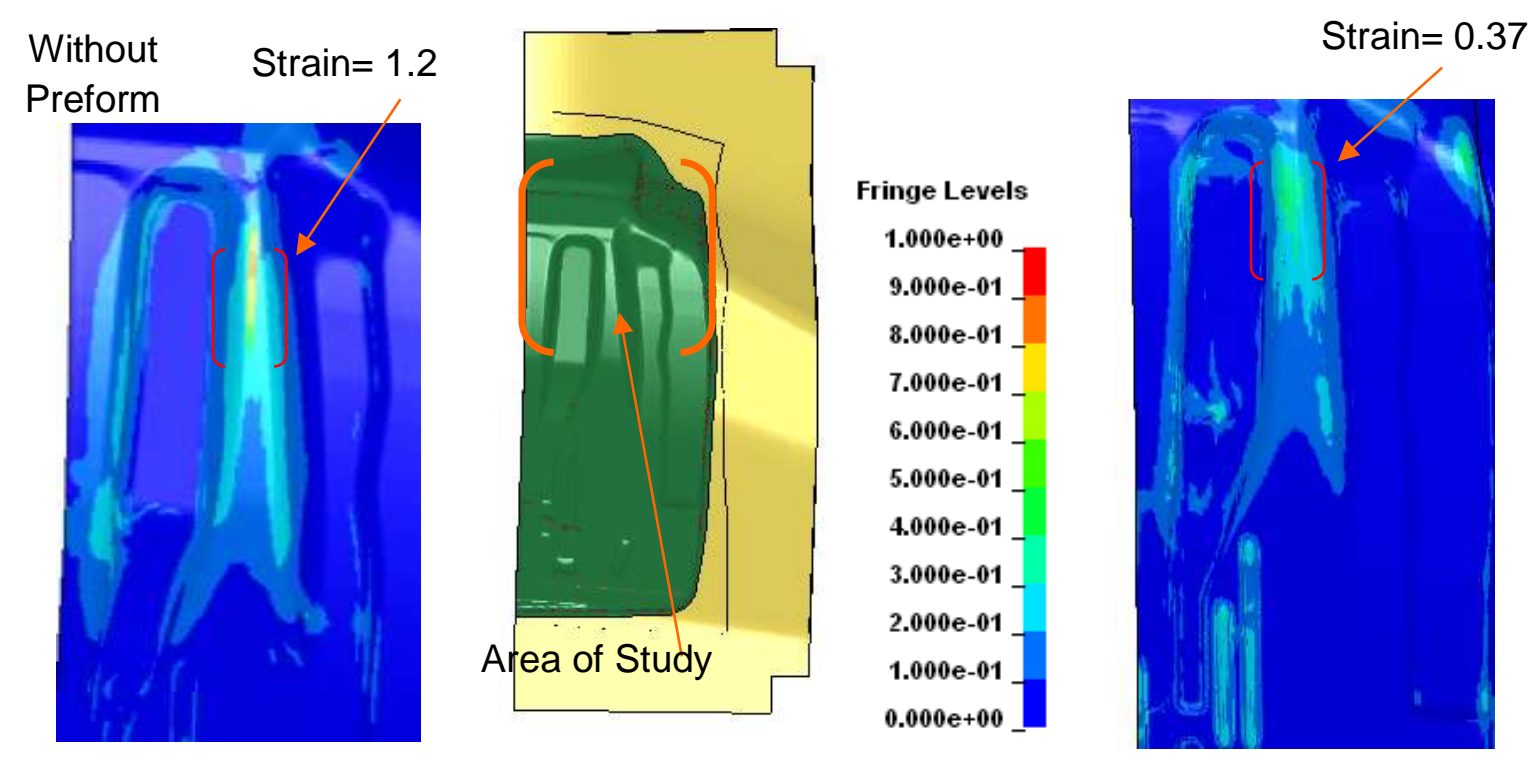

Figure 1.2.1. Comparison of modeling results of one-step (left) and two-step (right) forming processes.

\section{Sub-task 1.3. Numerical model of the blank formation and die loading process}

\section{Initial Finite Element Modeling}

The finite element analysis of loads applied to the die during the EHF process was developed with the analysis being done within LS DYNA. Finite element modeling is a first step in this analysis, the output of which is used as an input in later steps such as fatigue analysis. The elastically deformable die was meshed with 3D solid tetrahedron elements. Other tool parts, such as blank holder, are simulated as rigid bodies. The blank is simulated with plastically deformable shell elements to which we apply a pressure pulse. The die is modeled with one integration point, four-node tetrahedron 3D solid elements (element formulation ELFORM=10) and elastic material (MAT_001). The blank is modeled with fully integrated shell elements (element formulation ELFORM=16) with 5 integration points through the thickness and transversely anisotropic elastic-plastic material model (MAT_037). Tool parts excluding the die are modeled with shell elements that are turned into rigid bodies by assigning them the rigid material model (MAT_020).

A standard penalty-based formulation of the contact algorithm was initially used (SOFT=0). In this contact, if a slave node penetrates through the master surface, a force is applied to it with magnitude proportional to the amount of penetration and to a stiffness factor $[k]$, where $k$ is:

$$
k=\frac{f_{s} K A^{2}}{V},
$$


for 3D solid elements, and

$$
k=\frac{f_{s} K A}{\max (\text { shell diagonal })},
$$

for shell elements, where $f_{s}$ is a scale factor (normally defaulted to 0.1 ), $K$ is the bulk modulus, $V$ is the volume and $A$ is the area of the master element that contains a segment through which penetration occurred.

The contact force is applied along the normal of the master surface and points outward of the body that is being penetrated. Besides the contact force a friction force is applied in a direction opposite to the tangent velocity if bodies in contact slide along each other. The friction in LSDYNA is based on the Coulomb model where the friction force $F$ is proportional to the normal force $f_{n}$ and the friction coefficient:

$$
F=\mu\left|f_{n}\right|
$$

In the utilized algorithm, we take into account the shell thickness for the blank by setting SHLTHK option to 1 . The same setting ignores the shell thickness of rigid bodies. It should be indicated that we use the one-way implementation for the contact of the blank and any rigid bodies and the two-way for the contact interface between the blank and the die. One-way implementation performs one pass through contact pairs in which it checks penetration of slave nodes through the master surface. The two-way variant, on the other hand, performs two passes where the first pass is the same as for the one-way version, and the second pass does the opposite and checks for penetration of master nodes against the slave surface. In our simulation we follow conventional recommendations and set the blank as slave and the die and other tool surfaces as master, but it only matters in the one-way implementation. In two-way implementation the choice is arbitrary. 


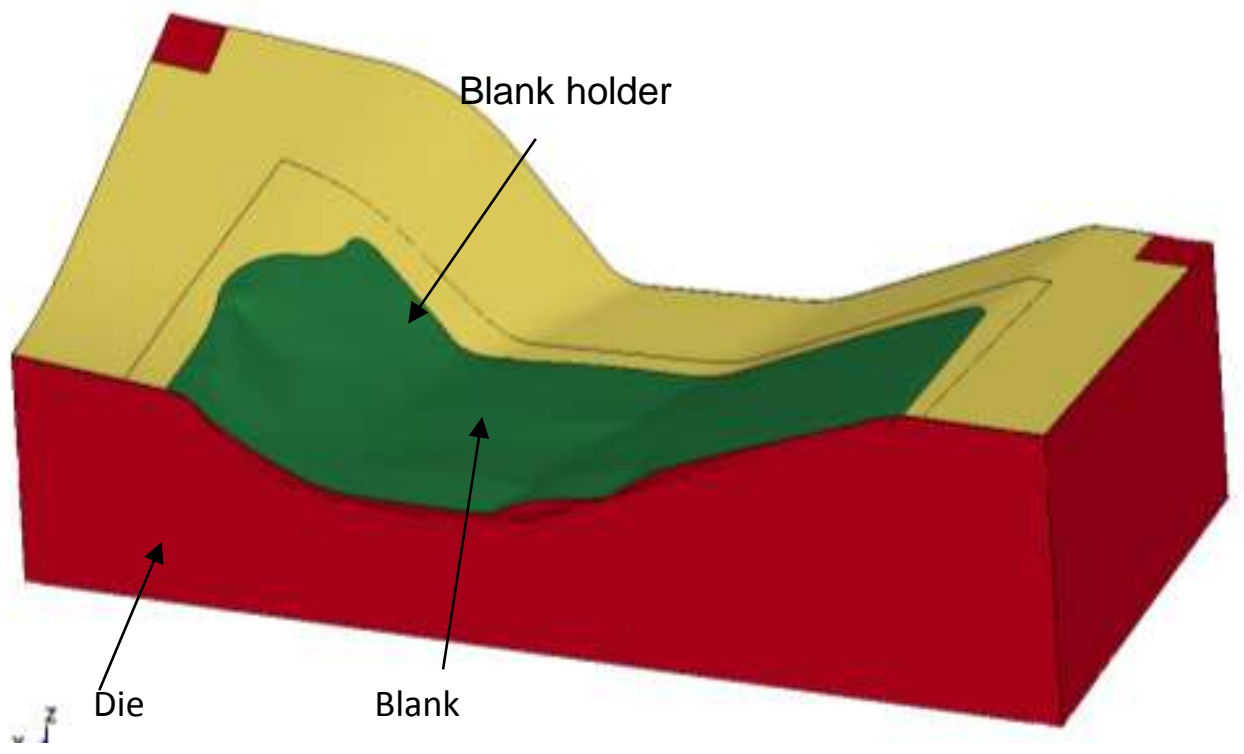

Figure 1.3.2. A simulation of the $\mathrm{EH}$ forming into an elastically deformable die.

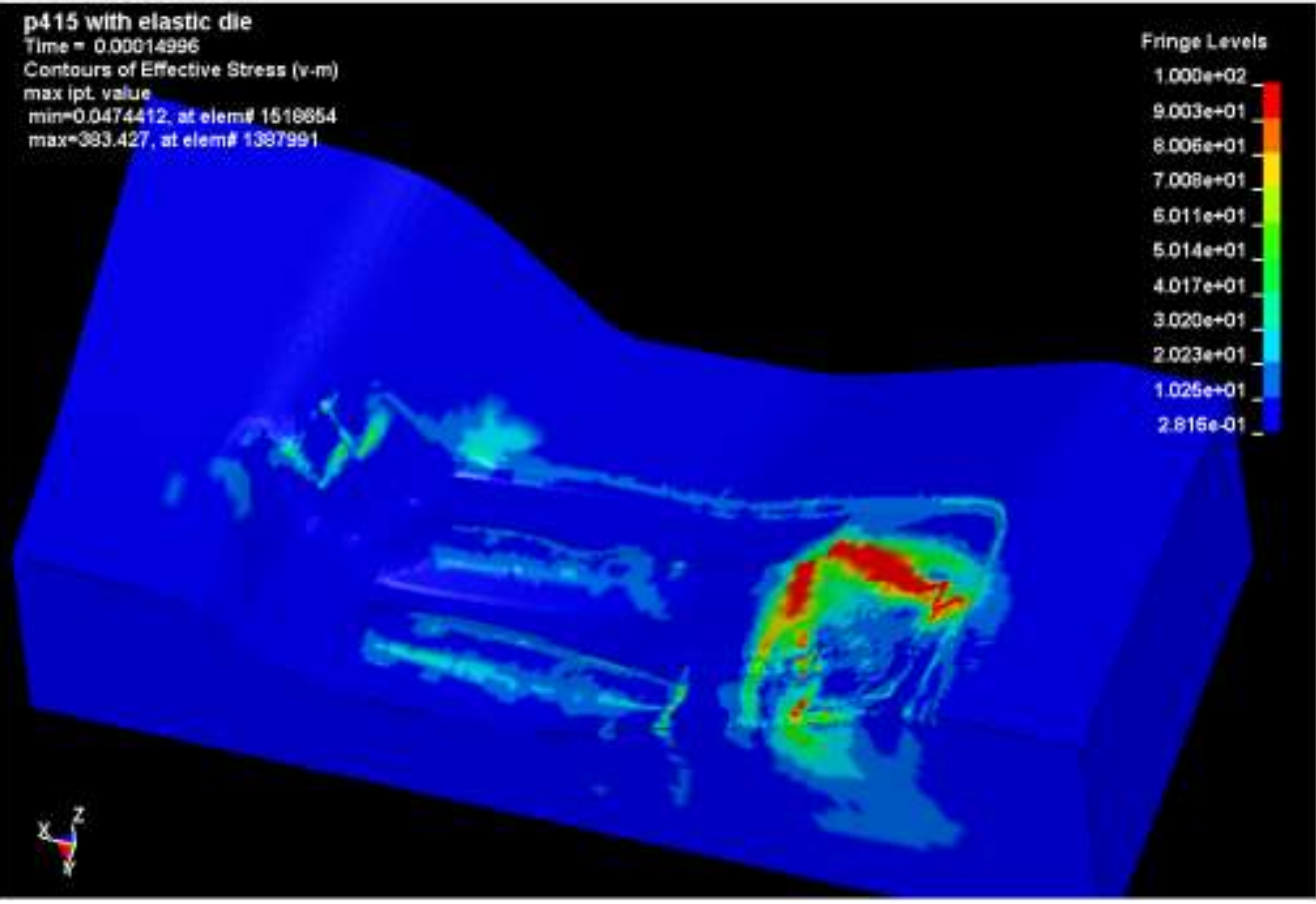

Figure 1.3.3. Von Mises stress (MPa) distribution in the die. 


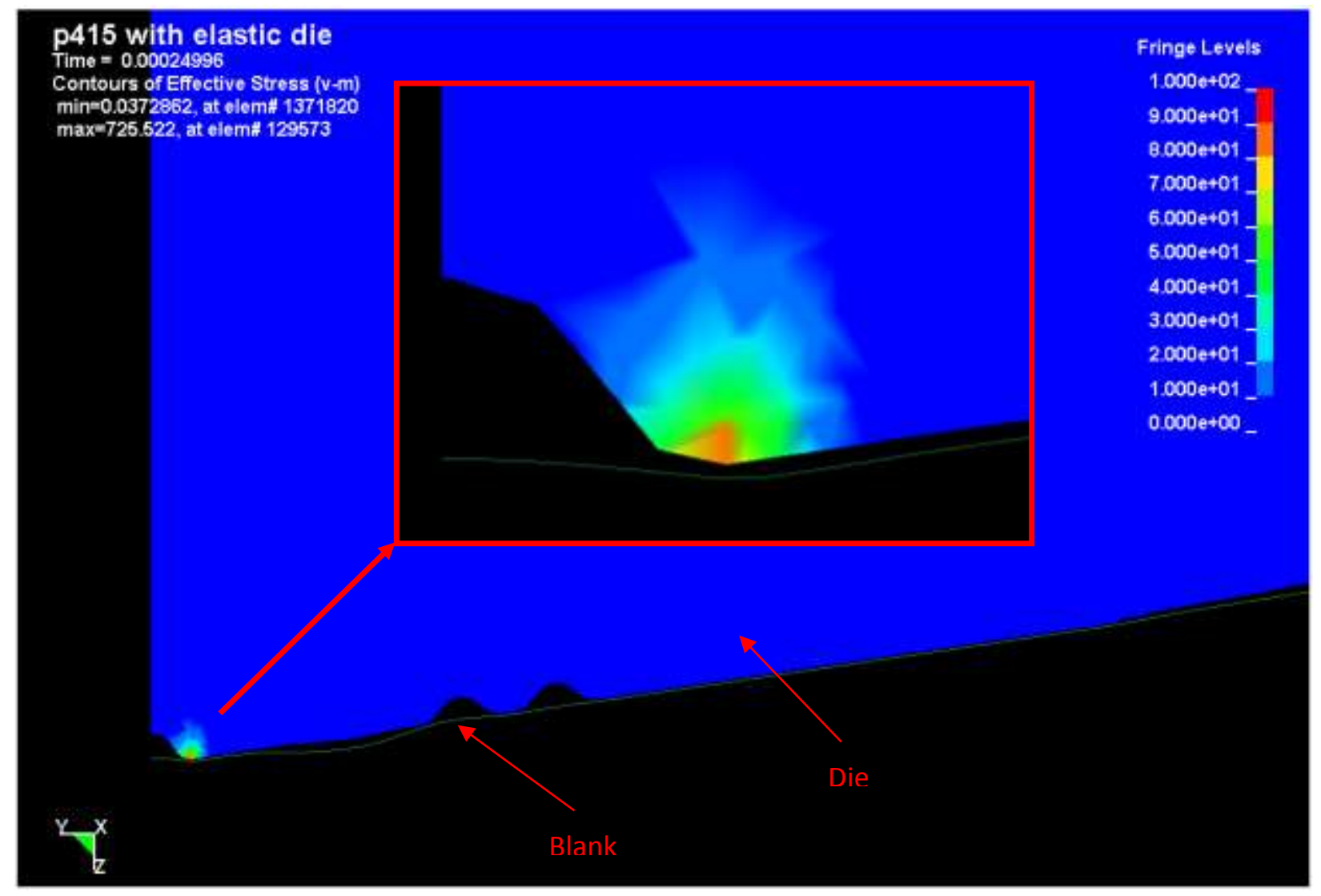

Figure 1.3.4. Von Mises stress (MPa) contour. Die cross-section through the highly loaded element at the moment of impact.

In Fig 1.3.2 the deformable die, shown in red, was meshed with tetra elements. The blank, shown in green, was meshed with shell elements and went through a gravity load and a binder closing step before the current simulation. The blank holder, shown in yellow, is a rigid body part that clamps the blank against the die. After the pressure pulse is applied to the blank, the blank accelerates and impacts the die. The Von Mises stress distribution for one of the time steps is shown in Figure 1.3.3.

In Figure 1.3.4 a die cross-section is shown through the highly loaded element at the moment of impact. This picture demonstrates that stresses are local and do not propagate very deep. Since different cross-sections look very similar to the one shown in Figure 1.3.4, it can be assumed that a surface treatment might work well in this situation and that there is no need for high strength material through the die thickness.

\section{Development of recommendations for die material for the EHF process.}

Review of potential failure modes for EHF tools in the context of tool material selection and design has been performed. The following failure modes were identified: chipping; wear (abrasive and adhesive) and galling; plastic deformation; cracking and mechanical fatigue. The EHF processes, especially when applied to forming of AHSS, are associated with: 
- High relative displacements of formed material at high velocities at contact with tool surface;

- High contact pressures;

- High speed and high energy impact of formed material against tool surface promoting local plastic deformation of the tool;

- Presence of corrosive media;

- Cyclic nature (several hundred thousand cycles of tool loading and unloading);

- Presence of hard abrasive inclusions in formed sheet material (martensite, bainite, carbides, etc.);

- Need for economically affordable tooling solution.

In order to understand the limits of tool material capabilities, rather severe conditions were created for their testing. The $38^{\circ}$ plane strain V-shaped insert was employed for testing die materials. Simulation shows that the blank impacts the die with velocity in excess of $300 \mathrm{~m} / \mathrm{s}$ near the vertex of the V-shape for the $1 \mathrm{~mm}$ DP980 blank material. Fatigue analysis of this die reveals severe damage at the bottom of the cavity with expectation of crack initiation after only the first few cycles. The stress distribution in the insert at the end of the EHF process is shown in Figure 1.3.5.Error! Reference source not found.1.3.6 shows cracks observed in the tool (case hardened 8620 steel with UTS $=1100 \mathrm{MPa}$ and Yield Stress $=775 \mathrm{MPa}$ ) after 32 discharges out of which only 5 used $1 \mathrm{~mm}$ DP980, and other 27 used thinner gages of lower strength alloys.

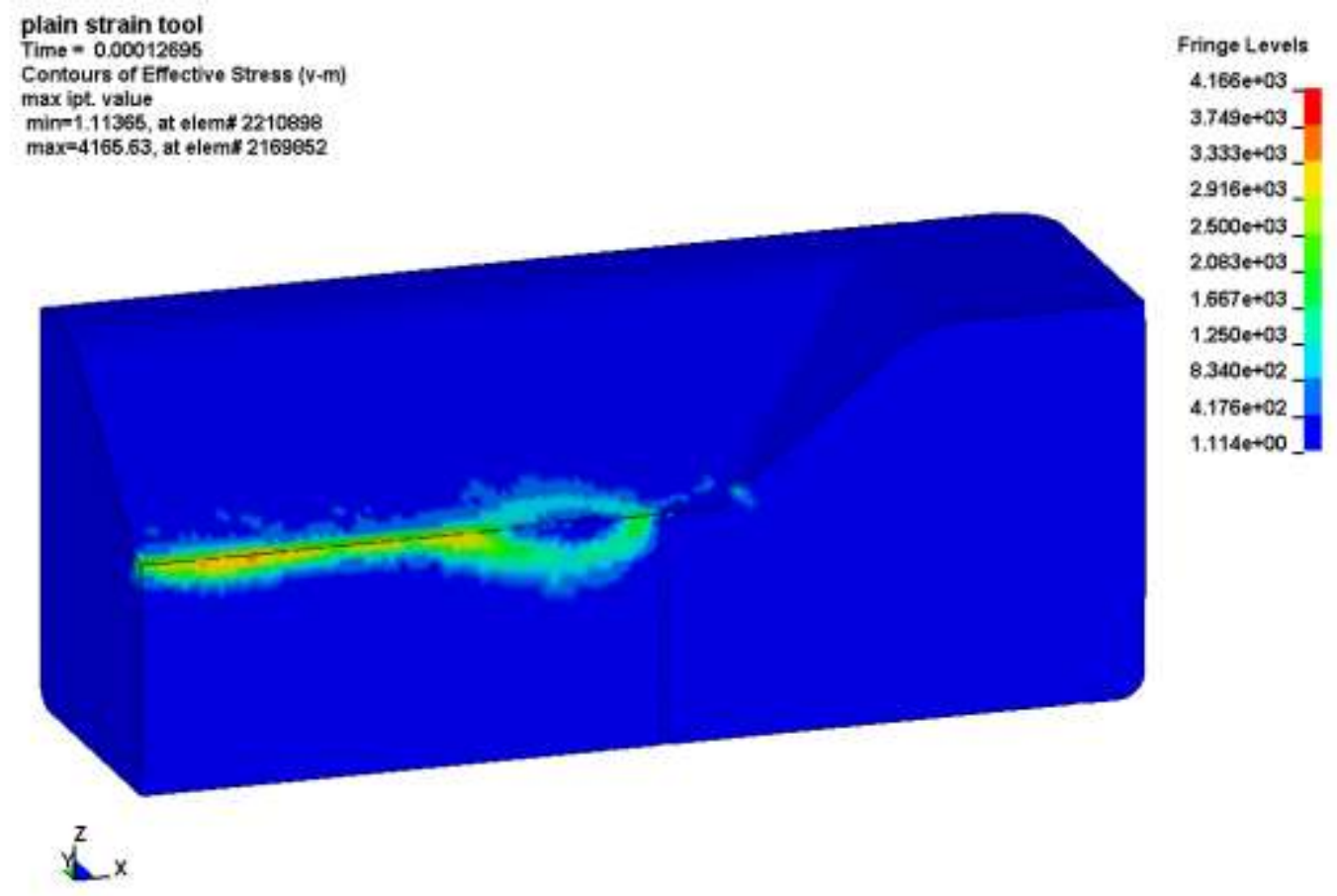

Figure 1.3.5. Distribution of effective stress in $38^{\circ}$ plane strain insert for EHF of $1 \mathrm{~mm}$ DP980 blank material. 


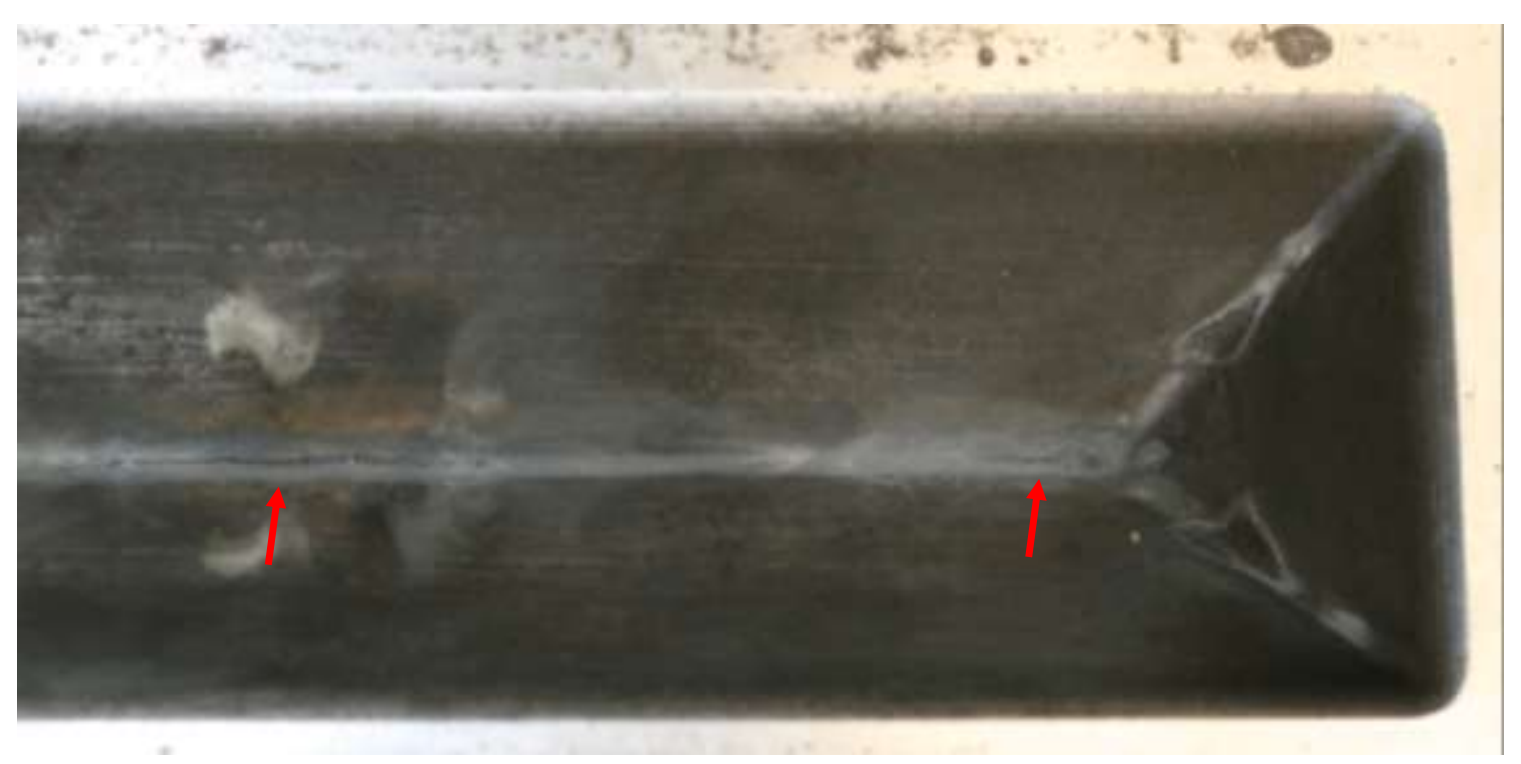

Figure 1.3.6. Cracks in $38^{\circ}$ plane strain insert.

Analysis of the accuracy of the loads on the die in the EHF process

After examining the results of numerical simulations, it was found that the contact algorithm and settings commonly used for metal forming simulations within LS-DYNA do not perform well in all $\mathrm{EH}$ forming simulations. Problems were noticed in cases where high speed impact $(\mathrm{V}>100$ $\mathrm{m} / \mathrm{s}$ ) was observed. These problems included significant penetration at the contact interface, abnormally high or low contact stresses, and significant dependency of contact stresses on the contacting bodies' mesh size and quality. To investigate this problem and find a possible solution, different contact algorithms and settings were tried in a simple simulation of two elastic square bars in a dynamic impact problem illustrated in Figure 1.3.7. The size of each bar was $10 \times 10 \times 100 \mathrm{~mm}$. The left bar had an initial velocity of $0 \mathrm{~m} / \mathrm{s}$ and the right bar had initial velocity of $100 \mathrm{~m} / \mathrm{s}$. The bars were meshed with different size (or the same size in some cases) hexahedron or tetrahedron (tetra) solid elements. In the case of hexahedrons, all element edges were the same length, forming cube elements with possible sizes of 1,2, and $2.5 \mathrm{~mm}$. Tetra mesh was obtained by splitting each hexahedron into 6 tetrahedrons. In all cases non automatic surface-to-surface contact was used ( ${ }^{*}$ CONTACT_SURFACE_TO_SURFACE card).

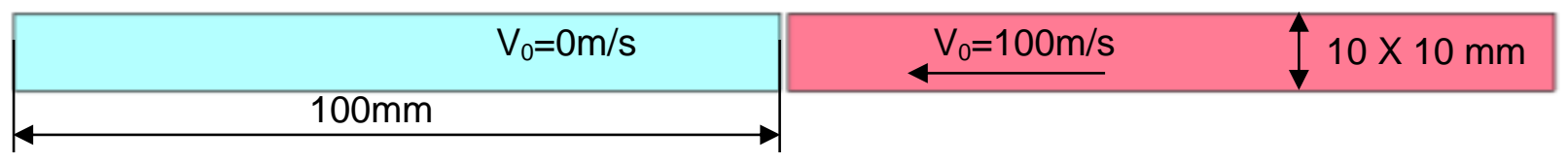

Figure 1.3.7. The model for contact algorithms evaluation in LS-DYNA. 
The contact stress in this case can be found by using the following equation:

$$
\sigma=\frac{\rho c v}{2},
$$

where $\rho$ is the material density, $c$ is the speed of sound in the bar material (for steel it is approximately $5000 \mathrm{~m} / \mathrm{s}$ ), and $v$ is the impact velocity. According to equation 1.3.1, for the steel bars and $100 \mathrm{~m} / \mathrm{s}$ impact velocity, the contact stress is about $2 \mathrm{GPa}$. The contact pressure reaches $2 \mathrm{GPa}$ immediately after the impact and stays unchanged for a period of time. The length of this period is determined by the time that it takes for the sound wave to travel from the impacted end of the bar to the opposite end and return back.

In addition to bar-to-bar impact numerical experiments reported above, sheet plate to bar impact simulations have also been performed. The setup of this simulation is shown in Figure 1.3.8.

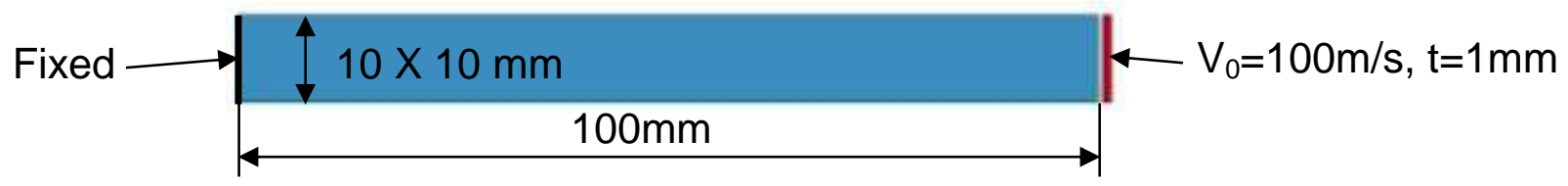

Figure 1.3.8. Sheet plate to bar numerical simulation model.

The sheet metal plate was chosen as a striker to more closely simulate conditions observed in the die impacted by the sheet metal blank. It can be seen that stiffness scale coefficient (SLSFAC) and mesh quality have a visible effect on the contact stress. Additionally, the influence of the penalty stiffness value option (PENOPT) and the constraint contact approach were studied for a $3 \mathrm{D}$ plane strain $34^{\circ} \mathrm{V}$-shaped die simulation shown in Figure 1.3.9.
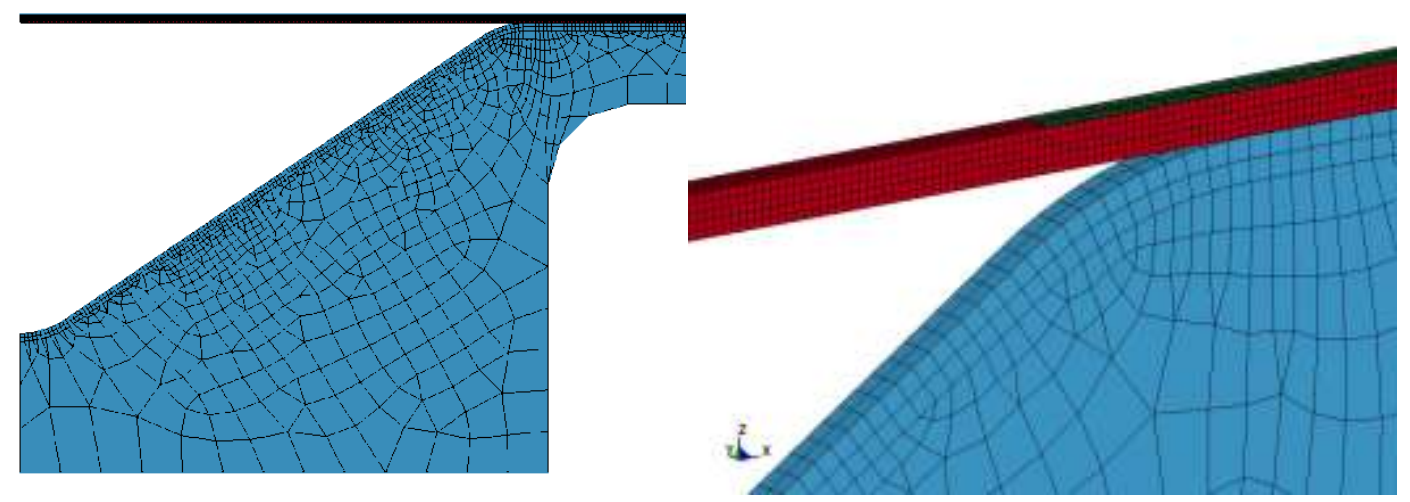

Figure 1.3.9. 3D plain strain $34^{\circ} \mathrm{V}$-shaped die simulation. 
It can be concluded that neither the PENOPT option nor the constraint contact can reduce the mesh size dependency of the contact pressure during the impact of the blank into the die. More work with LSTC, creator of LS-DYNA, was conducted to find a solution to this problem.

Some interesting effects have been noticed during deformation of the blank under a high speed pulsed pressure load. It was observed that the contact propagates in a wave-like fashion with plastic bending in entrance and exit zones as shown in Figure 1.3.10.

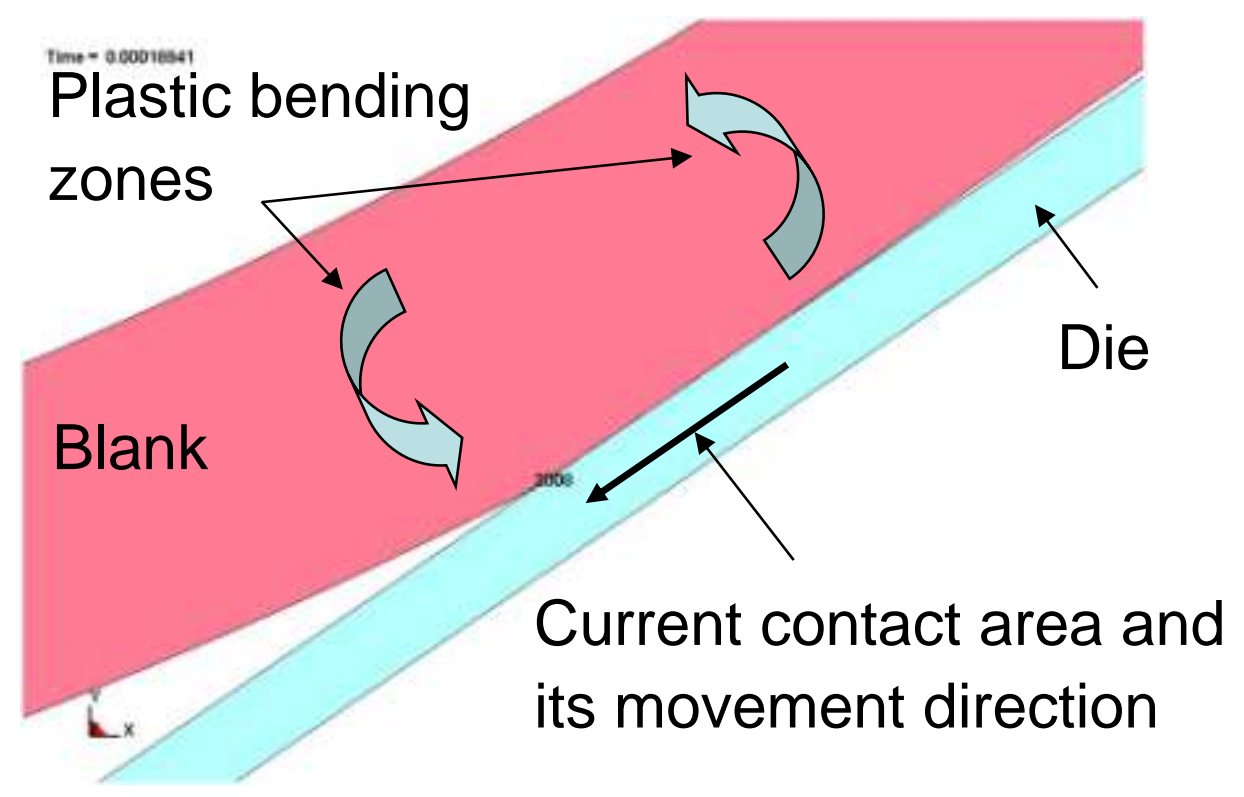

Figure 1.3.10. Development of contact between the blank and the die.

Obviously this effect does not occur if the blank moves perpendicularly to the die surface which rarely takes place. It also does not occur in the vicinity of symmetry axis or symmetry planes. One important consequence of the wave-like contact propagation is a significant reduction of the impact speeds. A graph in Figure 1.3.11 plots normal to the die surface component of velocity of some arbitrarily chosen node of the blank in the $34^{\circ}$ conical die $2 \mathrm{D}$ axisymmetrical simulation. The circle on the graph marks the moment of the impact. It can be seen that the node is decelerated at a very high rate shortly before the impact. 


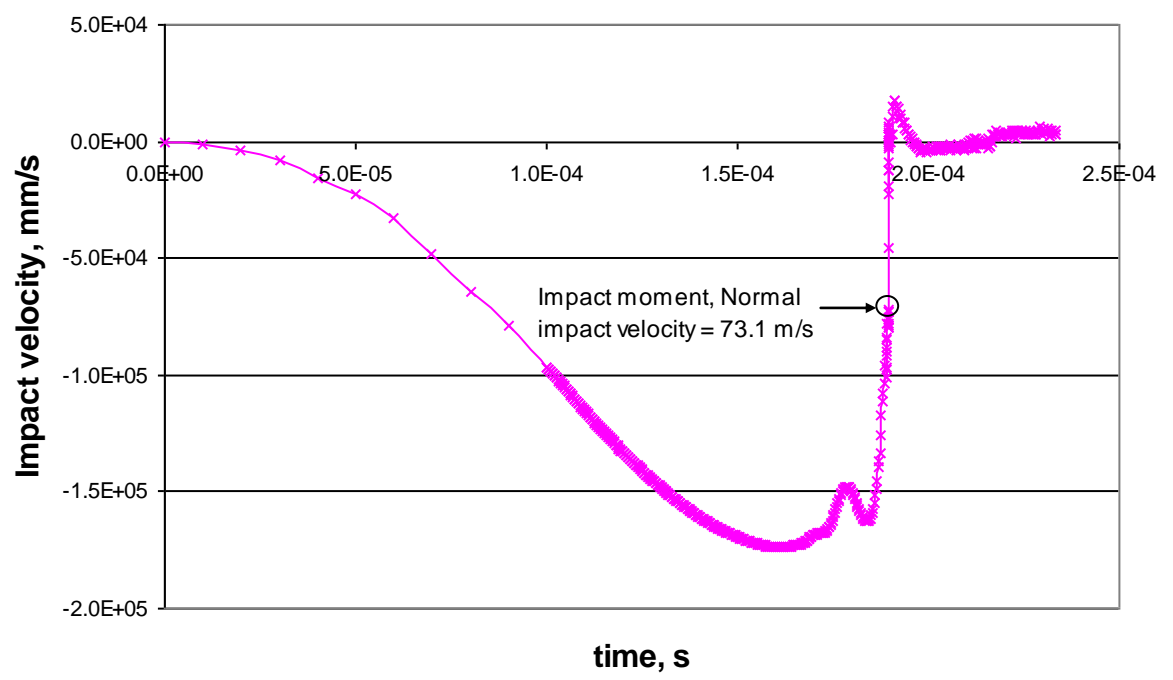

Figure 1.3.11. Normal to the die surface component of velocity of a typical blank node.

The wave-like contact propagation effect emphasizes the importance of impact velocity estimation. To be more specific, we need to estimate the normal component of the blank velocity relative to the die at each point at the moment of impact. Knowing the impact speeds distribution would allow quick identification of the critical areas on the die. Since no required functionality has been found in existing post-processors, a new code that works with LSPREPOST, pre- and post-processor for LS-DYNA created by LSTC, has been created. The program calculates maximum normal impact velocity seen by each surface element of the die throughout all loading stages and saves the result in an ASCII file which can be read and fringed by LS-PREPOST. The program bases its calculations on the blank nodal velocities and separation vectors calculated by LS-PREPOST, and currently only supports shell elements of the blank.

To assess the mesh effect on the contact stress a bar-to-bar impact was further investigated. The elements at the same location on the contact surfaces (both the master and slave sides) were selected. Five types of mesh were investigated. Two critical locations were considered: the central elements and the side elements on the contact surface. To indicate the mesh effect on the contact stresses, the average contact stresses (minimum principal stress) were calculated and analyzed. Results show that the calculated average stresses in all of the mesh types except $2.5 \mathrm{~mm}$ by $2.5 \mathrm{~mm}$ brick elements, correspond to the theoretical value of $2000 \mathrm{MPa}$. It is also noted that the oscillation of the stress curve is in a reasonable range, with an average of 2000 MPa. In all the meshes, the mesh of $1 \mathrm{~mm}$ by $1 \mathrm{~mm}$ has showed the most stable results with a smallest oscillation of the stress. The simulation also showed a larger variation of the stress values for the tetrahedral mesh compared to the brick mesh, which went through investigation with LSTC, the makers of LS-DYNA. 
Furthermore, two parameters were investigated, namely VDC (viscous damping coefficient) and bulk viscosity (Q1 and Q2). The parameters can be changed in KEYWORDS in LS-DYNA: (i) ${ }^{*}$ CONTACT_SURFACE_TO_SURFACE, and (ii) ${ }^{*}$ CONTROL_BULK_VISCOSITY.

For VDC, a range of numbers was tested: $1,10,20,30,40$, and 80 . It indicated that the calculations did not run properly if VDC $>30$, and negative volumes were generated in solid elements. So two more tests were run with VDC $=22$ and 26 (between 20 and 30 ). The results show that the best VDC value should be between 20 and 26 .

Identifying the recommended parameters for impact numerical simulations

An elastic bar to rigid wall impact was simulated. The analytical contact stress value is $\sigma=\rho c v$, where $\rho$ is the material density, $c$ is the elastic wave speed in material of the bar, and $v$ is the impact velocity taken as $100 \mathrm{~m} / \mathrm{s}$ here. The bar is meshed with solid elements of $0.27 \mathrm{~mm}$ sides. The simulation shows values in agreement with the analytical solution. The elastic wave stress is the same on the impacting surface and at the free lateral surface where the strain values are close and limited in both locations to follow the linear elastic law.

Figures 1.3.12 through 1.3.14 describe an elastoplastic bar to rigid wall impact. The simulation shows that the reported values are as expected; the flat impact on the surface results in a plastic wave with a stress value of $4 \mathrm{GPa}$. At an element on the free surface the stress is approaching the yield stress of the elastoplastic steel used (335.1 MPa). This behavior of elastic and plastic wave stresses was later traced and confirmed in V-die impact upon choosing the correct contact parameters and other controlling factors in the FEA simulations. Other factors like strain hardening and strain rate effects will actually control the yield strength in the real test material and the free-to-deform elements will show the new yield strength values.

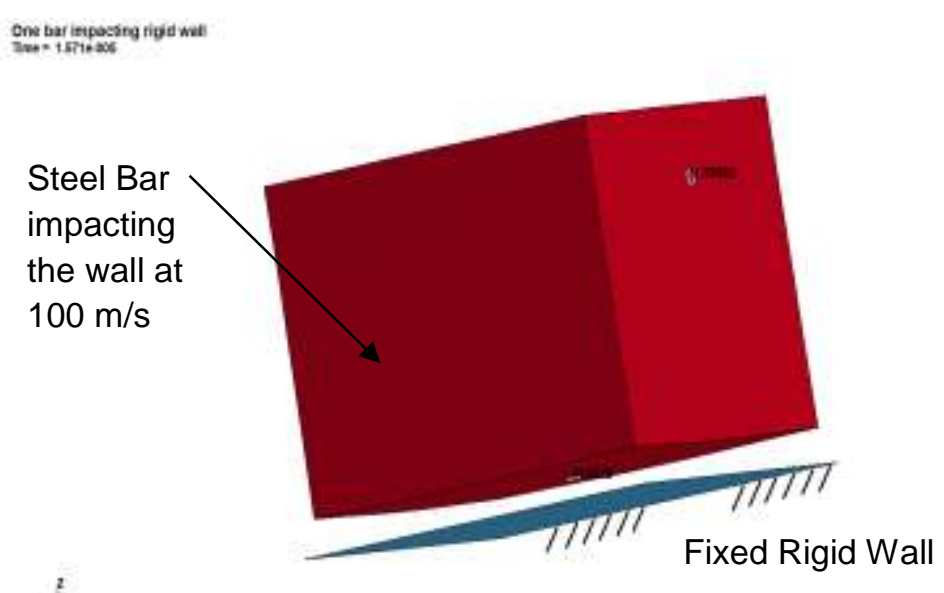

Figure 1.3.12. Bar impact into a rigid wall 


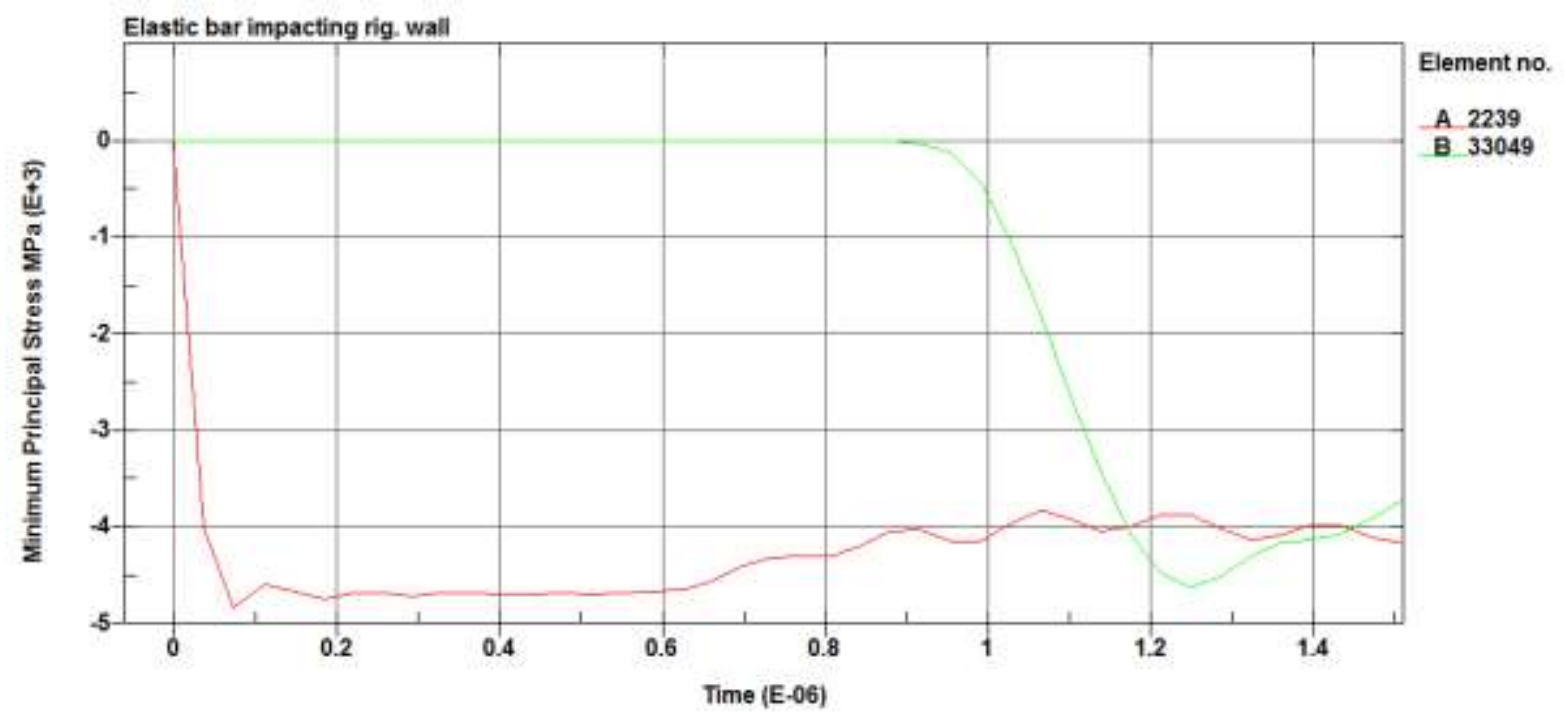

Figure 1.3.13. Stress values in elastic bar impacting a rigid wall: red line - stress in the center of the contact surface; green line - stress on the side surface of the bar. See elements in Figure 1.3.12.

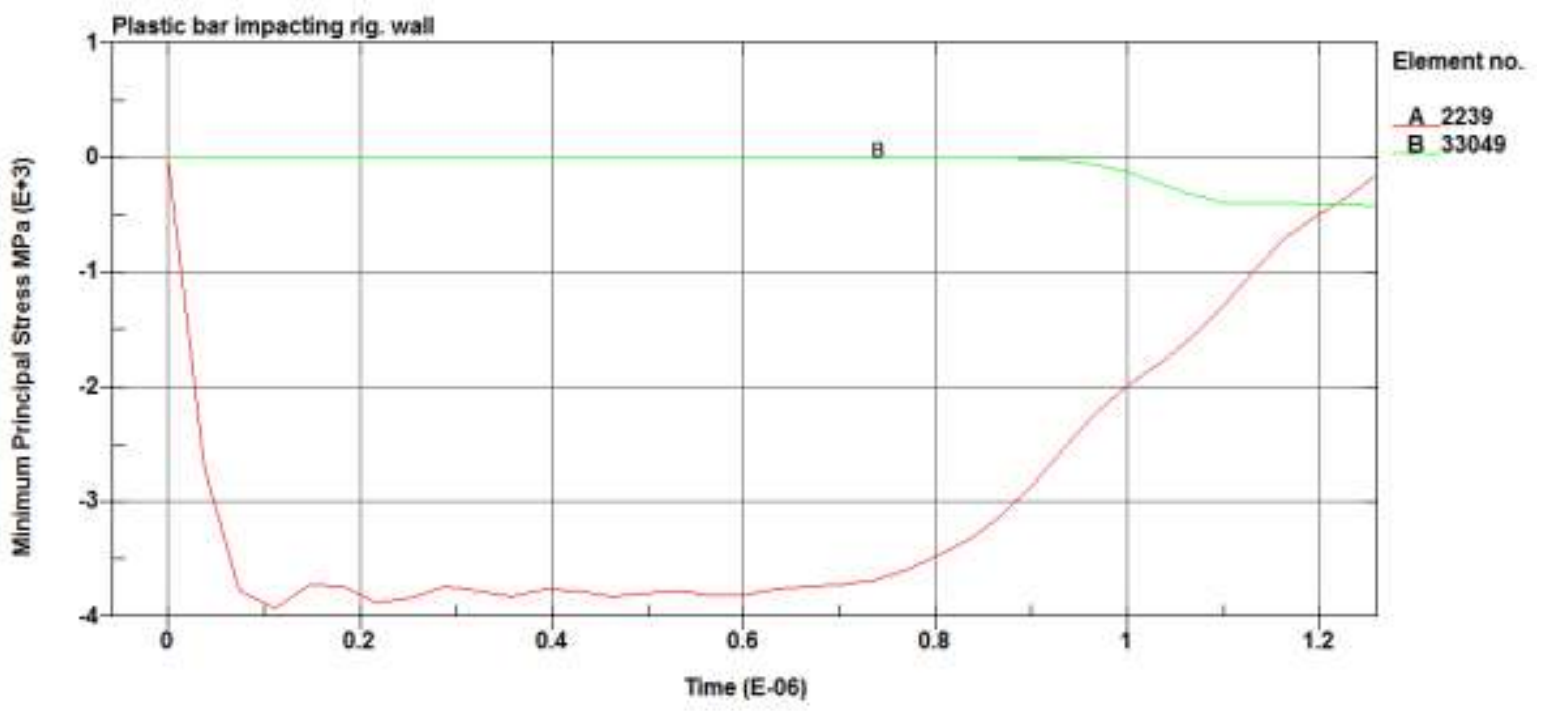

Figure 1.3.14. Stress values in elastoplastic bar impacting a rigid wall: red line - stress in the center of the contact surface; green line - stress on the bar's side surface. See elements in Figure 1.3.12.

Previous studies on the wave propagation shows that in a code such as LS-DYNA, which belongs to a family of 'Hydro' codes, bulk viscosity is essential to treat shocks. Generally, the LS-DYNA program has the capability (performed by default) to automatically detect the shocks and treat them by adding a pressure term that is based on the element dimension, density, strain-rate and some user-defined coefficients (Q1 and Q2). The current simulations modeled 
recommended that the input card *CONTROL_BULK_VISCOSITY be used. Figures 1.3 .15 and 1.3.16 show how a very stable contact has been established in an elastic bar to bar impact. This stable contact provided a ground for the contact to be used in the targeted V-die simulation and actually showed very reliable behavior. No mesh sensitivity is noticed, and contact stresses are the same in both bars. Penetration was minimized and solution stability enhanced. Stress value is in agreement with the analytical solution of $\sigma=1 / 2 \rho \mathrm{cv}$.

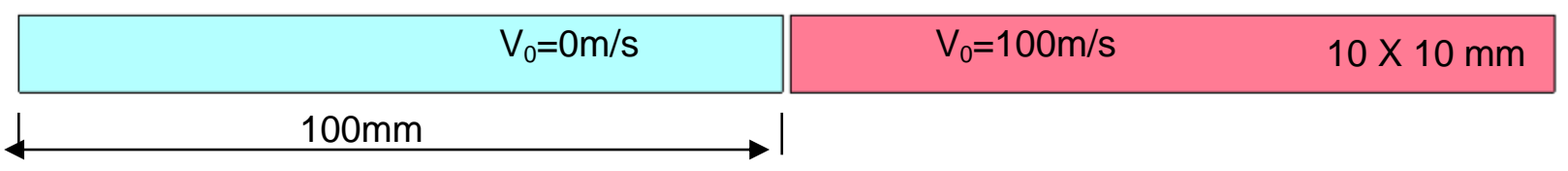

Figure 1.3.15 Bar-to-bar impact schematic.
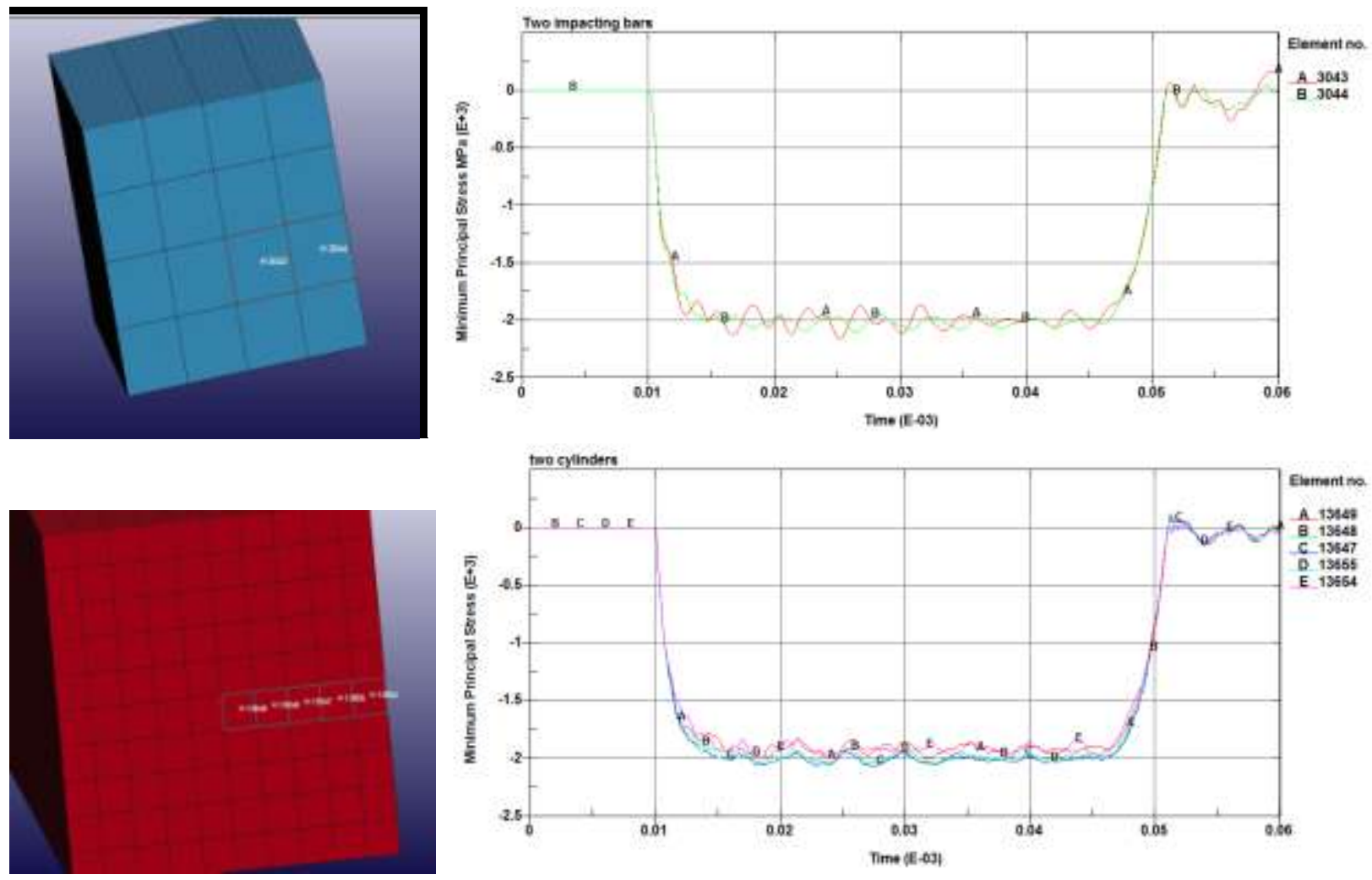

Figure 1.3.16. Two impacting elastic bars, both meshed with solid elements and different size mesh. $\underline{\mathrm{V} \text {-die Simulation in EHF process }}$ 
As a continuation on die failure analysis in the high speed process of electrohydraulic forming, contact stresses between the die and blank were thoroughly investigated. This analysis is necessary with regards to die durability, manufacturing reliability, quality control, process capabilities and safety. The die used in this study was a $38^{\circ} \mathrm{V}$-shaped channel die that produces plane strain loading conditions in the blanks that it forms. A finite element predictive model was created to accurately describe stresses affecting the die during the EHF process.

The recommended parameters from the previous numerical simulations, along with the physical behavior of blank and die during elastic and plastic waves, were then utilized in the V-die impact simulation in order to prove correctness and repeatability of stresses in the die. A $38^{\circ} \mathrm{V}$-die is used in an EHF simulation to form a blank of DP-500. Different mesh sizes of the blank were used. The solution was numerically stable. Only minimized penetration was observed with reference to previously reported results. The max stress in the die never exceeded the max physical ceiling of $\sigma=1 / 2 \rho c v$ with the max values toward the $V$ corner. Since the blank is impacting the die in a "kink" mode, the flat impact was observed only in the $V$ corner of the die where the contact stress is substantially higher than in other areas of contact. The results of the simulation are shown in Figures 1.3.17 and 1.3.18.

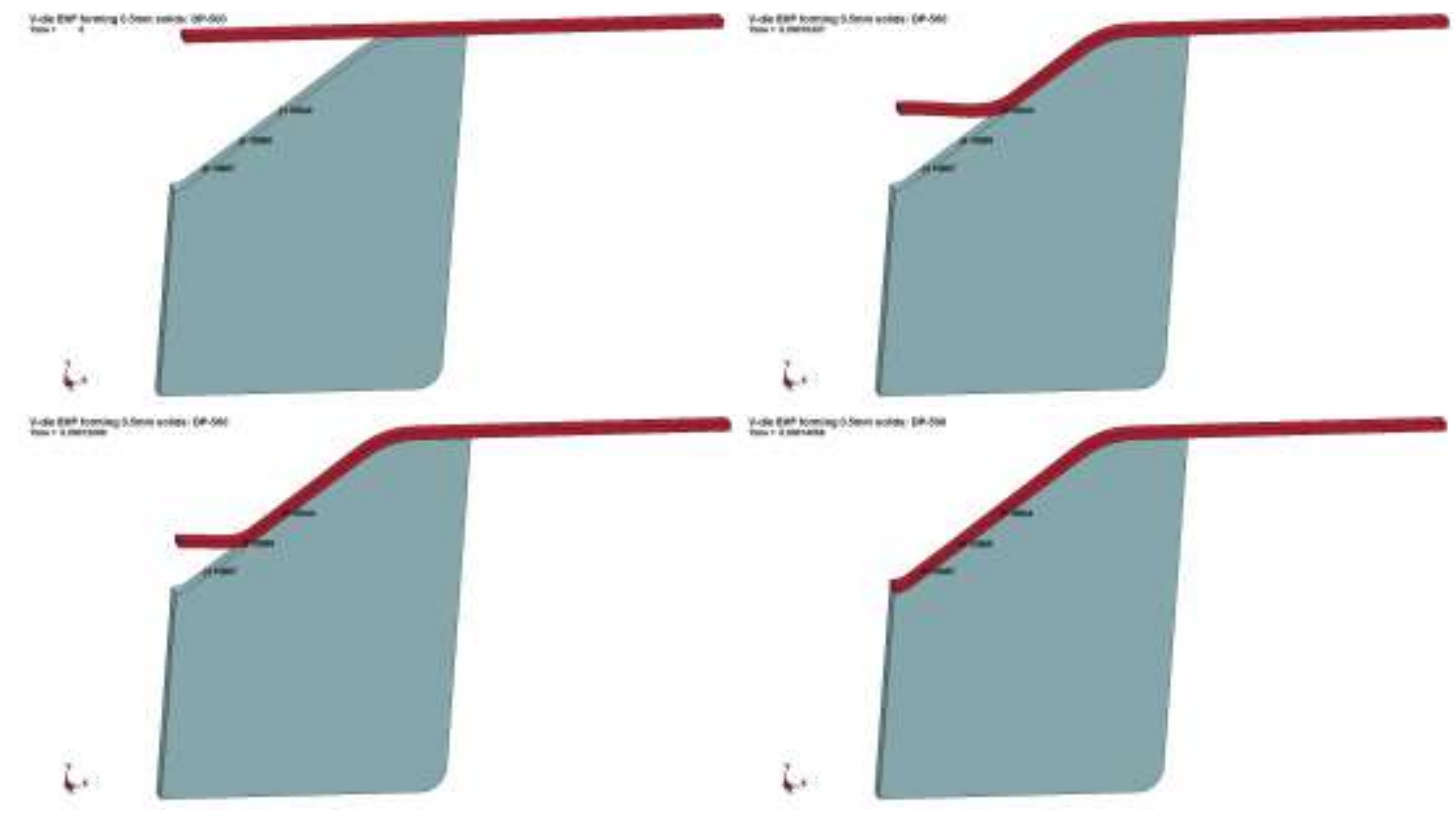

Figure 1.3.17. Simulation of electrohydraulic forming of a DP-500 blank into a V-shape die. 


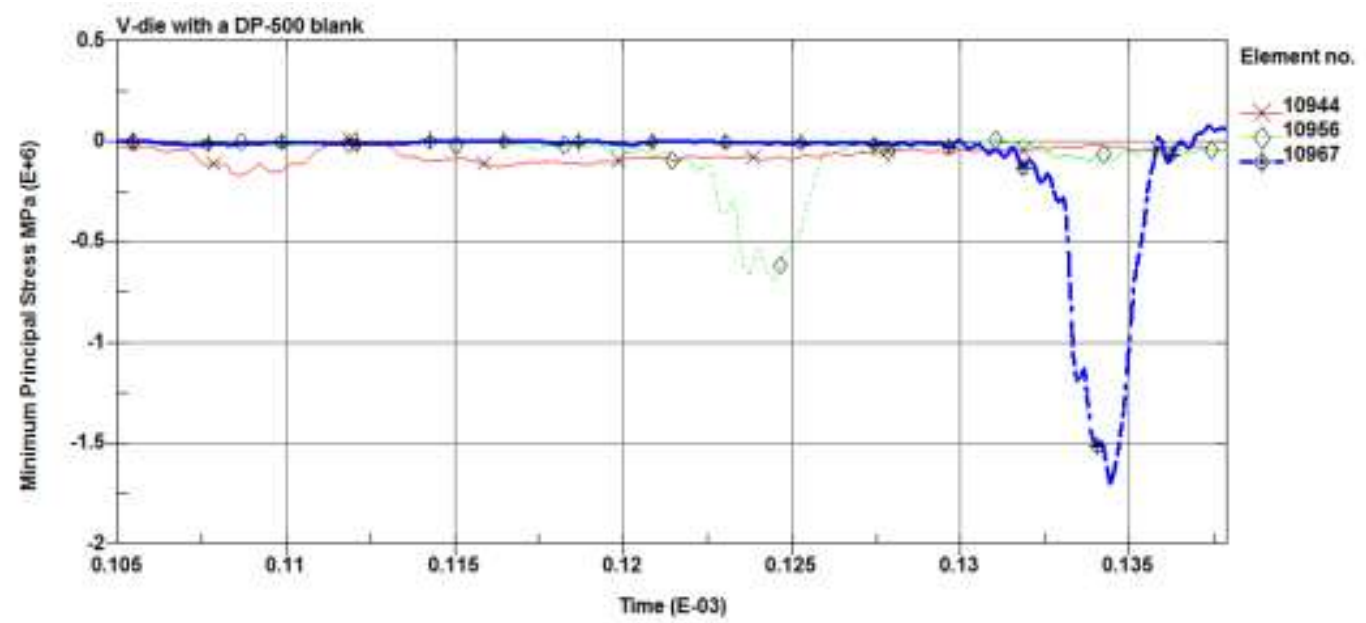

Figure 1.3.18. Stresses in the elements shown in Figure 1.3.17 along the contact surface in the V-die during EHF.

Fracture of the experimental V-shape die is shown in Figure 1.3.19 in the corner of the die. This failure mode was confirmed for all four investigated die materials (see Task 4).
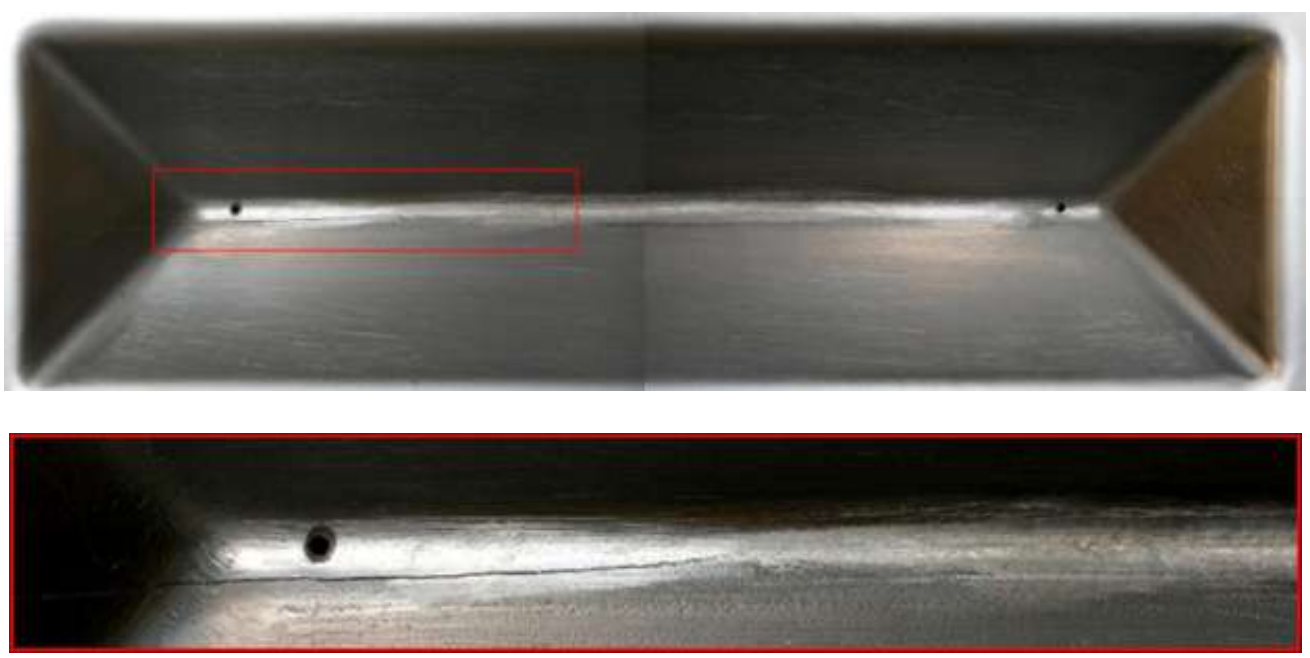

Figure 1.3.19. View of tool steel B V-shape die after a series of EHF forming cycles.

Additional work was done on refining the methodology of numerical modelling of contact stresses during pulsed forming of sheet metal blanks into the die cavity. These contact stresses define the choice of the die material and, therefore, are ultimately important. 
In the following simulations, A $38^{\circ} \mathrm{V}$-shaped die was chosen for contact stress prediction. Only a narrow section was simulated to reduce the computation time, with a symmetry plane condition imposed on both sides of the section. To further reduce the simulation time, the symmetry of the tool was taken into account and only the right hand side was included in simulation. The bottom nodes of the die were fixed as well as the right most nodes of the blank. The latter was done to represent the locking condition. The binder was modeled with rigid shell elements and was fixed in space with a small clearance above the blank. The die was modeled with elastic material and the blank was modeled with elastic-plastic material.

Figure 1.3.20 below demonstrates the quality of the mesh of both the blank and the die. A layer that extends for one $\mathrm{mm}$ from the die impacted surface was modeled with uniform mesh of hexahedral elements. The uniform layer of the die and the entire blank were refined in successive simulations from $0.5 \mathrm{~mm}$ down to $0.05 \mathrm{~mm}$ hexahedral elements.
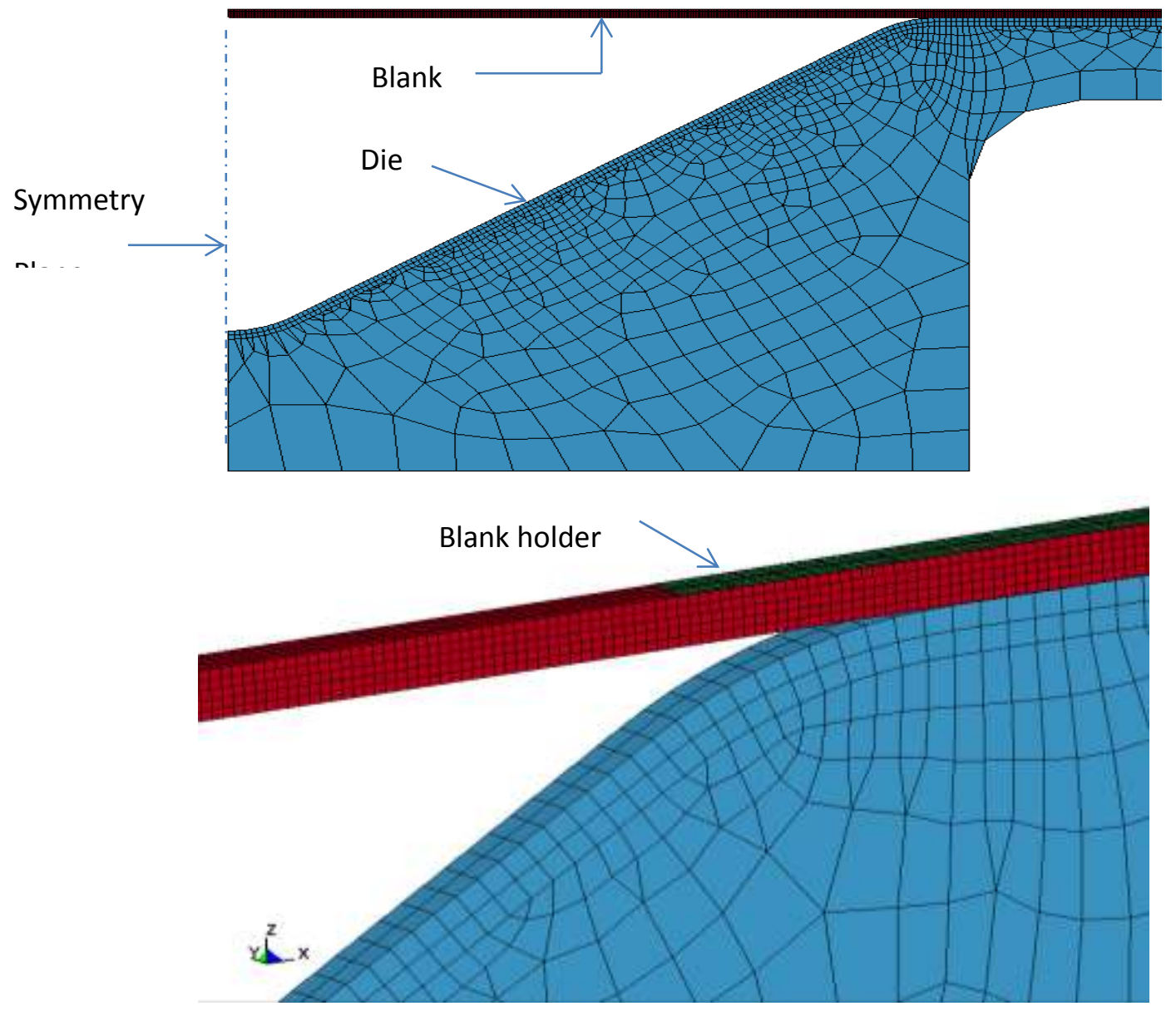

Figure 1.3.20. 3D plain strain $38^{\circ} \mathrm{V}$-shaped die simulation. 
Theoretically, the ideal mesh should be uniform in all directions and get convergence for the critical values of the problem. However, it should be fine enough to give accurate results, so that further refinement dramatically runs up the cost of computing with negligible improvement in accuracy. To investigate this, a $34^{\circ} \mathrm{V}$-shape die was used in an EHF simulation to form a blank of DP500 $1 \mathrm{~mm}$ sheet. The dominant stress at the first peak is in the horizontal direction, causing high tensile stresses at the base of the $\mathrm{V}$, and at a time well before the blank impacts the base of the $\mathrm{V}$. Unlike the first stress peak, the dominant stress in the second peak is in the vertical direction, causing high compressive stresses when the blank reaches the corner, or the base of the $\mathrm{V}$. The stress levels and types are indicated in Figure 1.3.21 below. The convergence values obtained for the model with $0.1 \mathrm{~mm}$ hexahedral elements for a DP 500 blank with a deformable elastic die are shown in Table 1.3.1 below.

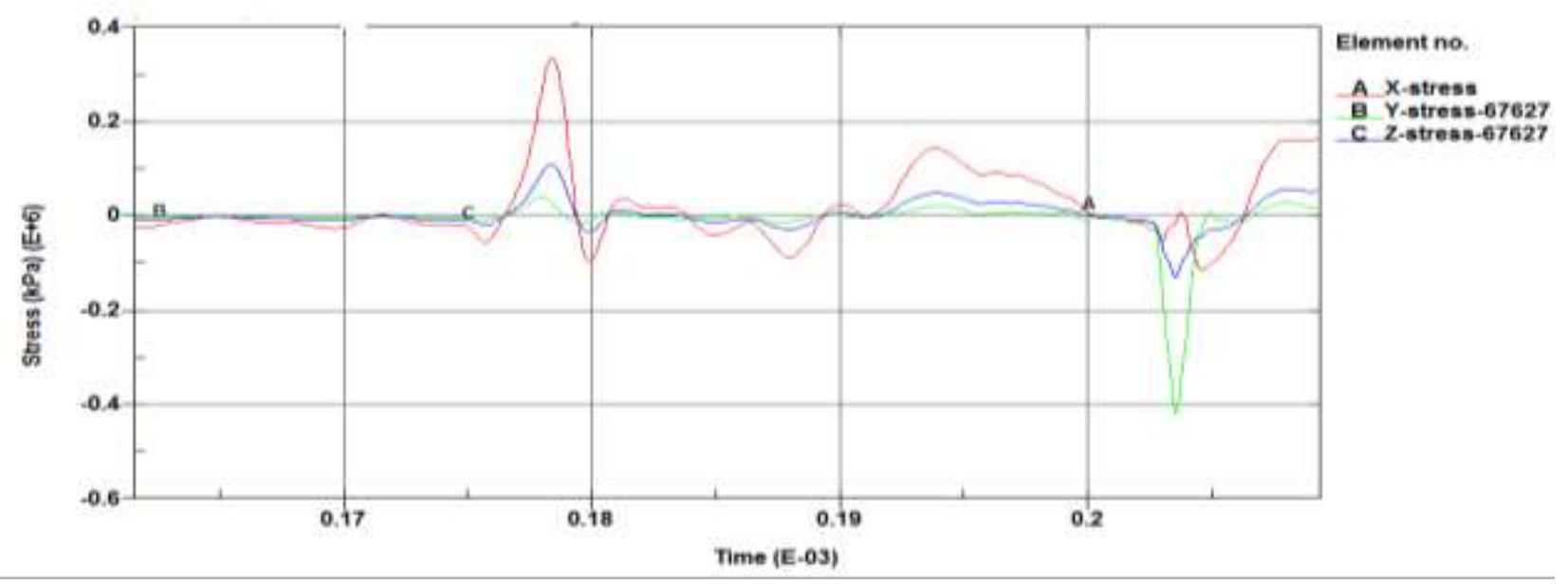

Figure 1.3.21. Contact stresses at the two stress peaks in the corner element.

Table 1.3.1. Convergence of the V-Die corner element von-Mises Stress.

\begin{tabular}{|l|l|l|l|l|}
\cline { 2 - 5 } \multicolumn{1}{c|}{} & \multicolumn{3}{l}{ Both blank and die element size } & $0.1 \mathrm{~mm}$ \\
\cline { 2 - 5 } \multicolumn{1}{l|}{} & $1 \mathrm{~mm}$ & $0.5 \mathrm{~mm}$ & $0.1 \mathrm{~mm}$ & $0.1 \mathrm{~mm}$ \\
\hline Channel(ALE) Size & $0.5 \mathrm{~mm}$ & $0.5 \mathrm{~mm}$ & $0.5 \mathrm{~mm}$ & $1 \mathrm{hr} 46 \mathrm{~min}$ \\
\hline Solution Time & $2.5 \mathrm{mi}$ & $4 \mathrm{~min}$ & $1004 \mathrm{MPa}$ & $1166 \mathrm{MPa}$ \\
\hline $\begin{array}{l}\text { v-M Stress at Die } \\
\text { corner that caused } \\
\text { tensile loading }\end{array}$ & $278 \mathrm{MPa}$ & $450 \mathrm{MPa}$ & $3247 \mathrm{MPa}$ & $3418 \mathrm{MPa}$ \\
\hline $\begin{array}{l}\text { v-M Stress at Die } \\
\text { corner that caused } \\
\text { compressive } \\
\text { loading }\end{array}$ & $367 \mathrm{MPa}$ & $500 \mathrm{MPa}$ & & \\
\hline
\end{tabular}




\section{Summary of task 1.3}

1. The contact stress numerical simulation was confirmed by analytical solutions for bar to rigid wall impact and bar to bar elastic and elastoplastic impact. The highest contact stresses occur in the areas where the surface impact takes place. In this case the impacting material cannot flow along the contact surface and the contact stress can be estimated by elastic impact analytical formula. For the areas where local impact takes place, the contact stress can be estimated as the material's yield stress for the corresponding level of strain and strain rate.

2. Analysis of contact stresses has been conducted in blank impact simulations driven by the EHF plasma channel transmitting pressure through the liquid. Issues arising from mesh-size sensitivity have been resolved. Convergence of contact stresses has been accomplished.

3. The numerical results were confirmed by the experimental study of $V$-shape die failure. The numerical model is now capable of predicting stresses in the dies for pulsed forming, which concludes the study on die materials within Sub-Task 1.3.

Sub-task 1.4 Process modeling validation for full scale automotive panel

Building the simulation of a multistage EHF process for the full-scale automotive part poses additional issues that must be addressed. The ALE solver in LS-DYNA has a few limitations that affect the size and calculation costs of the problem:

1. The Eulerian mesh for water, vacuum, and plasma can have only hexa elements preferably of the same size and shape, which means the mesh must be uniform throughout the whole area. The uniformity of a mesh is not a requirement by LS-DYNA, but practically speaking it is the only way to build a mesh for an arbitrary configuration. Besides, a model with uniform mesh is more numerically stable. That way, the overall volume of the chamber together with element size defines the total number of ALE elements.

2. The size of the elements of the deformable Lagrangian blank, which contacts the ALE liquid, must be not less than about 0.6-0.7 of the size of ALE space elements. It also cannot vary too much. In fact, the blank also has to be meshed almost uniformly.

3. The vacuum ALE elements add the same calculation costs as any other ALE elements.

With these limitations, the smallest element of the blank defines the size of ALE elements and, taking into account the uniformity of ALE elements, defines the total number of ALE elements. Considering the fact that all sub-chambers in the designed configuration are connected with each other by gaps under the walls (and in fact are the parts of one big chamber), the model has to include all the elements from all the sub-chambers for each discharge.

All these factors together lead to a very substantial size of the numerical model in terms of the number of elements and calculation costs, and this necessitates a search for a compromise 
between precision of the simulation and realistic simulation time when using available computers. Currently, the achievable element size for the blank for this problem lies in range of 3-4 mm. In this case, the number of ALE elements is about 0.7 million which in turn gives the running time for one simulation of about 7 to 20 hours (depending on stage of the process) using the available cluster computer which includes 48 high speed processors.

It should also be noted that additional effort is required to remove unnecessary vacuum elements outside of the chamber volume. The method of building an ALE mesh is to create a prism meshed with hexa elements, which prism contains the area between the chamber and the die as an inscribed figure. That way, there can be initially up to $50 \%$ of space outside of chamber filled with unnecessary vacuum elements, with all of them contributing to the calculation costs. With running time for the simulation as mentioned before, it is necessary to remove these elements from the model. To do this, additional software was created that automatically detects unnecessary elements and removes them from the input file.

The last issue related to the simulation of a multistage EHF process is how to pass the results of simulation of one discharge to the simulation of the next discharge with maximum reuse of existing input files. The working procedure is as follows:

- The blank is isolated in the separate input file, which is attached to the simulation input using *INCLUDE card. The ALE mesh for the water, vacuum, and channel is also isolated in a separate file and attached in the same manner.

- Since is not known exactly how long it will take to establish the deformations in all the sub-chambers, each run requires periodical checking of the results. Once the blank reaches the steady state, the simulation of the particular discharge can be stopped.

- The next step is to prepare the input for the next simulation. It can be done in the LSDYNA interactive post processor (Isprepost). The blank mesh in the last state must be saved as "Dynain ASCII." The created file is a regular LS-DYNA input file which just replaces the blank's input file in the next simulation.

- The ALE mesh for each sub-chamber is practically the same, with the only difference being the position of the channel elements, which is easy to change since this mesh is isolated in the separate file.

- The rest of the input remains the same. With two files replaced (new blank and ALE mesh) the input is ready to start again. This algorithm is repeated with the required order of discharges until the blank completely fills the die.

The designed discharge chamber and the results of simulation, conducted in accordance with the procedure described above, are presented in Task 7 - Demonstration of EHF of a full scale automotive panel application. 


\section{Task 2. Electrode system and discharge chamber design and development}

Electrode durability is key to commercialization and high volume production using EHF systems. Chief among the concerns in electrode development is the documented tip erosion and potential insulation damage that occurs with every discharge. Electrical breakdown of insulation has been a problem in older electrode designs, and although subsequent refinements improved life and mechanical damage from the EHF pressure pulses, the insulator materials have now proved to be just as important as the conductor materials.

Sub-task 2.1. Electrode erosion study [2-1]

\section{Electrode System Failure Modes}

In EHF, the large currents carried by the electrodes, the erosive high temperature plasma and, lastly, the extreme pressures acting at the electrode tips (2-3 GPa) [2-2] all act to shorten the electrode life in a variety of ways. An early chamber design is shown in Figure 2-1, and the insulation failure modes, in order of increasing severity, are shown in Figure 2-2. The first failure type, asymmetric erosion, is a result of the electrode orientation in the chamber design of Figure 2-1 and the pressure wave emanating from the inter electrode gap. The second failure type, observed in Figure 2-2b, can also be tied to the chamber design/electrode orientation, but can also happen because of reflected pressure waves and lack of lateral electrode support. Most electrodes fail as a result of the last failure mode, erosion, shown in Figure 2-2c, and subject of this paper. Plasma arc erosion is largely responsible for increasing inter-electrode gaps such that a functional system failure occurs - a faulty discharge or no discharge at all.

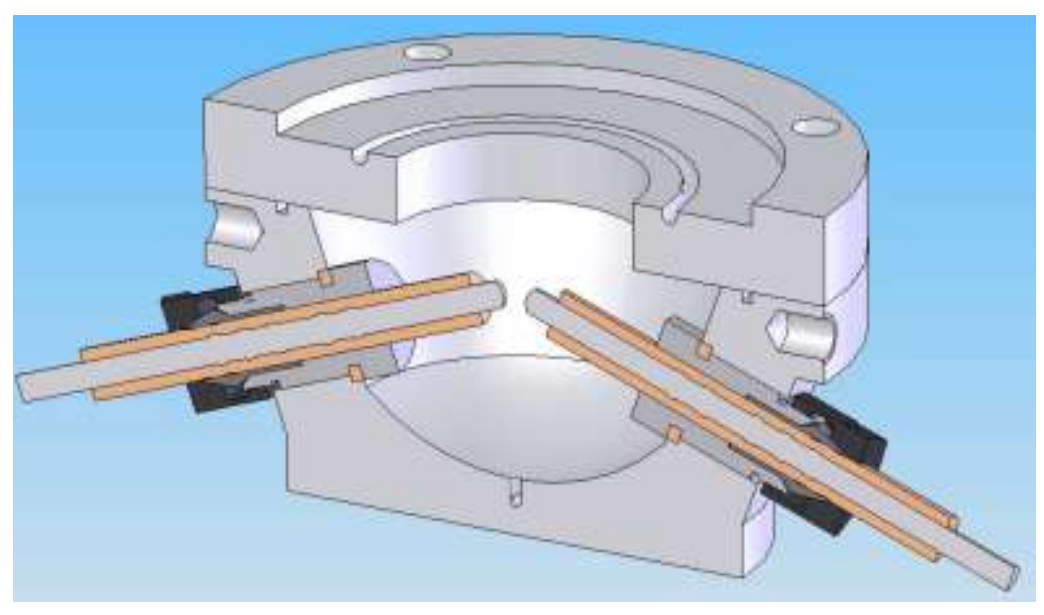

Figure 2-1. Early Electrode System Design.

Before testing After 120 pulses 


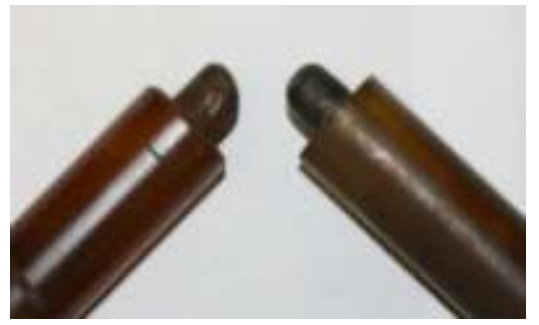

(a)

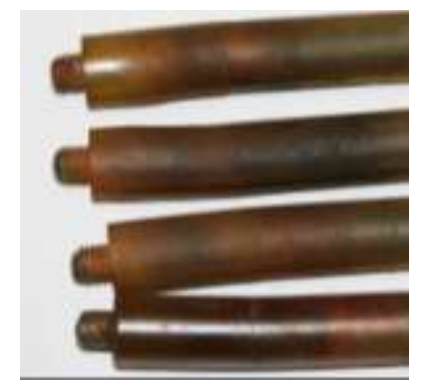

(b)

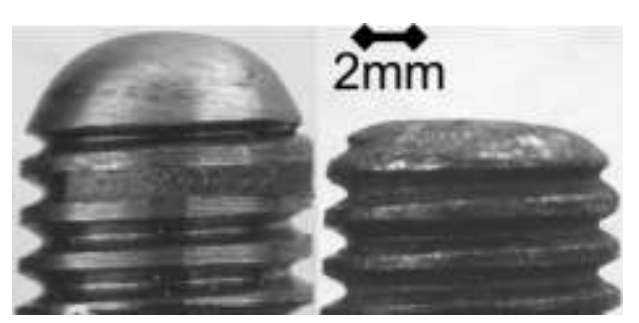

(c)

Figure 2-2. Electrode Failure Modes: (a) asymmetric erosion; (b) bending; and (c) erosion.

\section{Electrode Testing Program}

A program was developed to investigate electrode erosion performance and improve it sufficiently so that EHF can be used in a production environment. The factors that influence electrode performance are material, tip shape, discharge energy. A dedicated chamber was developed with which to perform accelerated testing and, as shown in Figure 2-3, the volume of the liquid in the chamber was quite small, roughly $1 / 10$ th the volume of a chamber used for producing a modest sized automotive panel. In this chamber, instead of a sheet metal blank and die, a thick steel plate was used to seal the chamber. Reducing the volume of the chamber and eliminating the sheet metal blank significantly increases the pressure levels and promotes faster accumulation of electrode damage. Thus this smaller and much more highly constrained volume significantly increases the pressures and forces in the chamber and accelerates the durability component of the test. In the design, directly opposed electrodes were used to (mostly) eliminate the bending failure mode observed in Figure 2-2b.

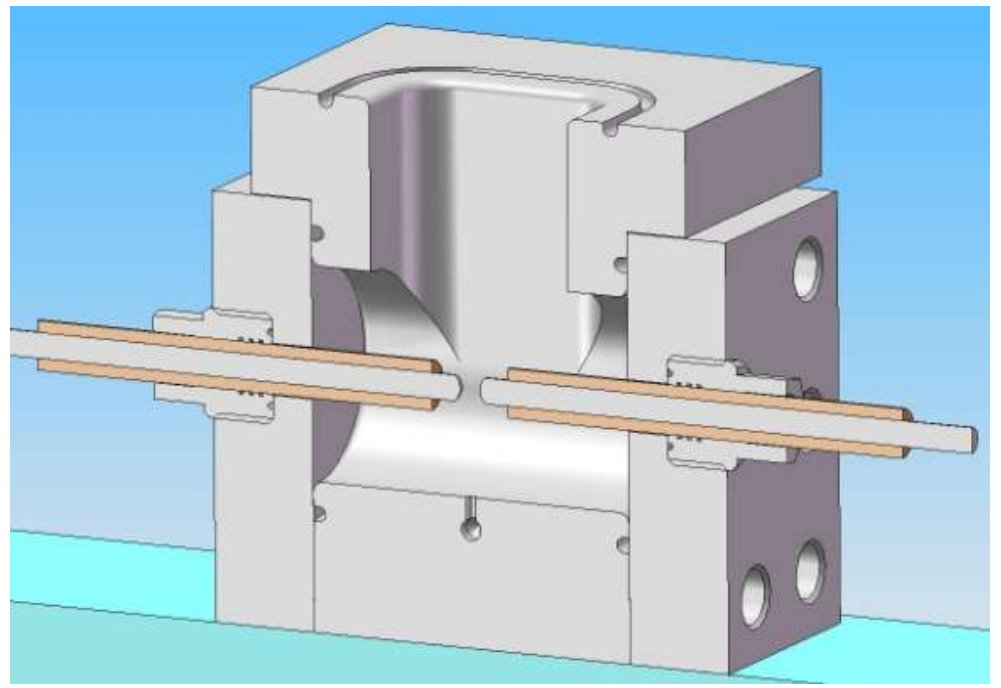

Figure 2-3. Cross section of 1.8 liter electrode testing chamber. A thick steel plate covers the top during testing. Not shown are the braces which anchor the electrode ends to the G-10 base plate and prevent electrode ejection. 
All tests employed a consistent $11 \mathrm{kV}(12.1 \mathrm{~kJ})$ pulse, selected to be an above average energy level used in automotive panel forming. In erosion testing 120 pulses were consistently discharged through each pair of electrodes (where possible) before test termination, and periodically the chamber was opened and the electrodes were inspected for damage. Up to 120 discharges were performed before test termination in erosion experiments. Throughout testing the current and voltage performance of the electrodes were monitored and recorded. At the end of the test the total electrode erosion and damage was documented.

Two pulse generators were used in this work. The primary unit was a $22.5 \mathrm{~kJ} / 15 \mathrm{kV}$ pulse generator capable of $100 \mathrm{kA}$ peak current. The secondary unit, used for the high durability test, was a $100 \mathrm{~kJ} / 25 \mathrm{kV}$ capable of $1.6 \mathrm{MA}$ peak current. This latter unit, when used, was reconfigured to deliver the same energy/voltage to the testing chamber as the first unit so that the equivalent energy was input, and this second chamber was only used in a single test to determine the maximum number of discharges that an electrode pair could survive. By maintaining energy and voltage equivalence it is expected that the amount of damage done to the electrodes is equivalent.

A series of different studies were conducted to examine the following effects: erosion in different electrode materials, the effect of different tip geometries, and the effect of entrained air in the chamber on the erosion rate - all were tested using the electrode testing chamber. A final test was performed where the inter electrode gap was varied and the effect on the EHF system performance was studied.

The electrode materials study examined several different materials for their suitability for use in an EHF chamber. It was believed that the refractory metals might offer the best performance because of their high melting point. M-184 tungsten alloy, commercially pure molybdenum, and a tungsten-copper powder metallurgy alloy used for spot welding electrodes, and a plain carbon cold rolled steel (SAE 1018) were studied.

The tip geometries examined were conical, hemispherical, and flat. The opposing electrode was always of the same configuration. Lastly, the effect of entrained air was studied using an electrode testing chamber with a special top that caused the experiment to maintain a consistent air volume. This last series of experiments was performed to understand whether cavitation, which would increase with increased air volume, would unduly affect the electrode life.

Figure 2-4 shows the electrodes that were used in the studies. Two variants of this design were used - one in which the insulation was interlocked with the electrode metal via a series of lands and another in which the interlock was achieved through a thread in the metal. The former is shown in the figure and was used in early experiments, especially in experiments with refractory metals, but the threaded design was used later and in the majority of experiments. Electrode tip shapes were simply cut into the electrode ends: hemispherical as shown, a flat tip was a simple 
right circular cylinder, and conical tips were right circular cones with an angle of $33.6^{\circ}$ measured against the electrode axis. The electrodes had a $12 \mathrm{~mm}$ diameter.

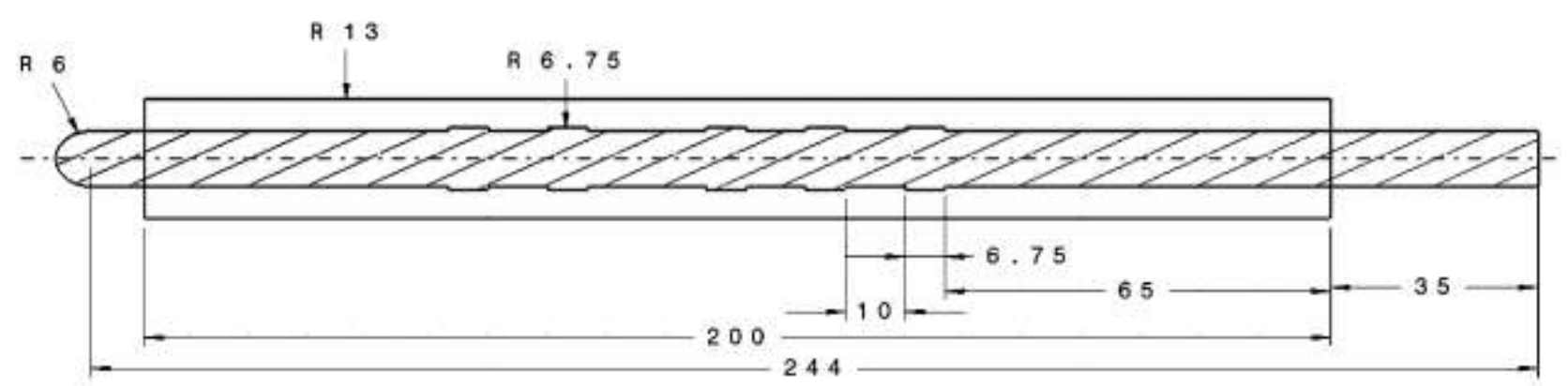

(a)

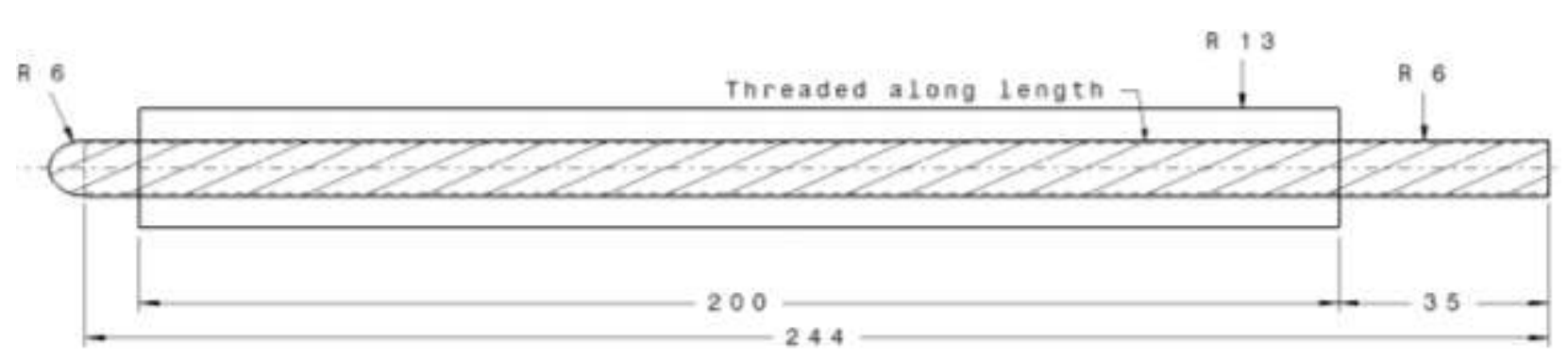

(b)

Figure 2-4. Electrode designs: (a) old and (b) threaded

A typical EHF discharge electrical characteristic is shown in Figure 2-5. The pulse generator applies the charge voltage (Vcharge) for roughly $1 \mathrm{~ms}$ before the formation of the plasma channel (at $\mathrm{t}=0$ ). The initial peak, Vmax, observed at the tool is not observed at the pulse generator capacitors but has been noted by other researchers [2-3]. During this time there is a leak down of the voltage so that, at the moment of discharge, the voltage $\left(\mathrm{V}_{\text {discharge }}\right)$ has dropped somewhat. This drop arises from leakage through the liquid medium between the exposed metal surfaces of the electrodes. Experiments with electrodes that had a significantly larger electrode surface area have shown that the leak down rate varies directly with the exposed electrode area. After $\mathrm{V}_{\text {discharge }}$ the plasma channel forms, current begins to flow, and the voltage rapidly drops to zero by the time the peak current is achieved. It is during this initial 30 ps period that most of the energy is delivered into the plasma channel and the pressure pulse is generated. The tail on the pulse is an indication of how long the plasma channel remains active before collapse. Electrical performance was recorded throughout the experiments and was used predominantly to determine whether discharges were either good or faulty. 


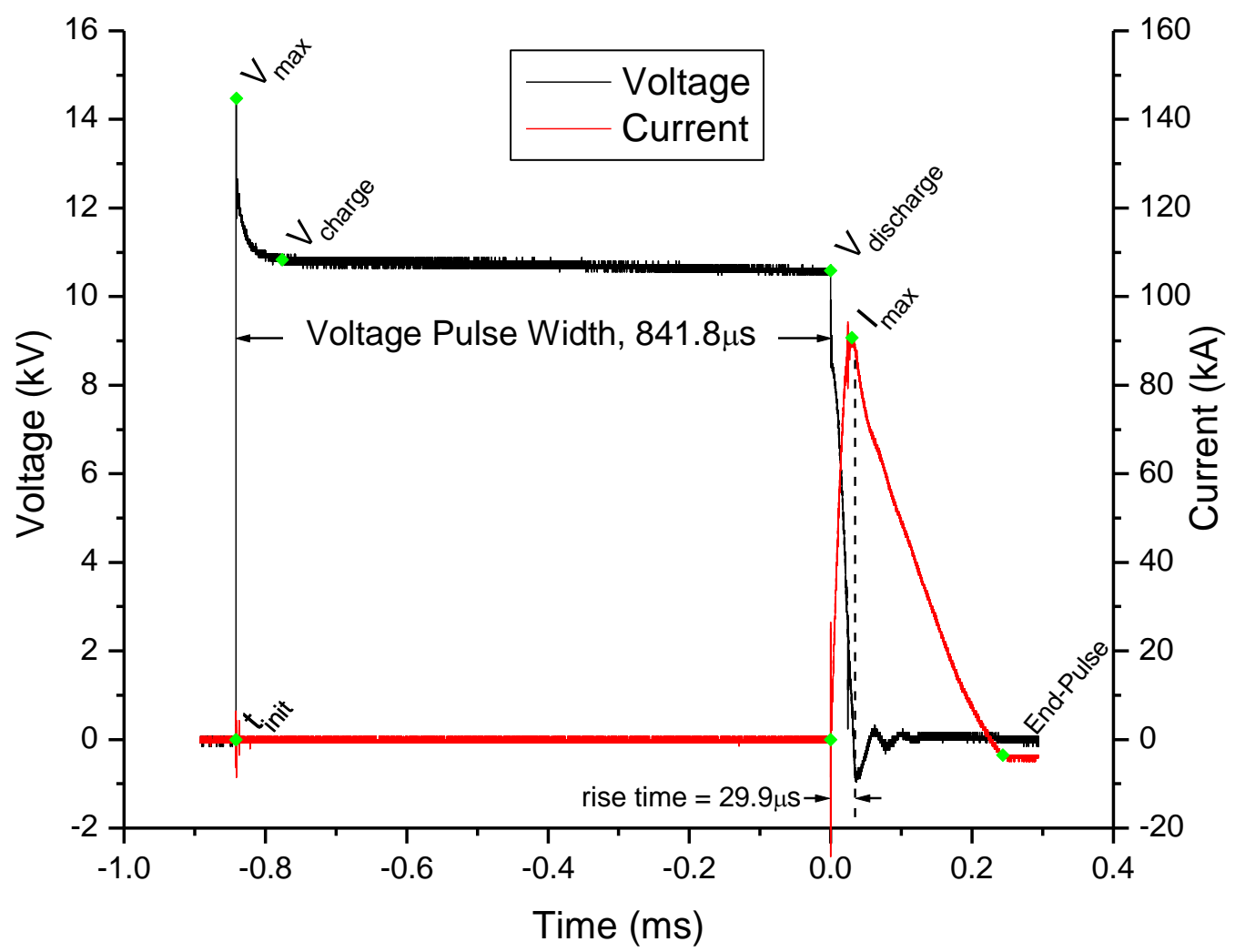

Figure 2-5. Typical $11 \mathrm{kV}(12.1 \mathrm{~kJ})$ electrical pulse showing initiation of the plasma channel at $\mathrm{t}=0$.

Results of Electrode Testing - Electrode Materials Tests

Electrode tests were conducted on SAE 1018 cold rolled steel, molybdenum, M-184 tungsten, and a copper-tungsten powder metallurgy alloy. The electrodes were constructed with hemispherical tips and tested in the chamber for up to 120 good discharges. Of these materials, only the steel survived the full 120 discharges. Molybdenum survived 15 discharges before the negative electrode fractured in a brittle fashion outside the chamber at the pulse generator attachment point (2-6b), likely as a result of the combination of residual stresses, a notch and the forces from the discharge. There was some evidence of plasma arcing across the crack faces the second failure (Figure 2-6c). The electrode broke two more times in a similar fashion after similar intervals, and the test was finally suspended - this behavior suggests somewhat stable crack propagation. The tungsten negative electrode broke in a similar fashion but after only a single discharge and the test was suspended (Figure 2-6a). The tungstencopper PM material broke inside the chamber under the insulation due to the bending forces after roughly 70 discharges. Volumetric erosion measurements were conducted on the first three materials shown in Figure 2-7. They indicate that the molybdenum showed significantly poorer erosion performance while the tungsten did not have enough discharges on it to be measured. The prohibitive material and machining costs associated with these refractory metals and their poor performance makes steel the clear choice. 


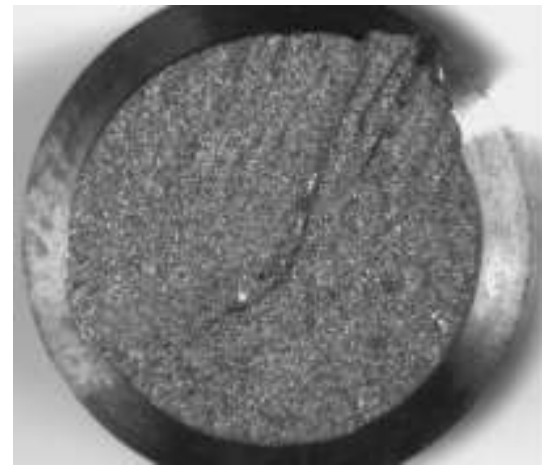

(a)

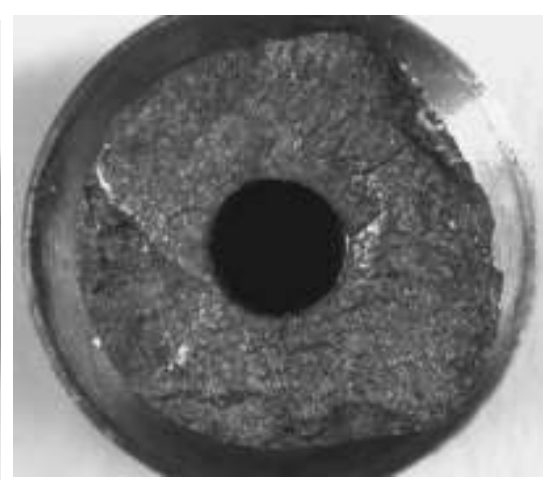

(b)

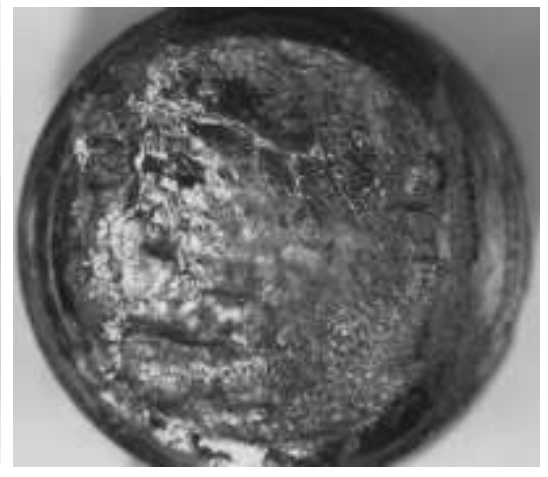

(c)

Figure 2-6. Brittle failures in the threaded region of the electrode external to the EHF chamber: a) tungsten failure and first molybdenum failure at end of turned machining center: c); second Mo failure showing fracture surface melting.

In addition to the total loss rates that were examined, the individual loss rates on both the ground and energized electrodes were recorded, and they are indicated by the +45 and the -45 hatching in Figure 2-7, respectively. In this and subsequent experiments the results consistently show the energized electrode loses nearly twice the volume of material when compared to the ground electrode.

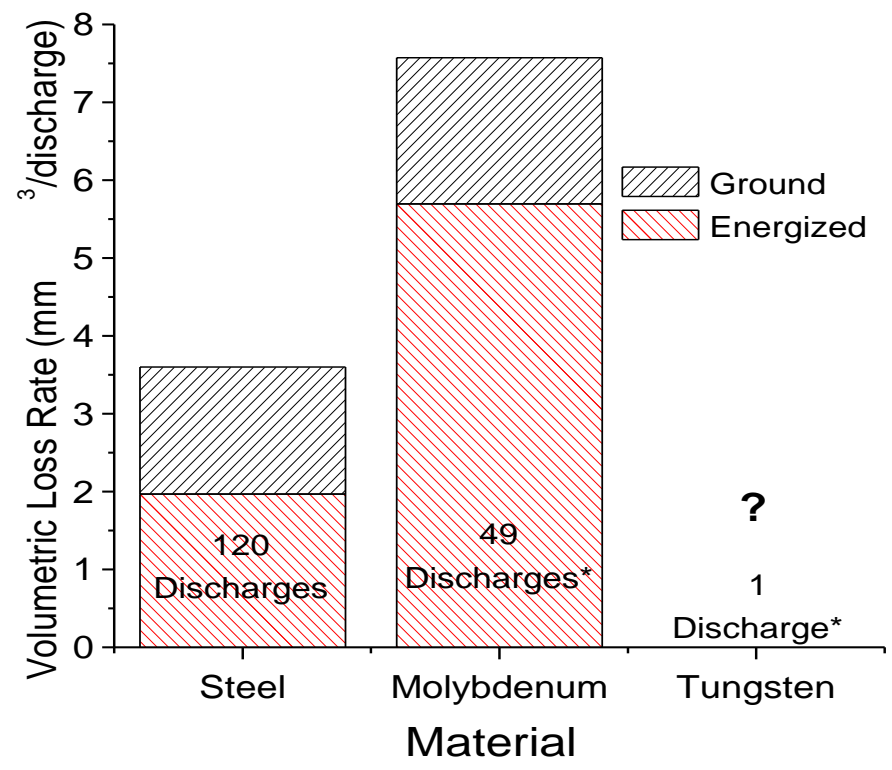

Figure 2-7. Volumetric erosion rate per discharge for electrode materials. Tungsten erosion after a single discharge was not measureable. 


\section{Electrode Tip Shape Tests}

A series of tests was conducted to determine optimum tip shape amongst the three selected: hemispherical, conical and flat. Initially the study was to focus on the efficiency of discharge/plasma formation and the optimal tip configuration (and combination) to achieve that goal. The electrode profile or tip shape can significantly influence the electrostatic fields set up after the voltage is applied to the electrodes and affects plasma channel formation and timing. The inter-electrode gap also influences these processes. Unfortunately, applying a modest numbers of discharges to the conical tip electrode pair results in rapid loss of electrode length through erosion and the subsequent increase in inter-electrode gap became the dominant concern.

Steel was used as the electrode material for these tests, and Figure 2-8a shows a bar graph indicating the volumetric loss rate for each of the tip shapes. It is believed that the volume lost is roughly constant regardless of the tip shape as suggested by Donaldson, et.al. [2-4]. For similarly configured electrode system tests (same tip/material/chamber), nominal erosion rate for both electrodes combined for steel is about $3.7 \pm 0.5 \mathrm{~mm}^{3} /$ discharge for tests with 120 discharges, and the hemispherical and flat electrode tests fall within reasonable bounds of this number. The stability of this number across a wide variety of tests suggests that the erosion process is not dependent on first order geometry. Testing on the conical electrodes had to be terminated early after only 10 discharges because the gap increase was too severe, and this relatively small number of discharges resulted in a low value of erosion with a high error. Figure $2-8 \mathrm{~b}$ shows the average electrode length loss rates for each electrode geometry. After only 10 discharges the pulses on the conical electrodes enough linear electrode length had been lost by both electrodes that the gap increased to the point where discharges became unstable and eventually could not be produced. The implication of this result is that geometric tip stability under erosion has greater impact on process stability than the electrical efficiency of the initial tip shape.

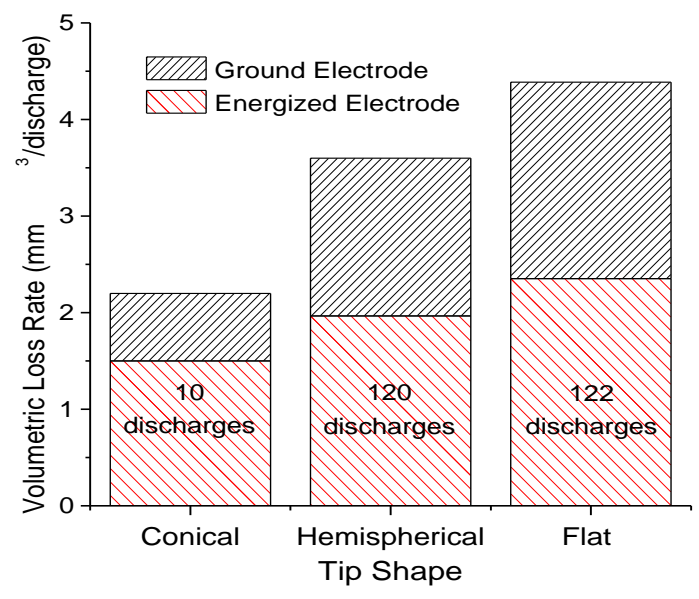

(a)

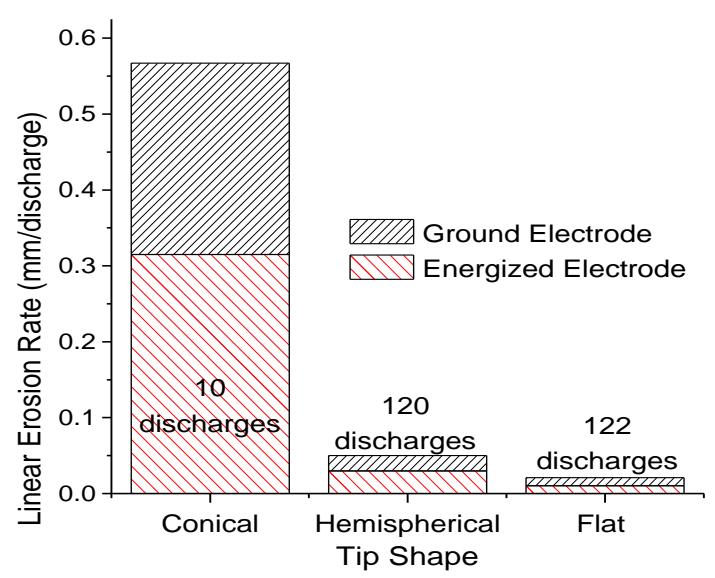

(b)

Figure 2-8. Linear and volumetric erosion rates of steel electrodes with different initial tip shapes. The graph at a) shows the volumetric loss rate and b) shows the data plotted against lost electrode length. 
Further, after considerable testing it was noticed that, regardless of the starting shape, orientation or function, the electrodes eventually all assumed a similar profile - as can be seen in Figure 2-9 - and this stable profile was achieved roughly half way through the required 120 discharges. Initially it was thought that the electrodes would hold their shape because the erosion was not that significant, but this was found to be incorrect. A stable profile would therefore provide the greatest overall process electrical stability and, thus the image profiles of Figure 2-9 were digitized, and the result is shown in Figure 2-10. The digital profiles were then normalized against the electrode radius, and the data analyzed in a number of different ways.

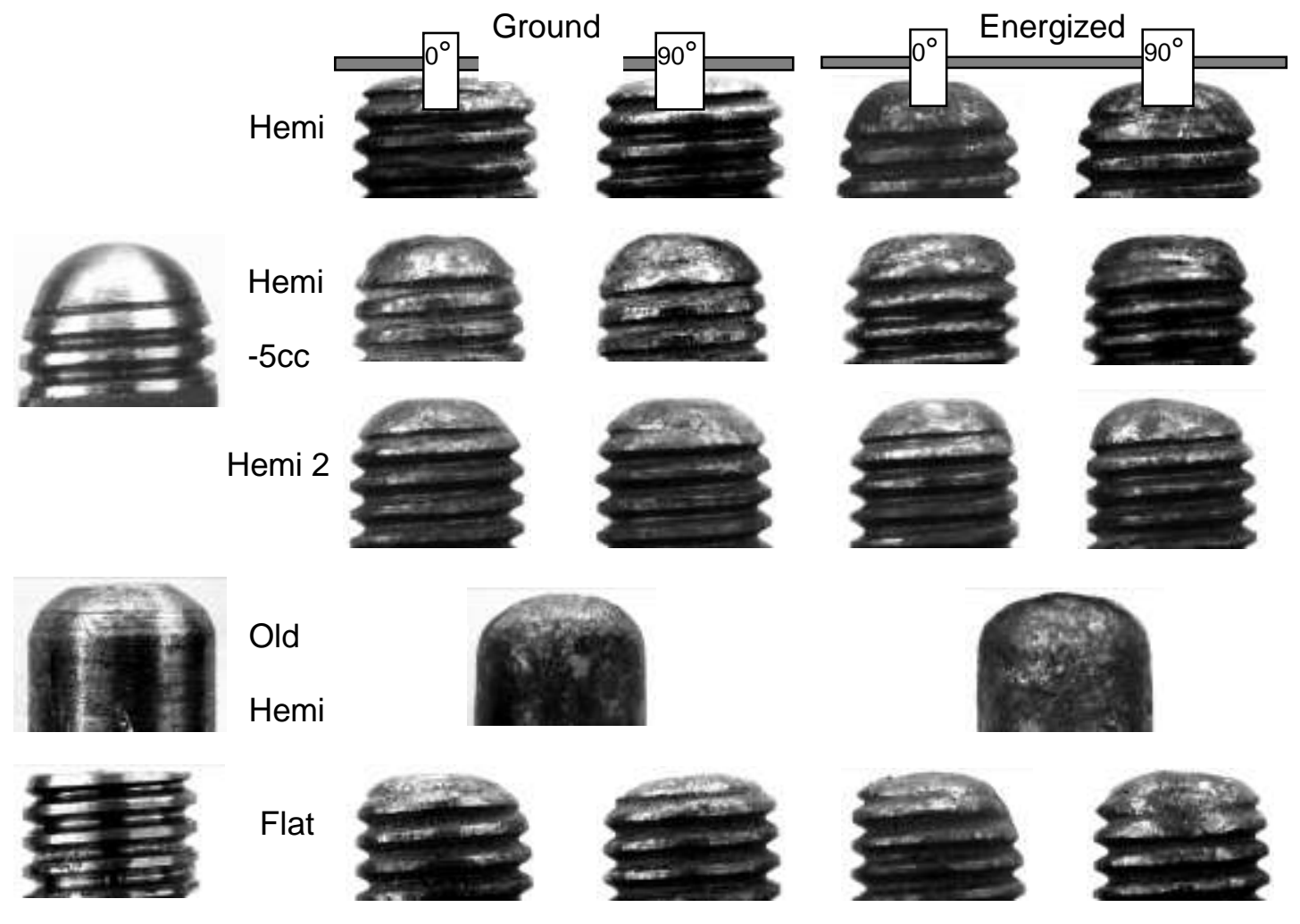

Figure 2-9. Initial and final electrode profiles after 120 pulses. With the exception of the "Old Hemi" electrode design from Figure $2-4 \mathrm{a}$, each electrode is shown in two orientations $\left(0^{\circ} \& 90^{\circ}\right)$. 


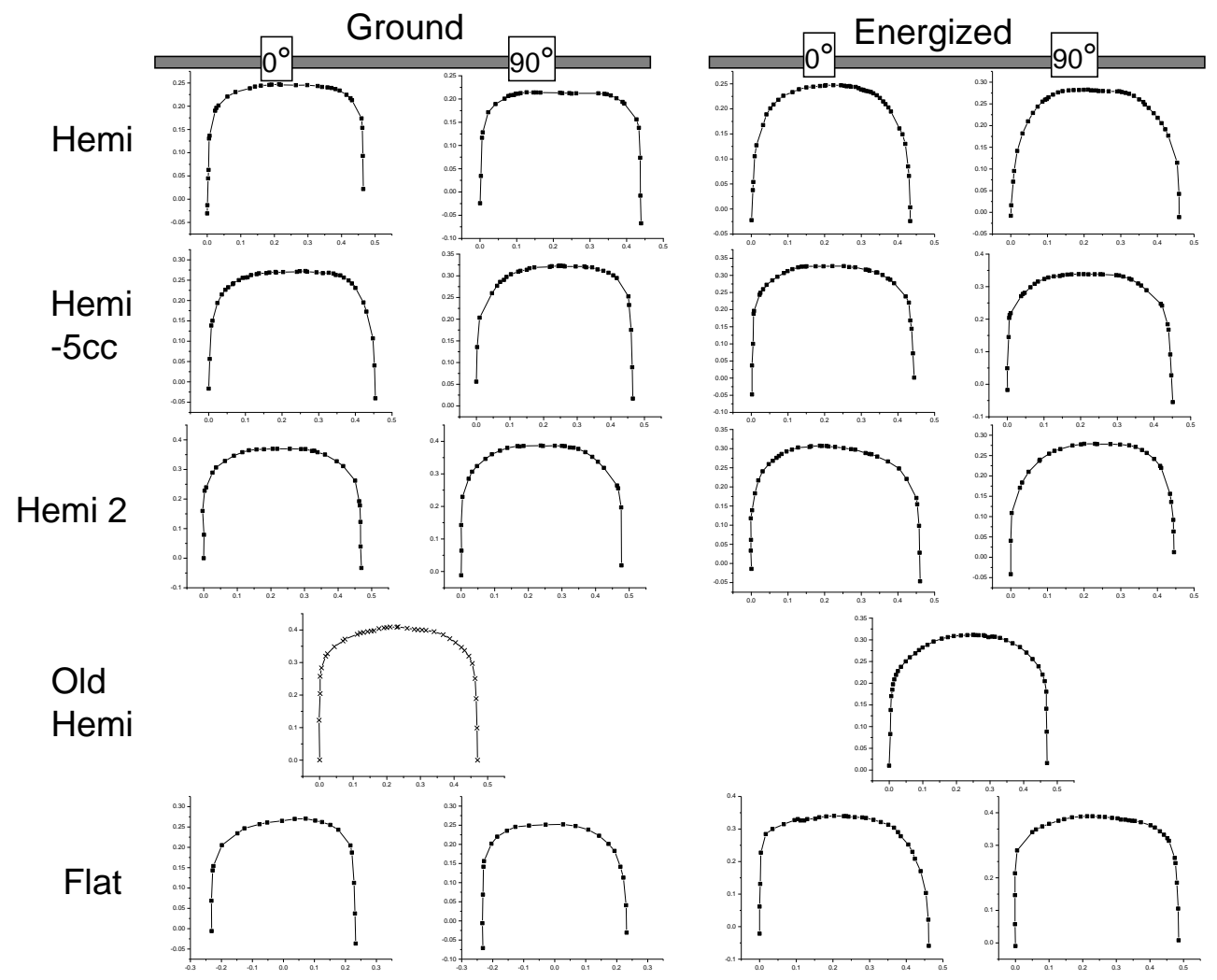

Figure 2-10. Digitized final electrode profiles after 120 discharges.
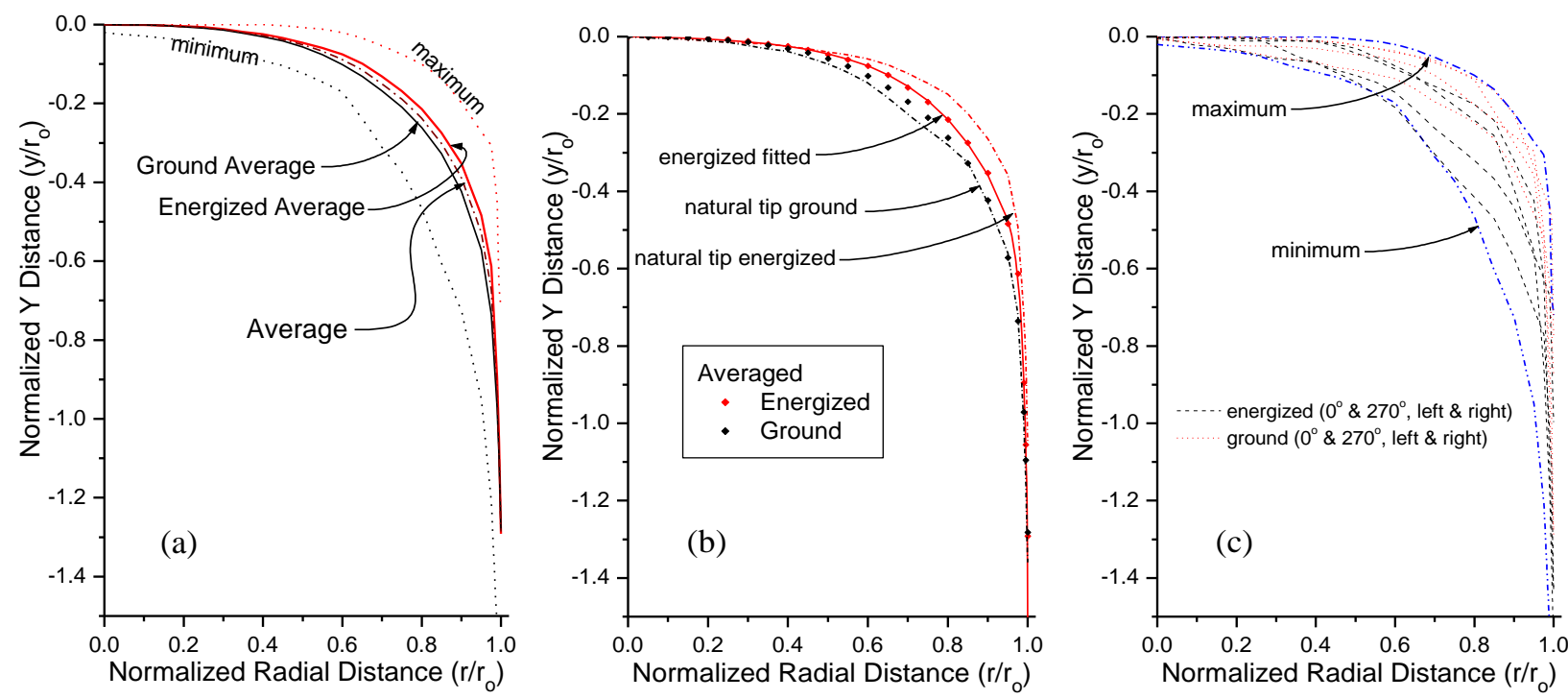

Figure 2-11. Electrode tip profiles: a) average and extremes, b) the fitted energized electrode equation, averaged energized and ground data, and averaged natural tip (energized and ground) data, and c) natural tip data (raw and maximum/minimum). 
In order to produce the profiles in Figure 2-10 the photographic profile was digitized, taking care to capture the profile only at the tips of the threads along the sides. The profile centerline was found, the data was split into right and left halves, the right half data reflected onto the left side, the two halves averaged into a single profile, and the highest $y$-value on the averaged profile was set to zero. This same procedure was then performed on the $90^{\circ}$ orientation, and that result was then averaged with the $0^{\circ}$ result. The electrode profiles were collected for the energized electrodes alone, averaged together, and the result is plotted in Figure 2-11a. The same process was repeated on the ground electrodes, and then, finally, the ground and energized profiles were averaged together to yield the "average" profile. Shown along with these profiles in Figure 2-11a are the minimum and maximum profiles which are an accumulation of the lowest and highest points from the raw profile data. Within the limited dataset, the maximum and minimum profiles establish a rough envelope of as-eroded profile shapes. The differences between the ground, energized and average profiles is relatively minor.

Because the volume loss is greatest on the energized electrode, its average profile was selected for further study. An equation of the form was fit to the profile, where $x(0 \leq x \leq 1)$ is the radial displacement from the electrode centerline normalized against the radius () and $y$ is the distance along the electrode's length also normalized against the radius - the origin is at the tip of the electrode at the centerline, as presented in Figure 2-11. The resulting parameters from the curve fit are presented in Table 2-1, and the results can be seen in Figure 2-11b.

Table 2-1. Electrode equation best fit parameters to energized electrode tip profile.

\begin{tabular}{|c|c|c|c|c|c|c|c|}
\hline$y_{0}$ & $A_{1}$ & $t_{1}$ & $A_{2}$ & $t_{2}$ & $A_{3}$ & $t_{3}$ & $\mathrm{R}^{2}$ \\
\hline-0.0038865 & 0.59789 & 0.19858 & 0.71285 & 0.01263 & 0.19676 & 0.00065 & 0.99993 \\
\hline
\end{tabular}

A set of electrodes was fabricated with the best fit tip profile. They were tested and the averaged tip profile results are shown in Figure 2-11b in comparison with the average energized and ground data. The averaged energized natural tip data falls above but reasonably close to the best fit line. The averaged natural tip ground data falls reasonably close to the nominal ground profile. Figure 2-11c demonstrates that the raw data (thin dotted and dashed lines) fall almost entirely within the bounds set by the maximum and minimum lines. Thus, the nominal profile tip (as defined by the curve fit) is both geometrically stable as discharges progress and thus maintains reasonably stable electrical performance.

\section{Influence of Entrained Air on Electrode Erosion}

A series of erosion tests were carried out using the electrode testing chamber fitted with a special closed volume lid that, because it was closed, maintained a constant level of air at the top of the chamber. The following tests were performed to determine if entrained air in the chamber lowered electrode erosion rates - it was thought that the additional air in the chamber would lower erosion rates. Figure 2-12 below shows the results of tests performed with no air, a small amount of air $(5 \mathrm{ml})$ and $500 \mathrm{ml}$ of air in the 1.8 liter electrode testing chamber. Given that the standard deviation in total electrode erosion rate (negative plus positive) combined is 
around $0.5 \mathrm{~mm}^{3} /$ discharge, it is fairly clear that there is no substantial effect on erosion of entrained air on erosion rates.

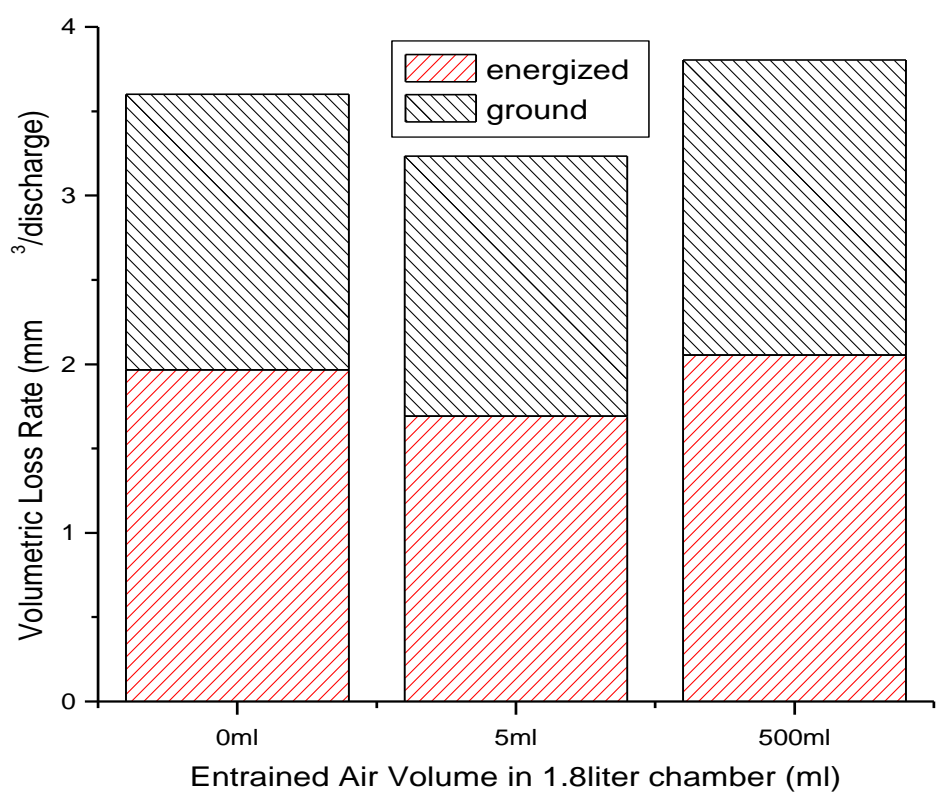

Figure 2-12. Effects of entrained air on electrode erosion over 120 discharges in the 1.8 liter chamber.

The Inter-Electrode Gap and EHF Process Stability

A study was executed to determine the influence that inter-electrode gap has on process stability where ten $11 \mathrm{kV}(12.1 \mathrm{~kJ})$ discharges were performed at each of three different gap settings - all other variables were held constant - and the transient process currents and voltages were measured at the tool. The experimental results showing the average voltage at the moment just before discharge and the peak current during the discharge are shown in Figure 2-13. Figure 2-5 shows how these variables were measured. As the gap increases, the average maximum current $\left(I_{\max }\right)$ and the average discharge voltage $\left(\mathrm{V}_{\text {discharge }}\right)$ both decrease. With the reductions in peak current and discharge voltage this means that about $10 \%$ of the energy available at process initiation is lost to leak down at the $20 \mathrm{~mm}$ gap setting. As mentioned before, the energy is lost during due to leakage between the exposed electrode surfaces from the moment the voltage is applied to when the plasma channel initiates. This gradual voltage leak down can be observed in Figure 2-5 between voltage application and discharge. This voltage loss is due to ionic transport in the working fluid, and the greater the conductivity of the fluid and the larger the exposed electrode surface area is the more rapid this loss rate is. The time between voltage application and plasma channel formation is termed the voltage pulse width, and it increases with increasing gap, as is indicated in Figure 2-13. The larger the gap, the longer is the voltage pulse width, and the greater is the voltage leak down. However, the $10 \%$ energy loss measured at the $20 \mathrm{~mm}$ gap can be compensated for by a small increase in the operating voltage. Even so, a simple adjustment upwards of Vcharge means that EHF system performance can be maintained. 


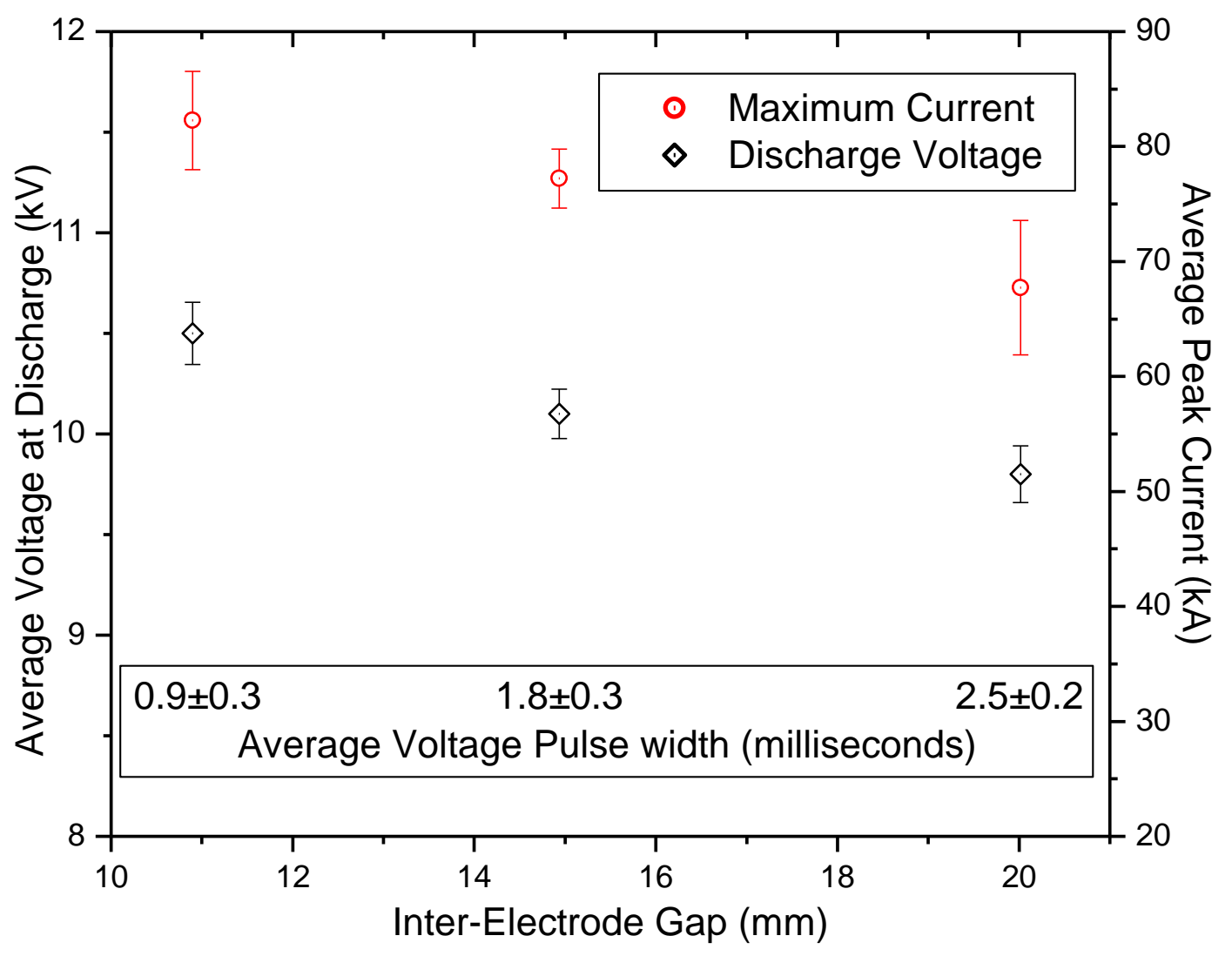

Figure 2-13. Electrical response of EHF discharges as a function of inter-electrode gap. The stability demonstrated in this set of tests indicates that the discharge current and voltage can be measured in a production system and serve as a criterion for adjusting the electrode position as well as serve as a monitor for the health of the system and the quality of the discharge.

\section{Sub-task 2.2. Develop robust electrode system}

Initially it was believed that metal erosion was the limiting factor in electrode life, however the initial Gate 1 bogey of 120 discharges showed that, in some cases, the insulation was beginning to break down. Subsequently, chief among the concerns in subsequent electrode development, was the potential insulation damage that occurs with every discharge. Electrical breakdown of insulation has been a problem in older electrode designs. Other insulation failure modes have included cracking from electrode bending arising from reflected pressure waves in older designs and chipping of insulation at the electrode tip observed in the 120 discharge erosion studies with square profile insulation. Bending damage to electrode and insulator was largely fixed by directly opposing the electrodes. However, frequent damage to the end of the insulation continued to be observed, such as that shown in Figure 2-14. 

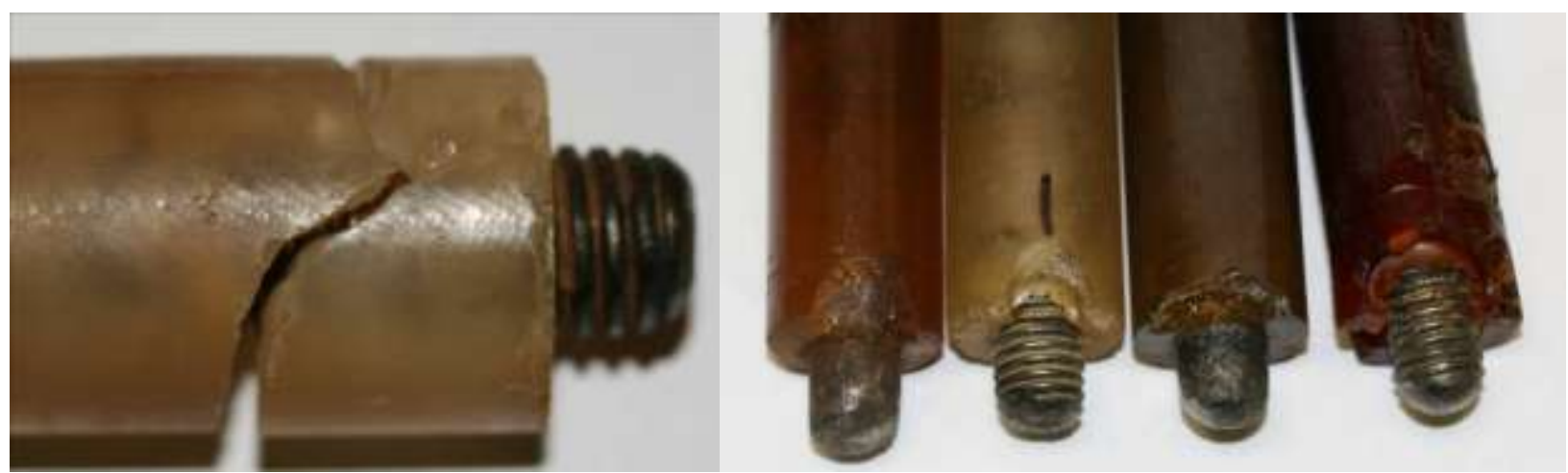

Figure 2-14. Typical (polyurethane) insulation damage observed on electrodes with square insulation profiles after 120 pulses. Note both chipping and separation of insulator from electrode shaft.

\section{Insulation Durability Improvement - Tip Morphology}

One of the first attempts to improve electrode insulation performance was to give the electrode insulation near the electrode tip a conical profile to reduce the stresses in the polyurethane. This modification significantly improved the durability out to 650 pulses, where the failure observed in Figure 2-15 was recorded. Both electrodes in this experiment demonstrated the same insulation failure mechanisms: circumferential crack around the point where the cone meets the outer diameter of the shaft, separation of the insulator from the electrode, and a final longitudinal crack.

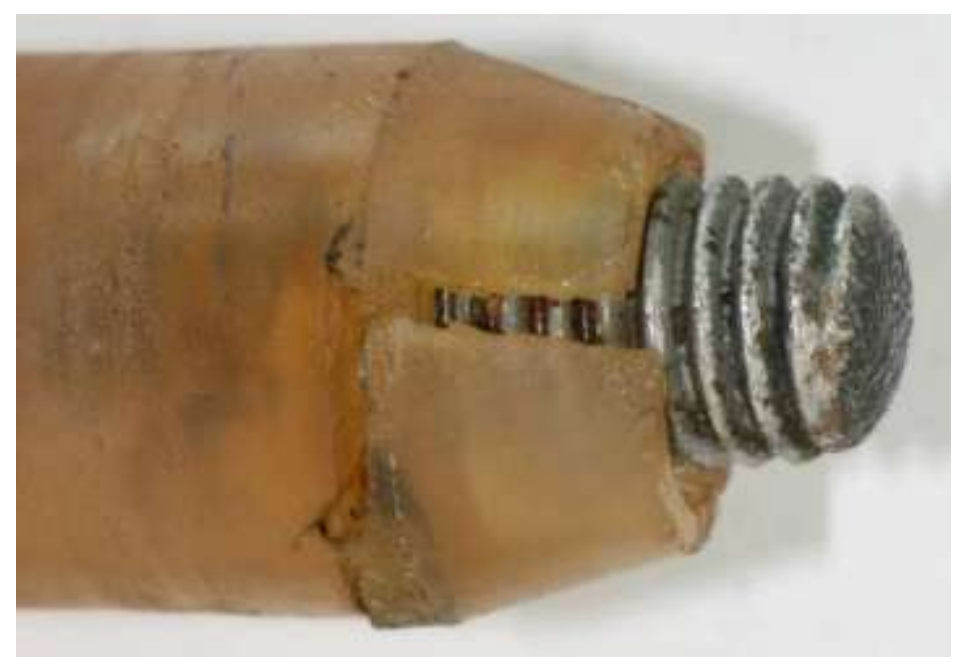

Figure 2-15. Typical (polyurethane) insulation damage observed on polyurethane electrodes with conical insulation profiles. After 120 pulses no significant damage was noted. This failure occurred after 650 pulses were applied. Note relative lack of insulation chipping. Failure observed in both electrodes includes circumferential cracking of insulation just behind the conical region, separation of insulator from electrode shaft, and final longitudinal failure of insulation. 
While this design was extremely successful in terms of Gate 2 - surviving 120 pulses - and can survive the operation of an EHF cell for a full shift of operation at low volumes, it was greatly desired to improve electrode life to the point where a pair of electrodes could survive a shift of production under high volume conditions. Thus, it was thought that a more durable, perhaps even non-insulating material, placed at the electrode tip might shield the insulation from significant pulse damage.

\section{Alternative Insulation Materials}

The success with this conical tip electrode insulation design naturally led to exploration of other insulating materials (other than polyurethane) used within the chamber.

The advantage of polyurethane is that it can be cast into a mold at room temperature, but the difficulty with most other candidate plastics is that they require either a high temperature cure and/or high molding pressures, which makes them difficult to work with in a research environment. In order to examine the performance of these materials in-situ, a special electrode system was designed so that these plastics could be bought in an as-cured state and machined into final form. The split-electrode system is shown diagrammatically in Figures 2-16 and 2-17.

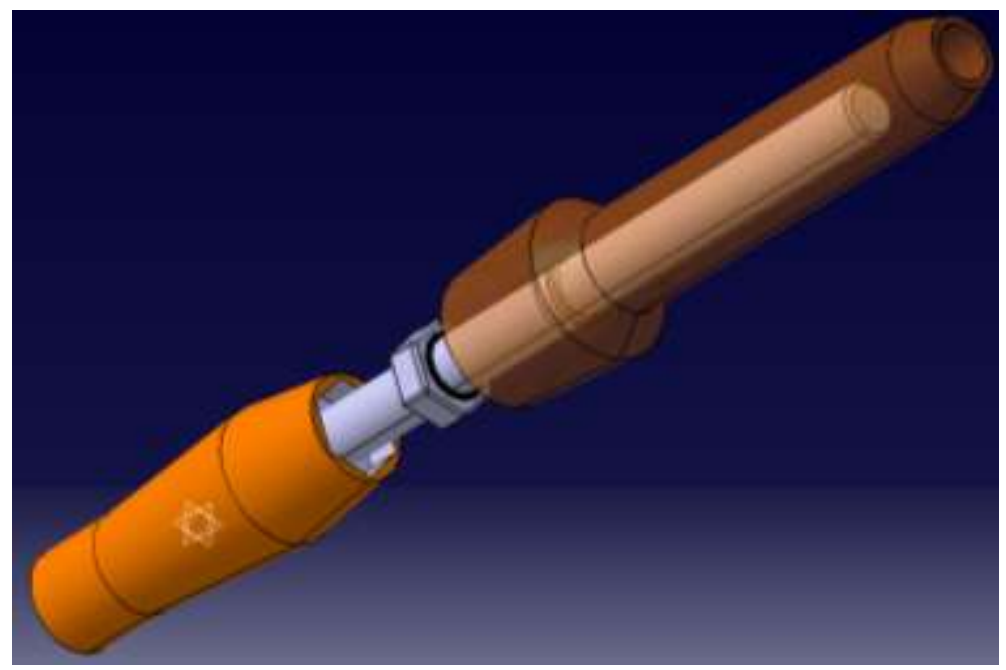

Figure 2-16. Exploded view of split electrode design.

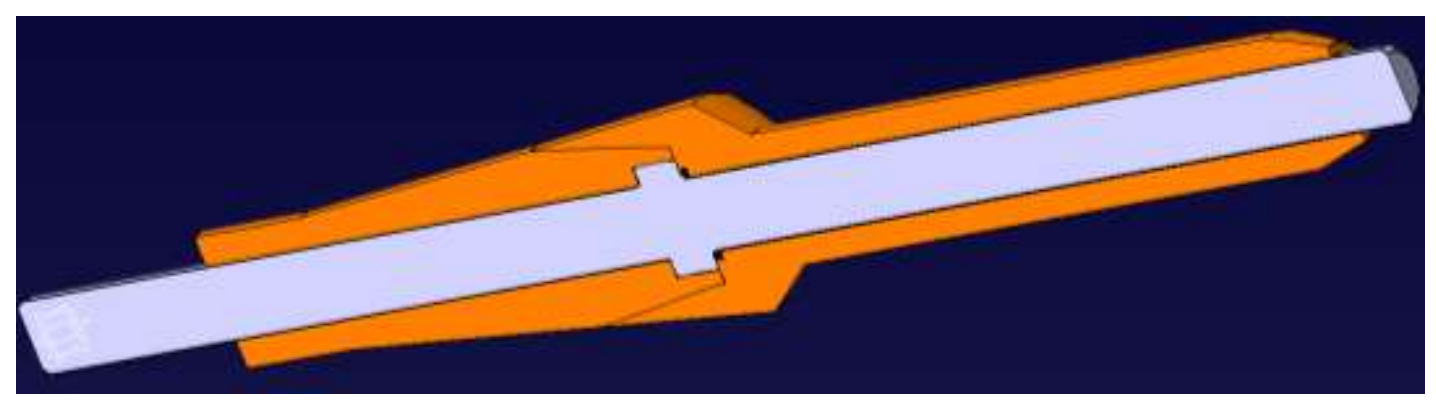

Figure 2-17. Cross-sectional view of assembled split electrode design. 
Again, all durability experiments took place at $12.1 \mathrm{~kJ}(11 \mathrm{kV})$ in the small 1.8 liter electrode testing chamber. This energy level has proven to date to be the average pulse energy used in forming panels. The large reduction in the volume of this chamber over that of production chambers has very significant effects on increasing chamber pressures, increasing erosion and presumably insulation damage. Thus tests made with this chamber yield very conservative electrode system durability estimates.

In these tests pulses are applied until either 2000 were successfully placed upon the system or insulation failure has been observed. Insulation failure was detected by one of two methods: 1) periodic inspections of the insulation inside the chamber every 100 discharges, and 2) observing any significant departures of the electrical performance from normal. If the latter condition was observed for two subsequent pulses, the chamber is opened and the insulation inspected.

The electrical performance was recorded for the first 10 pulses to establish a baseline, and thereafter for 5 subsequent pulses out of every 100 until failure. Pulses not recorded were, however, continuously monitored by the operator. The initial inter-electrode gap was set at $10 \mathrm{~mm}$, and the gap was checked during insulation inspections. For consistency, if the gap exceeded $13 \mathrm{~mm}$, the electrodes were advanced to re-establish the inter-electrode gap at $10 \mathrm{~mm}$. The other materials tested in the new electrode system include ultra-high molecular weight polyethylene, polycarbonate, acetyl, and polysulfone. The results of the insulation testing are summarized in Table 2-2 along with photos of the failures.

\section{Polysulfone:}

After only 46 pulses, the polysulfone completely failed at the point where the insulation enters the chamber - see Table 2-2 below. The failure was both mechanical and electrical with discharges occurring between the chamber wall and the electrode shaft straight through the insulation. The insulation failure, both electrical and mechanical, happened identically on both electrodes.

\section{Polycarbonate:}

Polycarbonate failed after 120 pulses in the chamber. Similar to but not as cataclysmic as the Polysulfone failure, both electrodes failed at the point of entrance into the chamber. The failure was both electrical and mechanical, as arcing to the chamber wall was evident.

\section{Acetyl:}

The acetyl fractured after 180 pulses in a fashion similar to the polycarbonate - at the point where the electrode enters the chamber, as seen in Table 2-2 below. This electrode failed in both a mechanical and electrical fashion. The other electrode failure was similar to the polyurethane failure in that it failed behind the bevel at the insulation tip and the tip was destroyed. 
Ultra High Molecular Weight Polyethylene (UHMW PE):

This material survived 700 pulses before significant plastic deformation and cracking was observed at the tips, as seen in Table 2-2. The damage observed is roughly equivalent to that of the polyurethane, and since the number of cycles is nearly the same, there is no compelling reason to use the UHMW PE over polyurethane.

Table 2-2. Insulation Testing Results.

\begin{tabular}{|c|c|c|c|c|}
\hline $\begin{array}{c}\text { Rank } \\
\text { Order }\end{array}$ & $\begin{array}{c}\text { Insulation } \\
\text { Type }\end{array}$ & $\begin{array}{c}\text { Pulses } \\
\text { to } \\
\text { Failure }\end{array}$ & $\begin{array}{c}\text { Failure } \\
\text { Location / } \\
\text { Failure Type }\end{array}$ & Visual appearance of failure \\
\hline \hline & $\begin{array}{c}\text { Ultra High } \\
\text { Molecular } \\
\text { Weight } \\
\text { Polyethylene } \\
\text { (UHMW PE) }\end{array}$ & 700 & $\begin{array}{c}\text { Tip / } \\
\text { Mechanical }\end{array}$ & $\begin{array}{c}\text { Tip / } \\
\text { Mechanical }\end{array}$ \\
\hline 3 & Polyurethane & 650 & $\begin{array}{c}\text { Chamber Entry } \\
\text { / Mechanical- } \\
\text { Electrical }\end{array}$ \\
\hline 4 & Polycarbonate & 120 & $\begin{array}{c}\text { Chamber Entry } \\
\text { / Mechanical- } \\
\text { Electrical }\end{array}$ \\
\hline 5 & Polysulfone & 46 & $\begin{array}{c}\text { Chamber Entry } \\
\text { / Mechanical- } \\
\text { Electrical }\end{array}$ \\
\hline
\end{tabular}

In the end the Ultra-High Molecular Weight Polyethylene (UHMW PE) and the polyurethane easily demonstrated the best performance among the group, but it was determined that the cost of creating UHMW PE electrodes far exceeded the minor life advantage found over polyurethane.

Insulation Protection Strategies

Since there were no insulation materials superior to polyurethane that were suitable for EHF applications, further research was dedicated towards attempting to protect the insulation tip from the pressure pulses emanating from the initial plasma formation. These experiments focused on placing a metal barrier between the pressure pulse and the edge of the insulation. 


\section{$\underline{\text { Nut welded to conical insulation tip electrodes - pressure side weld }}$}

In the first set of experiments conical profile insulation tip electrodes were taken and a reduced thickness nut was welded against the insulation cone to shield it from pressure pulses - as seen in Figure 2-18. The welds were placed on the side of the nut closest to the pressure pulse - but only after insulation was first cast onto the electrode shaft. The resulting electrodes failed after only 480 pulses (Figure 2-19) - a significantly shorter life than that observed for the conical insulation tip electrode alone.

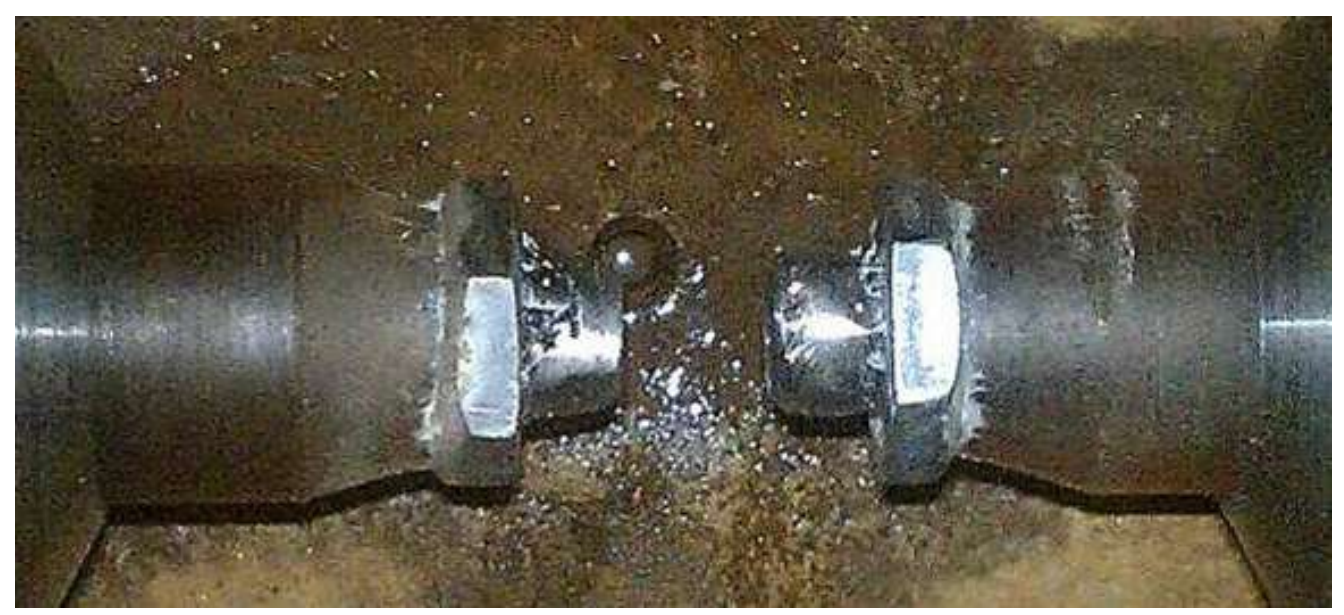

Figure 2-18. Half nuts welded to conical insulation tip electrodes, as seen before testing. Note that the welds were placed on the electrode tip side (not the insulation side) and some damage occurred to the insulation as a result of welding.

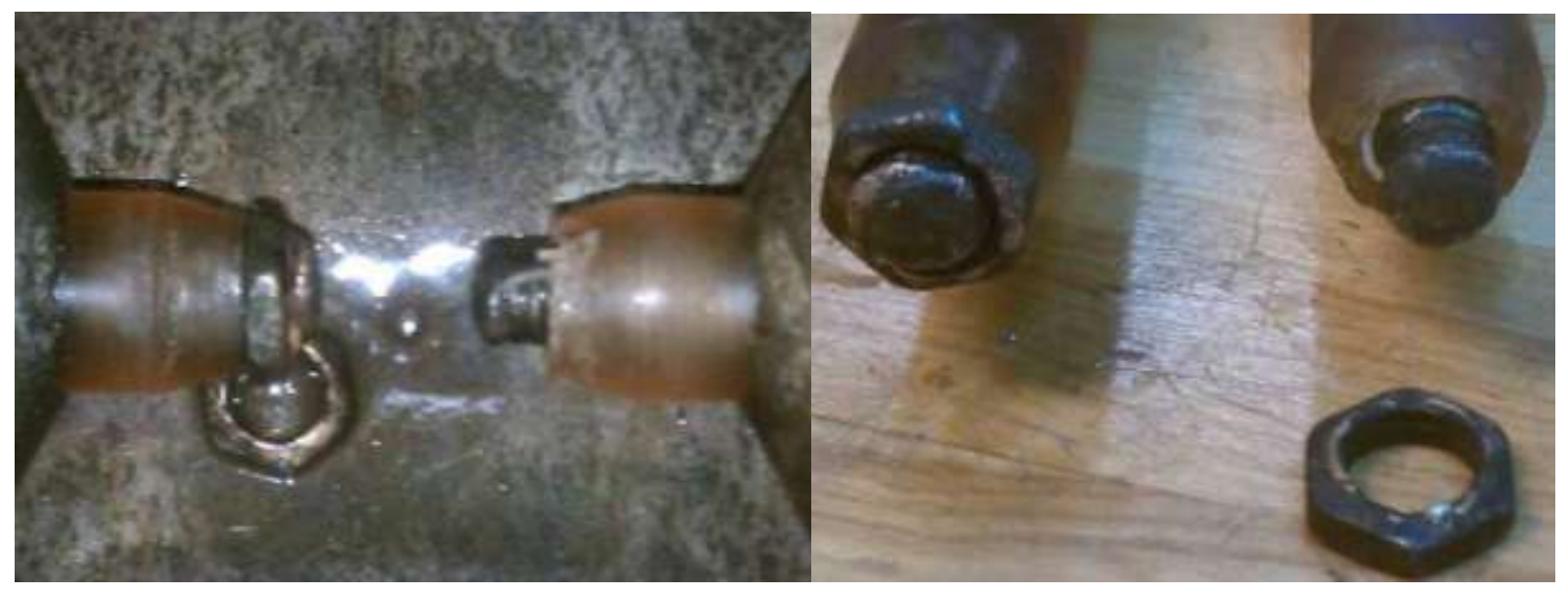

Figure 2-19. Results of test of electrodes with welded nuts as insulator protection - weld erosion and failure after 480 pulses. Both nuts were loose as significant damage occurred to threads after weld failure. 


\section{Custom machined nut welded to electrodes - insulation side weld}

The next experiment placed the welds on the insulation side of the shield, well away from the direct influence of the pressure pulse. In this case, custom machined shields were designed to protect the entire diameter of the insulation and to deflect the pressure pulse up and away into the chamber but still used the same conical shape that was successful in the test shown in Figure 2-15. The shield was welded from behind and the polyurethane then cast directly over the weld, creating a seamless joint between shield and insulation. The electrodes are shown both before and after testing in Figure 2-20. Even though significantly more pulses could have been applied before insulation degraded to an unusable level, the electrode test was suspended after 1000 pulses to preserve the insulation damage observed and documented in Figure 2-21.

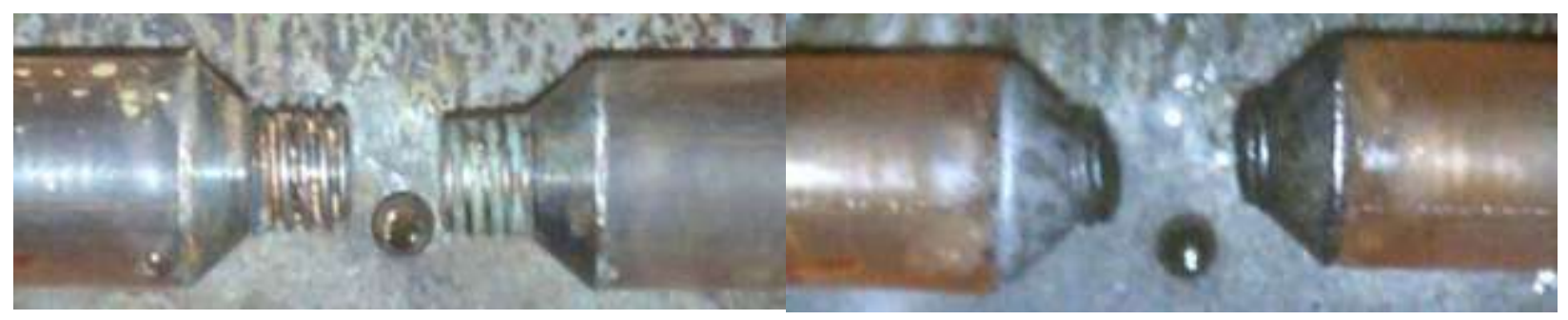

Figure 2-20. Results of electrodes test with machined insulation protection tip. Weld placed behind protector and insulation cast on after welding, as seen at left. Photo at right shows the electrodes in the chamber suspended after 1000 pulses were applied.

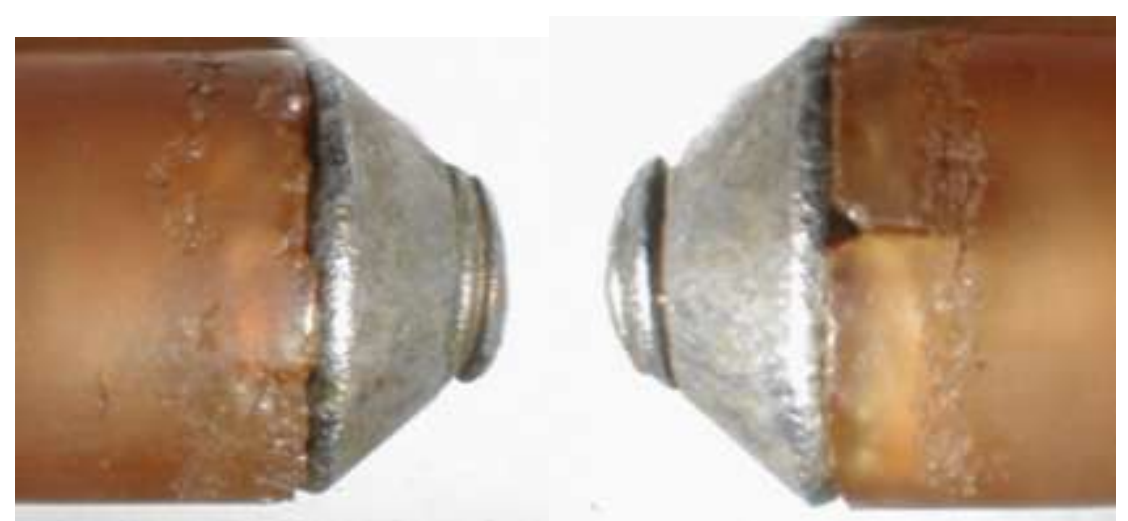

Figure 2-21. Detail of damage observed in test of machined and welded insulation electrode protection tip. Note general insulation erosion, chipping, and longitudinal crack formation.

1000 cycles in the electrode testing chamber represents roughly a $1 / 2$ shift worth of operational life under high volume production. Although visibly damaged, some operational life is clearly left, and the welds appeared undamaged. 
Tip protection integrated into electrode rod - no weld

A final experiment was performed using a new electrode design with the electrode tip shield and electrode rod machined as one and the insulation then cast on to the rod/shield. This test was performed in the 1.81 electrode testing chamber. While the test results, pictured in Figure 2-22, show that 2000 discharges produced significant but predictable erosion in the metal portion of the electrode, the insulation damage was confined to minor chipping with no significant cracking observed.

\section{Electrode Durability Summary}

Electrode erosion has been studied and it has been shown that the volumetric erosion rate is largely independent of tip shape but is a function of chamber volume. Tip shape has great influence on linear erosion rates: sharp conical tips have the worst (highest) erosion rates and flat cylinders having the best (lowest). As a result of a detailed study, an optimal tip shape was defined that minimized linear erosion, and the need for electrode system to be adjustable to maintain inter-electrode gap and optimum system performance was identified. In summary, electrode experiments discussed in earlier reports have shown that, with respect to erosion, the electrode rod durability has clearly exceeded the gate 2 requirement to survive 120 discharges.
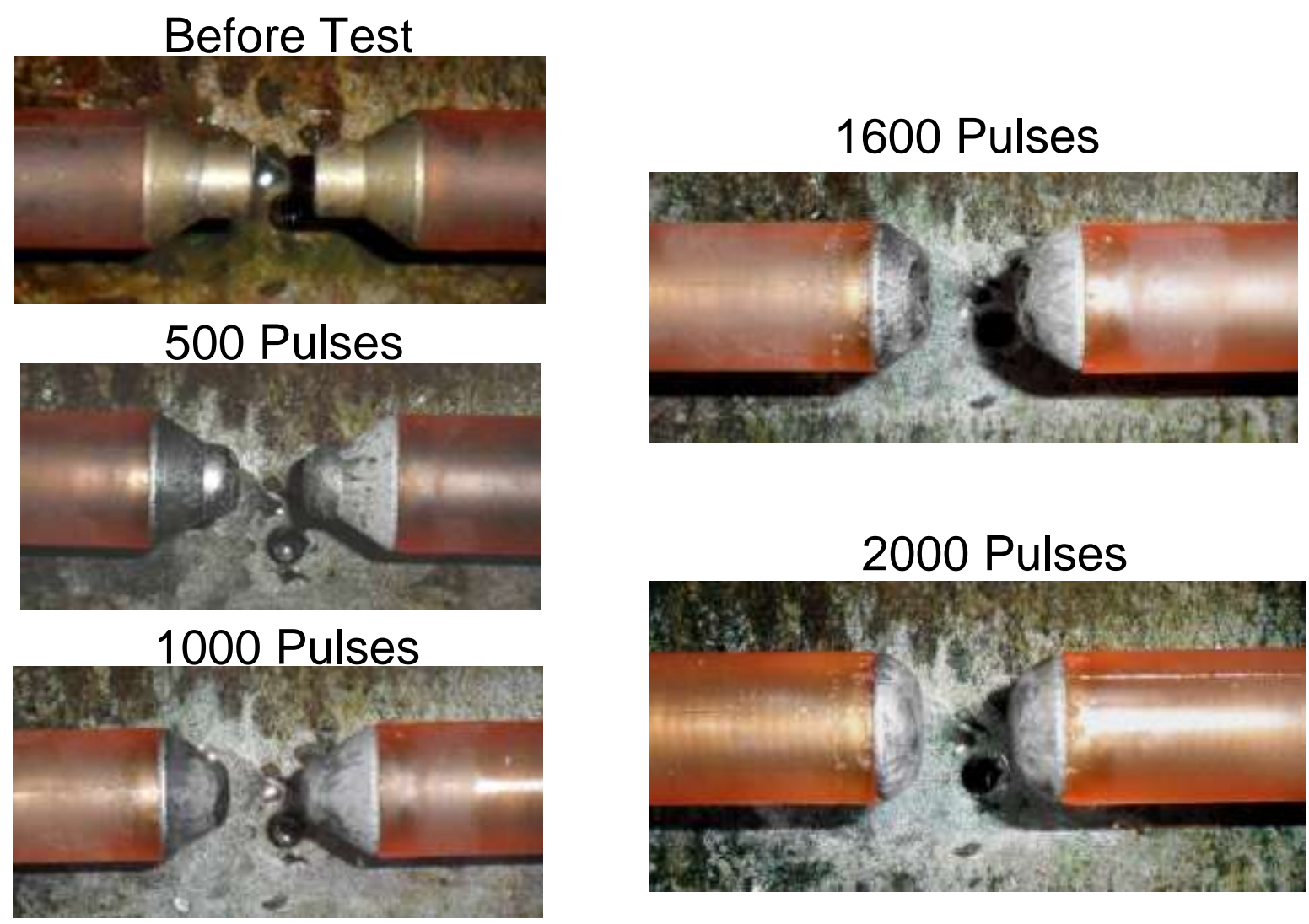

Figure 2-22. Results of durability testing on electrode with integrated protective shield. Electrode system lasted 2000 pulses - a life sufficient for the electrodes to survive a single day of production at high volume. 
An overall robust electrode system design has been successfully tested that meets the system requirements: seals water in under pulsed pressure, protects electrode from the mechanical forced induced by shock waves, conducts electricity safely in and out of the chamber, maintains electrode position within chamber under shock loading, and allows advancement of the electrodes to maintain proper inter-electrode gap.

\section{Electrode System Design}

After having achieved the required and desired electrode erosion performance benchmark for 2000 successful discharges with only minor performance degradation, an advanced electrode system suitable for production applications was developed and is the subject of US patent application 12/940235.

The electrode system is shown below in Figure 2-23. It has several components specifically designed to reduce and/or eliminate failure modes. The steel electrode has polyurethane cast about it and the holder fits the electrode hand-in-glove. The long conical surface that runs most of the length of the electrode serves to gradually shunt the forces in the electrode into the holder. The holder is threaded into the adapter and locked with a nut. The adapter serves to transfer the forces coming out of the holder and transmit them into the wall. The major improvements in this design over the previous generation are: 1) the conical support surface on the adapter serves to more effectively support the electrode from side loads and reduces the axial pressures borne by the holder; 2) the conical surface in the polyurethane insulation at the electrode tip acts to gradually deflect the pressure blast (and is far less likely to chip like the old version); 3) The electrode gap is easily adjusted by threading the holder farther into the adapter; 4) the forces in the chamber which try to eject the electrode are completely passed through to the holder rather than transferred through the length of the electrode and into a stanchion.

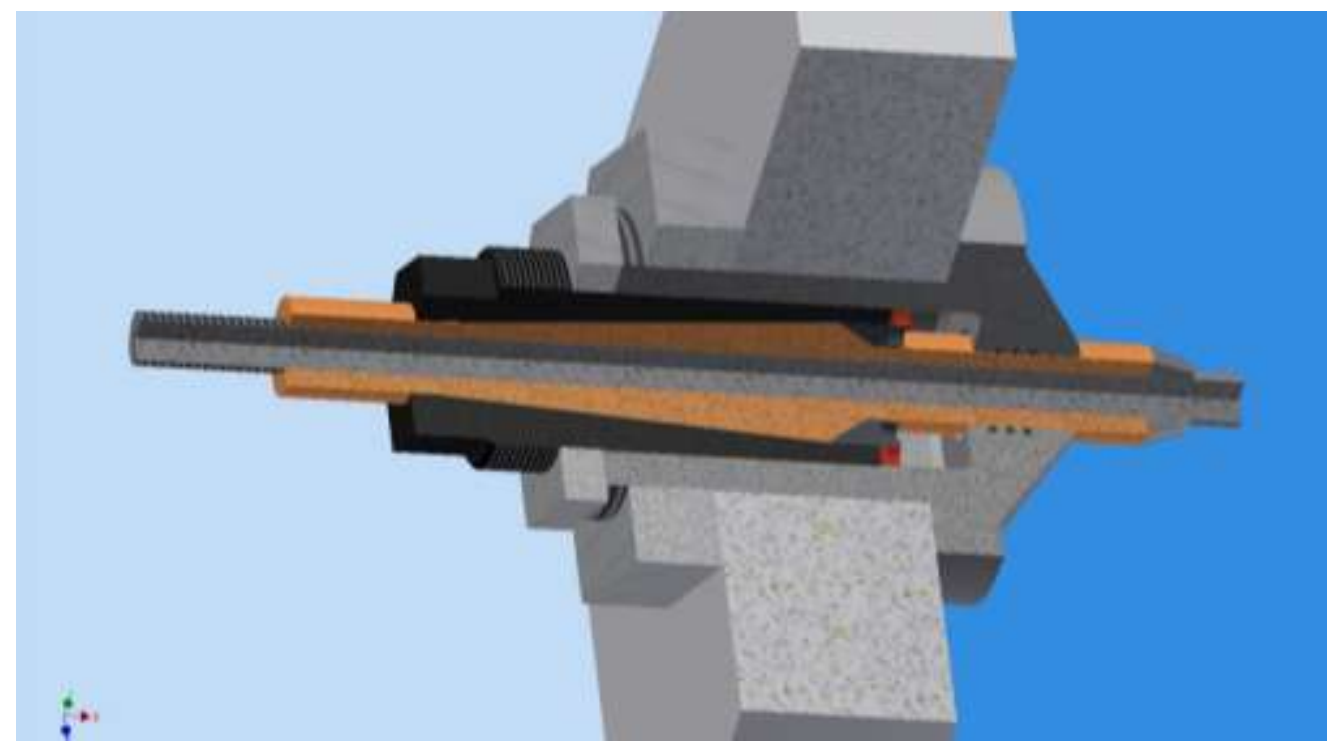

Figure 2-23. Cross-section of new electrode system showing the new system inserted into the chamber wall. 
This design, combined with the electrode testing chamber, has survived in excess of 20000 discharges through the course of electrode testing with occasional rubber seal changes. The electrodes were advanced and replaced successfully many times and the process parameters monitored.

\section{Electrode Monitoring System}

The performance of the electrodes is critical to the operation of the EHF cell. Short of a water leak in the chamber there are two eventualities which need to be detected and could cause poor performance: too large an inter-electrode gap, which causes general performance degradation, and insulation breakdown and shorting somewhere within the electrical delivery system electrodes are usually the problem. These problems and others can be detected through the pulse electrical characteristics.

Inter-electrode gap - Because of the deterministic nature of the electrode erosion rates and consistent electrode geometry it is anticipated that electrode adjustments may be made at a regular rate to maintain performance. Further, a too-large inter-electrode gap can be easily detected visually from the control booth when the chamber is opened. However, as a process check the electrical behaviors observed in Figure 2-13 are used to bound the problem. The current and voltage responses are fairly stable at the $20 \mathrm{~mm}$ gap level: the changes in current and voltage are small compared to the range of the signal and especially when compared to the variability in these measurements. The only reliable measure is the voltage pulse width (dwell) before breakdown which changes by almost a factor of two (from 0.9 to $2.5 \mathrm{~ms}$ ). The $0.2 \mathrm{~ms}$ noise level observed in these measurements is acceptable.

Bad Pulses - It makes sense that the longer the plasma channel remains active the more powerful the forming pulse is. In Figure 2-13 the current pulse reaches its peak in roughly $30 \mu \mathrm{s}$ - a time primarily determined by overall system inductance, and once it has reached the maximum the remaining period encompasses the expansion and collapse of the plasma channel. It then follows that an asymmetric pulse is preferable. Thus, a symmetric pulse is an indication of an early channel collapse, and has been correlated with misfires in the chamber where the positive electrode arcs to the chamber wall. A long series of these can indicate that there is an insulation failure in the electrode and maintenance is necessary.

Electrical Faults - A shortening of the pulse current rise time indicates that system inductance has changed and implies that a short has occurred somewhere in between the chamber and the pulse generator. The output of a second current sensor (ragowski) placed at the output of the pulse generator can be compared output of the one on the positive electrode, and any difference detected indicates a parasitic parallel breakdown path which must be repaired/eliminated. 
Algorithms for Determination of Critical Process Electrical Parameters

The discharge event marks the breakdown of the water in between the electrodes and the beginning of plasma channel formation. Again, note the predictable (and substantial) noise pulse that occurs in the current signal at the discharge initiation in Figure 2-24. The moment of discharge is usually simple to isolate, whether in current or voltage, because a large instantaneous and simultaneous value change is detectable in either channel, and, normally, it is detected by looking for sudden changes in slope. In a small minority of analyses this technique fails, and an alternative method in which three 40ns moving averages, each separated by $50 \mu \mathrm{s}$, are used as an edge detector that moves forward in time. When the angle that the averages make with respect to each other is minimized $\left(<<180^{\circ}\right)$, then the central average reliably locates the moment of discharge formation.

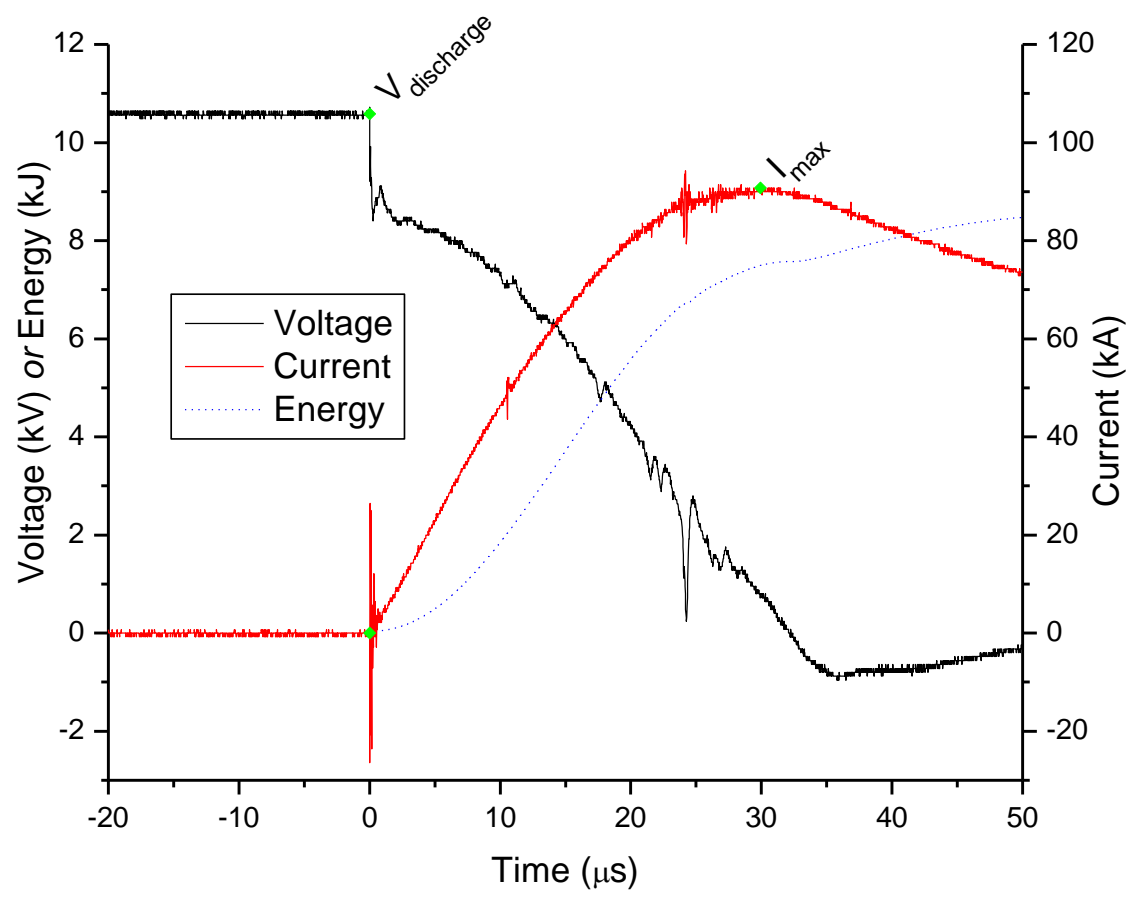

Figure 2-24. Detail of the moment of discharge.

Because of the amount of noise, the analysis of the current signal is far more problematic and required a more careful treatment. In this case, two $1.7 \mu$ s wide running averages separated by $3.4 \mu \mathrm{s}$ are swept across the current pulse starting at $\mathrm{V}_{\text {discharge. }}$ The width of the average was determined by finding the widest observed noise pulse out of a large dataset $-1.7 \mu \mathrm{s}$. The general vicinity of the current peak is then obtained when the value of the left hand average exceeds the right. Because noise spikes are commonly found in the current pulse, a very careful study of the peak region (peak location $+/-3.4 \mu \mathrm{s}$ ) is performed by the algorithm, and any data which greatly exceeds normal slew rates is ignored. The highest value of current is located in the remaining data. If a number of points share this maximum value, then the center of moment or centroid is taken as the peak location - this has the advantage of accurately locating the peak in datasets where noise occurs exactly at the peak. If no peak is located, then the acceptable slew rates are doubled and analysis is performed over again. The peak detection 
algorithm always calculates the value of the maximum using "valid" raw data and not the averaged data. The value of the average at the peak can be substantially altered by a meanshifted noise pulse, but the algorithm correctly locates the value and position of the maximum current.

From a process diagnostic standpoint calculating the pulse asymmetry is important because it is reflective of discharge quality, thus making determination of the end-of-pulse important. Symmetric pulses have been identified as "bad" pulses which do not impart significant deformation to the blank. However, determining end-of-pulse presents a challenge because of both the gentleness of the transition of the pulse to a zero (or flat) current condition and the ever-present noise spikes. Unfortunately, because of this difficulty, a fairly complex method is used to calculate the end-of-pulse. In the first step, very similar to previously described methods, two $5.8 \mu$ s wide moving averages separated by $7.5 \mu$ s are used to determine the general location of the end of pulse. Pulse end detection is complete when a combination of conditions are met, including: (1) the left hand average must be less than $3500 \mathrm{~A}$, (2) both the left average and the raw current signals be at a smaller current than the right hand average, and (3) the raw current be less than the raw current measured $15 \mu \mathrm{s}$ later (flatness/noise condition). This method usually over-predicts the length of the pulse, and, even with this fairly restrictive set of conditions, the location of the end-of-pulse requires refinement because of noise spikes near it that throw the determination off. Once this first rough endpoint is established, a scan for noise events is performed starting halfway between the current peak and the proposed end location. The position of any noise spikes found in this interval are logged and are used in a reverse time scan starting $15 \sqsupset \exists$ s past the proposed endpoint or last noise point (whichever is later). A cumulative average of the after-pulse rest current baseline is established and compared to the raw data signal. A second running average is kept which deliberately excludes data within noise spikes. A new endpoint is established if the difference of the cumulative average $(\bar{I})$ with both the running average $(\dot{\bar{I}})$ and the instantaneous raw signal $\left({ }^{I(t)}\right)$ exceeds either $275 \%$ or $75 \%$ of the current quanta ( $I_{\text {quanta }}$, the data resolution of the oscilloscope in amps), depending on whether the analysis location is within a noise spike or not, respectively. Written perhaps a bit more clearly, an endpoint is established when $(\dot{\bar{I}}-\bar{I} \geq \varphi) \cap(I(t)-\bar{I} \geq \varphi)$, where $\varphi=2.75 I_{\text {quanta }}$ if processing within a spike and $\varphi=0.75 I_{\text {quanta }}$ elsewhere.

A great deal of effort has been made in these algorithms to measure the curves based on the raw signals rather than overall signal averages. In part, this is because averages smooth out real transitions which are sharp (see the averaged current at the start of the current pulse in Figure 2-5 compared to the raw) and are fooled by spikes which have means substantially above or below that of the true signal (see tail or peak of pulse in Figure 2-5). Analyzing an averaged pulse signal usually results in process parameters which are both time and value shifted. 


\section{Algorithm output}

At the end of the analysis, the algorithm outputs the data, truncated to within $50 \sqsupset \mathrm{s}$ of the start and end of the voltage and current pulses, which contains the raw time, voltage, and current signal along with labels indicating the locations of $t_{\text {init }}, V_{\max }, V_{\text {charge }}, V_{\text {discharge }}, I_{\max }$ and end of pulse. The time base of the events is rewritten so that 0 is at the moment of plasma channel formation. Optionally, it can output an averaged current profile based on a $3.4 \sqsupset \mathrm{s}$ window. The algorithm also outputs some further simple analysis including the height of the current pulse, the time from discharge to current peak ( $100 \%$ rise time), the overall width of the current pulse, width of the voltage pulse, and a measure of the current pulse asymmetry factor, which when calculated with the time zero set at the moment of discharge formation is simply tEnd Pulse $/ t_{\text {rise }}$. Lastly, the program optionally outputs the cumulative energy spent in the chamber. All of these parameters can be seen in Figure 2-25.

One last note in all of this: a significant shift in system-wide inductance which changes the timescale of these events will require reassessment of the many time-constants discussed above. It is expected that the basic nature of the algorithms will be unchanged. The techniques described above will have to be substantially modified/replaced in the case of pulse generator without a crowbar (or diode clamp) across the outputs such as our Pulsar pulse generator unit.

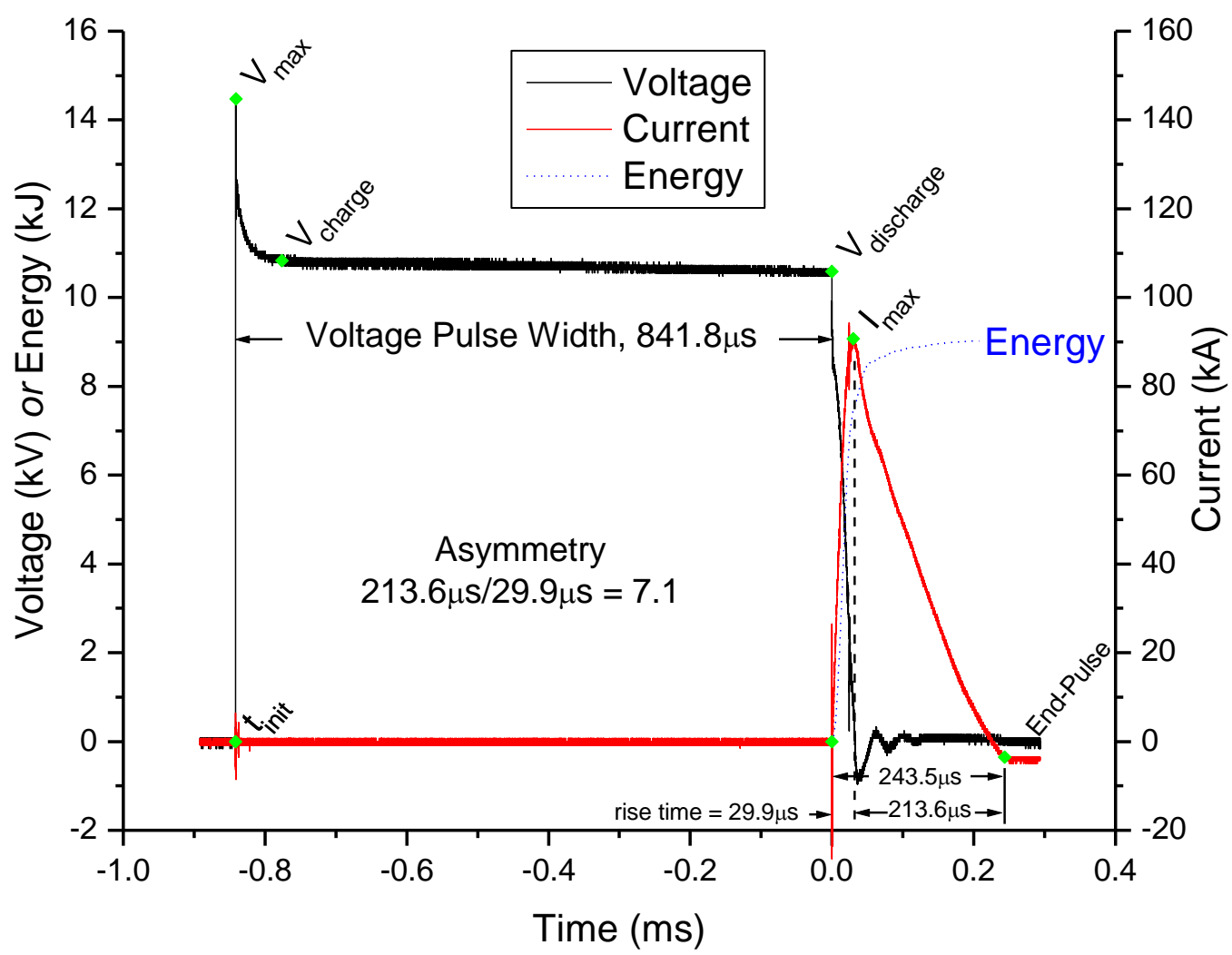

Figure 2-25. Graph showing full analysis of EHF electrical signals, including $t_{\text {init, }}, V_{\max }, V_{\text {charge, }}$ $V_{\text {discharge }} I_{\max }$, end of pulse, $100 \%$ rise time, pulse width and asymmetry factor. 
Task 2 Summary

EHF electrode erosion was explored for a number of different materials and it was determined that low carbon steel provided the best performance. For a given material and energy, it was discovered that, regardless of electrode configuration, the volume of material eroded was constant and the resulting stable eroded tip shape was consistent. Studies of electrode discharge parameters indicated that the discharge behavior was stable across a wide range of inter-electrode gaps. Ultimately these studies indicated that the process was stable and controllable across a wide input range, and that the process health can be monitored though the discharge electrical characteristics. Durability of the electrodes exceeds the one production shift requirement.

Electrode durability as a system was explored through the EH forming, trimming and calibration applications. A correlation between the parameters of the discharge and the electrode system performance was established. The variation of the discharge parameters was examined in conjunction with changing conditions inside the chamber, and based on this understanding criteria have been developed for electrode adjustment and for process health monitoring. A rapid electrode adjustment procedure has been developed and demonstrated. The schedule of electrode adjustment providing stable chamber performance has been formulated. 


\section{Task 3. Demonstration of EHF process automation and equipment at production rates}

\section{Sub-task 3.1. Develop an efficient water filling/evacuation system for the EHF chamber}

Fluid Handling System (FHS) - Specific benefits of the system

The final design specifications for the FHS were very broad and included capabilities for high pressure sheet hydroforming. The FHS includes a complex valve and sensor system designed for system flexibility and a high fluid transfer rate, up to 108 GPM, establishing a completely closed recycled water system. All of these specifications had the underlying intent of achieving 1) a semi industrial system, 2) rapid cycle time, and 3) maintaining a consistent environment within the chamber with a closed loop system. Figure 3.1.1 shows the entire circuit diagram of the fluid handling system.

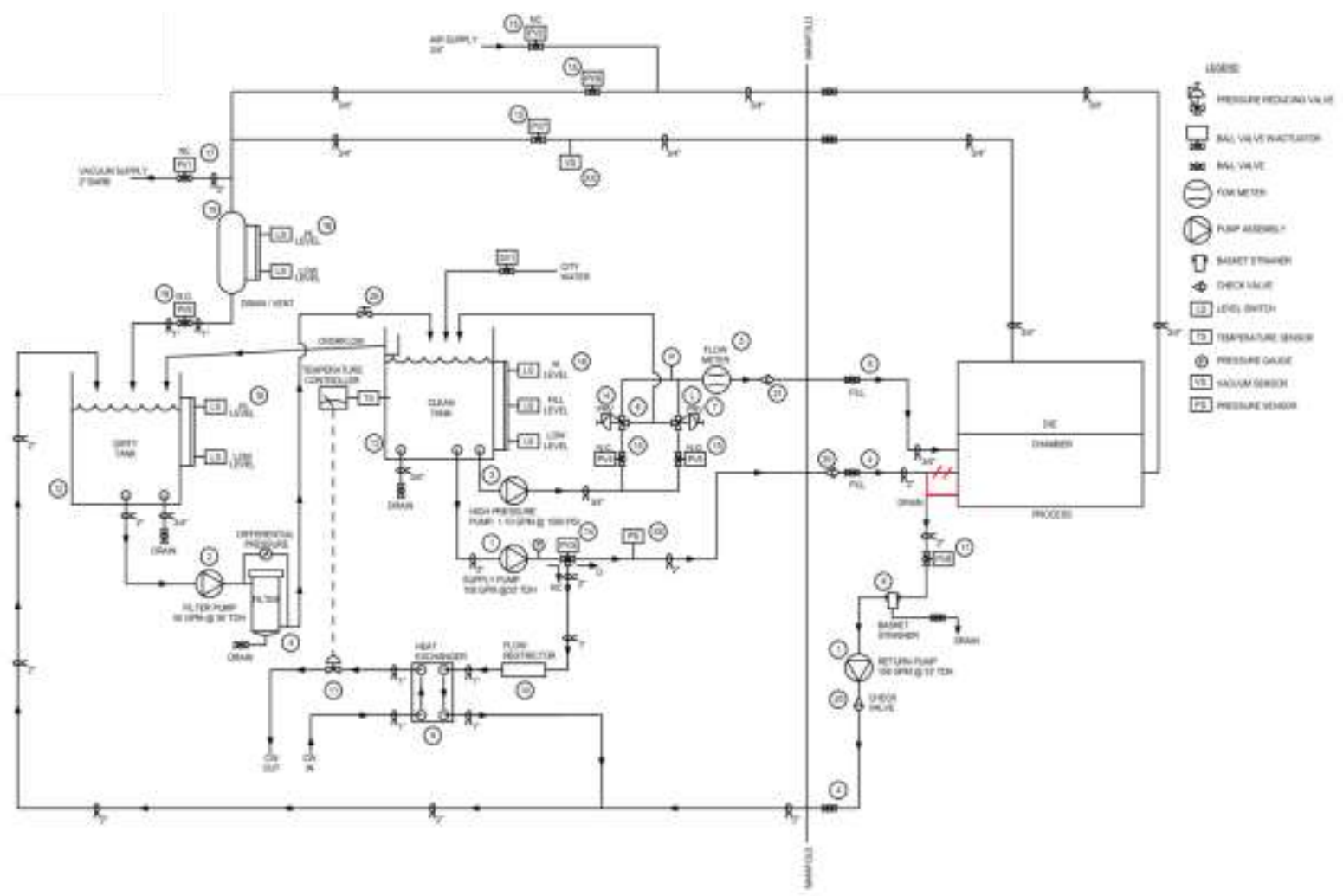

Figure 3.1.1. Fluid Management System (FHS) circuit diagram.

The function of the low pressure (15 PSI), high volume (108 GPM) circuit is to rapidly fill and drain the chamber. Initially the chamber is filled to a known volume using the high volume circuit. It is also used once the part is fully formed to drain the chamber. Flow meters for 2" pipe have a very low resolution of $\pm 100 \mathrm{~mL}$ requiring the experimentally determined time constants for both draining and filling. 
A high pressure (1500 PSI), 15 GPM circuit is used to replenish the fluid volume between pulses. The flow sensors attached to the high pressure circuit have a resolution of $\pm 5 \mathrm{~mL}$ and $3 / 4$ " pipe. The fine resolution and control of the high pressure circuit provided an opportunity to closely measure the progress of part formation between pulses. When the sheet metal blank is deformed by an EHF pulse, that volume displacement must be rapidly compensated by fluid to prevent part oil-canning. The second function of the high pressure circuit is used for sheet hydroforming. During hybrid forming, high quasi-static pressure is first applied to the blank as an initial forming step, and the part is then fully formed through subsequent EHF pulses. The advantage of the hybrid forming is that it reduces the number of pulses required and thus the significant reduction of cumulative time required to charge the pulse generator.

Packaging the FHS in the automation cell and optimizing the locations of the subsystems to achieve high cycle was a challenge. Figure 3.1.2 shows the overall layout of the test cell. In the upper right corner of the diagram is the heart of the system pictured in Figure 3.1.3. It is a two skid system that incorporates the high pressure pump, high volume fill pump, 100 gal. clean tank, and the $100 \mathrm{gal}$. dirty tank. The system has a separator tank for removing the hydroforming fluid from the vacuum system and is used during the evacuation of the chamber and die. Finally, to rapidly drain the chamber, air pressure is applied to the chamber to push the fluid out of the chamber.

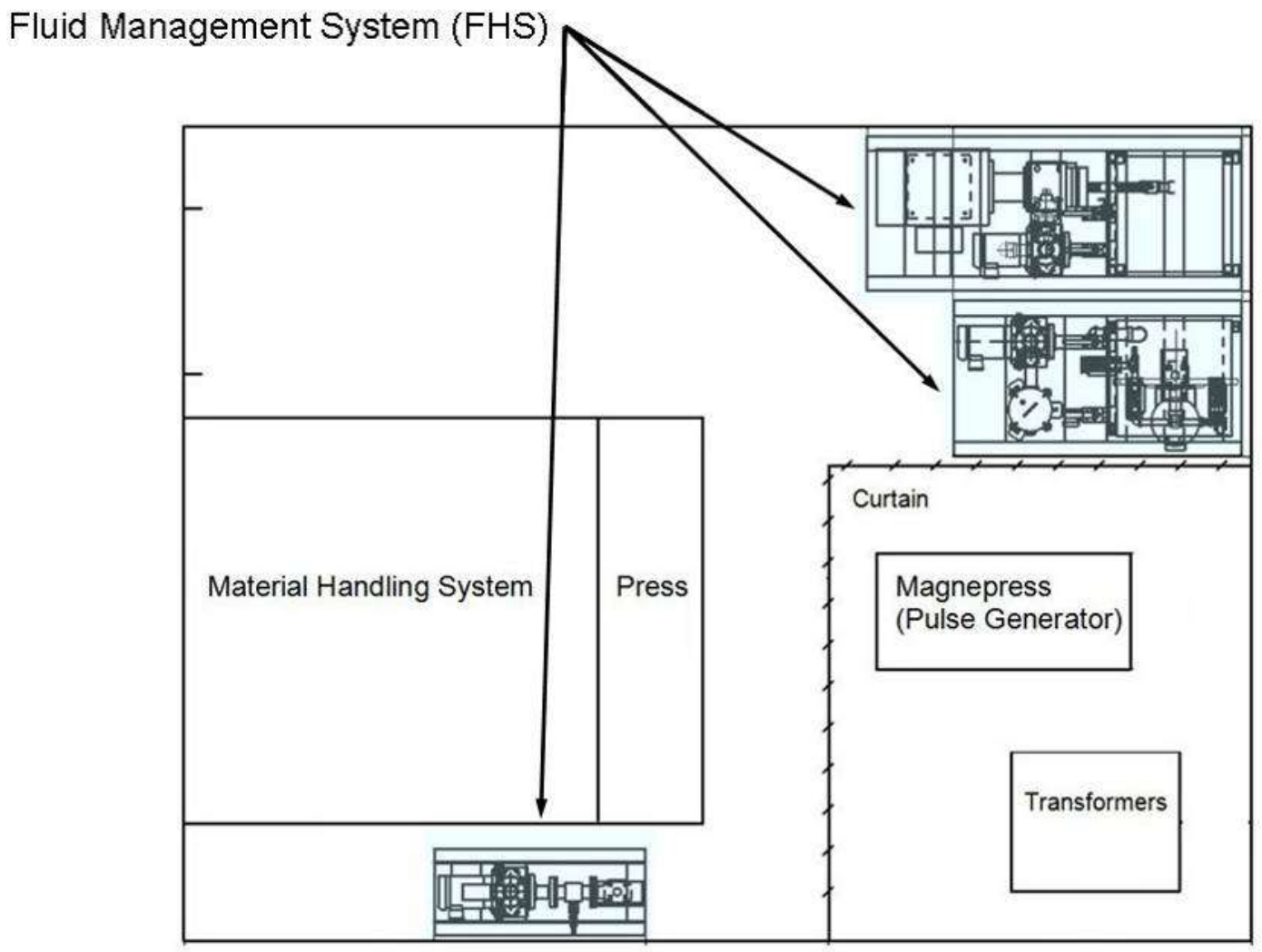

Figure 3.1.2. Automation room layout with the Fluid Management System (FHS). 

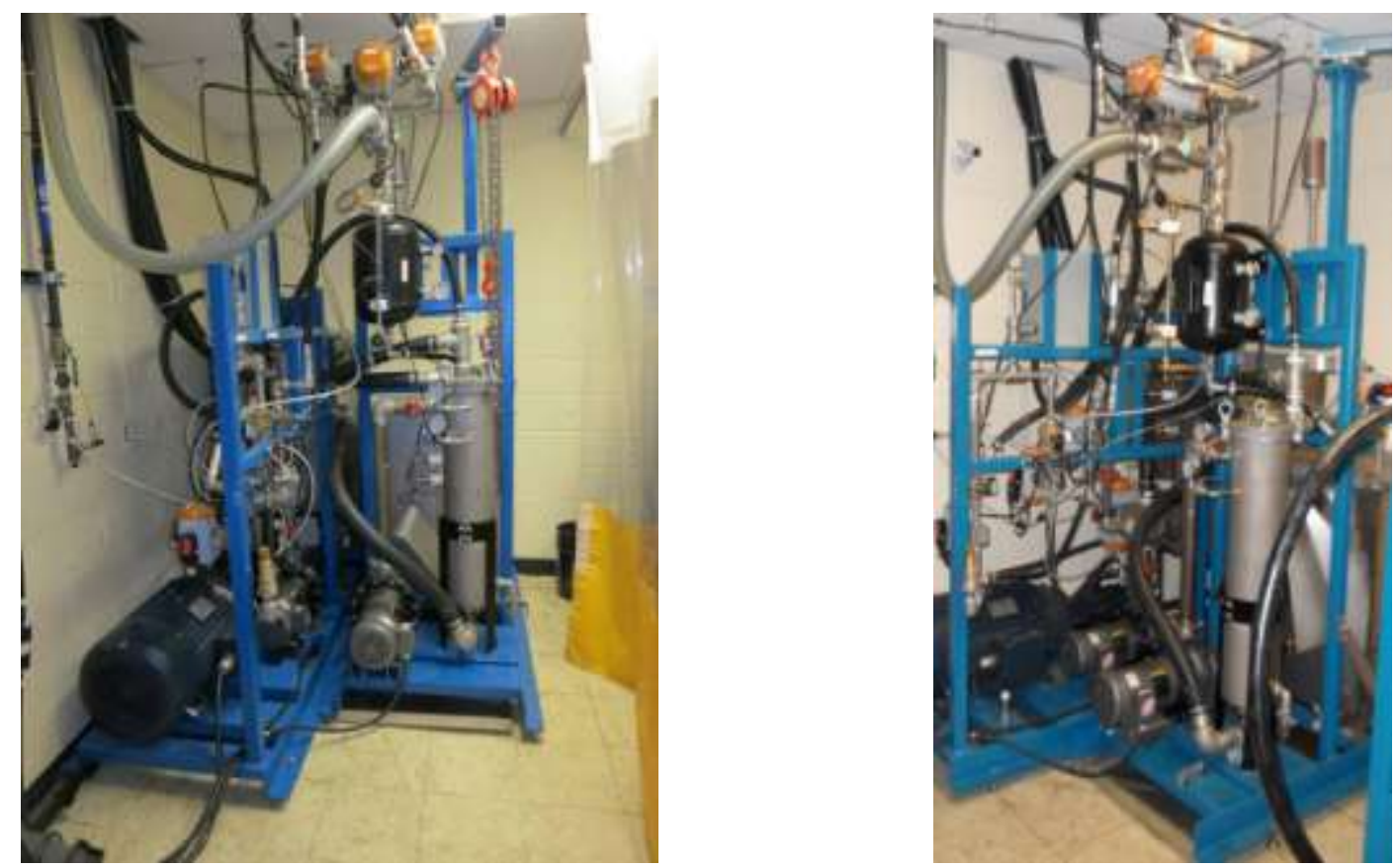

Figure 3.1.3. The two-skid, primary portion of the Fluid Handling System.

The third skid incorporated in the fluid handling system is shown in the bottom of the diagram of Figure 3.1.2 and pictured in Figure 3.1.4. It provides functionality for opening and closing vacuum, pressure, drain, and fill lines as close to the chamber as possible. The fill and drain lines are 2" in diameter, $30 \mathrm{ft}$. in length and routed up through the ceiling and down to the main skids. A significant amount of fluid is required to fill or drain back to the core fluid handling system. For efficiency, the final valves for filling, draining, pressure, and vacuum are located on the third skid as close to the chamber as possible.

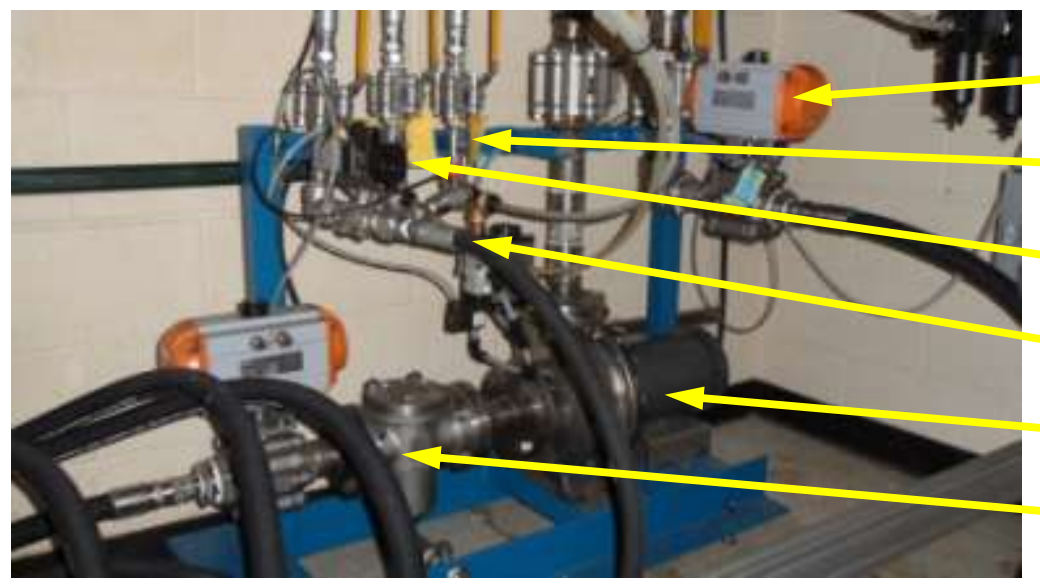

Low pressure /high volume fill

Vacuum

Air pressure

High pressure /low volume fill

Drain pump

Drain and screen filter

Figure 3.1.4. The third Fluid Handling System skid with the drain pump, fill manifold and valves for vacuum, high pressure fluid, low pressure fluid, air pressure, and vacuum. 
Maintaining a consistent environment within the chamber with a closed loop system was a challenge due to the rate the fluid in the chamber is fouled, and the overall volume of fluid being moved at a steady rate. During early experimentation we found that the particulate in the fluid impacted the blank, degrading the final part surface quality. It is possible to protect the blank from the electrode debris with a diaphragm. However, evacuating the chamber between parts is sufficient. Additionally, the debris would rapidly damage the pumps and valves in the fluid handling system. Figure 3.1.5 shows fluid samples collected from the FHS. Bottle 1 is water taken from the EHF chamber after 50 pulses with no filtration. Bottle 2 is from the chamber after seven pulses with no filtration. Bottle 3 is water from the FHS clean tank after filtration between parts and over 7000 pulses. The filtration process utilizes a bag filter with magnetic assist.

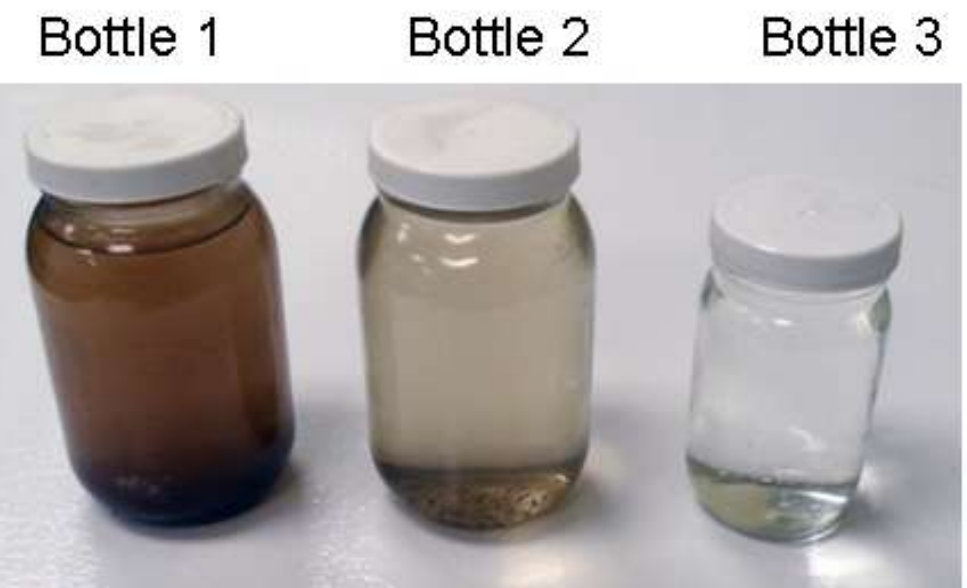

Figure 3.1.5. Fluid samples from FHS: Bottle 1 - 50 pulses, Bottle 2 - 7 pulses, and Bottle 3 7000 pulses with filtration.

A combination bag filter with a magnetic rod insert was found to be the optimal configuration for fluid filtration. The primary contaminate in the fluid is the erosion of the electrode tips and collects on the magnetic rod shown in Figure 3.1.6. Collecting the metallic slivers on the magnetic rod significantly extended the life of the cellos bag. Over 1000 parts were formed to collect the amount of debris on the magnetic rod.

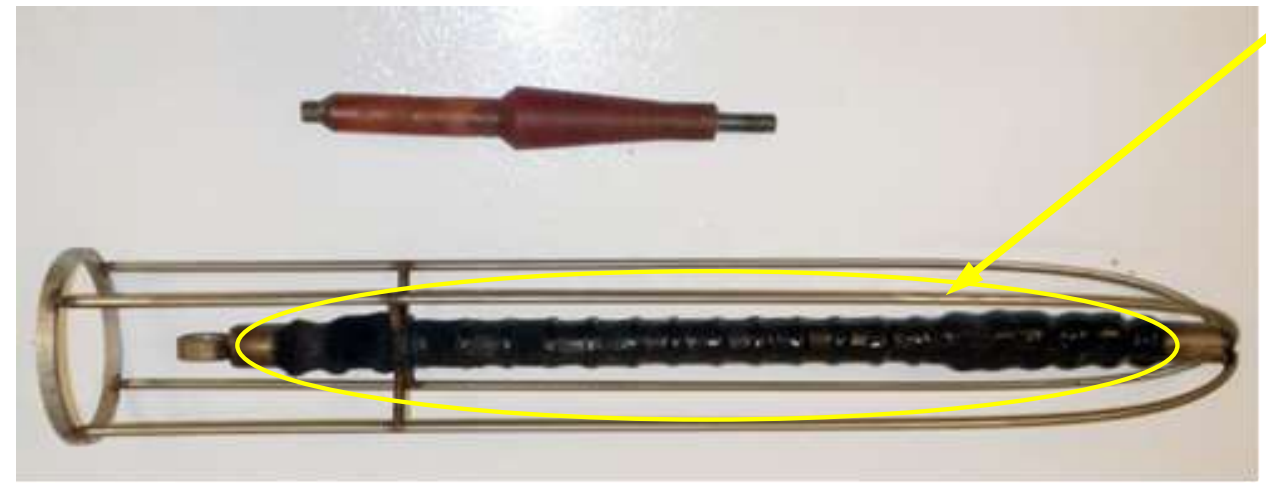

Ferrous electrode erosion collected on the magnetic assist rod.

Figure 3.1.6. The magnetic rod in the center of a cellulose bag filter that collects the ferrous electrode erosion. 
One factor initially not considered was the room humidly generated by the Fluid Handling System and the risk of having the high voltage pulse generator in the same local space. To avoid any risk causing a fault within the pulse generator an industrial grade dehumidifier capable of extracting 270 pints of water per day was installed. The dehumidifier eliminated any noticeable humidity change and the water collected was pumped into the FHS dirty tank.

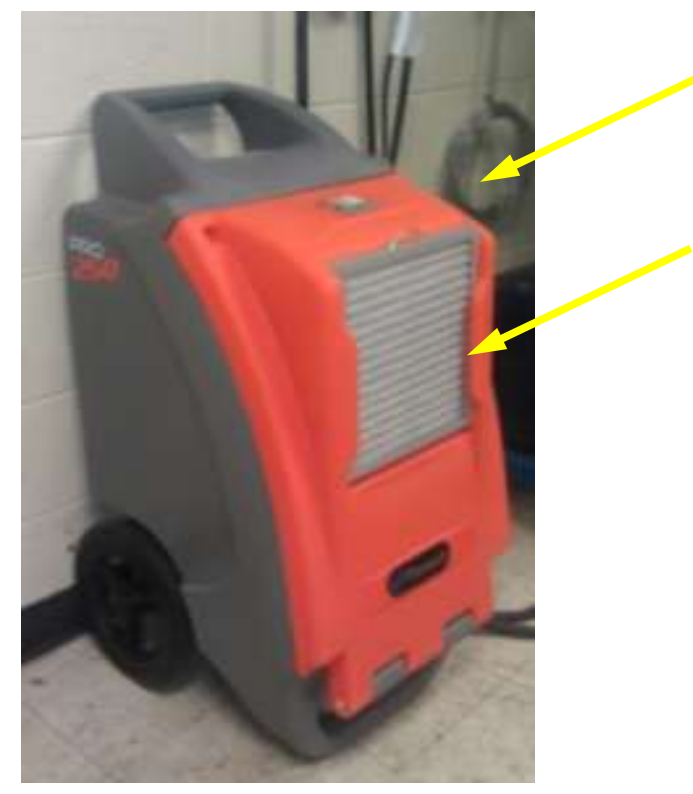

Water collected is recycled by pumping it into the Fluid Handling System's dirty tank

Edison Pro 250 commercial dehumidifier

Figure 3.1.7. Edison Pro dehumidifier capable of removing 270 pints of water per day.

Summary of Task 3.1: Develop an efficient water filling/evacuation system for the EHF chamber.

A Fluid Handling System (FHS) was developed to rapidly manage the electrohydraulic forming process that requires evacuating the chamber of any air, rapidly filling with fluid, refilling between pulses and then draining the chamber. The system is capable of filling and draining the chamber at 108 GPM and the actual times is limited by the size of the orifices in the chamber wall. The system has a highly controllable high pressure circuit that can precisely fill the void after a pulse and measure it at a resolution of $+/-5 \mathrm{~mL}$.

The flexibility designed into the system enables initial bulk deformation of the blank through sheet hydroforming up to $1500 \mathrm{PSI}$. The initial bulk deformation reduces the number of pulses required to form the part.

The system is a closed loop capable of continuously circulating water through the chamber while pulsing. The filtration system is capable of running for over a week with two shifts per day without maintenance. 


\section{Sub-task 3.2. Study of sealing materials}

A functional sealing system is of clear importance to the implementation of EHF in production. Most extant sealing systems seal between parts where their relative motion is limited and the surfaces are ground and polished leaving minimal roughness [3-1]. The challenge in EHF is that the sheet metal that is drawn across the seal has a relatively rough surface and further has the potential to be wrinkled during the draw. After an extensive literature search, it was determined that there were no systems or even data extant for this kind of application, and the effort rapidly became one of research and development.

Simple Seal Analysis and Development - Finite Element Analysis of Simple Seals

Figure 3.2-1 shows an LS-DYNA model of a standard rubber seal in a groove under EHF conditions with an Advanced High Strength Steel (AHSS) being drawn across the top.

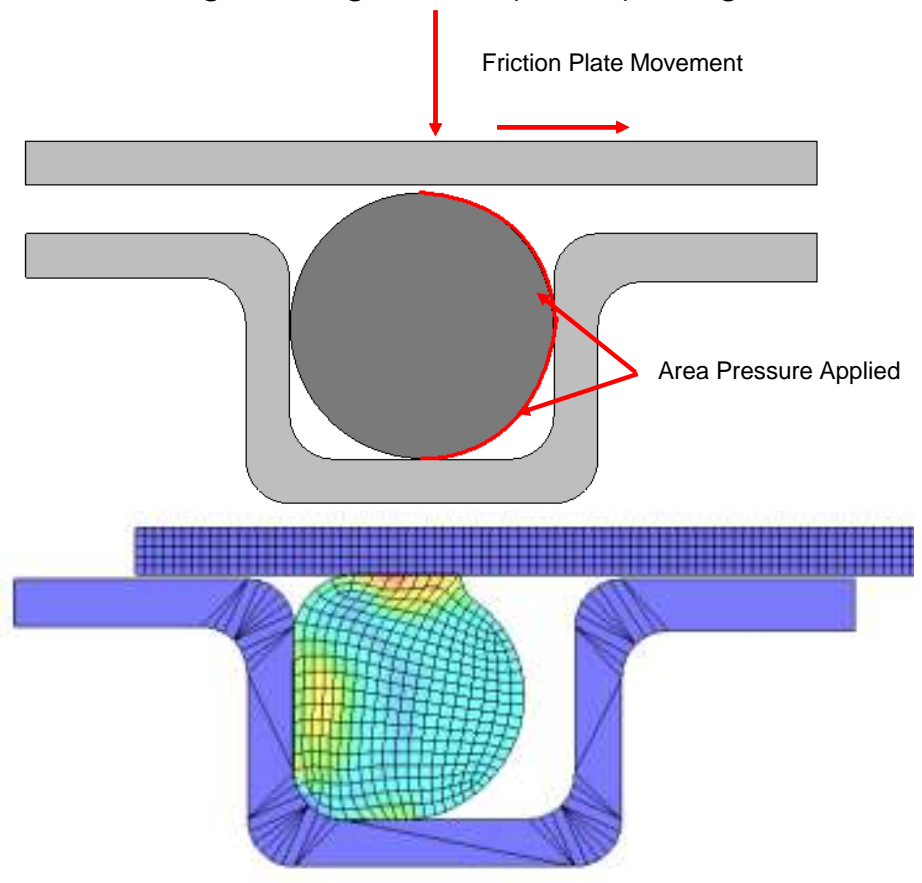

Figure 3.2-1. Round seal simulated using explicit code LS-Dyna.

Several seal models were created and simulated using the commercial, explicit finite element (FE) code LS-DYNA as well as the implicit code FE MSC MARC. Note that the model captures the expected seal behavior and shape - first the seal is crushed into the groove and pressure is applied through the fluid at right as the sheet is drawn across the seal - as the seal is flattened, displaced leftward in the groove, and the top is sheared in the direction of sheet motion. The peak von Mises stress in the seal of $145 \mathrm{MPa}$ was predicted. This peak stress is identical with the pressure that the seal can effectively contain. 
It was determined that, given the large strains, more complex materials models needed to be used, and the Mooney-Rivlin model was selected. Care was taken to develop the constants that drive the model directly from the materials themselves rather than textbook values, necessitating materials testing including compression and tension testing to failure. Further, the tests were conducted to identify the coefficients of friction specifically involved with these materials and surfaces. The resulting model shown in Figure 3.2-2 was run in MARC.

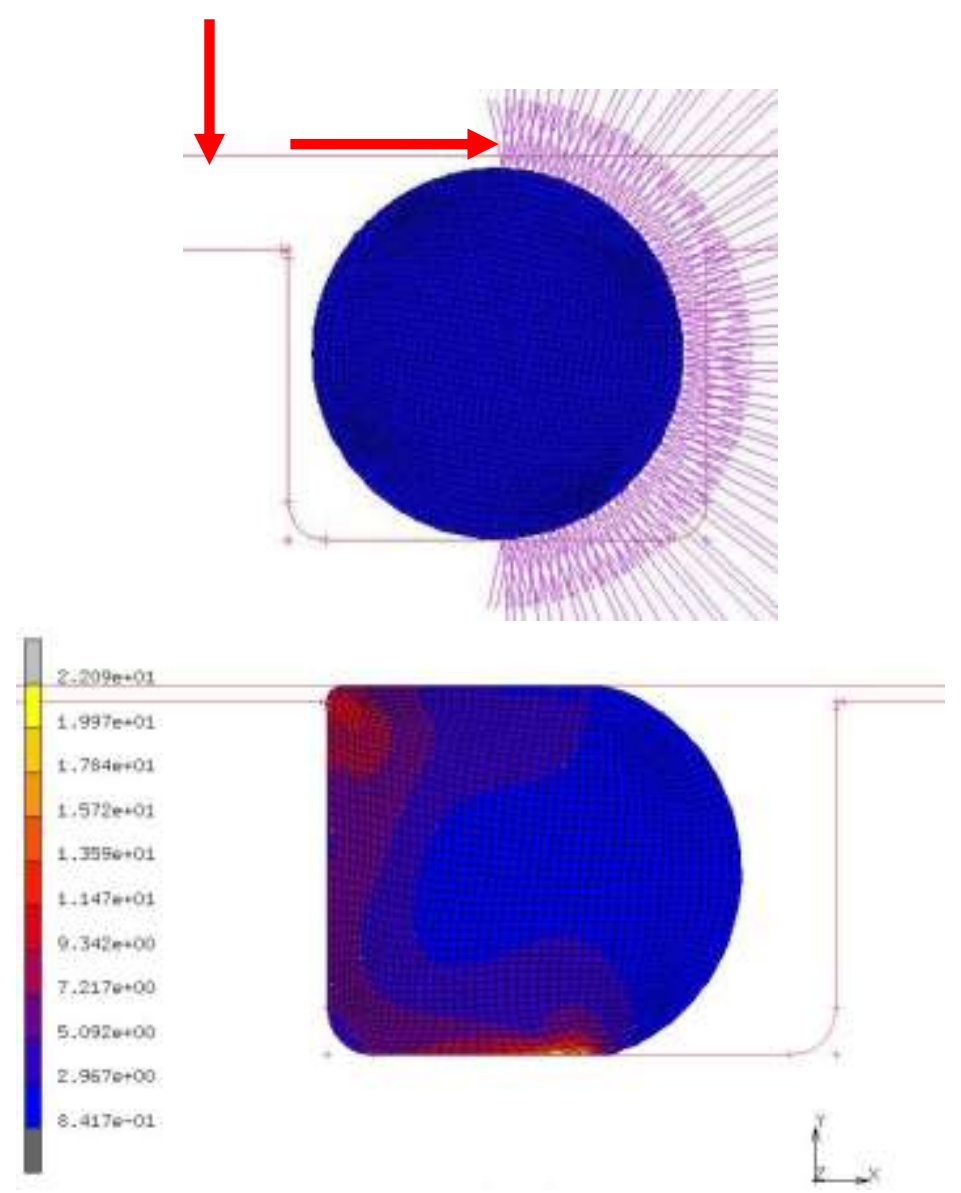

Figure 3.2-2. Round seal simulated using implicit code MARC and employing the Mooney-Rivlin material model.

The models successfully predicted the single groove forces and transverse seal deflections. The forces required to fully compress the seals were validated experimentally, and the contact forces were validated using pressure film.

Results from FEA analysis and validation were used to design a system that would test these seals under wear/fatigue conditions. Different seal materials of the same dimensions were simulated, and the resulting reaction forces on each type of seal were used to predict the maximum force required for squeezing the seal entirely. 


\section{Experimental Testing of Simple Seals}

A cyclic testing system for testing these seals was designed and fabricated. The movement of friction plate each time the seal squeezes would test the seal in abrasion and fatigue life. This system was designed, installed, and calibrated such that would satisfy the drawing of sheet metal into a die cavity, generating abrasive wear data on different seals. The tool for the seal wear study includes two $5000 \mathrm{lb}$. hydraulic actuators mounted into a three platen die shoe system with removable groove plate and a friction plate with a sheet metal surface that simulates the drawing of the blank. A plate with two similar groove channels was designed for each specific cross-section seal, and wear data were recorded. A schematic of how the system works is shown in Figure 3.2-3, where the movement of the groove plate is such that it satisfies the fatigue testing of the seal. The actual system is pictured in Figure 3.2-4, and the plate carrying the seals is shown in Figure 3.2-5.
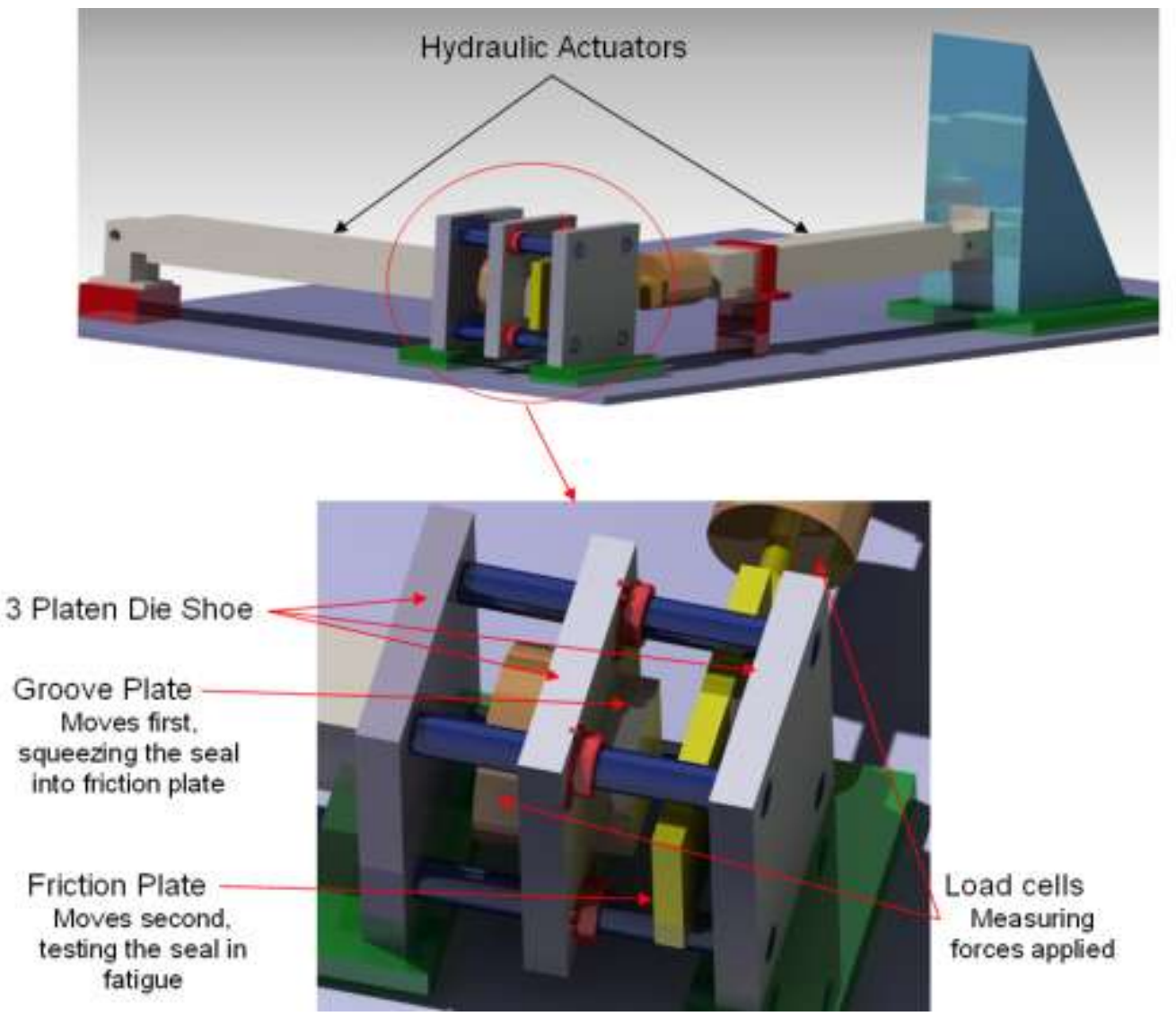

Figure 3.2-3. Diagram of the testing fixture for cyclic loading of sealing. 


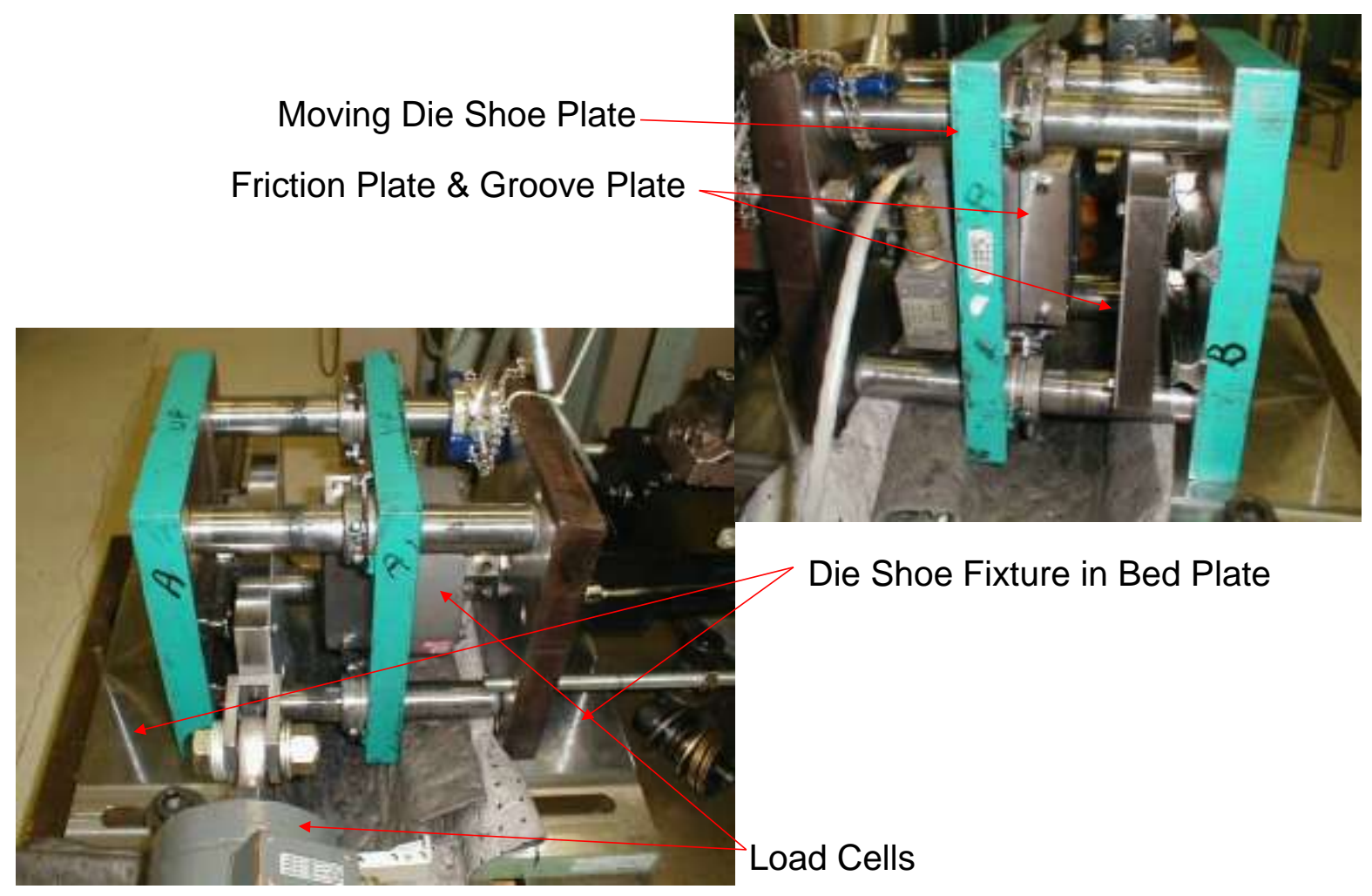

Figure 3.2-4. Actual fixture for cyclic loading of seals.

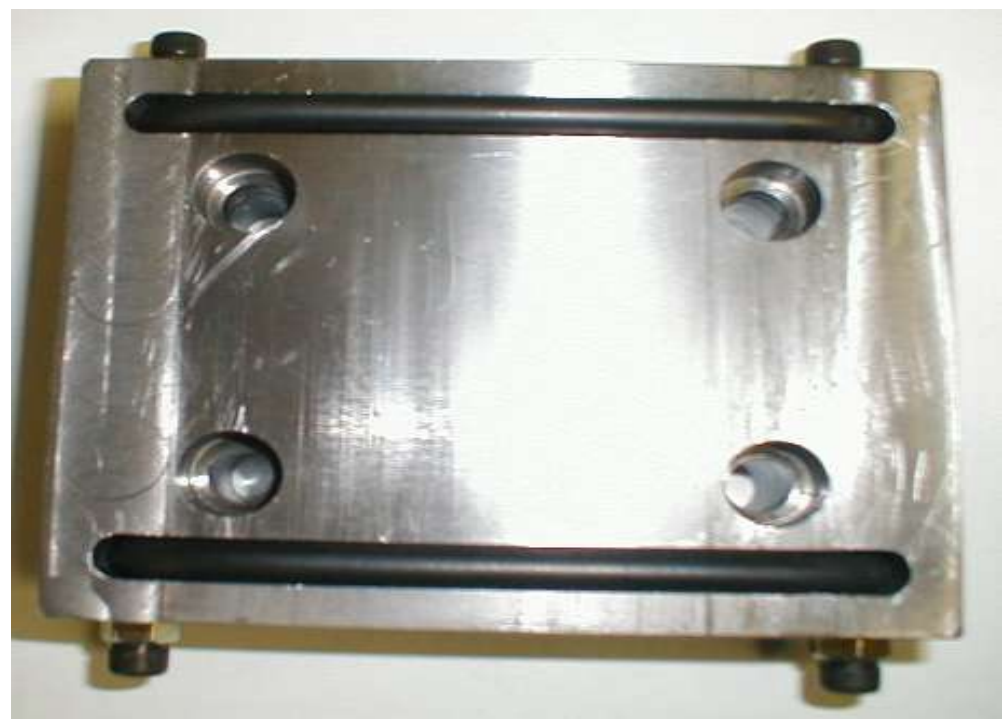

Figure 3.2-5. Groove plate carrying two rubber seals for wear testing. 
The seal-carrying (or groove) plate carries two seals and the actuator stroke was set such that the travel of one did not cross the track of the other. The cycle was set so that the seals only saw frictional motion in the drawing direction. The clamping load on the plate was set at 2000lbs, a load equivalent to that expected during drawing for this seal length, and all seals were tested for 2000 cycles. Tests on seals were conducted in dry as well as lubricated conditions. The weights of seals before and after the tests were taken with a microgram balance and the difference used as wear indicator for the tests. The cross-section diameter of the seal tested on both grooves was recorded before and after installation in order to determine stretching during installation of seals in each test. The results of testing on $1 / 4$ " diameter neoprene rubber seal material are shown in Figure 3.2-5.

Under dry conditions the seal wear is significant after 2000 cycles. Of 20 seals tested there was an average about $1.87 \%$ of the seal mass was lost but the standard deviation on the tests was with a standard deviation of $0.6 \%$. A high of $3.3 \%$ and a low of $0.72 \%$ mass were also observed in the unlubricated condition. The extent of the seal mass loss under this condition was too great and too variable to use in a production environment. However, a set of 20 tests were conducted where a forming lubricant drip was applied to the test plate, and under this lubricated condition (Figure 3.2-5), the mass loss drops to an average of $0.1 \%$ with a standard deviation of $0.05 \%$-- a far more acceptable and reliable behavior.

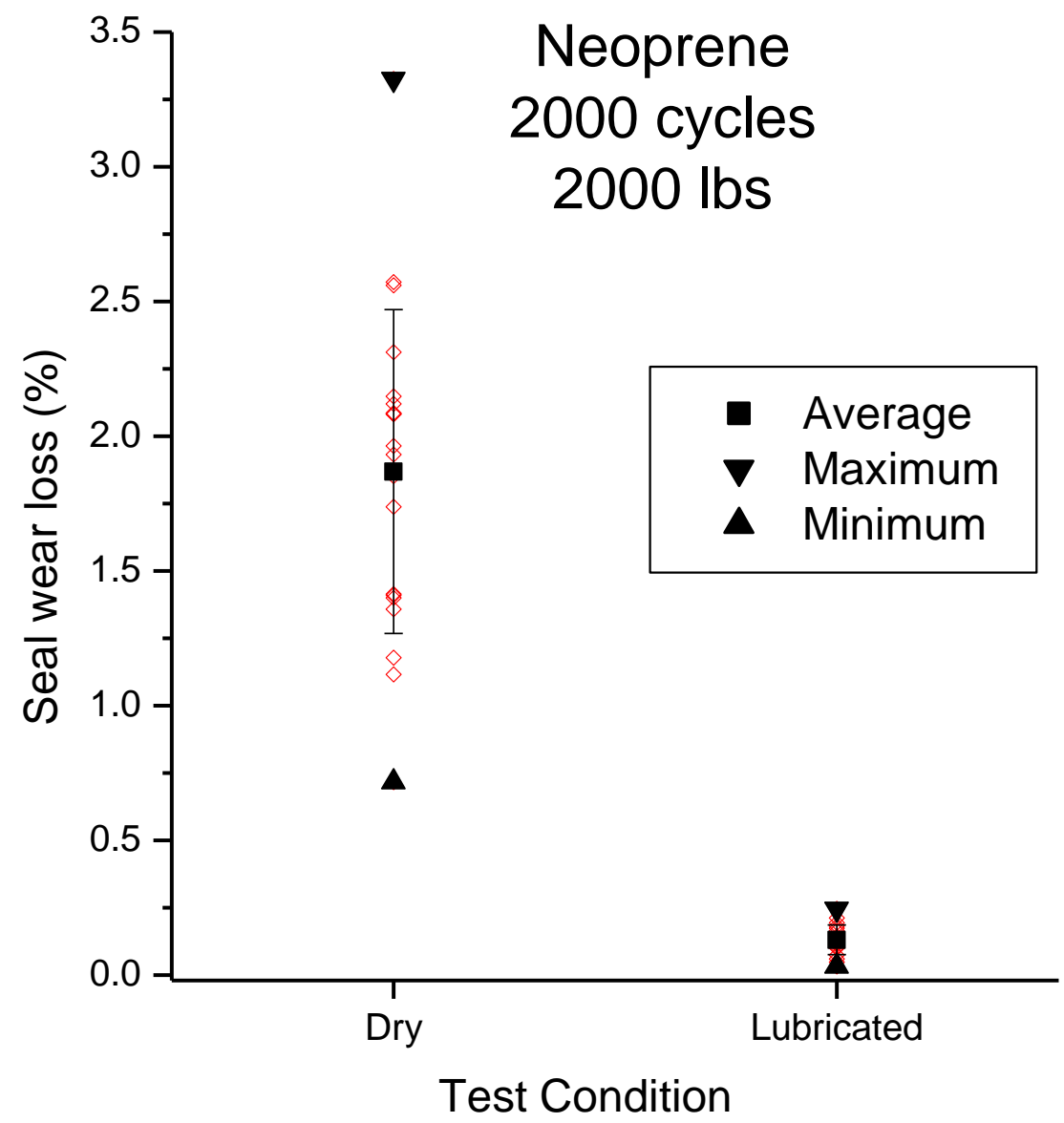

Figure 3.2-6. Neoprene seal wear expected under EHF forming conditions. 
Hybrid Seal Development

Even given the success of the lubricated standard seals, it was felt that in high volume production cases a more durable seal was necessary. The idea of the hybrid seal places a metal seal backed by a rubber "spring" in contact with the sheet. The seal groove was designed such that the ratio of cross-section area between both seals and groove channel equals $89 \%$. The overall idea behind the concept is shown in Figure 3.2-7 where a deep groove contains a $1 / 4$ inch neoprene seal at the bottom and a 9/32 inch SS303 stainless steel seal on top. The rubber seals the groove sides and bottom and to the metal seal on top, while the metal makes the seal to the AHSS sheet. The results of modeling are shown in Figure 3.2-8, and the model confirms that there is sufficient sealing force (29MPa) provided by the design for EHF applications.

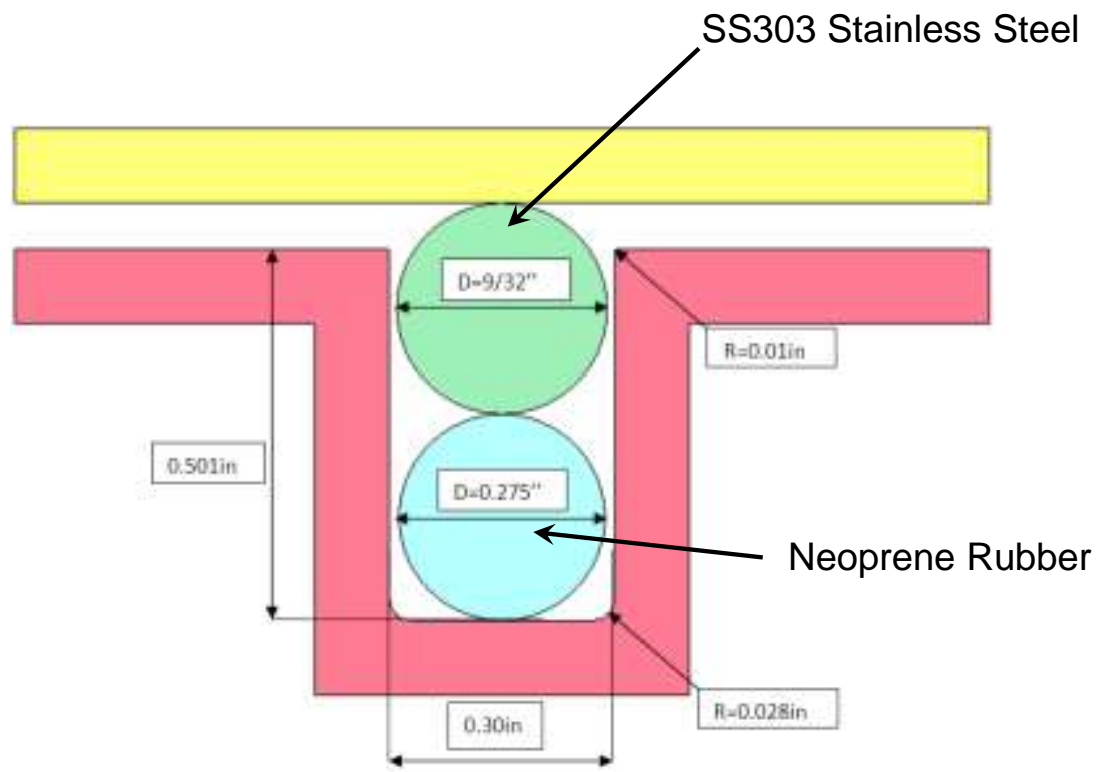

Figure 3.2-7. Hybrid metal seal with $9 / 32$ inch metal seal atop $1 / 4$ inch rubber seal on bottom in a 0.30 inch wide groove.

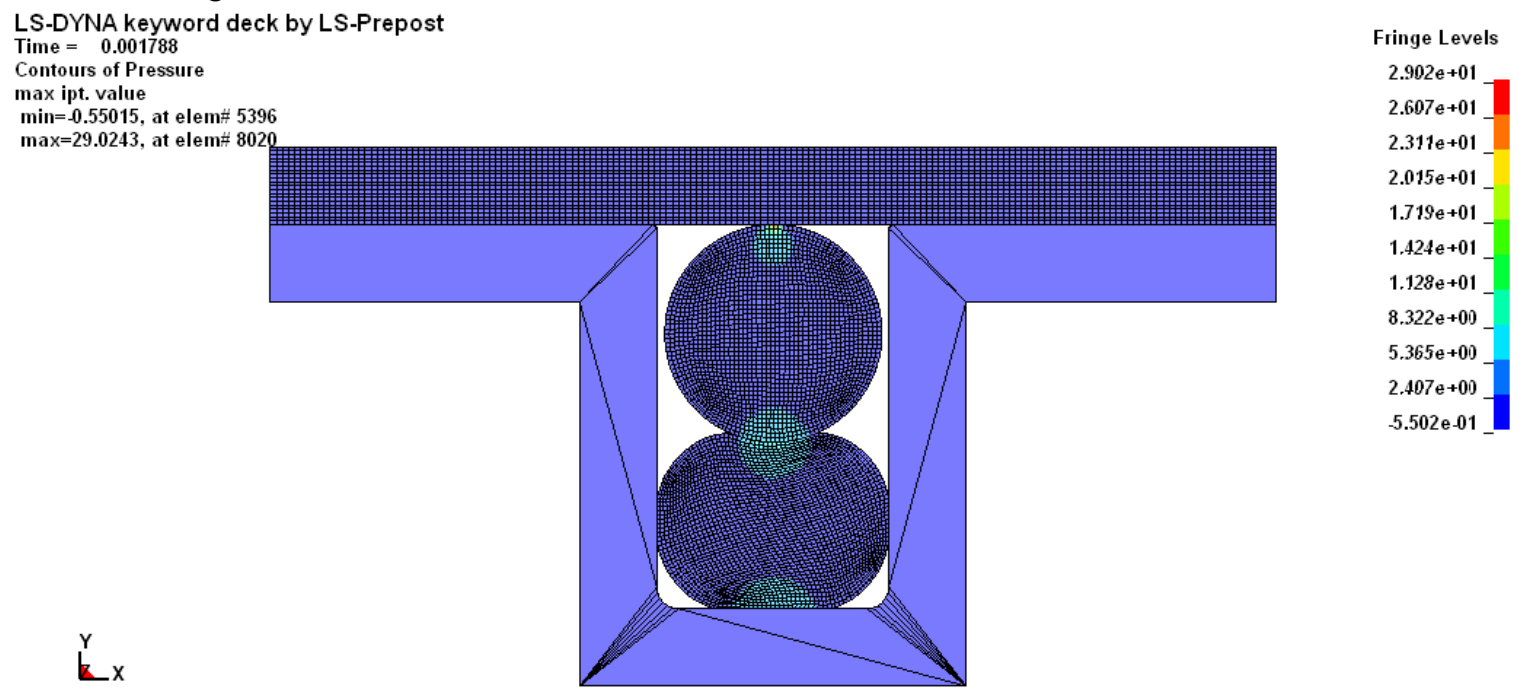

Figure 3.2-8. Hybrid metal seal model indicating a contact force of $29 \mathrm{MPa}$ at the sheet interface. 
A specific groove plate with a single groove channel but otherwise similar to Figure 3.2-5 was manufactured for testing the hybrid seal in the cyclic loading structure. The groove plate was attached to the seal wear system where cycling loading conditions were simulated to generate wear data.

Again, tests on seals were conducted under dry as well as lubricated conditions, and once again the (upper) seal mass was used as a wear indicator. Under high loading conditions, the weight of the rubber seal was recorded as signs of wear were observed. The initial test conditions were the same as the monolithic neoprene testing described above. The results, seen in Figure 3.29 , plot the wide range of loss amounts on a semi-logarithmic graph against clamping load.

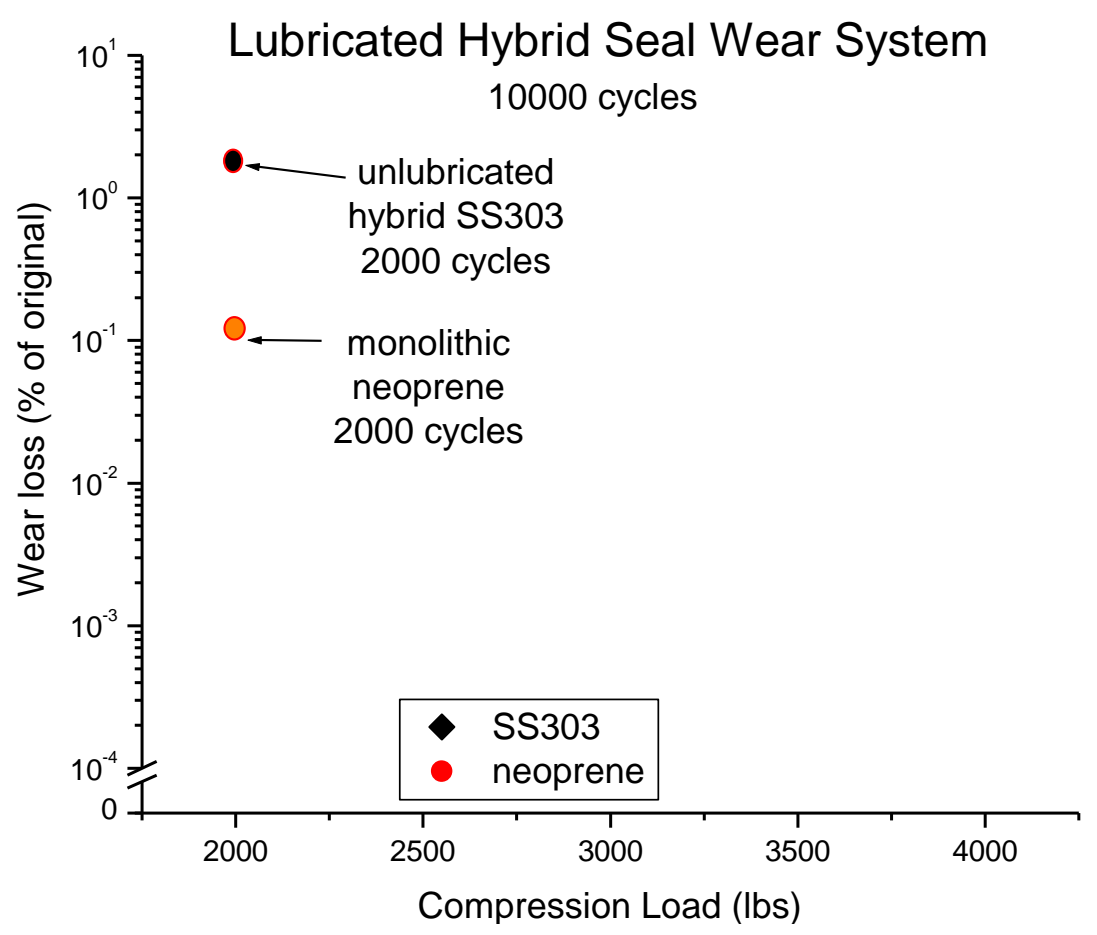

Figure 3.2-9. Results of unlubricated and lubricated testing of hybrid seal at a variety of compression loads.

At moderate loads (2000lb) and 2000 cycles of testing under lubricated conditions, very low wear was observed for metal seals, and the rubber seal on the bottom was preserved with no sign of wear. The dry testing condition of metal seal resulted in severe wear and deformation. It was concluded that dry testing conditions are not appropriate for the hybrid seal system and subsequently only lubricated testing conditions were tested. Because of the low wear rate on the metal seal, more cycles were induced under lubricated condition testing. The maximum number of cycles was eventually run up to 10,000 to get a measurable change, and consistently low wear data were subsequently observed from the test results. Furthermore, the clamping load was increased to $4080 \mathrm{lb}$ and, while wear as well as pinching signs were observed in the rubber seal, the same low wear rates were maintained in the metal seal. Since this seal exhibits excellent wear properties for EHF applications, further testing of this seal design was pursued. 


\section{Seal Pressure Testing}

A tool for pressure testing the seals under high hydrostatic pressures was designed and can be seen in Figure 3.2-10. This tool was placed in a 150 ton press and liquid pressures up to 48 $\mathrm{MPa}$ were placed on the seals.

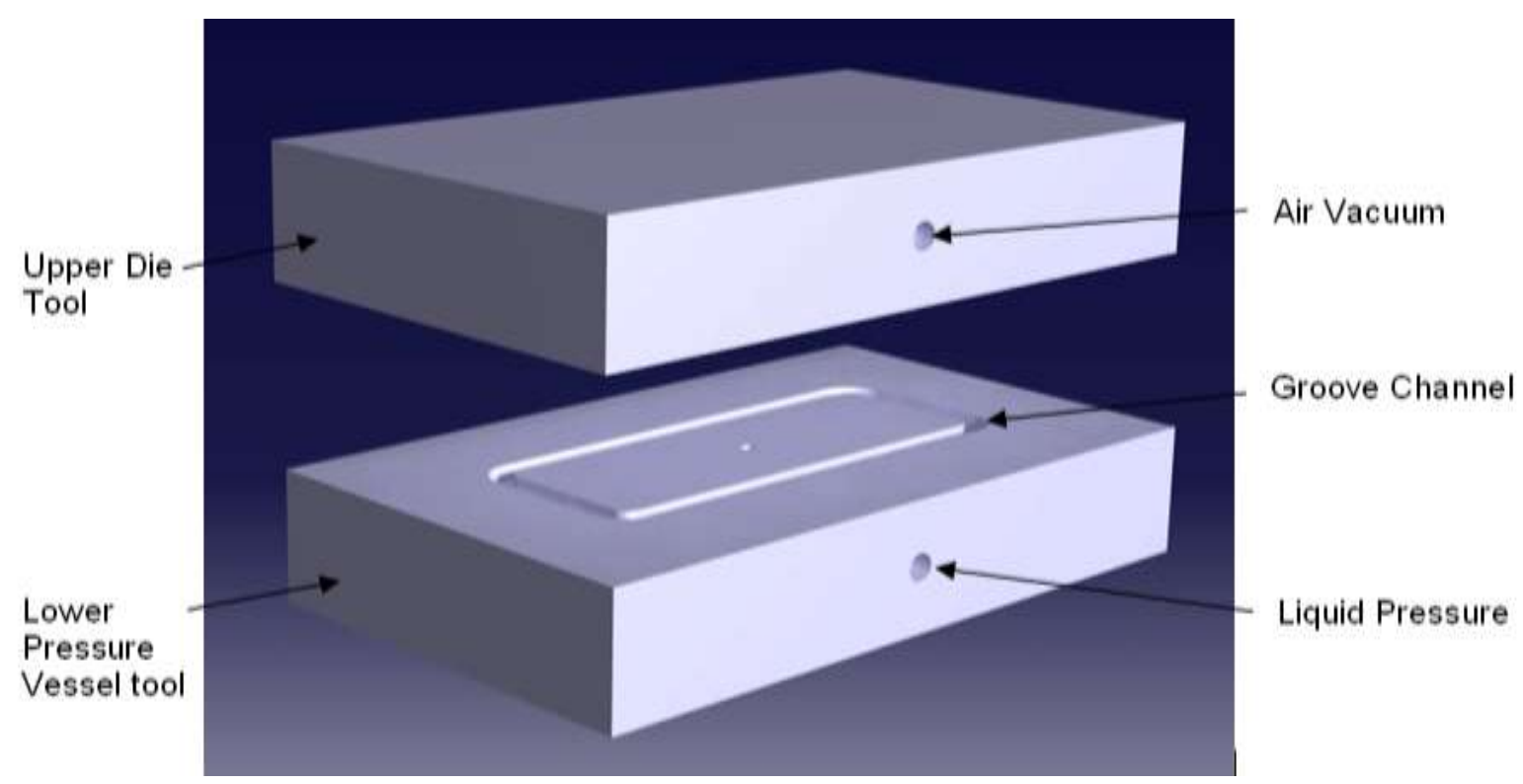

Figure 3.2-10. Tool for testing seals under hydroforming pressures.

The monolithic neoprene seal was tested first and withstood the maximum pressure of $48 \mathrm{MPa}$, the maximum pressure of the pump at the time. The pressure was cycled 100 times with no sign of failure. When removed from the tool the seal had suffered some extrusion damage, as seen in Figure 3.2-11.

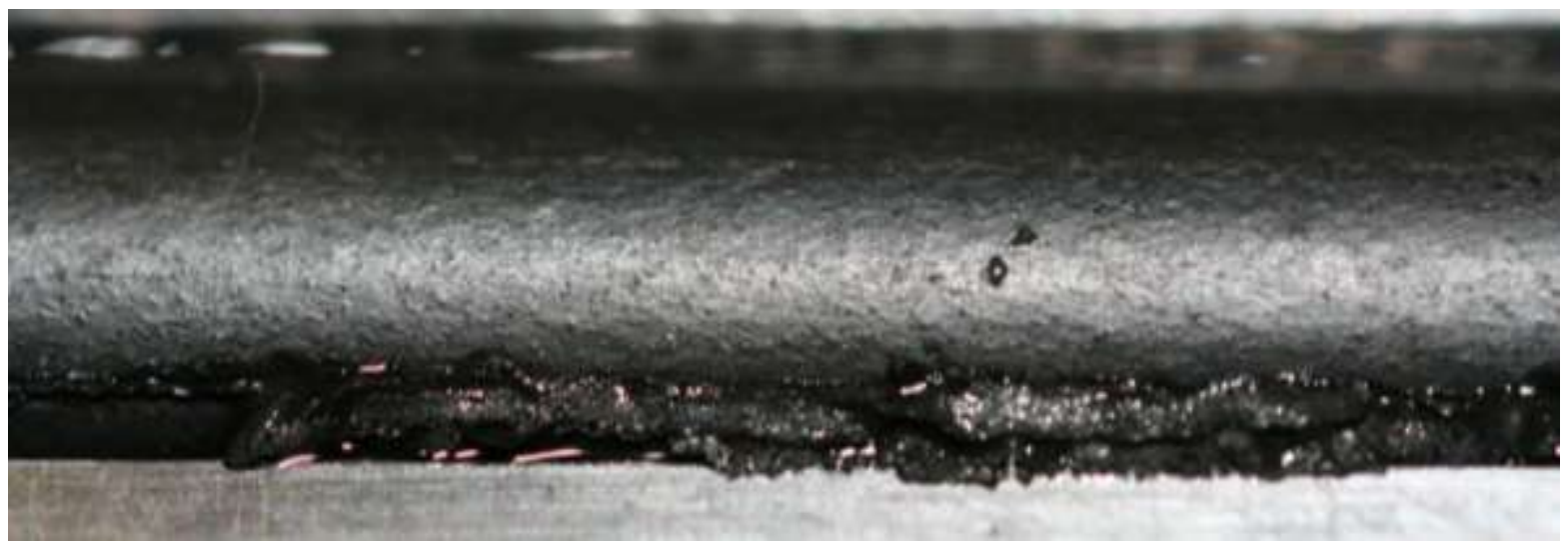

Figure 3.2-11. Extrusion damage during pressure testing of the monolithic neoprene seal. 
The hybrid seal was tested successfully up to $28 \mathrm{MPa}$ before leakage occurred - a pressure close to that predicted by finite element analysis and sufficient to withstand the short intense pressure pulses observed in EHF.

Summary of Task 3.1 - Study of Sealing Materials

Standard neoprene rope seals and a new hybrid seal design were studied to determine their suitability for EHF applications. The standard (monolithic) neoprene seal was found to be suitable for low volume production applications, but only under lubricated conditions because of its wear properties. Although less able to seal against very high hydrostatic pressures, the hybrid stainless/neoprene seal was found to be suitable for high volume production. U.S. Patent number 8136378 and P.R.C. patent CN201799515 was awarded for the hybrid seal and other EHF related seal designs. 


\section{Sub-task 3.3. Integrated computer controlled EHF system}

\section{Process Automation - System Integration Overview}

The overall automated cell system design can be found in Figure 3.3.1. The system includes the Master PLC, material handling gantry, hydraulic press, fluid handling system and the pulse generator. The Labview data acquisition and analysis system, described in detail in Task 5, provides high speed data acquisition of pressure/electrical signals and high level diagnostics regarding pulse and forming quality, and pulse-by-pulse/system-by-system data logging.

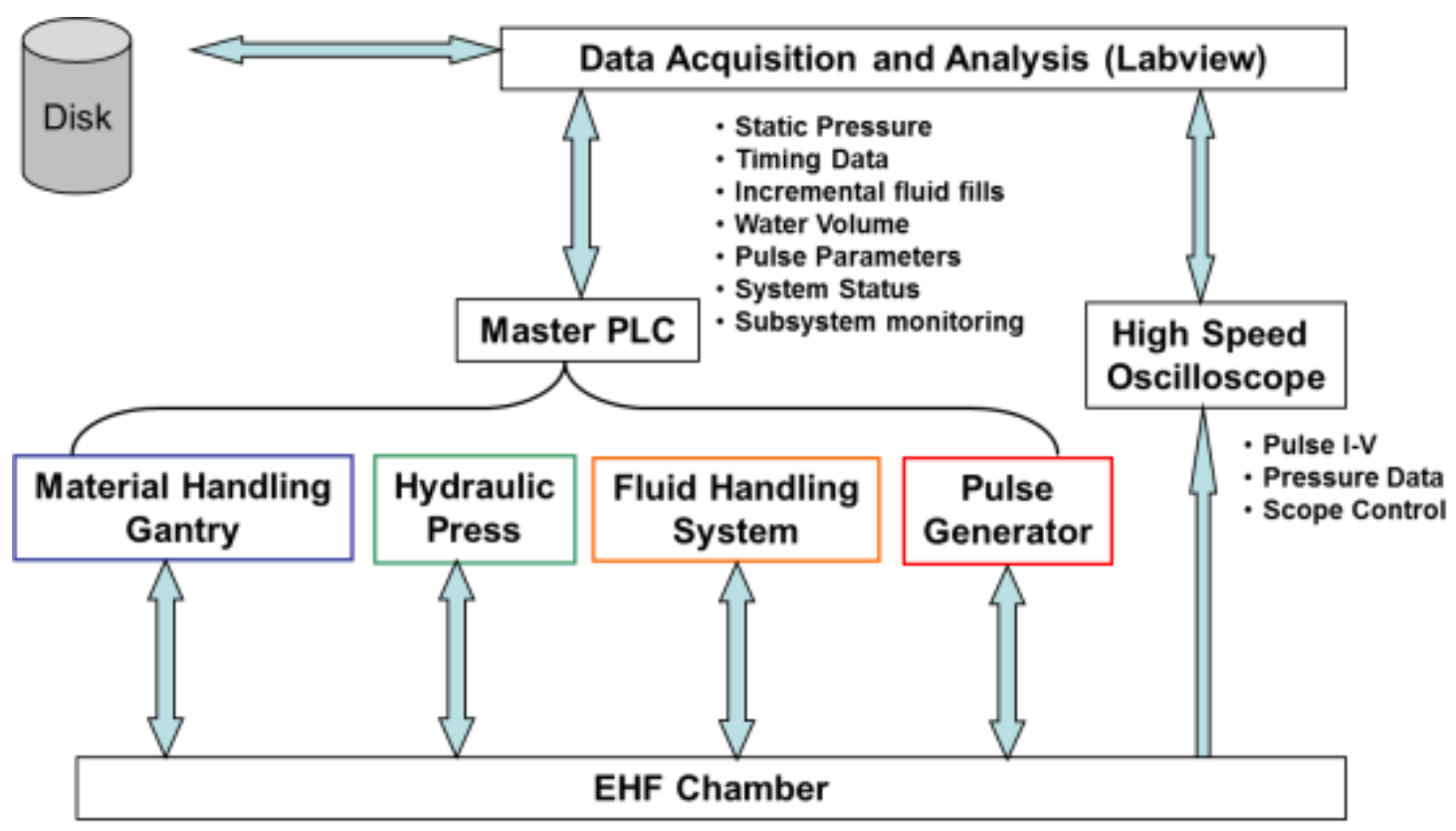

Figure 3.3.1. Shows the Master PLC and subsystem control diagram.

The Master PLC controls the subsystem PLC's on the material handling gantry and pulse generator, and it directly controls every aspect of the operations of the hydraulic press and fluid handling subsystems. In addition, the overall system and each subsystem have safety requirements that must be enforced by the Master PLC.

The complexity of integrating a complete control system for diverse subsystems including hydraulic press controls, automated material handling, fluid handling system (FHS), and 15kV pulse generator cannot be understated. The gantry, fluid handling system, and pulse generator each came with their own Programmable Logic Controller (PLC) with no brand commonality, and all systems required direct drive relay logic control of one level or another.

One of the biggest challenges was noise isolation during pulse generator operation. The electromagnetic noise combined with the voltage and current levels used in the system can induce very high secondary currents and voltages in secondary systems. Since these 
subsystems required physical interconnection for the purposes of robust communication, Ethernet or analog/digital communication was carried out over fiber-optic lines.

\section{Material Handling Gantry}

The gantry, pictured in Figure 3.3.2, is a simple pick and place $x$ - and $z$ - axis robot with vacuum/pressure actuated end-of-arm tooling capable of picking up panels/blanks from anywhere between the legs and in the press volume. The system is automatically de-energized by the master PLC when personnel are in the room. A part presence sensor helps set fault conditions when the system runs out of blanks or when unable to properly pick a part or blank.

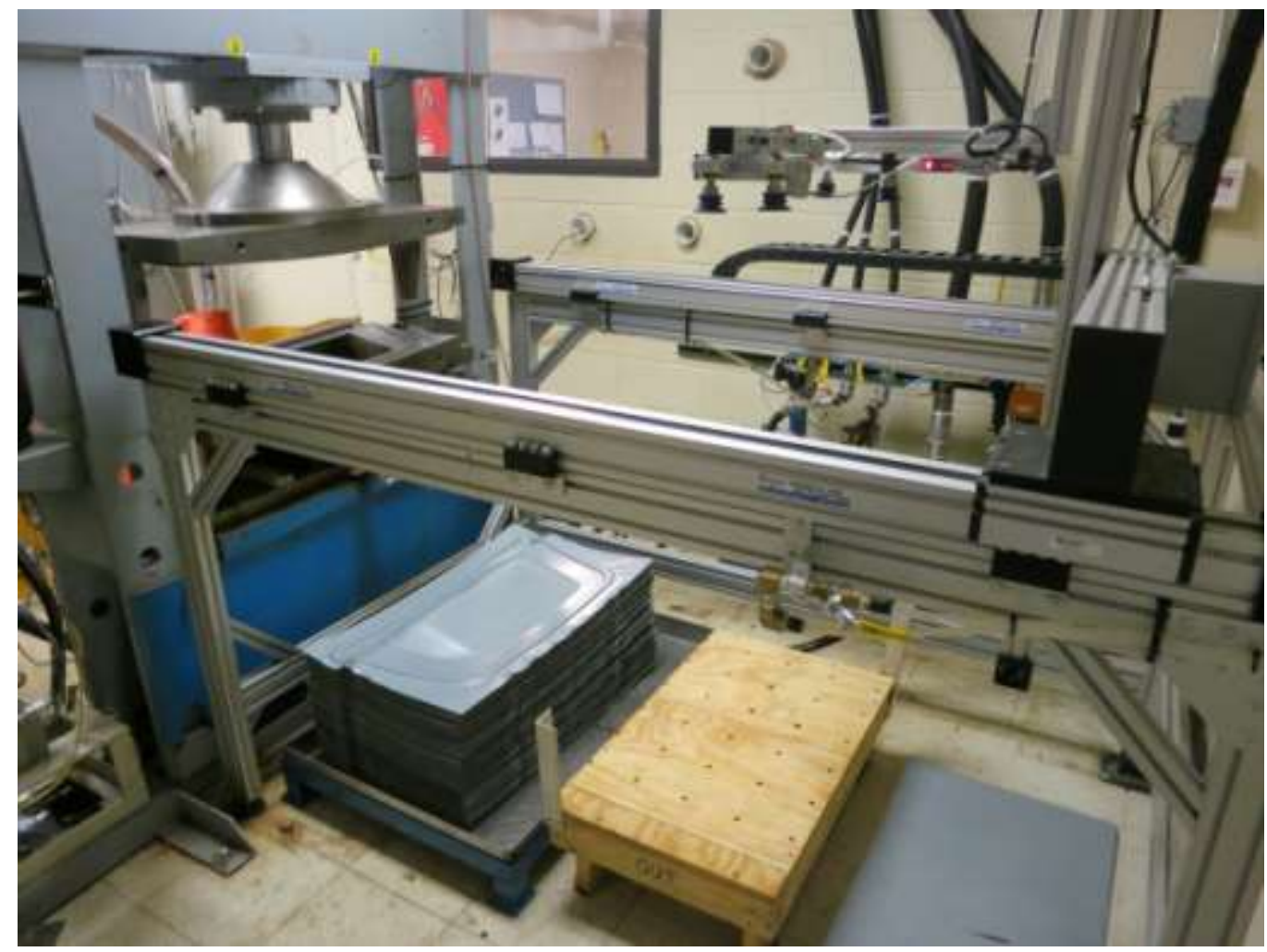

Figure 3.3.2. Material Handling Gantry. Input blanks are placed on wood pallet and finished parts are placed on the stack in the pan.

The subsystem has its own PLC and can be independently manipulated. The communications between the master and gantry PLC's are handled over a fiber backbone Ethernet to minimize EM noise. Control is maintained through command codes and data written directly to the gantry PLC.

\section{Hydraulic Press}

The original hydraulic press pictured in Figure 3.3.3 was significantly upgraded to vastly improve opening/closing speeds and to allow for remote control by the master PLC. A laser displacement sensor (to inform the PLC of the current actuator state) as well as pressure 
sensors (to indicate whether full tonnage has been developed) were added for process control/monitoring in order to function in an automated environment without intervention. The upgrade included a 30 GPM pump backed by a 5 gallon accumulator that can close the press in less than two seconds and a 5 GPM high pressure circuit that, once fully closed, can rapidly achieve the full 100 Tonne capability of the press.

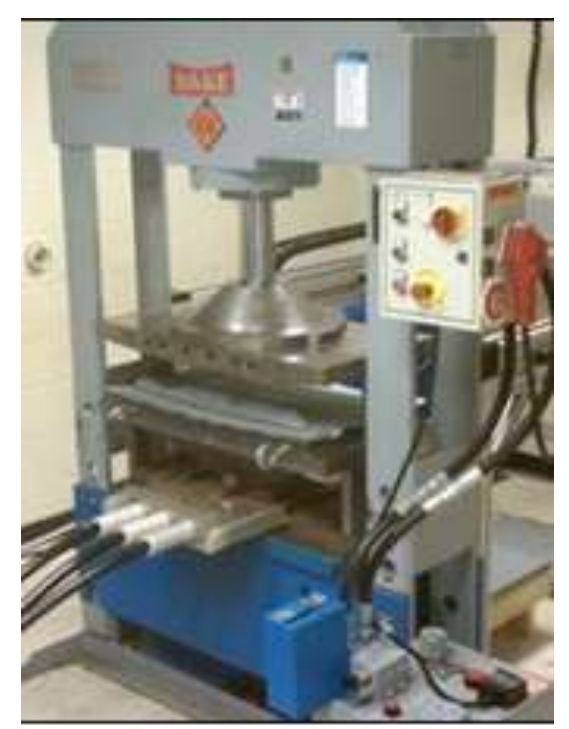

Figure 3.3.3. Original press now upgraded with new pump, manifold, and controls. See Figure 3.3.6 for a picture of upgraded press.

This new system is entirely operated by the PLC, which can remotely start and operate it within predefined safety limits. The unit is disabled by the PLC when not in operation or when personnel are in the cell. Lastly, its operation is tightly interlocked with that of the gantry to prevent damage to the gantry.

\section{Fluid Handling System}

A Fluid Handling System (FHS) was developed to rapidly manage the electrohydraulic forming process that requires evacuating the chamber of any air, rapidly filling with fluid, refilling between pulses and then draining the chamber. The system is capable of filling and draining the chamber at 108 GPM and the actual time is limited by the size of the orifices in the chamber wall. The system has a highly controllable high pressure circuit that can precisely fill the void after a pulse and measure it at a resolution of $+5 \mathrm{~mL}$. The system has the capability to statically hydroform the blank at pressures up to 1500 PSI. Lastly, it has a filtration system capable of running for over a week with two shifts per day without maintenance. However, the fluid handling system is covered in great detail in section 3.1 and the reader is referred there for more details and photographs.

Furthermore, like the hydraulic press, this very complex system is entirely controlled by the master PLC. As such it is in control of the system under potentially dangerous situations. These situations mostly occur during pressurization: 1500 psi hydroforming pressure, 100 psi 
air used to blow out the chamber, and 0 psi vacuum placed on the die side - all of which must be relieved in the case of a system fault and before the master PLC released the door locks for operator entry. This safety function is tightly linked with that of the hydraulic press operation. An example of the hydroforming function is given in Figure 3.3.4 where a part was partially formed through the application of hydrostatic pressures.

a)

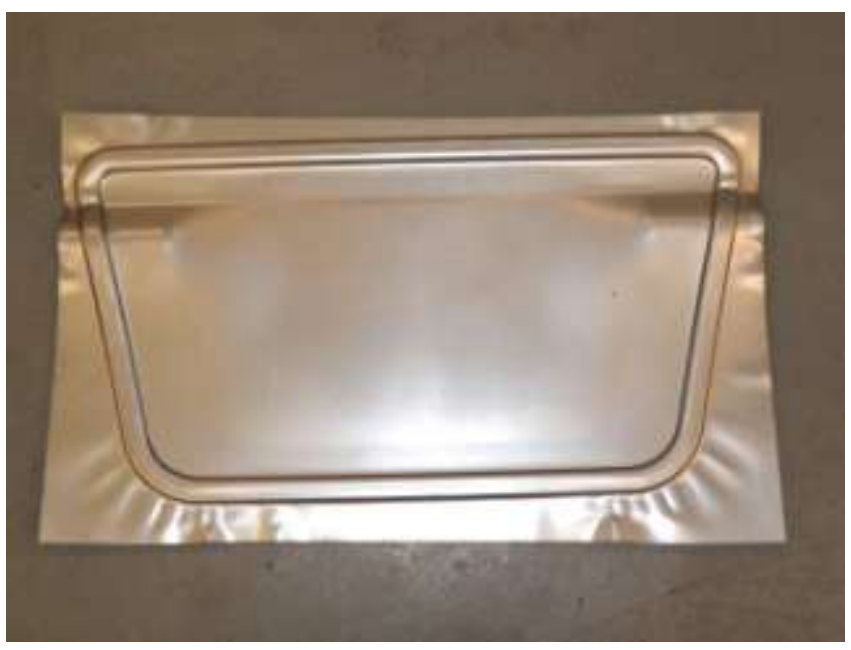

b)

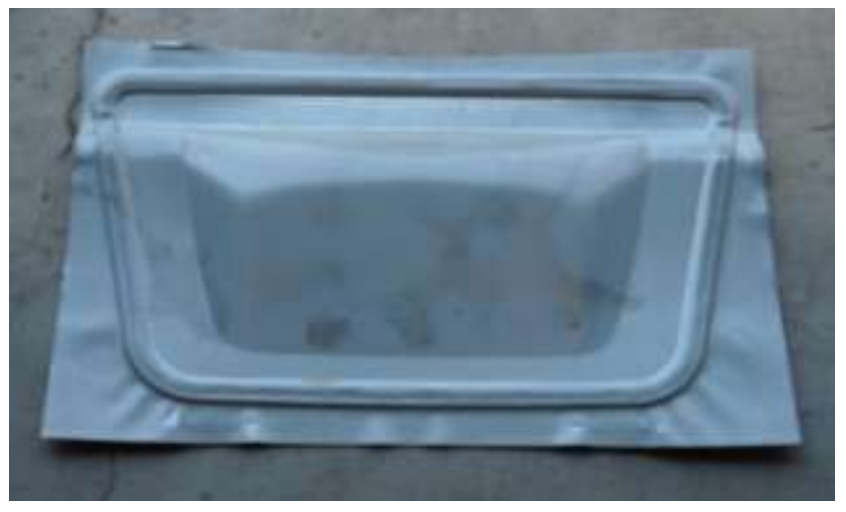

Figure 3.3.4. Blank a) as-clamped and b) conventionally but incompletely hydroformed at 300 psi.

\section{Pulse Generator}

The pulse generator is an off the shelf item manufactured by IAP Research, with the addition of

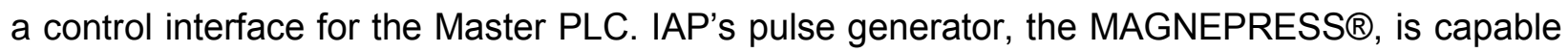
of delivering a pulse of $15 \mathrm{kV}$, at $100 \mathrm{kA}$, and at $22.5 \mathrm{~kJ}$. More information is available on IAP's public website: www.magnepress.com. The analog interface added to the pulse generator's PLC allows the Master PLC to control the charging voltage, initiate a pulse, trigger an e-stop, and detect any faults. A digital extension to the IAP control panel allows the master PLC to initiate and detect faults. 


\section{Complete System}

The complete system is pictured, as much as possible, in Figure 3.3.5 and shows all four major subsystems: press, pulse generator, fluid handling system, and materials handling system. The master PLC controls everything in the cell except for the room lights. It smoothly integrates all of the subsystems, senses all possible faults, and shuts the systems down safely before any personnel can enter the cell. It monitors flow volumes and can thereby detect a fully formed panel, or it can blindly follow the exact recipe entered into the master PLC.

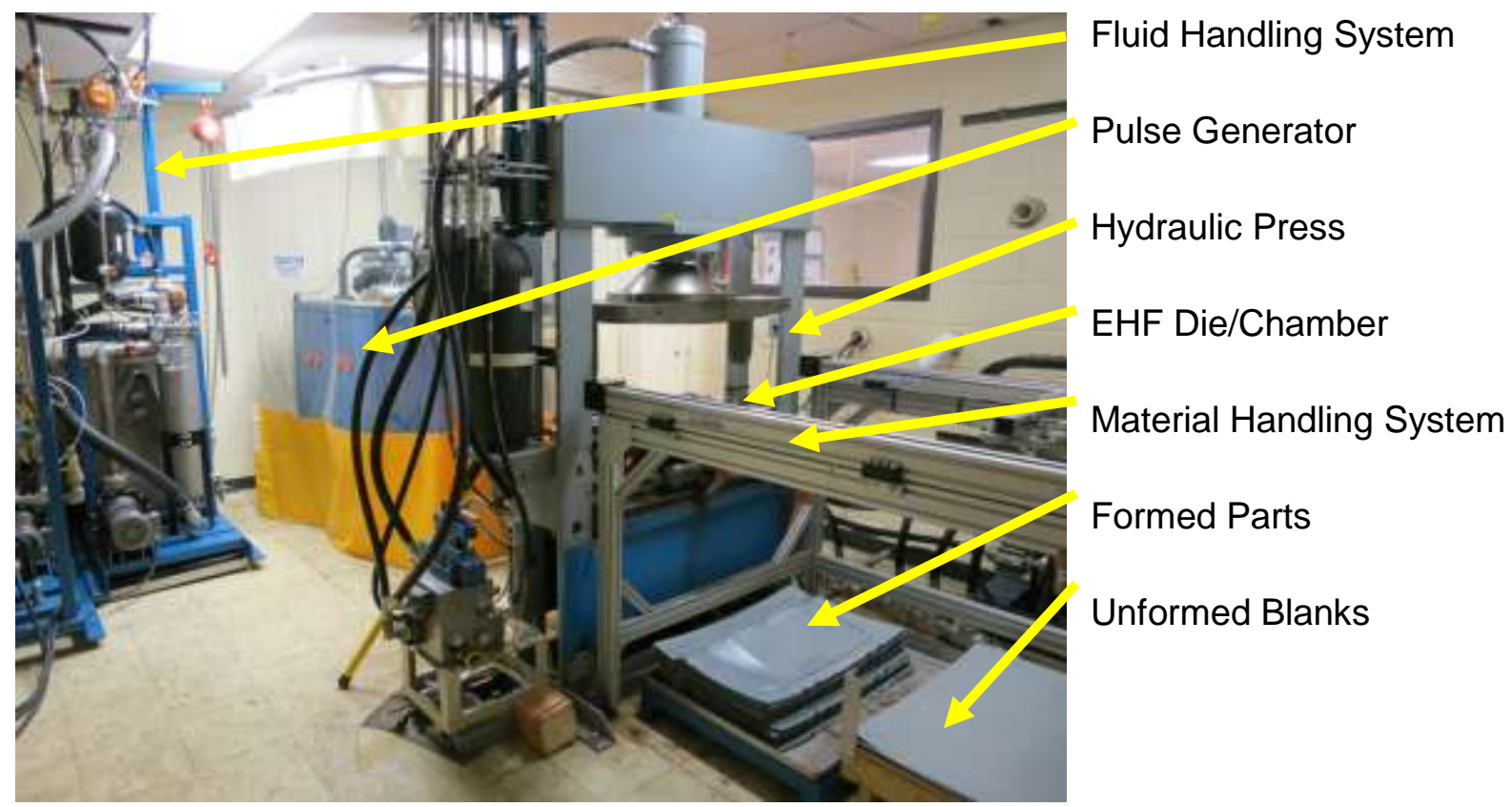

Figure 3.3.5. EHF Automation Cell.

\section{System Operation/Part Formation Process}

Once the safety interlock requirements have been met (cell evacuated, doors locked, operating modes of pulse generator and Master PLC switched), the Master PLC commands the gantry PLC to pick up an unformed blank and place it into the die/chamber. It commands the press to close and waits until it reaches full pressure, and then fills the chamber and evacuates the die. It fires the pulse generator and monitors the progress of blank deformation throughout multiple discharges. It then vents the die and blows compressed air into the chamber to hasten draining. Once the chamber is empty, the press is opened, and the material handling system removes the part and places it in the finished part stack. A process flow chart of the logic in this process is presented in Figure 3.3.6, and time sequence photographs of the process are shown in Figure 3.3.7. 


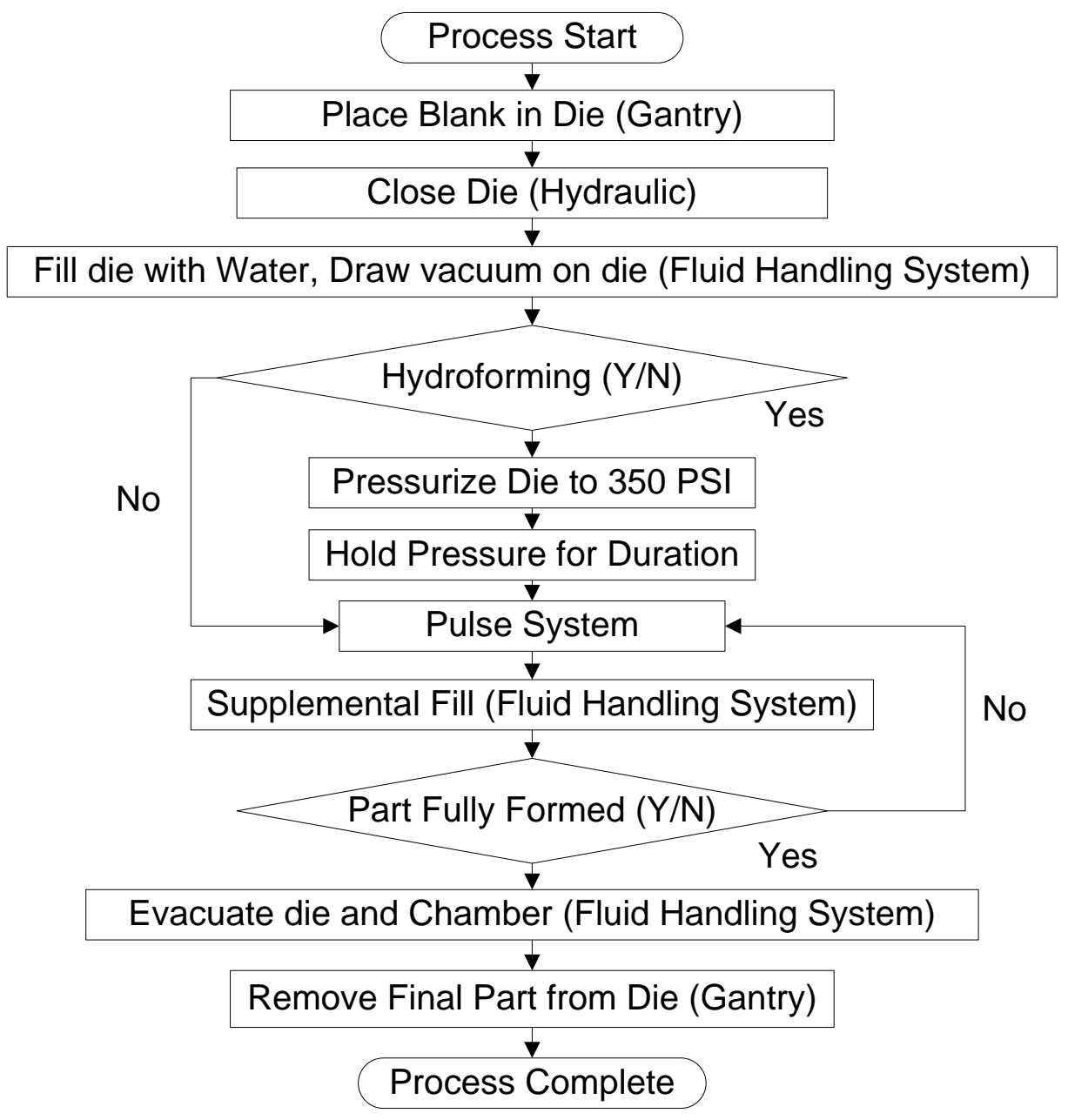

Figure 3.3.6. Flow chart for the EHF cell showing automation control steps.

\section{$\underline{\text { Conclusion }}$}

Shown in Figure 3.3.8 is a panel fully formed by the automation running the EHF cell. The cell, and especially the master PLC, was able to sense, manipulate and control all of the subsystems and reliably produce a fully formed blank. It needed operators only to install fresh blanks, remove fully formed parts, and start the system. Maintenance of electrodes and the fluid handling system were only occasionally necessary. 


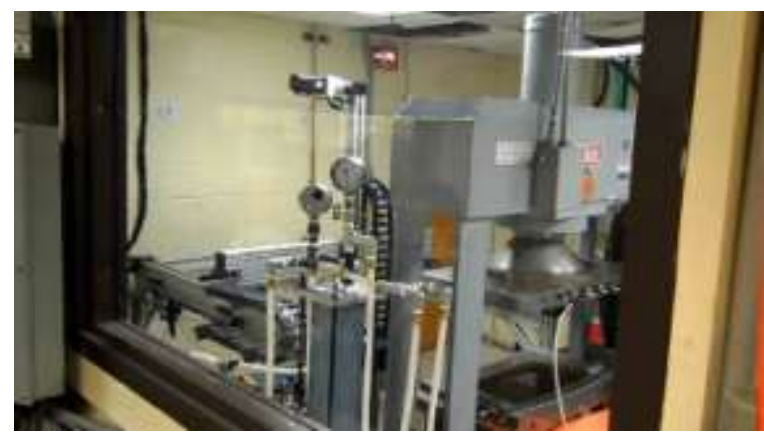

Step \#1: Gantry retrieves part blank from stack

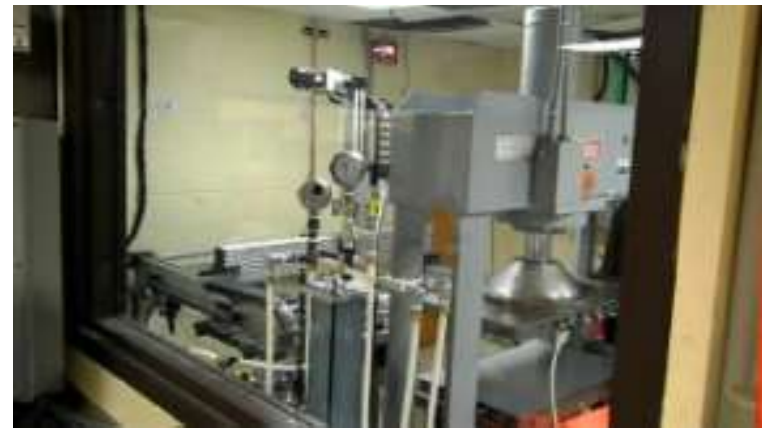

Step \#3: Press is closed

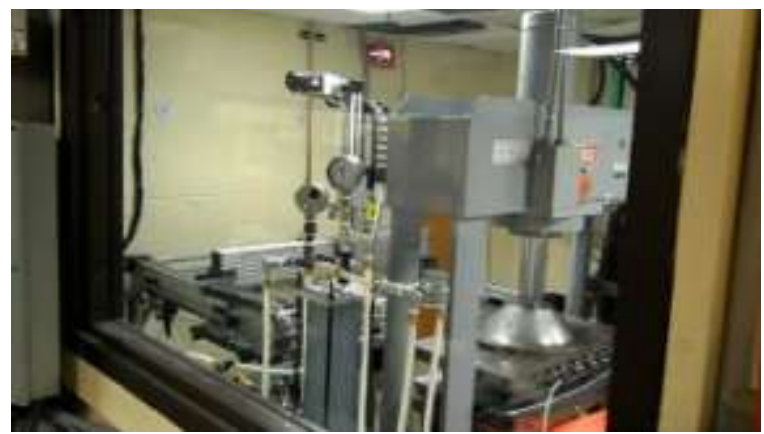

Step \#5: Prior to opening press, water is drained down and vacuum is released.

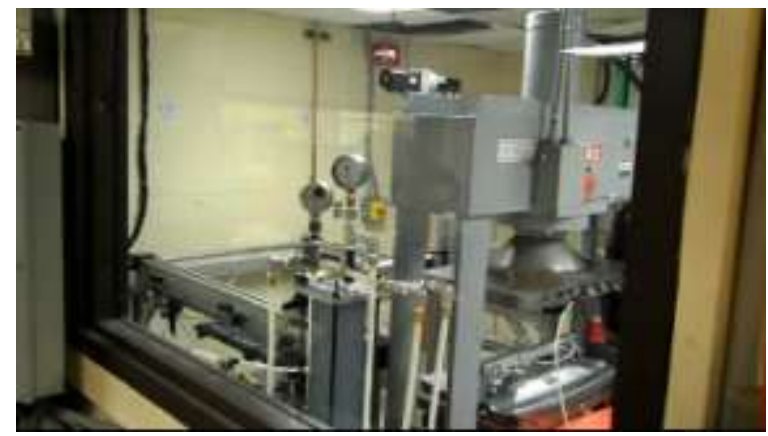

Step \#7: Gantry retrieves fully formed part from die/chamber

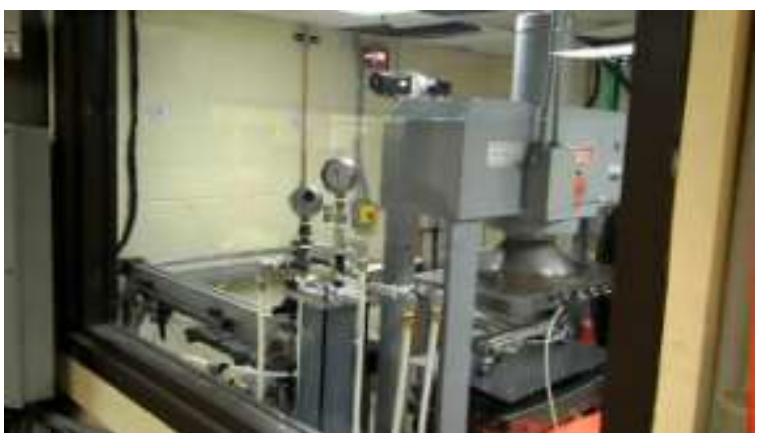

Step \#2: Gantry places blank in die/chamber

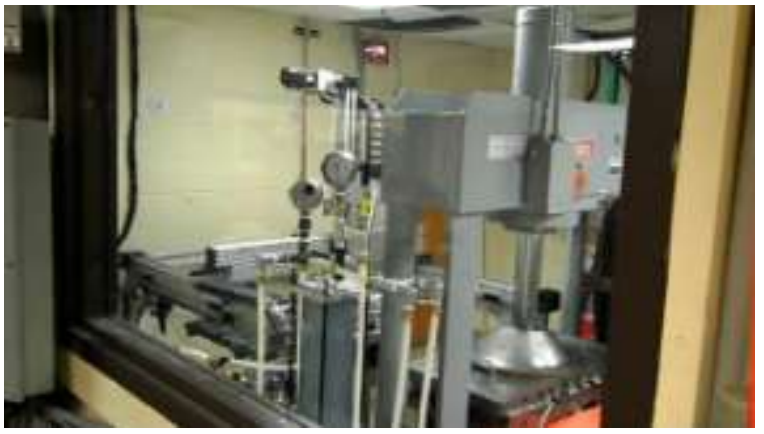

Step \#4: Chamber is filled with water, vacuum is drawn on upper die, and EHF pulses are applied.

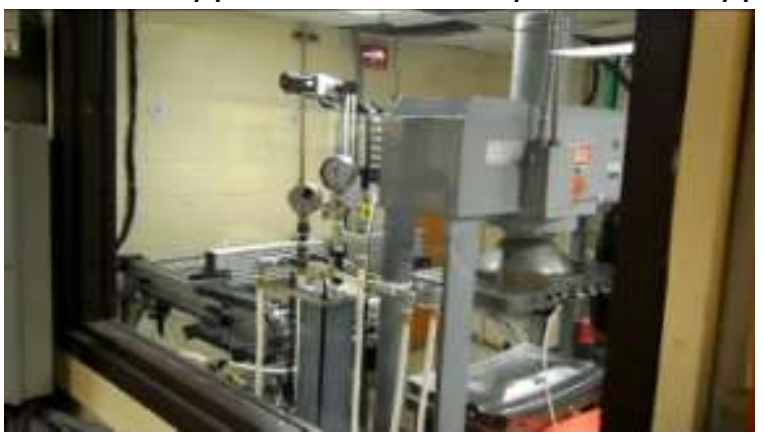

Step \#6: Fully formed part is ready for pickup

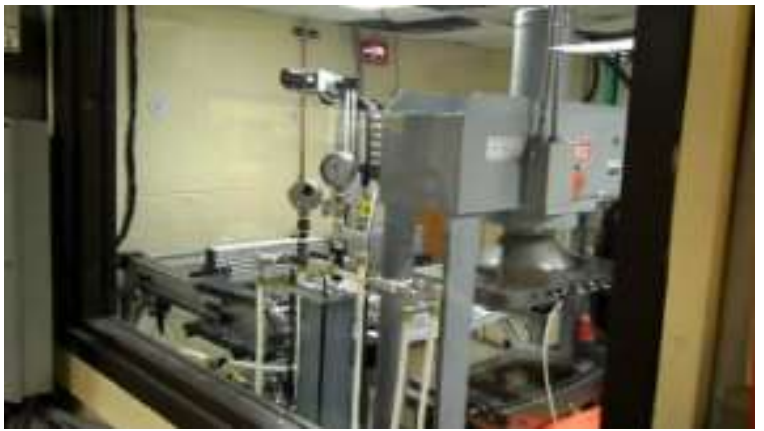

Step \#8: Gantry places part on stack of completed parts.

Figure 3.3.7. EHF Automation Part Production. 


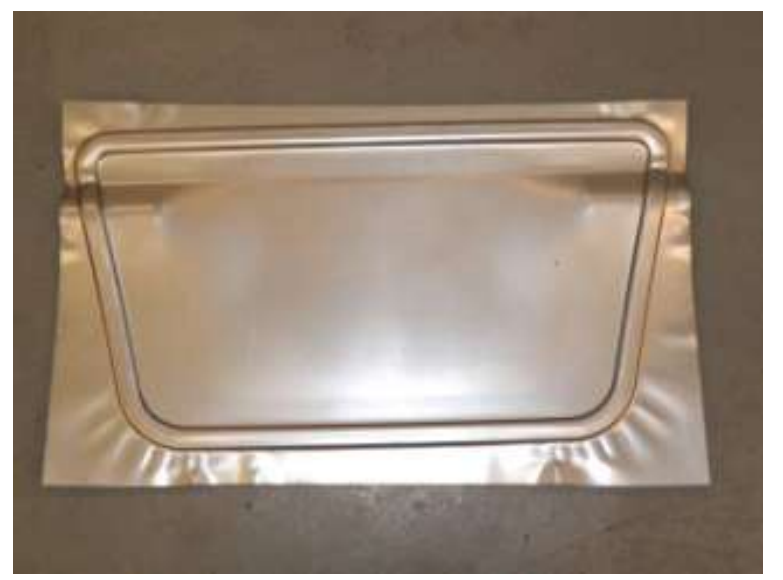

a) As-clamped blank without forming

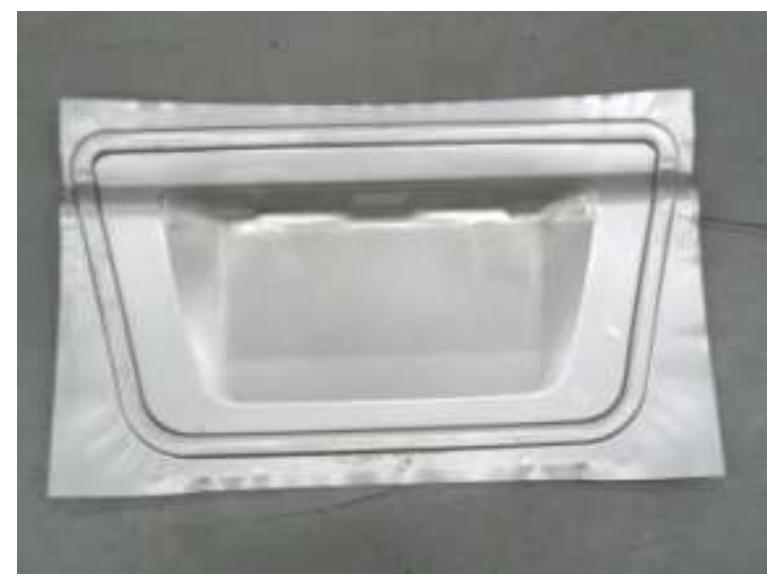

b) Blank fully formed though EHF

Figure 3.3.8. Blank shown a) as-clamped in the press, and b) fully formed by EHF automated cell. 
Task 4. Electrohydraulic sheet metal forming process enhancement and optimization

Sub-task 4.1. Develop integrated preforming process.

Improving vehicle fuel economy is a constant goal within the automotive industry. One method for accomplishing this goal is to incorporate light-weight materials into the vehicle body structure [4-1]. Aluminum alloys and advanced high strength steels are among the materials that are currently seeing increased usage and implementation in weight reduction efforts. One of the barriers to further implementation of these materials is their relatively low formability in sheet forming operations as compared to mild steels and conventional high strength steels. High velocity forming methods have been known for decades and have been shown to have the potential to increase sheet metal formability beyond conventional limits [4-2 to 4-5]. The various methods of high-velocity forming can be grouped into three general categories based on the source of the forming energy and based on how that energy is transferred into the motion of the blank: Explosive Forming, Electromagnetic Forming (EMF), and Electrohydraulic Forming (EHF). Explosive forming involves the use of an explosive charge arranged in order to accelerate a sheet metal blank into a one-sided tool. EMF involves storing electrical energy in a bank of high voltage capacitors and releasing that energy through a multi-turn coil positioned in close proximity to the blank being formed. The rapid increase of current in the coil induces an eddy current in the blank near to it, and the repulsive force between the two currents accelerates the blank toward the tool. EHF also involves storing electrical energy in a bank of high voltage capacitors, as does EMF. With EHF however, the energy is released in the form of an electrical current that arcs across a pair of electrodes submerged in water. The rapid heating and expansion of the plasma channel between the electrodes creates an intense pressure wave in the water, which then accelerates the blank toward the tool.

The vast majority of the research work on sheet metal formability using high velocity forming was done by starting with a flat blank and then using only one pulse of energy to form that blank into its desired shape [4-6 to 4-8], which sometimes was a die with specific geometry and at other times was simply free-forming into an open window. However, the panels which make up a vehicle body structure can be very large stampings, and forming parts of this size using only one pulse of energy would be very challenging to the point of being unfeasible, due to the amount of energy required and also dimensional control issues. The dimensional control issues would arise from the fact that at excessively high forming energies, the sheet metal has a tendency to impact the tool surface and then bounce back, sometimes to a considerable distance, thus negating any advantages of using a single high energy pulse. This bounce-back effect has been observed and described in previous research on high velocity forming methods with simple and symmetrical die geometries such as cones and cylinders [4-9, 4-10]. When considering the higher degree of geometrical complexity that is present in large automotive stampings, coupled with the tight dimensional tolerances, it becomes clear that forming such panels with only one pulse of energy is simply not possible. Furthermore, besides the issues with dimensional control, using only one high energy pulse to form large panels also presents serious problems in terms of tool damage and tool durability due to the excessively high speed 
impact between the sheet and the die [4-11]. Therefore, the best approach for manufacturing large automotive panels using high velocity forming would be to use a series multiple pulses or multiple forming increments, with the final few pulses done at an energy sufficient to fill any sharp details of the die but not enough to cause any bounce-back from the die surface. When considering these requirements, EHF presents multiple advantages as compared to EMF and explosive forming for potential application in high volume production of automotive panels. The chief advantages of both EHF and EMF as compared to explosive forming would be more precise control over both the amount of energy to be released in the discharge and also the rate of energy release, not to mention the simple fact that these methods do not require the storage of explosive materials in a manufacturing environment. The main advantage of EHF over EMF for an application involving multiple forming pulses is rooted in the fact that EHF uses a fluid as a pressure transmitting medium between the electrodes and the blank. Because of the presence of this fluid, the electrode gap may be positioned at a significant distance away from the workpiece (from 10 to $40 \mathrm{~cm}$, approximately) and yet still be able to deliver a pressure pulse sufficient to plastically deform the blank. Therefore, even with a series of multiple forming increments, all of the forming steps could be done in one EHF tool as long as fluid is able to back fill into the space opened up by each forming increment. With EMF, on the other hand, it is necessary to position the coil in close proximity to the blank $(<5 \mathrm{~mm})$ in order to deliver sufficient forming pressures. Therefore, in order to form a panel with multiple EMF pulses, multiple coils with unique geometry would be required to complete all of the forming increments, with part transfer or tool transfer automation between each increment. For any forming process or joining process that involves relatively smaller parts that can be formed or joined using only one pulse of energy, EMF would arguably be a better choice than EHF because EMF would not require any tools to be drained or filled with water as a step within each cycle, a process that would inevitably add to the cycle time. However, as stated previously, for the large sheet metal parts used in automotive production, EHF seems to be the most feasible, for the reasons described above.

One of the main factors that has limited more extensive implementation of high velocity forming methods in general, and EHF in particular, was longer process cycle times as compared to conventional stamping. The cycle times of conventional stamping processes will vary based on the size of the part being produced and the type of press used but is generally in the range of one part every 2 to 12 seconds. The majority of EHF processes that were implemented in various industries in the past had a cycle time in the range of one to two minutes per part. The factors that drive the longer cycle times of EHF as compared to conventional stamping would be the need to fill and drain a fluid reservoir for each part formed, the need to evacuate air from the forming cavity once the tools are closed, and also the time needed to charge the capacitor banks. While some smaller parts can be formed using only one EHF pulse, larger automotive panels would require multiple forming pulses as described previously, and additional process time would be associated with each individual pulse for charging the capacitor banks and backfilling the fluid reservoir with water.

A solution which naturally lends itself to reducing the cycle time of EHF is to combine it with a static pressure hydroforming preforming step. To form a large automotive panel using multiple 
EHF forming increments would require anywhere from four to eight pulses, whereas incorporating a hydroforming preforming step could replace the initial pulses and would necessitate only one to three pulses of EHF at the end to finish forming the panel. A similar process used in the aerospace industry in the 1960s was described by Sandford [12] in which a static pressure preforming step was combined with either EHF or explosive forming to form plate-type parts, although this work did not contain any quantitative data on improved formability. More recent patent literature also describes various hybrid conventional / highvelocity forming processes, with the main innovations being additional details about proper pressure sequencing and water flow control [4-13], and also methods of combining conventional stamping with EMF [4-14]. Vohnout and Daehn [4-15] reported formability improvements beyond conventional limits for 6111-T4 and 5754-O aluminum in high velocity forming after a quasi-static preforming step. In their work, the preforming strain was accomplished in a uniaxial tensile mode rather than a biaxial bulging mode, and the high strain rate forming was applied using a pulse of EMF. Liu et al. [4-16] also reported formability improvements beyond conventional limits for 5052-O aluminum in high velocity forming after a quasi-static preforming step. In their work, the preforming strain was applied in a biaxial mode using the hydraulic bulge test and the high strain rate forming was again applied using EMF. In both cases the results were promising from a manufacturing point of view because the introduction of significant amounts of quasi-static prestrain before additional forming steps naturally raises questions about the amount of ductility remaining in the metal for subsequent operations. However, neither of these works involved testing with advanced high strength steels, and neither involved the use of EHF to apply the high velocity forming step. The aim of this work is to report the results of formability testing of dual phase steels in conventional forming conditions, in EHF forming conditions using a single EHF pulse, and in EHF forming conditions after applying a hydroforming preforming step, and furthermore to analyze the EHF process through numerical modeling. The results of this work will contribute to developing the knowledge and understanding necessary to implement a hybrid hydroforming-EHF process in medium to high volume production in the automotive industry.

Regarding the effort to develop a hybrid HF-EHF process, two new pressure vessels were designed and fabricated, and were used in experiments involving different levels of static preforming pressure. These vessels are shown in Figures 4.1.1 and 4.1.2 below, along with the dies that were used in conjunction with the vessels to pre-form blanks using static pressure. Because the pressure vessels were fabricated from solid steel and do not include any electrodes, the vessels enabled high static pre-forming pressures, potentially up to 9000 psi, without the possibility of leaks at the electrode seals or force-out of the electrodes. Preliminary experiments on our first generation electrode design have confirmed that these electrodes can withstand 2000 psi of static water pressure without any leakage or force-out, but at 2200 psi the electrodes did exhibit a moderate leak as well as partial force-out from the chamber. Nextgeneration electrode systems have been designed to withstand higher levels of static pre-form pressure, and these systems are on schedule to be tested as part of future experiments. But, while the next-generation electrode systems are still in the process of being developed, the pressure vessels described heretofore will be sufficient for investigating the effect of different levels of static pre-forming pressure on material formability and process robustness. 


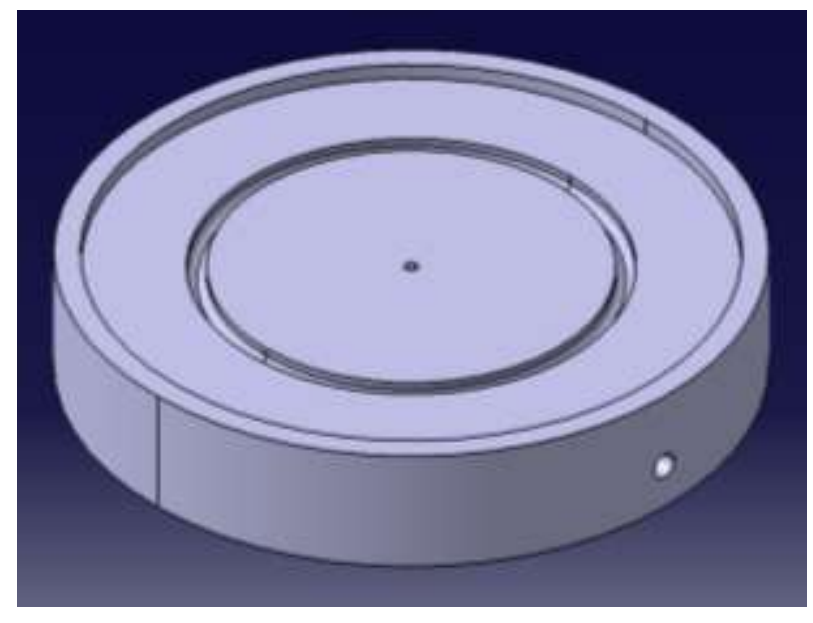

(a)

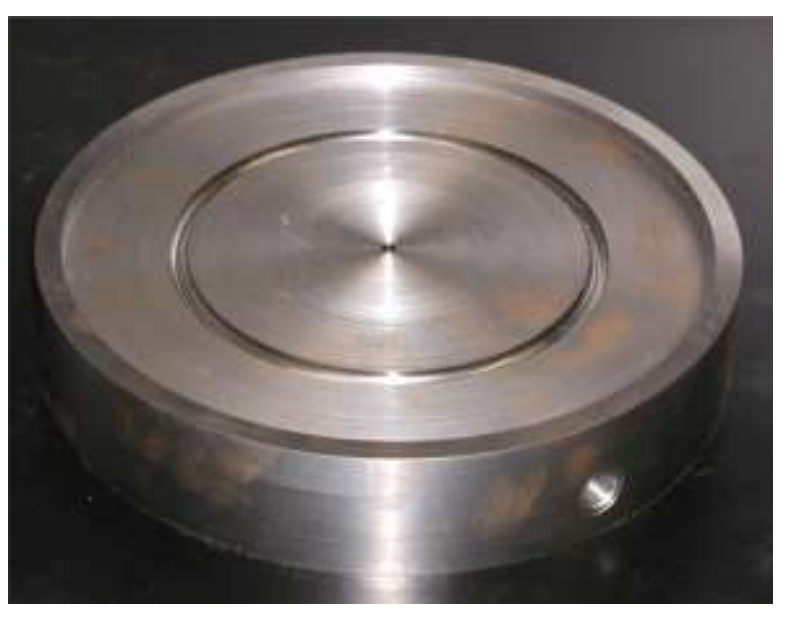

(b)

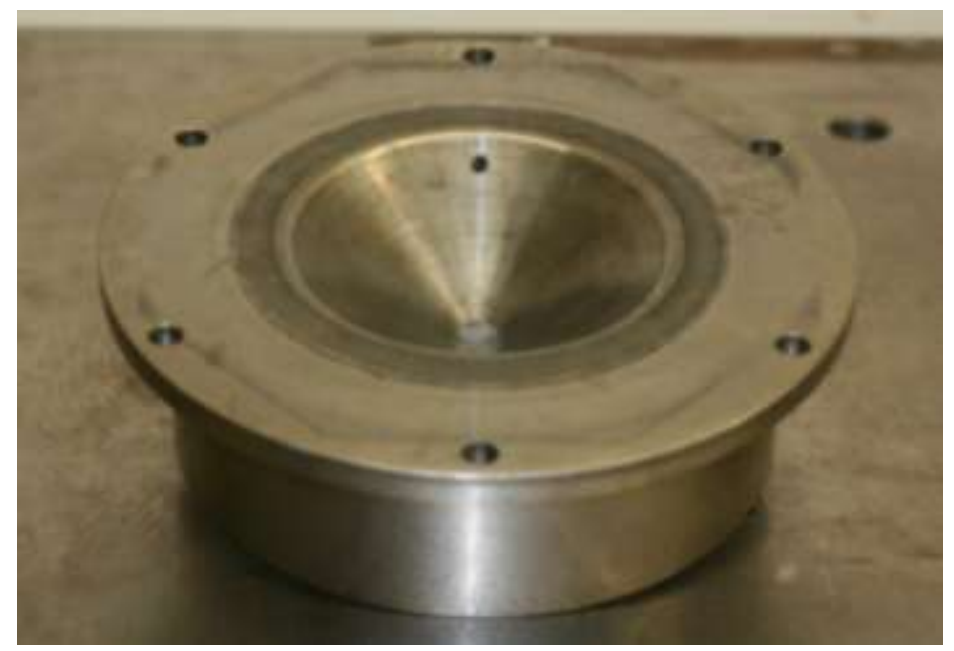

(c)

Figure 4.1.1. Round pressure vessel and accompanying die for forming conical samples in a state of biaxial strain: (a) CAD design of the round pressure vessel, (b) the fabricated vessel, and (c) the existing conical die (shown upside down) which mates with the round pressure vessel. 


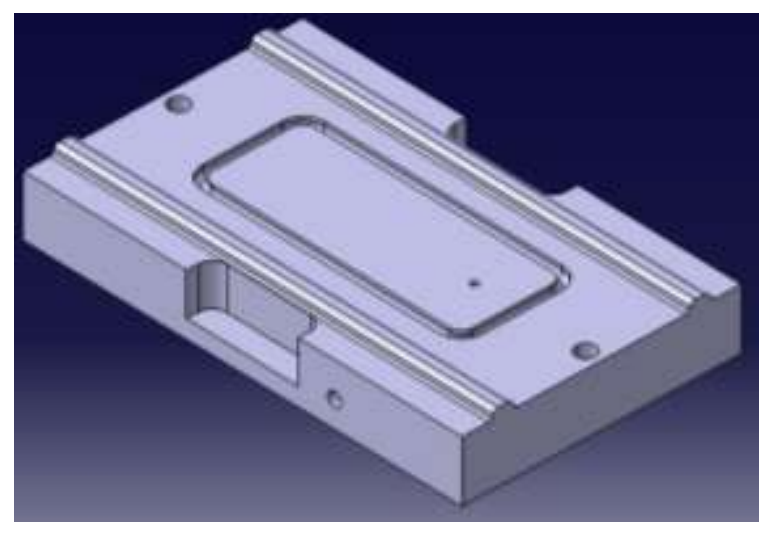

(a)

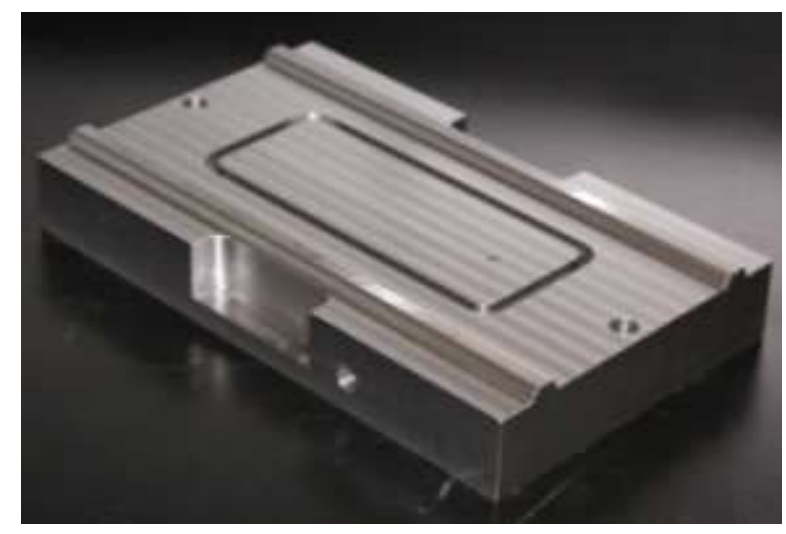

(b)

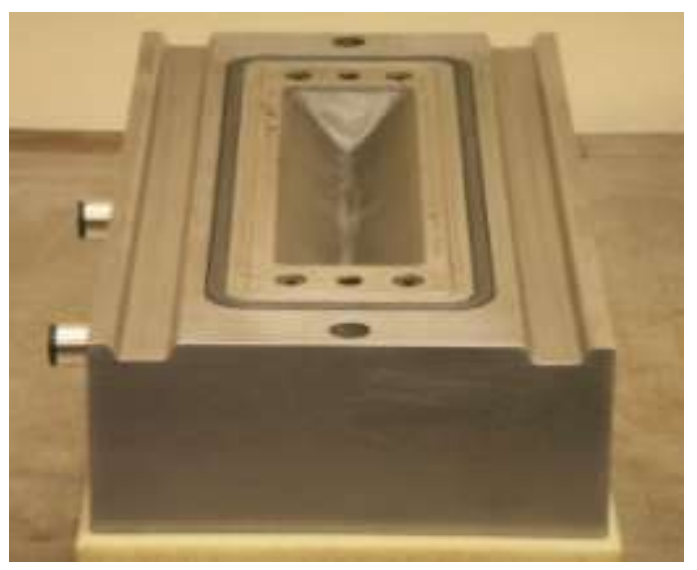

(c)

Figure 4.1.2. Rectangular pressure vessel and accompanying die for forming elongated channel samples in a state of plane strain: (a) CAD design of the rectangular pressure vessel, (b) the fabricated vessel, and (c) the existing elongated channel die (shown upside down) which mates with the rectangular pressure vessel.

Experiments were conducted in order to test the effects on sheet metal formability of a hybrid static-pressure hydroforming and electrohydraulic forming process. The goal was to determine whether the initial performing step at conventional strain rates would create excessive amounts of plastic deformation in the sheet metal, and thereby limit the benefits of available from the final forming steps at high strain rates. Photographs of some of the DP980 $(1.0 \mathrm{~mm})$ test specimens are shown in Figures 4.1.3 and 4.1.4 below. The same experiments were also run using DP780 at $1.0 \mathrm{~mm}$ and HSLA 350 at $1.0 \mathrm{~mm}$, and photographs of these samples are shown in Figures 4.1.5 through 4.1.8. The formability results for all three materials are shown in Figures 4.1.9, 4.1.10, and 4.1.11 below. All three graphs show the same promising trend, which is that a significant improvement in formability as compared to conventional forming limits is still available with EHF even after high levels of pre-forming. 


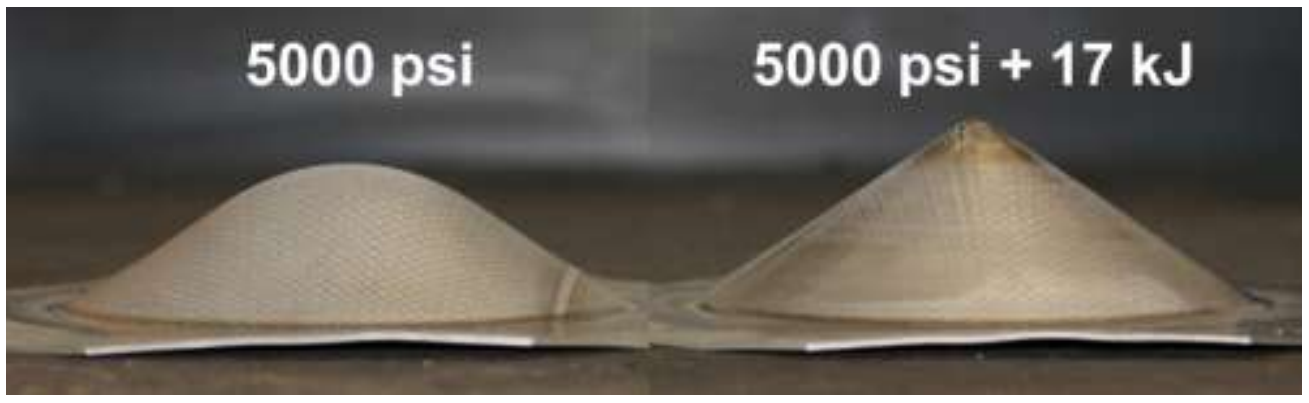

Figure 4.1.3. Test specimens of DP980, $1.0 \mathrm{~mm}$, used in pre-forming experiments. These specimens were formed into a conical die in biaxial strain. The specimen on the left was formed using only quasi-static water pressure at $5000 \mathrm{psi}$. The specimen on the right was pre-formed at $5000 \mathrm{psi}$ and then finally formed using one EHF pulse at $10.5 \mathrm{kV}(17 \mathrm{~kJ})$.

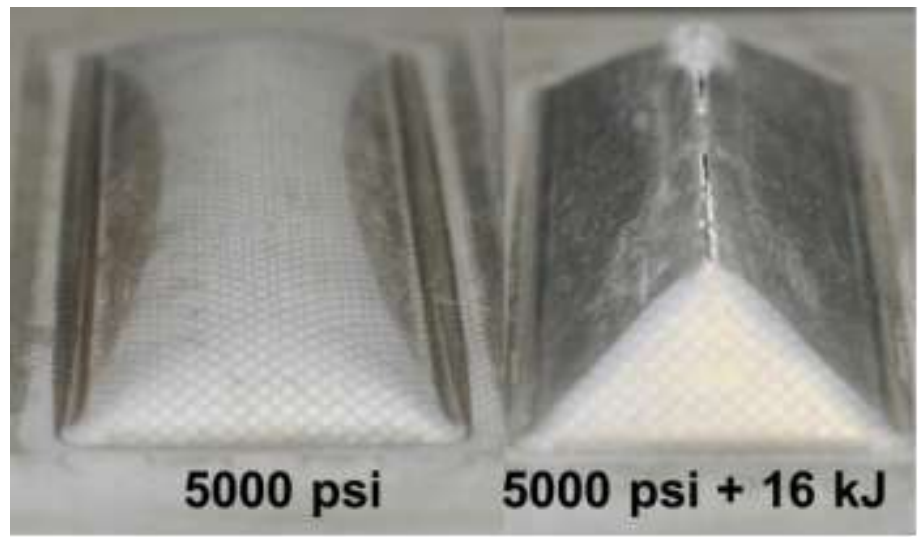

Figure 4.1.4. Test specimens of DP980, $1.0 \mathrm{~mm}$, used in pre-forming experiments. These specimens were formed into a v-shaped channel die in plane strain. The specimen on the left was formed using only quasi-static water pressure at 5000 psi. The specimen on the right was pre-formed at $5000 \mathrm{psi}$ and then finally formed using one EHF pulse at $10 \mathrm{kV}$ (16 kJ).

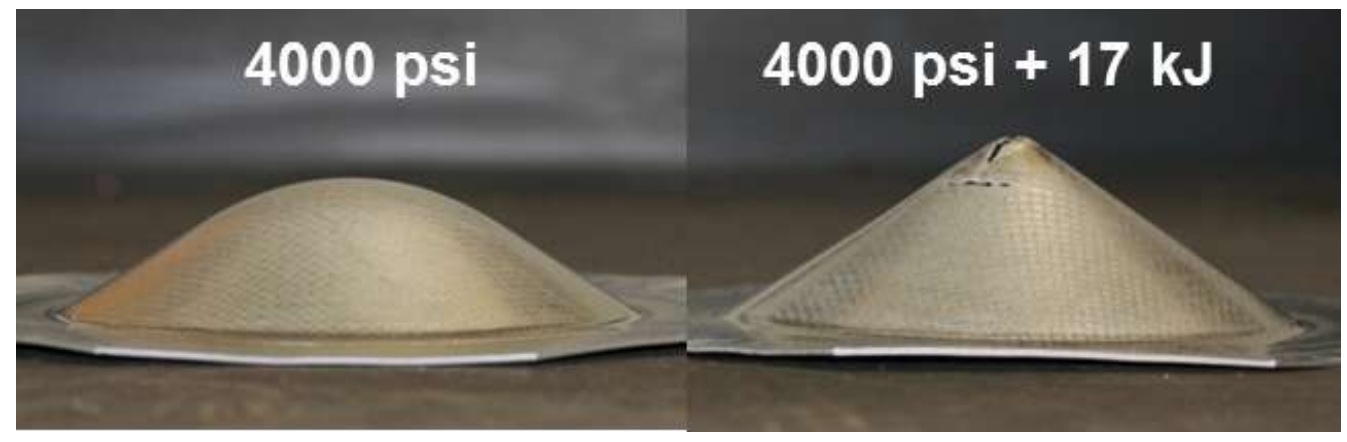

Figure 4.1.5. Test specimens of DP780, $1.0 \mathrm{~mm}$, used in pre-forming experiments. These specimens were formed into a conical die in biaxial strain. The specimen on the left was formed using only quasi-static water pressure at 4000 psi. The specimen on the right was pre-formed at $4000 \mathrm{psi}$ and then finally formed using one EHF pulse at $10.5 \mathrm{kV}(17 \mathrm{~kJ})$. 


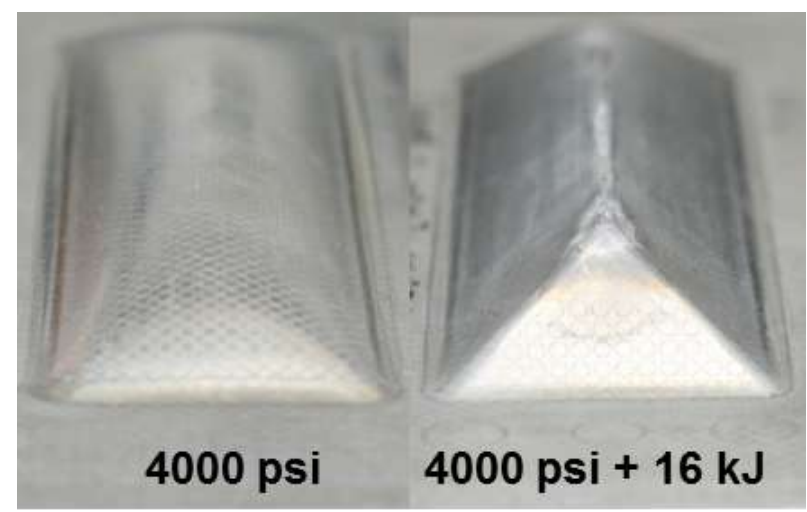

Figure 4.1.6. Test specimens of DP780, $1.0 \mathrm{~mm}$, used in pre-forming experiments. These specimens were formed into a v-shaped channel die in plane strain. The specimen on the left was formed using only quasi-static water pressure at 4000 psi. The specimen on the right was pre-formed at $4000 \mathrm{psi}$ and then finally formed using one EHF pulse at $10 \mathrm{kV}$ (16 kJ).

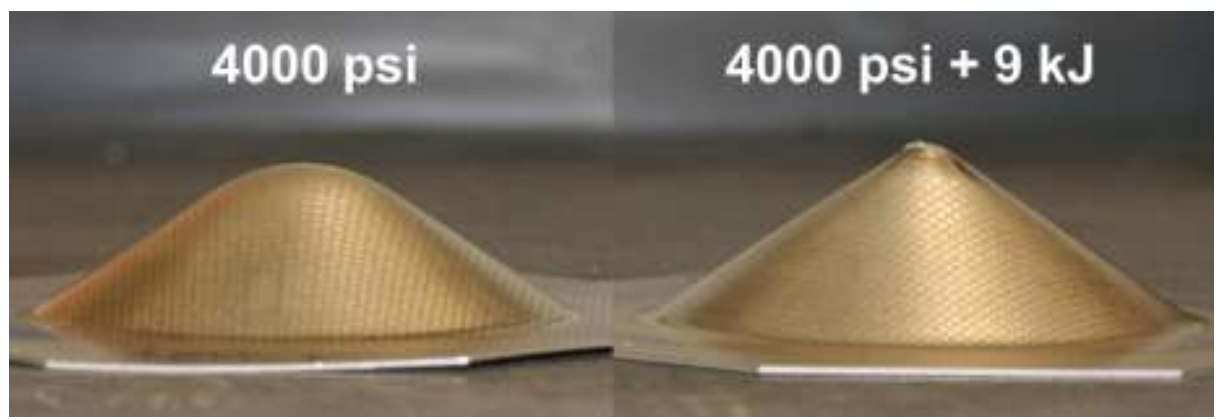

Figure 4.1.7. Test specimens of HSLA 350, $1.0 \mathrm{~mm}$, used in pre-forming experiments. These specimens were formed into a conical die in biaxial strain. The specimen on the left was formed using only quasi-static water pressure at 4000 psi. The specimen on the right was pre-formed at 4000 psi and then finally formed using one EHF pulse at $7.5 \mathrm{kV}(9 \mathrm{~kJ})$.

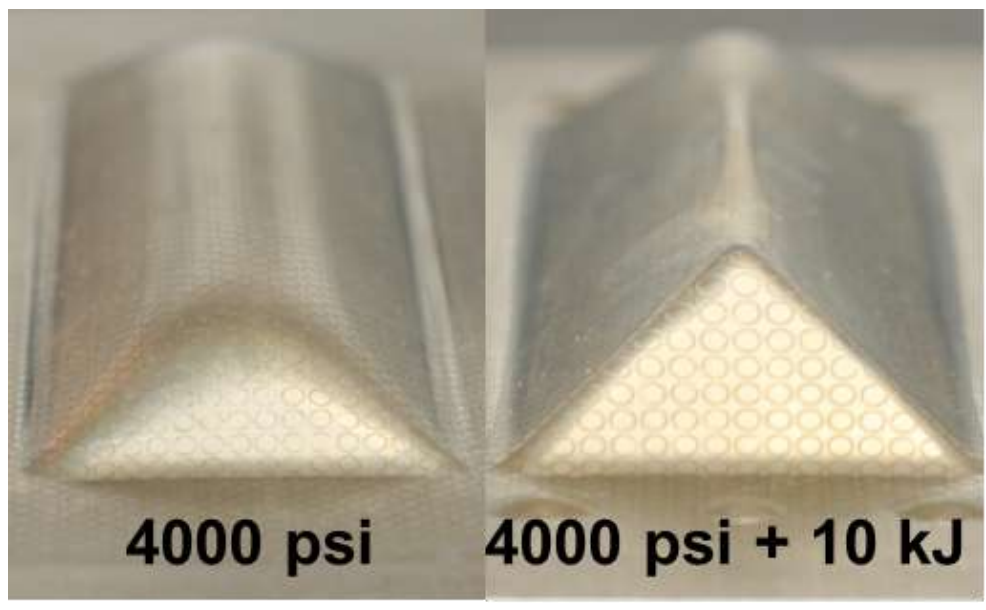

Figure 4.1.8. Test specimens of HSLA 350, $1.0 \mathrm{~mm}$, used in pre-forming experiments. These specimens were formed into a v-shaped channel die in plane strain. The specimen on the left was formed using only quasi-static water pressure at 4000 psi. The specimen on the right was pre-formed at $4000 \mathrm{psi}$ and then finally formed using one EHF pulse at $8 \mathrm{kV}(10 \mathrm{~kJ})$. 


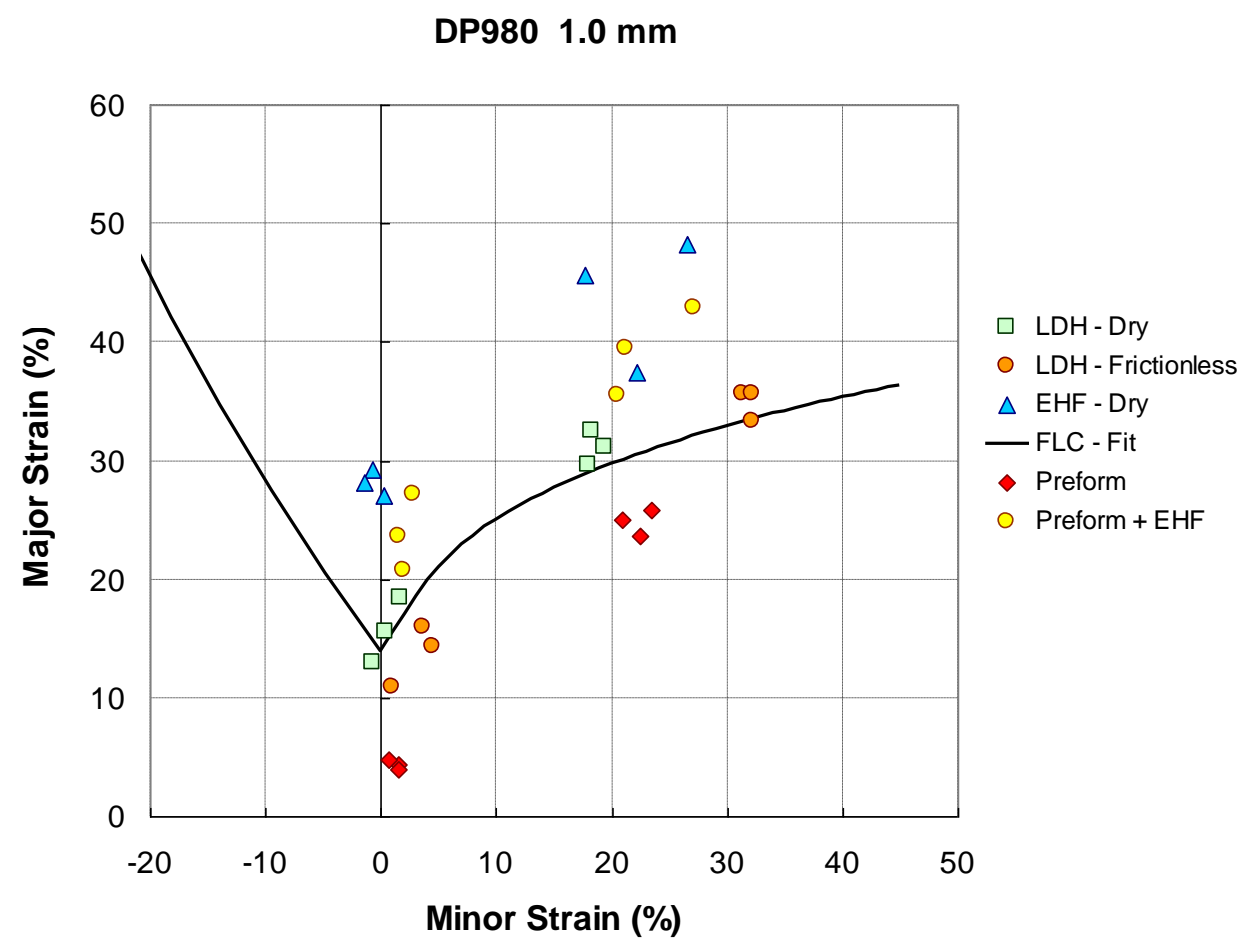

Figure 4.1.9. Experimental results of the effect of static pre-forming on formability of DP980, 1.0 $\mathrm{mm}$, in EHF.

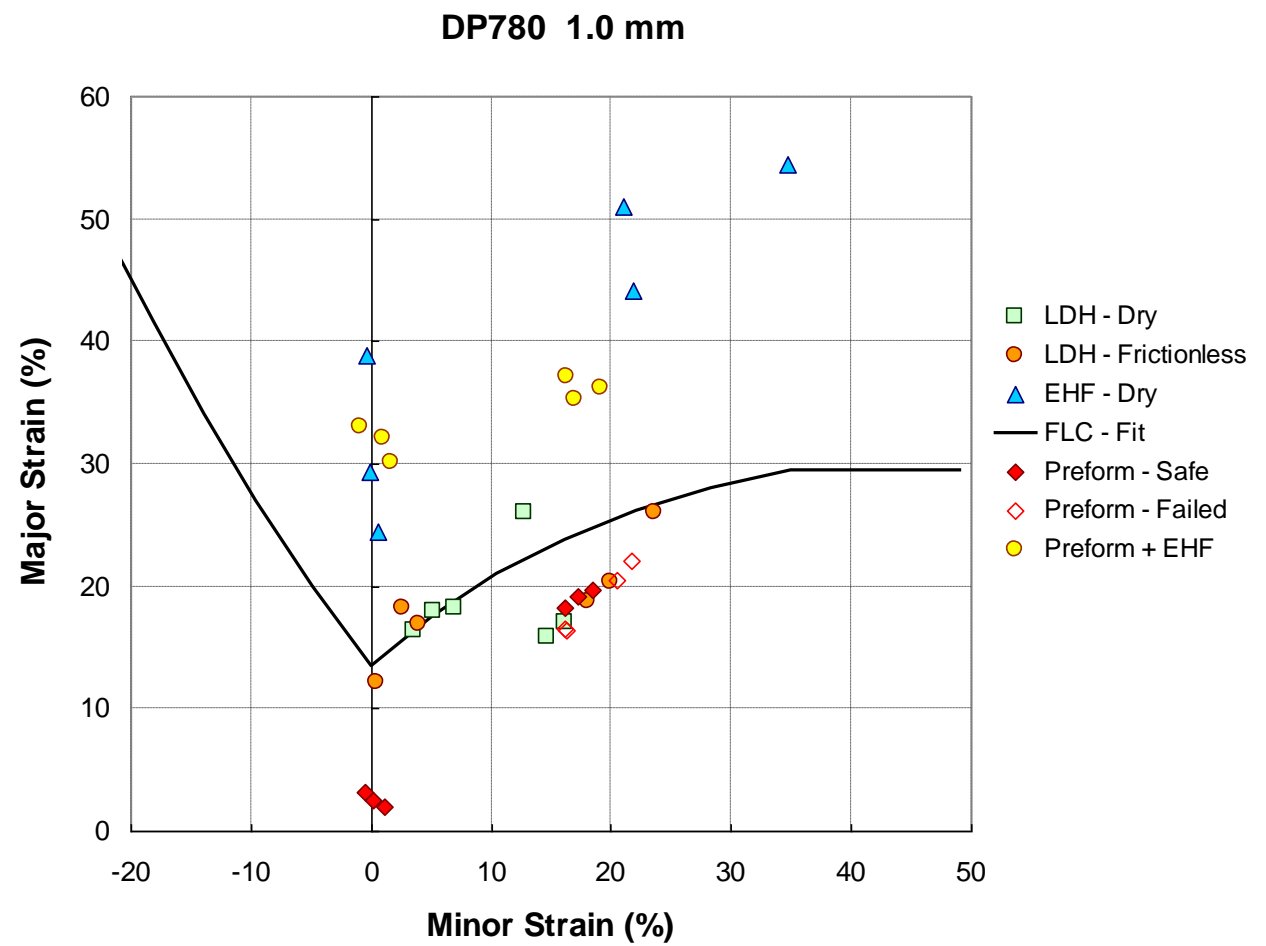

Figure 4.1.10. Experimental results of the effect of static pre-forming on formability of DP780, $1.0 \mathrm{~mm}$, in EHF. 
HSLA $3501.0 \mathrm{~mm}$

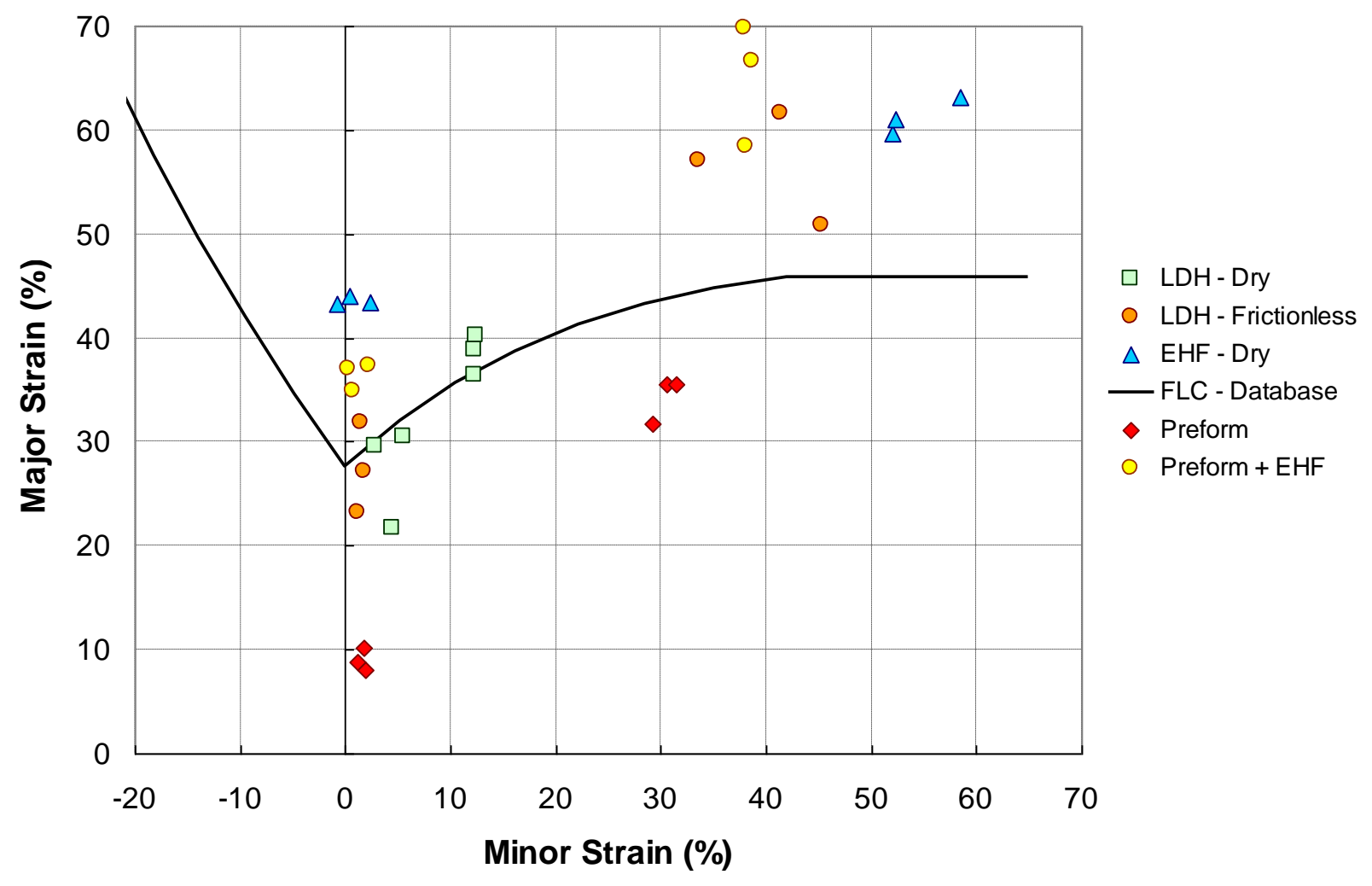

Figure 4.1.11. Experimental results of the effect of static pre-forming on formability of HSLA 350, $1.0 \mathrm{~mm}$, in EHF. 
Sub-task 4.2. Define the die material and lubricant appropriate for EHF.

\section{Evaluation of Die Materials in EHF Conditions}

Four different alloys were selected for evaluation as possible tool materials for EHF dies: (1) D6510, a nodular cast iron, (2) S0050A cast steel, (3) DC53 tool steel, and (4) SLD-Magic tool steel. Four v-shaped channel dies were fabricated, one from each material, for the purpose of testing in EHF conditions. After fabrication, each die was then heat treated and surface treated. Surface treatment consisted of nitriding followed by ion nitriding in order to create a thin and wear resistant layer of chromium nitride. The goal of the initial nitriding treatment was to create a moderately hard but well-anchored support layer which would allow better adhesion of the outermost chromium nitride layer. A summary of the mechanical properties of each material before heat treatment, after heat treatment, and after each surface treatment, is shown in Table 4.2.1. A summary of the chemical compositions of these alloys is shown in Table 4.2.2. The mechanical properties before heat treatment are nominal and were obtained from background literature on each material. The mechanical properties after heat treatment were obtained by performing hardness tests on blocks of material which were put through the same heat treatments and surface treatments as the dies. All four of the materials were evaluated using micro-hardness measurements, optical microscopy and scanning electron microscopy.

Table 4.2.1. Summary of mechanical properties of the die materials under evaluation.

\begin{tabular}{|c|c|c|c|c|}
\hline & D6510 & S0050A & DC-53 & SLD MAGIC \\
\hline Description & $\begin{array}{l}\text { Pearlitic Ductile } \\
\text { Cast Iron }\end{array}$ & $\begin{array}{l}\text { Alloyed Cast } \\
\text { Steel }\end{array}$ & Tool Steel & Tool Steel \\
\hline Producer, or Manager of Standard & NAAMS & NAAMS & Daido Steel & Hitachi Metals \\
\hline Hardness (before heat treatment) & $190-248 \mathrm{BHN}$ & $170-229 \mathrm{BHN}$ & $225 \mathrm{BHN}$ & $255 \mathrm{BHN}$ \\
\hline Tensile Strength (before heat treatment) & $570 \mathrm{MPa}$ & $585 \mathrm{MPa}$ & $\sim 750 \mathrm{MPa}$ & $\sim 850 \mathrm{MPa}$ \\
\hline Yield Strength (before heat treatment) & $350 \mathrm{MPa}$ & $310 \mathrm{MPa}$ & - & - \\
\hline Elongation (before heat treatment) & $4 \%$ & $16 \%$ & - & - \\
\hline Bulk Hardness (after heat treatment) & $350 \mathrm{HV}(30 \mathrm{HRC})$ & $420 \mathrm{HV}$ (35 HRC) & 850 HV (63 HRC) & $815 \mathrm{HV}(61 \mathrm{HRC})$ \\
\hline Thickness and Hardness of Nitrided Layer & $160 \mu \mathrm{m}, 660 \mathrm{HV}$ & $200 \mu \mathrm{m}, 890 \mathrm{HV}$ & $120 \mu \mathrm{m}, 1449 \mathrm{HV}$ & $120 \mu \mathrm{m}, 1438 \mathrm{HV}$ \\
\hline Thickness and Hardness of CrN Layer & $\begin{array}{l}5.2-5.5 \mu \mathrm{m} \\
2650 \mathrm{HV}\end{array}$ & $\begin{array}{l}4.4-5.2 \mu \mathrm{m} \\
2800 \mathrm{HV}\end{array}$ & $\begin{array}{c}5.2-5.6 \mu \mathrm{m} \\
3115 \mathrm{HV}\end{array}$ & $\begin{array}{c}4.5-4.6 \mu \mathrm{m} \\
3090 \mathrm{HV}\end{array}$ \\
\hline Nominal Hardness (after heat treatment) & $55 \mathrm{HRC}$ & $55 \mathrm{HRC}$ & $62 \mathrm{HRC}$ & $62 \mathrm{HRC}$ \\
\hline Tensile Strength (after heat treatment) & $\begin{array}{c}\sim 1000 \mathrm{MPa} \text { (bulk) } \\
>1750 \mathrm{MPa} \text { (surface) }\end{array}$ & $\begin{array}{c}\sim 1125 \mathrm{MPa} \text { (bulk) } \\
>1750 \mathrm{MPa} \text { (surface) }\end{array}$ & $\begin{array}{c}\text { > } \\
\text { (bulk) }\end{array}$ & $\begin{array}{c}\text { > } 1750 \mathrm{MPa} \\
\text { (bulk) }\end{array}$ \\
\hline
\end{tabular}


Table 4.2.2. Summary of the chemical composition of the die materials under evaluation.

\begin{tabular}{|l|c|c|c|c|c|c|c|}
\multicolumn{2}{c}{} & C & Si & Mn & Mo & Ni & Cu \\
\hline D6510 & $3.0-3.8$ & $2.0-2.4$ & $0.3-0.6$ & $0.35-0.50$ & $0.5-1.5$ & $0.35-1.0$ & 0.12 max. \\
\hline S0050A & $0.4-0.5$ & $0.2-0.5$ & $0.9-1.2$ & $0.35-0.50$ & & & $0.8-1.1$ \\
\hline DC-53 & 0.95 & 1.0 & 0.4 & 2.0 & & & 8.0 \\
\hline SLD-Magic & $0.7-1.6$ & $0.5-3.0$ & $0.1-3.0$ & $0.25-0.85$ & $0.3-1.5$ & $0.1-1.0$ & $7.0-13.0$ \\
\hline
\end{tabular}

\begin{tabular}{|l|c|c|c|c|c|c|}
\multicolumn{1}{l}{ (continued) } & \multicolumn{1}{c}{ S } & \multicolumn{1}{c}{ P } & Mg & Al & W \\
\hline D6510 & 0.02 max. & 0.05 max. & $0.04-0.06$ & & & \\
\hline S0050A & 0.05 max. & 0.045 max. & & 0.15 max. & & \\
\hline DC-53 & & & & 0.3 & & \\
\hline SLD-Magic & $0.01-0.12$ & 0.05 max. & & $0.0-0.7$ & $0.1-0.7$ & $0.5-1.7$ \\
\hline
\end{tabular}

Source Data: $\quad$ D6510 - The NAAMS Global Standard Components - www.naamsstandards.org

S0050A - The NAAMS Global Standard Components - www.naamsstandards.org

DC-53 - International Mold Steel, Inc. - www.imsteel.com

SLD-Magic - United States Patent Application No. US2009/0120540 A1

Testing of each die material in EHF conditions was done according to the following methodology. Every forming cycle started with a flat blank, and the blank was fully formed into the die cavity using one pulse of energy. Figure 4.2.1 shows photographs of the four die inserts, the insert holder, and the EHF chamber used in the testing. Blanks were formed into each die at increasing levels of energy until cracks were observed in the die. The overall dimensions of each of the four die inserts, in terms of $\mathrm{L} \times \mathrm{W} \times \mathrm{H}$, were $230 \mathrm{~mm}$ by $70 \mathrm{~mm}$ by $45 \mathrm{~mm}$. The blank material, forming energy, and voltage for each pulse used in the experimental program are shown in Table 4.2.3. The forming conditions used in these experiments were designed to be much more aggressive than what would be expected in a production embodiment of EHF, and this was done in order to accelerate the testing and to get meaningful results in a relatively short period of time. The factors which made this testing so aggressive were the following: (1) the blanks were always fully formed using one EHF pulse, rather than being formed incrementally using multiple EHF pulses, and (2) the relatively small volume of the EHF chamber used, combined with the high energy levels of each pulse, created tremendous impact forces on the die surface with each pulse. 


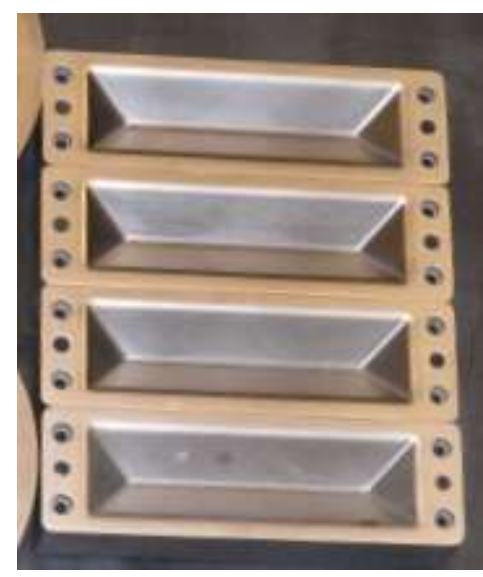

(a)

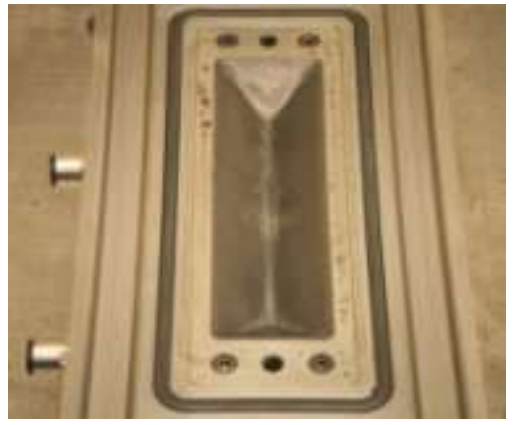

(b)

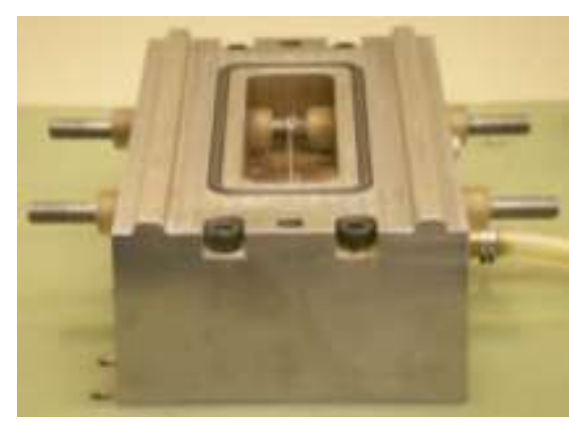

(c)

Figure 4.2.1. Tooling used to evaluate die materials in EHF conditions. (a) die inserts fabricated from D6510, S0050A, DC53, and SLD-Magic, (b) insert holder, and (c) EHF chamber.

Table 4.2.3. Sequence of EHF pulses used to evaluate each die material.

\begin{tabular}{|c|c|c|}
\hline $\begin{array}{c}\text { Voltage } \\
(\mathbf{k V})\end{array}$ & $\begin{array}{c}\text { Energy } \\
(\mathbf{k J})\end{array}$ & Blank Material \\
\hline 7.07 & 8.00 & DP500 - 0.7 mm \\
\hline 7.50 & 9.00 & $"$ \\
\hline 7.91 & 10.00 & HSLA 350 - $1.0 \mathrm{~mm}$ \\
\hline 8.29 & 11.00 & $"$ \\
\hline 8.66 & 12.00 & DP590 - 1.0 mm \\
\hline 9.01 & 13.00 & $"$ \\
\hline 9.35 & 14.00 & DP780 - $1.0 \mathrm{~mm}$ \\
\hline 9.68 & 15.00 & $"$ \\
\hline 10.00 & 16.00 & DP980 - $1.0 \mathrm{~mm}$ \\
\hline 10.31 & 17.00 & $"$ \\
\hline 10.61 & 18.00 & $"$ \\
\hline 10.90 & 19.00 & $"$ \\
\hline 11.18 & 20.00 & $"$ \\
\hline 11.73 & 22.00 & $"$ \\
\hline 12.25 & 24.00 & $"$ \\
\hline 12.75 & 26.00 & $"$ \\
\hline 13.23 & 28.00 & $"$ \\
\hline
\end{tabular}




\section{Evaluation of D6510 nodular iron}

Results of the evaluation of D6510 nodular iron are presented in Figures 4.2.2 to 4.2.4. Figure 4.2.2 shows the results of microhardness measurements for D6510 nodular iron in the Vickers scale obtained at $50 \mathrm{~g}$ load. The hardness of the outer CrN layer was measured at $5 \mathrm{~g}$ load, and was found to be $2650 \mathrm{HV}$. The ion-nitrided diffusion zone has a thickness of $160 \mu \mathrm{m}$ with the microhardness ranging from $660 \mathrm{HV}$ to $511 \mathrm{HV}$. The slight decrease in hardness right under the $\mathrm{CrN}$ layer is the result of decarburization, which occurs during flame hardening of the tools. The average hardness of the bulk tool material is $350 \mathrm{HV}$. Optical micrographs for D6510 nodular iron are presented in Figures 4.2.3a, 4.2.3b and 4.2.3c; these images are obtained at 100X, 500X and 1000X magnification correspondingly. The SEM image shown in Figure 4.2.3d was obtained at a magnification of $3000 \mathrm{X}$ and clearly shows the CrN layer with a thickness varying from 5.2 to $5.5 \mu \mathrm{m}$. Figure 4.2.4 presents photographs of the $\mathrm{D} 6510$ nodular iron $\mathrm{V}$-shape die after a series of EHF forming cycles. The first pulse in the series had an energy of $8 \mathrm{~kJ}$, and this pulse energy was gradually increased to the final pulse energy of $26 \mathrm{~kJ}$, in accordance with the schedule in Table 4.2.3. As we can see from the magnified area in Figure 4.2.4, severe wear and erosion led to the development of shallow micro-cracks at the bottom of the die.

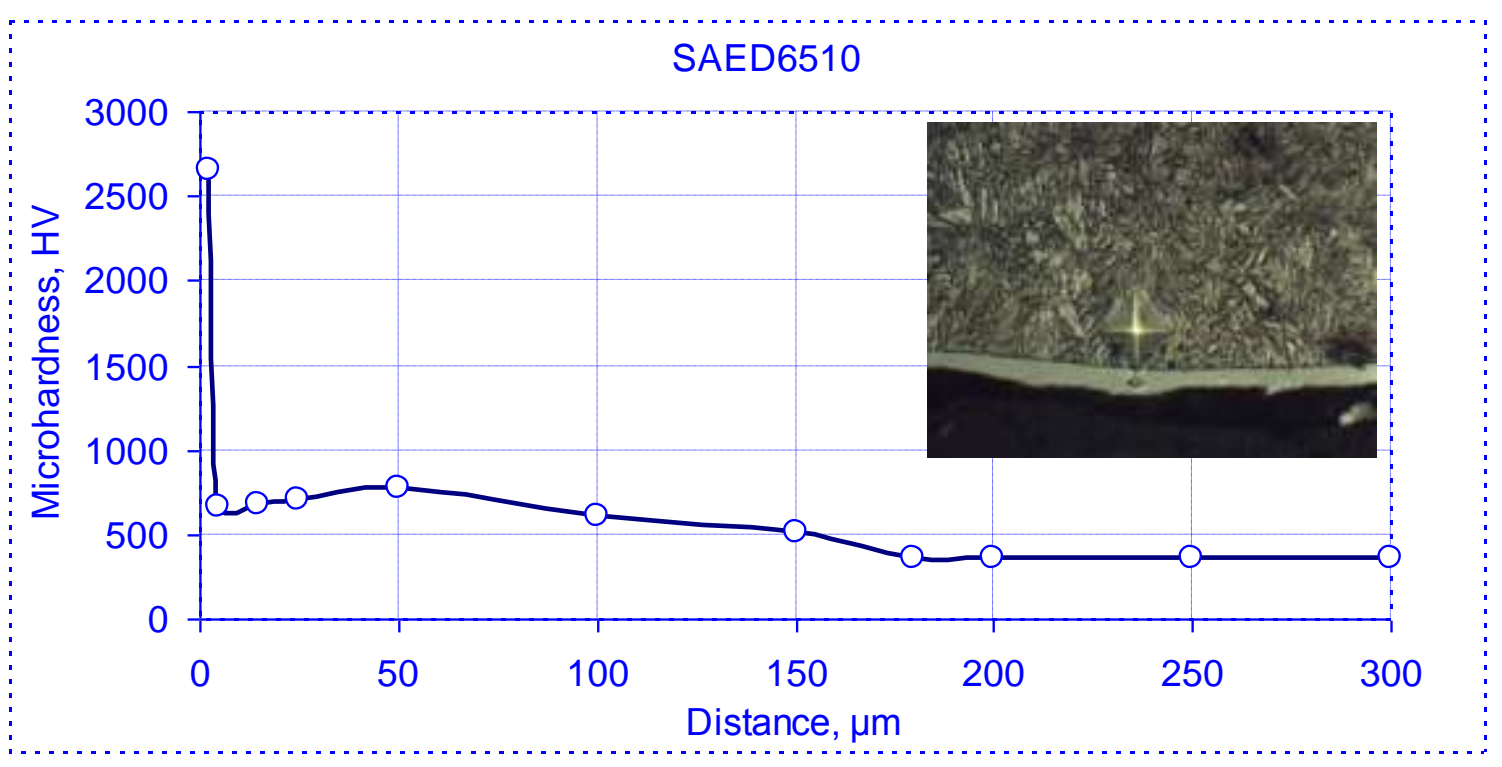

Figure 4.2.2. Results of microhardness measurements for D6510 nodular iron. 


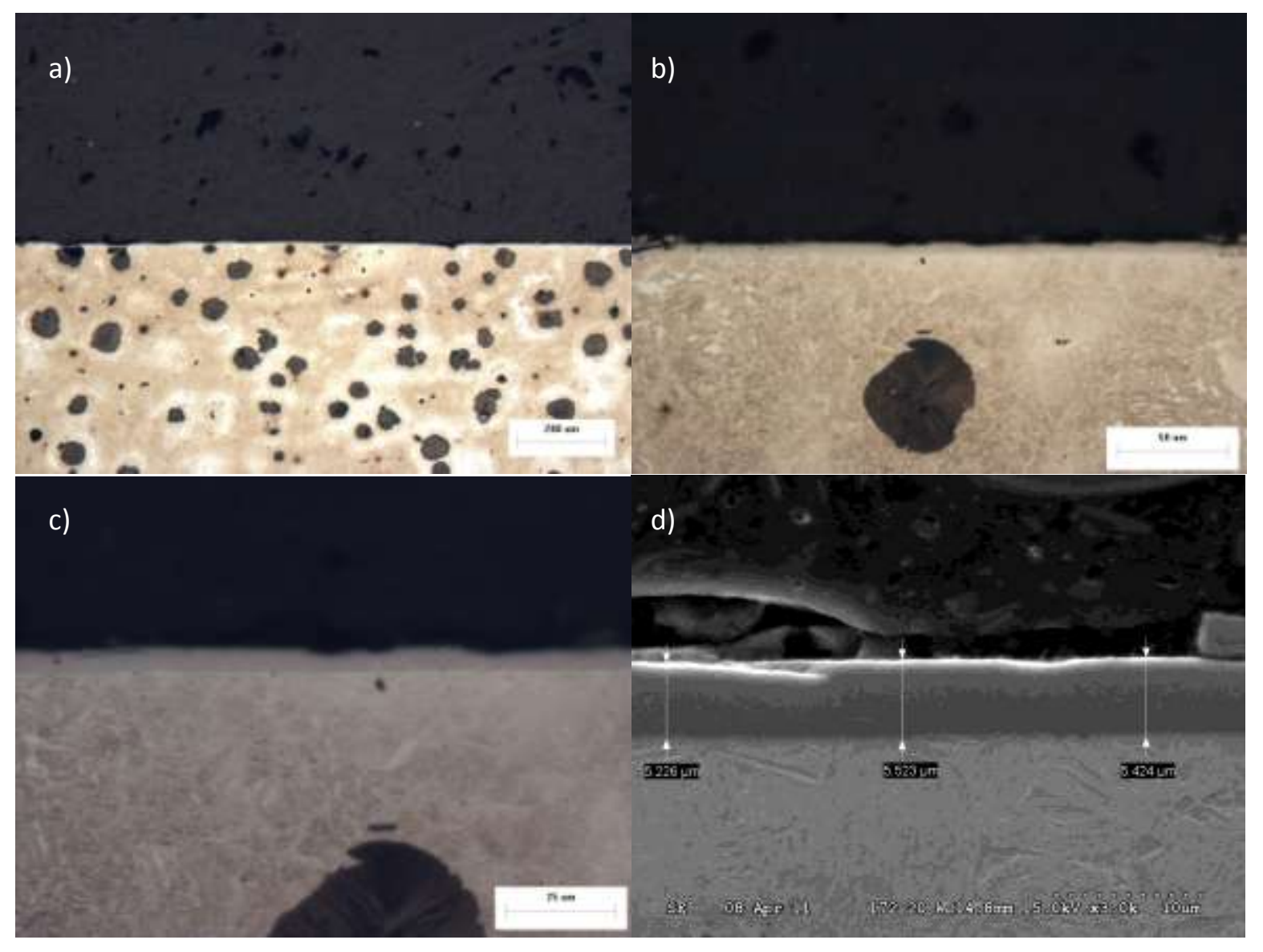

Figure 4.2.3. Optical micrographs for D6510 nodular iron; a) 100X, b) 500X, c) 1000X, d) 3000X SEM image; $2 \%$ Nital etch. 

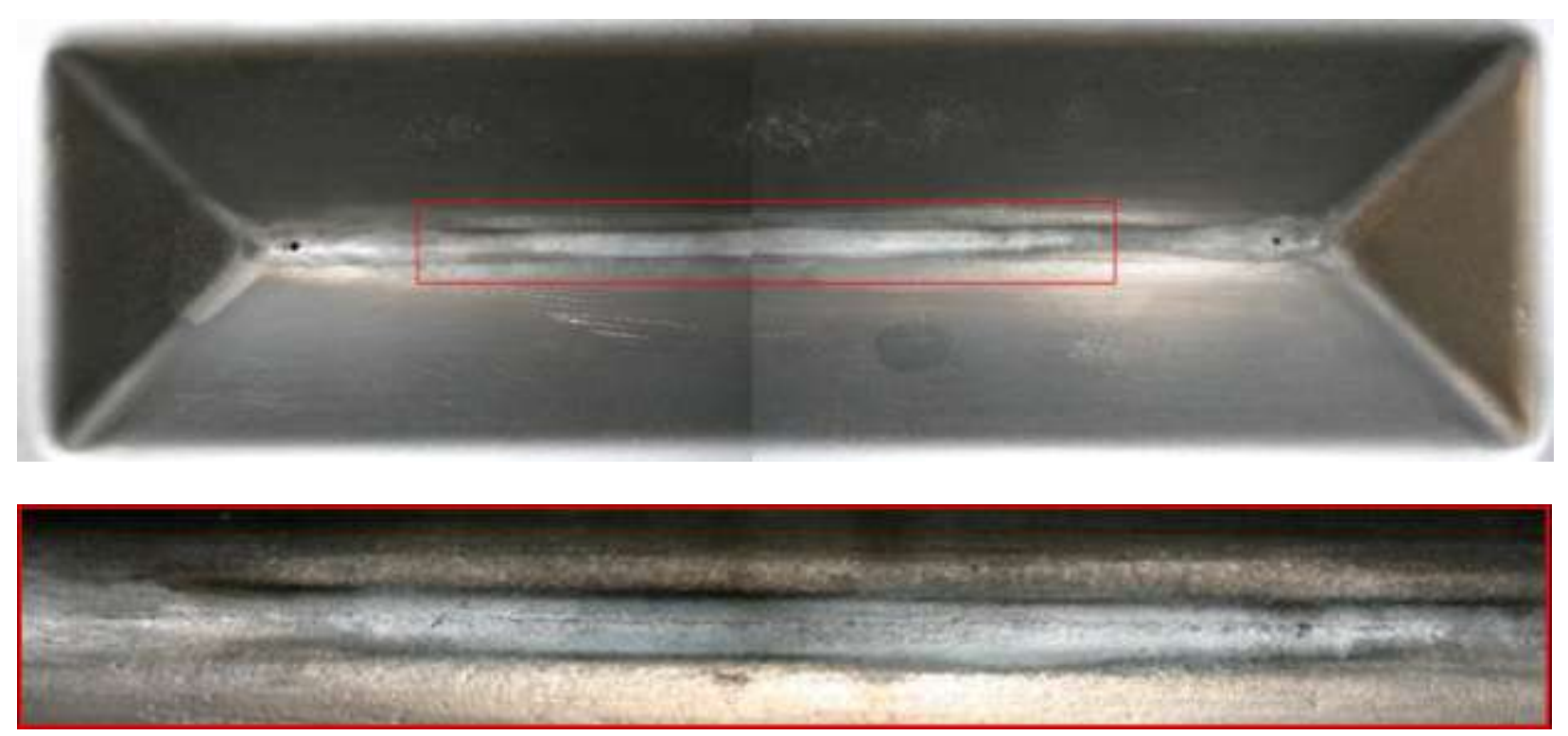

Figure 4.2.4. View of D6510 nodular iron V-shape die after a series of 16 EHF forming cycles. The first pulse in the series had an energy of $8 \mathrm{~kJ}$, and the final pulse had an energy of $26 \mathrm{~kJ}$.

\section{Evaluation of S0050A cast steel}

Results of the evaluation of S0050A cast steel are presented in Figures 4.2.5 to 4.2.7. Figure 4.2.5 shows the results of microhardness measurements for S0050A in the Vickers scale obtained at $50 \mathrm{~g}$ load. The hardness of the outer CrN layer was measured at $5 \mathrm{~g}$ load, and was found to be $2800 \mathrm{HV}$. The ion-nitrided diffusion zone has a thickness of $200 \mu \mathrm{m}$ with microhardness ranging from $890 \mathrm{HV}$ to $651 \mathrm{HV}$. The slight decrease in hardness right under the $\mathrm{CrN}$ layer is the result of decarburization, which occurs during flame hardening of the tools. The average hardness of the bulk tool material is $420 \mathrm{HV}$. Optical micrographs for the S0050A tool steel are presented in Figures 4.2.6a, 4.2.6b and 4.2.6c; these images were obtained at 100X, 500X and 1000X magnification correspondingly. The SEM image shown in Figure 4.2.6d was obtained at a magnification of $3000 \mathrm{X}$ and clearly shows the CrN layer with a thickness varying from 4.4 to $5.2 \mu \mathrm{m}$. Figure 4.2.7 presents photographs of the S0050A steel V-shape die after a series of EHF forming cycles. The first pulse in the series had an energy of $8 \mathrm{~kJ}$, and this pulse energy was gradually increased to the final pulse energy of $28 \mathrm{~kJ}$, in accordance with the schedule in Table 4.2.3. As we can see from the magnified area in Figure 4.2.7, severe wear and erosion led to the development of shallow micro-cracks at the bottom of the die. 


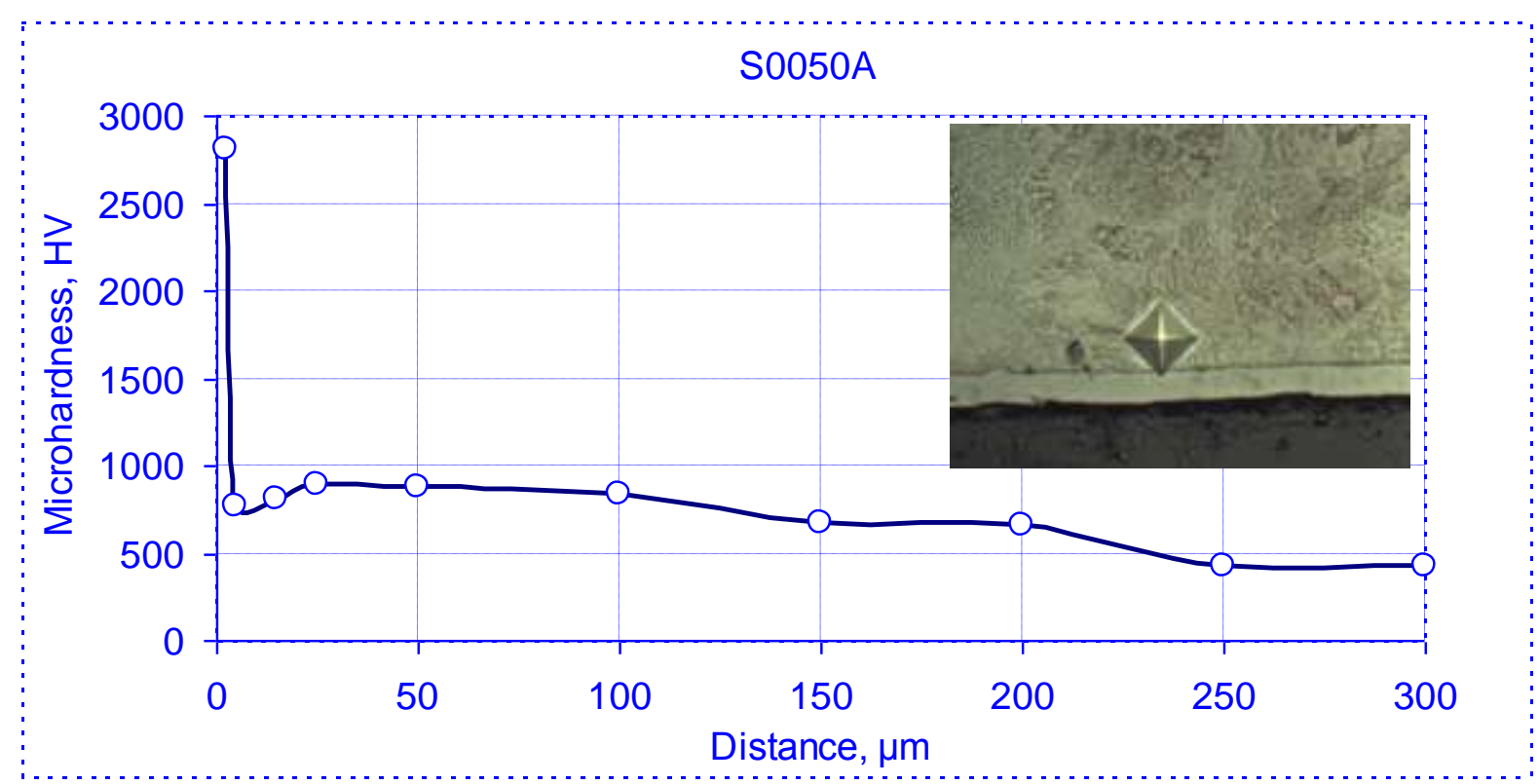

Figure 4.2.5. Results of microhardness measurements for S0050A steel.

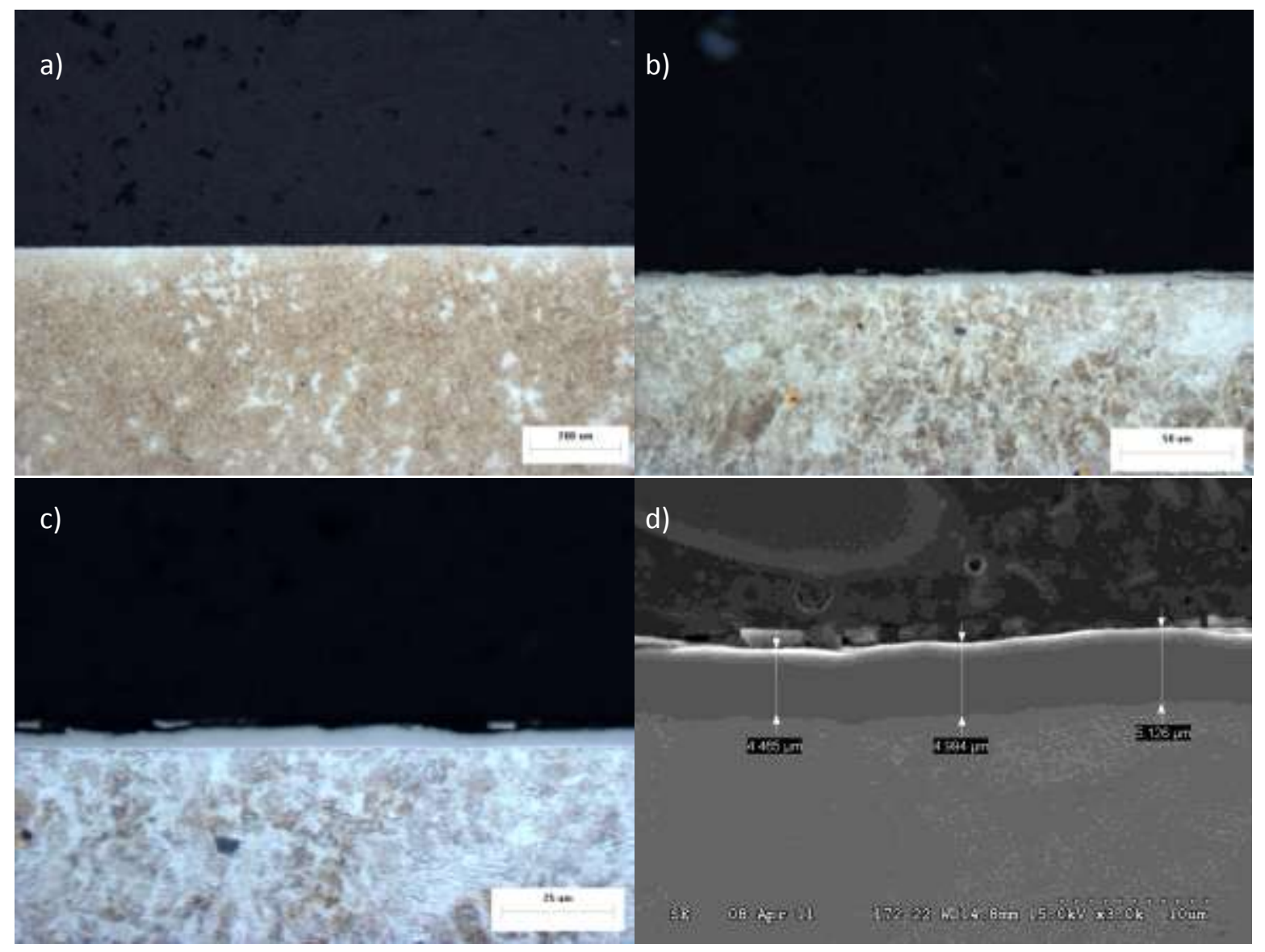

Figure 4.2.6. Optical micrographs for S0050A steel; a) 100X, b) 500X, c) 1000X, d) 3000X SEM image; $2 \%$ Nital etch. 

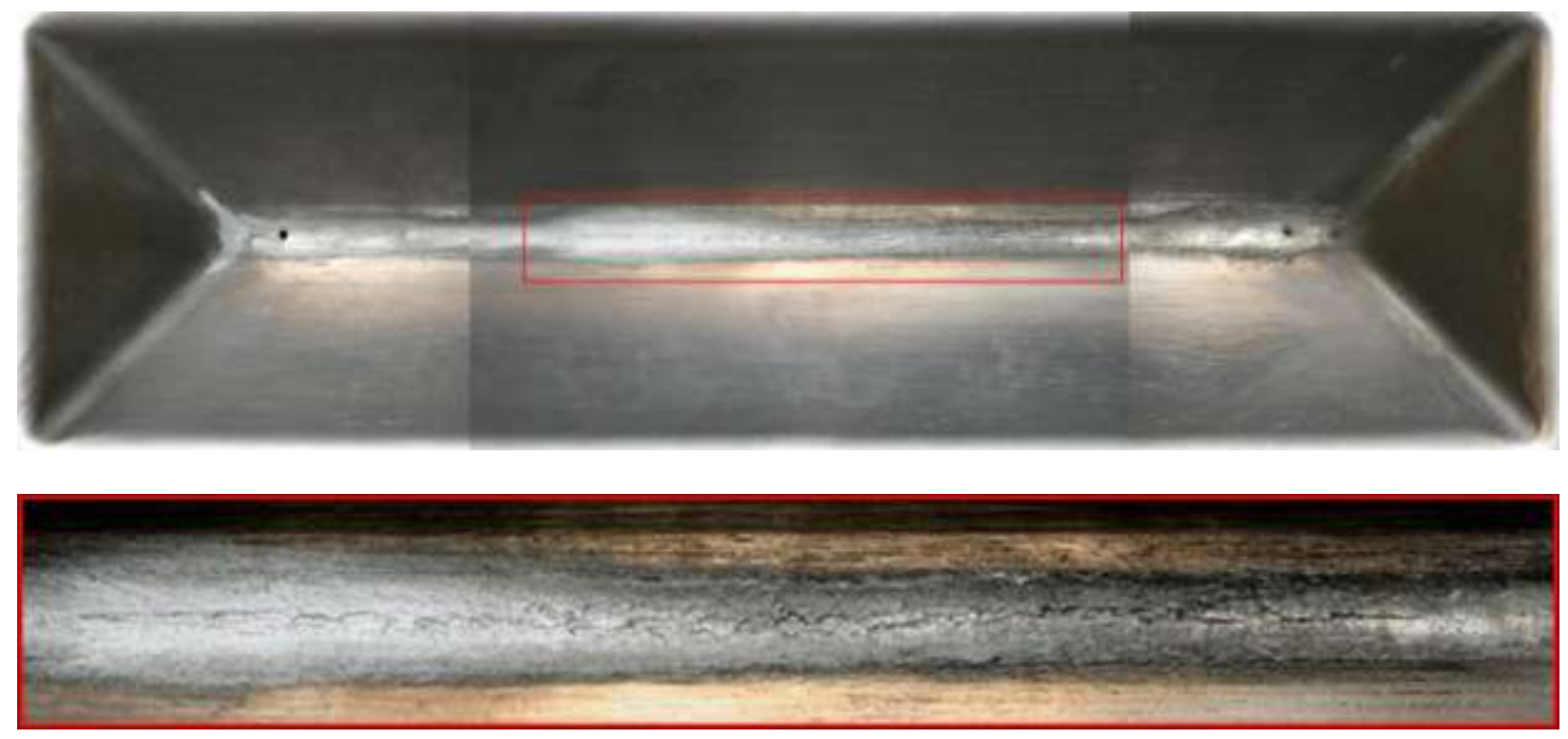

Figure 4.2.7. View of S0050A steel V-shape die after series of $17 \mathrm{EHF}$ forming cycles. The first pulse in the series had an energy of $8 \mathrm{~kJ}$, and the final pulse had an energy of $28 \mathrm{~kJ}$.

\section{Evaluation of DC53 tool steel}

Results of the evaluation of DC53 tool steel are presented in Figures 4.2.8 to 4.2.10. Figure 4.2.8 shows the results of microhardness measurements for DC53 tool steel in the Vickers scale obtained at $50 \mathrm{~g}$ load. The hardness of the outer $\mathrm{CrN}$ layer was measured at $5 \mathrm{~g}$ load, and was found to be $3115 \mathrm{HV}$. The ion-nitrided diffusion zone has a thickness of $120 \mu \mathrm{m}$ with the microhardness ranging from $1449 \mathrm{HV}$ to $1000 \mathrm{HV}$. The average hardness of bulk tool material is $850 \mathrm{HV}$. Optical micrographs for DC53 tool steel are presented in Figures 4.2.9a, 4.2.9b and 4.2.9c; these images were obtained at $100 \mathrm{X}, 500 \mathrm{X}$ and $1000 \mathrm{X}$ magnification correspondingly. The SEM image shown in Figure 4.2.9d was obtained at a magnification of 3000X and clearly shows the $\mathrm{CrN}$ layer with a thickness varying from 5.2 to $5.6 \mu \mathrm{m}$. Figure 4.2 .10 presents photographs of the DC53 tool steel V-shape die after a series of EHF forming cycles. As we can see from the magnified area in Figure 4.2.10, some wear and erosion is present on the die surface, but the primary failure mode was a sharp, deep, through-thickness crack that occurred after a forming pulse at $17 \mathrm{~kJ}$. The first pulse in the series had an energy of $8 \mathrm{~kJ}$, and this pulse energy was gradually increased to the final pulse energy of $17 \mathrm{~kJ}$, in accordance with the schedule in Table 4.2.3. 


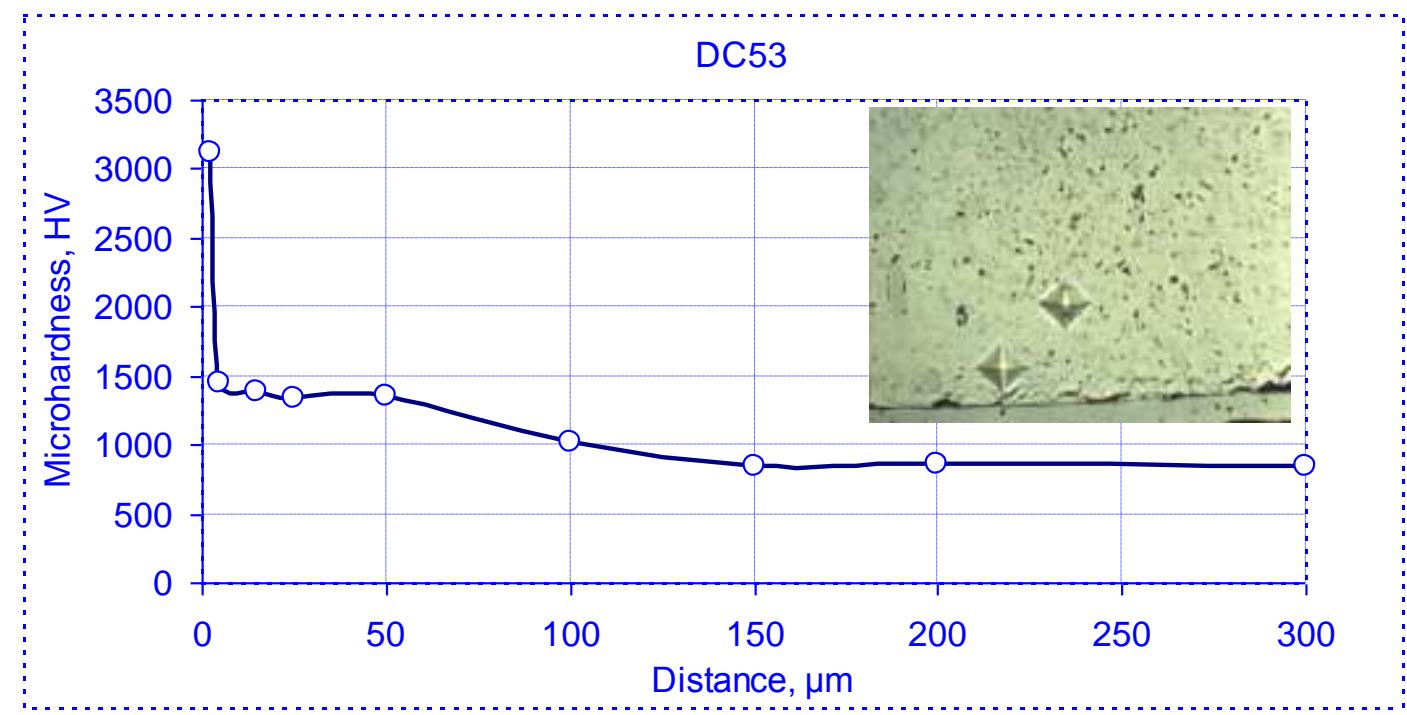

Figure 4.2.8. Results of microhardness measurements for DC53 tool steel.

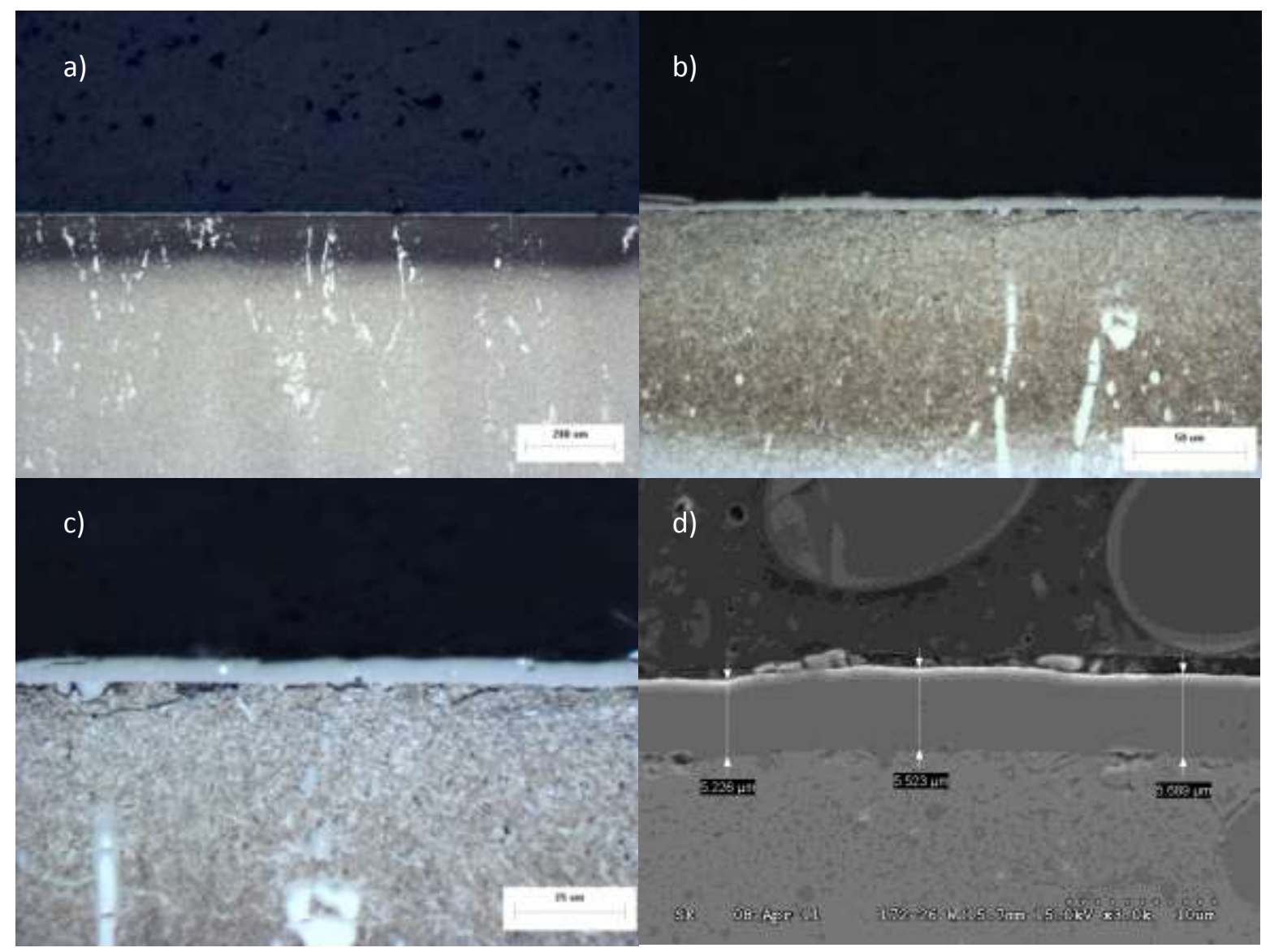

Figure 4.2.9. Optical micrograph for DC53 tool steel; a) 100X, b) 500X, c) 1000X, d) 3000X SEM image; $2 \%$ Nital etch. Note the presence of large clusters of carbides. 

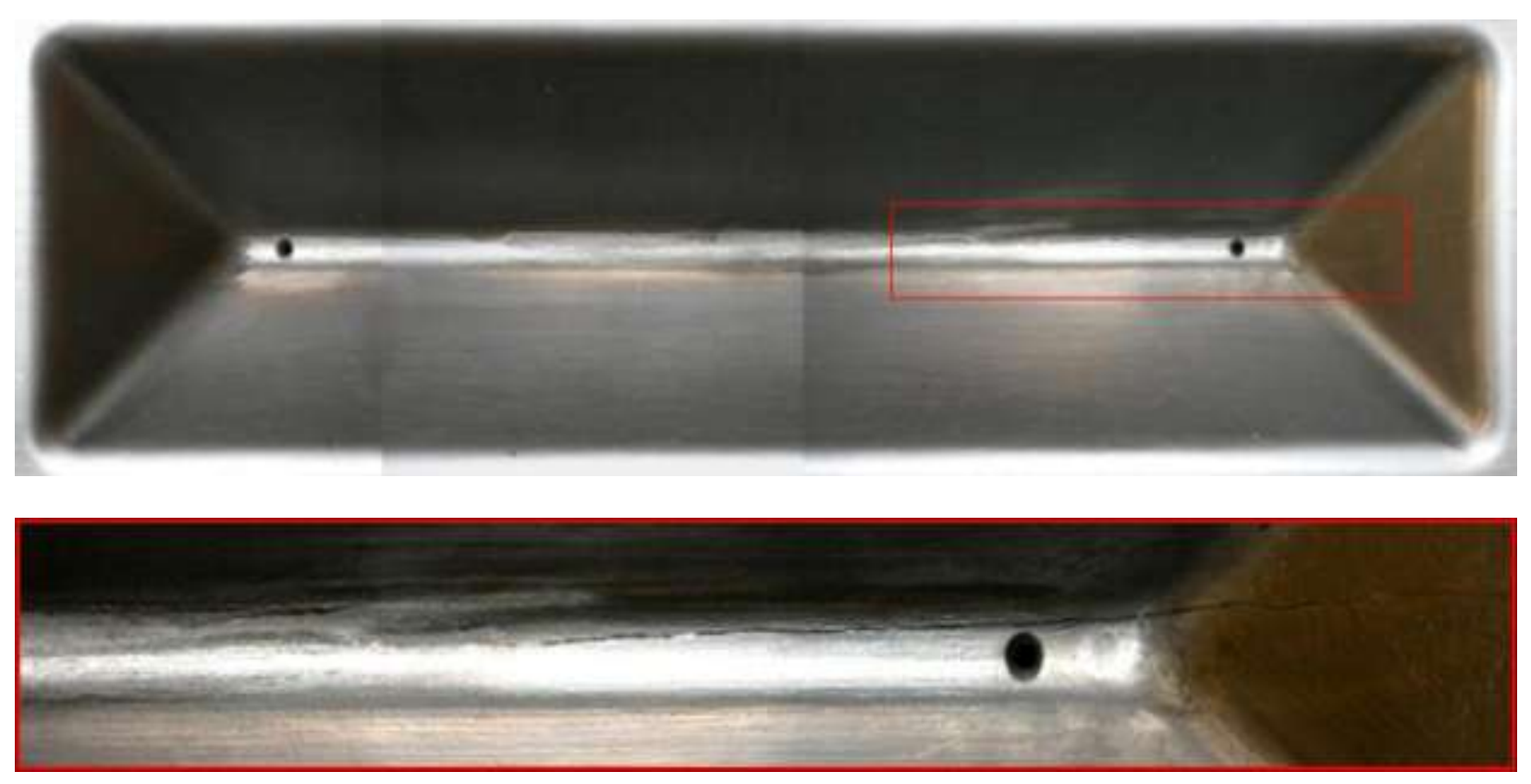

Figure 4.2.10. View of DC53 tool steel V-shape die after a series of ten EHF forming cycles. The first pulse in the series had an energy of $8 \mathrm{~kJ}$, and the final pulse had an energy of $17 \mathrm{~kJ}$.

\section{Evaluation of SLD Magic tool steel}

Results of the evaluation of SLD Magic tool steel are presented in Figures 4.2.11 to 4.2.13. Figure 4.2.11 shows the results of microhardness measurements for SLD Magic tool steel in the Vickers scale obtained at $50 \mathrm{~g}$ load. The hardness of the outer $\mathrm{CrN}$ layer was measured at $5 \mathrm{~g}$ load, and was found to be $3090 \mathrm{HV}$. The ion-nitrided diffusion zone has a thickness of $120 \mu \mathrm{m}$ with the microhardness ranging from $1438 \mathrm{HV}$ to $1197 \mathrm{HV}$. The average hardness of the bulk tool material is $815 \mathrm{HV}$. Optical micrographs for SLD Magic tool steel are presented in Figures 4.2.12a, 4.2.12b and 4.2.12c; these images were obtained at 100X, 500X and 1000X magnification correspondingly. The SEM image shown in Figure 4.2.12d was obtained at a magnification of 3000X and clearly shows the $\mathrm{CrN}$ layer with a thickness varying from $4.53 \mu \mathrm{m}$ to 4.6 $\mu \mathrm{m}$. Figure 4.2.13 presents photographs of the SLD Magic tool steel V-shape die after a series of EHF forming cycles. The first pulse in the series had an energy of $8 \mathrm{~kJ}$, and this pulse energy was gradually increased to the final pulse energy of $18 \mathrm{~kJ}$, in accordance with the schedule in Table 4.2.3. As we can see from the magnified area in Figure 4.2.13, similarly to the DC53 material, the series of high energy impacts did cause slight wear and erosion. However, as with the DC53, the primary failure mode was a sharp, deep, through-thickness crack that occurred after a forming pulse at $18 \mathrm{~kJ}$. 


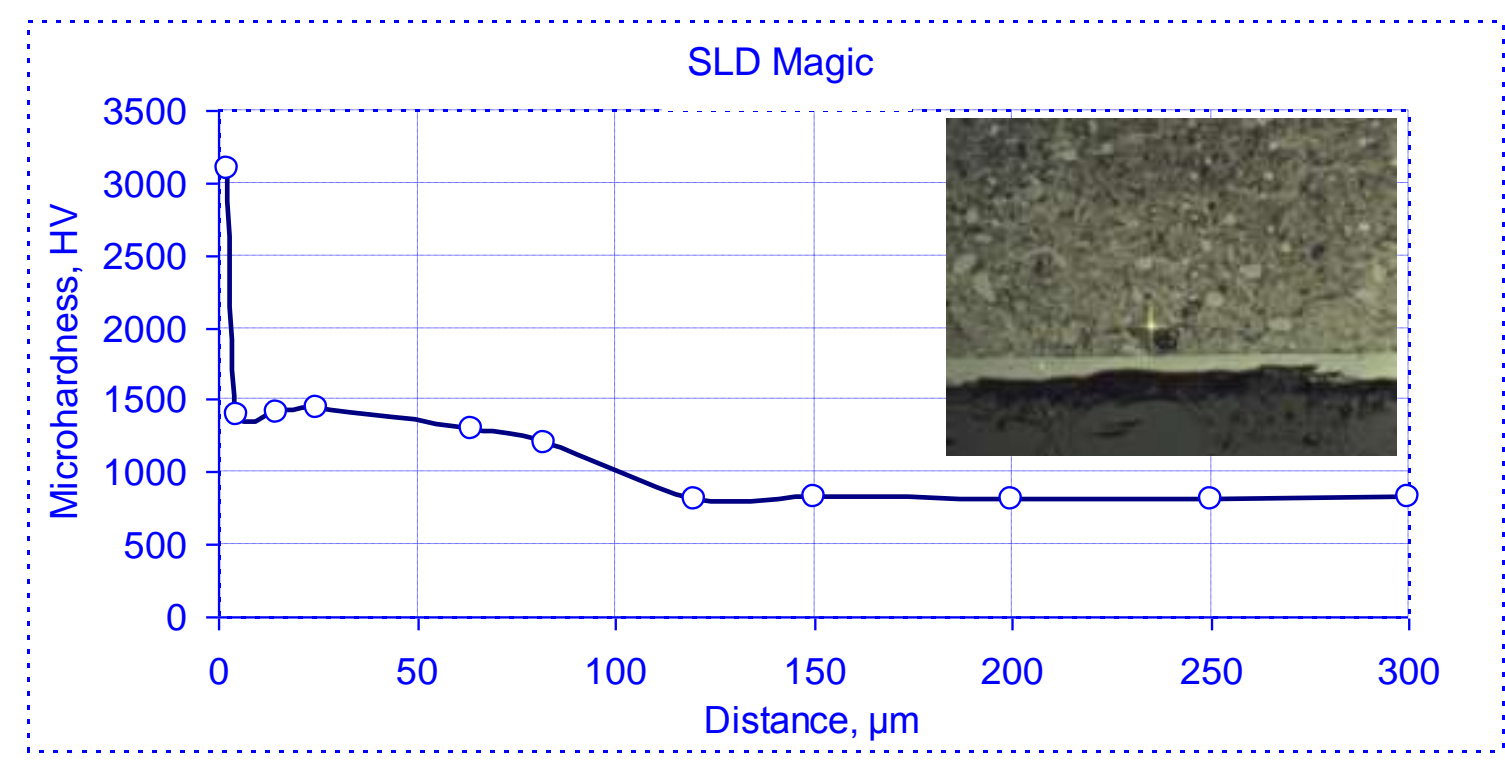

Figure 4.2.11. Results of microhardness measurements for SLD Magic tool steel.

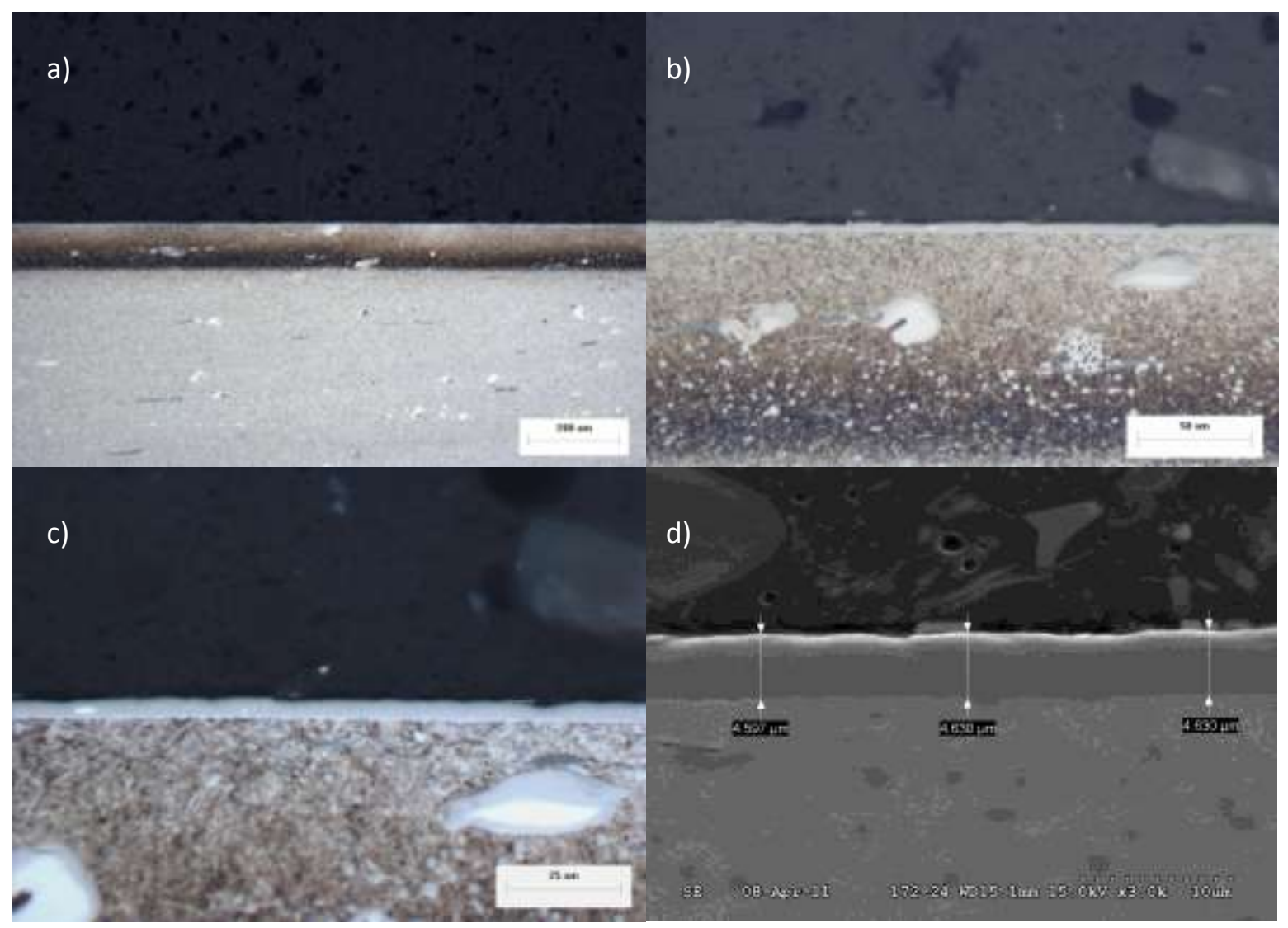

Figure 4.2.12. Optical micrograph for SLD Magic tool steel; a) 100X, b) 500X, c) 1000X, d) 3000X SEM image; $2 \%$ Nital etch. Note: carbides well dispersed. 

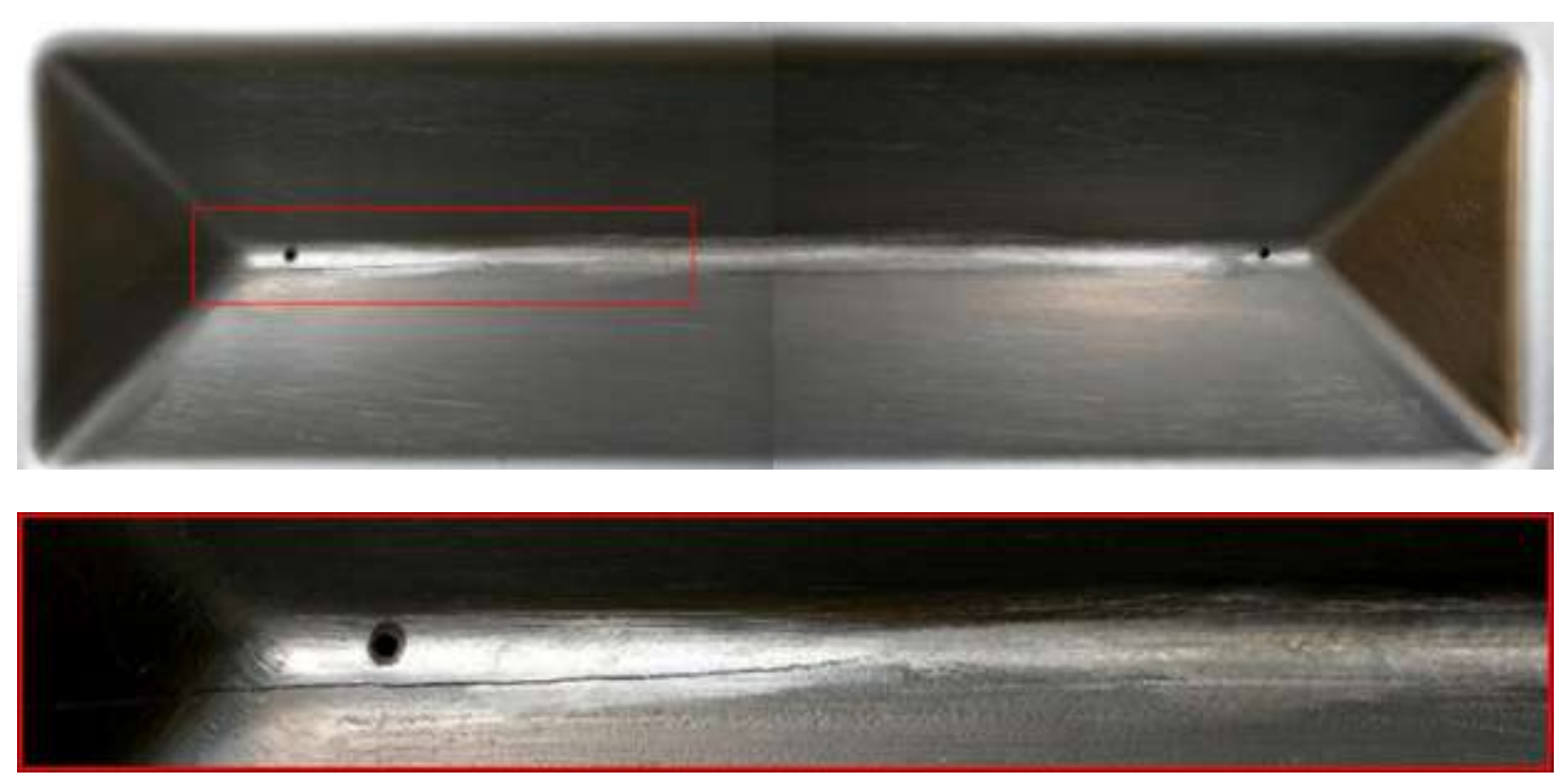

Figure 4.2.13. View of SLD Magic tool steel V-shape die after a series of $11 \mathrm{EHF}$ forming cycles. The first pulse in the series had an energy of $8 \mathrm{~kJ}$, and the final pulse had an energy of $18 \mathrm{~kJ}$.

\section{Conclusions Regarding Die Materials in EHF Conditions}

1. The materials with lower hardness, higher ductility, and higher fracture toughness, D6510 and S0050A, outperformed the high-alloyed and high wear-resistant alloys, DC53 and SLD Magic, in terms of the maximum pulse energy that the tools could withstand before tool failure.

2. The results obtained suggest that when selecting materials for EHF tooling, the primary criterion should be high impact fracture toughness, followed by high wear resistance.

3. Both D6510 and S0050A are Ford-approved materials for stamping dies, and are both relatively inexpensive when compared to higher-alloyed tool steels.

4. Recommendations for die-processing: (1) The target hardness after heat treating should not exceed 48 HRC. (2) The selection of a die-surface treatment should be made in light of the material selection, and would most likely be ion nitriding. 


\section{Evaluation of Lubricants in EHF conditions}

Testing of various lubricants in actual EHF forming conditions was conducted in the following way. The lubricants tested were Quaker 6130, and Quaker 61-MAL-HCL-1. Quaker 6130 is an amber colored liquid, and its main components are mineral oil and other petroleum distillates. Quaker 61-MAL-HCL-1 is a dark green translucent liquid, and its main components are also mineral oil and other petroleum distillates. The experiments consisted of drawing a blank of a known width into a die cavity using one pulse of EHF pressure, and then measuring the final width of the blank after forming, with a greater change in width indicating a larger draw-in length and therefore better performance from that lubricant. The blanks used in this experiment were DP590 steel, $1.0 \mathrm{~mm}$ thick, with a hot-dip-galvannealled coating. The blanks were cut to16.5 inches long by 5.25 inches wide. The lubricants were applied to each side of the blanks, at a coating weight of $1 \mathrm{ml}$ per $600 \mathrm{sq}$. in., which is consistent with Ford's specifications for lubricant weight on incoming blanks. This coating weight specification resulted in the application of 0.15 $\mathrm{ml}$ of lubricant being applied to each side of our blanks. The lubricant was dripped onto each blank using a volumetric pipette, and then spread evenly across the blank surface using a foam roller. Photographs of the EHF chamber and die used in this experiment are shown in Figure 4.2.14 below. This EHF chamber is equipped with three different electrodes positioned along its length, with the discharge occurring between the electrode tip and the chamber wall. For the lubricant experiments, all forming was done using a single pulse through the central electrode. This tooling lends itself well to experiments involving drawing metal, due to the long length of the chamber and die cavity, which allows the blank to flow in at the center. Photographs of the blanks before and after forming are shown in Figure 4.2.15 below.

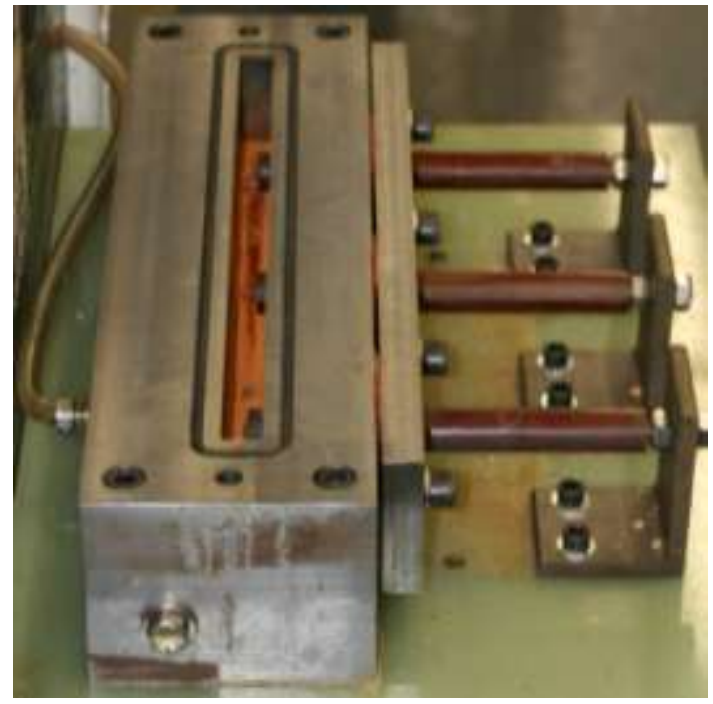

(a)

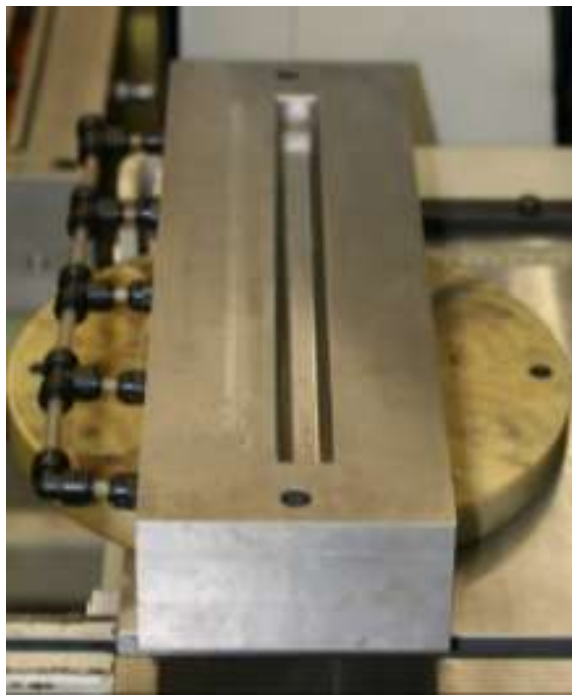

(b)

Figure 4.2.14. The EHF chamber (a) and die (b) used for lubricant testing. The die cavity is in the shape of an elongated U-channel, which allows for material to be drawn in near the central portion of the die. 


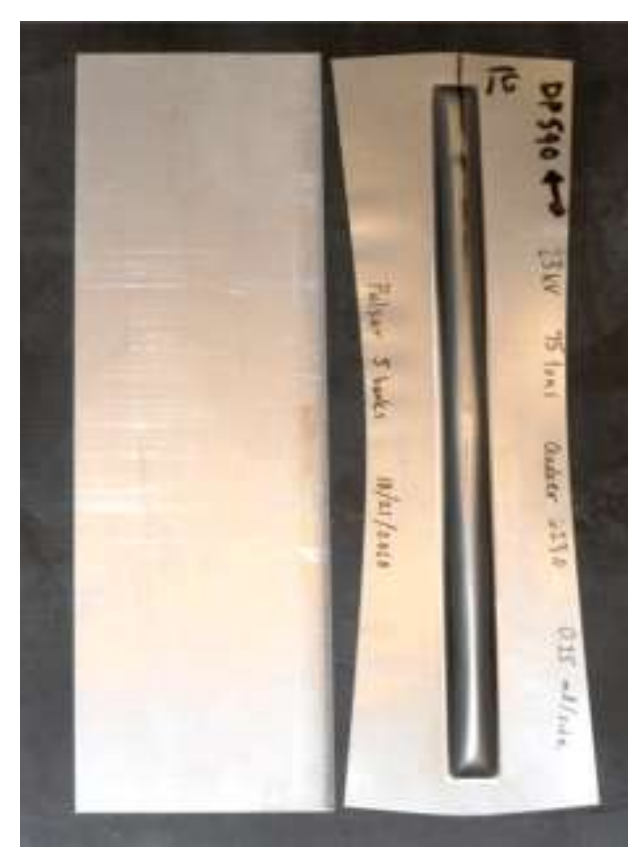

Figure 4.2.15. Photographs of blanks before and after forming. The draw-in length for each formed specimen was computed by subtracting the final width (measured at the middle of the blank) from the initial width.

Testing was done using two different levels of clamping force provided by a hydraulic press, 150 tons and 75 tons, and at three different lubrication conditions: (1) no lubricant, (2) 6130, and (3) $61-M A L-H C L-1$. The first tests were run at 150 tons of clamping force and were qualitative in nature. These tests were designed to determine, for each lubrication condition, how strong of a forming pulse the blanks could survive without splitting. Three blanks were formed at each condition in order to assess repeatability. For the unlubricated blanks, it was found that at forming energies below $15 \mathrm{kV}(22.5 \mathrm{~kJ})$, the blanks would survive, but at a forming energy of 15 $\mathrm{kV}$ the blanks were consistently splitting, as shown in Figure 4.2.16. Then, the same forming energy, $15 \mathrm{kV}(22.5 \mathrm{~kJ})$, was used to form blanks lubricated with 61-MAL-HCL-1. Now the blanks were consistently flowing into the die without splitting, confirming the positive impact of this lubricant in EHF conditions. Similar tests were run using Quaker 6130, and for this lubricant, still with 150 tons of clamping force, it was found that the blanks could consistently be formed at $16 \mathrm{kV}(25.6 \mathrm{~kJ})$ without splitting. Therefore, at 150 tons of clamping force, both the 6130 and the 61-MAL-HCL-1 showed improved performance compared to testing with no lubricant, with the 6130 showing slightly better performance at these conditions than the other lubricant. A summary of the test results at 150 tons in shown in the bar chart in Figure 4.2.17, comparing the amount of draw-in length measured from safely formed blanks at each condition. As expected, both of the test conditions involving lubrication showed greater draw-in lengths compared to the unlubricated condition. 


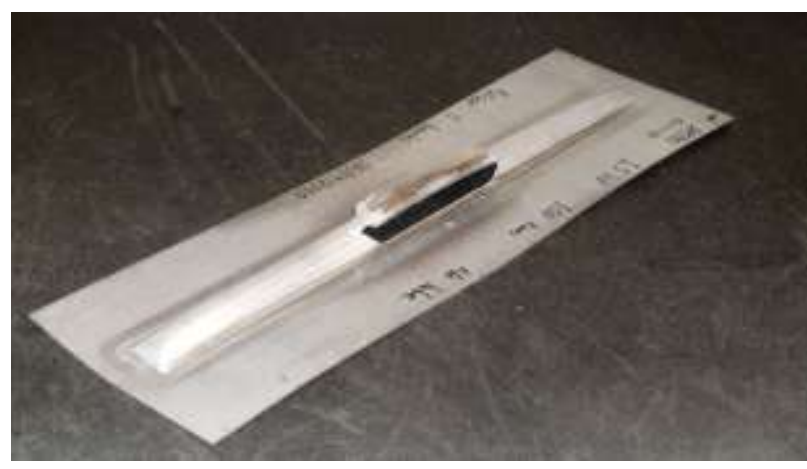

(a)

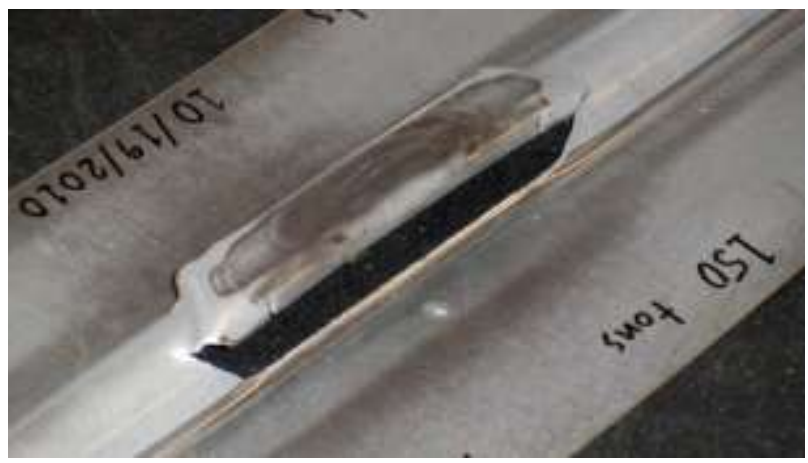

(b)

Figure 4.2.16. Photographs of blanks with splits arising due to inadequate lubrication: (a) full view of the blank, (b) close-up view of the split area.

\section{Lubricant testing at 150 tons clamping}

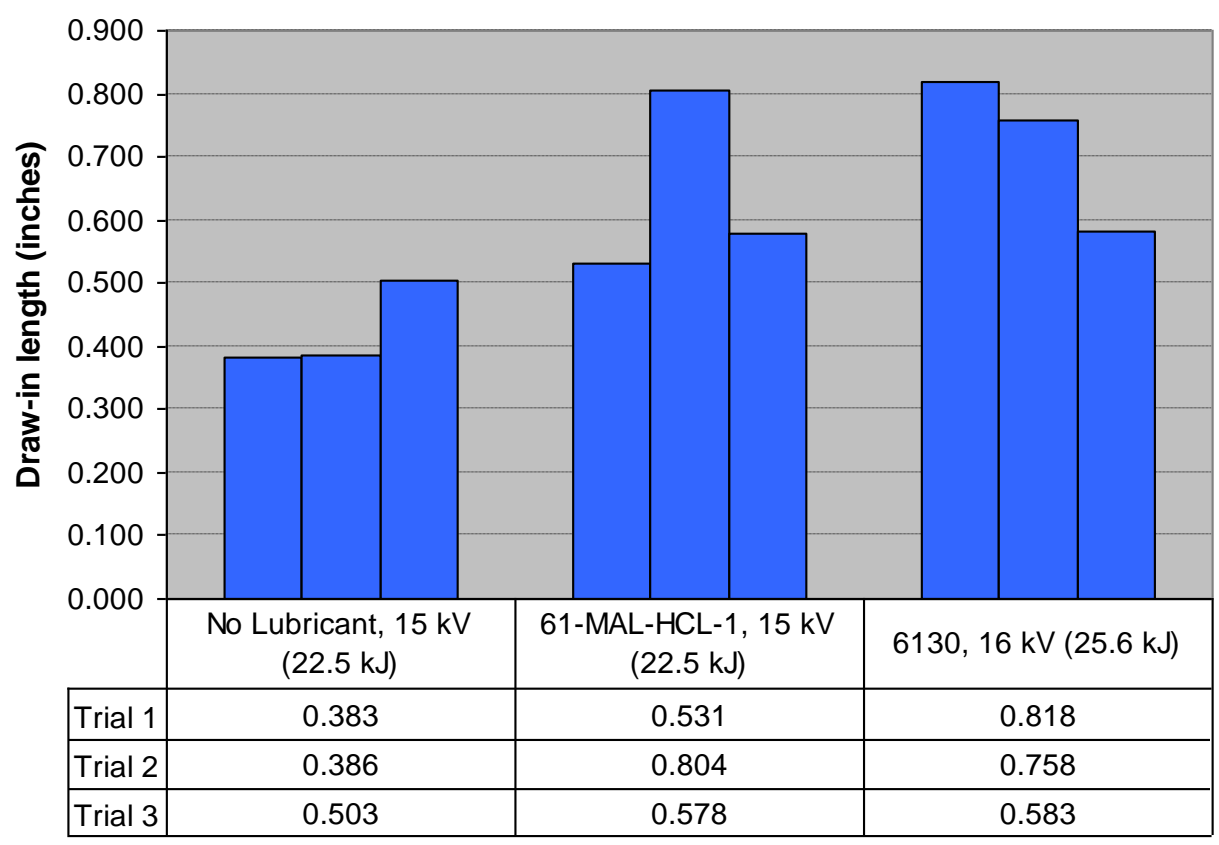

Figure 4.2.17. Summary of lubricant test results at a clamping force of 150 tons. Draw-in length was measured at the middle of each specimen after forming. 
Testing was also done at a lower clamping force, 75 tons. In these tests, a single pulse energy was selected $(13 \mathrm{kV}, 16.9 \mathrm{~kJ})$ and used consistently across all three lubrication conditions in order to make more fair comparisons of draw-in length. As with before, three blanks were formed in each condition. The results of these tests are shown in Figure 4.2.18. Again, as expected, both lubricants showed an improvement in draw-in length as compared to the unlubricated condition. At the lower clamping force, however, the Quaker 61-MAL-HCL-1 showed slightly better performance than the Quaker 6130, in terms of the average draw-in length (1.072 in. vs. 0.939 in.). Perhaps the most important conclusion which can be drawn from these lubricant experiments thus far is that conventional stamping lubricants have been shown to be compatible with the high velocity nature of electrohydraulic forming, due to the absence of any undesirable effects such as burning, smoking, etc., and that the lubricants do make a positive impact as compared to unlubricated forming.

Lubricant testing at 75 tons clamping and $13 \mathrm{kV}(16.9 \mathrm{~kJ})$

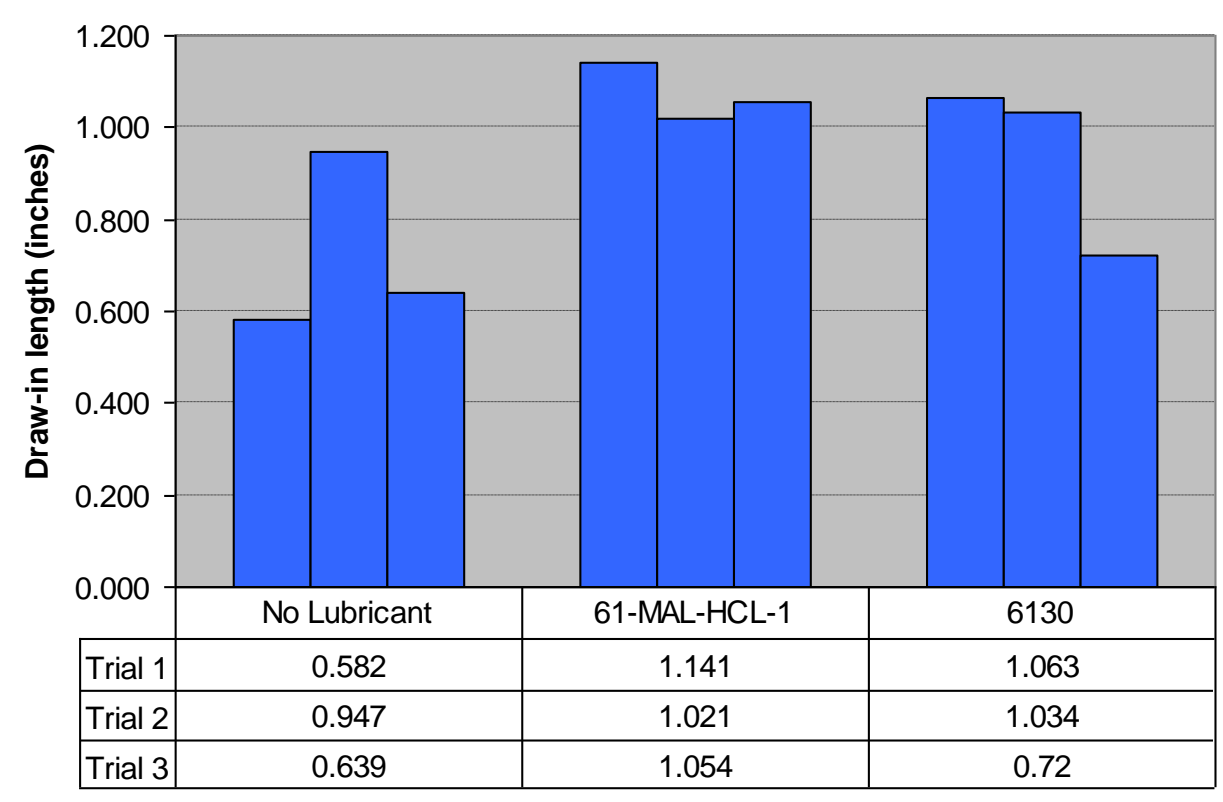

Figure 4.2.18. Summary of lubricant test results at a clamping force of 75 tons. Draw-in length was measured at the middle of each specimen after forming. 


\section{Task 5. Demonstration of EHF for production conditions}

Sub-task 5.1. Conduct preliminary testing of the automated EHF system.

Improved Chamber Design

The original chamber had enhanced ports added to it to improve flow rates and reduce cycle time, but a new optimized chamber was manufactured for the EHF cell. The design of the new chamber can be found in Figure 5.1.1. It represents a significant reduction in volume over the original chamber to improve pulse efficiency. Being designed via FEA, it provides a substantial improvement of the pressure distribution at the sheet metal surface for corner filling operations. The new chamber has significantly enlarged porting in line with greatly increased water/air flow, and it has incorporated within it the improvements in the electrode system. Lastly, the sealing was redesigned based on research from task 3.2. Figure 5.1.2 shows both the old and new chambers.

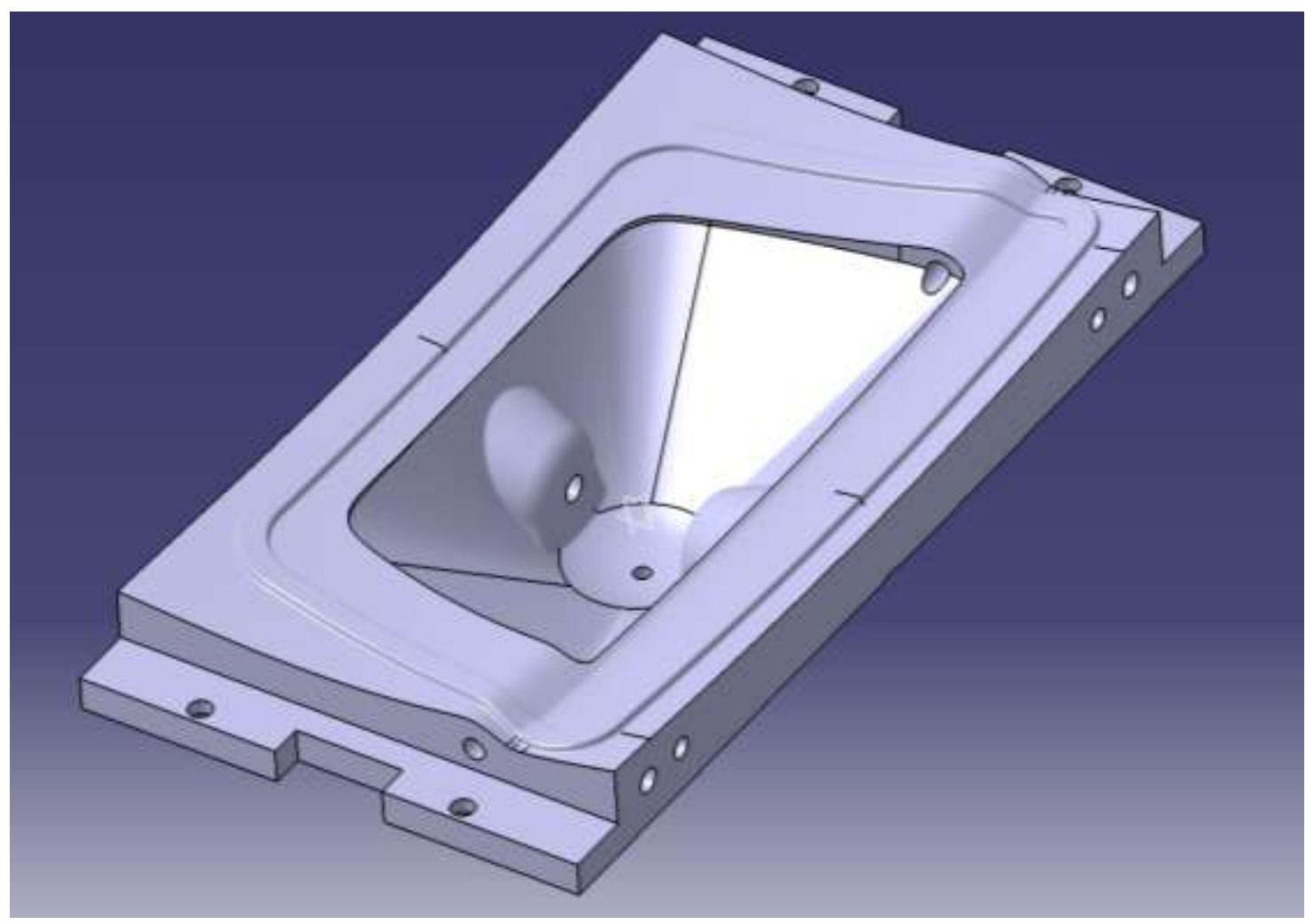

Figure 5.1.1. New FEA-designed chamber incorporating electrode system, sealing system, and water management design improvements. 

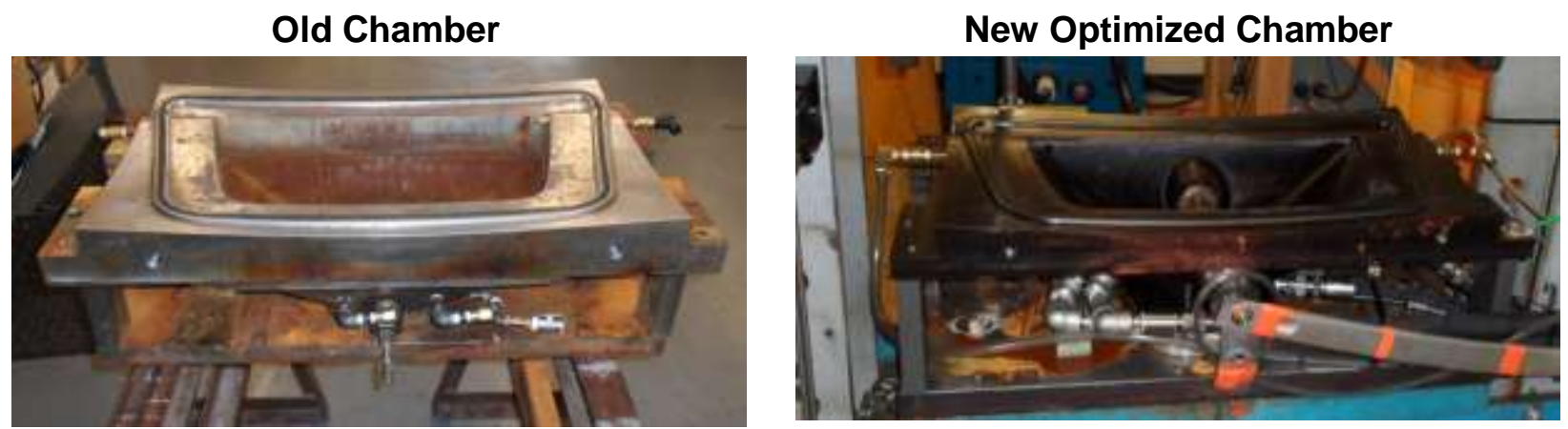

Figure 5.1.2. The new chamber optimized to decrease cycle time by reducing the volume from 19 to 11 liters and enlarged porting.

\section{Cycle Time}

The largest portion of the cycle time is pulse generator charging which requires 56 seconds per part, compared with the remainder of the cycle taken up by part handling (13.3 seconds), press open/close (15.6 seconds), and fluid handling (24.4 seconds). A replacement charger was identified which was capable of charging the IAP pulse generator in 1 second but cost $\$ 70 \mathrm{k}$. Due to budgetary constraints, this fast charger was not implemented. Further, the fluid handling time can be reduced by increasing the diameter of the drain and fill lines from $3 / 4$ " to 2 ". Figure 5.1.3 details the timing required to form one part.

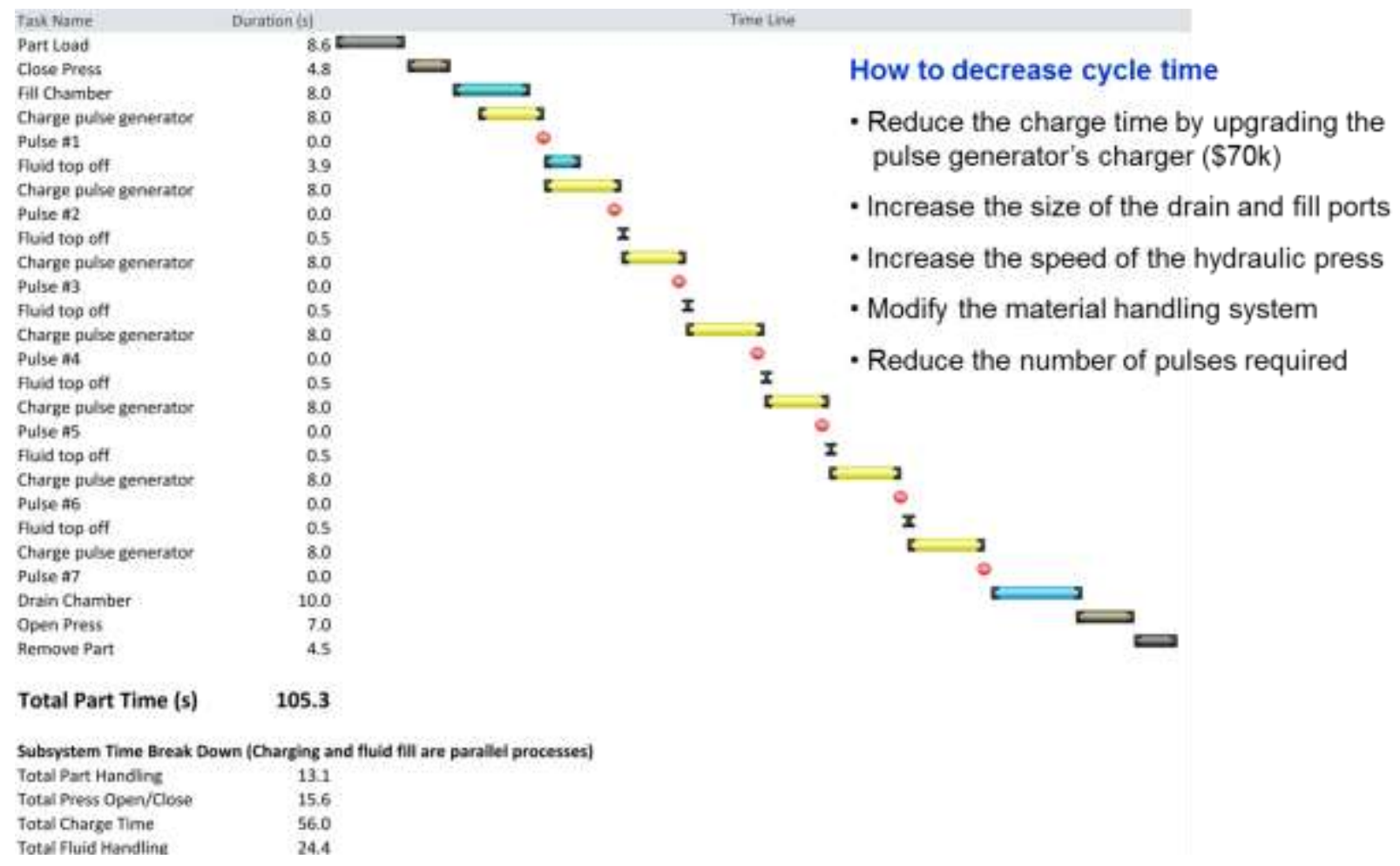

Figure 5.1.3. Typical cycle time based on the timing collected with the data acquisition system. 


\section{Electrostatic Modeling}

With the new chamber we found that about $50 \%$ of the pulses discharged to the chamber wall, and therefore we initiated an electrostatic modeling study to better understand the physics of the behavior. The analytical effort focused on the development of a proper finite element model of the new license pocket chamber and verification of proper convergence in an electrostatic analysis. Experimental work proceeded with electrical investigation of the operating characteristics of the license pocket chamber.

Several versions of the model were developed. The first two, shown in Figure 5.1.4, were too large to manipulate easily and took too many hours to solve. Because of the sensitivity to boundary conditions and a need to rapidly iterate, the model in Figure 5.1.5 was developed. This new model solves in just under an hour and contains minor improvements over the first two, predominately the inclusion of 3D solid elements for the sheet metal blank, instead of using shell elements or simply none at all.
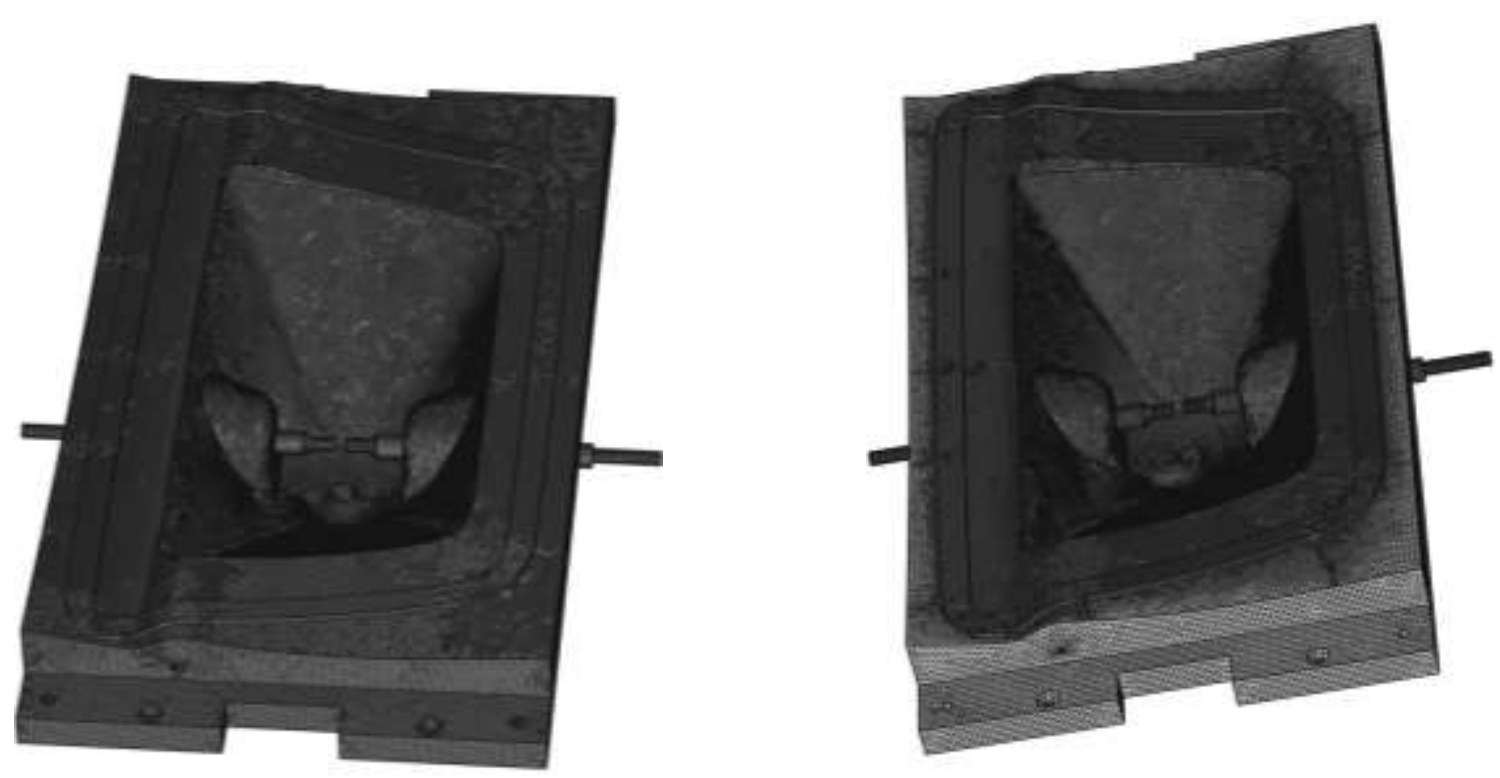

Figure 5.1.4. Initial Finite Element Models with 8.9 million (left) and 4.9 million elements (right). 

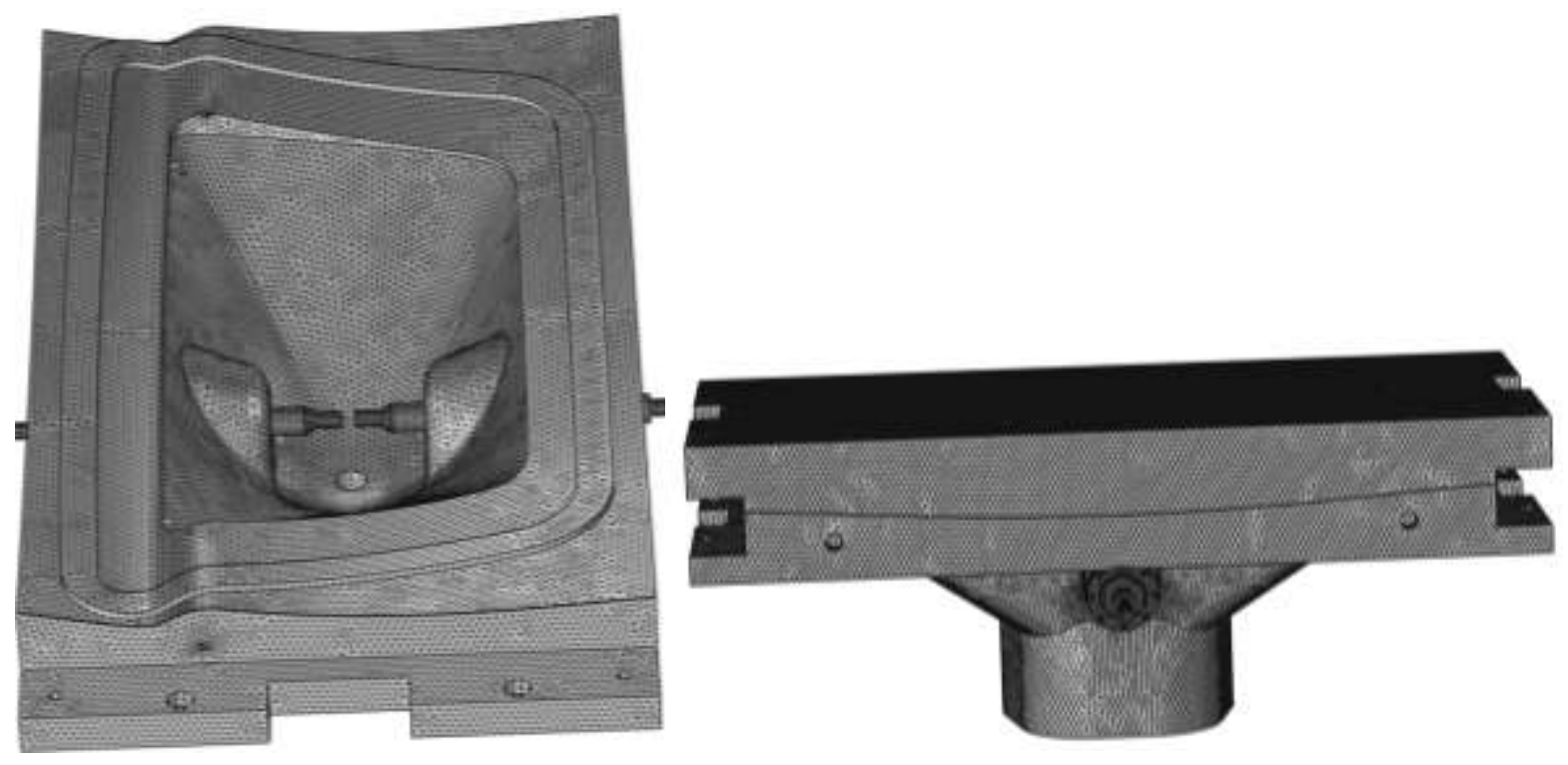

Figure 5.1.5. Working Finite Element model with 2.9 million elements. The side view shows the die closed on the sheet metal blank and chamber.

Simulations to verify the new model against that of the conical chamber were run, and a vertical slice through the chamber is shown in Figure 5.1.6 demonstrating the voltage in the ungrounded state (left) and under grounded operation (right). Note that the ungrounded simulation correctly predicts the floating voltage $(6 \mathrm{kV})$ in the chamber walls and the lessening of the voltage gradients. The electric field distribution in Figure 5.1.7 tells a similar story in that the electrical fields in the ungrounded chamber are roughly half that of the grounded chamber, meaning that unwanted arcing is far less likely to occur.

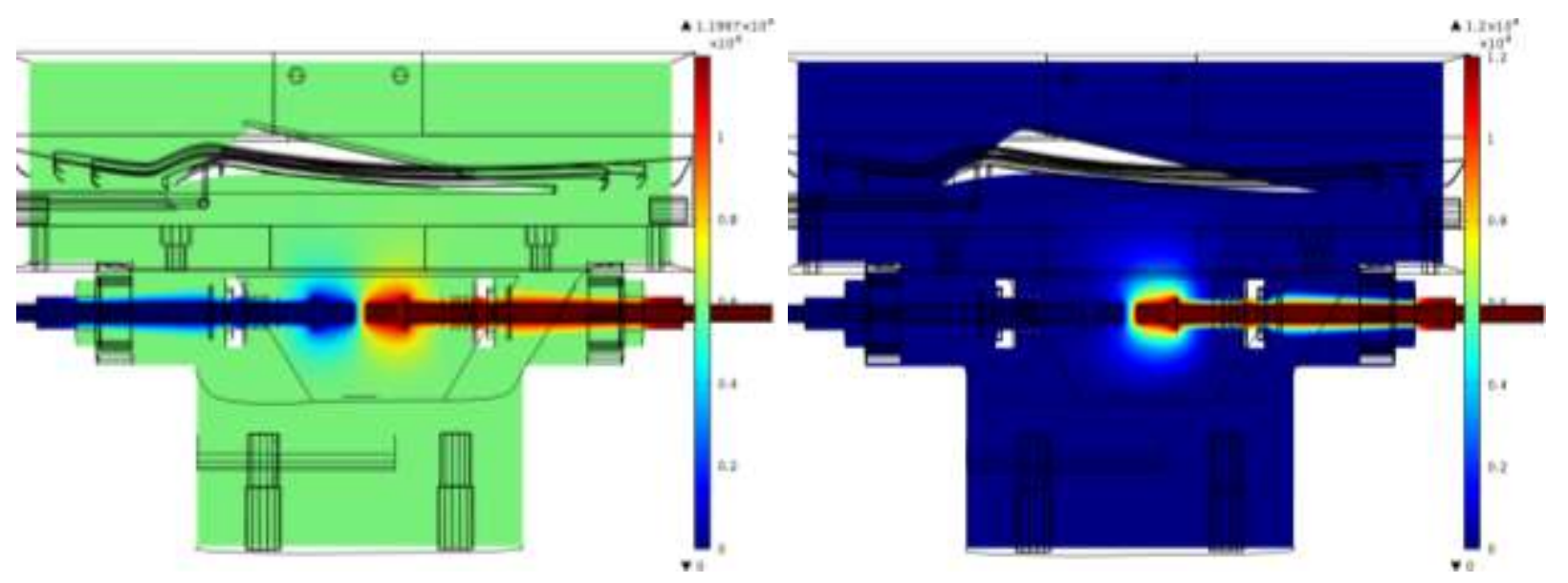

Figure 5.1.6. New License Pocket Chamber simulations showing the voltage (V) performance in the ungrounded (left) and grounded conditions (right). 


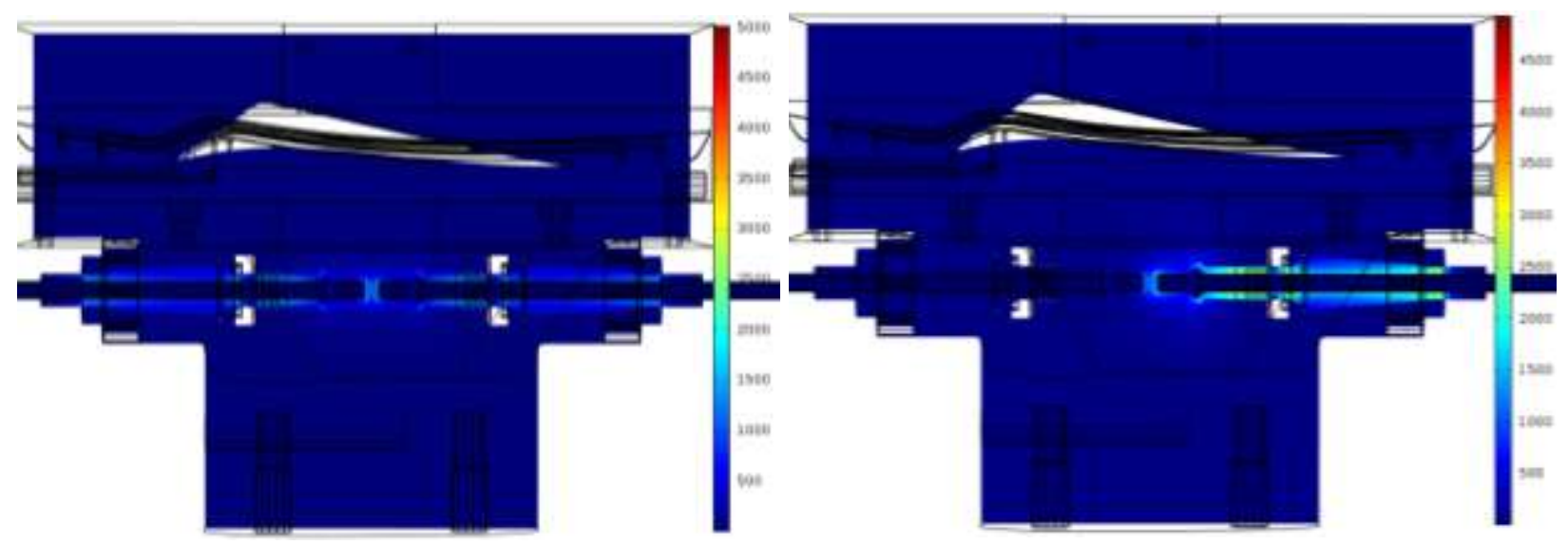

Figure 5.1.7. New License Pocket Chamber simulations showing the electric field distribution $(\mathrm{V} / \mathrm{m})$ in the ungrounded (left) and grounded conditions (right).

A synopsis of the failure modes for the new license chamber is as follows. During testing it was found that with some frequency the positive electrode would arc back to the chamber wall immediately surrounding the electrode. When the electrode was further inserted the arc would flash to the bottom of the chamber. Figure 5.1.8 illustrates some of the witness marks from the arcing and the arc paths.

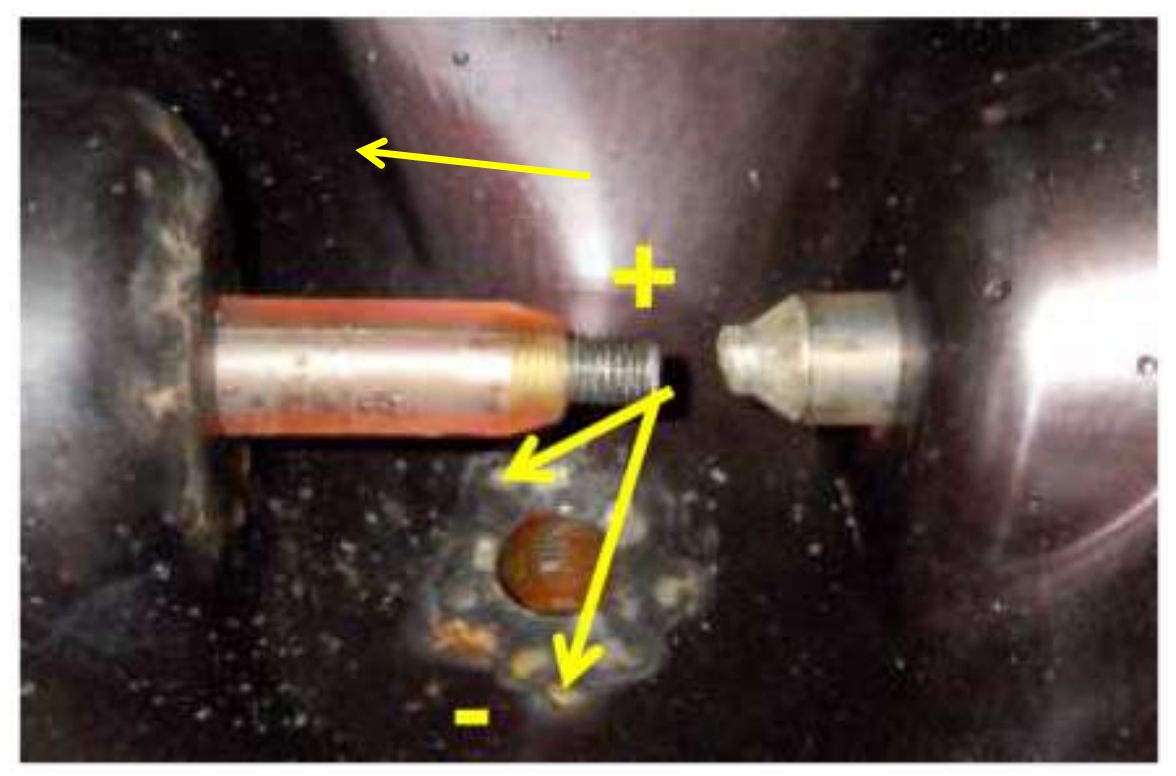

Figure 5.1.8. Photograph showing arcing witness marks on both chamber wall and chamber floor. The electrodes in this photo were offset from center to eliminate wall arcing which, while successful as a local strategy, was replaced by arcing to the floor. 
One explanation for the arcing is shown in Figure 5.1.9, where the model shows charge accumulation along the electrode insulation. In the ungrounded condition the charge build-up is more extensive but less intense than it is in the grounded condition. It is clear that more intense conjugate charges accumulate along the electrode shaft insulation, so in the case of the positive electrode, a strong negative charge distribution is found behind the electrode tip and leading to the wall. Although this charge accumulation does not explain arcing to the chamber floor it is suggestive of the possible failure modes to the wall. A 3-D view of the negative space charges surrounding the positive electrode shaft in the grounded chamber is shown in Figure 5.1.10. Note that the charge actually extends into the metal in the chamber wall. The existence of this charge provides a pathway for the discharge back to ground and eventually the pulse generator.

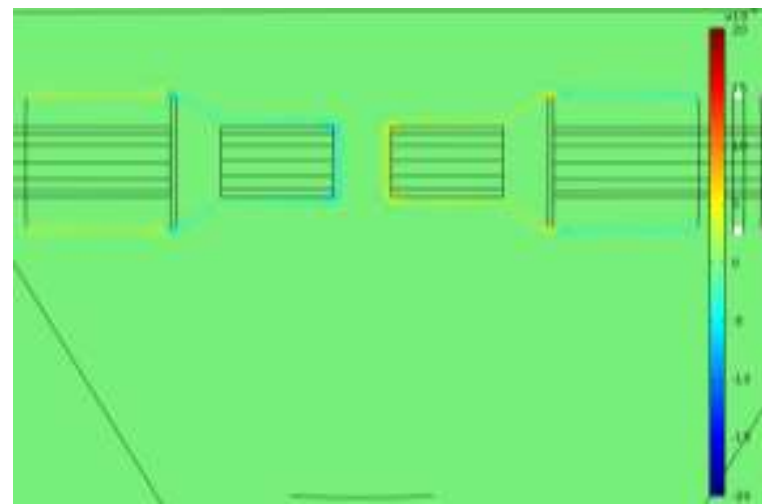

(a)

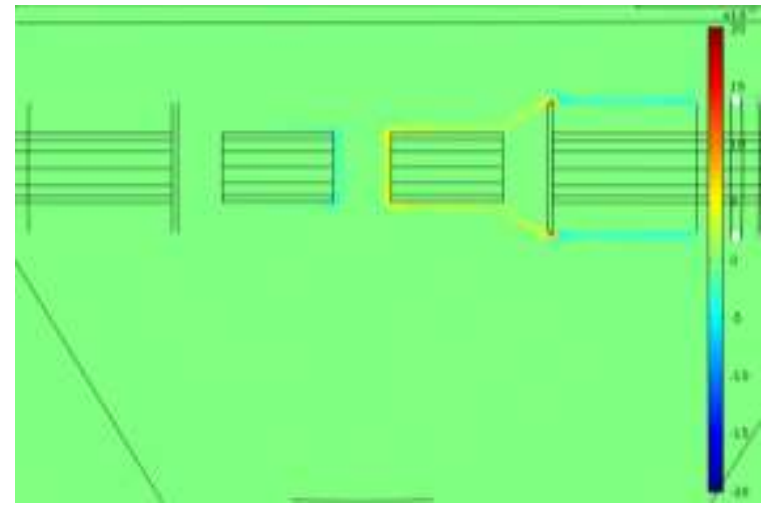

(b)

Figure 5.1.9. New License Pocket Chamber simulations showing the space charge density $\left(\mathrm{C} / \mathrm{m}^{3}\right)$ distributions immediately surrounding the electrodes in the ungrounded (left) and grounded conditions (right).

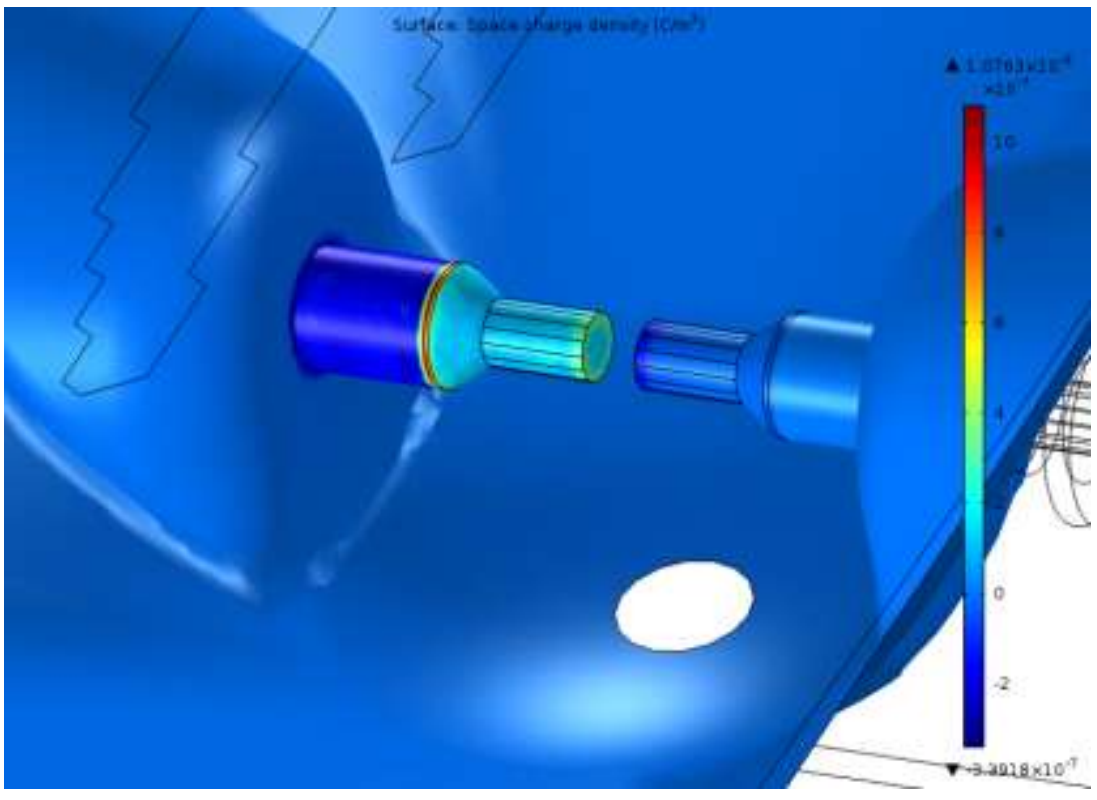

Figure 5.1.10. Three dimensional view showing accumulation of negative charge on the surface of the positive electrode in the grounded chamber and running into the chamber wall. 


\section{Experimental Testing}

Initial experimentation showed that with the standard electrodes the arcs would start at the positive electrode and go back to the portion of the chamber wall surrounding the positive electrode. Reducing the inter-electrode gap did not significantly improve electrode performance, and biasing the gap toward the negative electrode side of the chamber only served to cause the arcing to move to the chamber bottom around the fill/drain hole (see 5.1.8). The metal-shieldedtip positive electrode was swapped with standard-tip electrodes but there was no change. Finally, swapping in an all-steel negative electrode with a large flat land and redesigning the chamber grounding system alleviated the bad discharges.

The chamber was heavily instrumented and a series of experiments were run to determine the failure modes under a variety of operation conditions. A new set of electrodes were placed in the chamber, and current sensors (Rogowski's) were placed around every ground return path while voltage probes were placed at critical locations in order to help with diagnosis.

The first test was with the chamber in "normal" operation: filled with tap water, a $10 \mathrm{~mm}$ interelectrode gap was centered in the chamber, and a common ground point on the load frame with heavy duty return straps was attached to the IAP, the chamber wall and the press frame. The results of the fifth pulse are shown in Figure 5.1.11. It should be emphasized that there is no current though the negative electrode meaning that the pulse went to the chamber wall, through the chamber and frame return straps and back through the IAP return - note that the sign of the waveforms is not an indication of current flow direction. Also, the relatively symmetrical shape of the pulse indicated a bad discharge. No current was observed returning through the actuator rod in this particular test, but, of 10, two showed current leaving through the actuator rod, and that trace is shown in Figure 5.1.12. All subsequent pulses in this configuration had the same result.

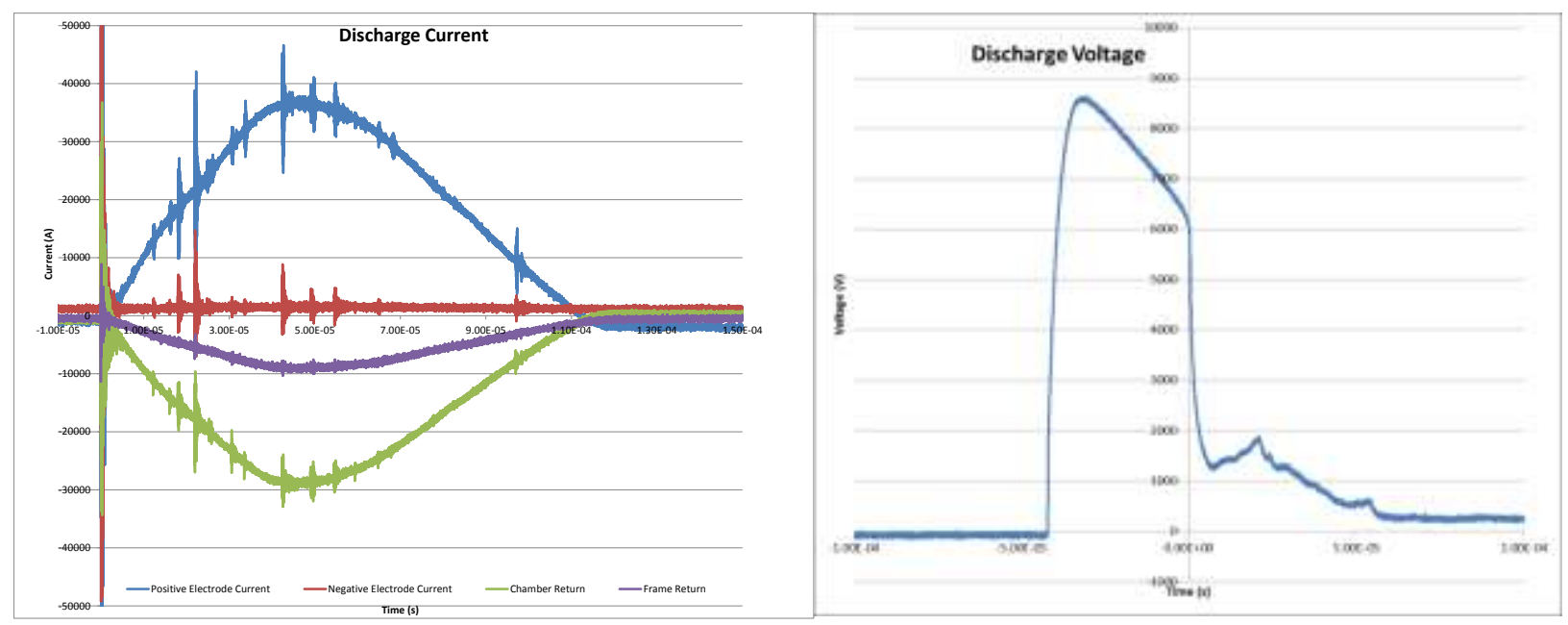

Figure 5.1.11. Bad discharge in license pocket chamber: Current profile (left) and voltage profile (right). Note lack of current returning through the negative electrode. 


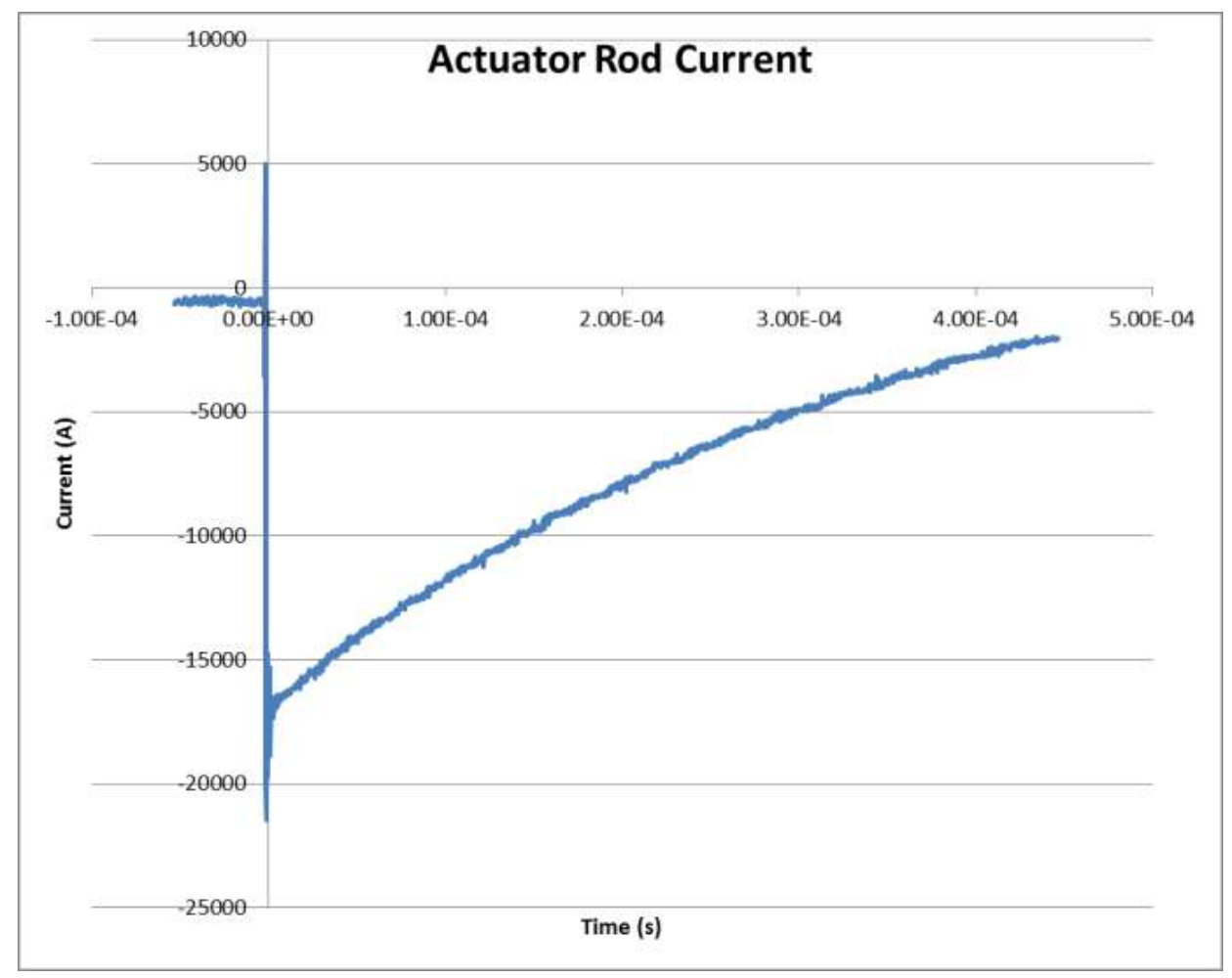

Figure 5.1.12. Bad pulse current leaving through actuator rod.

\section{Isolated Chamber Experiments}

The tool/chamber was isolated using G-10 between the press frame and actuator, and the strap between the IAP return, and the grounding node on the press frame was removed. All pulses in this configuration were normal and arced to the negative electrode. Obviously, no current was witnessed travelling through the actuator under these conditions. The correct operation of the chamber under these conditions also matches the experimentally observed behavior in the conical chamber as well as that predicted by the results of modeling. Unfortunately, insulating the chamber from the press is difficult in production so another solution was sought.

\section{Large Metal Negative Electrode Experiments}

It is understood that the most efficient electrode systems are those in which a conically tipped electrode is faced against a large flat - providing the positive electrode in our case with a large target. Thus, it was felt that replacing the standard insulated negative electrode with an all metal electrode with large surface area would help discharges find their way to the negative rather than the chamber wall. An all-steel electrode was mounted into the chamber in intimate electrical contact with the chamber as a whole. A picture of the chamber with this electrode configuration is shown in Figure 5.1.13. The grounding system was restored, and the G-10 isolation removed. This effort was successful, from the perspective that good discharges occurred to the new electrode reliably, as can be seen in Figure 5.1.14. The figure demonstrates the fact that this electrode configuration sets up multiple parallel return paths. One can see that the return paths through the chamber and frame straps are slightly active 
because the negative electrode is in intimate contact with the chamber wall and these other paths, while longer and somewhat higher resistance, are valid but in this case do not indicate a discharge to the chamber walls. The observed currents through the actuator rod were also negligible. In summary, the behavior of the chamber was consistent, good, and reliable using the all-metal electrode.

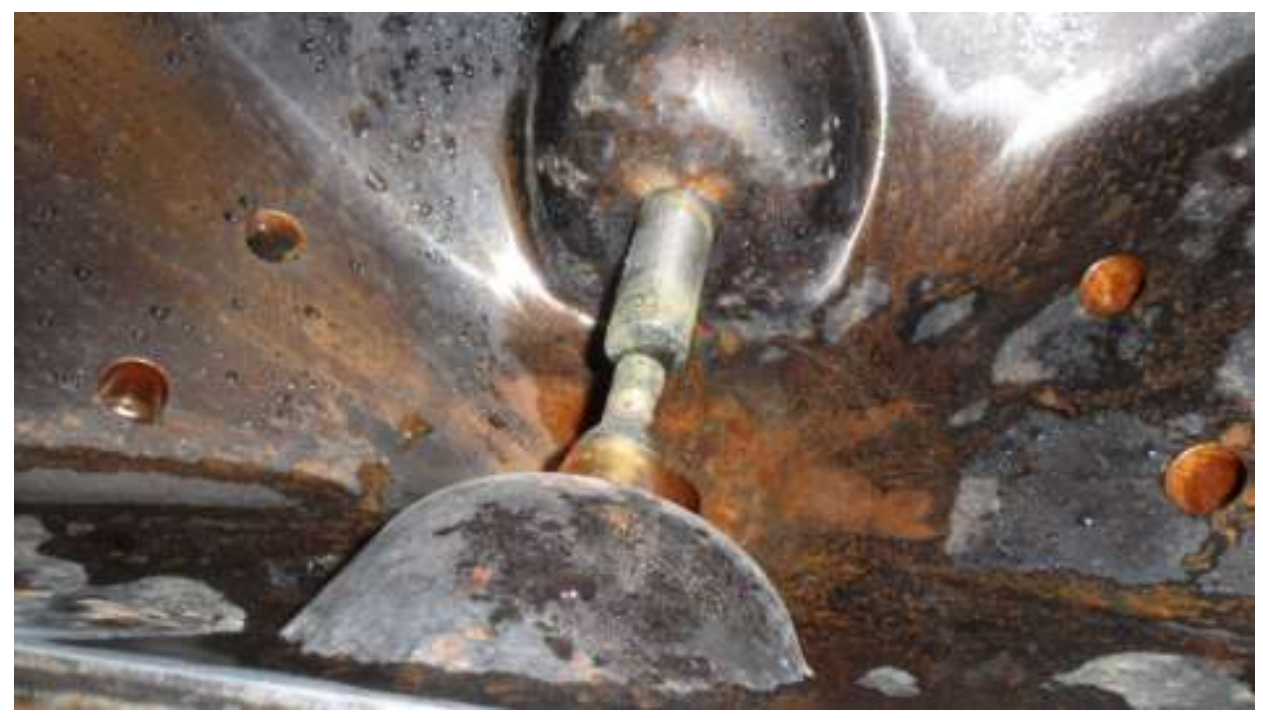

Figure 5.1.13. All-steel negative electrode with large cross-section installed in chamber.
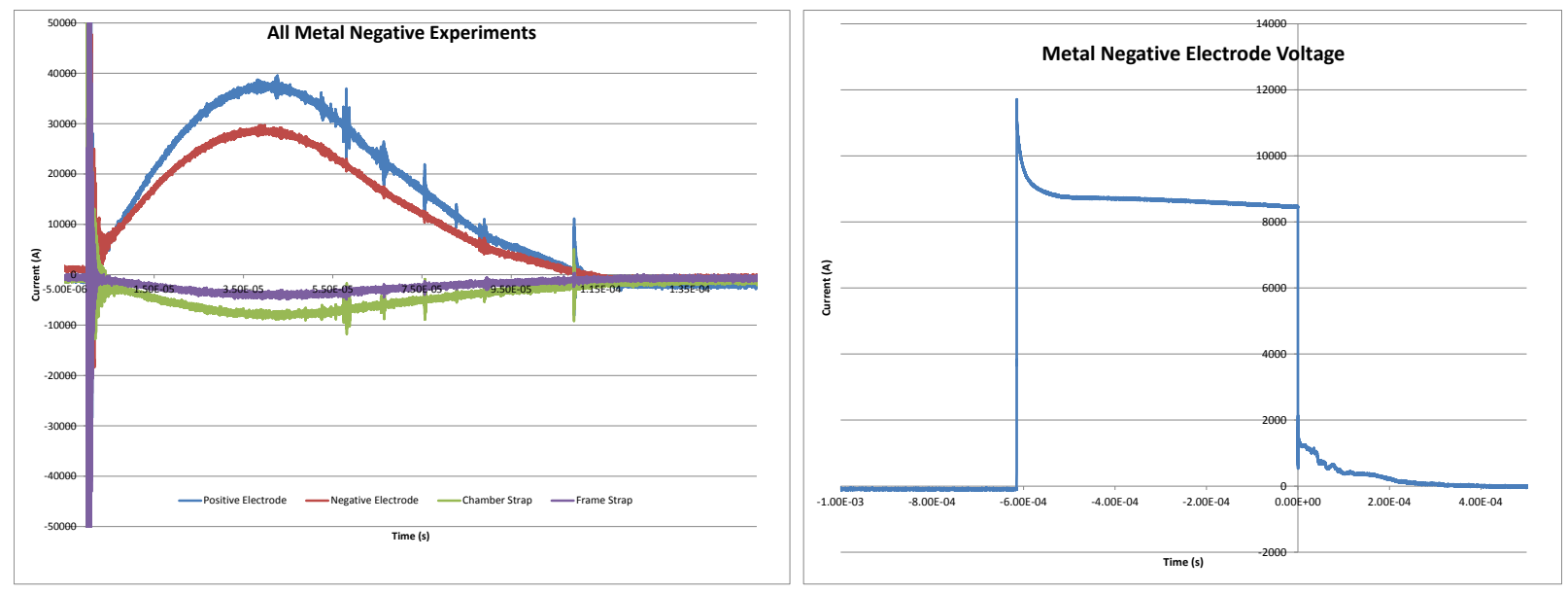

Figure 5.1.14. Discharge using all steel negative electrode. Current profile on the left and voltage profile on the right. Note asymmetric waveform shape. 


\section{Data Acquisition}

A Labview-based data acquisition system was developed to collect the pulse wave forms and monitor the overall system. The main screen of the data acquisition system is pictured in Figure 5.2.1. The system captures and logs information from the Air and Water Management, Gantry, Hydraulic Press, Hydroforming, and pulse generator subsystems, and the master controller. Monitoring all subsystems provides in-depth fault analysis capability, and the ability to determine the true cause of an improperly formed part. This system can be used to determine inefficiencies in timing, inadequate delivery of pulse energy, insufficient forming volume and other possible faults.

Most importantly, the data acquisition system provides the capability to monitor the performance of the electrodes through changes in the pulse waveforms. As the electrodes wear they erode back, increasing the distance between the electrodes. As the gap increases, the formation of plasma becomes less efficient.

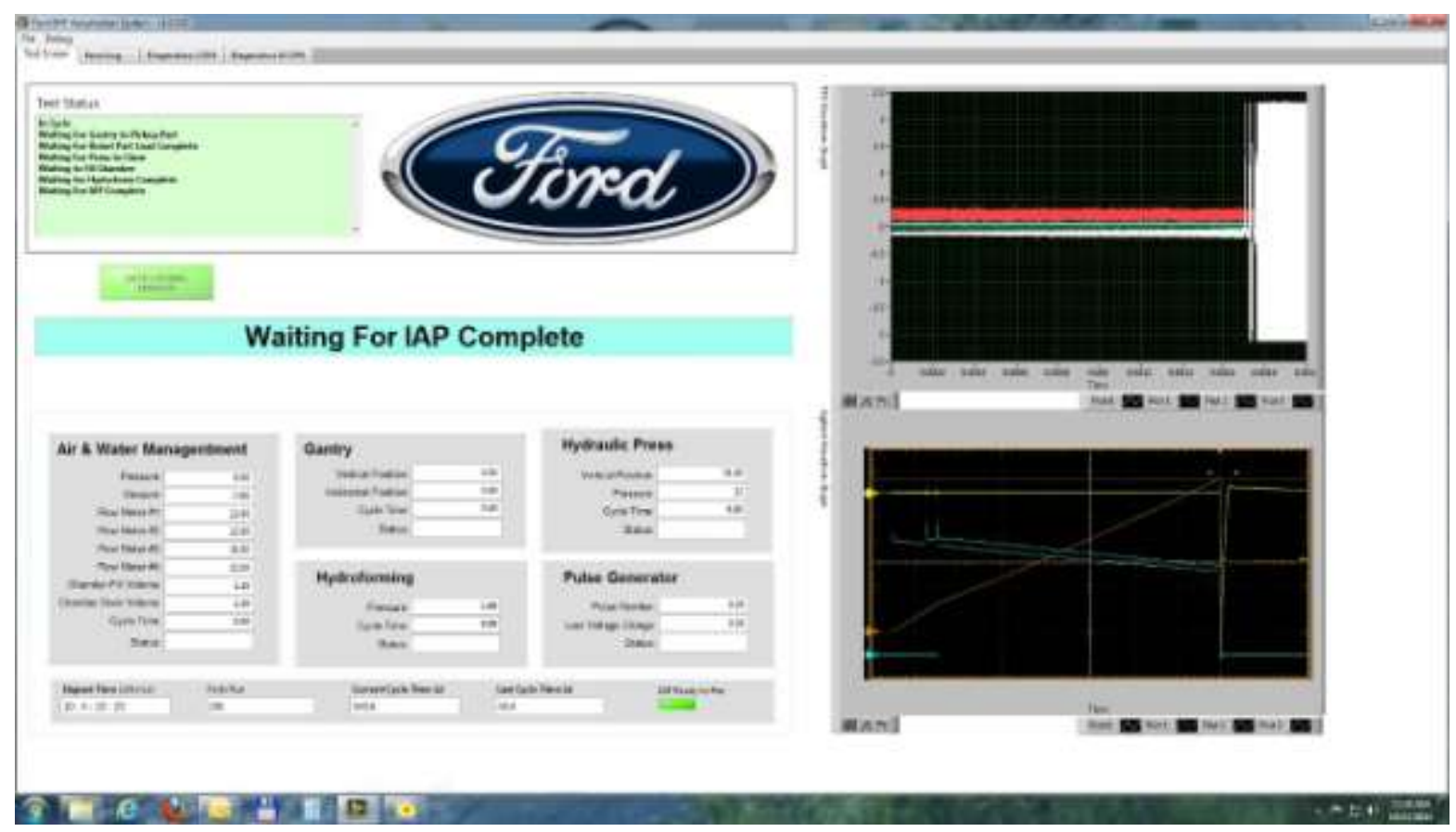

Figure 5.2.1. Labview based data acquisition system for capturing data from the automation cell.

The Fluid Handling System is engineered to operate indefinitely with monthly maintenance as a closed system capable of filtering the fluid to 10 micron, 100 GPM fluid flow rates, and to provide high pressure sheet forming capability. Additionally, the overall Labview data acquisition 
system was integrated into the Master PLC Fluid Handling System and collects overall operational system data. The system has produced over 1200 parts with the Automation Cell and the system is capable of running an 8 hour shift without maintenance. Figure 5.2.2 shows the 1200 parts formed in the automation cell.

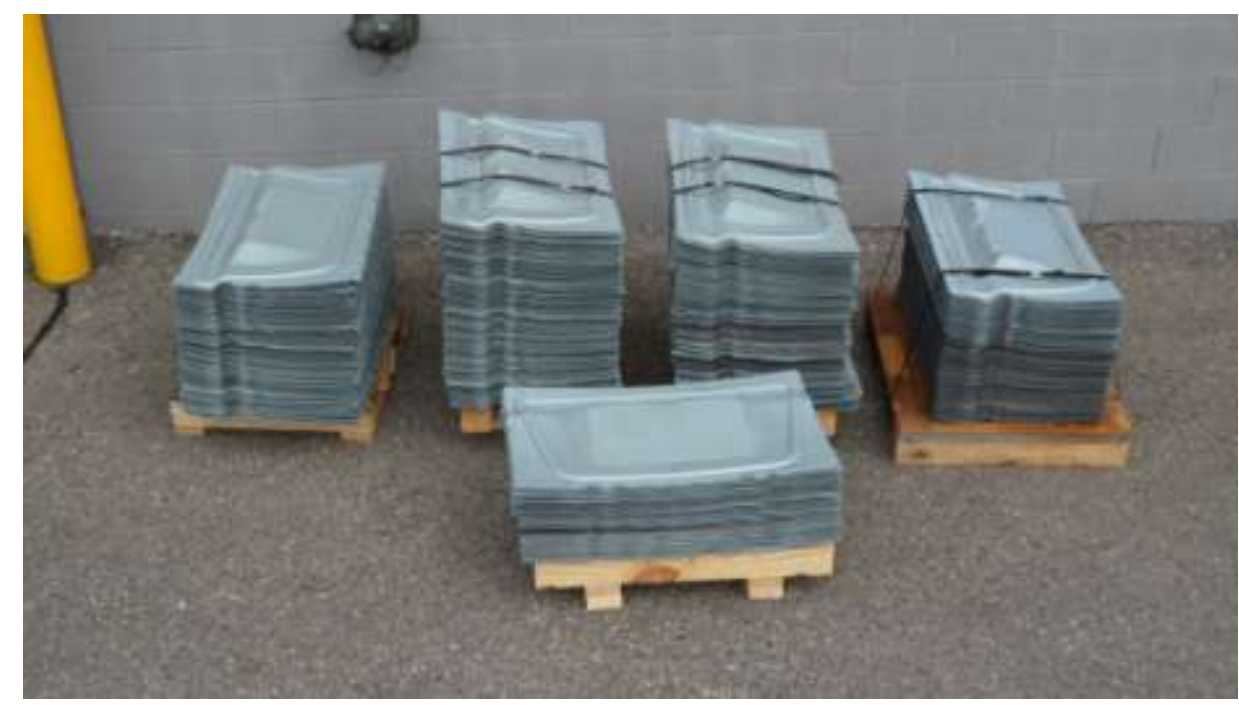

Figure 5.2.2. Over 1200 formed parts from the automation cell.

\section{Next Generation Pulse Generator Development}

The slowest system in the automated cell is the pulse generator. The amount of time required to charge and discharge the pulse generator seven times is roughly 56 seconds. A further limitation is the $100 \mathrm{kA}$ maximum peak current that our present generator can handle - a higher peak current will allow forming of larger parts with fewer pulses. Lastly, the current pulse generator, designed for magnetic forming, has inefficiencies which waste energy and reduce process capability. To address these issues it was deemed necessary to work with IAP to design a custom pulse generator for EHF. The following requirements were identified: (1) $<2$ second single pulse charging time; (2) $150 \mathrm{kA}$ peak current capability; (3) $<10 \mathrm{nH}$ system inductance and (4) $50 \mathrm{kV}$ capability. IAP has identified components, drawn up plans, and experimentally validated recently developed fault isolation technology. Conducted estimation indicated that this approach will reduce the pulse forming part of the process cycle time by roughly one third.

In order to develop an understanding of the maintenance and total life cycle performance of the next generation pulse generator, individual components were assembled and tested, specifically the charger, capacitors, triggering module and switches. A simple circuit with the triggering module, capacitor and a spark gap switch was assembled for aggressive, accelerated discharge testing. The initial testing required eight seconds to charge the capacitors, and the manufacturer's specification was one second. IAP Research worked with the manufacturer of 
the charger, NWL Transformers, to identify and resolve the programming issue. After the PLC programming was updated, we were able to consistently conduct discharges with a one-second charging time, as required for a production environment. The charger is shown in Figure 5.2.3. During the charging process, the PLC monitors the charging of the capacitors (5.2.4), and, once charged to the set voltage, triggers the spark gap switches (Figure 5.2.5) to initiate the pulse. The configuration being evaluated has two parallel capacitor banks with a separate trigger and spark gap switch for each module. This configuration allows for a pulse delivered in parallel or in series.

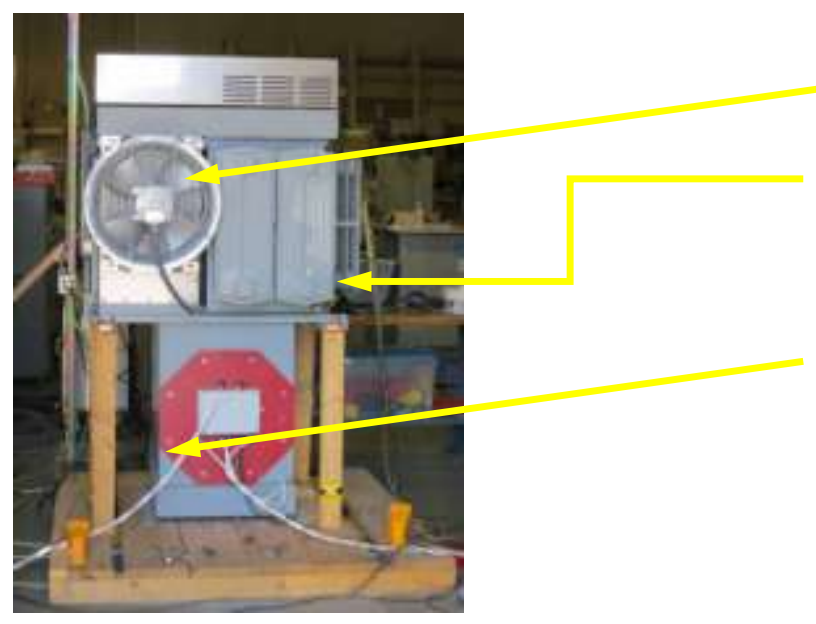

Cooling fan

Heat exchangers

High voltage wiring to charge the capacitors (800 mA)

Figure 5.2.3. NWL Transformer's $50 \mathrm{kV}$ charger is designed for continuous pulsing, and according to the manufacturer, has extended life exceeding significantly the life of other components.

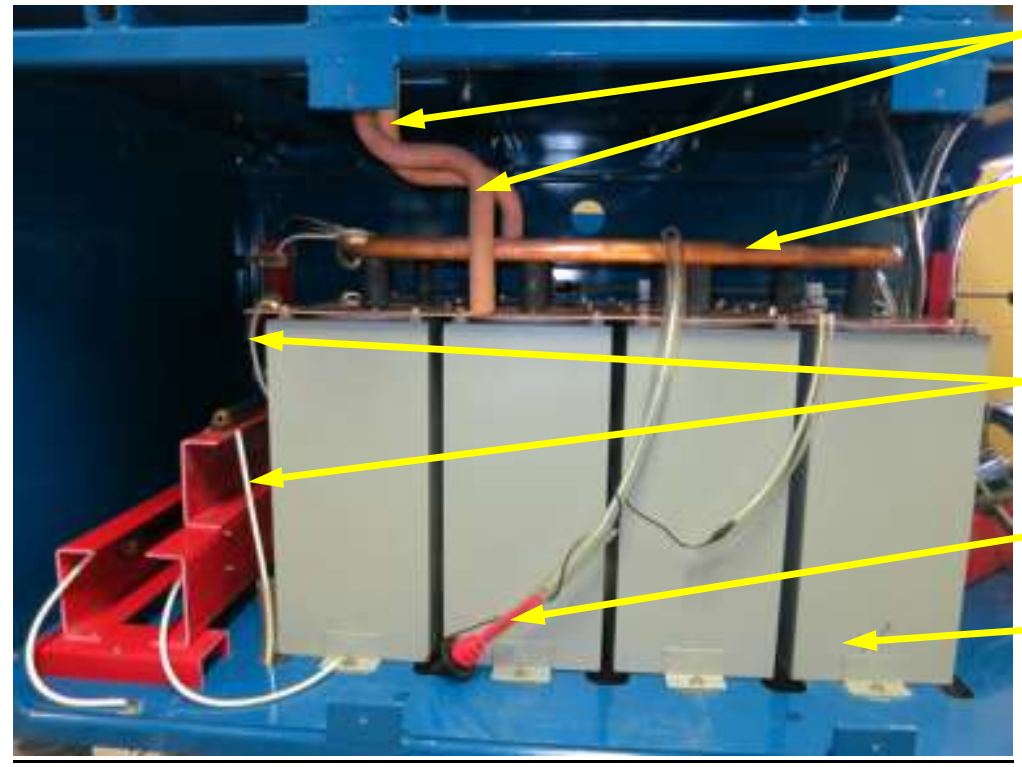

Electrical conductor to spark gap switch

Corona Shield

Negative Buss

High voltage charging wires from the charger $(800 \mathrm{~mA})$

Voltage divider probe

(8) Capacitors - two rows

Figure 5.2.4. Capacitor bank of one of the two modules connected to individual spark gap switches and pulsed in parallel. 


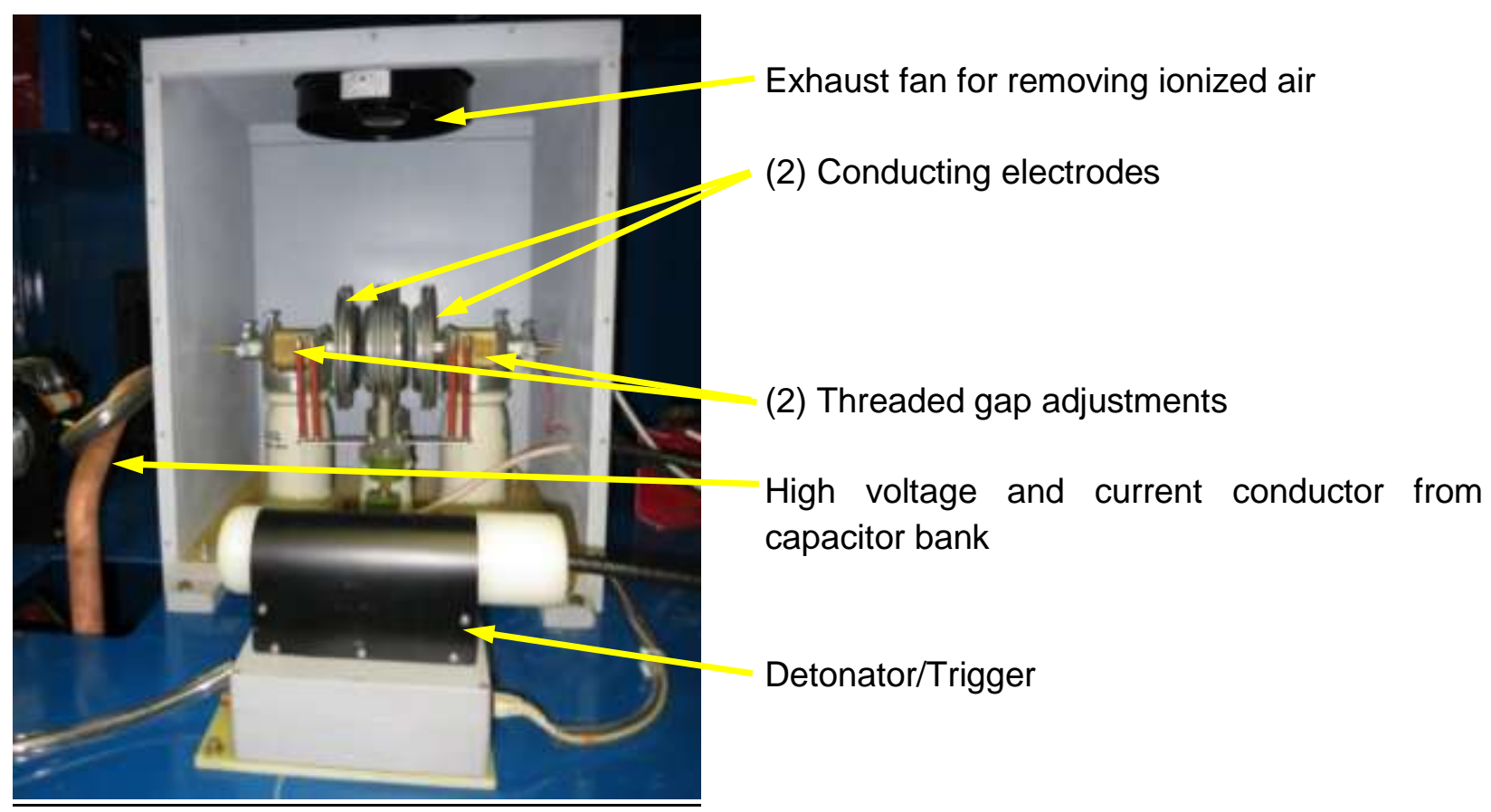

Figure 5.2.5. The high voltage spark gap switch ( $25 \mathrm{kV}$ to $50 \mathrm{kV})$ and trigger box.

While working through the design process, the spark gap switch was the primary concern. The manufacturer of the switch reported very high life cycles, estimated as $1.16^{\star} 10^{8}$, and very consistent functionality. The specifications called for a minimum of $25 \mathrm{kV}$ to form the plasma channel, but in our testing it would form semi-consistently at $23 \mathrm{kV}$ and consistently at $25 \mathrm{kV}$. One of the switches required some minor calibration of the spark gap, and then both switches performed with the same characteristics. After the initial calibration, over 2500 pulses were formed without any misfires. The manufacturer of the switches confirmed that the amount of visible erosion on the surface of the electrodes, as shown in Figure 5.2.6, is typical for this amount of usage. 


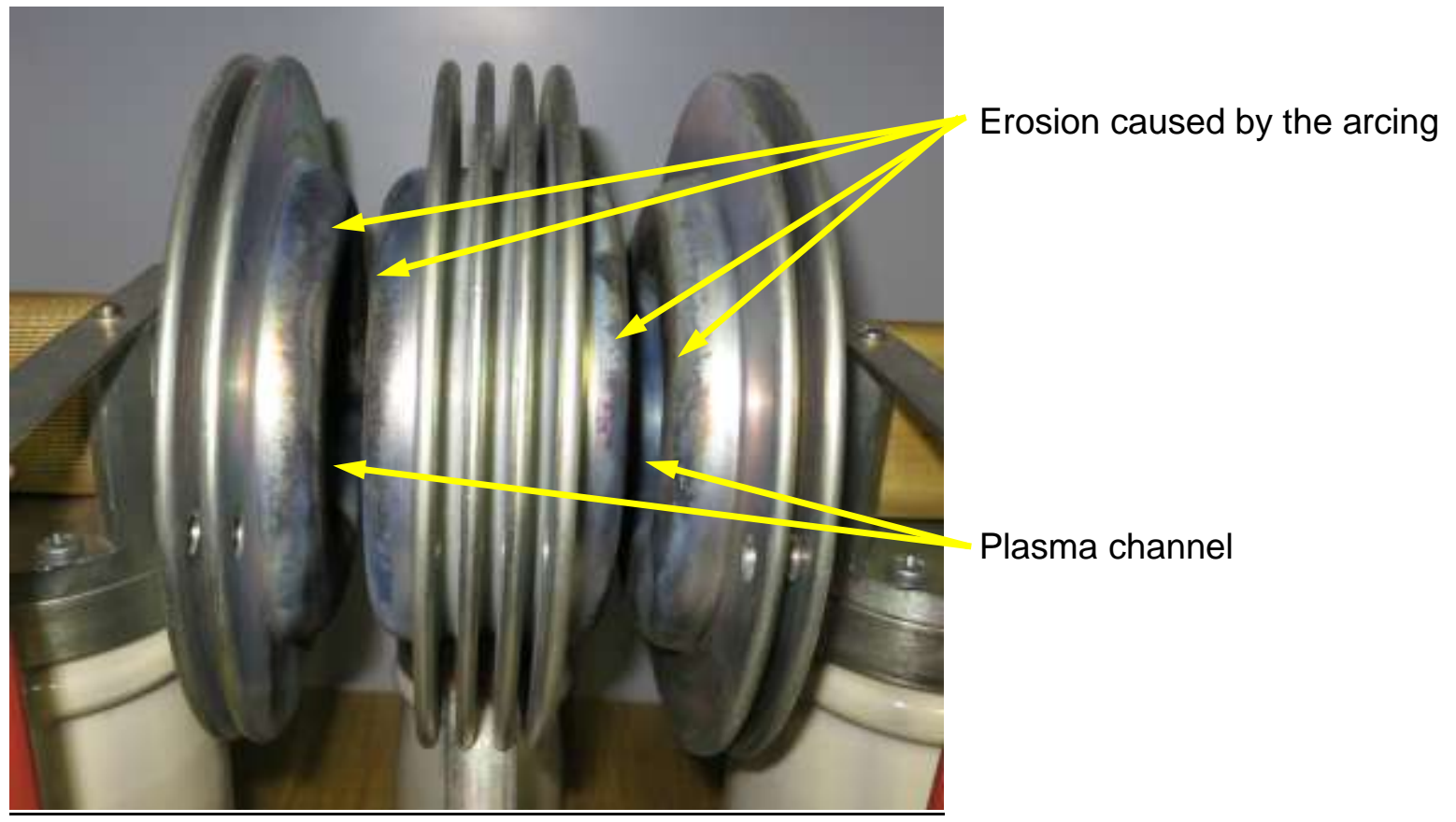

Figure 5.2.6. Erosion on the electrodes of the high voltage spark gap switch that carries up to $50 \mathrm{kV}$ and $75 \mathrm{kA}$.

Next Generation Pulse Generator Anticipated Maintenance

The pulse generator design developed in conjunction with IAP Research meets the production rate of one pulse per second without any apparent thermal concerns. The components purchased to perform the validation study are performing to the manufacturer's specifications and the process requirements.

IAP's conclusion is that minor maintenance of the electrodes is required every 15.5 hours operating at $1 \mathrm{~Hz}$ (60,000 pulses). Minor maintenance includes removing two access panels and wiping the electrode contact surfaces down with isopropyl alcohol. Major maintenance of the electrodes is required every 600 hours $(2,000,000$ pulses). Major maintenance requires that electrodes are lightly sanded and then the spark gap is adjusted. 
Task 6. New applications for EH stamping technology

\section{Sub-task 6.1. EH trimming process}

Traditional trimming processes require accurate alignment of the shearing edges within approximately $10 \%$ of the sheet metal thickness or less, as recommended by Smith [6-1]. If the cutting clearance is allowed to grow due to tooling wear, insufficient tool stiffness, or poor alignment of trim blades, these conditions will most likely lead to burrs on the edges of the trimmed panels, which can create problems during flanging and hemming operations and may also require expensive de-burring procedures.

A number of studies have been conducted on the effect of punch velocity on the quality of the resulting sheared surface in sheet blanking and bar cropping operations. The early results published by Johnson and Slater [6-2] and Davies and Dhawan [6-3] indicated improvement in the quality of the blanked surface with an increase of punch velocity up to $9-12 \mathrm{~m} / \mathrm{s}$ for a variety of materials. Breitling [6-4] studied the effect of punch velocity up to $4 \mathrm{~m} / \mathrm{s}$. He points out that the change in material behavior in shearing processes can be attributed to the effect of temperature, which is especially relevant in the case of steel workpieces. Due to the higher thermal conductivity of copper and aluminum, the advantages are less pronounced for these materials, but they are still measurable. Das and Sadollah [6-5] analyzed the effect of axial force and impact velocity during the cropping of billets, and indicated that an increase in impact speed does improve the quality of the billet.

Pulsed sheet metal forming technologies such as Electromagnetic Forming (EMF), Explosive Forming (EF) and Electrohydraulic Forming (EHF) have been known for a number of decades. A number of studies published in the literature indicated that in pulsed forming processes the velocity of the blank can be an order of magnitude higher than the punch velocities employed in the high speed die shearing experiments discussed above. A very detailed review of pulsed forming technologies was reported by Psyk et al. [6-6] and Mynors and Zhang [6-7]. The significantly lower cost of one-sided dies was one of the major advantages which drove the initial implementation of pulsed forming technologies in low volume industrial and defense applications. From a tooling simplicity point of view, pulsed forming technologies were similar to the rubber forming process initially developed by Guerin [6-8], and were also similar to a variety of quasistatic hydroforming processes described by Singh [6-9]. However, employment of quasistatic hydro-piercing often leads to the generation of burrs and hanging slugs, as reported by Singh [6-9]. Quasistatic hydro-piercing processes require special measures to avoid these defects due to the almost immediate relief of pressure as soon as the workpiece begins to separate. Also, it should be noted that quasistatic hydro-piercing processes require rather expensive investments into the clamping presses, since quasistatic fluid pressure needs to be counteracted along the entire surface of the workpiece. 
Pulsed forming processes may also be applied to hole piercing operations, which can be incorporated into the same die as the EF or EHF forming processes. Examples of such operations were reviewed in early manufacturing studies described by Bruno [6-10] and Davies and Austin [6-11]. The direct electromagnetic cutting of sheets and tubes is mentioned in Belyy et al. [6-12]. In general, EMF and the corresponding electromagnetic pressure are applicable to a variety of shearing operations. However, cutting processes usually require higher pressure levels than forming processes and, therefore, negatively impact the life of electromagnetic coils, which adds to the cost of any EMF-based cutting process. Golovashchenko [6-13] analyzed an EMF shearing process applied to trimming of aluminum alloys and copper tubes by using a compression coil and two internal mandrels with shearing edges. The Finite element analysis of electromagnetic cutting of tubes presented by Golovashchenko [6-14] indicated that with the increase of the radial displacement of the tube, two regions of fracture are developing. One mode of fracture originates on the external surface and is caused by bending and tension along the meridian of the tube. A second mode of fracture originates from the internal surface, close to the edges of the mandrel. These fractures propagate toward each other. As a result, sections of the tube are separated from each other.

The direct electromagnetic cutting of sheets was investigated by Uhlmann and Scholz [6-15], comparing it to conventional quasistatic shearing. In the case of conventional shearing within rather small clearances, the cutting process is characterized by shearing and consequent shear fracture, while in the case of electromagnetic cutting, the cutting process is characterized by bending and subsequent cracking. Accordingly, the resulting geometries of the sheared edge differ significantly. In the case of electromagnetic cutting, no shear zone is present, but there is a sizeable rollover zone.

A combination of EHF forming and trimming, as well as EMF forming and trimming, was discussed by Gafri and Livshiz [6-16]. In this analysis, pulsed forming processes enable substantial savings by combining forming and shearing processes in one tool. In traditional press stamping, these operations are performed in separate stamping dies. However, forming and shearing processes usually require different levels of pulsed pressure, which is particularly important for the processes where liquid serves as a transmitting media between the pressure source (explosion or high voltage discharge in liquid) and the blank. In a fluid-based pulsed forming process, if the forming process is performed in several discharges, there is always a possibility that shearing will initiate before the forming process is completed, resulting in a loss of dynamic pressure while the pulsed forming process is still ongoing, and also creating a condition in which it is difficult to generate enough pressure in any subsequent discharges. EMF processes do not have this problem. However, only parts of very simple geometry can be formed in one EMF discharge, while parts of more complex geometry would require several coils in order to form them with EMF. Also, EMF processes can be employed for shearing or trimming of rather thin blanks with moderate mechanical strength and good electrical conductivity.

Another potential industrial application of electromagnetic shearing technology was demonstrated in Schäfer et al. [6-17]. In this work, electromagnetic compression was applied to 
forming and cutting in one pulse. Kallee et al. [6-18] discussed the possibility of using EMF to pierce holes in press hardened steels, where the conventional process of die piercing had difficulties due to intensive wear of cutting tools. However, no details regarding the use of drivers or coil durability were provided. The objective of this paper is to present the newly proposed electrohydraulic channel trimming process for trimming of AHSS and UHSS automotive parts, to demonstrate the benefits of this process, to explain the blank separation mechanism based on numerical modeling of the process and to illustrate the quality of the resulting trimmed edges.

The current development work on $\mathrm{EH}$ trimming is targeting a high-intensity electrohydraulic pressure pulse capable of shearing heavy gauge AHSS and UHSS for automotive applications. The design of an EH trimming chamber suitable for trimming automotive panels, which may have several meters in perimeter, was driven by the following considerations: (1) in order to have longer life and lower minimal maintenance of the electrode system, the discharge energy should be as low as possible; (2) the energy of the compressed liquid in the chamber can be calculated as an integral of pressure by volume of the chamber, or as a product of an average pressure multiplied by the volume of the chamber. From this perspective, the highest pressure can be accomplished by employing a chamber with the smallest volume. In order to achieve maximum pressure on the blank surface, the discharge channel should be as close to the blank surface as possible. Another consideration which was taken into account while developing the concept of the new process was that during bar impact into a rigid wall, the stress wave propagates along the length of the bar, delivering the same level of stress to all the points of the bar. In a similar way, the pressure wave in a liquid-filled tube propagates through the tube delivering the same level of pressure to all areas of the tube. All these considerations were taken into account when the initial concept of the channel chamber was proposed. A schematic view of the tool in cross-section is shown in Figure 6.1.1. 


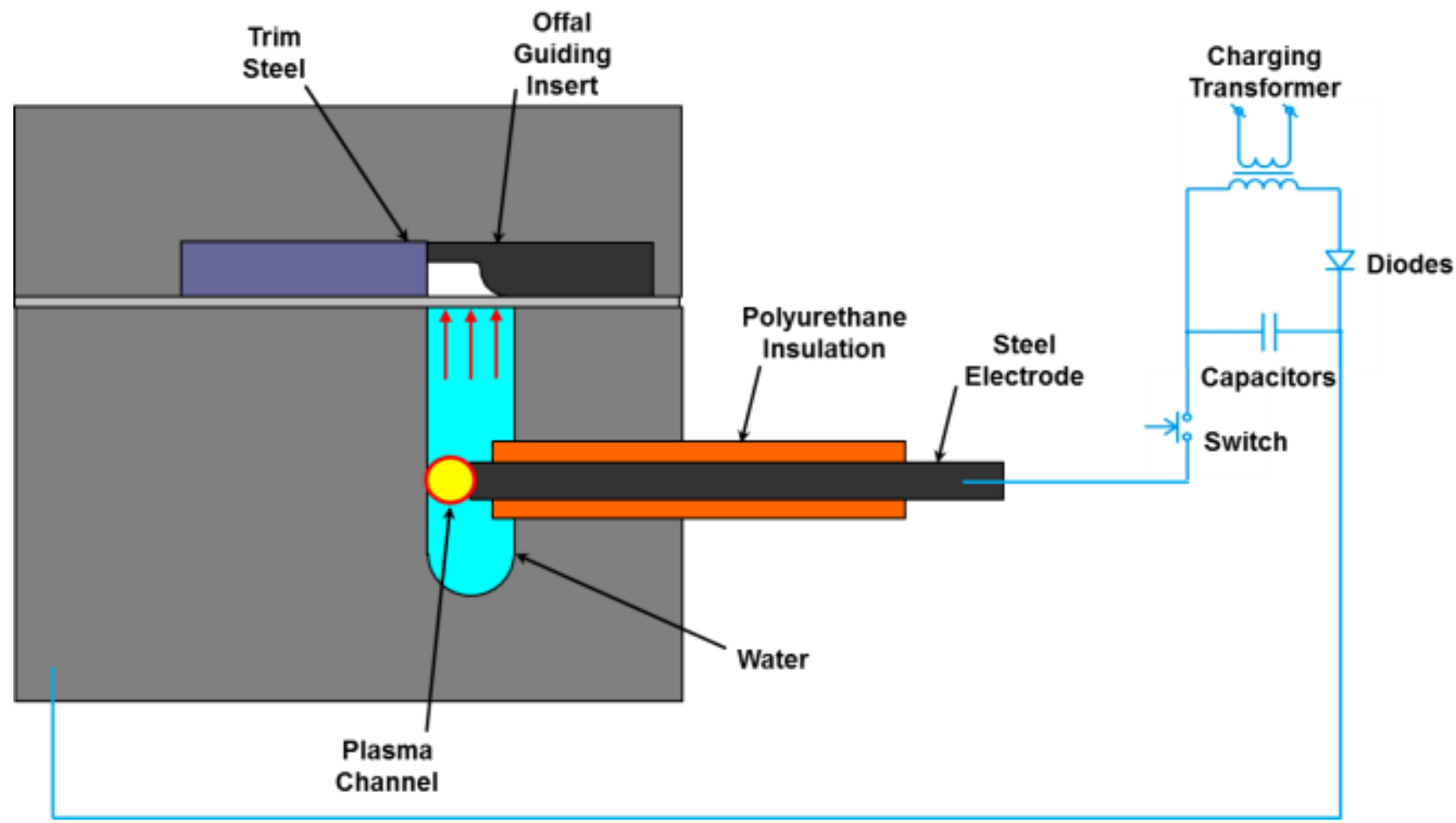

Figure 6.1.1. A cross-sectional, schematic view of the channel chamber connected to the pulsed-current generator during electrohydraulic trimming.

The channel chamber has one electrode which is connected to the energized output of the pulse generator. The insulation of the electrode must be sufficient to prevent a short-circuit discharge to the chamber wall. The ground output can be connected to the bulk of the chamber, serving as a simple ground electrode, as shown in Figure 6.1.1. The cavity of the chamber is filled with water before the beginning of the process. When using small-scale, laboratory tooling of simple geometry along with flat sheet metal blanks, filling the chamber by hand from a pitcher is sufficient and is less complicated than using pumps and valves. When tooling geometry becomes more complicated, with the trim line matching the perimeter of a complex, threedimensional automotive stamping, then an integrated system of pumps and valves must be used to properly fill and drain the fluid channels. In this case, air from the channels must first be evacuated using a vacuum pump, and then the channels can be filled with water until the full volume and all the geometrical features are completely filled. The air-evacuation step is necessary because if any air pockets are present in the channels, this air will be rapidly compressed and heated by the $\mathrm{EH}$ pressure pulse, and this compression and heating will consume a significant amount of energy within the process, decreasing the amount of energy which reaches the sheet metal blank. The blank is clamped in between the chamber and the trimming tool to avoid material inflow from the flanges to the area between the trimming insert and the offal guiding insert. 
Once the EH trimming tool is filled with a sufficient volume of liquid, the high voltage can be applied to the electrode to generate the desired shockwave. Unlike the EH forming processes, the $\mathrm{EH}$ trimming process should be performed in one discharge per designated area of trimming. No vacuum is necessary in the offal cavity behind the blank, since the offal cavity is not expected to be filled completely, as it is in EH forming. Once the blank begins to trim and separate, any air that was trapped in the offal cavity can then move into the space opened up below the trimmed blank.

The desired $\mathrm{EH}$ pressure pulse should have a rather short duration and high amplitude in order to avoid the situation which often happens in quasistatic hydro-piercing, where pressure begins to drop rapidly as soon as the separation begins. Also, a more intense pressure pulse would result in faster acceleration of the blank and in turn would cause the prevailing dynamic mode of deformation in the blank. The trimming insert was fabricated from cold rolled, oil-hardenable flat plate which was heat treated to HRC 62. The shearing edge of the trimming insert should be as sharp as possible to avoid possible burrs, which might start occurring after some number of cuts.

The electrode configuration employed in this study was very simple: a steel threaded rod had polyurethane insulation cast around it in a special mold capable of providing a minimal amount of air bubbles left in the insulation. As one might expect, because the volume of the fluid channel is so small and because the positive electrode discharges directly to the chamber wall, the durability of the tooling in terms of both fatigue life and erosion of the channel wall could become an issue if not properly designed. Because the EHT process is still new and under ongoing development, these issues were not addressed in the early proof-of-concept tooling designs. The experimental tooling discussed in this report has undergone several hundred pulses without any significant damage, which is a promising result. However, if EHT tooling were to be designed for a production environment and a planned life of several hundred thousand discharges, then a strategy would have to be developed to address the erosion of the channel wall, such as removable inserts of steel or tungsten. Furthermore, the fatigue life of the entire tool itself would need to be assessed using numerical modeling of the loads on the chamber.

During the discharge process, the pressure wave that is formed accelerates the material of the blank against the sharp trim edge into the cavity between the trimming insert and offal guiding insert, thus trimming the sheet. Further analysis of the $\mathrm{EH}$ trimming process, including experimental results and numerical modeling, will be described in the following paragraphs. 


\section{Experimental Tooling and Equipment used for EH Trimming}

The design of the first generation, laboratory-scale electrohydraulic trimming tool is shown in Figure 6.1.2. It consists of a narrow fluid channel machined into a rectangular steel block. The fluid channel is $20 \mathrm{~mm}$ wide, $60 \mathrm{~mm}$ deep and $381 \mathrm{~mm}$ long. The chamber was originally designed with three electrodes evenly spaced across the length of the channel, in order to study the effect of the electrode position on the performance of the process. However, after several rounds of experiments, it became clear that the tool could have been designed with just one electrode instead of three, as will be explained later. Each electrode is press-fit inside the chamber to prevent the leakage of water from around the electrodes. In addition to press fitting, additional sealing is also desirable, and this can be accomplished through seal grooves and rubber O-rings positioned on the internal surface of the electrode holes, or through other sealing measures at the outer junction between the electrode and the chamber. The laboratory chamber is mounted on a $50 \mathrm{~mm}$ thick insulation plate. In order to restrain the axial force on the electrodes which occurs during the discharge and to prevent them from being forced out of the chamber, they are supported in the axial direction by L-brackets bolted to the insulation plate.

It should be indicated that the initial intuitive design of the channel chamber illustrated in Figure 6.1.2 underestimated the capabilities of the process. The length of the cut resulting from one discharge at the central electrode is sufficient to cover the whole chamber, assuming that the optimized offal guiding geometry is used. Therefore, all of the trimming experiments described in this report were conducted using only one discharge through the central electrode. As stated above, the second and third electrodes in the tool proved to be unnecessary due to the efficiency of the process. However, the design of this tool could be still be improved by increasing the length of the fluid channel to the maximum length of sheet that could be trimmed by one pulse through one electrode. Based on the initial experiments on $\mathrm{EH}$ trimming, this length was shown to be at least $400 \mathrm{~mm}$.

The one-sided trimming die is positioned on top of the channel chamber. Accurate alignment of the die and the chamber is accomplished by two guide pins. The trim blades were identical to those used in a previous study on conventional trimming, in order to have full correlation of the trimming tool materials and fabrication procedure for both processes. Three trim blades were mounted inside the trimming die together with one offal guiding insert. The shape of the offal guiding insert was varied during this experimental study to alter both the height and the width of the cavity above the discharge channel. The different offal guiding inserts (shapes A, B and C) are shown in position in the EH-trim tool in Figure 6.1.3 below. With offal guiding inserts $A$ and $B$, the available vertical space above the blank in the offal cavity was $11.2 \mathrm{~mm}$. The difference between inserts $A$ and $B$ is in the horizontal gap that these inserts create in the offal cavity. Insert A created a horizontal gap of $20 \mathrm{~mm}$, while insert B created a horizontal gap of $10 \mathrm{~mm}$. Offal guiding insert $C$ restricted the size of the offal cavity even further, by creating a horizontal gap of $10 \mathrm{~mm}$ and a vertical gap of only $4.5 \mathrm{~mm}$ of available space above the blank in the offal cavity, as shown in Figure 6.3. 


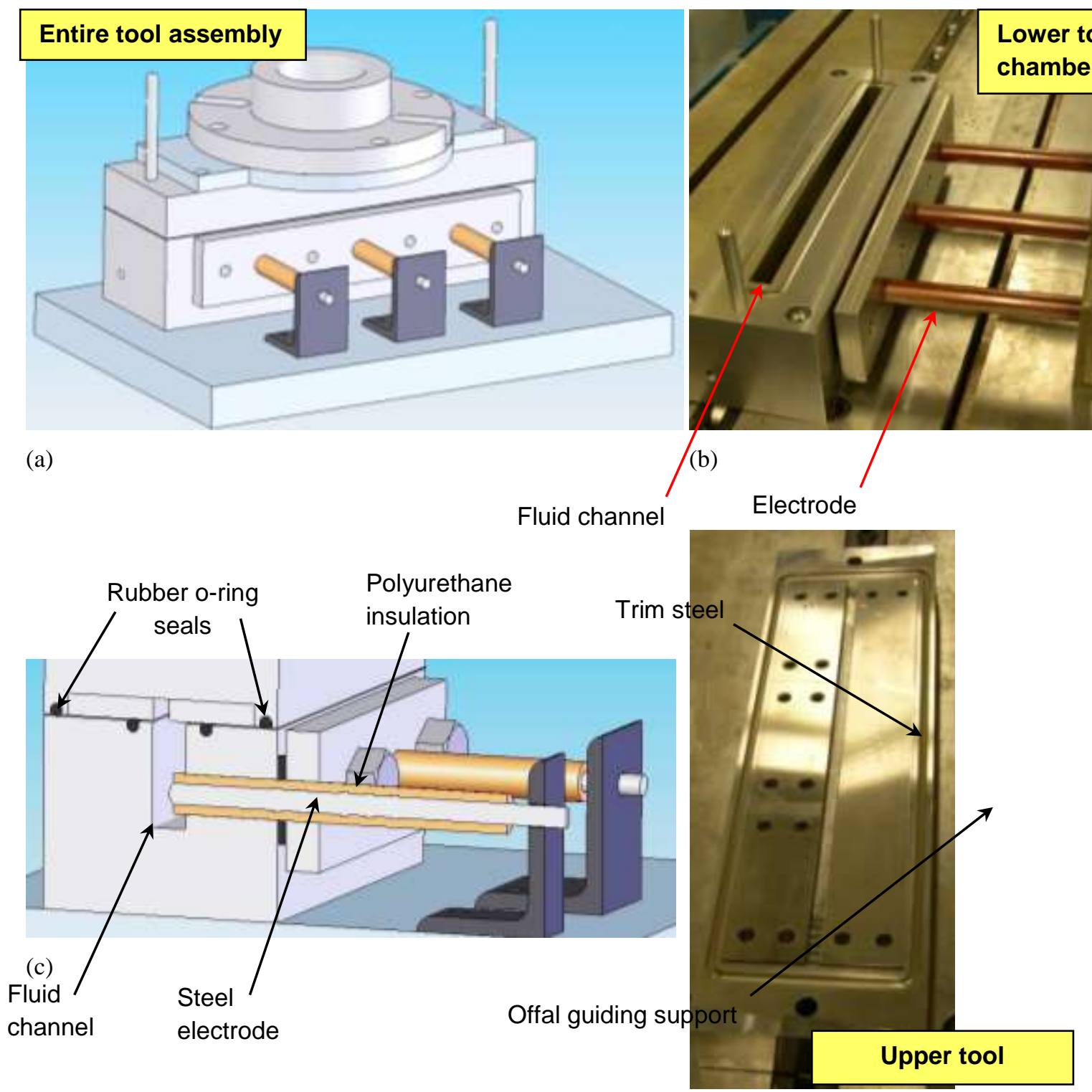

(d)

Figure 6.1.2. Laboratory tool for electrohydraulic trimming: (a) CAD model of the assembled tool; (b) channel chamber with three electrodes; (c) cross-section of the assembled tool; (d) upper half of the tool with sharp trimming edge and offal guiding support. 


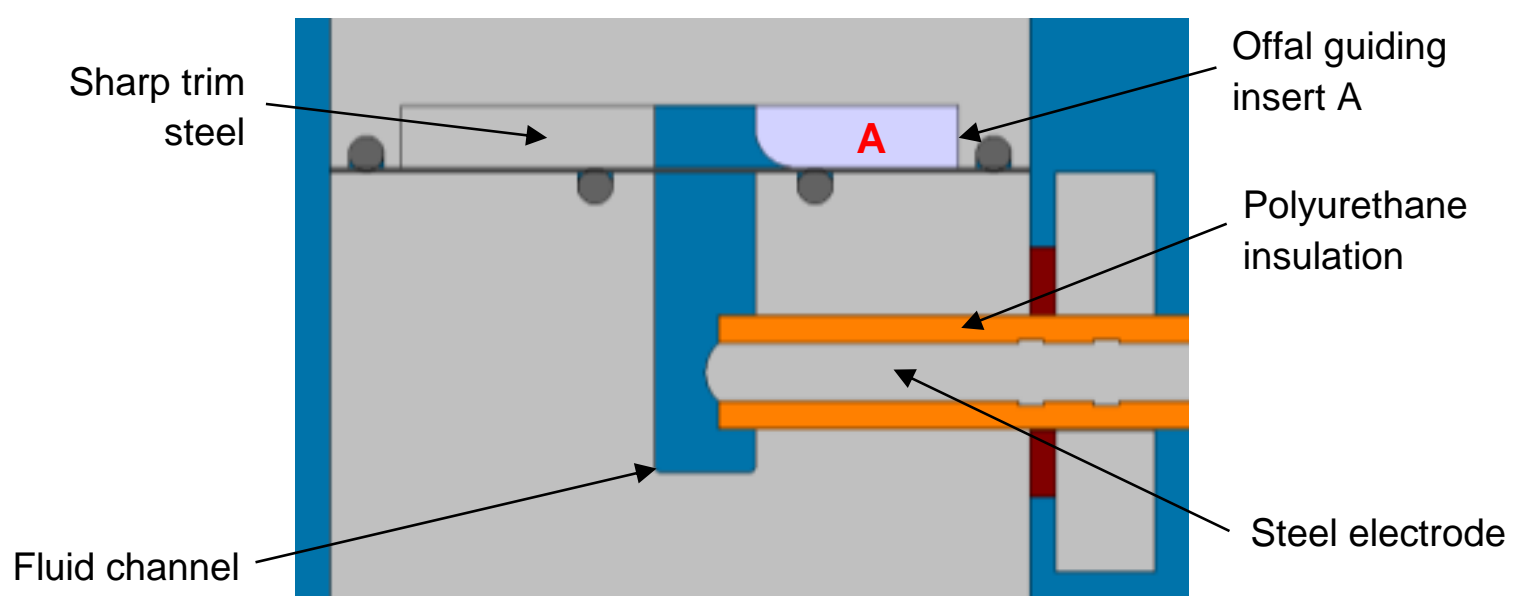

(a)

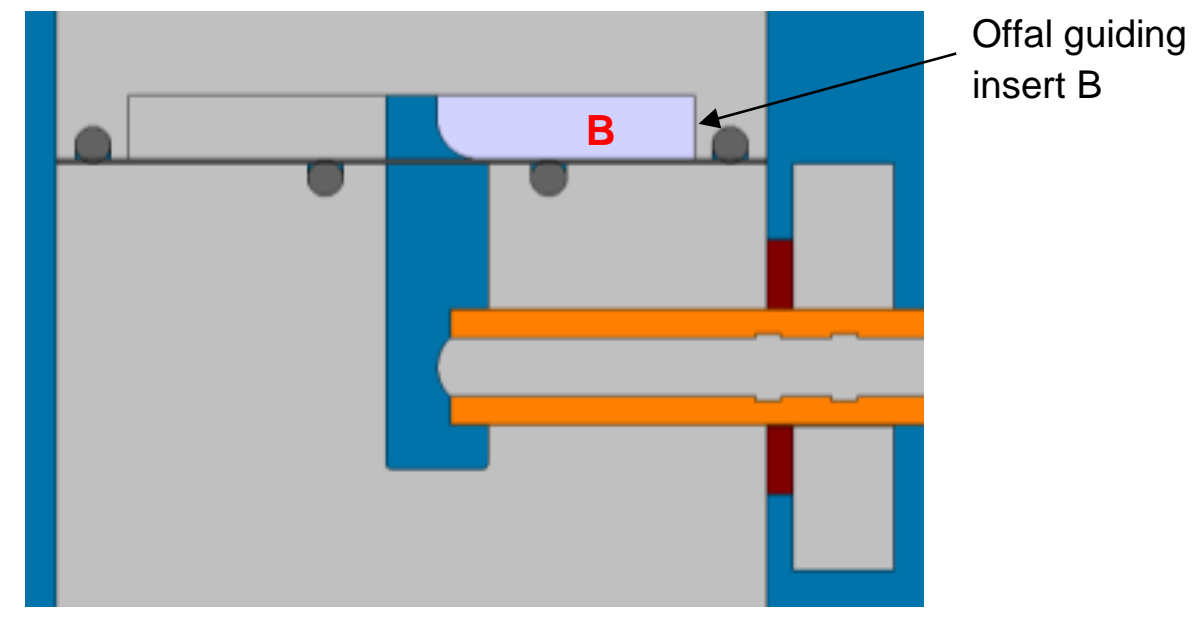

(b)

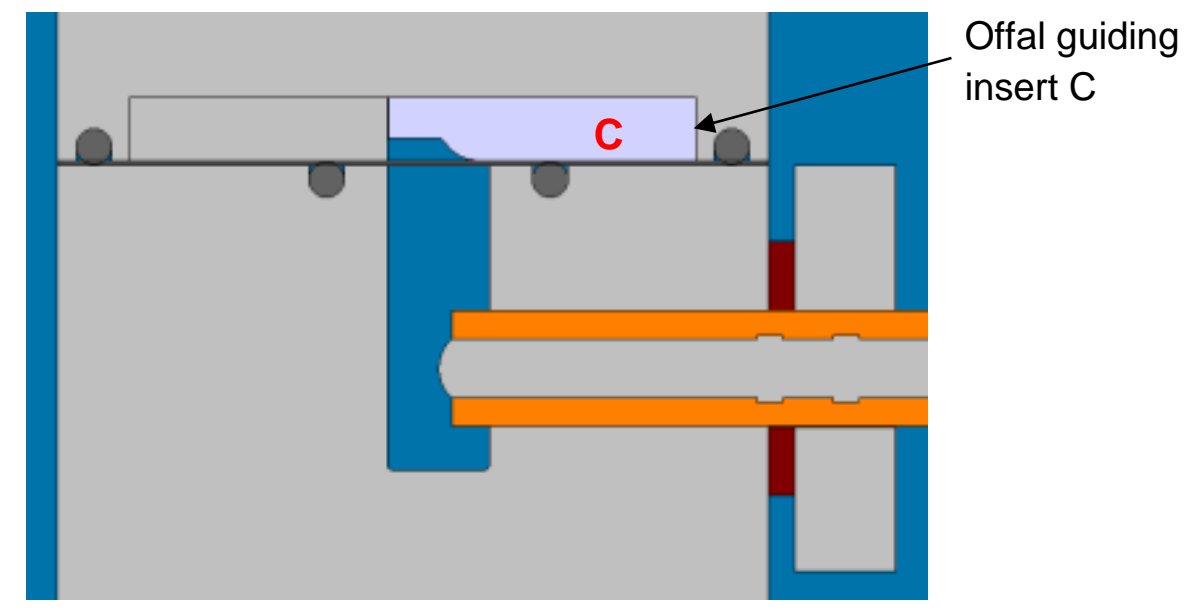

(c)

Figure 6.1.3. Schematic of EH-trimming tool with three different offal guiding insert designs. 
A 100kJ Pulse generator was employed in this experiment. It had a capacitance of $320 \mu \mathrm{F}$, a maximum voltage of $25 \mathrm{kV}$, and an internal inductance of $20 \mathrm{nH}$. The pulse generator was composed of eight blocks of capacitors, with each block connected to the discharge circuit through its own individual vacuum switch. Electrical energy was stored in high voltage capacitors charged through a transformer and a set of diodes. A set of vacuum switches closes the circuit and delivers high voltage stored in each module of capacitors to the electrodes. The parameters which define the efficiency of the EHF process are the mutual position of the electrodes, the electrical properties of the liquid, the charged voltage, the capacitance of the circuit, the inductance and resistance of the equipment and connecting cables, the volume of the chamber, and the distance between the discharge channel and the blank. At the beginning of the discharge process the electrical resistance of the channel between the electrodes drops by several orders of magnitude and the electric current grows rapidly. As electrical energy is deposited into the plasma channel, it expands extremely rapidly, and this expansion generates the shock wave in the liquid and the corresponding high pressure which enables electrohydraulic processes to be useful in industry.

\section{Results of Experiments on EH Trimming}

The first experiment compared electrohydraulic trimming with guiding inserts of shapes $A, B$ and $\mathrm{C}$, which create three different offal cavities above the blank. The difference between these three offal cavities is in terms of the horizontal gap and the vertical gap, as shown in Figure 6.1.3. The comparison was made by trimming blanks of DP590, $1 \mathrm{~mm}$ thick, against each offal guiding insert at the same pulse energy of $9 \mathrm{~kJ}$ at $7.5 \mathrm{kV}$. Then, the trimmed length of each blank was measured. Measurement results showed that for insert $A$ the trimmed length was 244 $\mathrm{mm}$, for insert $B$ the trimmed length was $251 \mathrm{~mm}$, and for insert $C$ the trimmed length was 381 $\mathrm{mm}$ or the full length of the fluid channel and trim blade. Photographs of the blanks trimmed in this first experiment are shown in Figure 6.1.4.

The reason that inserts $A$ and $B$ showed lower performance than insert $C$ is that the cavity volume above the fluid chamber and the blank was too large, allowing much of the energy of the discharge to be converted into plastically deforming the offal metal instead of trimming the blank at the sharp trim edge. Furthermore, pressure from the EHF pulse was able to dissipate into this open volume, rather than propagating horizontally along the trim edge. Another key observation regarding inserts $A$ and $B$ is that the trimming results are nearly the same, which indicates that the energy efficiency of the $\mathrm{EH}$ trimming process is much more sensitive to the vertical gap than the horizontal gap. By limiting the vertical displacement of the blank to a level that is no more than what is truly necessary for trimming, the energy efficiency of the process is improved, as seen in the results from insert $\mathrm{C}$. In other words, by limiting the displacement of the metal after it has separated from the trimmed edge, less energy is wasted in further deforming this scrap metal, and more energy and pressure is therefore being applied to trim the rest of the blank. One important factor that was taken into consideration in the design of this insert was to make sure that the displacement distance of the trimmed scrap was not too small. It was estimated that for the process to be successful, the available space above the blank must be at least three times the blank thickness. At this point, this recommendation was developed based upon experimental observations. This recommendation is preliminary and needs further analysis, 
since it may be sensitive to the particular tooling dimensions and equipment parameters employed in this work. However, limiting the displacement of the offal generally helps to concentrate the strain in the area of the shearing edge of the tool. In the case of insert $C$, the gap above the blank had a height of $4.5 \mathrm{~mm}$. With a blank thickness of $1 \mathrm{~mm}$, this tool geometry therefore fits within the design guidelines.

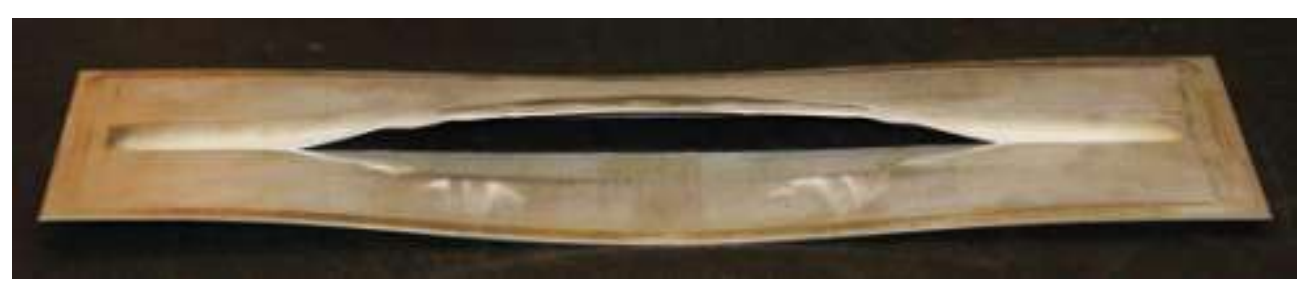

(a)

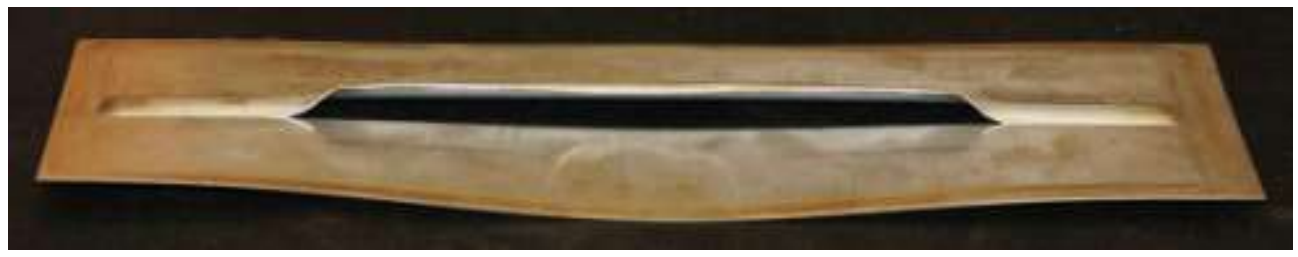

(b)

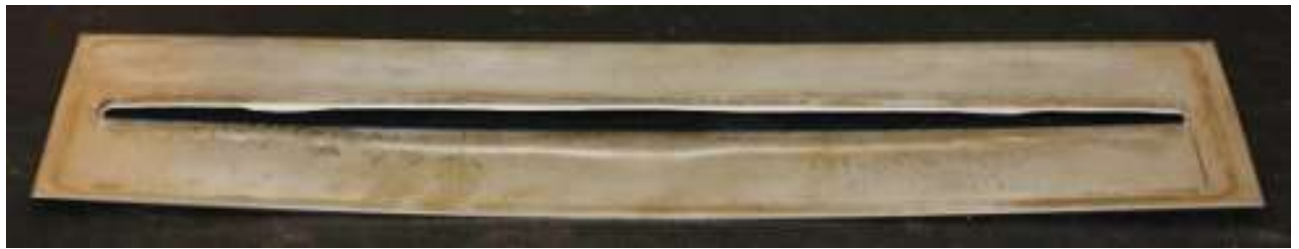

(c)

Figure 6.1.4. Electrohydraulic trimming of $1 \mathrm{~mm}$ thick DP590 blanks using a pulse energy of 9 $\mathrm{kJ}$ at $7.5 \mathrm{kV}$ : (a) sample trimmed with offal guiding insert A shown in Figure 6.1.3a; (b) sample trimmed with offal guiding insert $B$ shown in Figure 6.1.3b; (c) sample trimmed with offal guiding insert $\mathrm{C}$ shown in Figure 6.1.3c. 
Another interesting observation from early experiments with $\mathrm{EH}$ trimming is the effect of reflected pressure waves. This effect is illustrated by the results shown in Figure 6.1.5. Figure 6.1.5a shows a section view of the $\mathrm{EH}$ trimming tool, revealing one electrode in the center of the fluid channel. The arrows represent the path of the pressure waves that result from a discharge through this electrode (a more detailed analysis of pressure wave propagation will be provided in the section on numerical modeling). Immediately after the discharge, fluid pressure builds up rapidly in the area surrounding the electrode, and the pressure waves propagate with spherical symmetry away from the initial plasma channel. Figure $6.1 .5 \mathrm{~b}$ shows a blank of $2 \mathrm{~mm}$ thick 4130 steel, partially trimmed by a $40 \mathrm{~kJ} \mathrm{EH}$ pulse at $20 \mathrm{kV}$. The effect of the reflected pressure waves is clearly visible, and can be seen by the areas of secondary trimming at the right-most and leftmost ends of the trim line. The area of trimming in the center of the blank was caused by the initial pressure wave which propagated directly upward and away from the initial plasma channel. The areas of trimming at the ends of the blank were caused by reflected pressure waves. These pressure waves initially started propagating outward away from the plasma channel, and when these waves reached the steel walls of the tool at the ends of the channel, the pressure would build up in the local area near the end walls of the channel, causing the blank to trim in the areas directly above the ends of the channel. Figure $6.1 .5 \mathrm{c}$ shows a blank of the same material and thickness, but fully trimmed by a $50.6 \mathrm{~kJ} \mathrm{EH}$ pulse at $22.5 \mathrm{kV}$. The curved regions of the offal indicate that these were the areas of the blank which were the last to trim and separate from the part side of the blank.

It should be noted that this mechanism of trimming, involving primary and secondary zones of trimming which eventually come together and meet, is commonly observed with heavy gauge sheets and high energy pulses, but is not normally observed with thinner gauge sheets and lower energy pulses. For example, the trimmed blanks shown in Figure 6.1.4 do not exhibit this effect of primary and secondary zones of trimming. The reason for this difference has to do primarily with the inertia that is involved in accelerating a thicker blank. Thicker blanks require a larger displacement at the trim edge in the time period of deformation prior to crack initiation, and thicker blanks would also require slightly more time for the crack to propagate through the thickness of the sheet. Furthermore, thicker blanks have a larger mass per unit of length compared to thinner blanks of the same material. In light of these facts, one can see that thicker blanks require a longer time interval to fracture the trimmed blank and open the chamber in the area of sheet metal blank fracture. Once the chamber volume is opened by the fractured blank, the blank will continue to trim along the length of the trim edge, and this can be described as an unloading wave in the material following behind the shock wave in the water. As the time interval and the distance between the unloading wave and the shock wave increases in a given set of experimental conditions, then the effects of this wave mechanism will be more pronounced on the trimmed blank. 


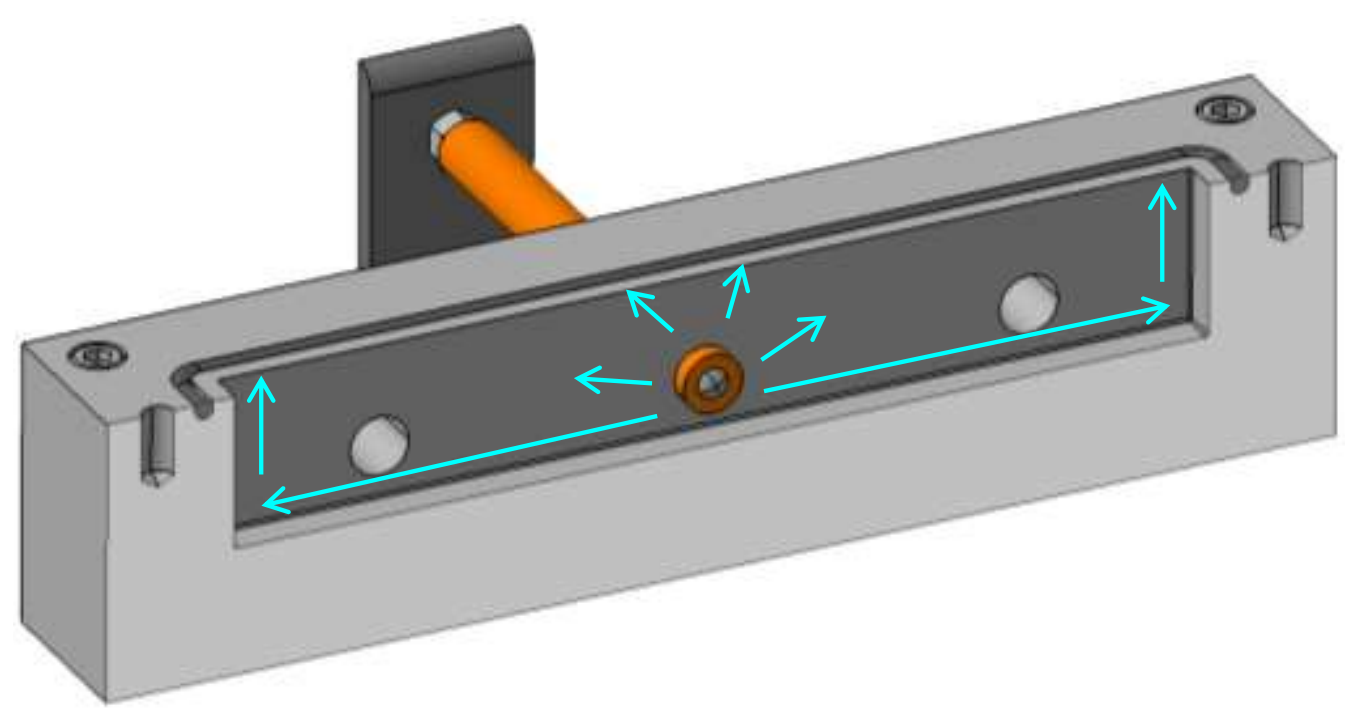

(a)

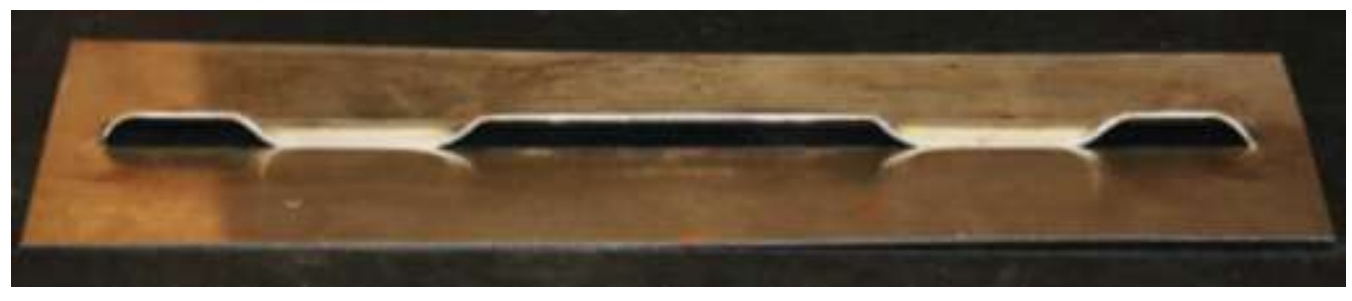

(b)

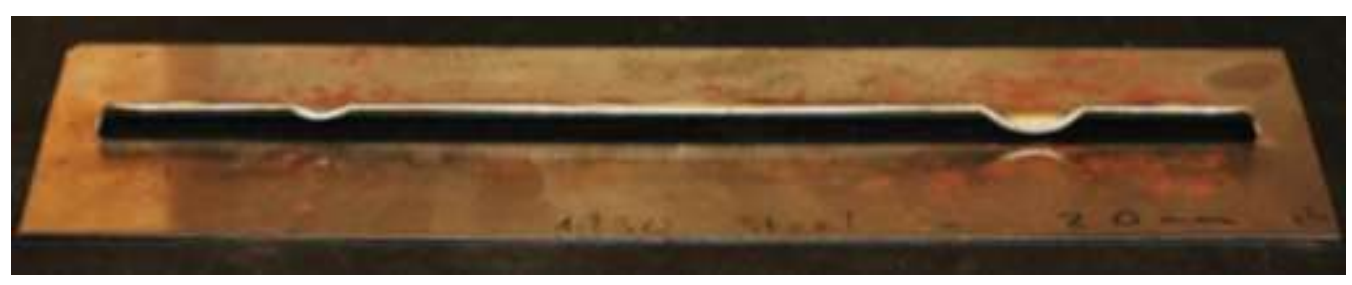

(c)

Figure 6.1.5. The effect of reflected pressure waves in EH trimming, illustrated by the results of trimming $2 \mathrm{~mm}$ thick 4130 steel: (a) schematic sectional view of the EH trimming chamber, showing one central electrode and the direction of pressure wave propagation after the discharge; (b) trimming results using a pulse energy of $40 \mathrm{~kJ}$ at $20 \mathrm{kV}$; (c) trimming results using a pulse energy of $50.6 \mathrm{~kJ}$ at $22.5 \mathrm{kV}$. 
Based on this initial study, we designed tools that would be used to further test and develop the EH-trimming process. The tools that were designed were (1) a conventional two sided tool that will form the candidate part to be studied, and (2) an EH-trimming tool that will trim the conventionally stamped panel using pulsed electrohydraulic pressure. The intent for these tools was to work with an actual stamped part. Therefore, the decision was made to work with the Front Floor Side Inner from the Ford Focus, but at 50\% scale relative to the actual panel in order to save on tooling costs. This part is shown in Figure 6.1.6 below. The design of the conventional stamping tool is shown in Figure 6.1.7 below. The tool features a female die on the lower half, and a punch and binder surface on the upper half that can move independently through the use of gas cylinders. The parts were formed from DP600 at $1.0 \mathrm{~mm}$ thick.

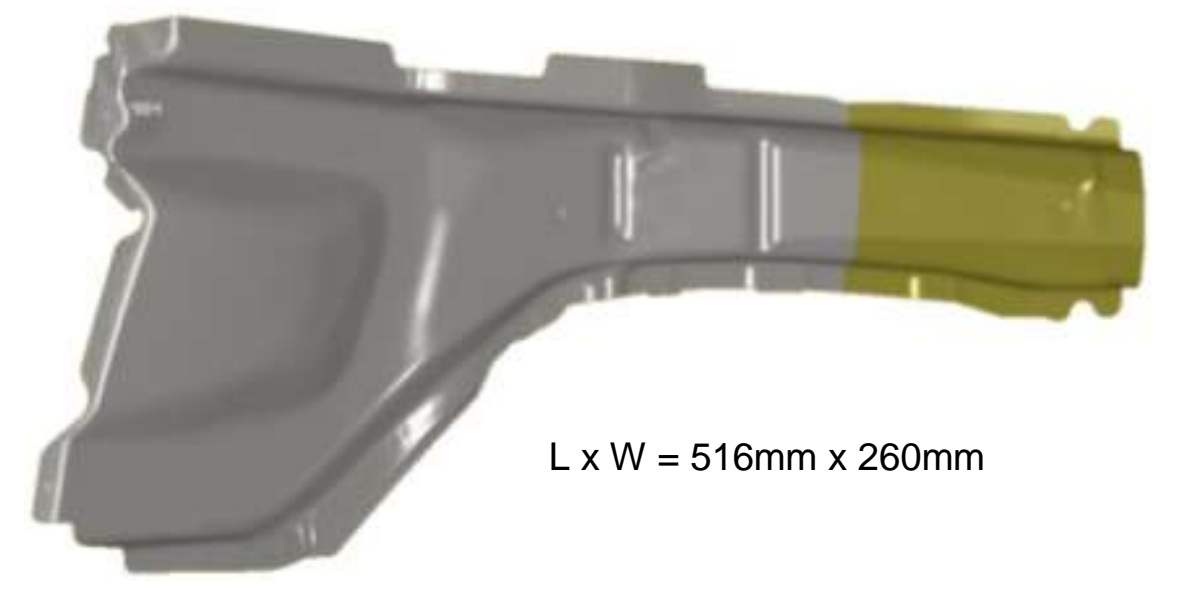

Figure 6.1.6. Candidate part selected for development and testing of EH-trimming. The part was at $50 \%$ scale.

The design of the $\mathrm{EH}$-trimming tool is shown in Figure 6.1 .8 below. This tool was designed to accomplish trimming along a complex, three-dimensional, closed-loop trim line; something never before demonstrated with the EH-trimming technology. Fluid channels of $20 \mathrm{~mm}$ width are positioned all around the part perimeter, with sharp trim steels positioned above the blank trim line, as in previous EH-trim tools. One new feature of this tool as compared to previous EH-trim tools is the use of separate sub-channels, or fluid channels with dividing walls between each sub-channel. Close examination of Figure 6.1.8 will reveal that four electrodes are positioned around the part perimeter, with each electrode in its own sub-channel, separated from the other sub-channels with dividing walls. The purpose of these walls is to enable the each individual segment of the trim line to be trimmed independently from the other segments. Without these walls, once one area of the trim line was trimmed, water from the fluid channel would begin to leak through the trimmed area of the blank, and this would cause problems for further trimming due to the loss of water in some channel areas and the flooding of water into the scrap support volume in other areas of the tool. Also, experiments have confirmed that even though dividing walls may be present in the fluid channels below the blank trim line, as long as the trimming knives follow the trim line in an uninterrupted and continuous fashion above the trim line then the inertia of the EH-trimming process will cause the blank to trim beyond a dividing wall, 
thereby allowing the different segments of the trim line to be connected for continuous trimming of the entire perimeter.

(a)

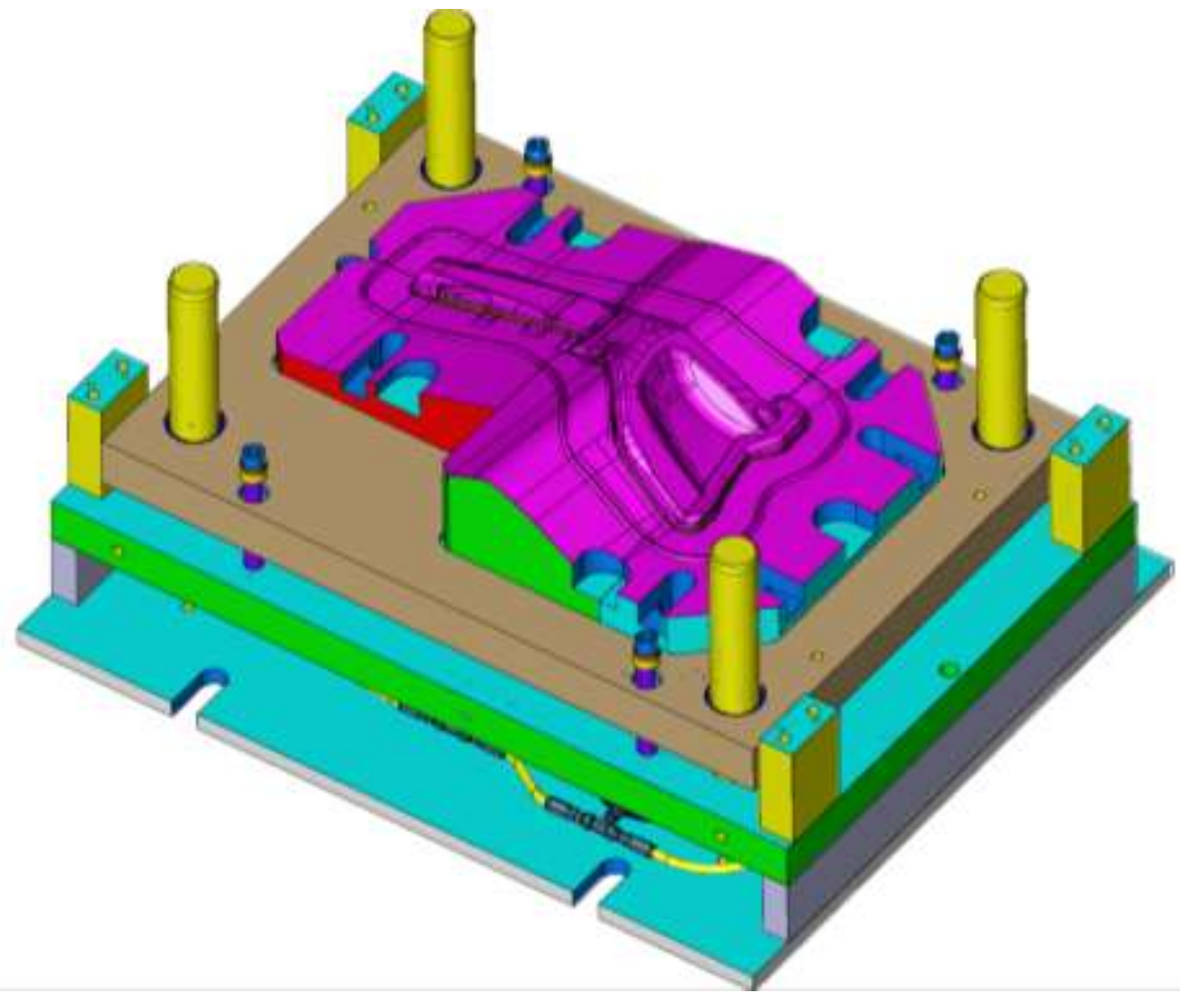

(b)

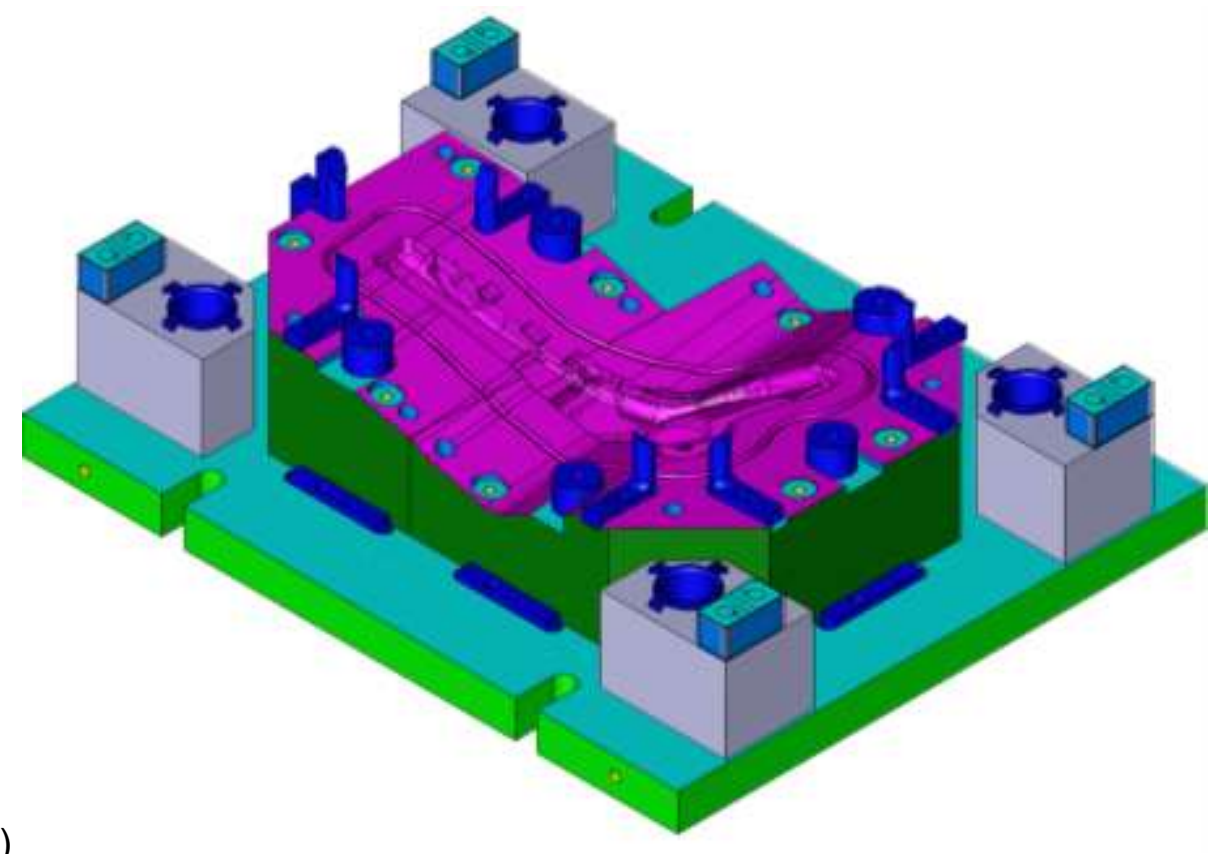

Figure 6.1.7. Conventional stamping tool for forming of the C346 floor side inner at $50 \%$ scale; (a) upper tool, including male punch, binder surface, gas springs, and guide pins, and (b) lower tool, including a female die, guides for blank positioning, guide pin bushings, and spacers for adjusting tool contact pressure and material drawing. 

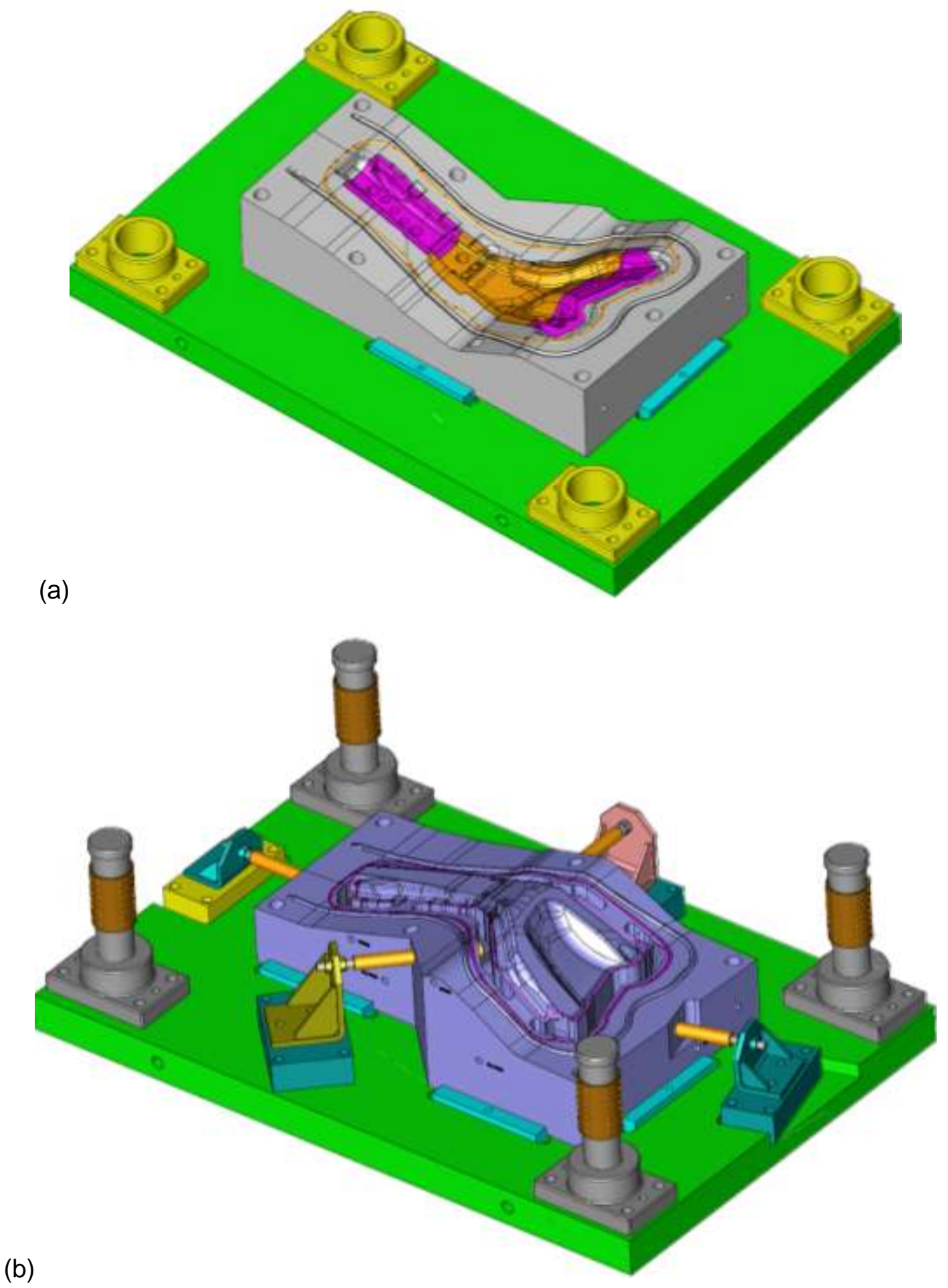

Figure 6.1.8. EH-trimming tool for the C346 floor side inner at 50\% scale. (a) Upper tool, including trim knives, scrap support, and guide pin bushings. (b) Lower tool, including chamber with sub-channels, electrodes, and guide pins. 
The lower half of the $\mathrm{EH}$ trimming tool which includes the fluid sub-channels is shown in Figure 6.1.9. The upper half of the EH trimming tool which includes the trim blades is shown in Figure 6.1.10. The majority of the machining was done with a standard 3-axis milling machine. Some of the more detailed and geometrically complex features of the tool, such as the inner seal groove and some features of the fluid channels, were machined using a 5-axis mill, which added some additional lead time to the fabrication process due to limited availability on such machines. A close-up view of one of the fluid channels is shown in Figure 6.1.11. The lower edge in the image, or the edge closer to the smaller seal groove, corresponds to the trim line of the panel. The transition between the inner wall of the channel and the base of the channel is visible as a radius, and this radius is important for reducing any stress concentration that would occur at this location due to the force from the pressure pulse. A close-up view of the edge of the trim blade is shown in Figure 6.1.12. The edge of the trim blade can be seen protruding above the surrounding area of the tool. This arrangement is in the tool by design and is necessary for proper function. The open space immediately adjacent to the trim edge can be called the offal guiding area or the offal space because it is in this space where the trimmed offal will move when it is separated from the part. This volume is kept relatively small by design, as previous experiments have shown that the energy efficiency of the $\mathrm{EH}$ trimming process is improved when the offal space is only as large as it needs to be to allow for proper separation in the sheet metal and no larger.

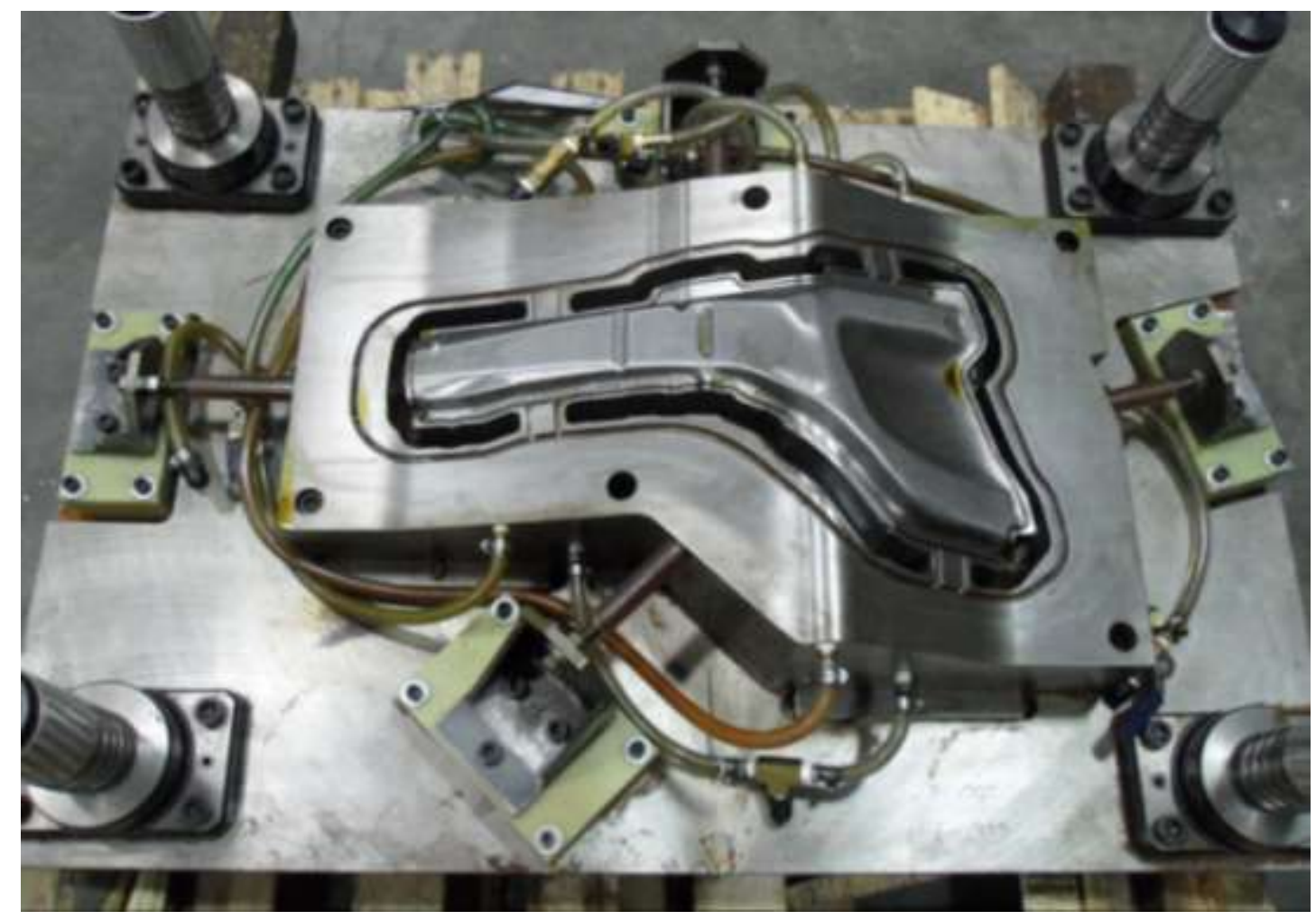

Figure 6.1.9. EH trimming tool, lower half, including four fluid sub-channels. 


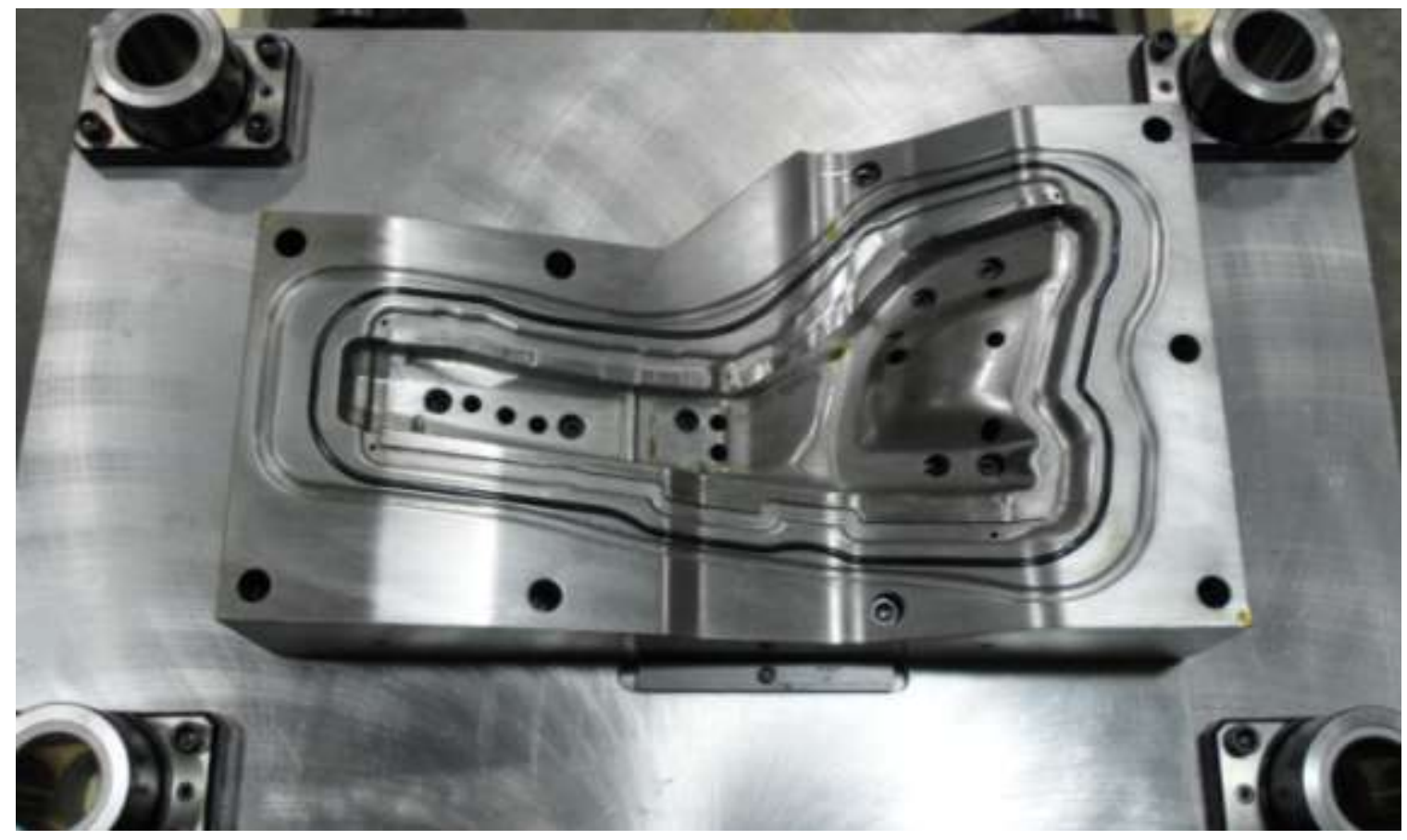

Figure 6.1.10. Upper half of EH trimming tool, including trim blades.

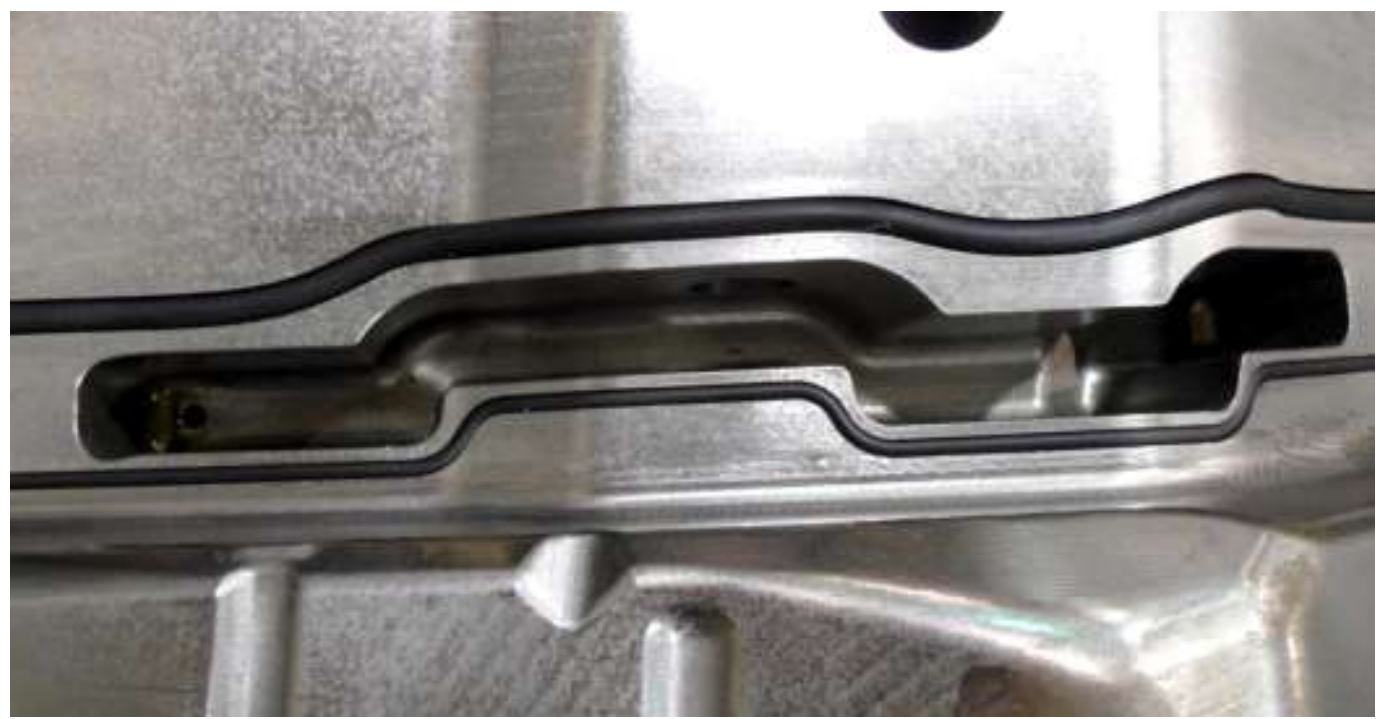

Figure 6.1.11. Close-up view of one of the fluid sub-channels. The lower edge in the image - the edge closer to the smaller seal groove - corresponds to the trim line of the panel. 


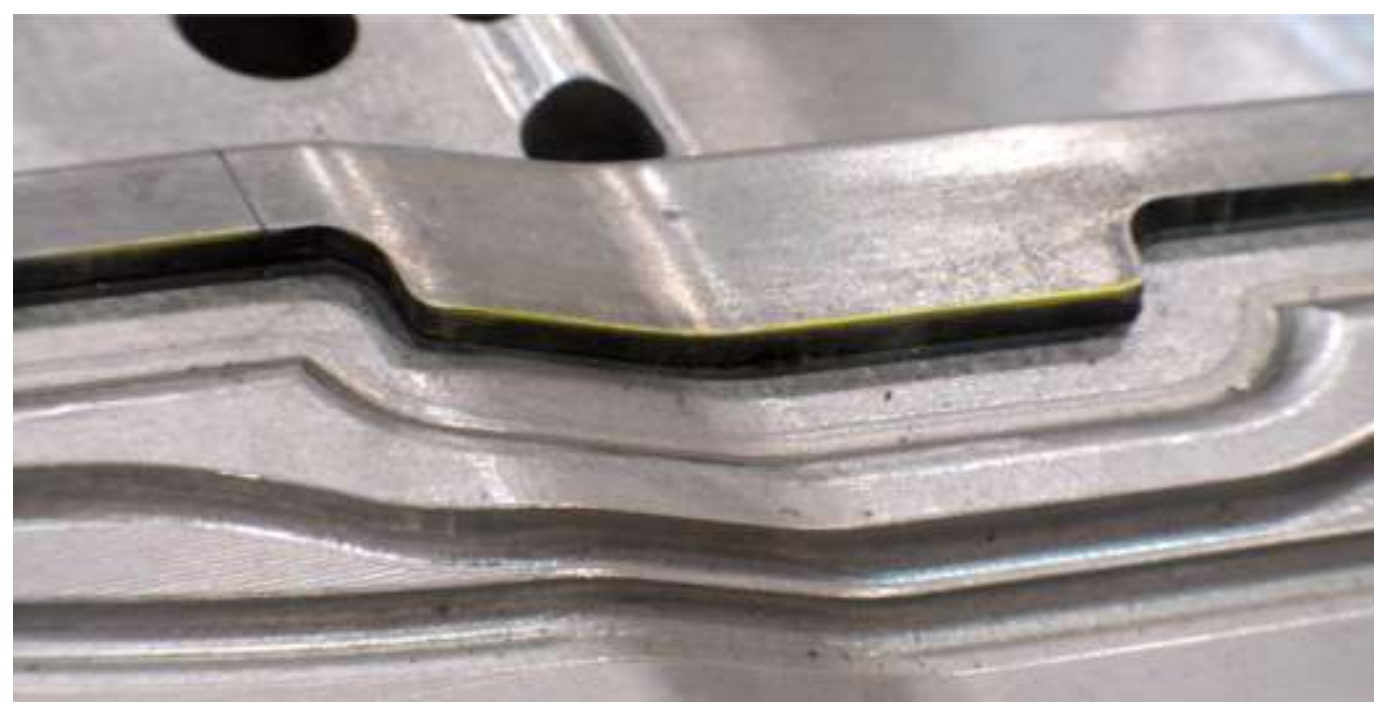

Figure 6.1.12. Close-up view of the edge of the trim blade (shown protruding above the offal guiding area or offal space). This region of the trim line corresponds to the central region of the fluid channel shown in Figure 6.1.11.

Significant progress was made on the EH trimming process during the third quarter of 2012 . The completed tool was delivered to our laboratory and was installed in the press for the first ever prove-out of this type of trimming technology using actual part geometry. After about one week of experimentation and optimization with the tool, we were able to successfully trim out the entire perimeter of the panel geometry. The process works by trimming one segment of the trim line at a time, with the part perimeter being divided into four segments in total. One $\mathrm{EH}$ pulse is used in each segment, using the particular energy level that is appropriate for that segment. Figure 6.1.13 shows a photograph of the trimmed panel, and Figure 6.1.14 shows the offal that is remaining after trimming. Figure 6.1 .15 shows a stack of trimmed panels revealing the absence of any burrs and a clean trim edge matching the fine details of the part geometry. Figure 6.1.16 shows the front nose of the floor side inner panel after being partially trimmed using one discharge within that sub-channel of the tool. The mechanism of EH-trimming can be seen in the photograph: the part-side of the metal is clamped in place, and the scrap-side of the metal is deformed upward and outward, against the sharp trim edge and away from the partside. 


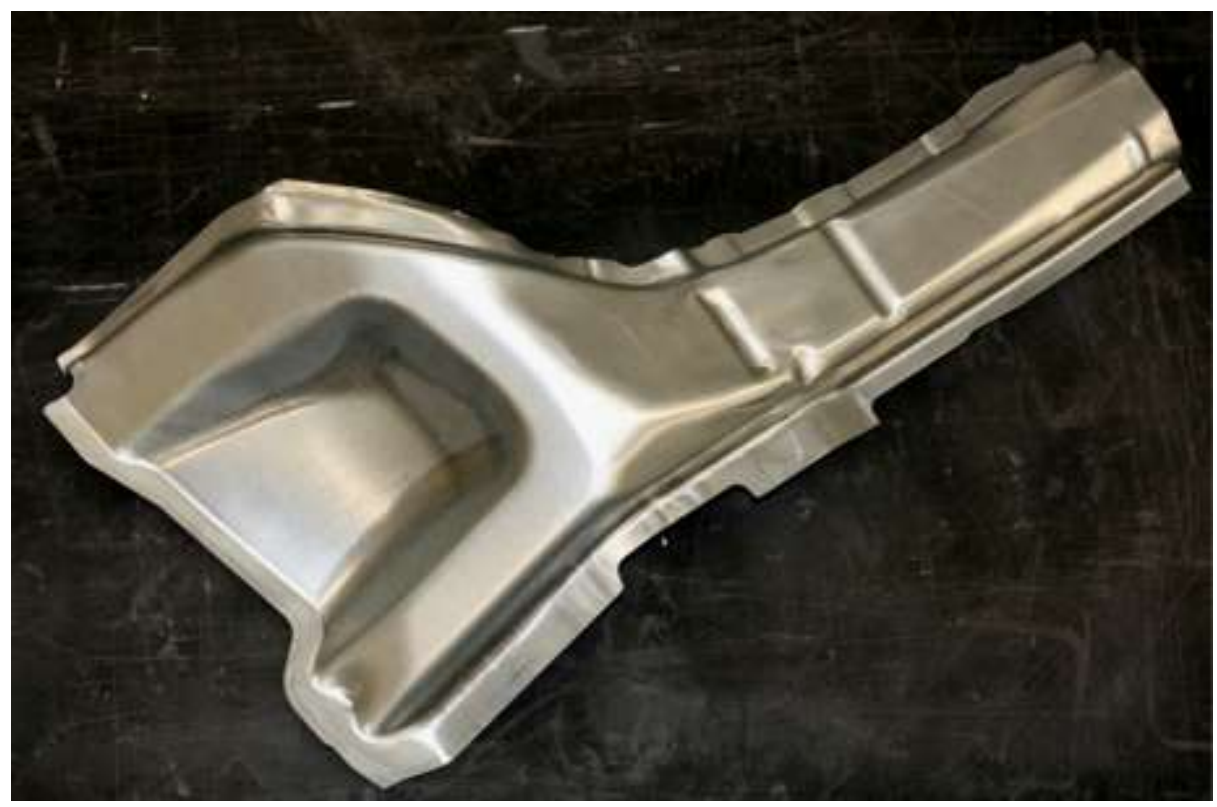

Figure 6.1.13. Front floor side inner panel, trimmed using $\mathrm{EH}$ trimming.

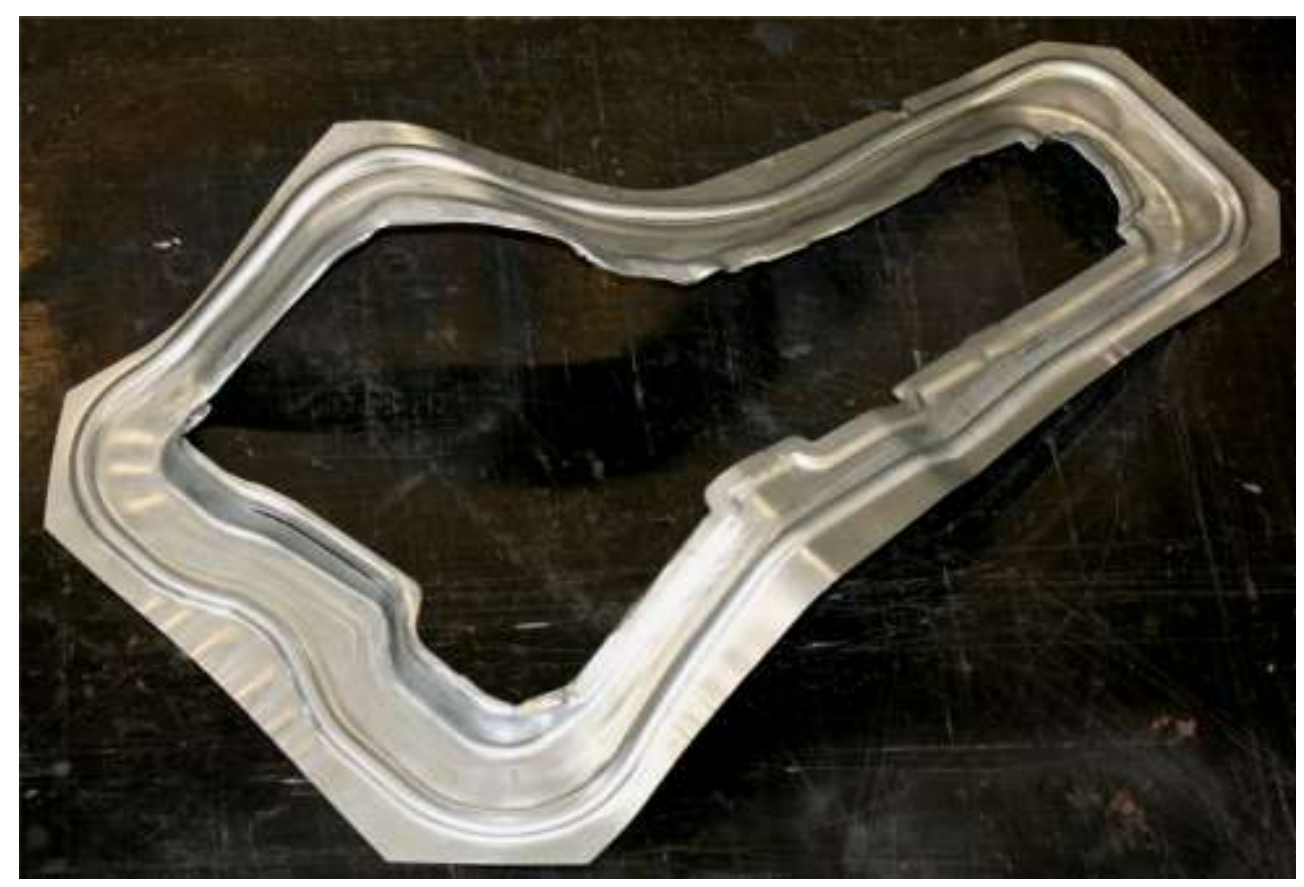

Figure 6.1.14. The offal resulting from EH trimming of the floor side inner panel. 


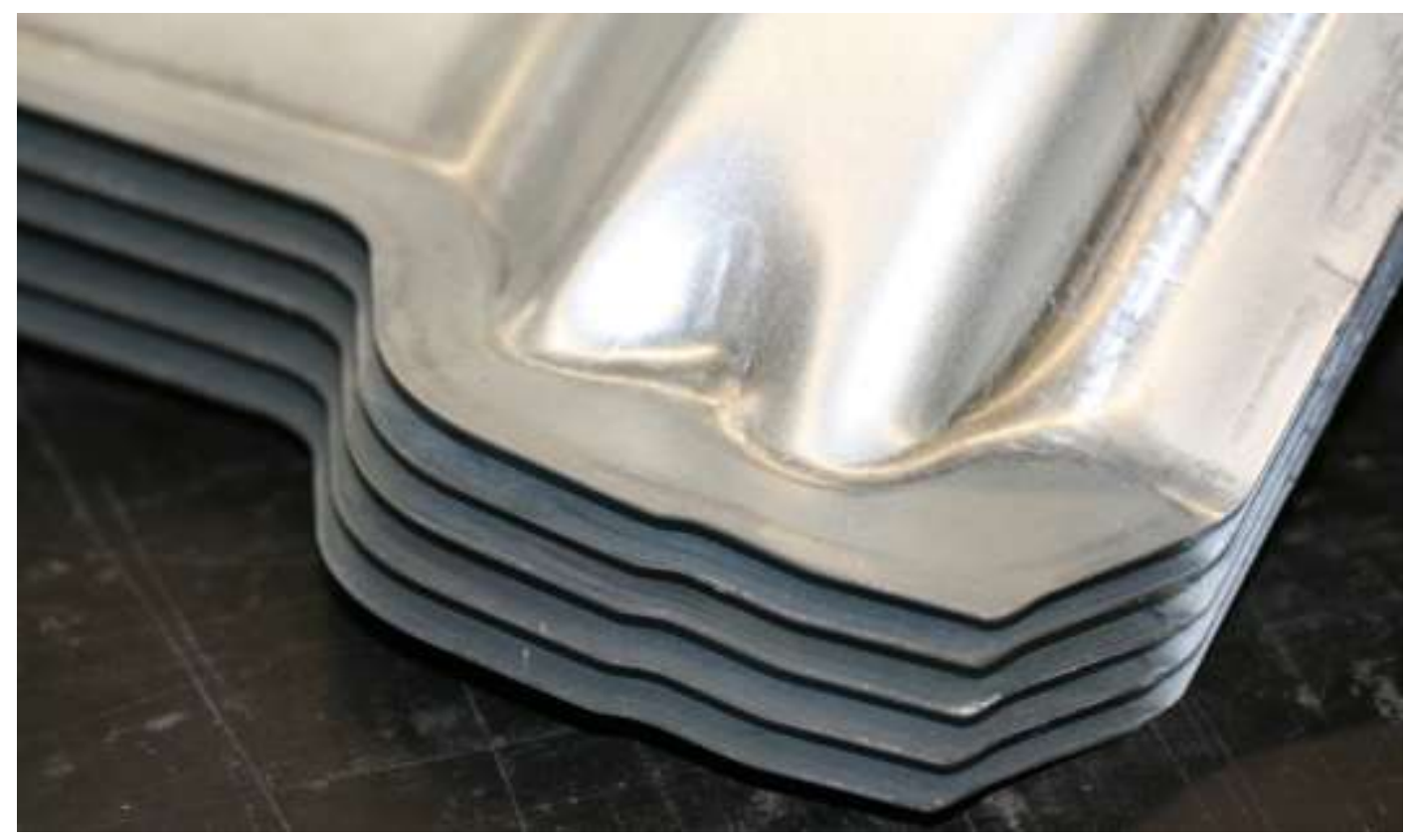

Figure 6.1.15. A stack of panels trimmed using EH trimming, revealing the absence of any burrs and a clean trim edge matching the fine details of the part geometry.

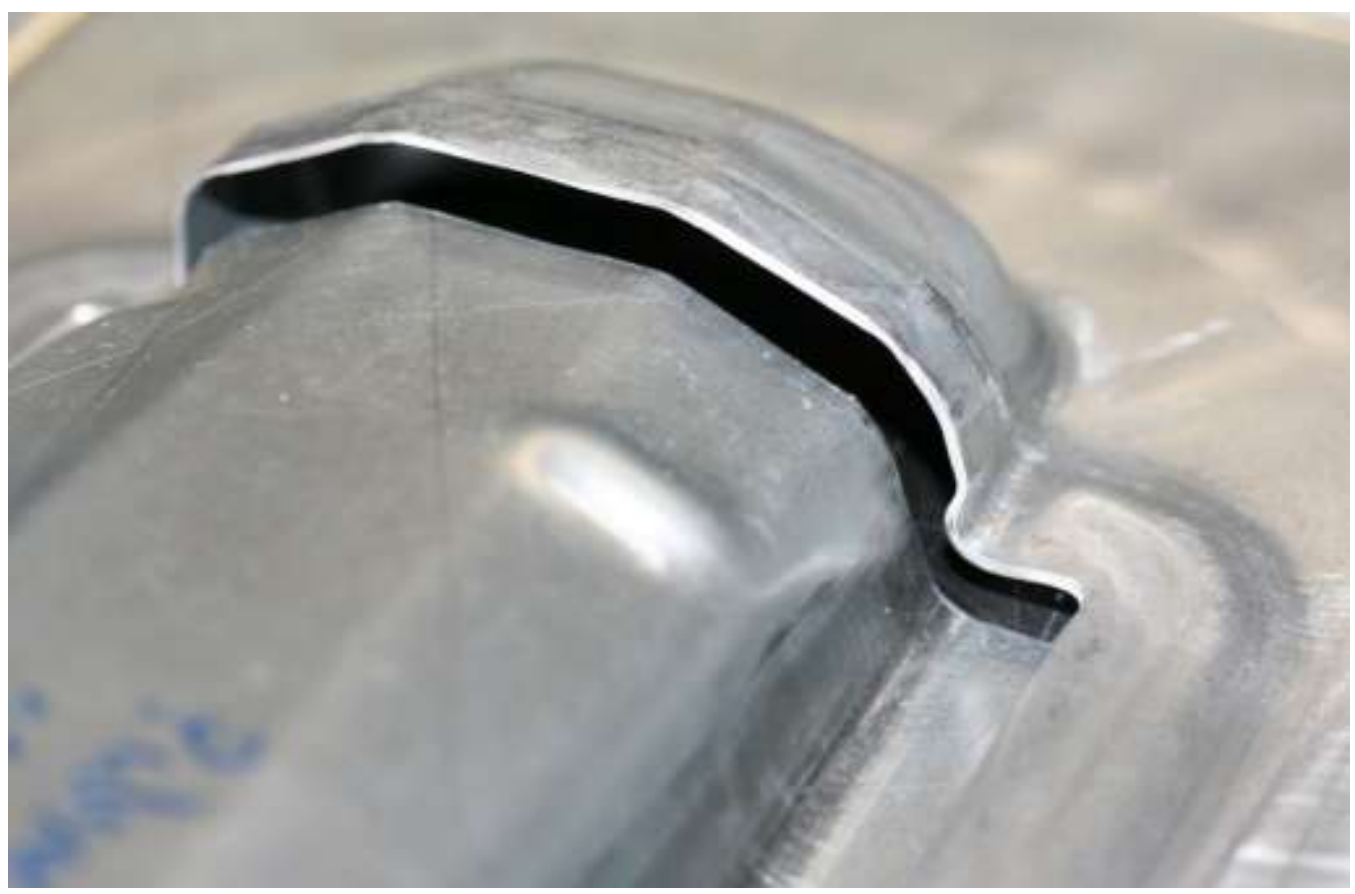

Figure 6.1.16. The front nose of the floor side inner panel partially trimmed using $\mathrm{EH}$-trimming. The mechanism of EH-trimming can be seen in the photograph: the part-side of the metal is clamped in place, and the scrap-side of the metal is deformed upward and outward, against the sharp trim edge and away from the part-side. 
Now that the concept of EH-trimming along a complex 3D trim line has been validated, it is natural to begin to think about the best approach for the first implementation of this technology. There are three main drivers behind the utility of the EH-trimming process: trim-edge quality, reduction of tooling costs compared to cam-trimming, and reduction of cycle time as compared to laser trimming. Whether or not EH-trimming is the right solution for a particular part will depend on the specifics of that part, such as material, complexity, planned production volume, etc. At the current time, the most likely choice for the first implementation of EH-trimming would be as a replacement for laser trimming in a low volume application. For example, for certain large panels, the cycle time for laser trimming can be ten minutes per part. Once the production volume of the part reaches a high enough level, the cost of an EH-trimming tool would be more than offset by the improvements in trimming cycle time. Another question which remains unanswered with EH-trimming has to do with the durability of the trim edge and also the durability of the tool itself, specifically the chamber with the fluid channels. A solution for solving any durability issues most likely could be found based on existing technologies. However, due to the simple fact that the EH-trimming process is a novel concept, the existing $\mathrm{EH}$-trim tools have only experienced several hundred discharges, and it would take additional time and testing before the discharge count would reach several thousand or tens of thousands.

As stated above, no truly long-term durability testing has been conducted with the $\mathrm{EH}$ trimming tool due to time constraints. However, some preliminary durability data can be reported based on the testing that was done during the limited timeframe of this project. The total number of discharges that was conducted in each of the four sub-channels of the EH trimming tool was 24 discharges. After these 24 discharges, the sharp trim edge was still intact in most areas of the $\mathrm{EH}$ trimming tool, but there were three small areas of the tool where the trim edge was damaged by chipping. The chipped edges are shown in Figure 6.1.17 and 6.1.18. The material used for the trim steels in the large EH trimming tool was air-hardened D2 tool steel, a material commonly used in conventional trim steels. The fact that this material chipped under $\mathrm{EH}$ trimming conditions indicates that a different tool material strategy should have been used. In future $\mathrm{EH}$ trimming tools, some slight changes to the tool geometry could be made to improve the life of the trim steels. If the design changes are made in combination with a better material for the trim steels, then there is a reasonable expectation that the trim edge durability issues could be addressed. Furthermore, the lack of true long-term durability data on the trim steels in $\mathrm{EH}$ trimming prevented us from developing a viable cost model for the $\mathrm{EH}$ trimming process. 


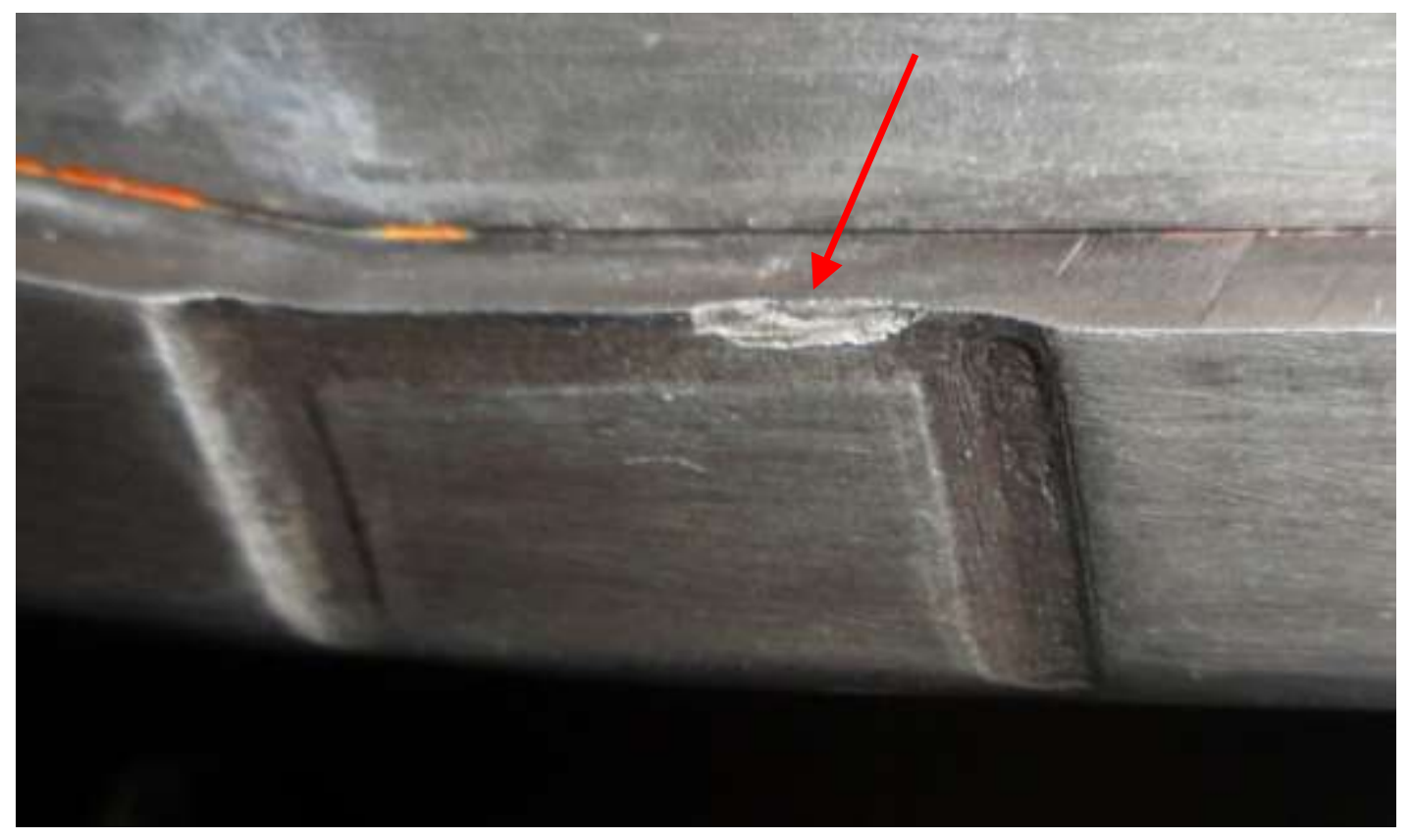

Figure 6.1.17. A small area of chipping on the sharp trim edge in the large $\mathrm{EH}$ trim tool.

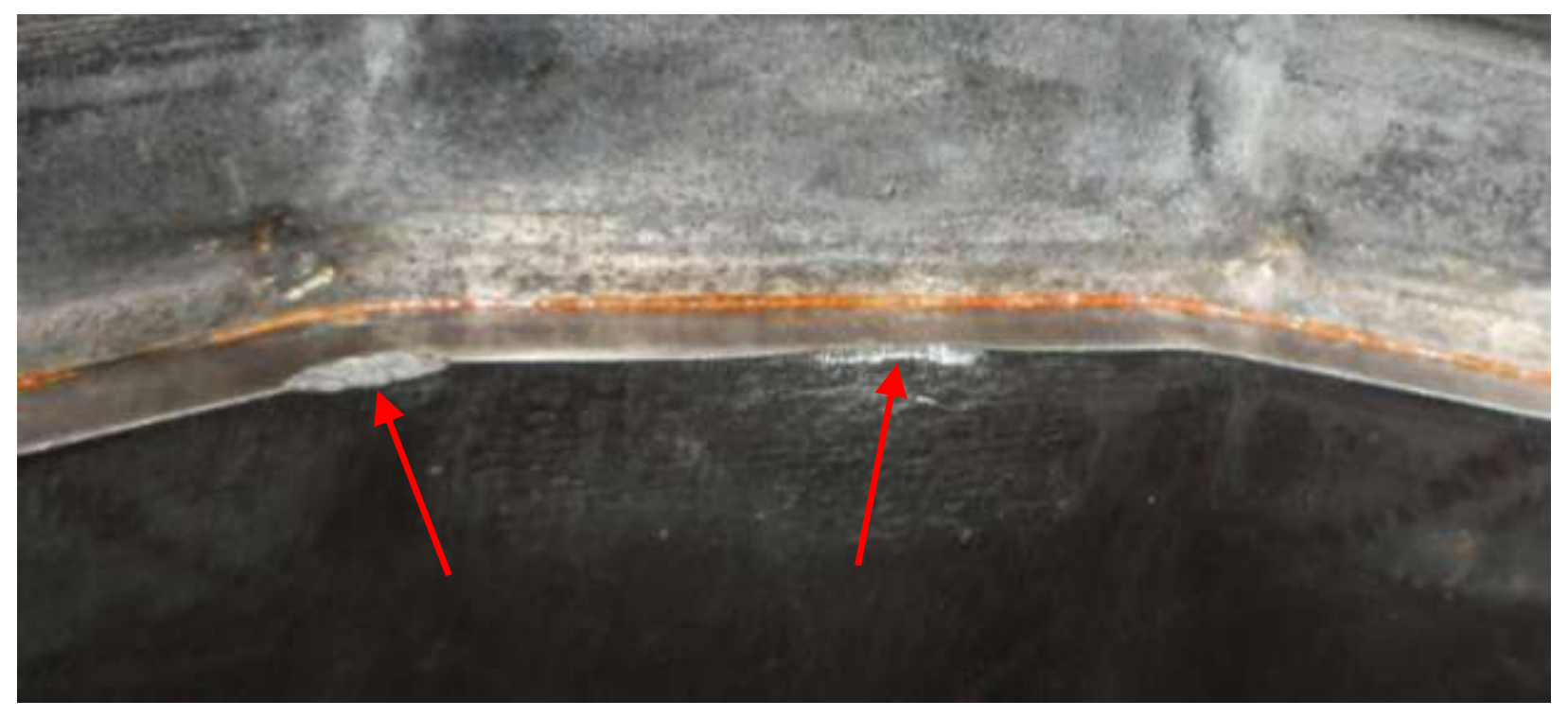

Figure 6.1.18. Two small areas of chipping on the sharp trim edge in the large EH trim tool. 
Electrohydraulic calibration (EHC) of springback is a novel method of removing springback from stamped sheet metal panels and is based upon the electro-hydraulic effect: a complex phenomenon related to the discharge of high voltage electrical current through a liquid. The EHC process involves clamping a stamped panel against a female die with the desired part shape and then applying several pulses pressure onto and through the thickness of the sheet, in a process somewhat similar to conventional coining operations. However, in EHC the pressure is applied by a fluid and through the use of the electrohydraulic effect, and not with a matching hard tool as done in coining. In this report, experimental results on small, flat coupons as well as large, complex parts will be presented.

The first experiments were simple in nature, and involved starting with flat coupons of dual phase steel. In order to highlight the effectiveness and the capabilities of the EH calibration process, the initial flat samples were made of the dual phase steel DP980, which represents the highest strength dual phase steel being used in industry today. The initial strips were fabricated from $1 \mathrm{~mm}$ and $1.4 \mathrm{~mm}$ thick DP980 sheet, and were $304.8 \mathrm{~mm}$ (12 inches) long and $50.8 \mathrm{~mm}$ (2 inches) wide. The experiment was conducted in three steps. Firstly, the sample was bent on a sheet metal brake where the sheet was clamped against tool with a radius of either $25.4 \mathrm{~mm}$ (1") or $12.7 \mathrm{~mm}$ (1/2"), and then bent to 90 degrees, as shown in Figure 6.2.1.

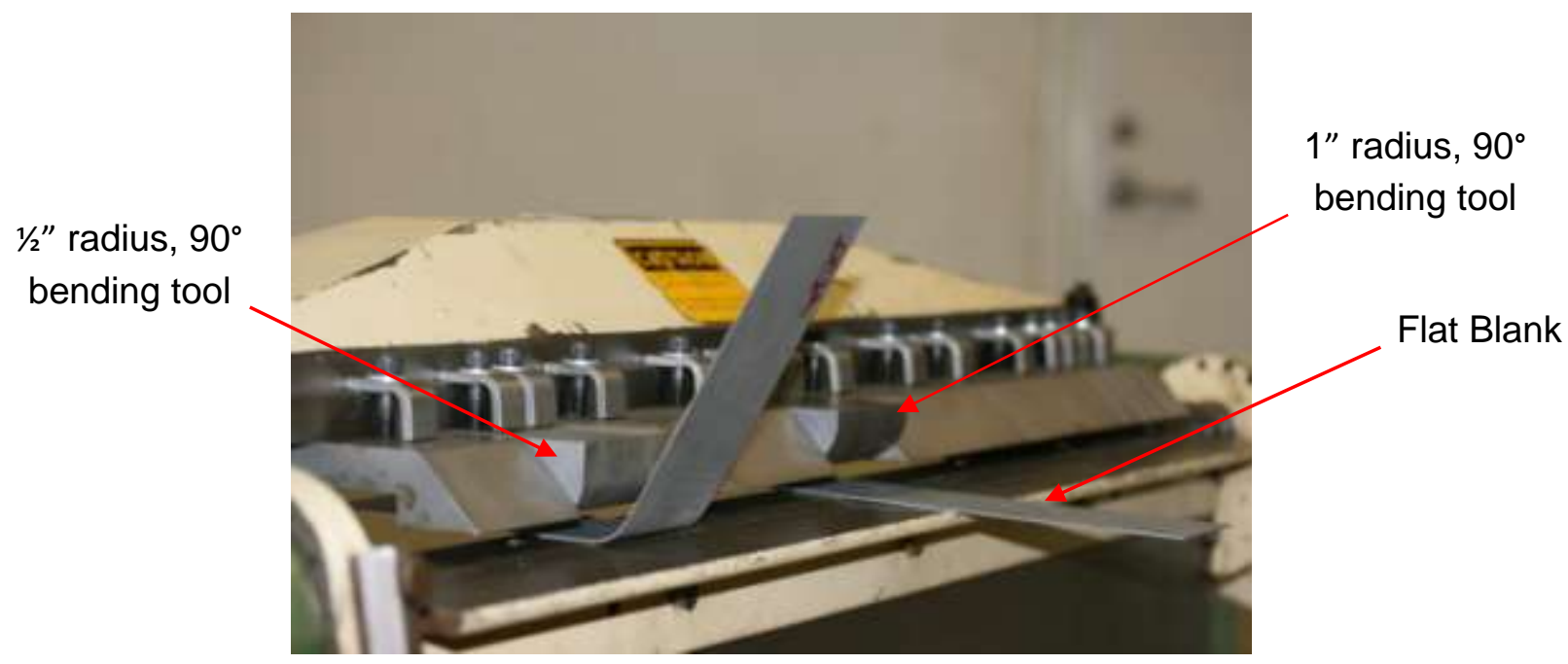

Figure 6.2.1. Bending of a flat strip on a sheet metal brake where the sample is clamped against a tool with a 1 " or $1 / 2$ " radius, bent to $90^{\circ}$, then released from the tool and allowed to springback.

Secondly, the strip was compressed between two flat steel plates using a $1.5 \mathrm{MN}$ hydraulic press. The difference between manual flattening with flat plate and flattening on a press was rather small. Nevertheless, press flattening was selected to ensure repeatability of the process. 
As expected, the press flattening operation did remove some of the springback, but not all, and the effect of the press flattening step is shown in Figure 6.2.2. Thirdly, the strip was then placed on the EH chamber shown in Figure 6.2.3 with the convex surface facing downward, toward the water-filled cavity in the chamber. The strip was then clamped flat between the $\mathrm{EH}$ chamber and a flat steel plate which in this case was acting as the calibration die. Also, as seen in Figure 6.2.3, an aluminum bridge wire was installed across the two electrodes before each pulse, in order to extend the length of the discharge channel beyond the capabilities of the electric breakdown length in the water. The length of the wire was $100 \mathrm{~mm}$, enabling more uniform pressure distribution along the surface of the blank compared to a typical breakdown distance of $10 \mathrm{~mm}$. The chamber was connected to a pulse generator with a capacitance of $320 \mu \mathrm{F}$, a maximum stored energy of $100 \mathrm{~kJ}$ and an internal inductance of $20 \mathrm{nH}$ (according to the pulse generator specifications). A discharge at $7 \mathrm{kV}$ and $7.8 \mathrm{~kJ}$ was found to be the most effective for removing springback from the DP980 sheets of both $1.0 \mathrm{~mm}$ and $1.4 \mathrm{~mm}$ thickness. After the EH calibration pulse was applied, the springback was eliminated, and the strip was flat. A comparison of an EH calibrated strip to a non-calibrated strip is shown in Figure 6.2.4.

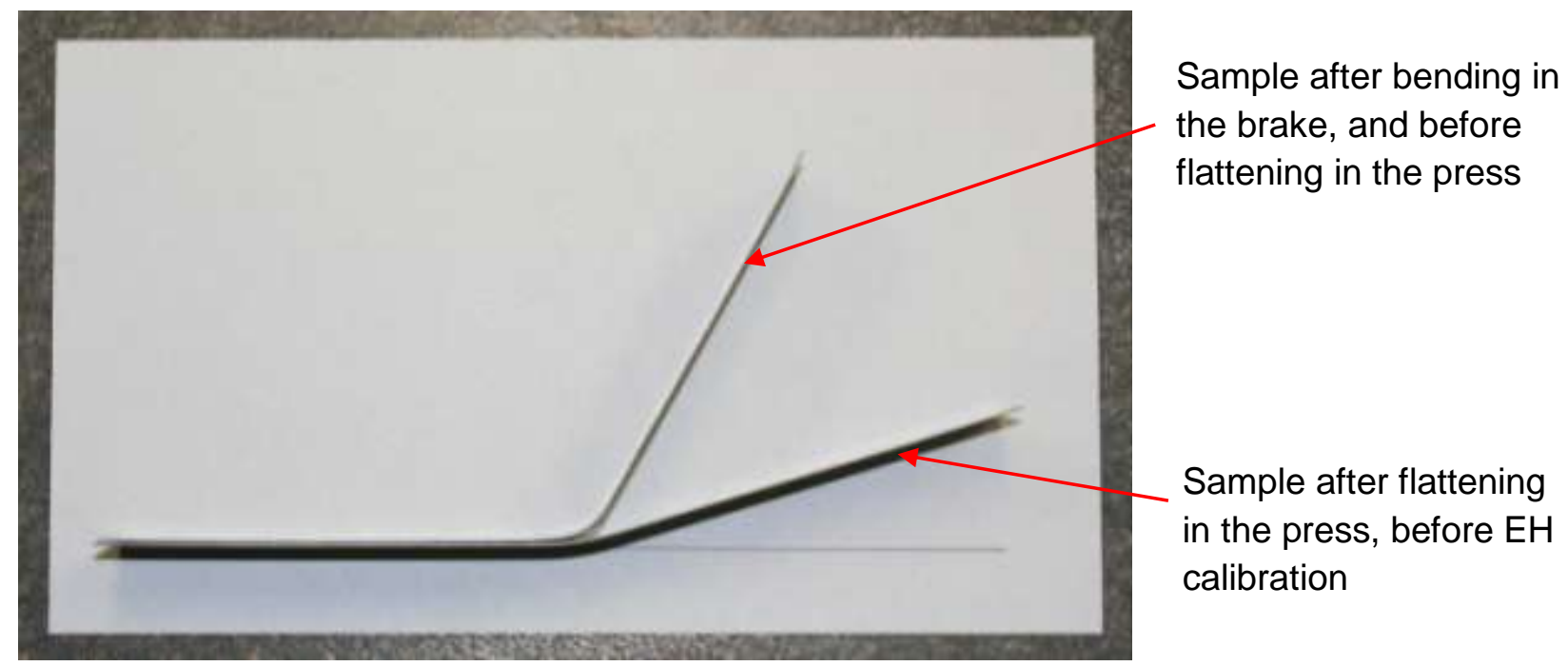

Figure 6.2.2. Comparison of DP980 samples before and after flattening in a $1.5 \mathrm{MN}$ hydraulic clamping press. As seen in the Figure, the hydraulic press was not able to remove all of the springback from the strip. 


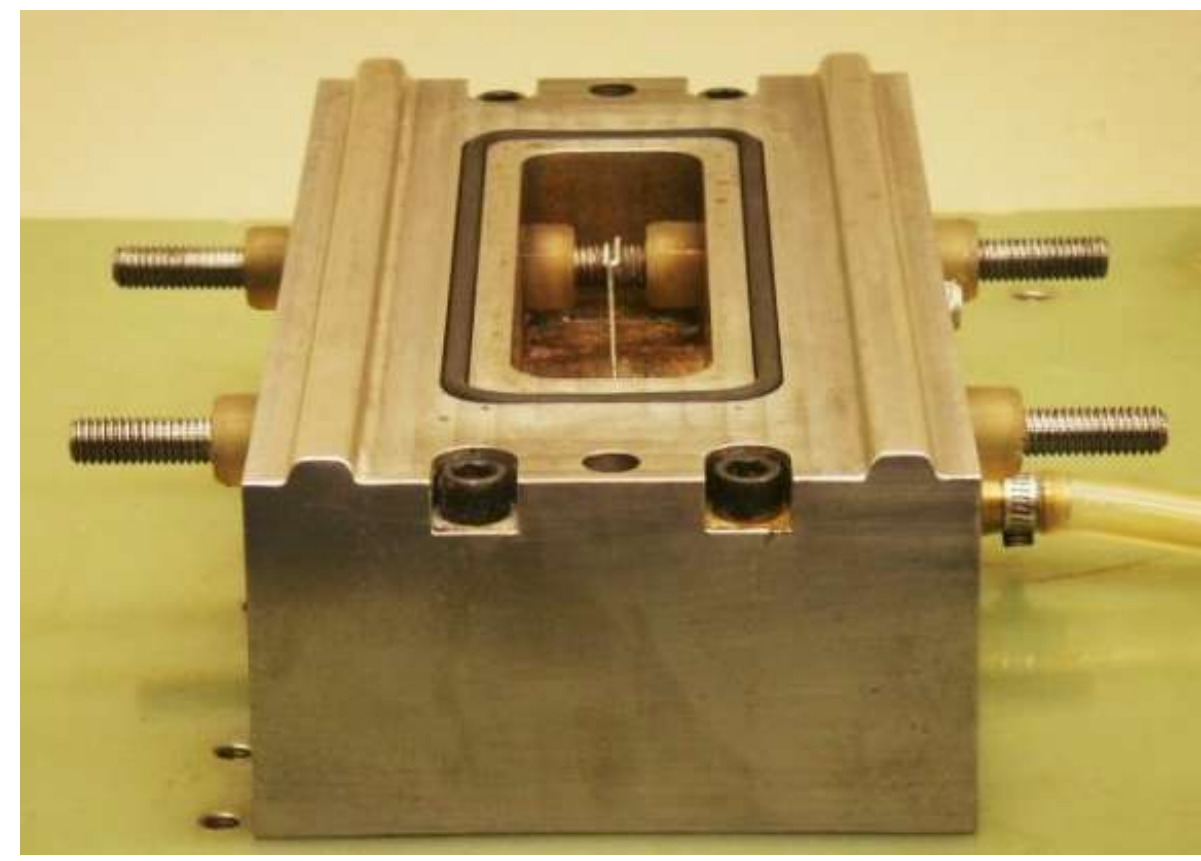

Figure 6.2.3. EH calibration chamber with electrodes connected by an aluminum bridge wire.

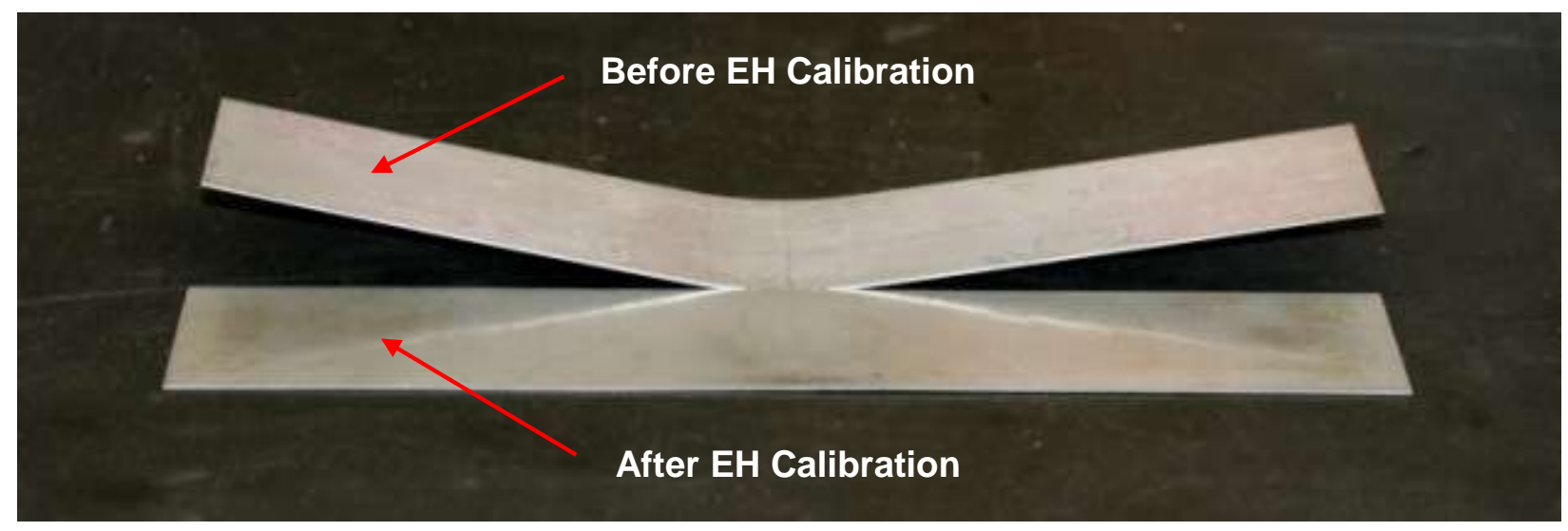

Figure 6.2.4. Comparison of a strip of DP980, $1 \mathrm{~mm}$ thick, bent over a 1 " radius and then flattened by a $1.5 \mathrm{MN}$ clamping press, to a strip then calibrated by one $\mathrm{EH}$ pulse at $7 \mathrm{kV}$ and 7.8 kJ.

Quantitative experimental results are presented in Figures 6.2.5 through 6.2.8, where the springback angle was measured for DP980 strips of both $1 \mathrm{~mm}$ and $1.4 \mathrm{~mm}$ thickness as a function of discharge energy and bending radius. Based on the experimental results, the following observations can be made. Lower thicknesses and larger bending radii result in a higher springback angle for a given blank geometry, after the bending and flattening steps described above. This effect can be seen by comparing the data points at the $0 \mathrm{~kJ}$ energy level 
in Figures 6.2.5 through 6.2.8, or in other words, the data points corresponding to initial bending and press flattening but without any $\mathrm{EH}$ calibration. For the data points corresponding to $\mathrm{EH}$ calibration, the general trend is that the springback is getting lower with the increase of the discharge energy. It was observed that most of the springback was eliminated using a relatively low discharge energy of $4 \mathrm{~kJ}$. For all combinations of bending radii and sheet thickness studied in this experimental work, a $7.8 \mathrm{~kJ}$ discharge produced a rather robust result of having springback very close to zero degrees. In a few cases the effect of "springforward" was observed, which can be thought of as the introduction of new residual stresses into the surface layer of the sheet, in a manner similar to shot peening. However, the magnitude of this distortion was rather minimal compared to the initial springback value. This effect can be minimized by limiting the amount of the discharge energy.

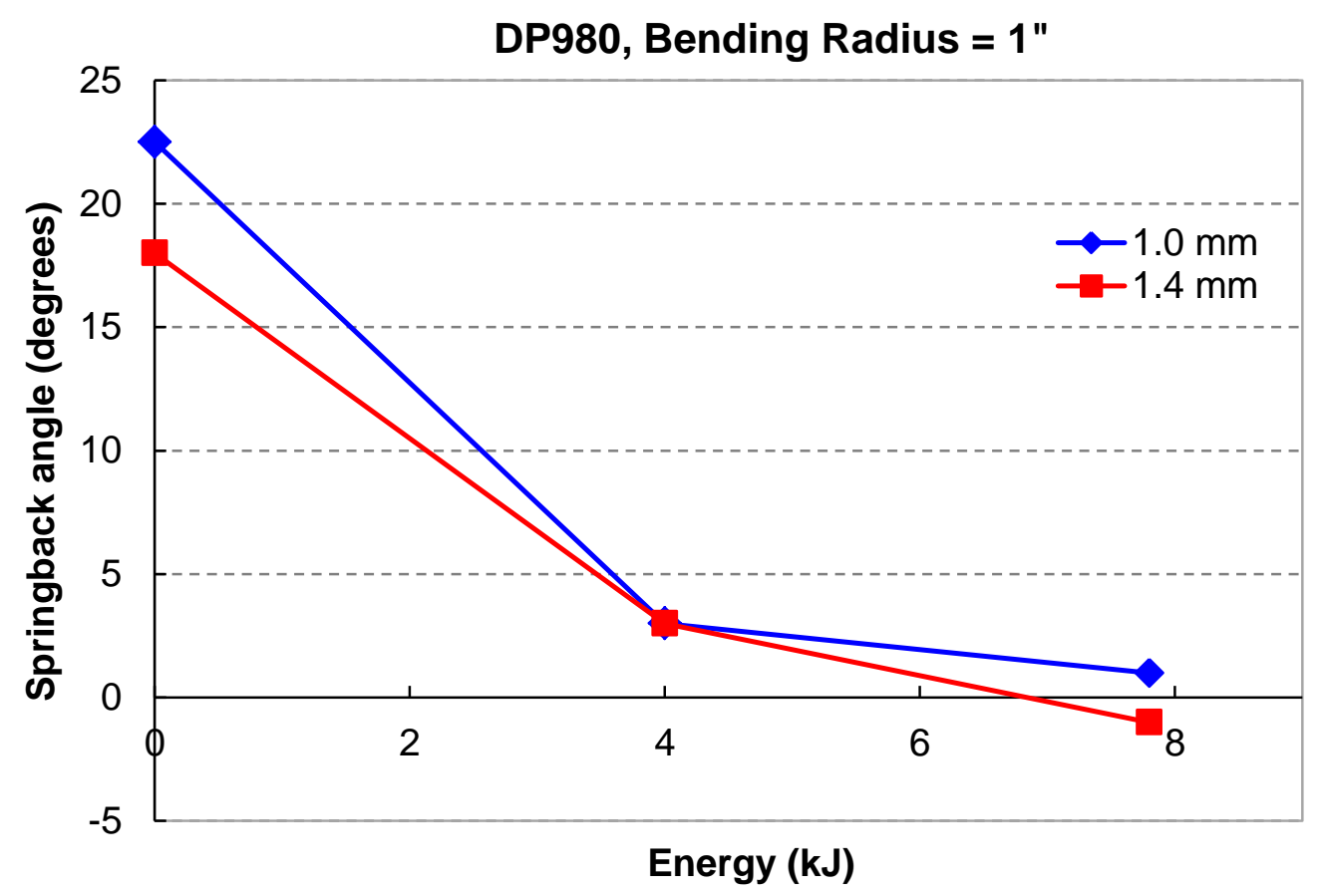

Figure 6.2.5. Experimental results of EH calibration of DP980 strips, in terms of springback angle as a function of discharge energy, using an initial bending radius of 1 ". 


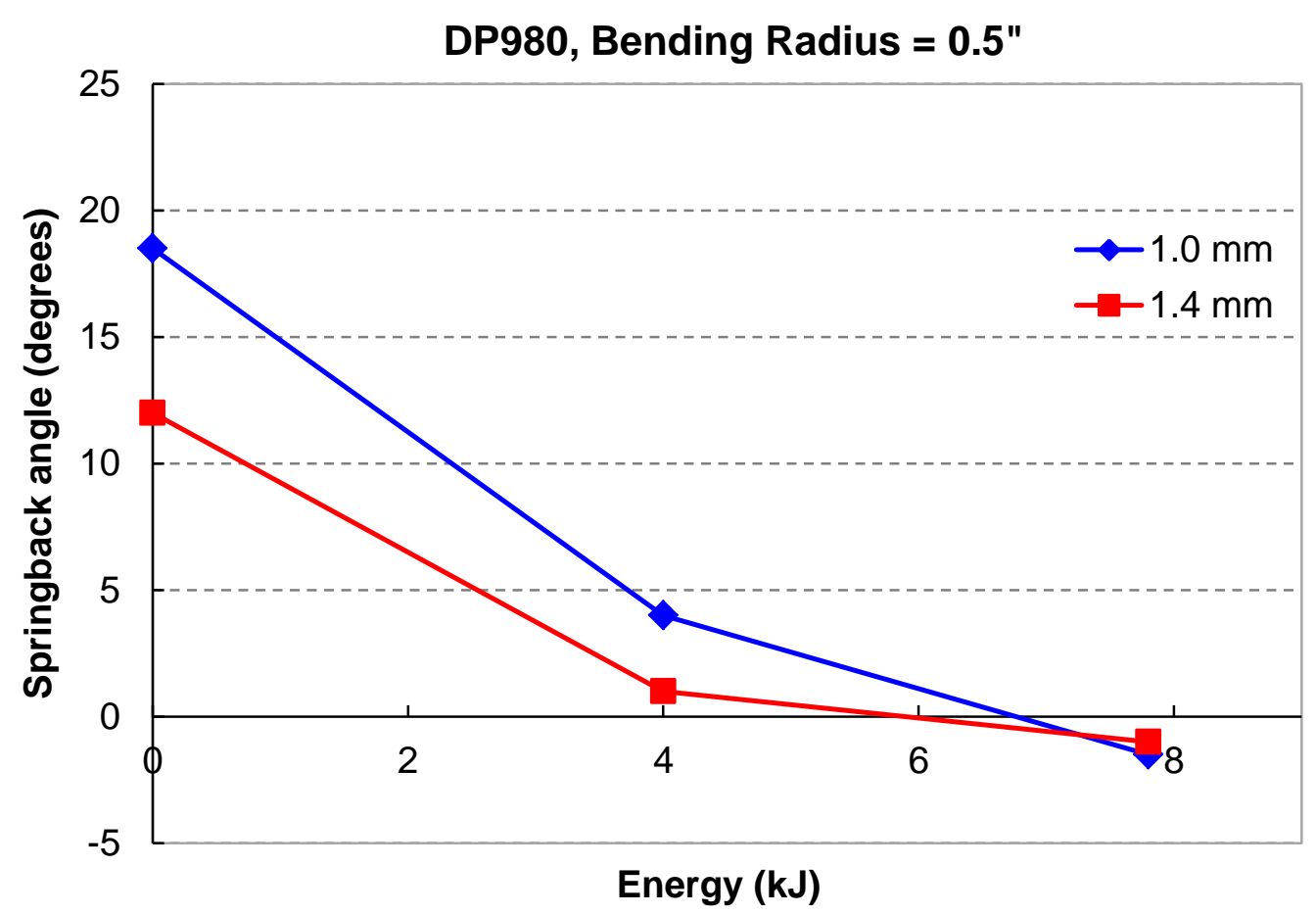

Figure 6.2.6. Experimental results of EH calibration of DP980 strips, in terms of springback angle as a function of discharge energy, using an initial bending radius of 0.5 ".

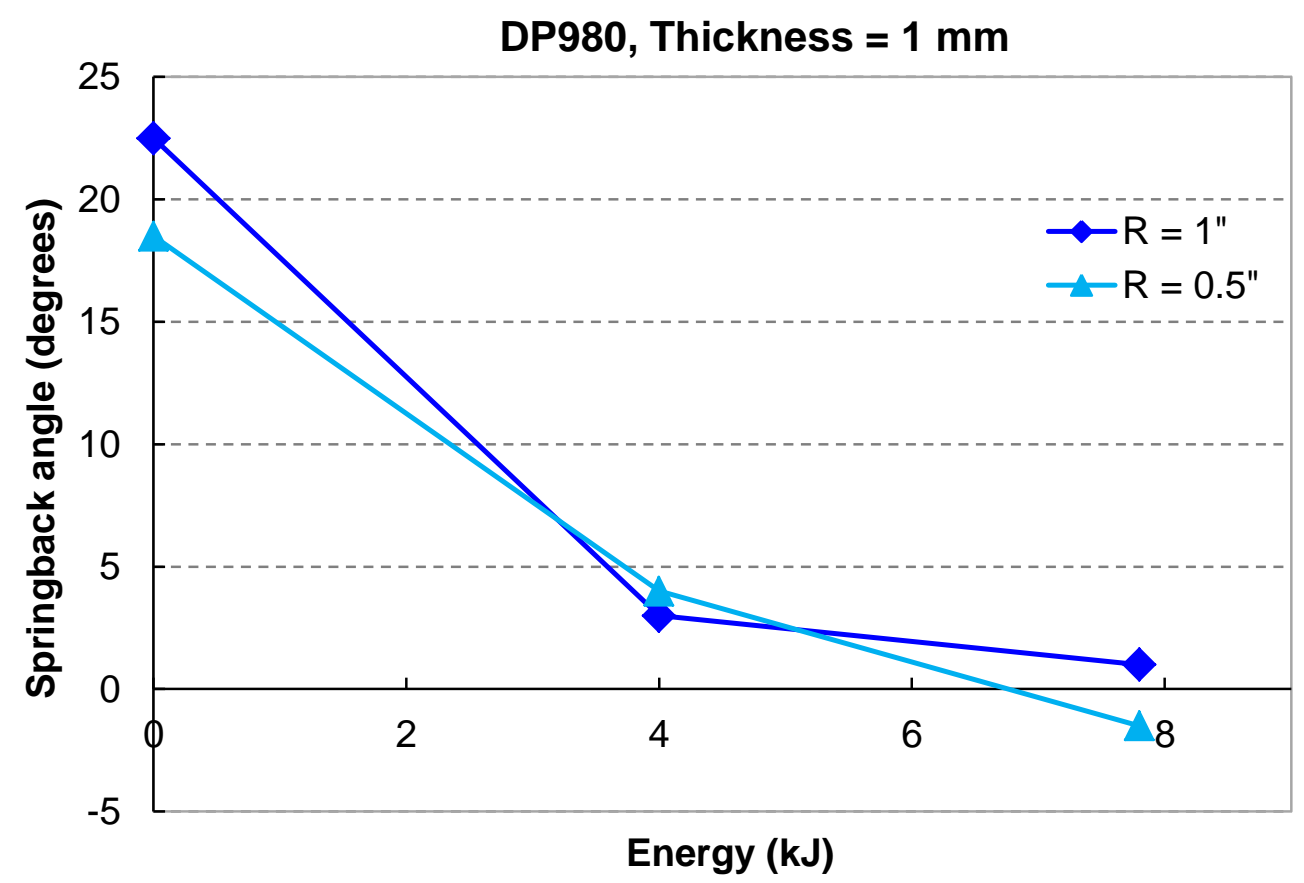

Figure 6.2.7. Experimental results of EH calibration of DP980 strips of $1 \mathrm{~mm}$ thickness, in terms of springback angle as a function of discharge energy. 


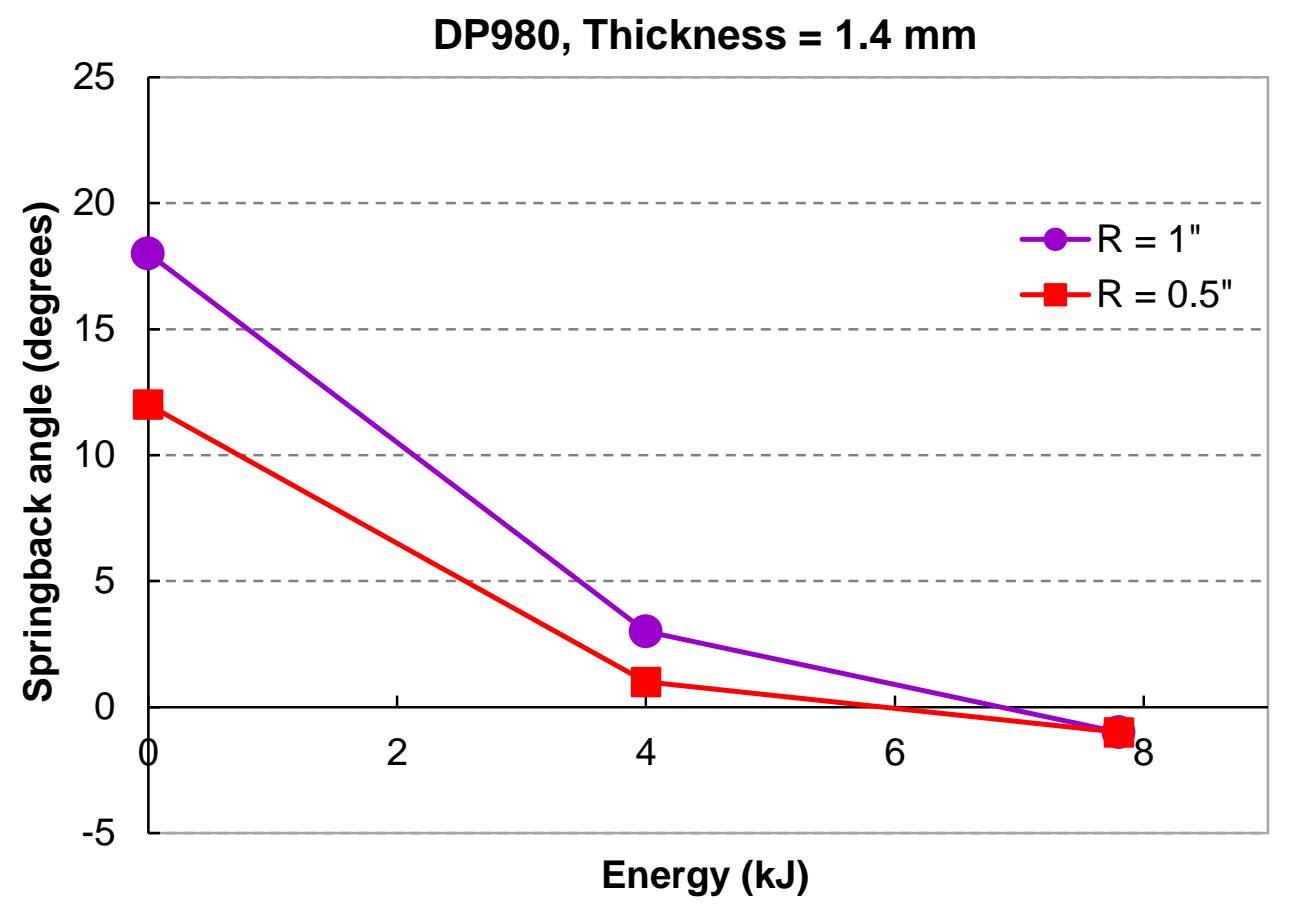

Figure 6.2.8. Experimental results of EH calibration of DP980 strips of $1.4 \mathrm{~mm}$ thickness, in terms of springback angle as a function of discharge energy.

Further validation of the $\mathrm{EH}$ stress relieving technology required demonstrating the process on a real automotive panel with complex springback modes. After reviewing a number of possible applications, a current production panel serving as a part of front rail structure was selected. The decision was made based upon the knowledge of the prior history of this particular panel, which required several tooling recuts to achieve the required dimensional accuracy in the formed panels. Furthermore, this part was also selected based on the suggestions of other colleagues in Ford Research, because it was expected to have a significant amount of springback away from the desired shape after it is stamped, and therefore would be a good candidate for $\mathrm{EH}$-calibration. The decision was made to fabricate a dedicated draw die to form the selected panel at $50 \%$ scale, and then to conduct EH trimming of the drawn panels followed by $\mathrm{EH}$ calibration of the trimmed panels. The selected panel was formed out of $1 \mathrm{~mm}$ thick DP590, a dual phase steel.

The chamber which was used in the $\mathrm{EH}$ calibration process was designed based upon the methodology of numerical simulation described in the earlier sections of this report. Since the blank is clamped to the die surface in EH stress relieving calibration, the displacements of the blank and the elasticity of the die surface were neglected in our simulation of the $\mathrm{EH}$ calibration process. Therefore, the $\mathrm{EH}$ discharge was simulated as occurring in a closed volume with no displacement of any fluid or sheet metal. Based on the assumption that no major distortion of the mesh occurs in a closed volume, a Lagrangian formulation of the problem was considered more appropriate. In order to create a numerical model of the $\mathrm{EH}$ calibration process, the 
following models were developed and later on coupled: (1) an electrical model of the discharge channel; (2) a model of the plasma channel; and (3) a model of the liquid as a pressure transmitting medium. Models 2 and 3 were developed using the capabilities of the LS-DYNA commercial code. Model 1 was developed based on basic electrical circuit analysis as well as experimental measurements of current waveforms. The key difference in this modeling effort was the design of the EH chamber being modeled, which consisted of multiple sub-chambers as part of one overall chamber. The objective of the modeling effort was to optimize the geometry of the sub-chambers so as to maximize the pressure pulse that reaches the surface of the sheet metal panel. In order to minimize the time necessary for model construction and computation, the initial discharge was simulated as a small diameter sphere positioned between two electrodes.

Since the selected part represented a channel of variable cross-section where springback occurs in every cross-section, it was considered necessary to apply stress relieving pressure over the entire surface of the trimmed panel. Since there is no analytical model available which would predict the stress relieving effect based on applied pressure parameters, the design of the chamber was developed through relying on the concept that the distribution of stressrelieving pressure can be adjusted during the experimental tryout if the chamber has multiple electrodes which can apply pressure to different areas of the blank independently. Initially, the multi-electrode approach in a single fluid cavity was given a preference in order to have a smooth distribution of pulsed pressure across the whole surface of the blank. The design of the chamber corresponding to this single-cavity concept is shown in Figure 6.2.9a.

A number of other chamber designs were developed based upon the concept of subdividing the single fluid cavity into several sub-chambers separated by internal walls. Based on prior experience with electrohydraulic processes this approach was pursued, because it was expected to produce a higher pressure amplitude on the blank surface as a result of the concentrating effect of the dividing walls. After multiple iterations of design and simulation, the design of the chamber shown in Figure 6.2.9b was selected. The new design had three subchambers, an overall reduction in height, and a more smooth shape in comparison with the single-cavity configuration of the initial design. 


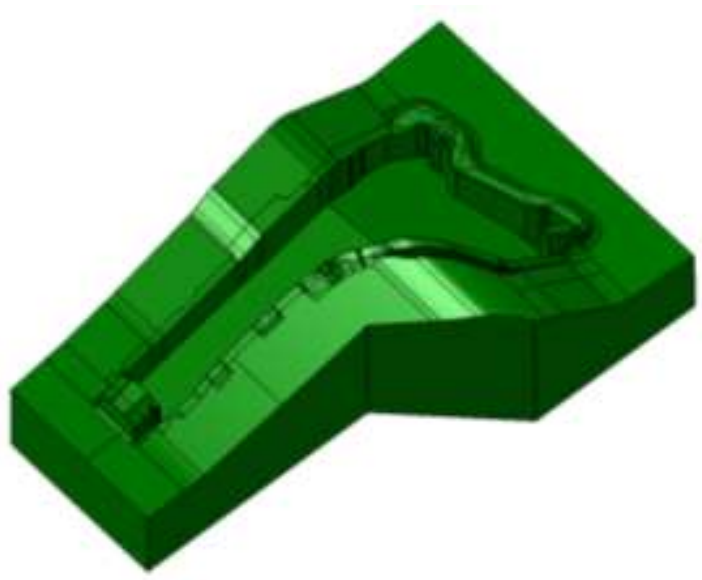

(a)

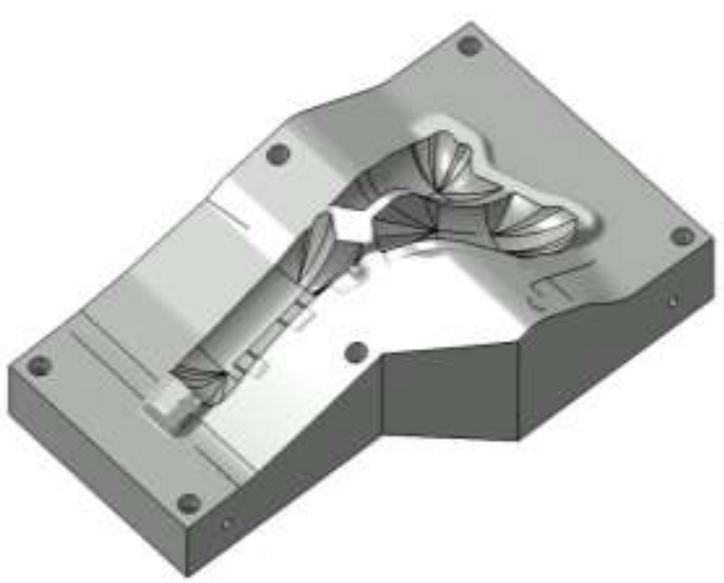

(b)

Figure 6.2.9. Geometry of the chamber for the single-cavity chamber concept (a) and for the multiple sub-chamber concept (b).

A comparison of the two configurations was made based upon the magnitude of the pressure pulse reaching the blank surface. Four points of observation, $A, B, C$, and $D$ were chosen on the surface of the blank as shown in Figure 6.2.10, and pressure values were compared for both chamber designs throughout the whole calibration process. The simulations for both configurations were conducted at the same level of discharge energy. The results of the simulation in terms of the pressure history at points $A, B, C$, and $D$ are shown in Figure 6.2.11 for the single-cavity chamber design (see Figure 6.2.9a for geometry), and are shown in Figure 6.2.12 for the multi-cavity chamber design (see Figure 6.2.9b for geometry).

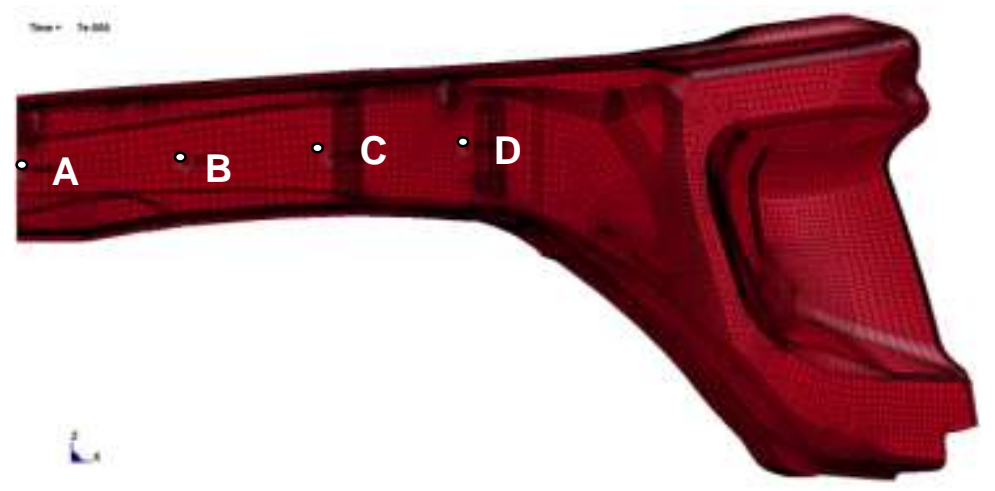

Figure 6.2.10. Points of observation on the surface of the blank. 


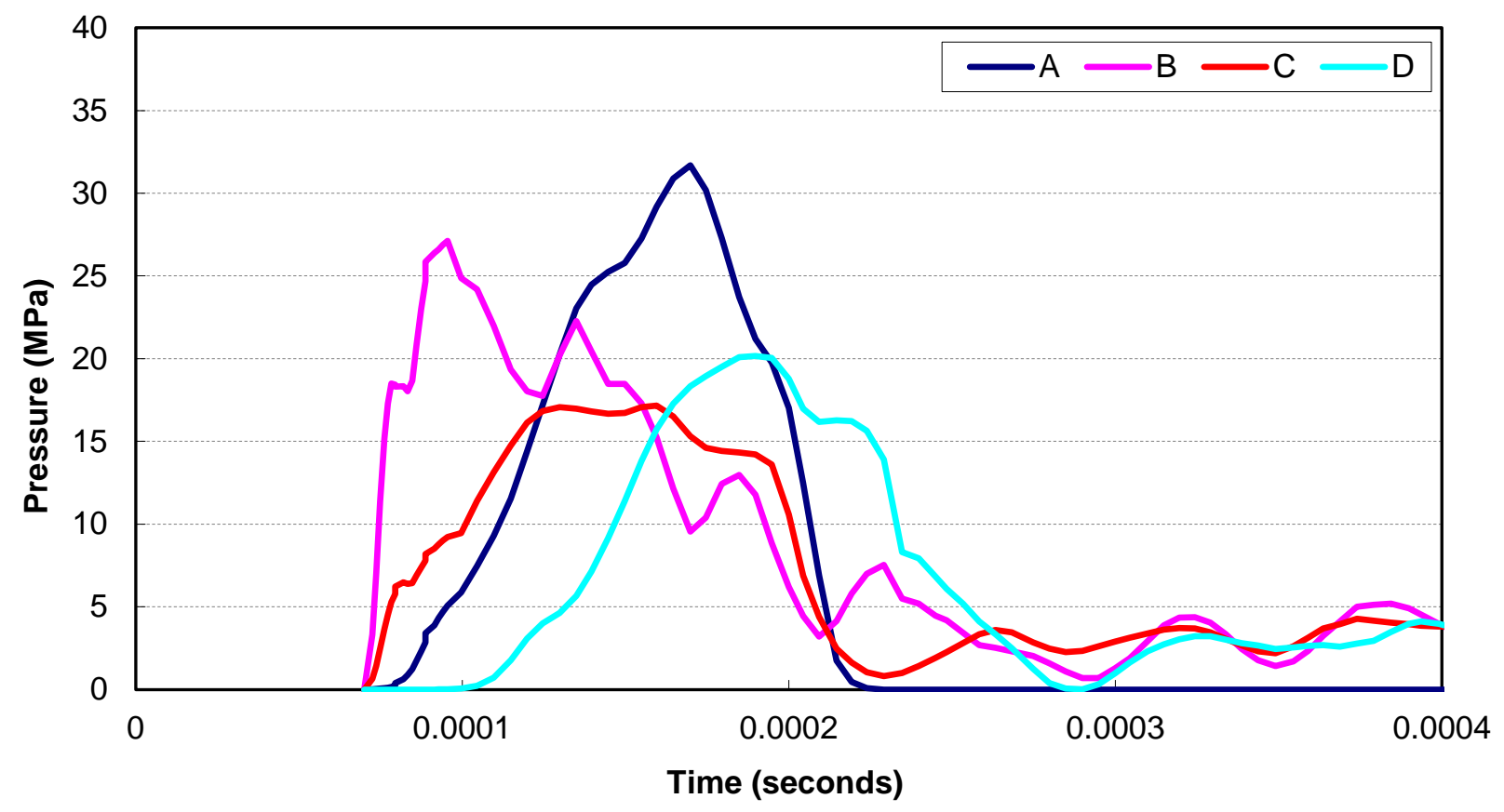

Figure 6.2.11. Pressure history after the discharge in the single-cavity EH calibration chamber.

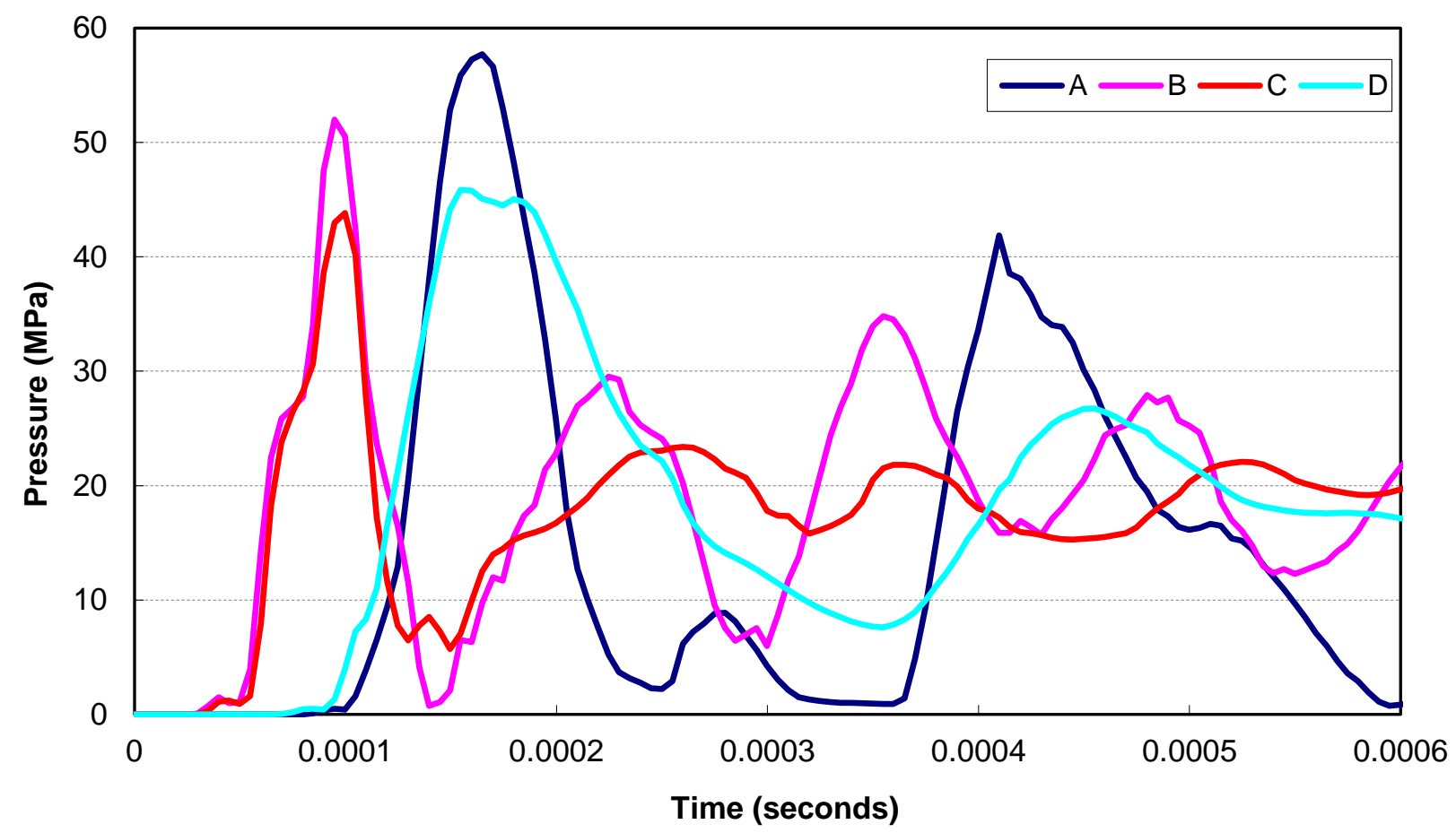

Figure 6.2.12. Pressure history during the $\mathrm{EH}$ discharge in the multi chamber design. 
A comparison of the two graphs clearly shows the difference in pressure. With the same total energy, the multi-chamber design produces much higher pressure peaks, which leads to the conclusion that less energy would be required in this chamber in order to produce the same calibration effect. Another added benefit of the sub-chamber concept in the multi chamber design is the presence of multiple pressure peaks, which occur due to reflection of the pressure waves off of the sub-chamber walls. Multiple pressure peaks also may reduce the number of discharges needed for calibration, and therefore have the potential to reduce the overall cycle time of the calibration process. The history of the pressure distribution on the surface of the blank within the first sub-chamber is shown in Figure 6.2.13. It can be observed that the pressure initially builds up in the area close to the electrode and then propagates toward the edges of the sub-chamber. The location of the electrode in each sub-chamber was determined based upon the goal of producing an optimized pressure pulse. Based on these considerations, the multi-chamber design shown in Figure 6.2.14 was chosen to be fabricated for further experimental study.

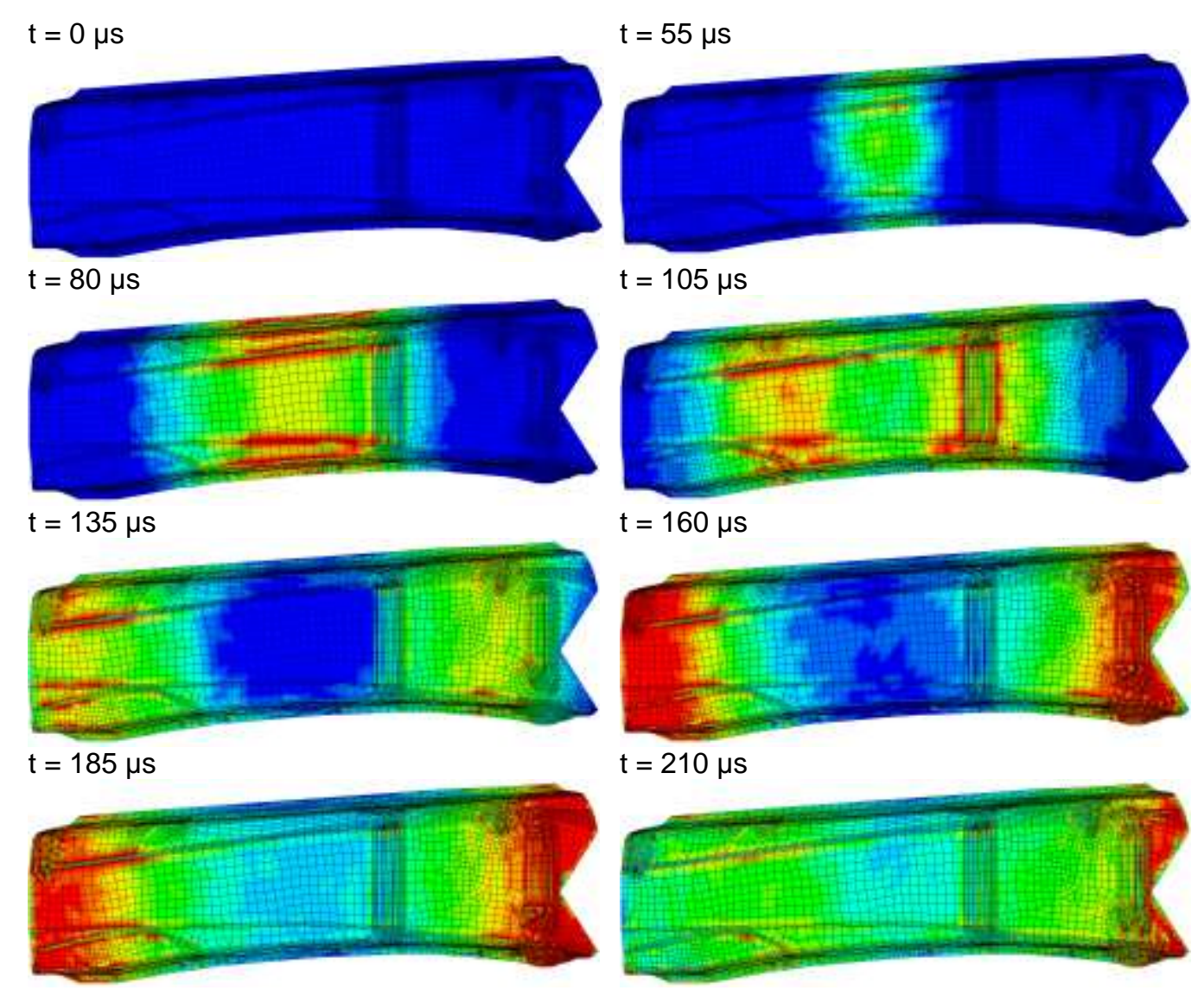

Figure.6.2.13. Propagation of pressure inside the first sub-chamber during the $\mathrm{EH}$ calibration process. 


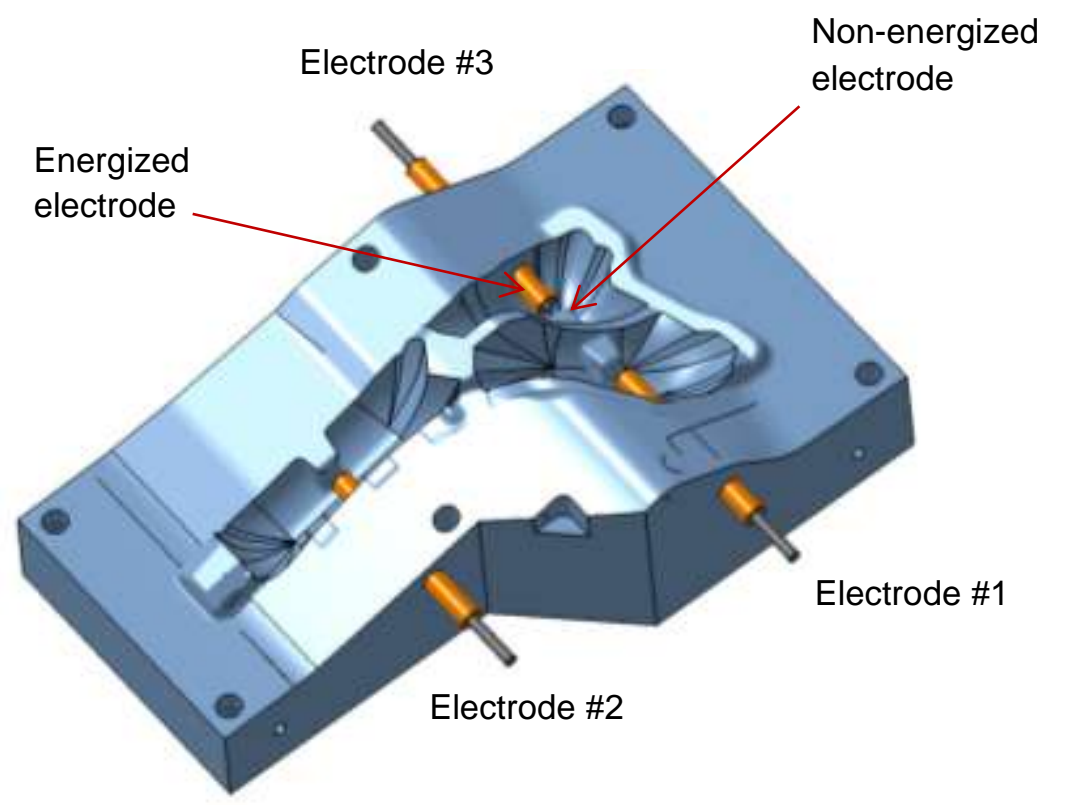

Figure 6.2.14. Optimized design of the chamber which was selected for fabrication.

Figures 6.2.15 through 6.2.17 show photographs of the finished EH calibration tool in a $1.5 \mathrm{MN}$ hydraulic press during the initial tryout process. Figure 6.2 .15 provides an overall view of the lower tool showing the three sub-chambers and the electrodes. Figure 6.2.16 provides a closeup view of the two sub-chambers near the back end of the part, showing the position of the energized electrodes and the ground (non-energized) electrodes. The polarity of the energized electrodes can be either positive or negative, and this process parameter is dictated by the internal design of the pulsed current generator. However, no matter whether the energized electrode is charged positive or negative, it is necessary that the energized electrode be disconnected from the ground by an insulation block of sufficient thickness and mechanical strength. The force applied to the energized electrode is directed along the axis of the electrode and away from the chamber, driven by the internal pulsed pressure during the discharge. The energized electrodes are mounted on steel brackets connected by bolts to a spacer block made out of high voltage insulation material. Figure 6.2.17 provides an overall view of the upper tool, which consists of a female die surface cut to the desired part geometry. 


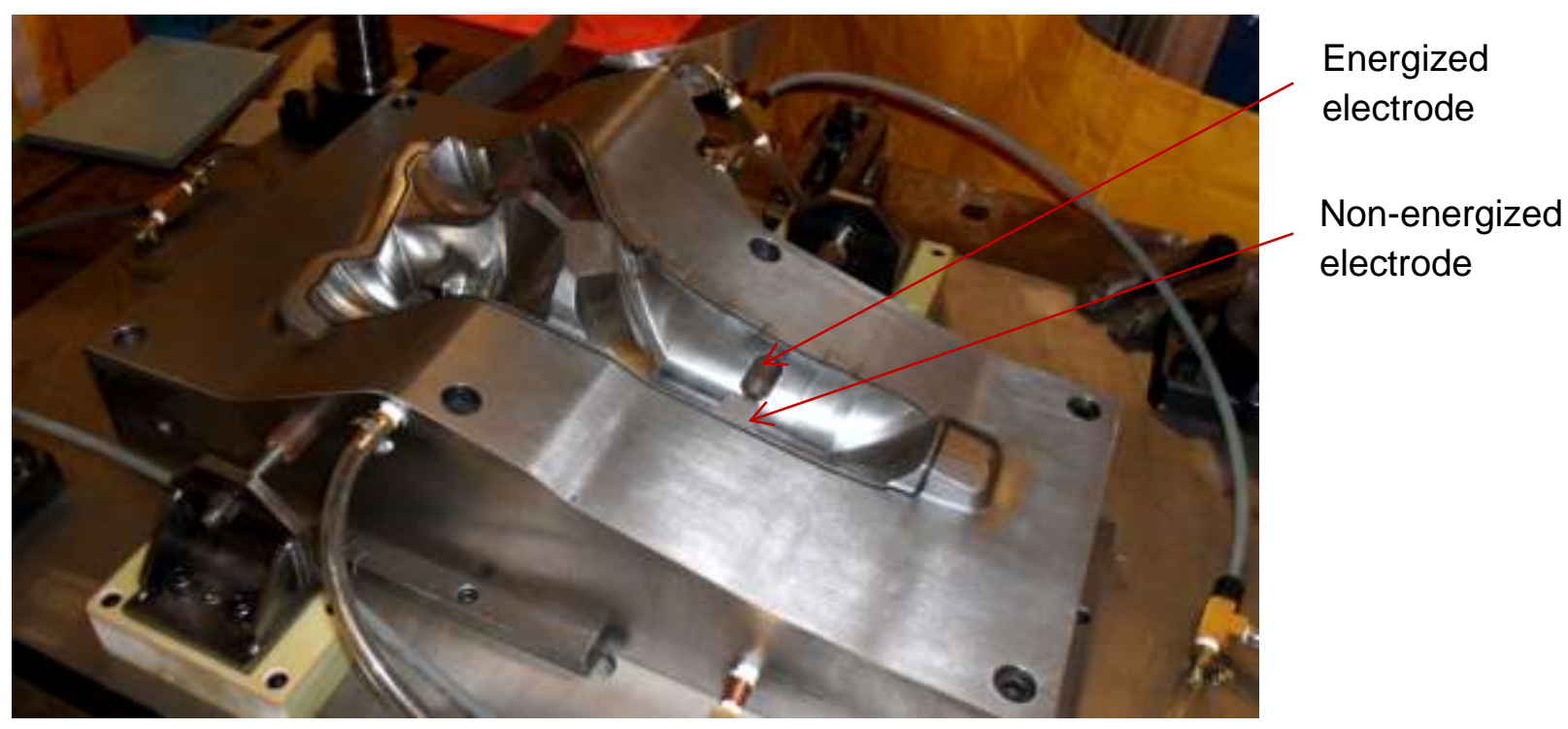

Figure 6.2.15. The lower half of the EH calibration tool, showing three sub-chambers and three electrodes.

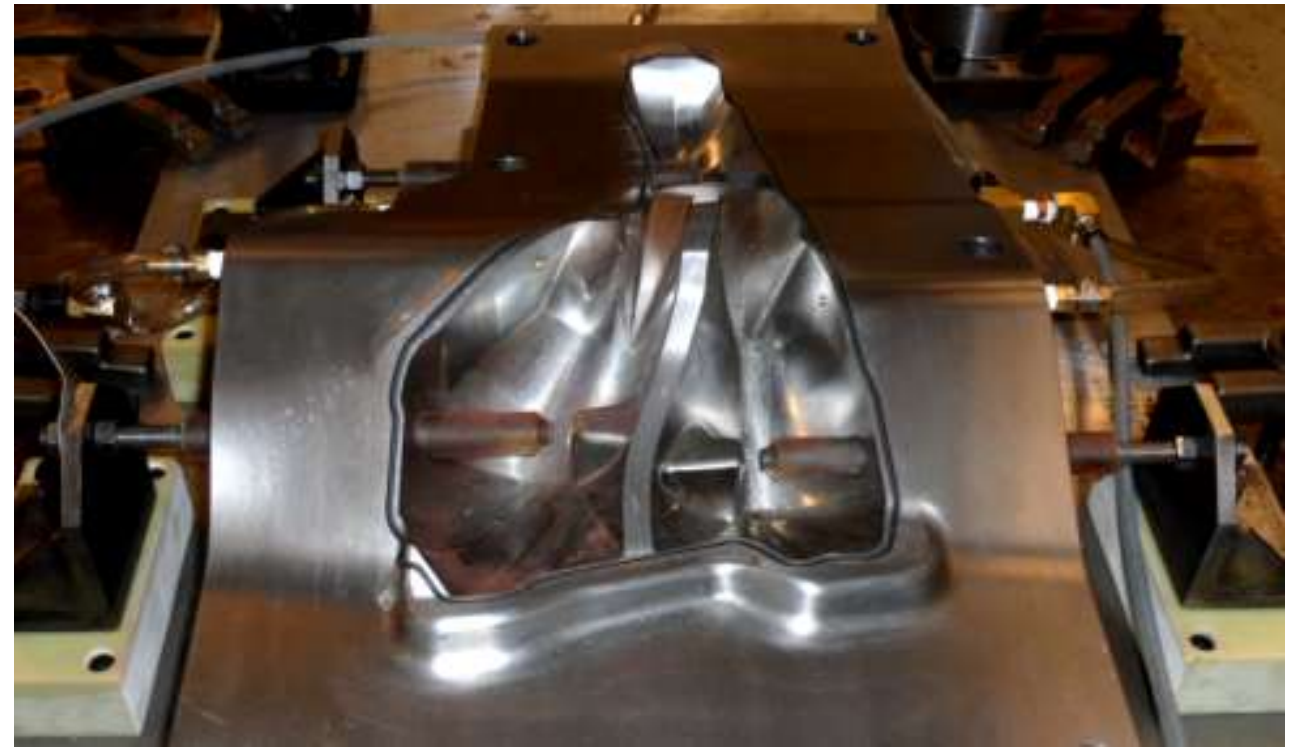

Figure 6.2.16. Close-up view of the two sub-chambers near the back end of the EH calibration tool, showing the position of the energized electrodes and the ground electrodes. 


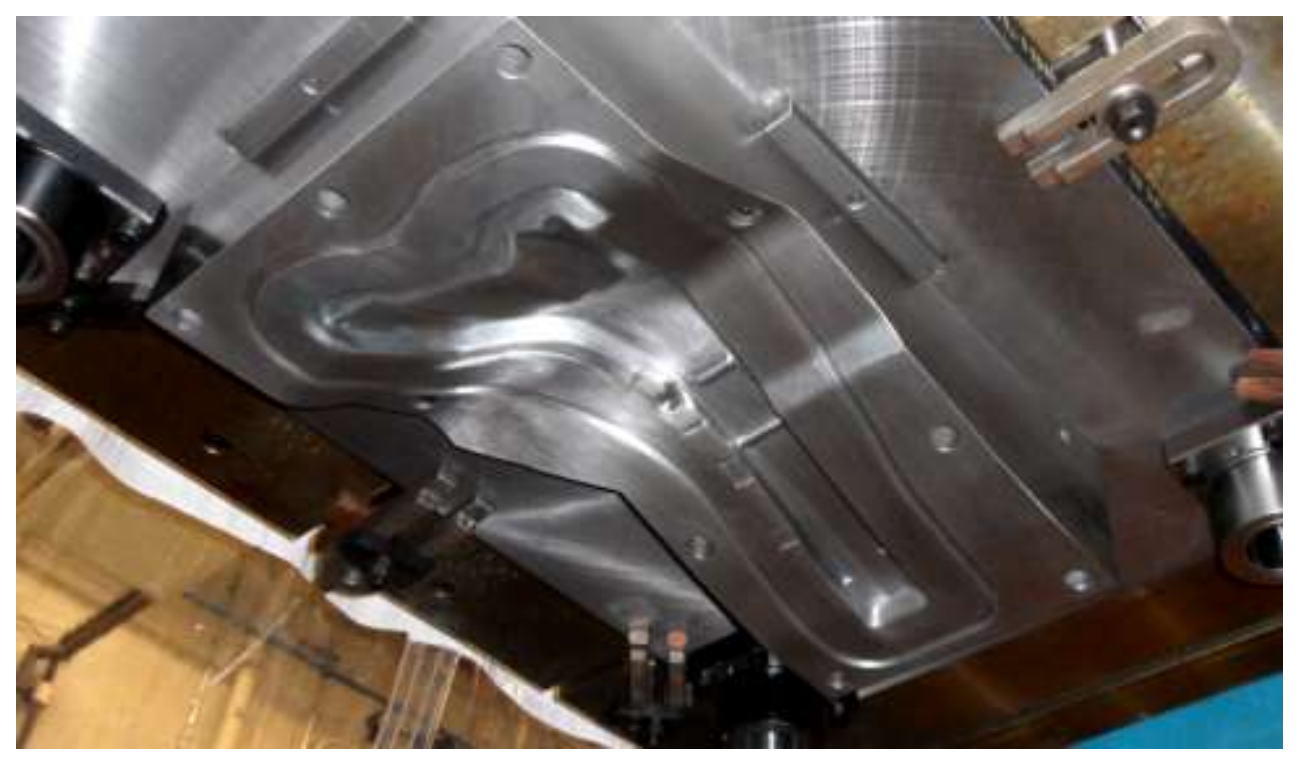

Figure 6.2.17. Overall view of the upper half of the EH calibration tool, which consists of a female die surface cut to the desired part geometry.

After a series of preliminary experiments, an appropriate sequence of pulses and pulse energies was defined which was able to remove virtually all of the springback from the experimental panels. The sequence of pulses consisted of one pulse in each sub-chamber, with each pulse done at $13 \mathrm{kV}$. This voltage level corresponds to an energy of $27 \mathrm{~kJ}$ when using a capacitor bank of $320 \mu \mathrm{F}$ capacitance. The sequence of calibration discharges was done in numerical order from electrode 1 to electrode 3, as shown in Figure 6.2.14. Figures 6.2.18 and 6.2.19 show a summary of the results of the calibration process in terms of removal of springback from the stamped panels. Figure 6.2.18a shows the panel resting on the lower tool before the calibration process was applied. The lower left flange can be seen deviating from the desired part geometry by a full $25 \mathrm{~mm}$, due to various modes of springback throughout the panel, such as bending modes and twisting modes. Figure 6.2.18b shows the panel again resting on the lower tool, but after the calibration process has been applied, and the panel is now virtually in complete contact with the lower tool. In Figure 6.2.18b, the deviation of the part from the desired geometry of the tool is less than $1 \mathrm{~mm}$ in all locations, which is an acceptable level of dimensional accuracy for interior automotive panels. Figure 6.2.19 shows the same EH calibration results as Figure 6.2.18, but from a different angle. 


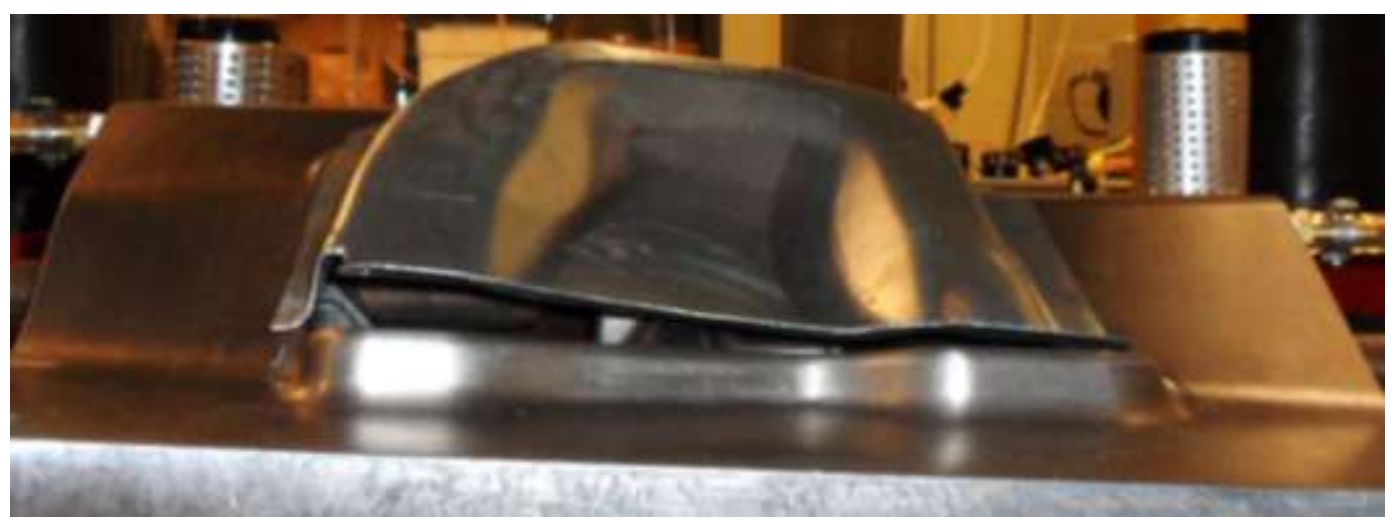

(a)

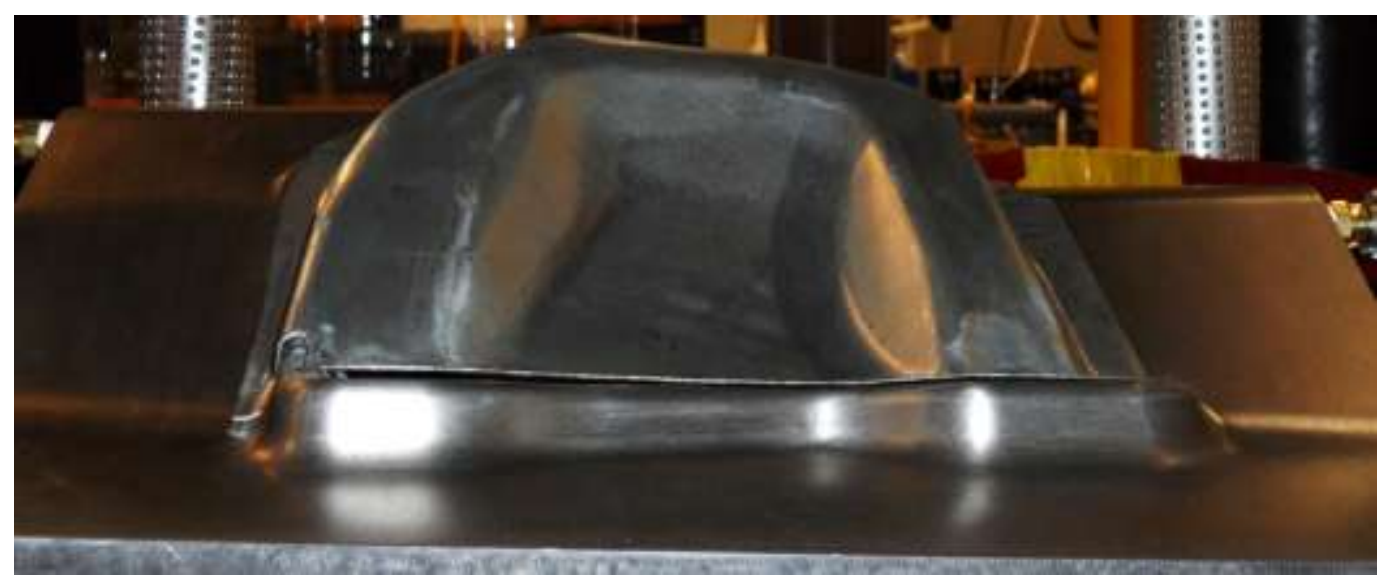

(b)

Figure 6.2.18. The stamped panel before (a) and after (b) the EH calibration process was applied. 


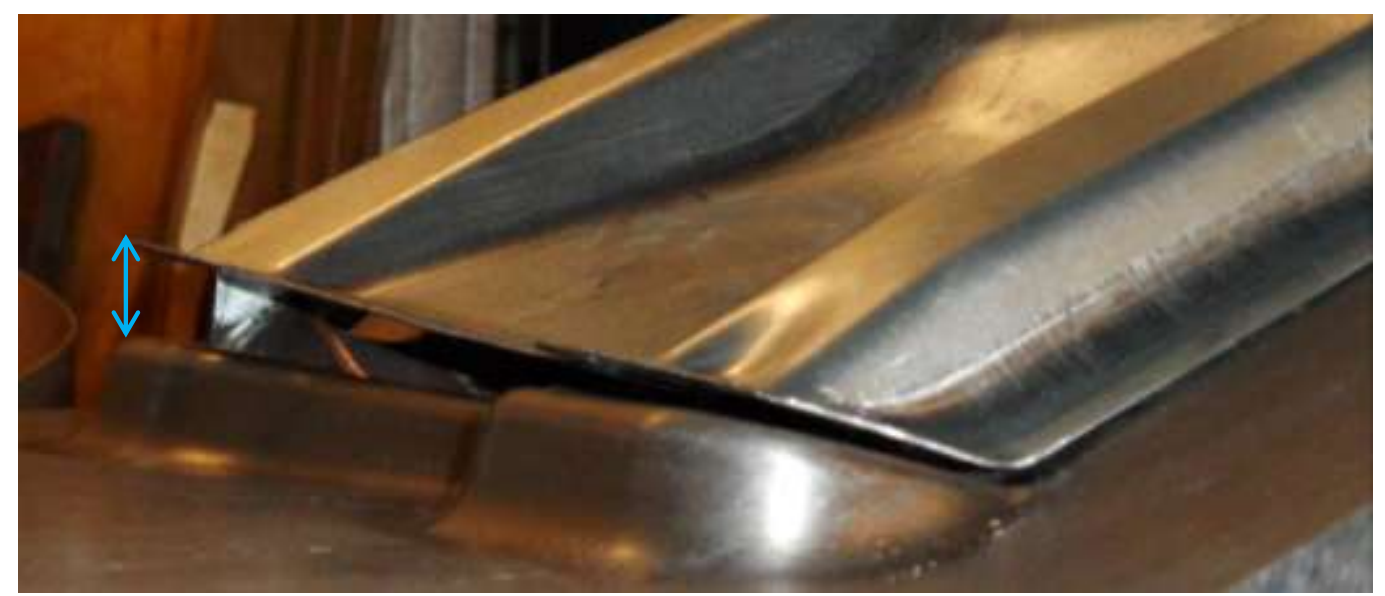

(a)

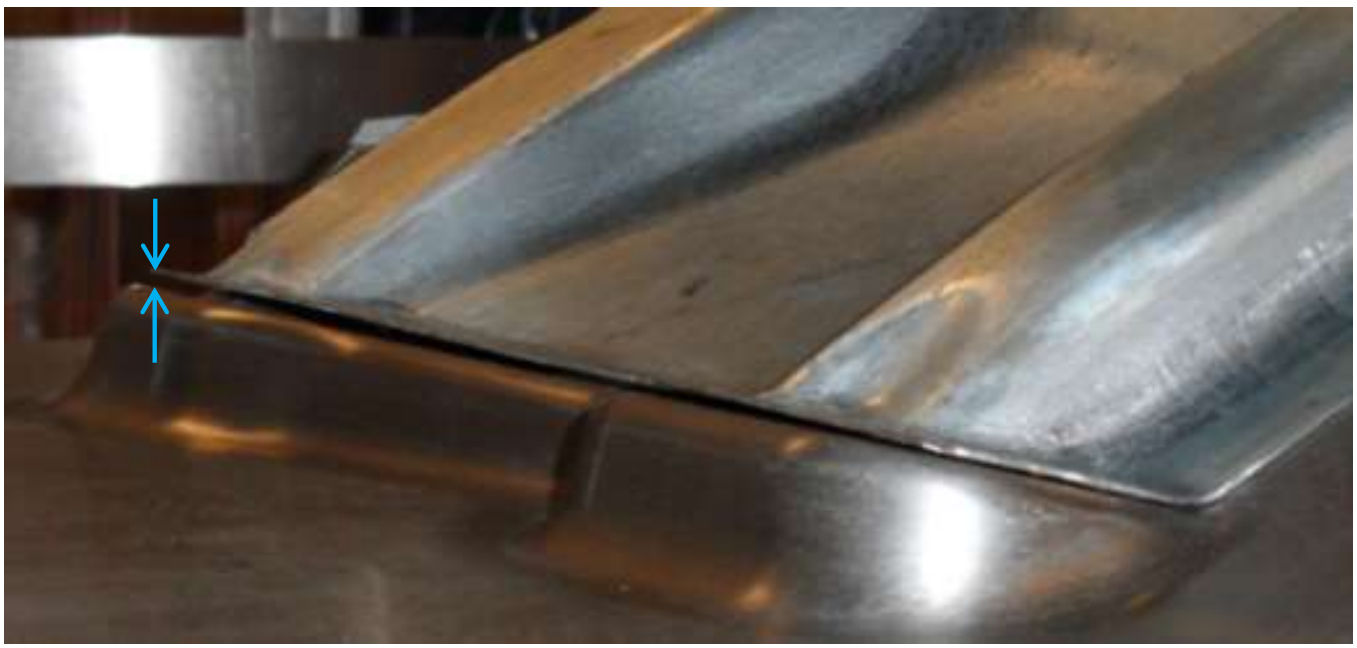

(b)

Figure 6.2.19. The stamped panel before (a) and after (b) the EH calibration process was applied. 
Task 7. Demonstration of EHF of a full scale automotive panel application

A dash panel was selected as the appropriate part for the experimental, full scale demonstration of the EHF process within Task 7 of the project. The selected panel was successfully formed in production using mild steel in conventional stamping. However, it was impossible to completely form this panel in conventional stamping using Advanced High Strength Steel DP 600 due to numerous wide open splits in multiple areas. In fact, this part is considered to be one of the most complex panels currently stamped at Ford.

In order to form this part using DP 600 without geometrical modifications, a novel forming process was proposed which includes two separate operations: the preforming process and the EHF final shape forming. The preforming process incorporates the following sequence of steps: (1) define a pre-formed shape for the central cavity. The stamping of this preform is the first step of the process, after the initial placement of the blank and its settling process due to gravity, and the closing phase between binder and addendum; (2) After the central portion has been preformed, the peripheral tools (left and right portion of the die) are coming down toward the die (die still stationary as well as pre-formed punch) for forming; (3) Gaining pockets are formed by quasistatic hydroforming by applying pressure from the die side and forming the central portion of the blank into the gaining pockets on the punch surface. When the preforming process is completed, the blank is moved from the preforming die to the EHF die and several discharges are applied to spread the sheet metal from the gaining pockets and fill the corners and local features of the dash panel shape.

The first task at hand was to define the location of the beads (around the part from the center of die opening line) and their equivalent restraining forces through multiple iterations of simulation. In order to not to be penalized by the calculation time during these several analyses, the contribution of each bead is represented by a line (center line of bead) associated to a restraining force. Multiple iterations were performed in order to define the optimum set of bead parameters with a suitable blank size for steps 1 and 2 of the process. Also, some modifications of the addendum surface were performed. The objective during the iterative process was to eliminate splits and excessive thinning as well as wrinkles into the part by allowing small wrinkles into the addendum and the binder. Applying very strong bead values can drastically reduce buckles and wrinkles on unsupported areas, but the central portion still often shows areas of splits on the wall due to the deep cavity. After multiple iterations on bead parameters, the preformed shape was able to be formed with reasonable buckles and no splits. Thinning was about 5 to $8 \%$, which leaves enough room for final forming (EHF) before necking. With the bead values determined during the preform stage, more adjustments needed to be done. In order to control the unsupported areas, double bead lines (locations and values) have been created.

For further optimization and refinement of the process in a way as close as possible to the planned physical tool, analyses have been performed using physical bead geometry for the single and double beads instead of the bead lines used during the initial feasibility study. Based 
on the restraining forces that were represented by the line beads, the "Bead Generator" module from DynaForm was applied to define all the geometries of the male (blank holder) and female (die) beads. Figure 7.1 shows the full die with physical beads. Performing the analysis with physical beads shows different results (formability) compared to line beads. Even though the calculation time is more intensive, as the use of physical beads (especially for the double beads case) is critical and more realistic, necessitating a higher number of elements and longer computation times, the extra time to optimize the bead geometry during the design phase saved time during the actual tryout phase. Some minor changes have been made in the preform shape and the bead geometry (male/female) in order to successfully form steps $1 \& 2$ while refining the simulation results from the line bead formulation to the physical bead. Multiple iterations were performed with the physical beads in order to define the optimum blank size and shape. The shape and location of the blank (especially in lower left area) is critical to the formability of preform. Figure 7.2 shows the optimized shape of the incoming flat blank.

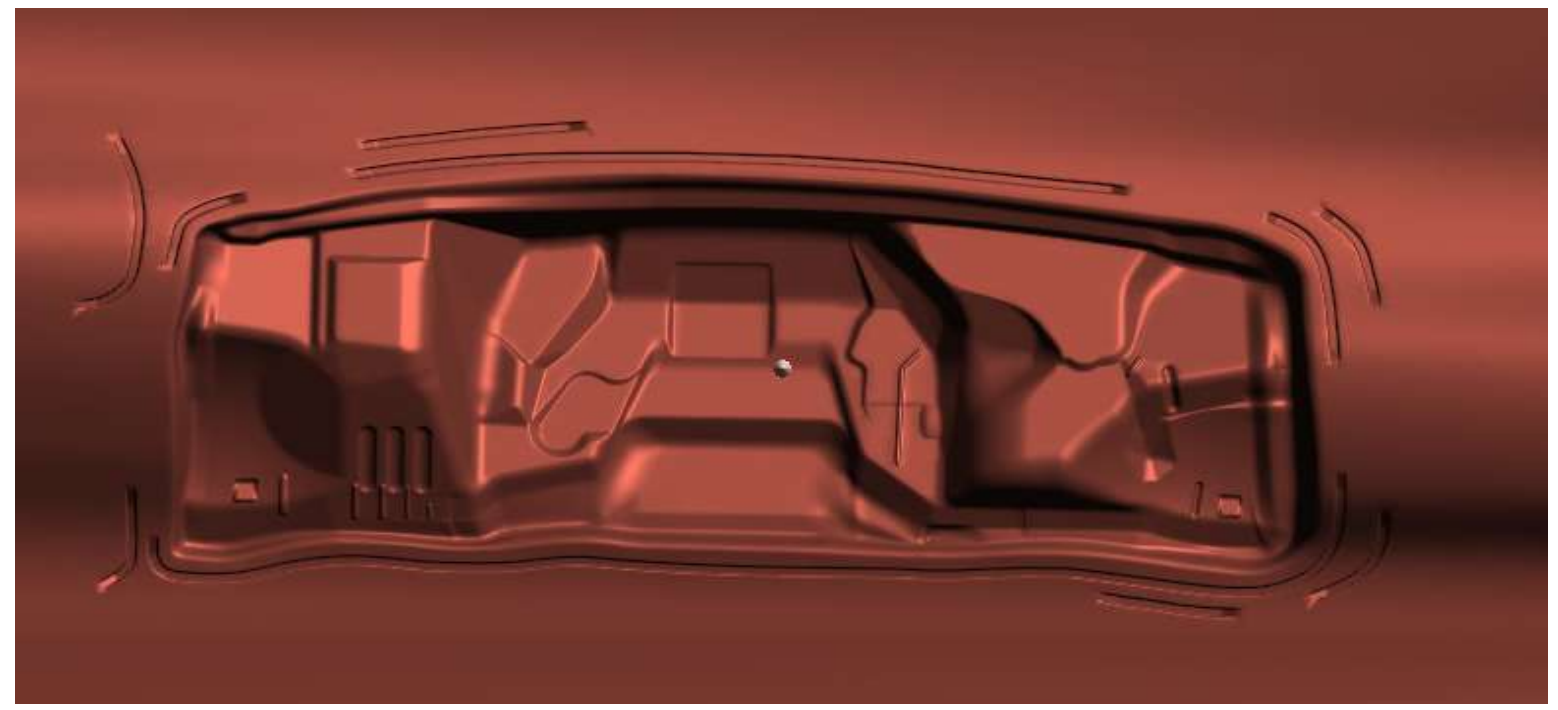

Figure 7.1. Final die with female beads. 


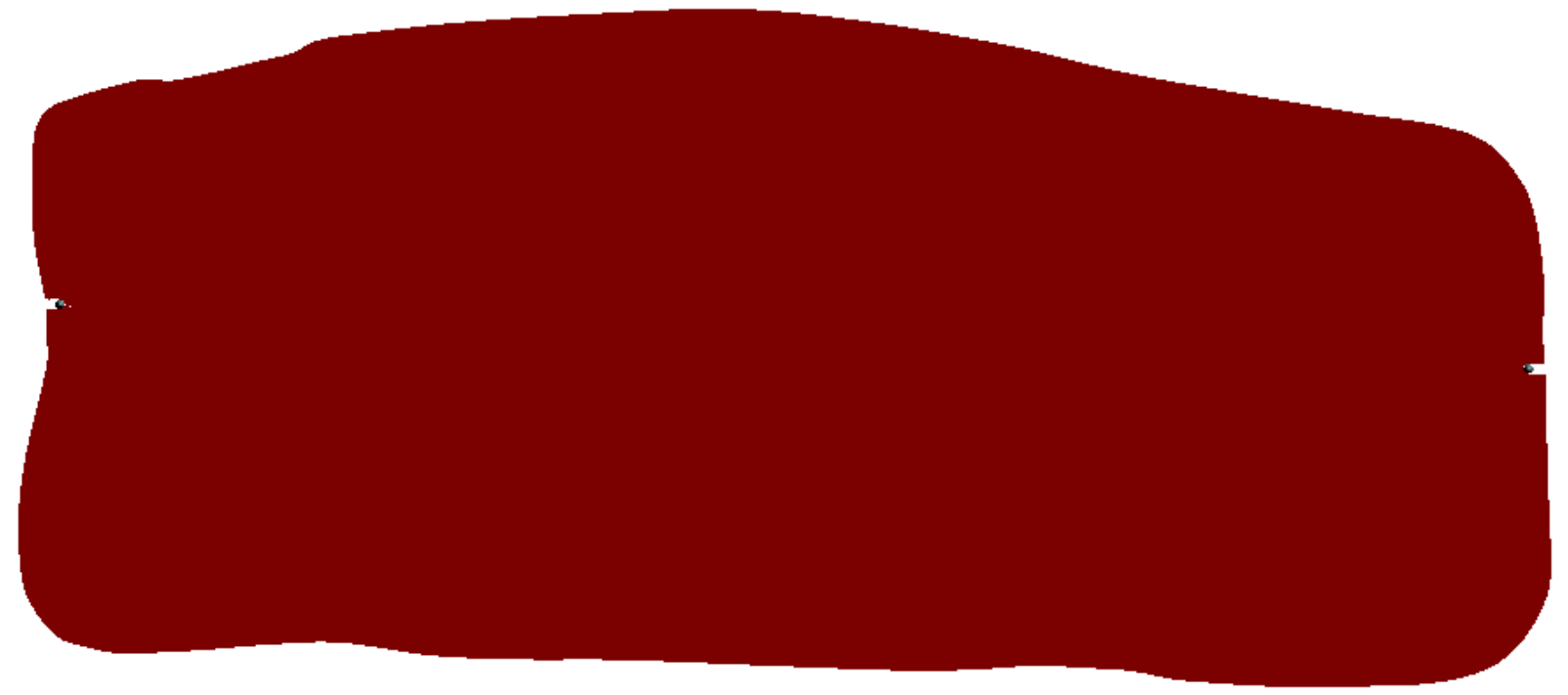

Figure 7.2. Final incoming blank shape.

In order to put and keep the blank in the optimum location, and also to have a stable blank gravity/holding phase, two locator pins were added to the tool design with alignment slots added to the blank. These pins and corresponding slots keep the blank more stable during the gravity phase and blankholder-clamping phase and maintain the proper location of the blank. It is important, since some areas are sensitive to the blank location and shape, and the drawing is critical for the formability. The slots are shown in Figure 7.2 on the narrow sides of the initial flat blank. Employment of these slots substantially improved the stability of the blank position within the preforming die during the die closing stage. The initial position of the blank inside the preforming tool is shown in Figure 7.3 while its position after the binder closing is illustrated in Figure 7.4.

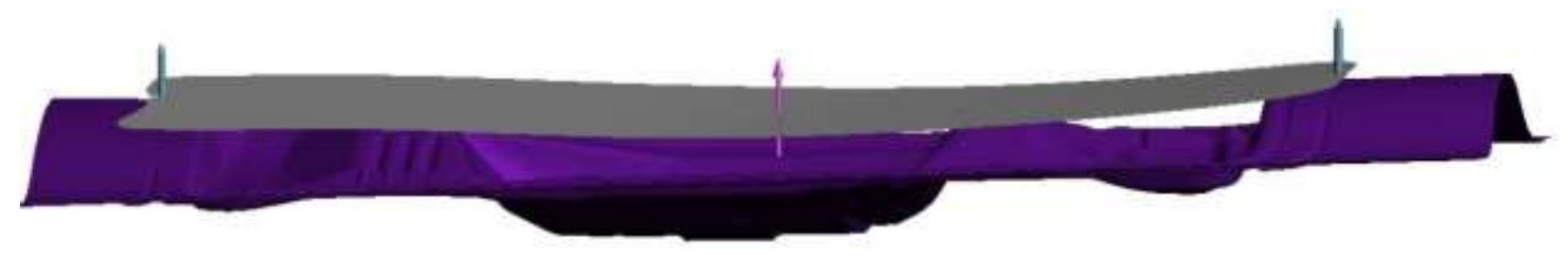

Figure 7.3. Blank location after gravity phase. 2 pins control the blank location. 


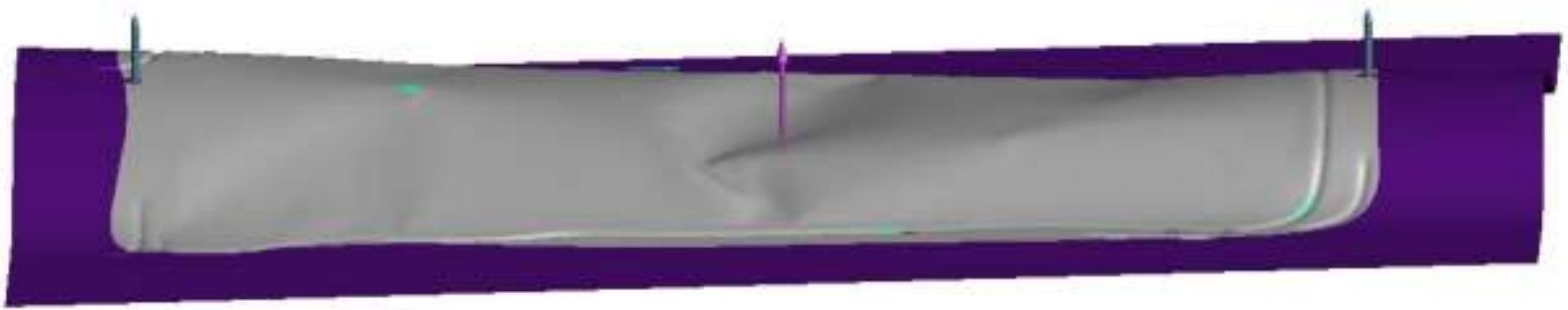

Figure 7.4. Blank location after the binder closing is completed.

Simulation of the overall process was performed using the physical beads configuration and optimum blank with 2 pins targeting stabilization of the blank position during gravity and binder closing stages. The simulation results indicated that we do not anticipate to have any splits or folding/doubling of the material. The wrinkles which appear on the upper left and lower left of the preform shape in Figure 7.5 are expected to be spread during the final step of the EHF process.

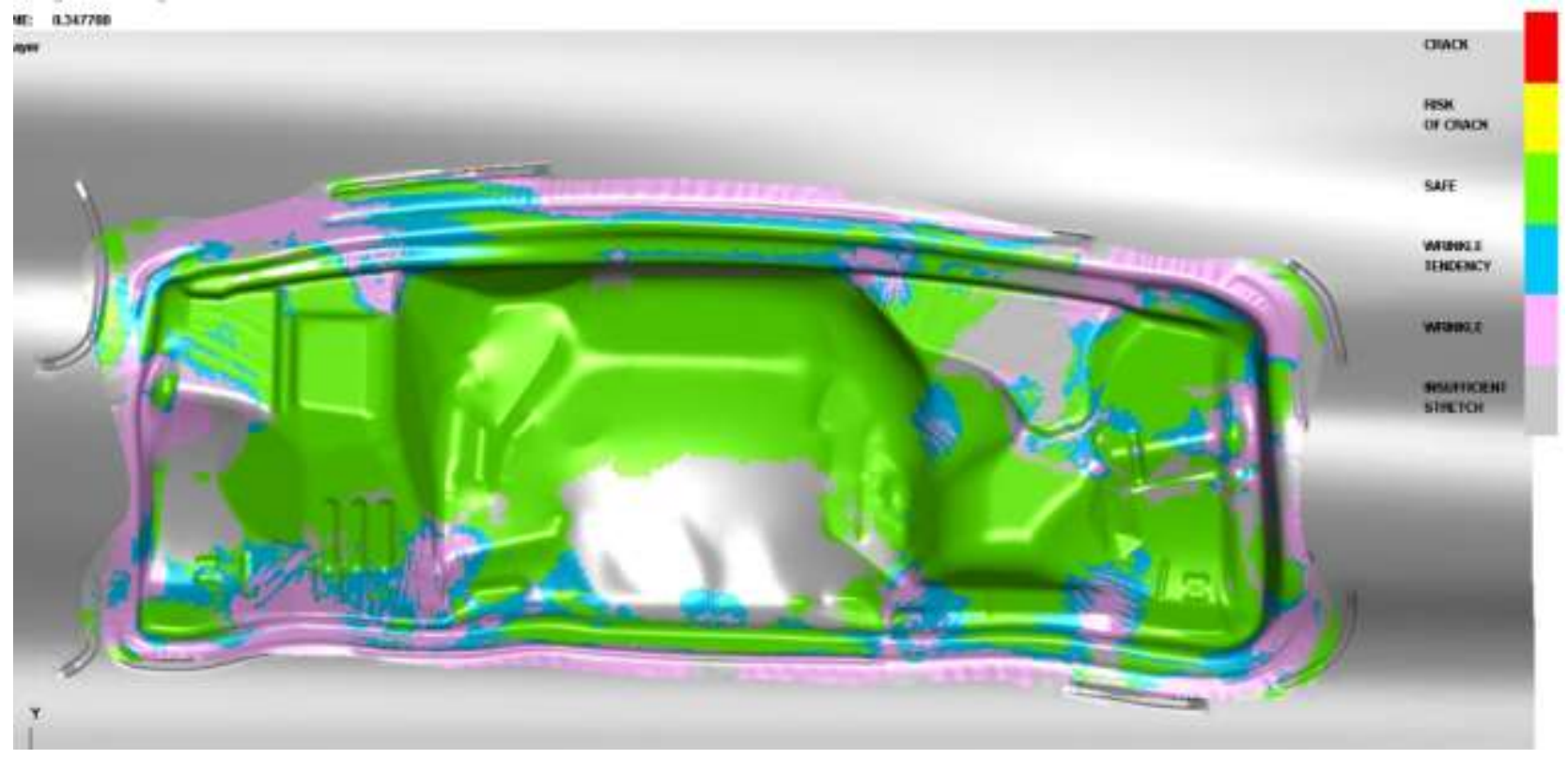

Figure 7.5. Final formability of the deformed blank after steps 1,2 and 3.

During the numerical study of the EHF process it was defined that the following factors influence the effectiveness of the EHF chamber: (1) the total volume of the chamber and the volume distribution between sub-chambers, (2) the distance between the discharge position (location of tips of electrodes) and the surface of the blank, and (3) the distance between the discharge 
location and the chamber walls, which influences the reflection of waves in the liquid towards the surface of the blank. Figure 7.6 shows the blank after preforming with the boundaries of subchambers in which individual electrode pairs are positioned.

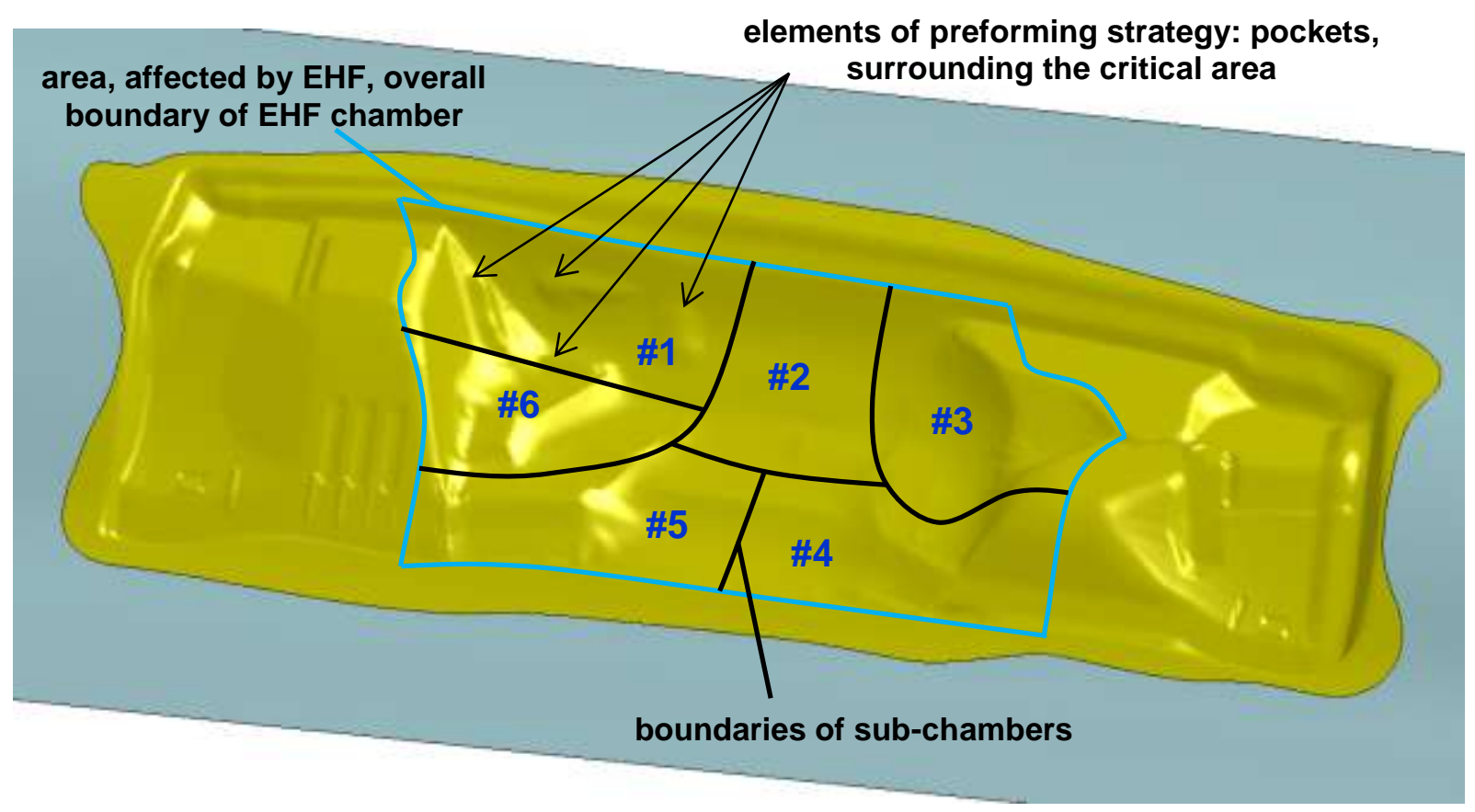

Figure 7.6. Preformed blank with EHF chamber layout.

The blue line marks the area which is not yet formed completely after the preforming and must be finished by EHF. Therefore, this line is the boundary of the overall EHF chamber. The approximate dimensions of this area are $900 \mathrm{~mm} \times 600 \mathrm{~mm} \times 200 \mathrm{~mm}$ which defines a significant volume. Apart from the volume, the distance between the discharge and the blank in such a large chamber might lead to significant losses of the pressure level. To solve this problem and also to optimize the direction of reflection of pressure waves, the chamber space was divided into six sub-chambers. Each of the sub-chambers affects only a specific part of the overall surface of the blank and has significantly less volume and distance from the blank than in one large chamber.

When planning the placement of walls for each sub-chamber, it is necessary to take into account that after the discharges the blank will move from the initial preformed position, thus increasing the clearances between the blank and the chamber walls. Larger clearances will lead to decreases in efficiency due to spreading of the pressure pulse and increased water flow through these clearances. To decrease the opening of the clearances, the subdivision of the chamber volume between the sub-chambers must be planned in such a way that the blank under the sub-chamber walls is already touching the die or at least very close to it. Such a configuration is shown in Figure 7.6 where the black lines represent the position of the sub- 
chamber walls. The Figure 7.7 shows the mesh of the chamber surface designed in accordance with the Figure 7.6. The sub-chamber's numeration is the same.

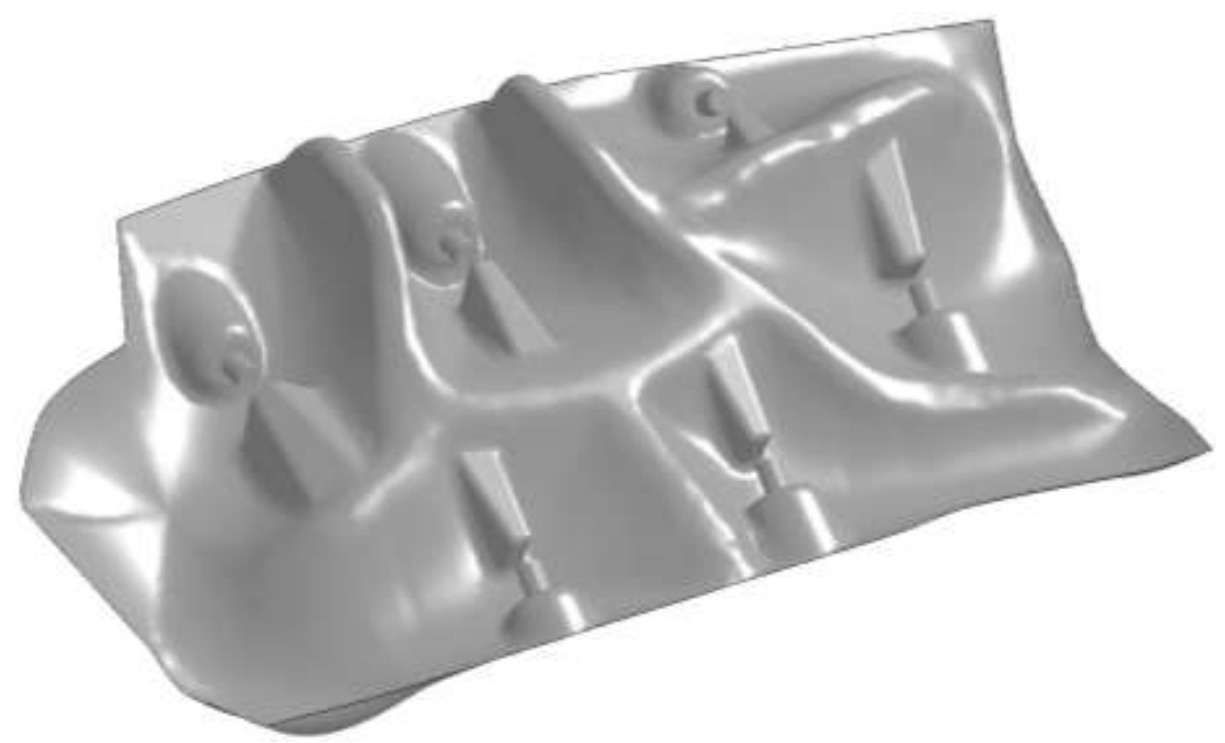

Figure 7.7. The EHF chamber final design.

The progress of deformation of the blank during the first discharge in the first sub-chamber is shown in Figure 7.8. The spreading of the pocket can be clearly observed. It also can be noted that deformation in the first sub-chamber is practically finished after about $200 \mu$ s even there is some deformation still going on in the adjacent sub-chambers. The evolution of the shape of the blank in the central area of the dash panel through all the steps of the proposed forming process is shown in Figure 7.9. It is visible that due to the inevitable pressure spreading to the adjacent sub-chambers and water flow through the clearances between the blank and the corresponding sub-chamber walls during each discharge, the formation of the blank also occurs outside the area corresponding to each individual sub-chamber. Therefore, the majority of blank's displacement into the die cavity happened during the initial two or three discharges. During the later discharges, the evolution of the shape of the blank is hardly visible. The number of discharges necessary to completely form the blank, as predicted by this model, is seven. In the experimental environment, some additional discharges may be required in each of the subchambers to account for some possible leaks of liquid through the seal and to calibrate the fine details of the shape. 
$0 \mu s$

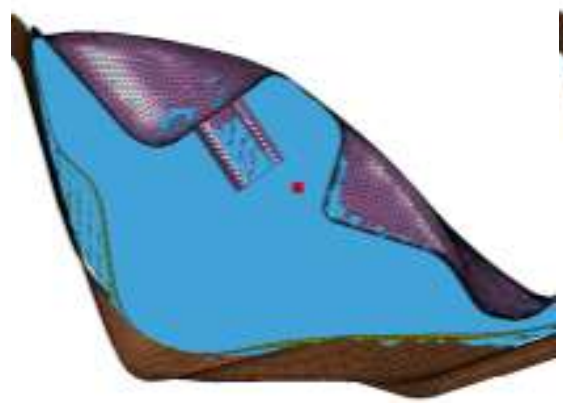

$75 \mu \mathrm{s}$

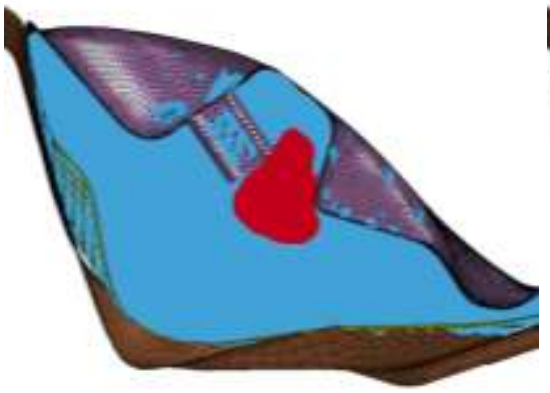

$200 \mu \mathrm{s}$
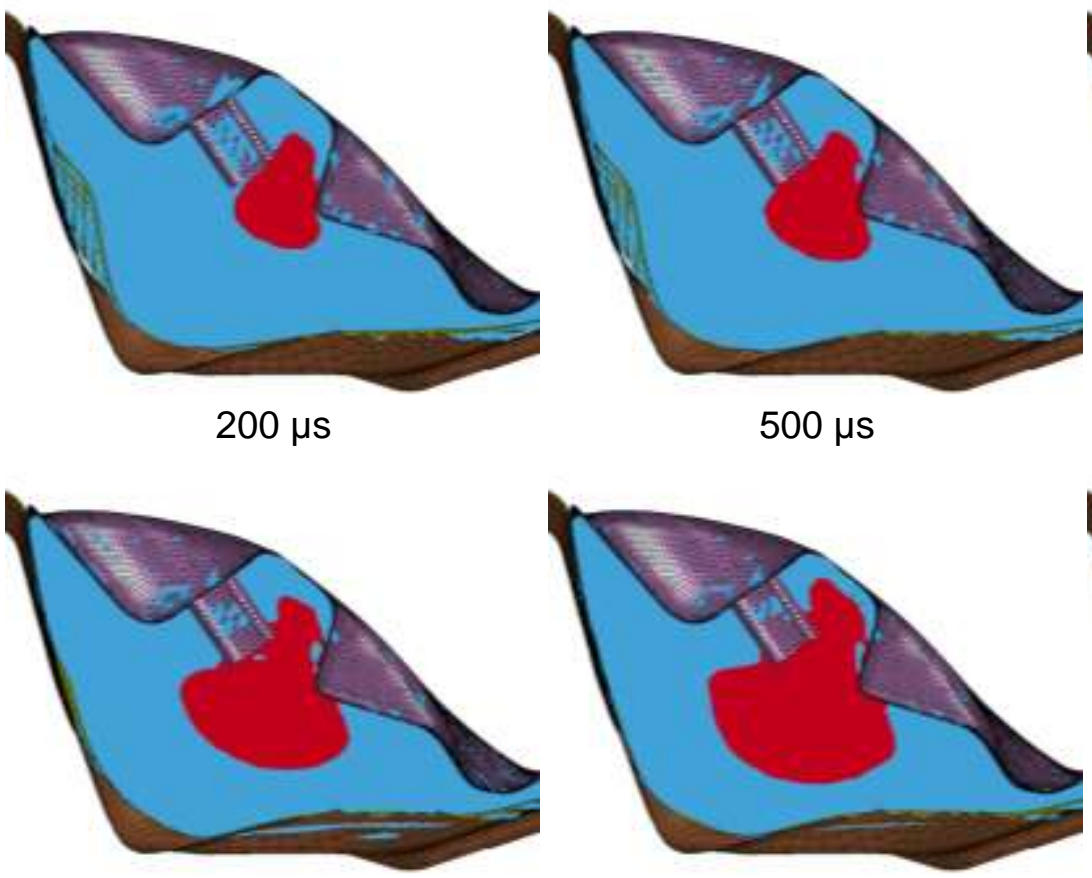

$500 \mu s$

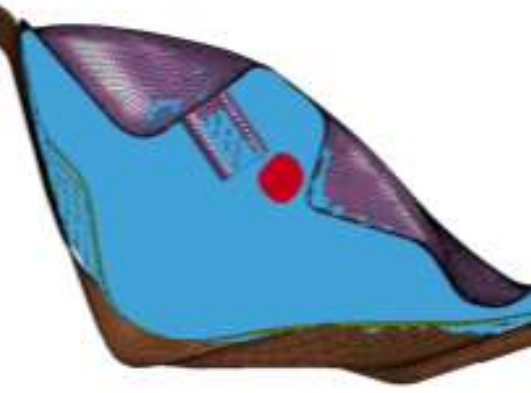

$100 \mu s$

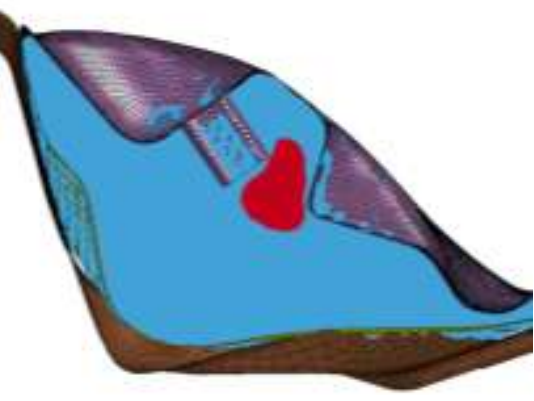

$150 \mu \mathrm{s}$

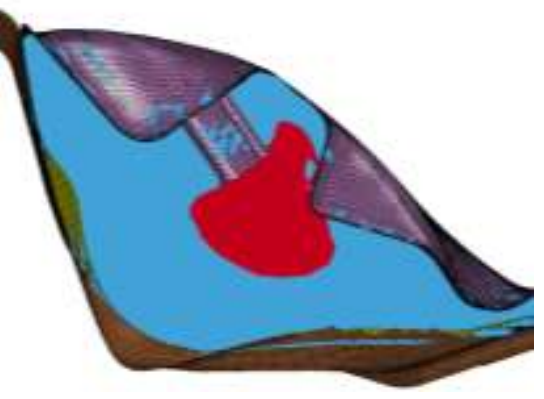

$1000 \mu s$

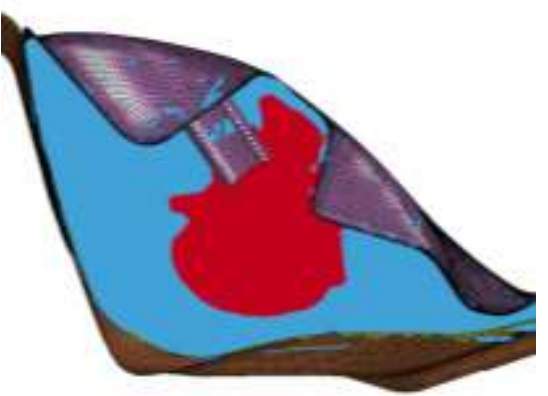

Figure 7.8. The shape of the discharge channel and deformation of the blank during the first discharge in sub-chamber \#1. 


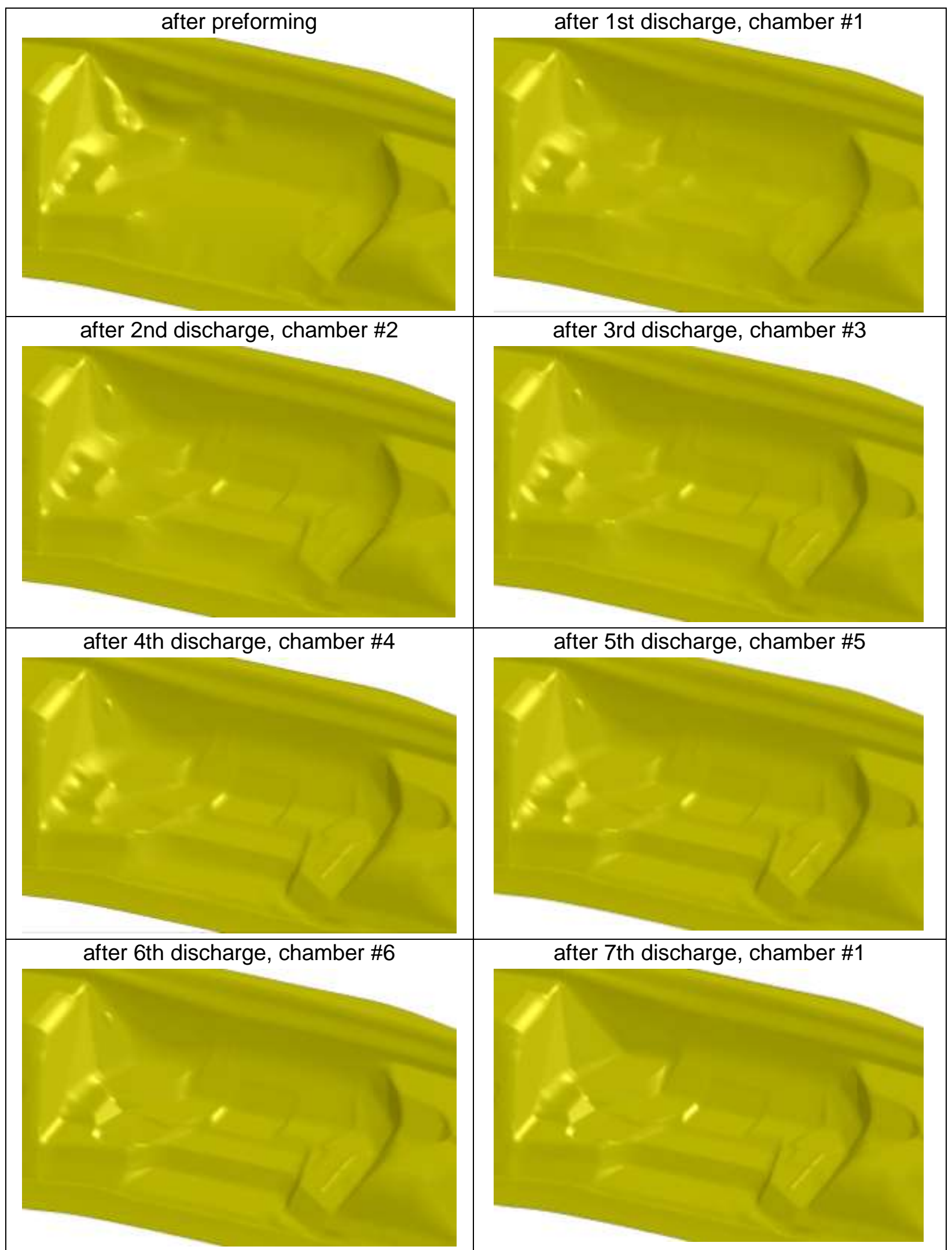

Figure 7.9. Evolution of the shape of the blank in the central area of the dash panel after each forming step and discharge. 
After the last EHF discharge, the blank is completely formed. Figure 7.10 shows the final formability of the part (central cavity) using pulsed $\mathrm{EH}$ pressure. Only local thinning was observed which is still considered to be safe, keeping in mind the substantial capabilities of the EHF process to address any issues with insufficient formability. The design of the pre-forming die is shown in Figure 7.11. This die forms the initial dash panel part shape with the sides of the blank fully formed and the center region partially formed.

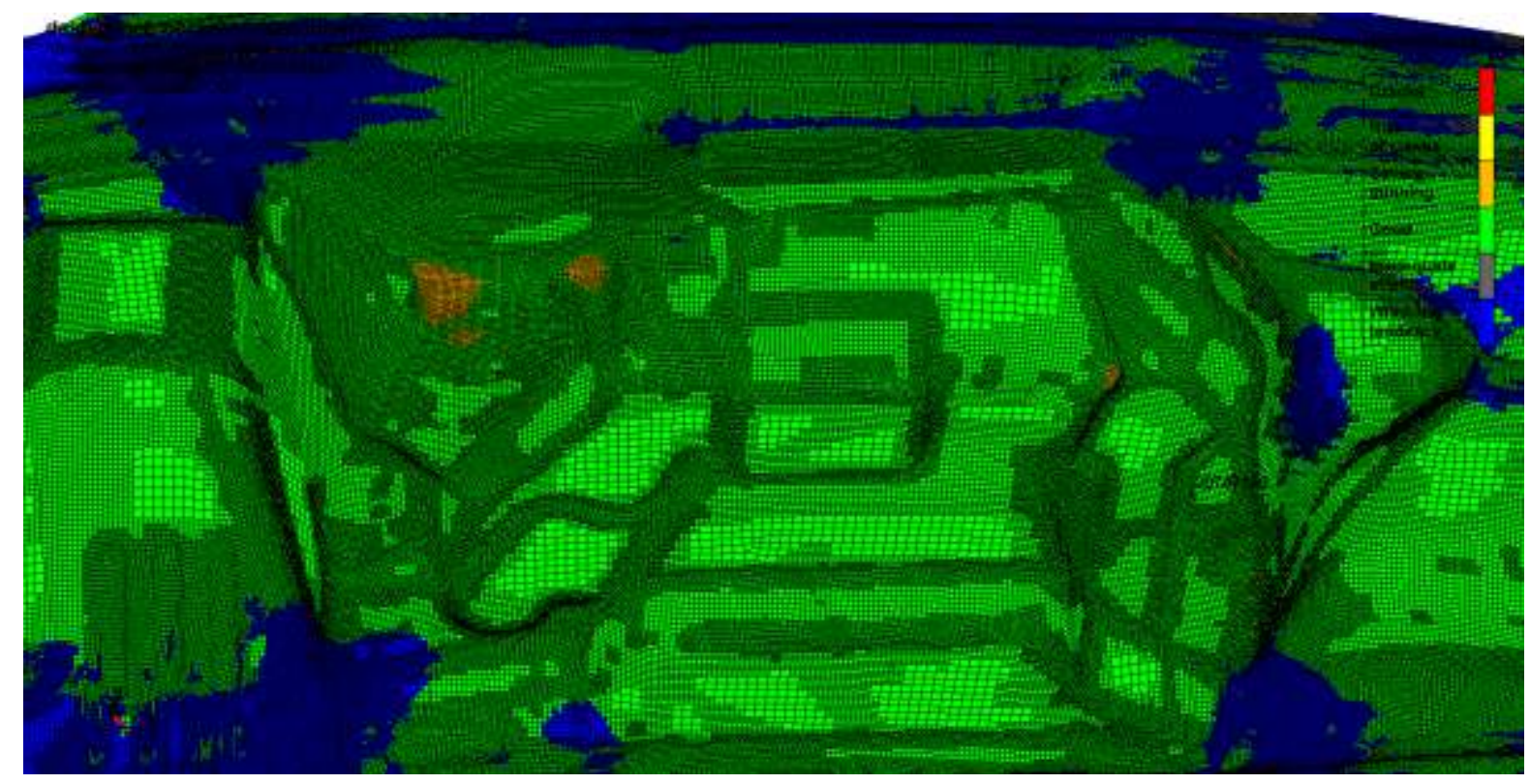

Figure 7.10. Formability map of the central cavity after pulsed pressure was applied, and the process was completed.

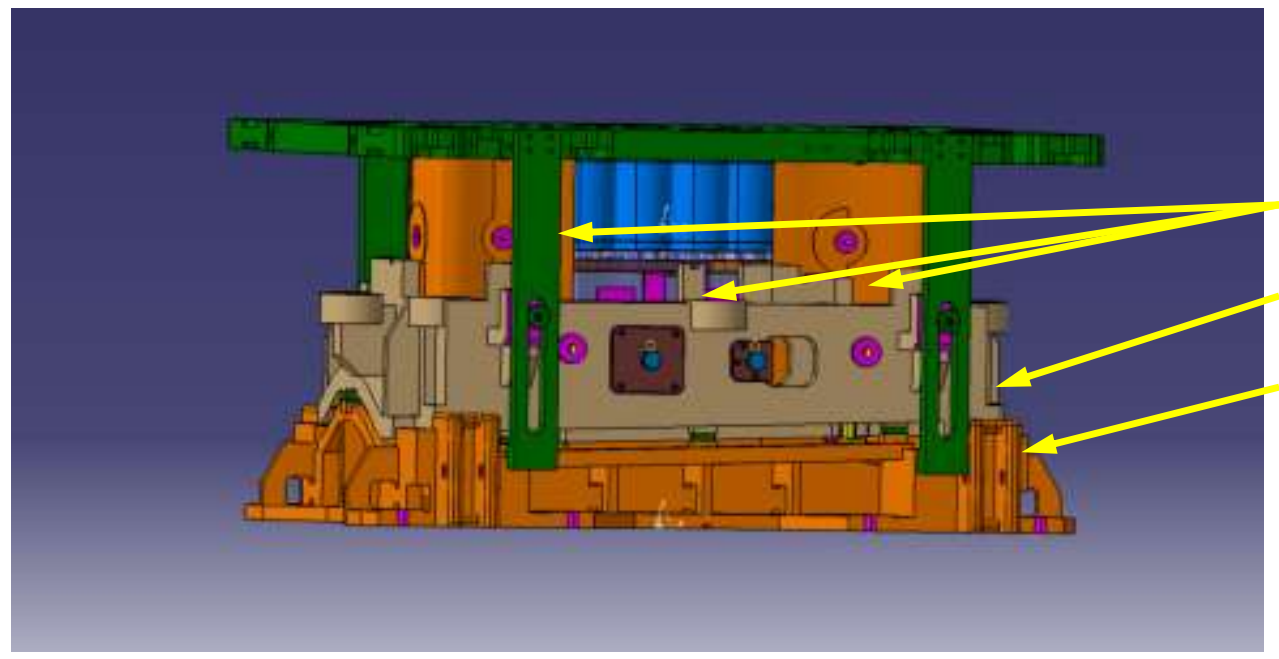

(3) Punches

Binder Ring

Die Cavity

Figure 7.11. The dash panel pre-forming die with center punch driven by nitrogen cylinders and two side punches attached to the upper die shoe. 
The pre-forming die was designed and fabricated as a triple action tool. At the beginning of the process, the flat blank is positioned inside the tool on top of the lower preforming die using guiding pins as shown in Figure 7.12. The function of the guiding pins is to control the position of the blank relative to the die surface, according to the design of the process defined through multiple iterations of numerical simulation. The flat blank of a defined shape was laser trimmed from the steel sheet. In order to accommodate the motion of the blank during the binder closing step, half-elliptical slots were cut on the flat blank. The first step was closing the binder. During this step the initially flat blank shown in Figure 7.13 was wrapping the binder surface and filling the drawbeads of the preforming die. The drawbead configuration is shown in Figure 7.14 on the upper binder surface. The drawbead design is the key for this preforming process, since it enables restrained material flow during central punch motion and substantially easier material flow during the motion of the side punches. The second step was the central punch stroke, during which the central portion of the blank is drawn into the die cavity. To accommodate this, the central punch was fabricated separately from the side punches.

\section{Upper die Sheet metal blank with locating holes}

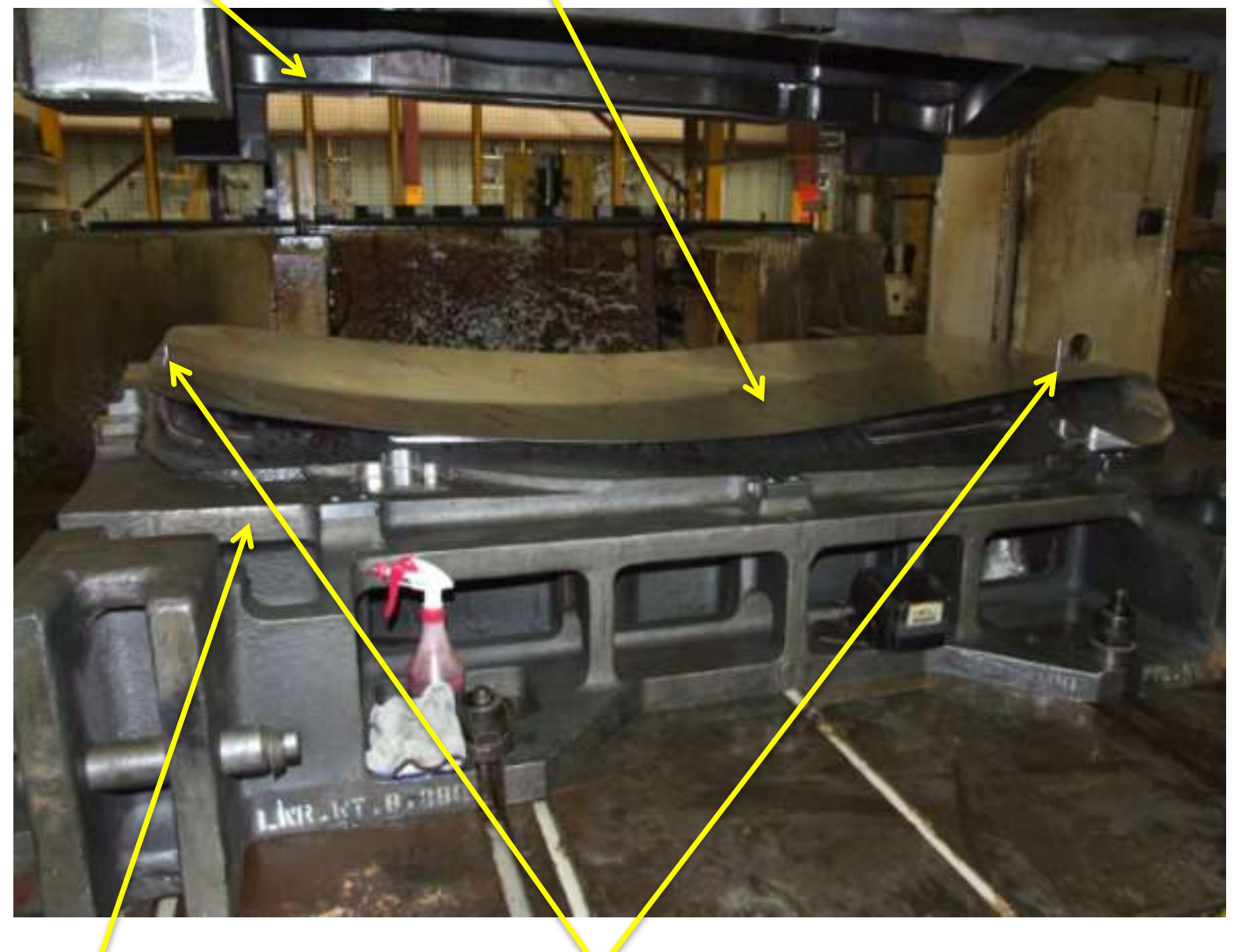

\section{Lower die}

Locating pins defining position of the blank

Figure 7.12. The preforming die in the assembled configuration, with the flat blank positioned by locating pins. 


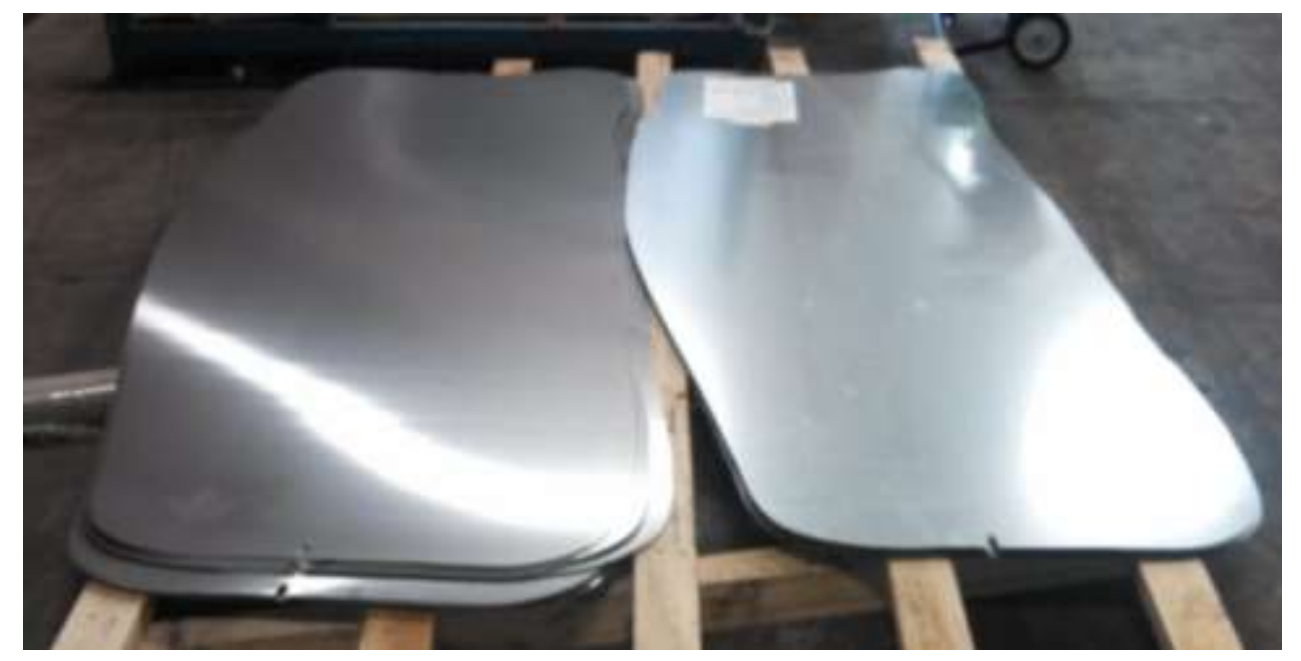

Figure 7.13. Individual flat blanks laser trimmed from $1.5 \mathrm{~mm}$ thick DP600 sheet.

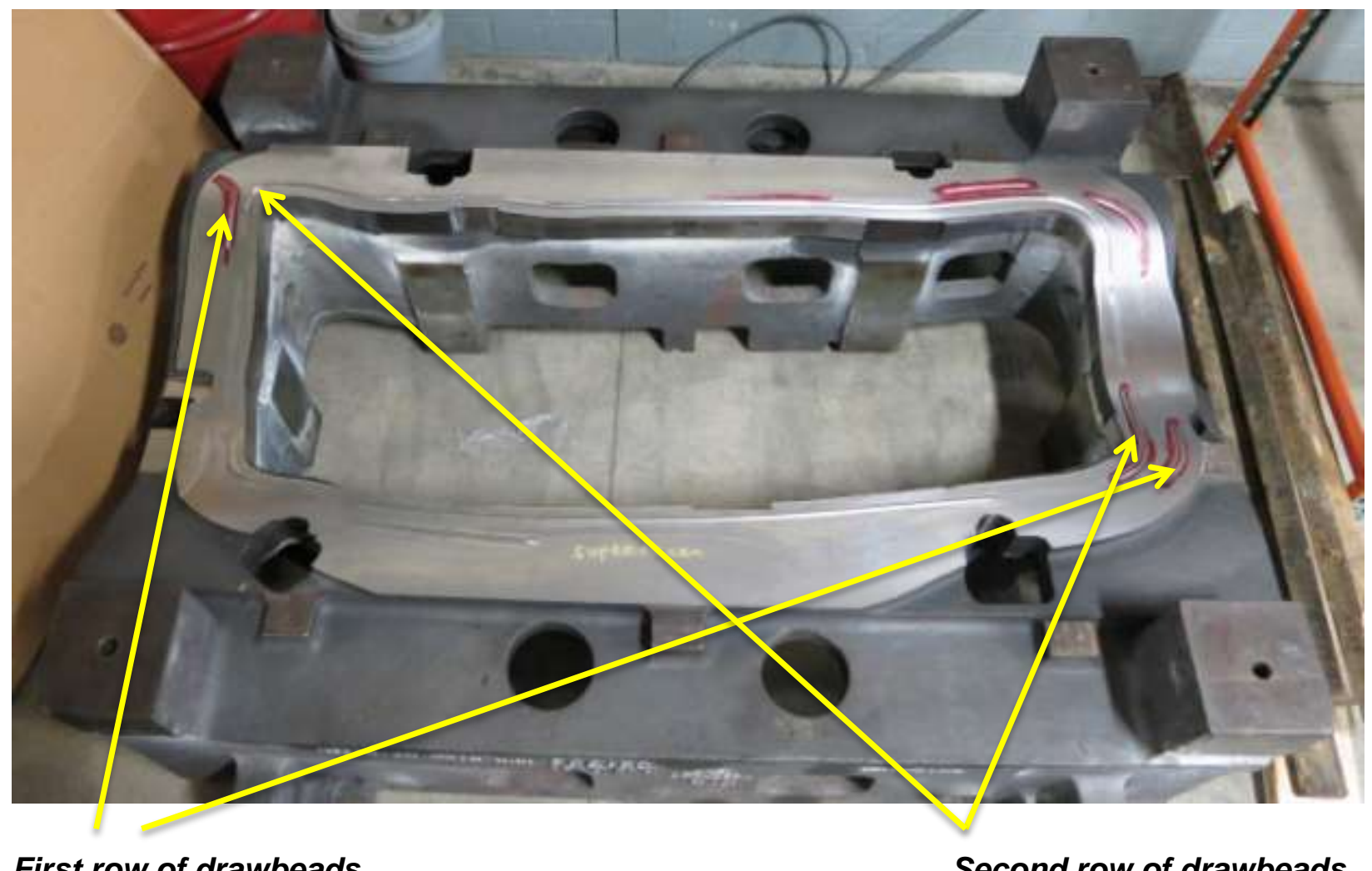

Figure 7.14. Upper binder of the preforming die with two rows of drawbeads enabling two different modes of the material flow during the stage of central punch drawing and during the stage of side punch drawing. 
The side punches and the nitrogen cylinders supporting the central punch in the assembled upper preforming die are shown in Figure 7.15. As mentioned above, the second action is the drawing of the central portion of the blank with the central punch. When the central punch reaches to its home position, the upper ram of the press still continues going down, compressing the cylinders positioned above the central punch and enabling the third action of engaging the side punches and filling the shape in the periphery of the panel. The assembled lower preforming die is shown in Figure 7.16.

The left side punch Nitrogen cylinders The central punch The right side punch

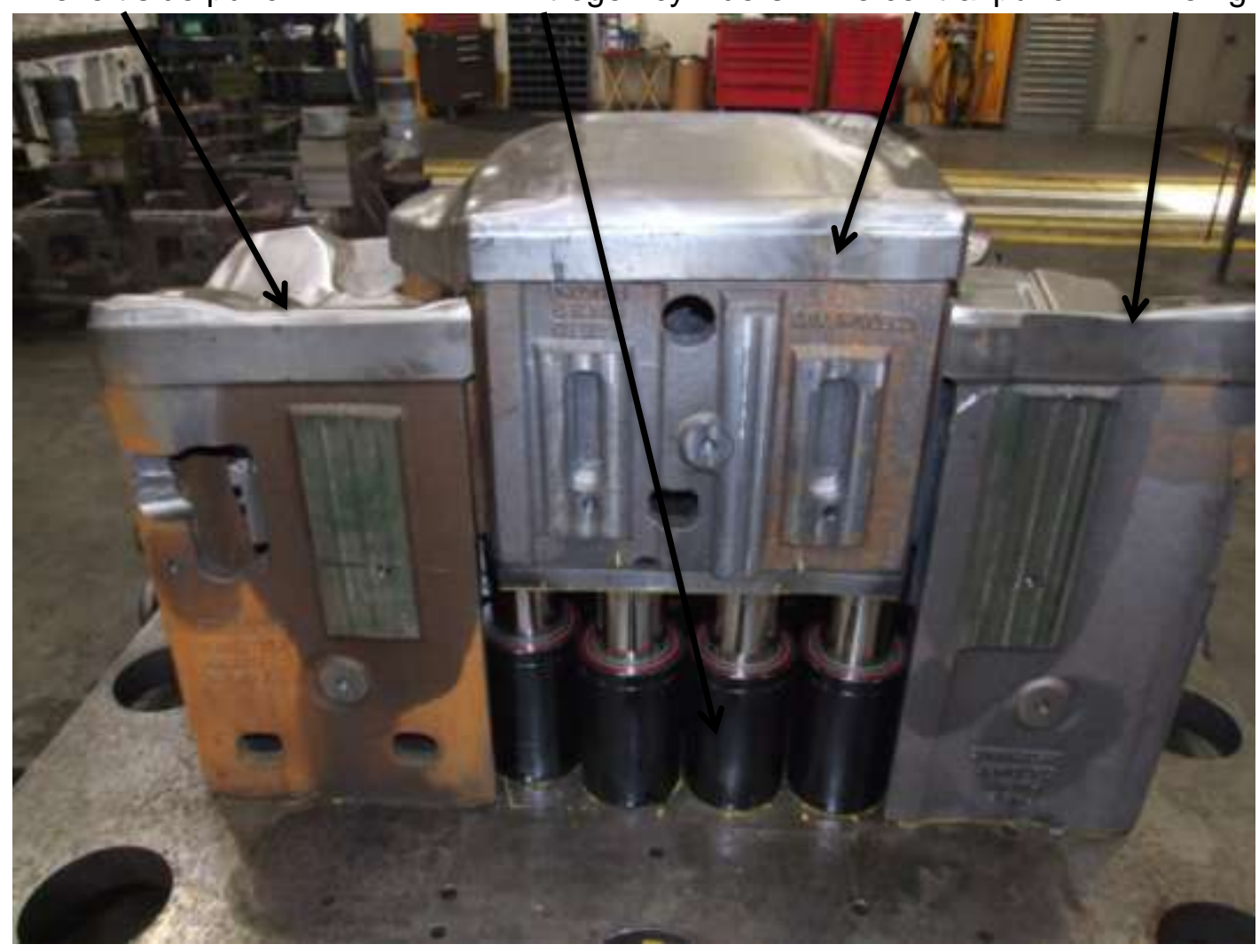

Figure 7.15. Assembled upper preforming die in upside down position. 


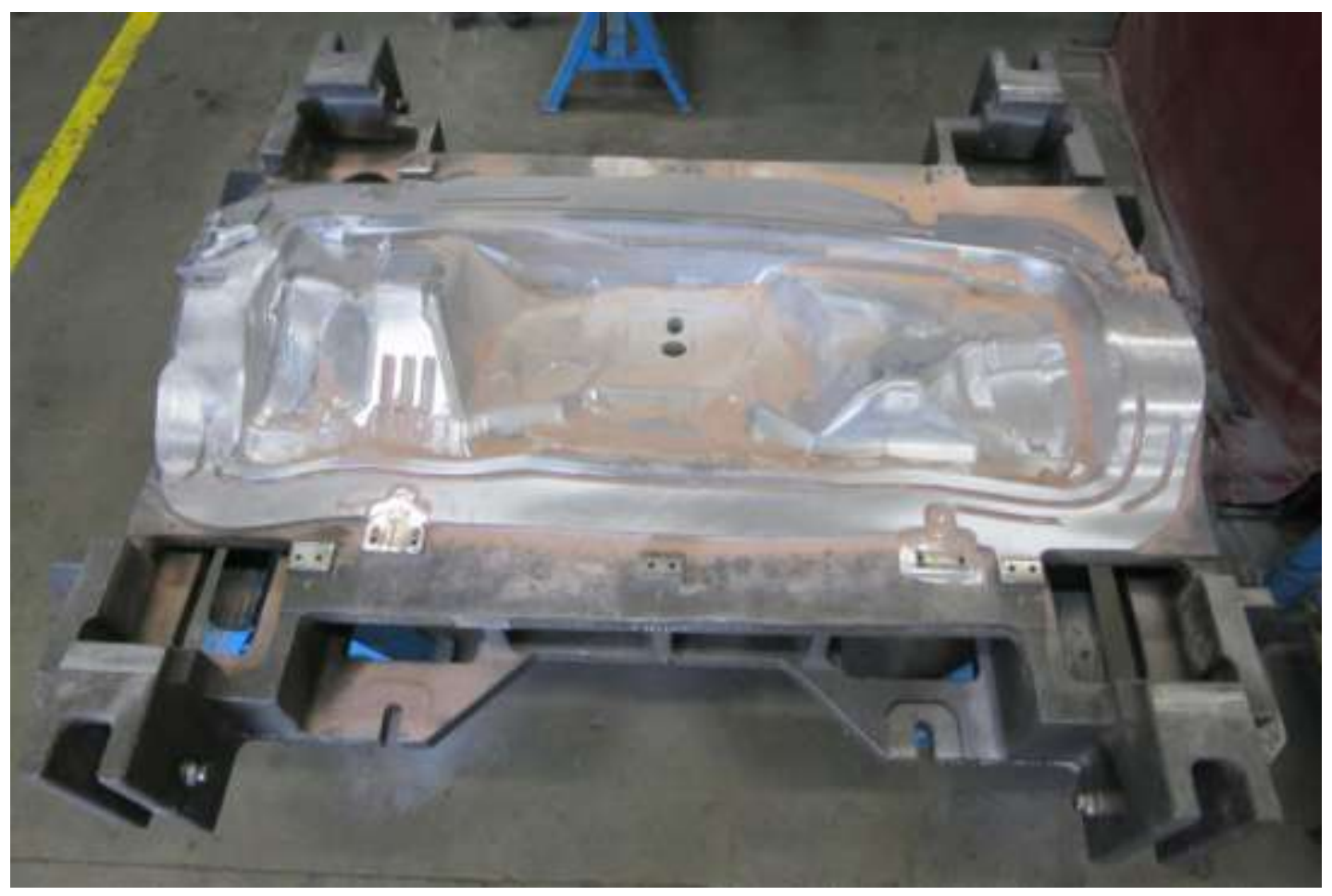

Figure 7.16. Assembled lower preforming die.

The initial tryout was performed at Amino North America Corporation. The press at Amino NA is equipped with a set of hydraulic cylinders providing up to 800 tons of binder force. The required binder force for the process was 200 tons. The preforming die installed in a double action hydraulic press at Amino NA is shown in Figure 7.17. The preformed blank is shown in Figure 7.18 as positioned on the preforming die in order to conduct measurements of blank in-flow.

The pre-forming die actions can be summarized in the following sequence:

- First, the binder closes around the periphery of the part, forming the blank between the drawbeads.

- Once the drawbeads are fully closed the center punch will start drawing the blank in the central region of the part.

- Once the central punch reaches its home position, the upper die will continue to travel downward, allowing the side punches to form the rest of the panel while the central punch is held in position by the nitrogen gas springs.

- After the side punches complete their stroke, the press stops and dwells with all punches in the home position.

- Water is then pumped into the metal gainer pocket region to reverse form the pockets. 


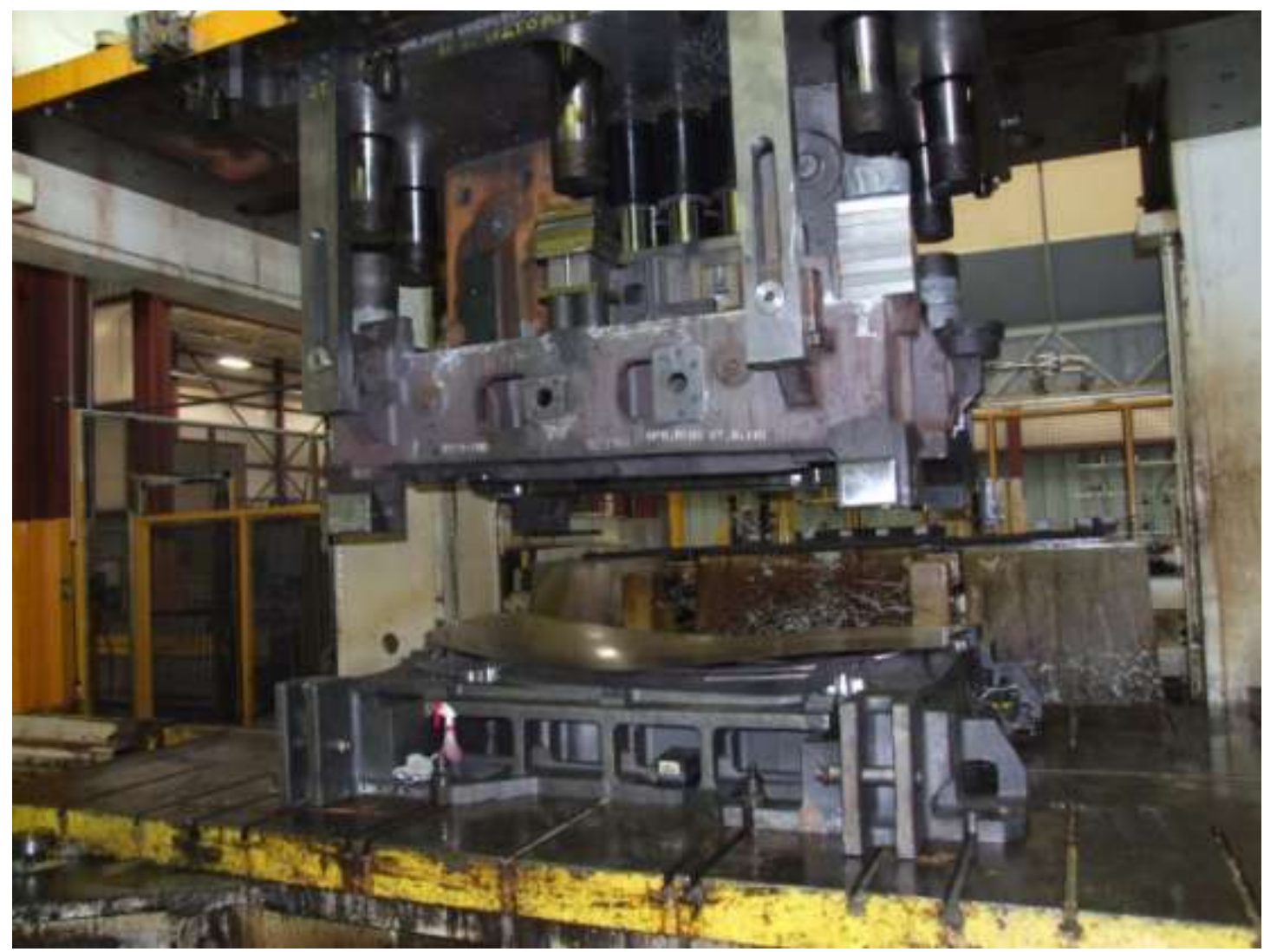

Figure 7.17. The assembled preforming die installed in a 3000 ton, double action hydraulic press at Amino NA during the tryout process.

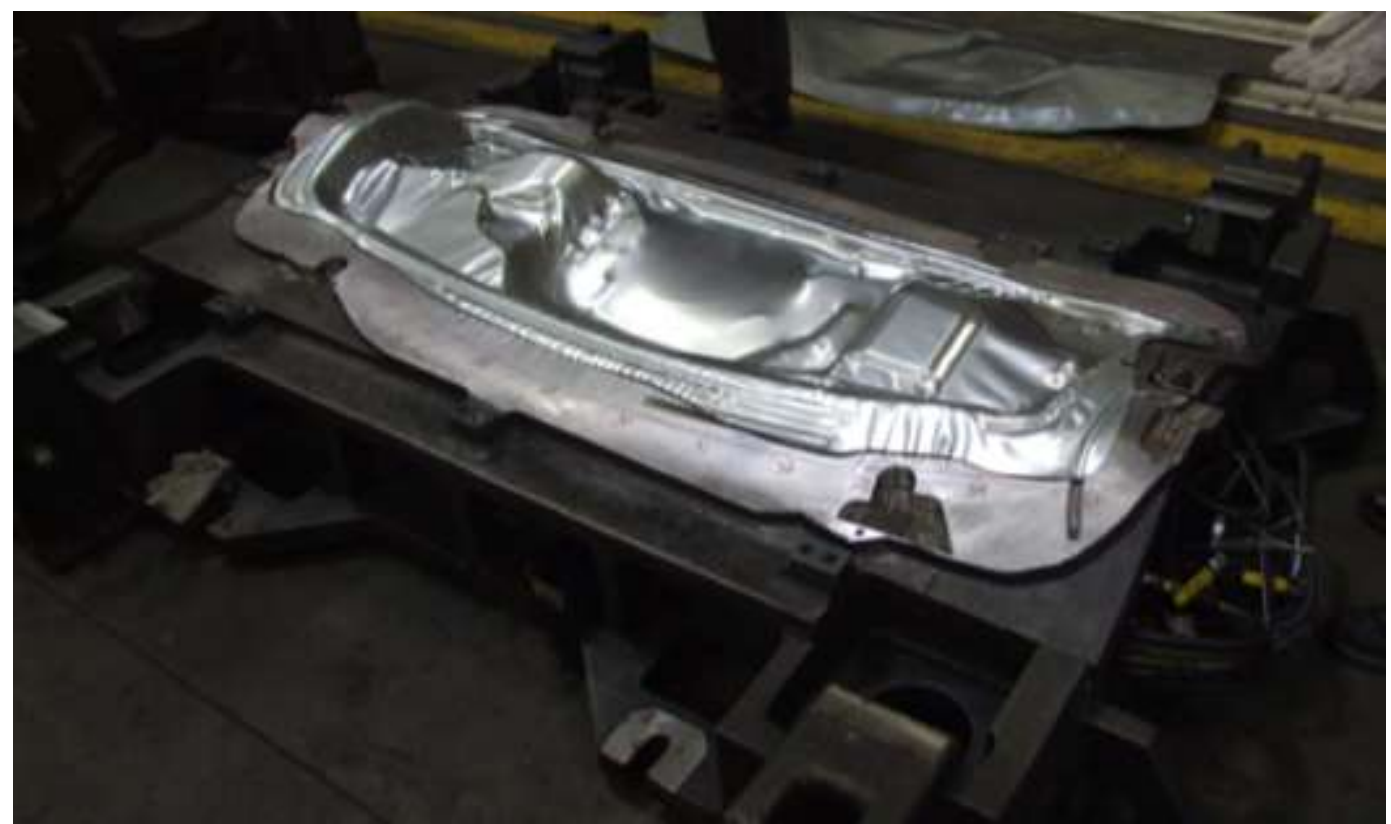

Figure 7.18. Preformed blank positioned inside the lower die during measurements of the material inflow into the die cavity. 
The EHF chamber and die for the final forming step are schematically shown in Figure 7.19. The EHF chamber is comprised of six sub chambers, each with an electrode, to increase and concentrate the forming pressure on the blank. The vertical height of the chamber is driven by the steep entrance angles of the electrodes required to reach optimized locations as determined by simulation. The electrodes are used to produce the plasma and subsequent water pulse that drives the sheet metal into the lower die. The lower die cavity is manufactured to the final part shape since the EHF process eliminates part spring back and thus eliminates the need for die compensation that is required by conventional stamping processes. The EHF chamber and the lower die cavity were machined from hot rolled steel billets to avoid issues with porosity that could initiate a fracture during the EHF pulses (Figures 7.20 and 7.21).

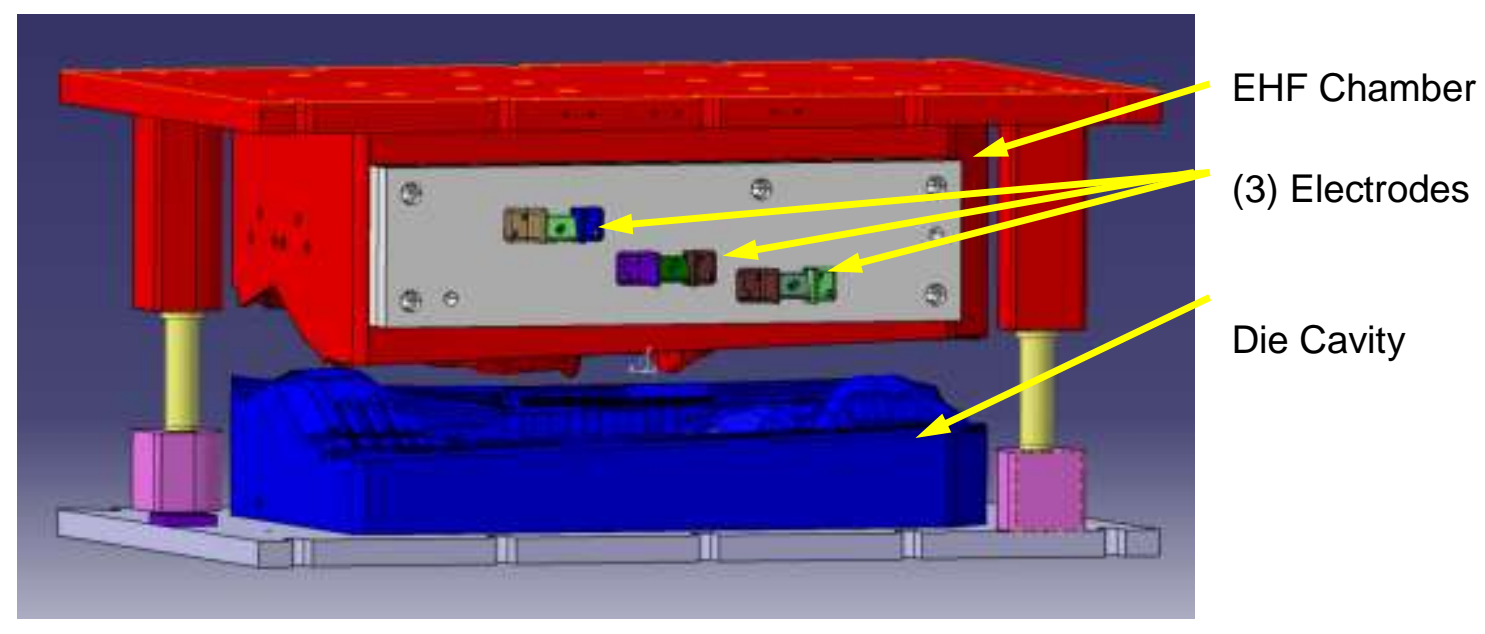

Figure 7.19. Final dash panel forming tool with the EHF fluid chamber on top and the lower die machined to the final part shape.

The EHF tooling includes the die shown in Figure 7.20 and the chamber shown in Figure 7.21. Figure 7.22 shows the EHF chamber and die assembly installed in a 150 ton hydraulic Beckwood press at the Ford R\&AE lab at Norwood. The sequence of experimental steps for the final forming stage using EHF was the following:

- The partially formed part is placed in the die and located by two guide pins.

- The chamber and die are closed.

- The chamber is filled with fluid.

- Each of the electrodes is pulsed sequentially.

The EHF tool in closed position is illustrated in Fig.7.23 while fully formed panel is shown in Fig.7.24. 


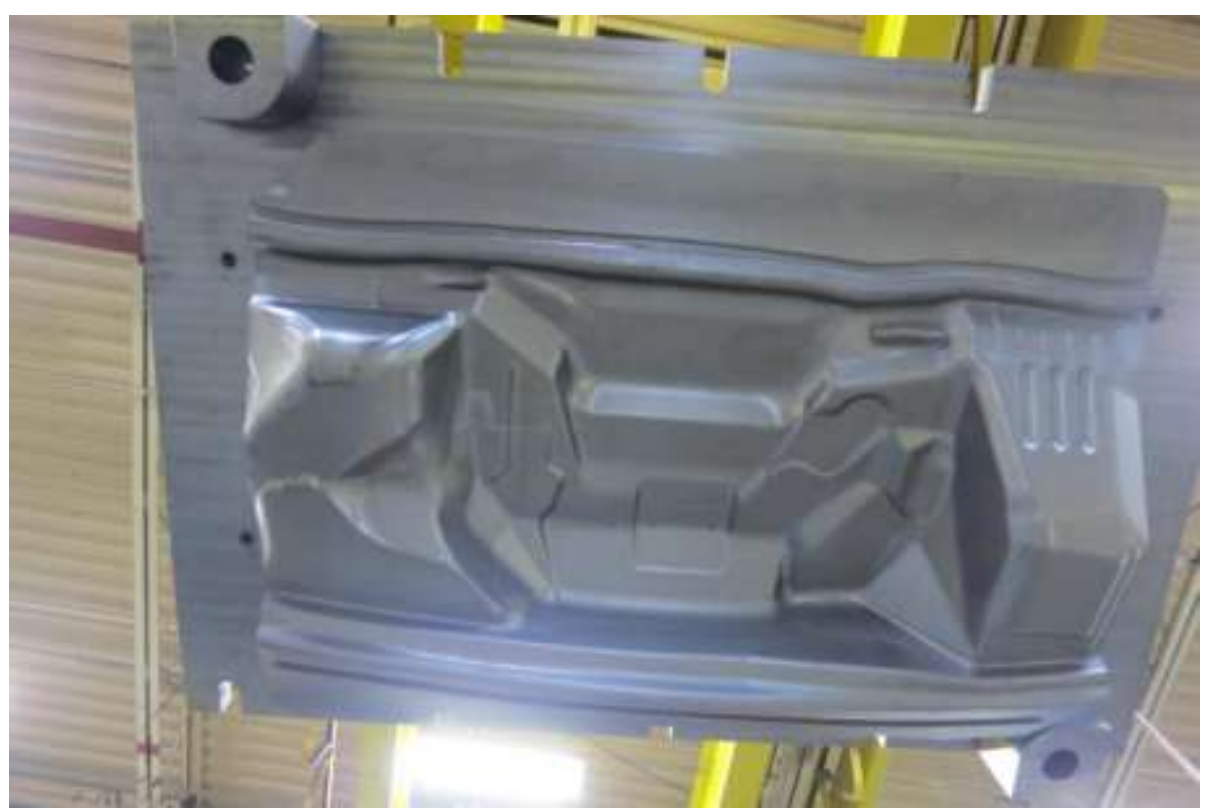

Figure 7.20. EHF die providing final shape forming.

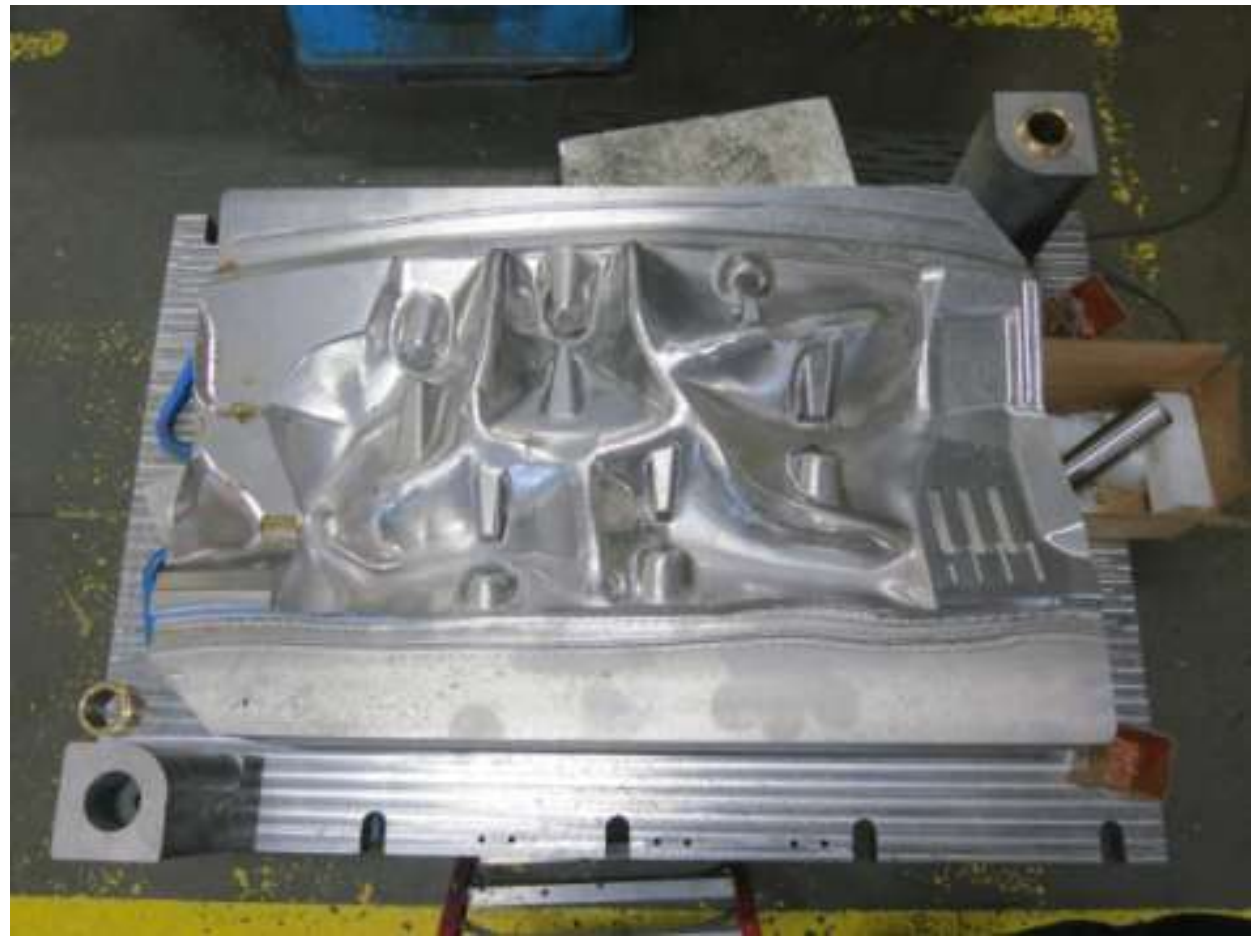

Figure 7.21. EHF chamber prior to the installation of electrodes. 
Cables connecting the electrodes to the Pulsar unit EHF chamber

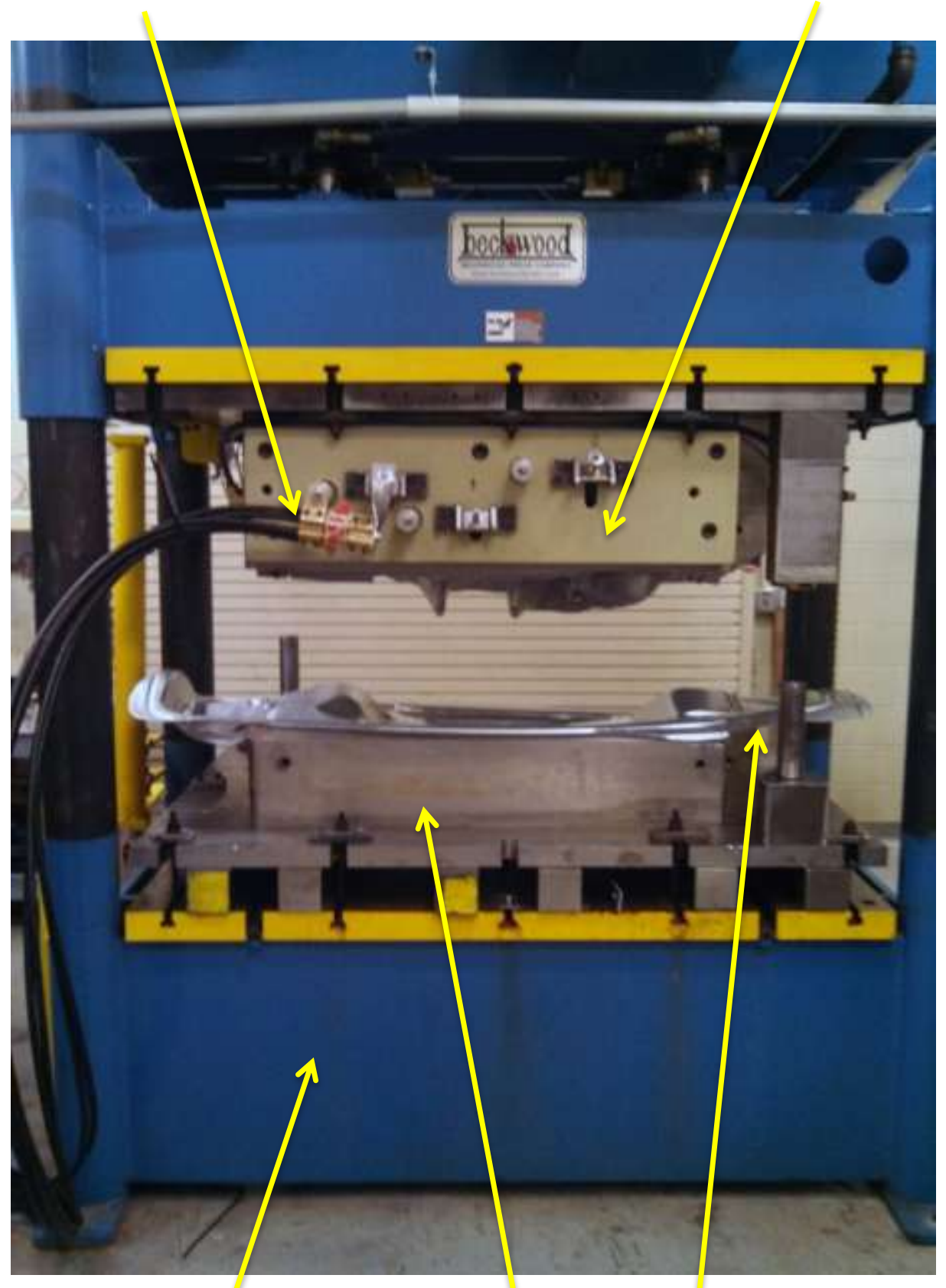

Beckwood 150 ton hydraulic press EHF die Preformed blank

Figure 7.22. EHF tooling in open position during the tryout process at the Ford Norwood lab. 


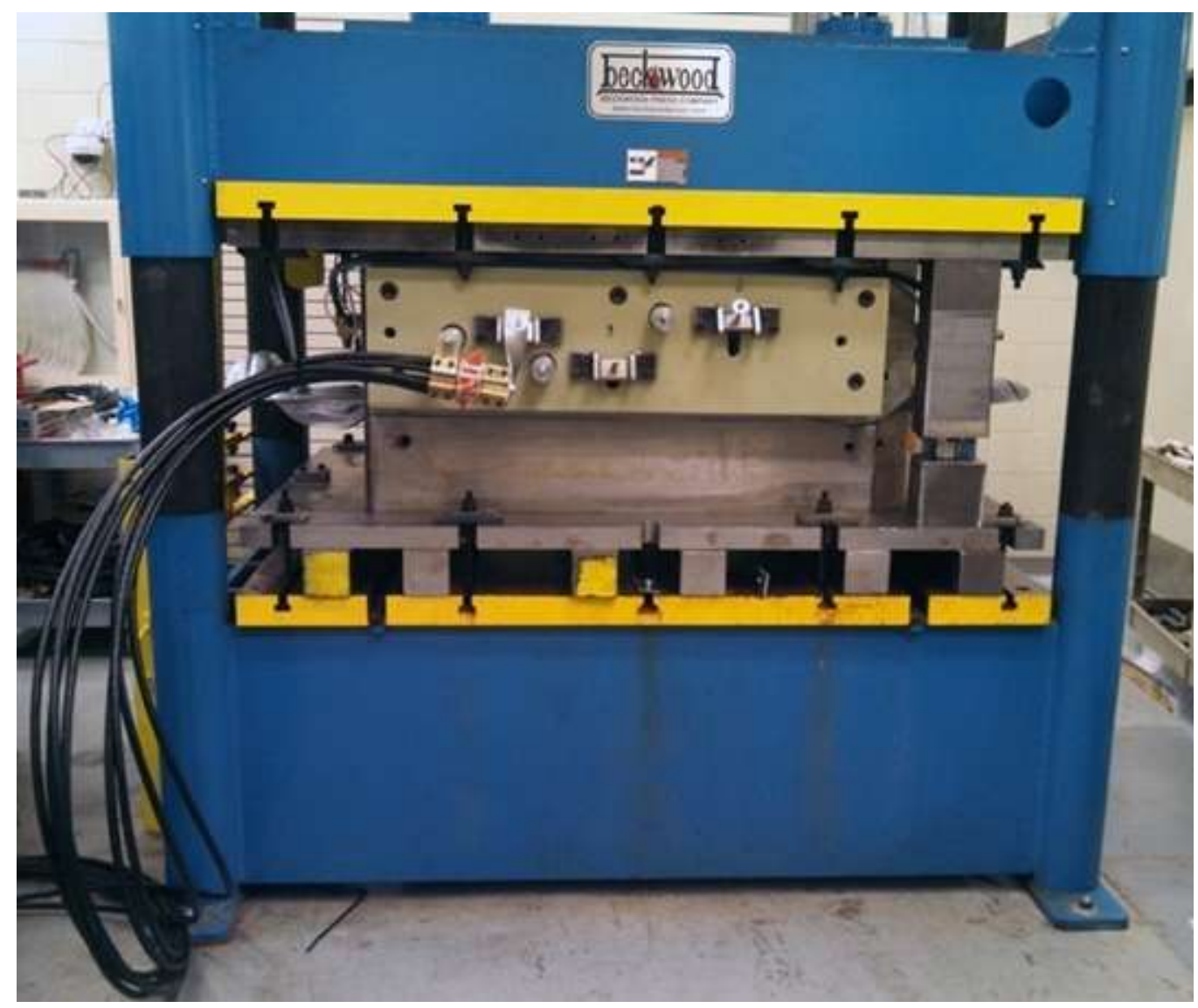

Figure 7.23. EHF tooling in closed position during the tryout process at the Ford Norwood lab. 


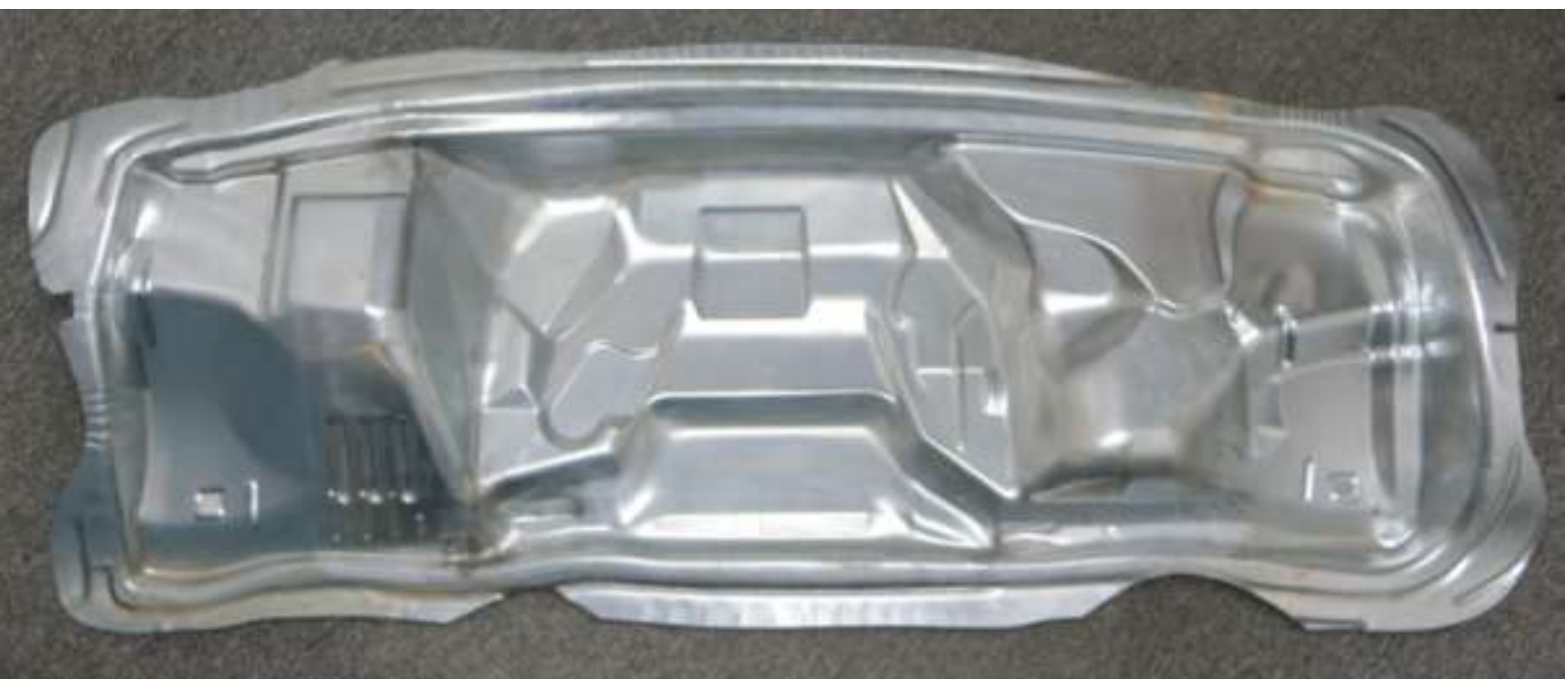

a)

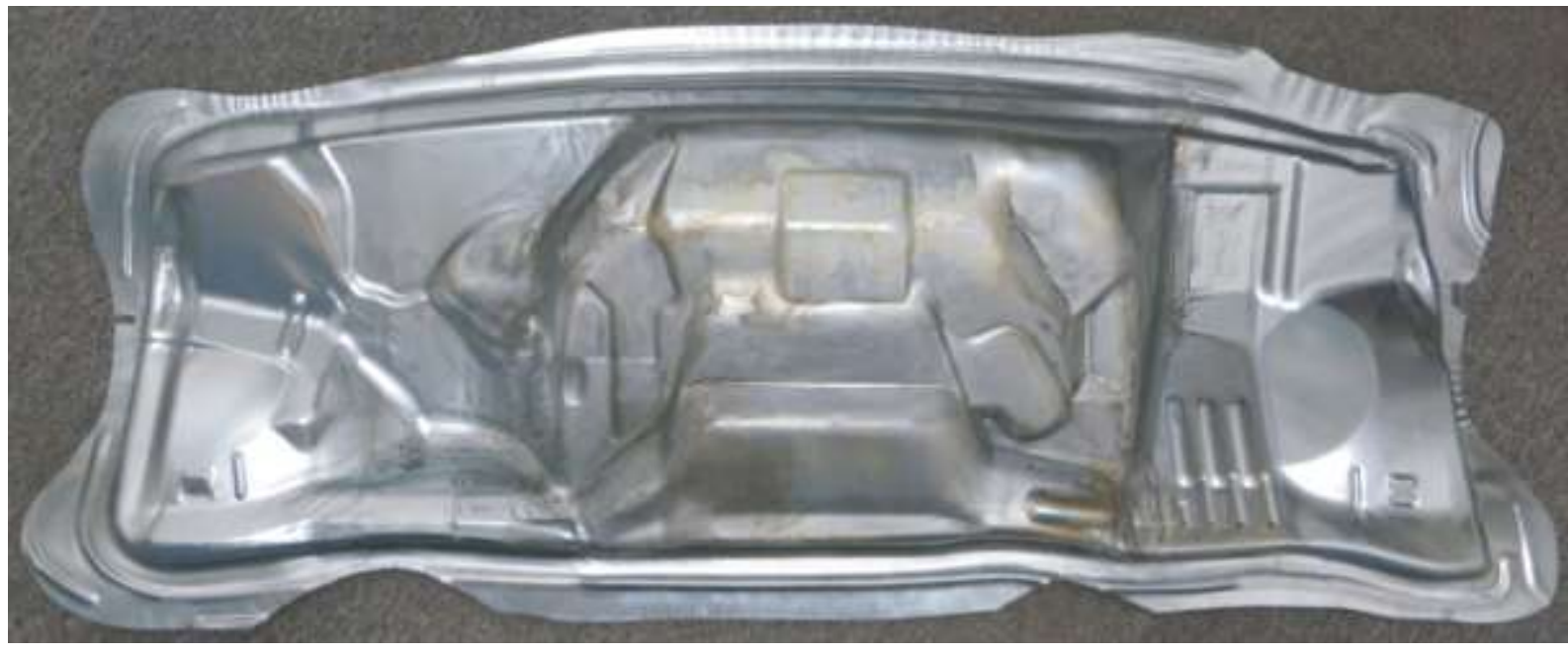

Figure 7.24. The fully formed dash panel (1.5 mm DP600) after EHF forming. a)Viewed from the EHF chamber side. b) Viewed from the die side. 


\section{Cost Model Development}

In order to understand the financial viability of Electrohydraulic Forming (EHF) in a high volume production setting and to explore the use of EHF within a plant, a pre-existing Ford cost model was modified to reflect EHF-assisted production of a light truck dash panel. The addition of EHF to conventional stamping allows the automotive industry to down-gage and lightweight using AHSS, reduce steel consumption and improve vehicle fuel economy. Actual production costs are far better understood with EHF and we can therefore provide the best production estimates for this hybrid process.

The starting point was an activity-based, conventional stamping cost model developed by the Ford cost modeling group, and as such the model itself and many of its inputs are Ford secret and cannot be divulged, and we cannot provide an accurate part cost. However, a conventional stamping model for the dash panel was developed first - per above, the actual conventional stamping process is not well modeled (but the EHF is) deliberately to mask the overall part cost - and then the additional steps required by EHF were added to generate a second model for EHF-assisted production. The two models are identical in every detail with the exception of the additional EHF steps. That the output of this analysis is not an accurate total part cost is not a problem since the difference in costs provides an accurate estimate of the incremental cost of adding EHF.

Two models were developed, one for the conventionally stamped dash panel, and one for the dash panel as described in the previous section. The models were targeted at a light truck dash panel with an annual volume of 600,000 units. The models take into account scrap, inflation rates, opportunity costs, straight line capital depreciation, and end of life capital salvage. Facility rates include depreciation, cost of capital, insurance, taxes, heating, electricity, maintenance, water, MRO, and vendor's services. Two 10 hour shifts are assumed per day, and assumptions are made as to break time and overtime rates. Unplanned downtime is factored in as well as direct and indirect labor for line operations. Reject rates, floor space requirements, and cycle times are also factored into the part cost.

\section{Advantages of EHF}

Because of the very short mechanical pressure pulse $(<1 \mathrm{~ms})$ the EHF clamping press can be of significantly smaller capacity than the conventional drawing press, and because EHF tooling is one-sided, tooling costs are significantly lower than conventional tooling. Further, the tooling can be cut to final surface geometry with no or very little die rework because the final calibration pulse in EHF results in minimal springback. The only unusual maintenance cost item is the EHF pulse generator, and this is given a higher rate based on experience with the automated cell. Lastly, the energy costs of running an EHF pulse generator to form parts are small compared the cost of operating the press to clamp the part. Although not employed in this model, one of the advantages of EHF is that a single press and tool replaces any number of drawing operations - one simply applies multiple pulses until the part takes the shape of the die. 


\section{Cost Model Inputs}

The common cost components are laid out in Table 7.1 below, and the EHF-related component costs are as-used in the cost model. Raw steel material costs are current market costs as provided by USS. EHF maintenance down time is factored in through a reduced workday and EHF electrode costs are bundled in with the consumables on a per panel basis.

Table 7.1. Typical Cost Model Inputs.

\section{Capital Cost / Depreciation}

\section{Equipment}

EHF Clamping press

Robot (each)

EHF machine (pulse generator)

Water-Air Management System (WAMS)

Conventional Trimming Press

\section{(cost \$)}

500000

50000

300000

200000

2000000

100000

500000

1200000

Conventional Trim Dies
Rate

$2.75 \%$

$2.75 \%$

$5.00 \%$

$2.75 \%$

$3.34 \%$

$3.34 \%$

\$9/sq.ft.

Floor Space

years depreciation

20

10

10

10

20

Press (all)

\section{5}

5

5

EHF machine (pulse generator)

Water-Air Management System

EHF Die \& Chamber

Trim Dies

$0.028 \mathrm{kWh} /$ part $20 \mathrm{~kW}$

$5 \mathrm{~kW}$

Robot

\section{Materials Costs}

Electrode Construction Cost

\$53.14/pair

Parts/electrode pair

2400

EDDQ steel (conventional)

$\$ 0.40 / \mathrm{lb}$

DP600 steel (EHF)

$\$ 0.49 / \mathrm{lb}$ 


\section{Process Models}

The conventional stamping process for the light truck dash panel is shown at the right of Figure 7.25. The process has been intentionally altered to prevent disclosure of Ford cost competitive information. A blanking operation (not shown) is factored into the cost. Otherwise, the process is fairly straightforward with a drawing, a trimming and a flange and restrike operation. The parts are made of $1.23 \mathrm{~mm}$ thick Extended Deep Draw Quality (EDDQ) steel, the only sufficiently formable steel from which the dash panel can successfully be conventionally fabricated.

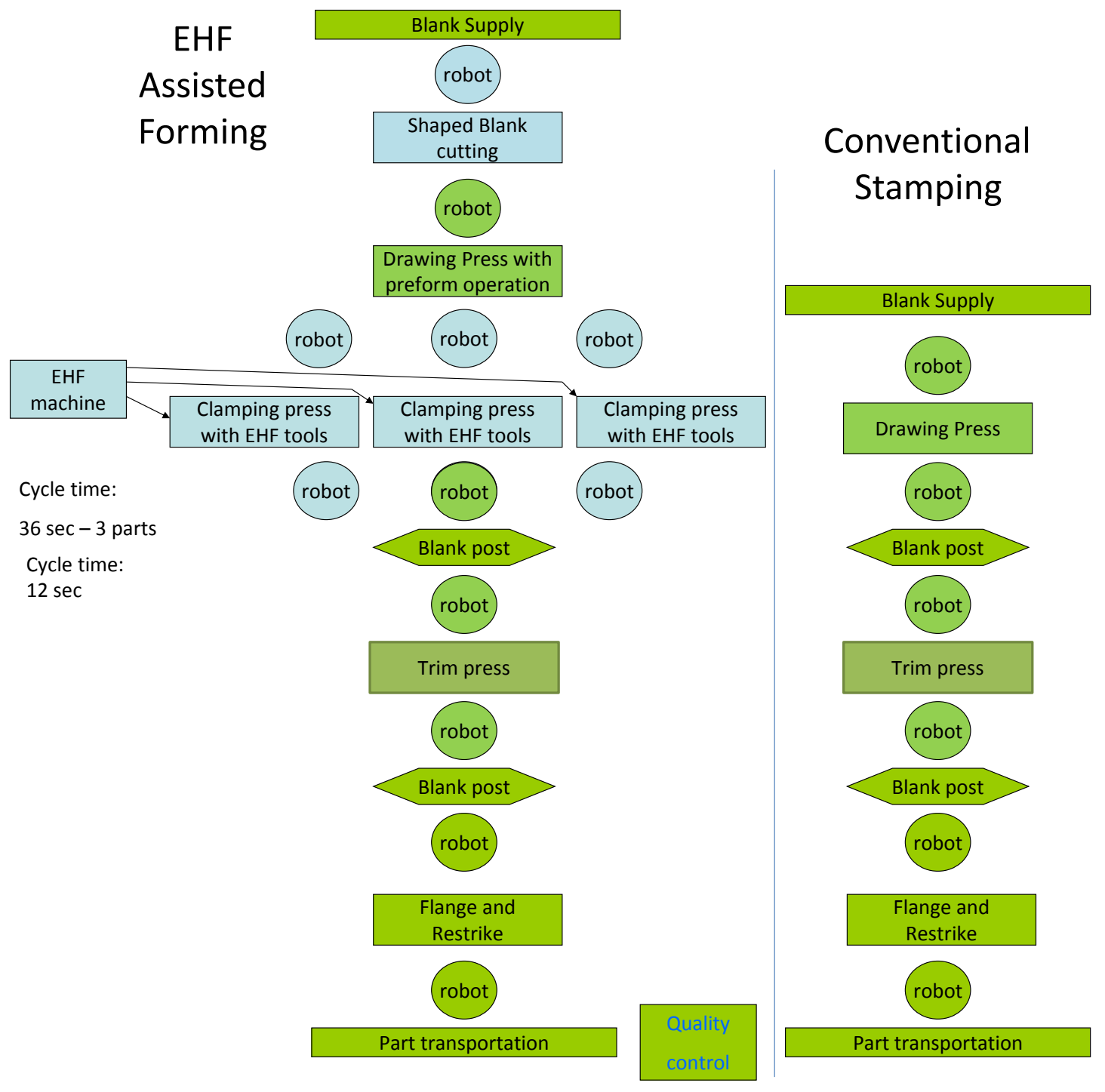

Figure 7.25. EHF-assisted conventional stamping (left) and conventional stamping processes as employed in cost models. 
The EHF model, shown at the left hand side of Figure 7.25, contains every step that the conventional stamping model does but it incorporates a shaped blank production step and an incremental charge to draw tooling costs of $\$ 100,000$ to cover the added complexity of pocket forming. Further down the line are the EHF pulse generator, three fluid handling systems (not shown) and three presses each with EHF tools and robots. Each EHF press requires 36 seconds to finish the corners of the deep pocket in the panel, and, with three EHF presses running in parallel, the process can be orchestrated in a way such that one pulse generator can be used for all three presses - the EHF stage can effectively produce a panel every 12 seconds. The possible gage/weight reduction assumed for this part is $20 \%$, per the original project proposal and recent studies in the literature [7-1], bringing the actual gage modeled down to $0.984 \mathrm{~mm}$.

\section{Cost Model Results}

Figure 7.26 shows the output of the cost model for the conventional stamping analysis. In this model the light truck dash panel cost is $\$ 13.69$ per panel. This compares to $\$ 13.78$ per panel using the EHF assisted production line shown in Figure 7.27. Note that in both models the biggest cost is material. 


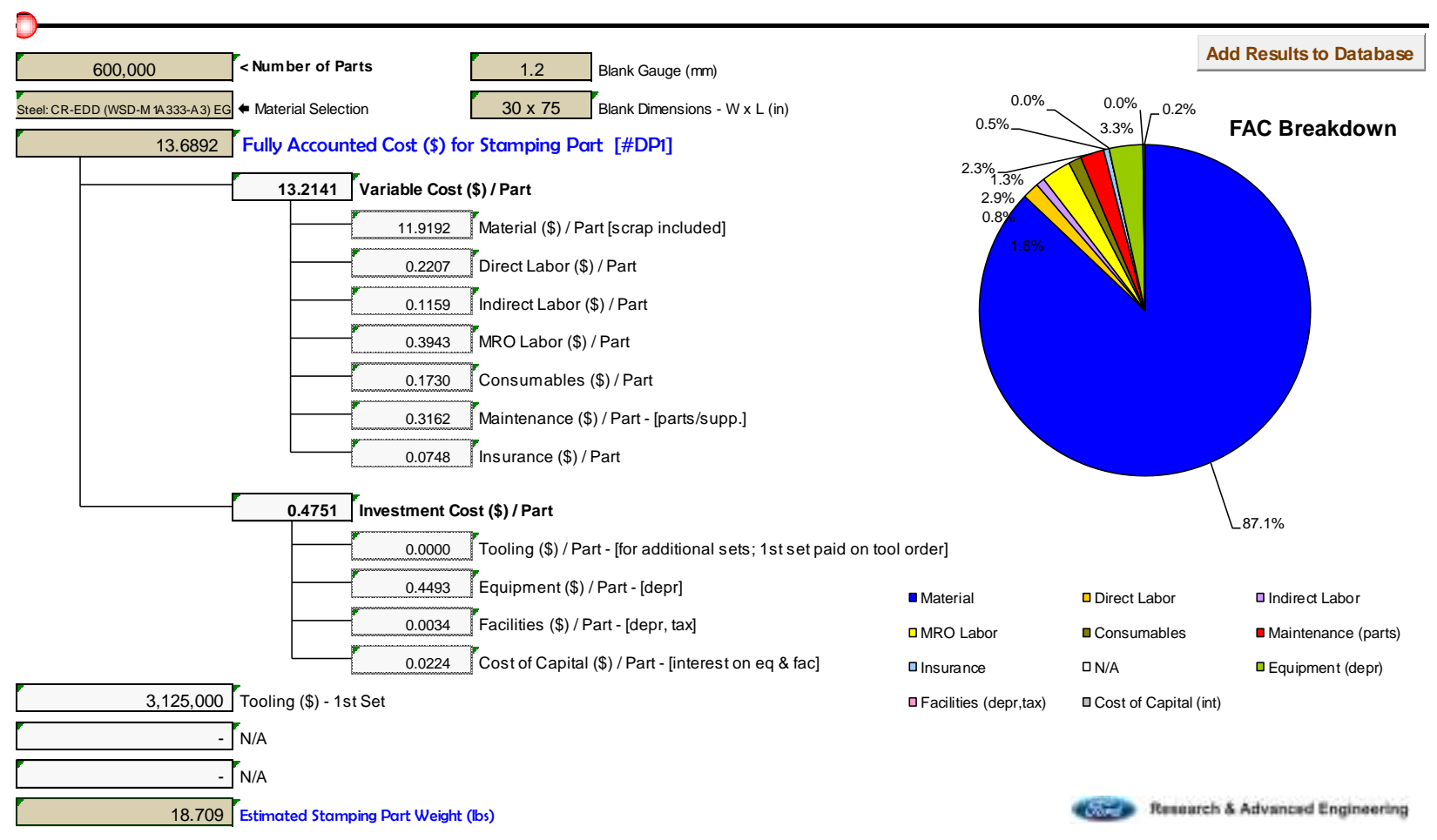

Figure 7.26. Output from conventional stamping cost model.

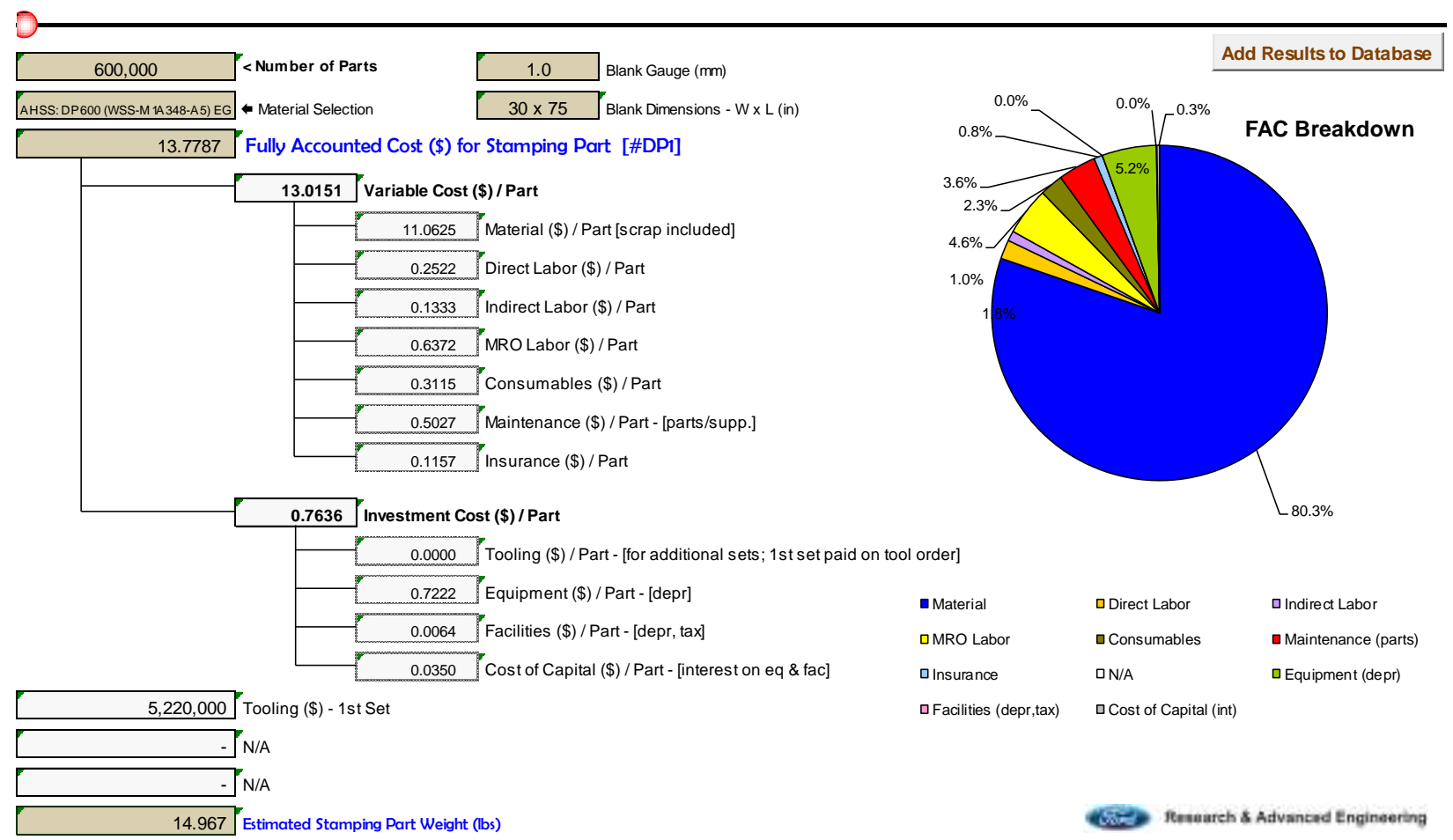

Figure 7.27. Output from EHF-assisted stamping cost model. 
With the exception of materials cost, the EHF-assisted fully-accounted cost is slightly more expensive in every category because, in this application, EHF is simply an addition step to an already existing process - the net increase in non-materials categories is about $\$ 1$ per part. However, in spite of the increased cost of DP600 (0.49/lb) over EDDQ $(0.40 / \mathrm{lb})$, the weight reduction resulting from a $1.23 \mathrm{~mm}$ gage change down to a $0.98 \mathrm{~mm}$ gage results in a cost reduction of $\$ 0.86$ per part. The net overall cost increase per part in adding an EHF step is $\$ 0.09$ per part. This small difference can be considered as a virtually cost neutral change.

From an automotive standpoint, the critical result is the weight savings that EHF has enabled. The conventionally stamped EDDQ part weighs $18.7 \mathrm{lbs}$ and the EHF-produced equivalent DP600 part weight $15.0 \mathrm{lbs}$ - an overall weight reduction of $3.7 \mathrm{lbs}$ per part. Thus, an overall $20 \%$ weight reduction yields a $0.5 \%$ cost increase. The cost penalty for this switch is $\$ 0.025 / \mathrm{lb}$, and recent research [7-2] indicates that, in the automotive industry, cost penalties of up to $\$ 2.72 / \mathrm{lb}$ are cost effective - a fact confirmed by internal Ford sources.

Thus, with a wide margin for error, the cost models indicate that the inclusion of an EHFassisted forming process into production makes perfect sense. 


\section{Accomplishments}

\section{Project accomplishments in relation to the project objectives}

The technical objectives of the proposed project included developing:

a) a predictive numerical design tool for EHF processes,

b) an electrode and discharge chamber equipment design suitable for automotive panel manufacturing,

c) a laboratory prototyping system and using the system for process development,

d) enhanced applications of the EHF process by demonstrating the ability to trim and calibrate formed parts, and

e) demonstrate one full-scale automotive prototype using the developed EHF process

All the technical objectives have been accomplished 100\%. In more detailed way the comparison of accomplishments to objectives can be presented through the Tasks/Milestones description in Table A1 and in Task/Subtask- Accomplishments format in table A2.

Table A1.

\begin{tabular}{|c|c|c|c|c|c|c|}
\hline \multirow{2}{*}{$\begin{array}{c}\text { Task/ } \\
\text { Milesto } \\
\text { ne } \\
\text { Number }\end{array}$} & \multirow[b]{2}{*}{ Title or Brief Description } & \multicolumn{4}{|c|}{ Task/Milestone Completion Date } & \multirow[b]{2}{*}{ Progress Notes } \\
\hline & & $\begin{array}{c}\text { Origina } \\
1 \\
\text { Planned }\end{array}$ & $\begin{array}{l}\text { Revised } \\
\text { Planned }\end{array}$ & Actual & \begin{tabular}{|c|} 
Percent \\
Complet \\
e \\
\end{tabular} & \\
\hline 1 & $\begin{array}{l}\text { Numerically predict pressure- } \\
\text { pulse in the electrode area }\end{array}$ & $12 \mathrm{~m}$ & $\begin{array}{c}04-01- \\
2010\end{array}$ & & $100 \%$ & $\begin{array}{l}\text { Numerical model predicted } \\
\text { pressure pulse in the } \\
\text { electrode area. }\end{array}$ \\
\hline 2 & $\begin{array}{l}\text { Complete demonstration of } \\
\text { robust electrode design to } \\
\text { exceed } 120 \text { discharges }\end{array}$ & $24 \mathrm{~m}$ & $\begin{array}{c}07-01- \\
2011\end{array}$ & & $100 \%$ & $\begin{array}{l}\text { Robust electrode design has } \\
\text { been demonstrated } \\
\text { exceeding } 120 \text { discharges. } \\
\text { Electrode life was extended } \\
\text { up to } 2000 \text { pulses. }\end{array}$ \\
\hline 3 & $\begin{array}{l}\text { Complete demonstration of } \\
\text { fully automated computer } \\
\text { controlled EHF cell with the } \\
\text { cycle time under } 3 \text { minutes }\end{array}$ & $24 \mathrm{~m}$ & $\begin{array}{c}07-01- \\
2011 \\
\end{array}$ & & $100 \%$ & $\begin{array}{l}\text { Automated EHF cell has } \\
\text { been demonstrated with the } \\
\text { cycle time of } 2 \text { min and } 20 \\
\text { sec. }\end{array}$ \\
\hline 4 & $\begin{array}{l}\text { Complete demonstration of a } \\
\text { hybrid HF-EHF process by } \\
\text { introducing static HF as a pre- } \\
\text { forming step in a single tool } \\
\text { automated cell. Accomplish } \\
\text { the cycle time of the hybrid } \\
\text { system under } 2.5 \text { minutes. }\end{array}$ & $24 \mathrm{~m}$ & $\begin{array}{c}07-01- \\
2011\end{array}$ & & $100 \%$ & $\begin{array}{l}\text { The hydroforming system } \\
\text { and EHF system have been } \\
\text { demonstrated. The cycle } \\
\text { time of the hybrid system } \\
\text { of } 2.5 \text { minutes was } \\
\text { accomplished. }\end{array}$ \\
\hline 5 & $\begin{array}{l}\text { Complete demonstration of EH } \\
\text { trimming (EHT) of AHSS. }\end{array}$ & $30 \mathrm{~m}$ & $\begin{array}{c}03-31- \\
2013 \\
\end{array}$ & & $100 \%$ & $\begin{array}{l}\text { Fabrication of EH trim } \\
\text { tool for Floor Side Inner }\end{array}$ \\
\hline
\end{tabular}




\begin{tabular}{|c|c|c|c|c|c|}
\hline & $\begin{array}{l}\text { Accomplish no-burr quality of } \\
\text { the sheared edge in a single } \\
\text { sided EHT die corresponding } \\
\text { to the quality of conventional } \\
\text { trimming with optimal } \\
\text { clearance in two-sided die }\end{array}$ & & & & $\begin{array}{l}\text { panel completed. No- } \\
\text { burr quality of the panel } \\
\text { demonstrated. Correction } \\
\text { of the trim die in one } \\
\text { local area of the trim line } \\
\text { and numerical simulation } \\
\text { of EHT has been } \\
\text { completed. }\end{array}$ \\
\hline 6 & $\begin{array}{l}\text { Complete calibration of parts } \\
\text { stamped from AHSS. } \\
\text { Eliminate } 80 \% \text { of springback } \\
\text { measured as a deviation angle } \\
\text { from the targeted surface. }\end{array}$ & $30 \mathrm{~m}$ & $\begin{array}{c}12-30- \\
2012 \\
\end{array}$ & $100 \%$ & $\begin{array}{l}\text { Completed calibration of } \\
\text { parts stamped from } \\
\text { AHSS. Eliminated vast } \\
\text { majority of springback } \\
\text { exceeding the } 80 \% \\
\text { target. }\end{array}$ \\
\hline 7 & $\begin{array}{l}\text { Complete full scale } \\
\text { demonstration of the EH } \\
\text { stamping process for one full } \\
\text { scale AHSS part with 9\% } \\
\text { weight savings potential }\end{array}$ & $36 \mathrm{~m}$ & $\begin{array}{c}06-30- \\
2013\end{array}$ & $100 \%$ & $\begin{array}{l}\text { Full scale demonstration } \\
\text { has been completed. }\end{array}$ \\
\hline
\end{tabular}

Table A2. Task/Subtask Schedule

\begin{tabular}{|c|c|c|c|}
\hline $\mathrm{N}$ & Subtask & Current stage & $\begin{array}{l}\text { Expected } \\
\text { completion } \\
\text { date }\end{array}$ \\
\hline 1.1 & $\begin{array}{l}\text { Numerical model of } \\
\text { pulsed pressure } \\
\text { generation process } \\
\text { (completed) }\end{array}$ & $\begin{array}{l}\text { An analytical tool capable of predicting pressure pulse as a } \\
\text { function of the discharge parameters has been developed. } \\
\text { This tool is being used for chamber design optimization } \\
\text { and analysis of blank formation }\end{array}$ & $03-2010$ \\
\hline 1.2 & $\begin{array}{l}\text { Optimal distribution of } \\
\text { strains in the blank in } \\
\text { two-step forming } \\
\text { process. } \\
\text { (completed) }\end{array}$ & $\begin{array}{l}\text { An analytical tool has been developed to predict the shape } \\
\text { of a preform leading to the final shape with minimum } \\
\text { stretching and without wrinkling. }\end{array}$ & $03-2010$ \\
\hline 1.3 & $\begin{array}{l}\text { Numerical model of } \\
\text { the blank formation } \\
\text { and die loading } \\
\text { process } \\
\text { (completed) }\end{array}$ & $\begin{array}{l}\text { Numerical model of the blank formation and die loading } \\
\text { process has been developed. }\end{array}$ & $06-2011$ \\
\hline 1.4 & $\begin{array}{l}\text { Process modeling } \\
\text { validation for full } \\
\text { scale automotive } \\
\text { panel (completed) }\end{array}$ & $\begin{array}{l}\text { Completed numerical simulation of preforming and EHF } \\
\text { forming of the panel for full scale demonstration. }\end{array}$ & $03-2013$ \\
\hline 2.1 & $\begin{array}{l}\text { Electrode erosion } \\
\text { study } \\
\text { (completed) }\end{array}$ & $\begin{array}{l}\text { Optimal profile of the electrode tip and material of the } \\
\text { electrode have been identified. }\end{array}$ & $03-2010$ \\
\hline
\end{tabular}




\begin{tabular}{|c|c|c|c|}
\hline 2.2 & $\begin{array}{l}\text { Develop robust } \\
\text { electrode system } \\
\text { (completed) }\end{array}$ & $\begin{array}{l}\text { Robust electrode design has been identified. The electrode } \\
\text { durability in accelerated test conditions is } 2000 \text { discharges. }\end{array}$ & $06-2011$ \\
\hline 3.1 & $\begin{array}{l}\text { Develop an efficient } \\
\text { water } \\
\text { filling/evacuation } \\
\text { system for the EHF } \\
\text { chamber } \\
\text { (completed) }\end{array}$ & $\begin{array}{l}\text { An efficient, fully automated water filling/evacuation system } \\
\text { for the EHF chamber has been developed }\end{array}$ & $06-2011$ \\
\hline 3.2 & $\begin{array}{l}\text { Study of sealing } \\
\text { materials } \\
\text { (completed) }\end{array}$ & $\begin{array}{l}\text { Developed seal design is incorporated into the EHF tool } \\
\text { designated for multi cyclic testing in EHF cell }\end{array}$ & $06-2011$ \\
\hline 3.3 & $\begin{array}{l}\text { Integrated computer } \\
\text { controlled EHF } \\
\text { system } \\
\text { (completed) }\end{array}$ & $\begin{array}{l}\text { Integrated computer controlled EHF system has been } \\
\text { developed. }\end{array}$ & $06-2011$ \\
\hline 4.1 & $\begin{array}{l}\text { Develop integrated } \\
\text { preforming process } \\
\text { (completed) }\end{array}$ & $\begin{array}{l}\text { Integrated preforming process has been developed and } \\
\text { validated. }\end{array}$ & $06-2011$ \\
\hline 4.2 & $\begin{array}{l}\text { Define the die } \\
\text { material and lubricant } \\
\text { appropriate for EHF } \\
\text { (completed) }\end{array}$ & $\begin{array}{l}\text { Testing of V-shape dies out of four materials currently used } \\
\text { for die fabrication has been completed. The window of die } \\
\text { material properties for EHF has been identified. Numerical } \\
\text { analysis predicted heaviest loaded area of the die where } \\
\text { fracture occurred. Die material and lubricant appropriate for } \\
\text { EHF have been identified. }\end{array}$ & $06-2011$ \\
\hline 5.1 & $\begin{array}{l}\text { Conduct preliminary } \\
\text { testing of the } \\
\text { automated EHF } \\
\text { system (completed) }\end{array}$ & $\begin{array}{l}\text { Preliminary testing of the automated EHF system indicated } \\
\text { that WAMS system operation represents the slowest } \\
\text { portion of the cycle time. Preliminary testing of the } \\
\text { automated EHF system has been completed. }\end{array}$ & $06-2011$ \\
\hline 5.2 & $\begin{array}{l}\text { Demonstration of the } \\
\text { automated EHF } \\
\text { process for specified } \\
\text { period of time } \\
\text { (completed) }\end{array}$ & $\begin{array}{l}\text { Analysis of interaction between the EHF discharge and the } \\
\text { control system of EHF cell components has been } \\
\text { completed. The working configuration of the EHF cell } \\
\text { computer controls cell has been identified. Electrostatic } \\
\text { analysis of EHF chamber designated for the cell has been } \\
\text { completed. Analysis of the effect of the discharges on the } \\
\text { clamping press performance has been completed. Testing } \\
\text { of EHF equipment components has been completed. }\end{array}$ & $06-2013$ \\
\hline 6.1 & $\begin{array}{l}\text { EH trimming process } \\
\text { (completed) }\end{array}$ & $\begin{array}{l}\text { The EH trimming tool has been fabricated. Initial } \\
\text { demonstration of } \mathrm{EH} \text { trimming process has been } \\
\text { completed. Modification of } \mathrm{EH} \text { trim tool to accommodate } \\
\text { the local imperfections and final demonstration of } \mathrm{EH} \\
\text { trimming has been completed. }\end{array}$ & $03-2013$ \\
\hline 6.2 & $\begin{array}{l}\text { EH springback } \\
\text { calibration process } \\
\text { (completed) }\end{array}$ & $\begin{array}{l}\text { Fabrication of the drawing tool and EH calibration tool for } \\
\text { validation of the EH calibration process of the Front Floor } \\
\text { Side Inner at 50\% scale has been completed. }\end{array}$ & $12-2012$ \\
\hline 7 & $\begin{array}{l}\text { Demonstration of } \\
\text { EHF of a full scale } \\
\text { automotive panel } \\
\text { application } \\
\text { (completed) }\end{array}$ & $\begin{array}{l}\text { Numerical simulation of the hybrid hydroforming - EHF } \\
\text { process for full scale panel application has been } \\
\text { completed. Design of the EHF chamber for the full scale } \\
\text { demonstration of the dash panel forming process has been } \\
\text { completed. The design of electrode reinforcement areas } \\
\text { has been completed. Full scale demonstration has been } \\
\text { completed. }\end{array}$ & $06-2013$ \\
\hline 8 & $\begin{array}{l}\text { Project Management } \\
\text { and Reporting } \\
\text { (in progress) }\end{array}$ & $\begin{array}{l}\text { Eighteen quarterly reports and six oral reviews have been } \\
\text { delivered. Final report has been completed. }\end{array}$ & $09-2013$ \\
\hline
\end{tabular}




\section{$\underline{\text { Commercialization activities }}$}

Project DE-FG36-08G018128 is included in the official project portfolio of Ford Research \& Advanced Engineering ( $R \& A E)$. All Ford $R \& A E$ projects are monitored and tracked via the Global Technology Development System (GTDS) to effectively manage the implementation process. At this moment, the internal Ford project is being continued jointly with Amino North America Corporation. After full completion of the Ford internal study, the project will be reviewed by the Management team, and the decision regarding the future of the EHF technology will be made. If the technology is approved for production implementation, a specific panel from a new vehicle program will be specified for the EHF process, and the technology will be further developed from the Concept Readiness stage to Application Readiness and Implementation Readiness stages of the GTDS process.

$\underline{\text { Publications in scientific/trade journals, conference proceedings: }}$

- S.Golovashchenko, A.Gillard, A.Mamutov, and R.Ibrahim "Pulsed Electrohydraulic Springback Calibration of Parts Stamped from Advanced High Strength Steel" Submitted for publication to Journal of Materials Processing Technology.

- S.Golovashchenko, A.Gillard, A.Mamutov, J.Bonnen and Z. Tang "Electrohydraulic Trimming of Advanced and Ultra High Strength Steels" Accepted for publication in Journal of Materials Processing Technology.

- J.Bonnen, S.Golovashchenko, S.Dawson and A.Mamutov "Electrode Erosion Observed in Electro-Hydraulic Discharges Used In Pulsed Sheet Metal Forming" Accepted for publication in Journal of Materials Engineering and Performance.

- S. Golovashchenko, A. Gillard, A. Mamutov. "Formability of Advanced High Strength Steels in Electrohydraulic Forming" Journal of Materials Processing Technology. Volume 213, Issue 7, July 2013, Pages 1191-1212.

- Gillard, S. Golovashchenko, and A. Mamutov "Effect of quasi-static prestrain on the formability of dual phase steels in electrohydraulic forming" Journal of Manufacturing Processes. Volume 15, Issue 2, April 2013, Pages 201-218.

- S.Golovashchenko, N. Bessonov, and A.llinich "Two-step method of forming complex shapes from sheet metal" Journal of Materials Processing Technology, Volume 211, Issue 5, May 2011, p. 875-885.

- R.Ibrahim, S.Golovashchenko, A.Mamutov, J.Bonnen, A.Gillard, and L.Smith "Analysis of contact stresses in high speed metal forming processes" Proceedings of 5th International Conference on High Speed Forming, Dortmund, 2012, p.93-102.

- J.Bonnen, S.Golovashchenko, S.Dawson, and A.Mamutov "Electrohydraulic sheet metal forming of aluminum panels" Proceedings of the TMS Light Metals 2012 Conference, Orlando, FL, pp.494-454.

- S.Golovashchenko, A. Mamutov, J.Bonnen, A. Gillard "Electrohydraulic Forming of Sheet Metal Parts" Proceedings of 10th International Conference on Technology of Plasticity, p.1170-1175. 
Patents

- S.Golovashchenko "Method and apparatus for pulsed forming, punching and trimming of tubular members" US Patent 8534107. Issue Date: Sep 17, 2013

- S.Golovashchenko " Hydromechanical Drawing Process and Machine" US Patent 8534106. Issue Date: Sep 17, 2013

- S.Golovashchenko and J.Bonnen "Seals for fluid forming tools" US Patent 8,136,378. March 19, 2012

- S.Golovashchenko "Method and tool for contracting tubular members by electrohydraulic forming before hydroforming," US Patent 7,905,129.

- S.Golovashchenko "Pulsed electro-hydraulic calibration of stamped panels," US Patent $7,827,838$.

- S.Golovashchenko "Electrohydraulic trimming, flanging and hemming of blanks" US Patent 7,810,366.

- S.Golovashchenko, A.Gillard, D.Piccard, A.llinich "Electrohydraulic forming tool and method of forming sheet metal blank with the same" US Patent 7,802,457.

- S.Golovashchenko, A.Gillard, D.Cedar, A.llinich, "Electrohydraulic forming tool" US Patent 7,516,634.

- S.Golovashchenko, V.Mamutov, A.llinich."Electrohydraulic forming tool having two liquid volumes separated by a membrane" US Patent 7,493,787.

Patent Applications Submitted to US Patent Office and currently in processing

- J.Bonnen and S.Golovashchenko "Method and Tool for Expanding Tubular Members by Electrohydraulic Forming". Patent application in filing procedure

- S.Golovashchenko, R.Narainen, A.Mamutov, A. Diveto, J.Bonnen and S.Dawson "Deep draw manufacturing process". Patent application in filing procedure

- S.Golovashchenko "Electro-Hydraulic Rivet and Method of Riveting and Welding Parts" US Patent Application 13/291,346. Filed Jan.19, 2012.

- J.Bonnen and S.Golovashchenko "Method and Apparatus for EHF" 13/590,506 Filed Aug.21, 2012.

- S.Golovashchenko "Apparatus and Methods Using Reduced Volume Electrohydraulic chambers for trimming and joining panels" US Patent Application 13/237,059 filed 9/20/2011.

- J.Bonnen, S.Golovashchenko, S.Dawson "Electrode assembly for electrohydraulic forming process" US Patent Application 12/940,235 filed11/5/2010

- S.Golovashchenko "Electrohydraulic forming process with electrodes that advance with a fluid chamber toward a workpiece," US Patent Application 12/915,110 filed 10/29/2010

- S.Golovashchenko "Method and Apparatus for Making a Part by First Forming an Intermediate Part that has Donor Pockets in Predicted Low Strain Areas Adjacent to Predicted High Strain Areas", US Patent Publication 20110179846. 13/045,878 filed 3/11/2011

- S.Golovashchenko "Method and Tool for Expanding Tubular Members by ElectroHydraulic Forming"US Patent Publication 20110067470. 
- S.Golovashchenko " Electrohydraulic Flanging and Trimming" US patent Publication 20100175447.

- S.Golovashchenko " Method of designing and forming a sheet metal part" US Patent Publication 20090272171 


\section{Conclusions}

\section{Scientific/technical findings of the project.}

1. Developed numerical model of the EHF process was validated experimentally at both coupon and component level. The numerical model was successfully applied to the design of the electrode system and multi-electrode EHF chamber for full scale validation of the process. The numerical model predicting stresses in the dies for pulsed forming was validated by the experimental study of the die insert failure for corner filling operation.

2. Electrohydraulic forming process and its major subsystems including durable electrodes, EHF chamber, water/air management system, pulse generator and developed process controls were validated to be capable to operate in fully automated computer controlled mode.

3. Novel processes of electrohydraulic trimming and electrohydraulic calibration have been demonstrated at the component reduced scale level.

4. Full scale demonstration of a hybrid conventional forming-EHF forming process demonstrated that one of the most complex sheet metal automotive parts, the dash panel, was formed in laboratory conditions from DP600 steel providing factor of two increase in material strength and enabling potential down-gauging of the panel by $25 \%$.

5. The economic feasibility of the developed EHF processes was demonstrated by developing a cost model of the process comparing it to the conventional stamping process. The cost model demonstrated that the developed process is cost neutral with the accuracy of accepted assumptions to the conventional stamping process but reduces the consumption of raw materials by $25 \%$ which has potential to reduce carbon footprint of produced cars and trucks when the technology gets implemented by reducing the energy embedded in vehicles and the energy consumed while producing automotive structures as well as the weight reduction of cars and trucks which is required to reduce fuel consumption.

\section{Potential Benefits Assessment of the Technology}

The objective of this project was to develop and deploy the EHF process as a near-net shape automotive panel manufacturing technology that simultaneously reduces the energy embedded in vehicles and the energy consumed while producing vehicles. The specific energy, environment, and economic benefits of the completed project result from EHF processing that:

- uses half of the tooling materials and energy compared to conventional stamping

- enables the down-gauging of steel panels in automotive structures by more than $25 \%$, which substantially reduces the energy embedded in vehicles and their carbon footprint by reducing the amount of embedded steel in the vehicle

- consolidates multiple conventional forming, blanking, and punching processes into a single EHF process, thereby eliminating machines through process consolidation.

Given these multiple benefits, developing and commercializing EHF technology has the potential to yield an energy savings of 16.38 TBtu/year, reduce greenhouse gas emissions by 
622 million pounds/year of $\mathrm{CO} 2$ and 16 million pounds/year of NOX and SO2 in U.S. transportation vehicle manufacturing by 2020 .

Commercialization Aspect of the Technology

Project DE-FG36-08G018128 is included in the official project portfolio of Ford Research \& Advanced Engineering (R\&AE). All Ford $R \& A E$ projects are monitored and tracked via the Global Technology Development System (GTDS) to effectively manage the implementation process. At this moment, the internal Ford project is being continued jointly with Amino North America Corporation. After full completion of the Ford internal study, the project will be reviewed by the Management team, and the decision regarding the future of the EHF technology will be made. If the technology is approved for production implementation, a specific panel from a new vehicle program will be specified for the EHF process, and the technology will be further developed from the Concept Readiness stage to Application Readiness and Implementation Readiness stages of the GTDS process.

\section{Barrier for the EHF technology implementation}

Necessity to acquire a substantial amount of new capital equipment for EHF implementation such as pulse generators and hydraulic presses, while the existing park of mechanical presses cannot be utilized for this technology. 


\section{Recommendations}

As it was indicated above the EHF technology is progressing through Ford Global Technology Development System (GTDS). It is anticipated that it will achieve Concept Readiness stage at the end of 2014 when Ford internal development of the technology is expected to be completed. Multiple discussions with Ford suppliers and Stamping Business Unit representatives indicated that the most realistic commercialization path goes through Ford Tier 1 supplier Amino North America where the tryout of the preforming process for the dash panel was performed.

The initial implementation should occur at the production facility where sheet hydroforming processes are routinely used. Another requirement is to have a relatively low volume pilot part which volume can be realistically accomplished using the existing stage of the technology. 


\section{References / Bibliography}

[B1] Chachin V. N., "Electrohydraulic Treatment of Engineering Materials," Science and Technology, Minsk, 1978.

[B2] Lane T., "Description of an Electrometer invented by Mr. Lane; with an Account of some Experiments made by him with it: In a Letter to Benjamin Franklin," Philosophical Transactions of the Royal Society, Vol. 57, 1767, p. 451.

[B3] Yutkin L.A., "Electrohydraulic effect." - Moscow-Leningrad:Mashgiz, 1955, p. 50.

Grove L. C. and Innis R. T., "Electrohydraulic Metal Forming System and Method," US Patent 3,253,442, 1966.

[B4]Hundley M.L., "Electro-hydraulic Flat Forming System," US Patent 3,491,564, 1970.

"Conductor Initiated Electrohydraulic Forming Process," GB Patent 1,294,240, 1972.

[B5] Daehn G., "High Velocity Sheet Metal Forming: State of the Art and Prognosis for Advanced Commercialization," Report published on the web: http://www.mse.eng.ohiostate.edu/ Daehn/hyperplasticity/stateofartrept/index.html

[B6] Guliy G.A., "Scientific Fundamentals of Discharge Pulsed Technologies," Kiev, Ukraine, 1990.

[B7] Balanethiram V. S. and Daehn G. S., "Hyperplasticity: Increased Forming Limits at High Workpiece Velocity," Scripta Metallurgica et Materialia, Vol.30, pp.515-520, 1994.

[B8] Rohatgi, A., Stephens, E.V., Soulami, A., Davies, R.W., Smith, M.T., 2011. Experimental characterization of sheet metal deformation during electrohydraulic forming. Journal of Materials Processing Technology 211, 1824-1833.

[B9] Rohatgi, A., Stephens, E.V., Davies, R.W., Smith, M.T., Soulami, A., Ahzi, S., 2012. Electrohydraulic forming of sheet metals: Free-forming vs. conical-die forming, Journal of Materials Processing Technology 212, 1070-1079.

[B10] Psyk, V., Risch, D., Kinsey, B.L., Tekkaya, A.E., Kleiner, M. 2011. Electromagnetic forming - A review. Journal of Materials Processing Technology 211,787-829.

[1.1.1] Arsentiev VV. To theory of developing of impulse discharge channel in liquid media. Electrical breakdown of dielectrics and semiconductors; 1964. p. 199-206.

[1.1.2] Kaklugin AS, Norman GE. Electrical conductivity of Non-Debye plasma. Thermal physics of high temperatures, vol. 11, No. 2. 1973. p. 238-44.

[1.1.3] LSTC. LS-DYNA Keyword User's Manual, Volume 12012 
[1.1.4] Balanethiram VS, Daehn GS. Hyperplasticity: increased forming limits at high workpiece velocity. Scripta Metallurgica et Materialia 1994;30:515-20.

[1.1.5] Imbert J, Worswick M, Winkler S, Golovashchenko S, Dmitriev V. Analysis of the increased formability of aluminum alloy sheet formed using electromagnetic forming. Society of Automotive Engineers, Paper No. 2005-01-0082; 2005.

[1.1.6] Rohatgi A, Stephens EV, Soulami A, Davies RW, Smith MT. Experimental characterization of sheet metal deformation during electrohydraulic forming. Journal of Materials Processing Technology 2011;211:1824-33.

[1.1.7] Rohatgi A, Stephens EV, Davies RW, Smith MT, Soulami A, Ahzi S. Electrohydraulic forming of sheet metals: free-forming vs. conical-die forming. Journal of Materials Processing Technology 2012;212:1070-9.

[2-1] J.J.F. Bonnen S.F. Golovashchenko, S.A. Dawson, and A.V. Mamutov "Electrode Erosion Observed In Electrohydraulic Discharges Used In Pulsed Sheet Metal Forming, To be published in Journal of Materials Engineering and Performance, DOI: 10.1007/s11665-013-0690-0

[2-2] V.N.Chachin, Electrohydraulic treatment of materials (Minsk: Science and Engineering, 1978), 183.

[2-3] A.M. Loske and F.E. Prieto, "The Influence of Electrode Shape on the Performance of Electrohydraulic Lithotripters," J. Stone Disease, V.5, No. 4 (1993).

[2-4] A.L. Donaldson, M. O. Hagler, M. Kristiansen, G. Jackson, And L. Hatfield, "Electrode Erosion Phenomena in a High-Energy Pulsed Discharge," IEEE Transactions On Plasma Science, Vol. PS-12, NO. 1, MARCH 1984.

[3-1] H. K. Mueller, B.S. Nau, Fluid Sealing Technology, Marcel Dekker, Inc., New York, 1998, pp. 484.

[4-1] Cheah, L., Heywood, J., 2011. Meeting US passenger vehicle fuel economy standards in 2016 and beyond. Energy Policy 39, 454-466.

[4-2] Psyk, V., Risch, D., Kinsey, B.L., Tekkaya, A.E., Kleiner, M., 2011. Electromagnetic Forming - A Review. Journal of Materials Processing Technology 211,787-829.

[4-3] Mynors, D.J., Zhang, B., 2002. Applications and capabilities of explosive forming. Journal of Materials Processing Technology 125-126, 1-25.

[4-4] Balanethiram, V.S., Daehn, G.S., 1994. Hyperplasticity: increased forming limits at high workpiece velocity. Scripta Metallurgica et Materialia 30, 515-520.

[4-5] Golovashchenko, S.F., 2007. Material formability and coil design in electromagnetic forming. Journal of materials Engineering and Performance 16, 314-320. 
[4-6] Imbert, J., Worswick, M., Winkler, S., Golovashchenko, S., Dmitriev, V., 2005. Analysis of the Increased Formability of Aluminum Alloy Sheet Formed Using Electromagnetic Forming. Society of Automotive Engineers, Paper No. 2005-01-0082.

[4-7] Rohatgi, A., Stephens, E.V., Soulami, A., Davies, R.W., Smith, M.T., 2011. Experimental characterization of sheet metal deformation during electrohydraulic forming. Journal of Materials Processing Technology 211, 1824-1833.

[4-8] Rohatgi, A., Stephens, E.V., Davies, R.W., Smith, M.T., Soulami, A., Ahzi, S., 2012. Electro-hydraulic forming of sheet metals: Free-forming vs. conical-die forming, Journal of Materials Processing Technology 212, 1070-1079.

[4-9] Golovashchenko, S., 1999. Numerical and experimental results on pulsed tubes calibration. Proceedings of the 1999 TMS Symposium "Sheet Metal Forming Technology", SanDiego, 117-127.

[4-10] Woodward, S., Weddeling, C., Daehn, G., Psyk, V., Carson, B., Tekkaya, A. E., 2011. Production of low-volume aviation components using disposable electromagnetic acutators. Journal of Materials Processing Technology 211, 886-895.

[4-11] Ibrahim, R., Golovashchenko, S., Mamutov, V., Bonnen, J., Gillard, A., Smith, L., 2012. Analysis of Contact Stresses in High Speed Sheet Metal Forming Processes. Proceedings of the International Conference on High Speed Forming, Dortmund, Germany.

[4-12] Sandford, J. E., Recent Advances Stir Interest In High Velocity Forming. Iron Age Metalworking International. October, 1970.

[4-13] U.S. Patent 7,802,457. Electrohydraulic Forming Tool and Method of Forming Sheet Metal Blank with the Same. Golovashchenko, S.F., Gillard, A. J., Piccard, D., and llinich, A. M., 2010.

[4-14] U.S. Patent 6,050,120. Hybrid Matched Tool-Electromagnetic Forming Apparatus. Daehn, G.S., Vohnout, V. J., and DuBois, L. 2000.

[4-15] Vohnout, V. J., Daehn, G. S., 2002. Effect of Quasi-static Prestrain and Eddy Currents on Limit Strains in Electromagnetic Pulse Forming of Two Aluminum Alloys. Aluminum 2002 Proceedings of the TMS 2002 Annual Meeting.

[4-16] Liu, D., Yu, H., Li, C., 2011. Experimental Observations of Quasi-Static-Dynamic Formability in Biaxially Strained AA5052-O. Journal of Materials Engineering and Performance 20, 223-230.

[6-1] Smith, D.A., 1990. Die design handbook, Society of Manufacturing Engineers, Dearborn, MI.

[6-2] Johnson, W., and Slater, R.A.C., 1966. Further experiments on quasi-static and dynamic blanking of circular discs from various materials. Proceedings of the Institution of Mechanical Engineers, 180 (3I), 163-181. 
[6-3] Davies, R., and Dhawan, S.M., 1966. Further developments in high-speed blanking of metals. Proceedings of 7th International Machine Tool and Design Conference, Birmingham, pp. 67-79.

[6-4] Breitling, J., 1998. The challenges and Benefits of High Speed Blanking. Dr. Ing. Dissertation, Universität Stuttgart. ISBN 3883552666.

[6-5] Das, M.K., and Sadollah, Z.T., 1979. Cropping billets with low aspect ratios. Proceedings of 20th International Machine Tool and Design Conference, Birmingham, pp.195-202.

[6-6] Psyk, V., Risch, D., Kinsey, B.L., Tekkaya, A.E., and Kleiner, M., 2011. Electromagnetic forming - A review. Journal of Materials Processing Technology 211, 787-829.

[6-7] Mynors, D.J., and Zhang, B., 2002. Applications and capabilities of explosive forming. Journal of Materials Processing Technology 125-126, 1-25.

[6-8] Guerin, H.E., 1940. The aviation rubber stamp. The Machinist 84, pp. 206-208 and 246248.

[6-9] Singh, H., 2003. Fundamentals of hydroforming. Society of Manufacturing Engineers, Dearborn, pp. 29-35.

[6-10] Bruno, E.J., 1968. High Velocity Forming of Metals. American Society of Tool and Manufacturing Engineers, Dearborn, MI, USA, pp. 73-108.

[6-11] Davies, R., and Austin, E.R., 1970. Electromagnetic forming. Developments in High Speed Metal Forming, pp. 253-270.

[6-12] Belyy, I.V., Fertik, S.M., and Khimenko, L.T., 1977. Spravochnik Po Magnitnoimpul' Snoy Obrabotke Metallov [Electromagnetic Metal Forming Handbook]. English translation by Altynova, M.M., available online at http://www.mse.eng.ohiostate.edu/_Daehn/ metalforminghb/index.html (accessed on April 11th, 2010).

[6-13] Golovashchenko, S.F., 1995. Electromagnetic impulse joining and shearing of Al-Cu and Al-Mg alloy tubes. Aluminum and Magnesium for Automotive Applications, Proceedings of Fall TMS Meeting, Cleveland, pp. 209-221.

[6-14] Golovashchenko, S.F., 1996. Impulsive electromagnetic tubes shearing process. Proceedings of the 5th International Conference on the Technology of Plasticity, Columbus, $\mathrm{OH}$, pp. 939-942.

[6-15] Uhlmann, E., and Scholz, M., 2003. Zerteilen von Aluminiumblechen durch Impulsmagnetfelder. Tagungsband zum 2. Kolloquium Elektromagnetische Umformung, Dortmund (Deutschland), S. 87-94.

[6-16] Gafri, O., and Livshiz, Y., 2004. Electromagnetic and/or electrohydraulic forming of a metal plate. US patent $6,708,542$.

[6-17] Schäfer, R., Pasquale, P., and Kallee, S., 2009. Industrial Application of the Electromagnetic Pulse Technology. PSTproducts GmbH, Alzenau, Germany, www.english.pstproducts.com. 
[6-18] Kallee, S., Schäfer, R., and Pasquale, P., 2010. Automotive Applications of Electromagnetic Pulse Technology (EMPT). PSTproducts GmbH, Alzenau, http://www.pstproducts.com.

[7-1] L. Cheah, J. Heywood, "Meeting U.S. Passenger Vehicle Fuel Economy Standards in 2015 and Beyond," Energy Policy, v. 39, pp 454-466, 2011.

[7-2] A. Brooker, J. Ward, L. Wang, "Lightweighting Impacts on Fuel Economy, Cost, and Component Losses," Society of Automotive Engineers, SAE 2013-01-0381, 2013. 